University of Rhode Island

DigitalCommons@URI

Open Access Master's Theses

2012

\title{
IMPROVING LOAD DISTRIBUTIONS IN CELLULAR MATERIALS USING STRESS TRAJECTORY TOPOLOGIES
}

Scott Breault

University of Rhode Island, scottbreault@gmail.com

Follow this and additional works at: https://digitalcommons.uri.edu/theses

\section{Recommended Citation}

Breault, Scott, "IMPROVING LOAD DISTRIBUTIONS IN CELLULAR MATERIALS USING STRESS

TRAJECTORY TOPOLOGIES" (2012). Open Access Master's Theses. Paper 100.

https://digitalcommons.uri.edu/theses/100

This Thesis is brought to you for free and open access by DigitalCommons@URI. It has been accepted for inclusion in Open Access Master's Theses by an authorized administrator of DigitalCommons@URI. For more information, please contact digitalcommons-group@uri.edu. 


\section{IMPROVING LOAD DISTRIBUTIONS IN \\ CELLULAR MATERIALS USING STRESS}

TRAJECTORY TOPOLOGIES

BY

SCOTT BREAULT

A THESIS SUBMITTED IN PARTIAL FULFILLMENT OF THE REQUIREMENTS FOR THE DEGREE OF

MASTER'S OF SCIENCE

IN

MECHANICAL ENGINEERING AND APPLIED MECHANICS

UNIVERSITY OF RHODE ISLAND 


\section{MASTER OF SCIENCE THESIS}

$\mathrm{OF}$

SCOTT BREAULT

\section{APPROVED:}

Thesis Committee:

Major Professor___ Martin H. Sadd

David G. Taggart

Richard J Vaccaro

Nasser H Zawia

DEAN OF THE GRADUATE SCHOOL

\section{UNIVERSITY OF RHODE ISLAND}

2012 


\begin{abstract}
The mechanical behavior of cellular topologies aligned with stress trajectories (ST) has been investigated. Two-dimensional stress trajectory topologies were generated for each of seven problems. The problems include: cantilever beam with shear end loading; two simply supported beams, one using the elasticity solution and an identical problem using the strength of materials solution; a disk under diametric compression; and a plate with a central stress free hole under equal biaxial, uniaxial and unequal biaxial loadings. The stress trajectory topologies were generated using MATLAB and the problems were simulated using Abaqus, a commercial finite element package. In each problem results from the stress trajectory topology were compared to other alternate topologies that came from random or uniform generation or from another problem.
\end{abstract}

The purpose of this study was to determine if stress trajectory cellular topologies would reduce the maximum and average stresses in comparison to control and uniform topologies. It is believed that a topology aligned with the stress trajectories will carry and distribute the stresses better than a random topology which often has localized areas of high stress. In the beam and disk problems, the topology effect was investigated by comparing the stress trajectory topology to a uniform topology. In the plate problems, the effect of element size in the ST topologies as well as the effect of the topology was investigated. The effect of the size of the element was investigated by comparing three different ST topologies of various densities. The effect of the topology was investigated by comparing the medium stress trajectory 
topology to two random topologies and the medium topologies of other plate problems.

For all cases it was found that stress trajectory topologies are better than other alternate topologies. The stress trajectory topology lowered the maximum and average stresses in the beam and disk problems. In the plate problems it was found that the size of the element has little effect as the maximum and average stresses were about the same. In looking at the different topologies for the plate problems it was found that the medium stress trajectory topology was the best of the five alternate topologies. The maximum and average stresses were lower than the random topologies and the stresses on the hole were much less than the other medium topologies. 


\section{ACKNOWLEDGMENTS}

I would like to thank my major professor, Dr. Martin H. Sadd, who has supported me throughout my thesis with his patience and knowledge of the subject. Without him this thesis would not have been completed or written. One simply could not wish for a better major professor.

I would also like to thank Dr. David Taggart and Dr. Richard Vaccaro, who were both gracious enough to take time out of their schedules to be on my thesis committee and help with any questions I may have had. I would also like to thank Dr. Lee for being the chair of my thesis defense.

Finally, I would like to thank my parents for supporting me throughout my studies at the University of Rhode Island and this thesis. I would especially like to thank my mother, Susan Breault, for helping me input the thousands of intersecting points into Abaqus. 


\section{TABLE OF CONTENTS}

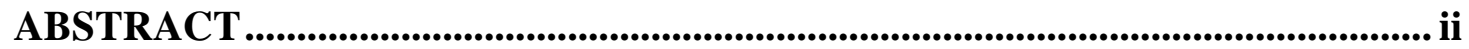

ACKNOWLEDGMENTS ..................................................................................... iv

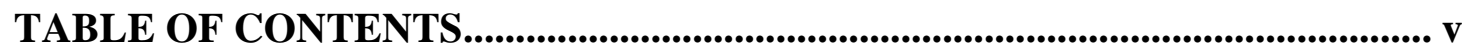

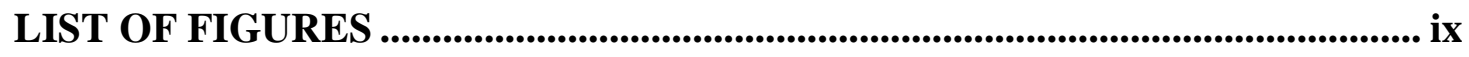

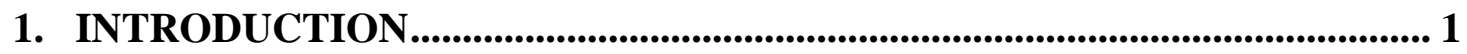

1.1 Cellular Materials ...................................................................................... 1

1.1.1 Geometries of Cellular Materials ........................................................... 2

1.1.2 Properties of Cellular Materials ............................................................. 4

1.2 Finite Element Analysis of Cellular Materials …....................................... 8

1.3 Stress Trajectories, Michell Structures and Applications to Cellular Materials 12

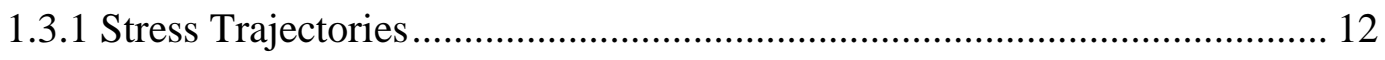

1.3.2 Michell Structures .......................................................................... 16

1.3.3 Application to Cellular Materials ......................................................... 18

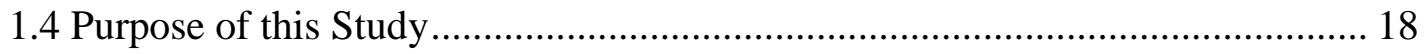

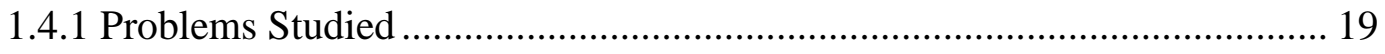

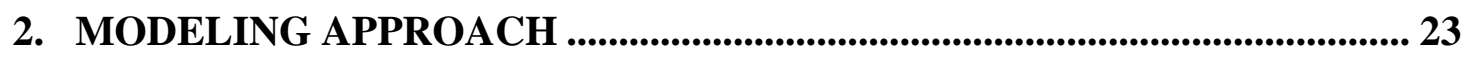

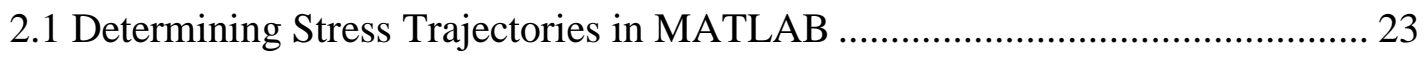

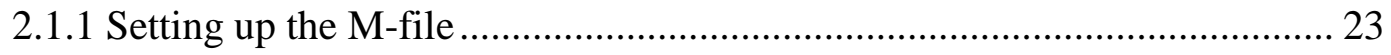

2.1.2 Generating the Stress Trajectories .................................................... 25

2.1.3 Solutions to Problems That Arose ........................................................ 26 
2.2 Establishing Finite Element Topologies Based on Stress Trajectories

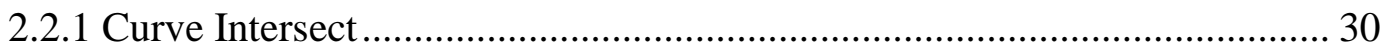

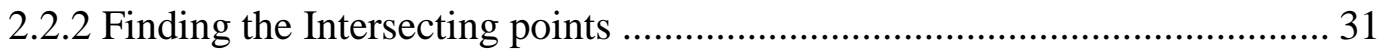

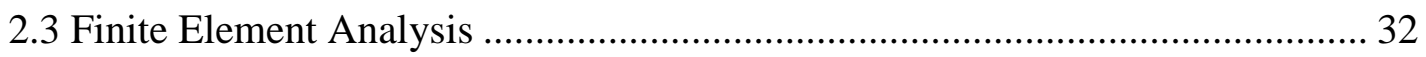

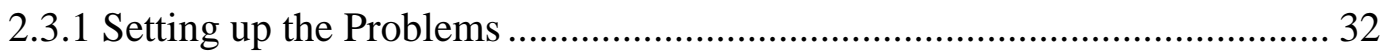

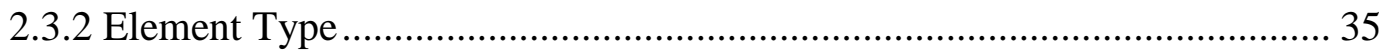

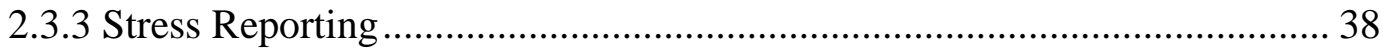

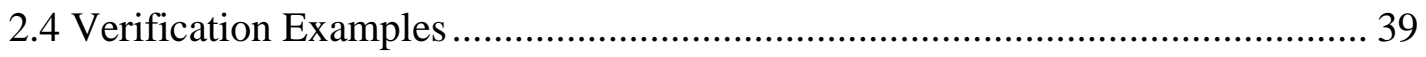

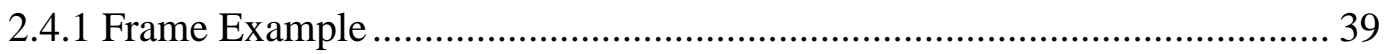

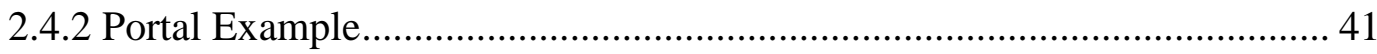

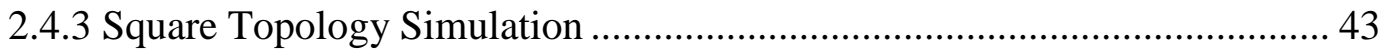

3. SIMULATION EXAMPLES AND RESULTS .......................................... 47

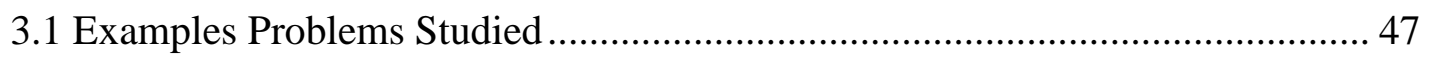

3.2 Cantilever Beam Problem ..................................................................... 47

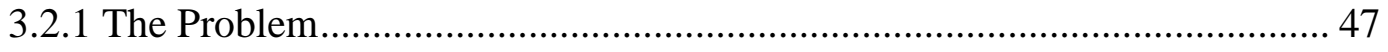

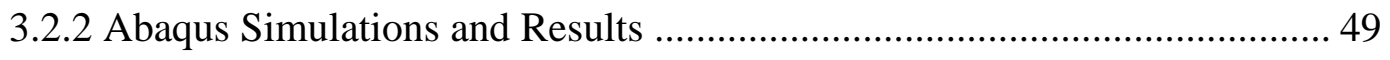

3.3 Simply Supported Beam Problems ........................................................... 52

3.3.1 The Problem....................................................................................... 52

3.3.2 Abaqus Simulations and Results ...................................................... 53

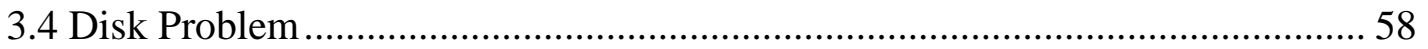


3.4.1 The Problem.

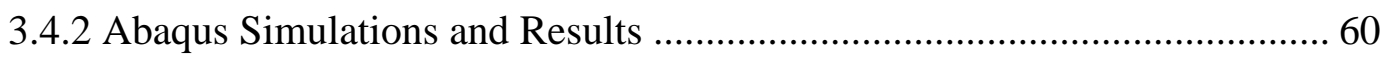

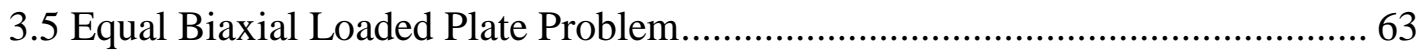

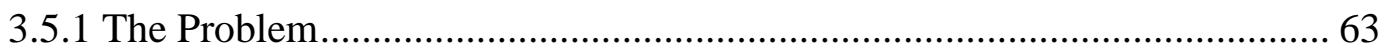

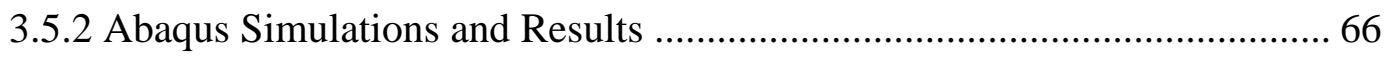

3.6 Uniaxial Loaded Plate Problem............................................................. 71

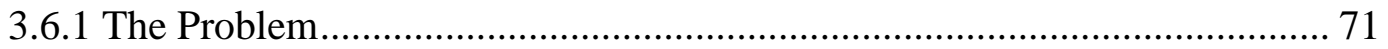

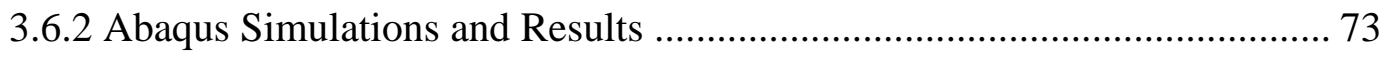

3.7 Unequal Biaxial Loaded Plate Problem ...................................................... 79

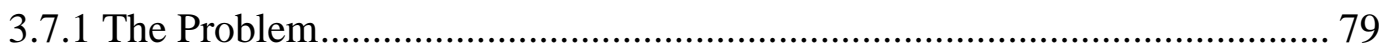

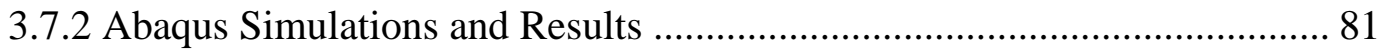

4. SUMMARY, CONCLUSIONS AND RECOMMENDATIONS ................... 87

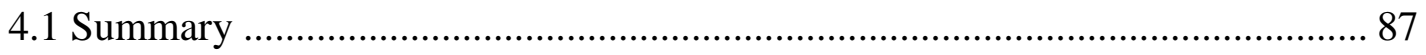

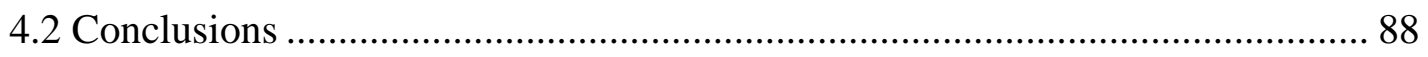

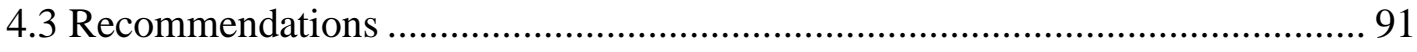

APPENDIX ............................................................................................................. 94

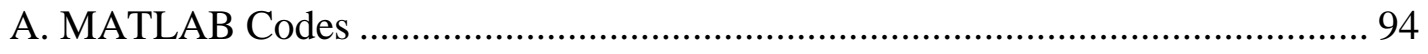

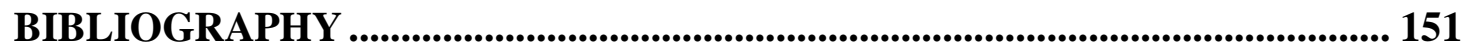




\section{LIST OF TABLES}

TABLE

PAGE

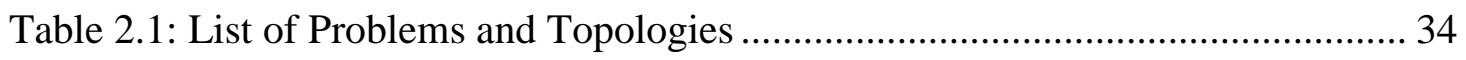

Table 2.2: Properties of Each Problem …………………......................................... 35

Table 2.3: Stresses in Each Member ............................................................................. 40

Table 2.4: Given Moments and Transverse Load ...................................................... 41

Table 2.5: Analytical vs Abaqus Stresses ................................................................... 42

Table 2.6: Analytical vs Abaqus Displacement ........................................................... 43

Table 2.7: Calculated Stresses in Vertical Elements................................................. 44

Table 3.1: Stress Trajectory Topology vs Control Topology ………………............... 50

Table 3.2: Topological Data for Simply Supported Beams Examples ....................... 53

Table 3.3: Topological Data for Equal Biaxial Loaded Plate Examples ..................... 65

Table 3.4: Maximum, Minimum and Average Stresses for the Three Topologies...... 67

Table 3.5: Maximum, Minimum and Average Stresses for the Five Topologies ......... 71

Table 3.6: Topological Data for Uniaxial Loaded Plate Examples .............................. 72

Table 3.7: Maximum, Minimum and Average Stresses for the Three Topologies...... 75

Table 3.8: Maximum, Minimum and Average Stresses for the Five Topologies ........ 78

Table 3.9: Topological Data for Unequal Biaxial Loaded Plate Examples ................. 79

Table 3.10: Maximum, Minimum and Average Stresses for the Three Topologies.... 82

Table 3.11: Maximum, Minimum and Average Stresses for the Five Topologies ...... 86

Table 4.1: Maximum and Average Stress for the Each Problem.................................. 91 


\section{LIST OF FIGURES}

FIGURE

PAGE

Figure 1.1: Periodic Structures of Cellular Materials .............................................. 2

Figure 1.2: (a) Cork, (b) Balsa, (c) Sponge, (d) Cancellous Bone, (e) Coral, (f) Cuttlefish Bone, (g) Iris Leaf, (h) Stalk of a Plant 3

Figure 1.3: (a) Open Cells, (b) Closed Cells, (c) \& (d) Thin Walled Cells, (e) \& (f) Wider Walled Cells, (g) \& (h) Open and Closed Cells Mixed............... 4

Figure 1.4: Example of Duocel ${ }^{\circledR}$ Aluminum Foam of Various Densities..................... 5

Figure 1.5: Property Comparison of Cellular Materials and True Solids .................... 7

Figure 1.6: Schematic of Honeycomb between Two Panels...................................... 8

Figure 1.7: Voronoi or Irregular Polygon Topologies ........................................ 9

Figure 1.8: Stress Components for Edges of Regular Honeycomb. ......................... 10

Figure 1.9: Cell Topologies Studied in Compression .......................................... 10

Figure 1.10: Notations Used for the Stress State ................................................ 13

Figure 1.11: Stress Trajectories and Stress Contours......................................... 15

Figure 1.12: Femur Head ............................................................................. 16

Figure 1.13: Principal Stress Trajectories in the Femur Head ................................ 16

Figure 1.14: Michell Structure for Chan Cantilever .......................................... 17

Figure 1.15: Flowchart of Procedure for Given Problems ...................................... 19

Figure 1.16: Cantilever Beam Sketch and Stresses.............................................. 20

Figure 1.17: Simply Supported Beam using Elasticity Stresses ............................. 20

Figure 1.18: Simply Supported Beam using Strength of Materials Stresses .............. 20

Figure 1.19: Disk Sketch and Stresses ........................................................... 21 
Figure 1.20: Equal Biaxial Loaded Plate Sketch and Stresses................................. 22

Figure 1.21: Uniaxial Load Plate Sketch and Stresses........................................ 22

Figure 1.22: Unequal Biaxial Loaded Plate Sketch and Stresses ............................ 22

Figure 2.1: Primary and Secondary Stress Trajectories in Cantilever Beam .............. 26

Figure 2.2: Stress Trajectories in a Cantilever Beam with Original Stresses ............. 27

Figure 2.3: Stress Trajectories and Stress Contours in Plate with Unequal Loading .. 28

Figure 2.4: Original Stress Trajectories of Uniaxial Loaded Case ........................... 28

Figure 2.5: Stress Trajectories in Uniaxial Loaded Case ........................................ 29

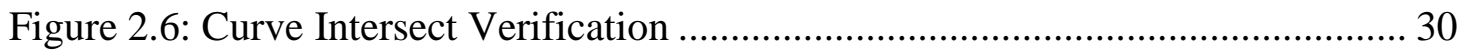

Figure 2.7: Intersecting Points of Uniaxially Loaded Plate ................................... 32

Figure 2.8: Abaqus Graphic of Uniaxially Loaded Cellular Plate ............................. 33

Figure 2.9: Full Uniaxially Loaded Plate ........................................................... 33

Figure 2.10: Diagram Demonstrating Frame Elements ..................................... 36

Figure 2.11: Cross-section after deformation Euler Bernoulli Beam Theory .............. 37

Figure 2.12:Cross-section after deformation Timoshenko Beam Theory .................. 37

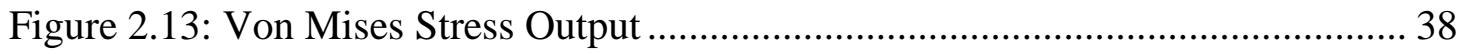

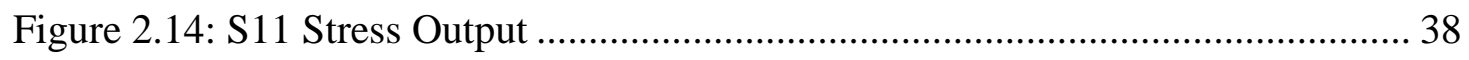

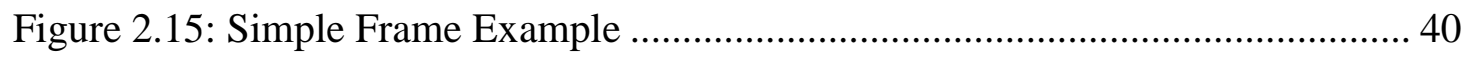

Figure 2.16: Portal Frame Geometry and Boundary Conditions ............................ 41

Figure 2.17: Free Body Diagrams of Side Members ......................................... 42

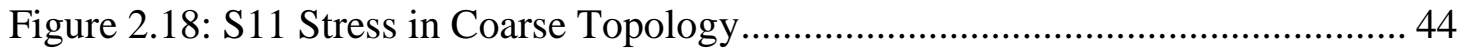

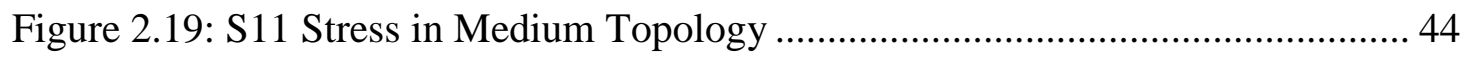

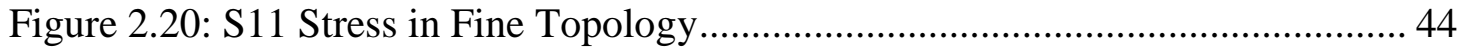


Figure 2.21: Path Where the Stresses Were Reported 45

Figure 2.22: Numerical vs Abaqus Stresses in Coarse Topology ............................. 45

Figure 2.23: Numerical vs Abaqus Stresses in Medium Topology .......................... 46

Figure 2.24: Numerical vs Abaqus Stresses in Fine Topology ............................... 46

Figure 3.1: Stress Trajectories for Cantilever Beam ............................................ 48

Figure 3.2: Abaqus ST Topology of Cantilever Beam ........................................ 48

Figure 3.3: Abaqus Control Topology for the Cantilever ....................................... 49

Figure 3.4: Stresses in Cantilever Beam with Stress Trajectory Topology ................ 49

Figure 3.5: Stresses in Cantilever Beam with Control Topology ............................. 50

Figure 3.6: Histograms of Stress for Cantilever Beam Problem............................... 51

Figure 3.7: Abaqus Topology of Simply Supported Beam using Elasticity Stresses .. 53

Figure 3.8: Abaqus Topology of Simply Supported Beam Using SM Stresses ......... 53

Figure 3.9: Control Topology for Simply Supported Beam ................................. 53

Figure 3.10: Stresses in Simply Supported Beam with Stress Trajectory Topology using SM Stresses .................................................................. 54

Figure 3.11: Stresses in Simply Supported Beam with Control Topology Using SM Loading

Figure 3.12: Stresses in Simply Supported Beam with Stress Trajectory Topology using Elasticity Stresses...... 55

Figure 3.13: Stresses in Simply Supported Beam with Control Topology Using Elasticity Loading ................................................................... 55

Figure 3.14: Stresses in Simply Supported Beam using SM Stresses ...................... 56 Figure 3.15: Frequency Plots for Simply Supported Beam using Elasticity Stresses.. 57 
Figure 3.16: Stress Trajectories of Disk in MATLAB ………………...................... 59

Figure 3.17: Abaqus Topology of Disk Problem ........................................................ 59

Figure 3.18: Abaqus Control Topology for Disk Problem .......................................... 60

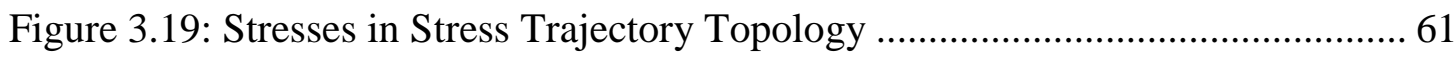

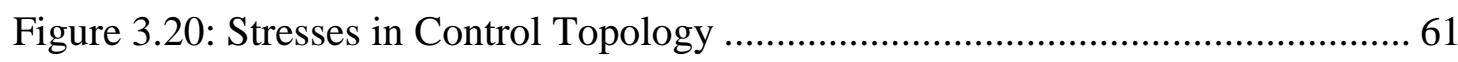

Figure 3.21: Stress Distribution in Disk Topologies................................................. 62

Figure 3.22: Coarse, Medium and Fine Topology of Equal Biaxial Loaded Plate...... 64

Figure 3.23: Random Topologies for Equal Biaxial Loaded Plate ………………....... 65

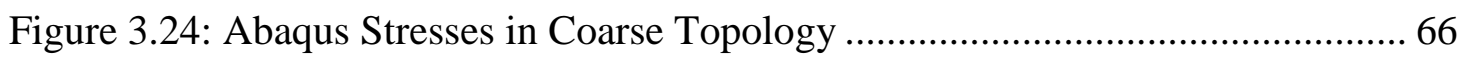

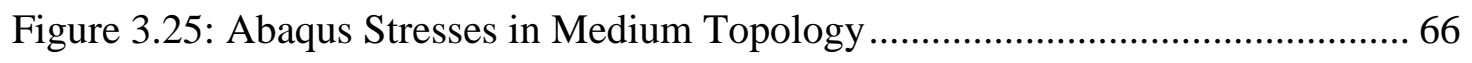

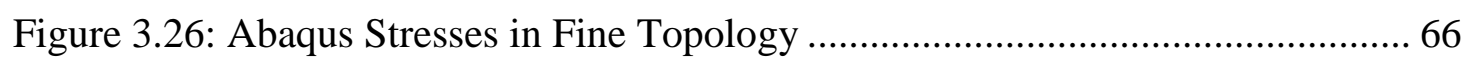

Figure 3.27: Stress Distribution in Coarse Topology ……………………................. 67

Figure 3.28: Stress Distribution in Medium Topology ……......................................... 67

Figure 3.29: Stress Distribution in Fine Topology ……….......................................... 67

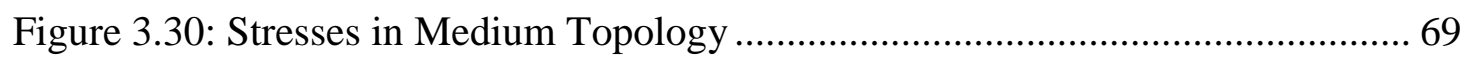

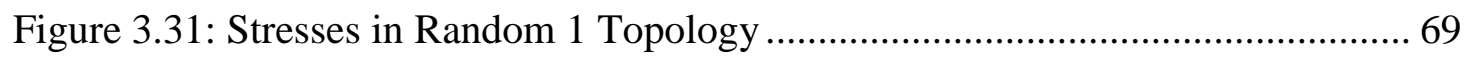

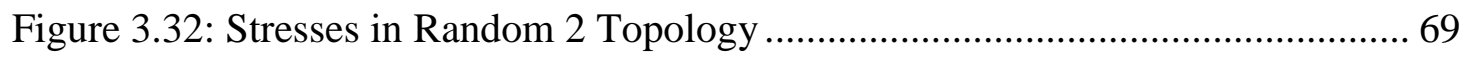

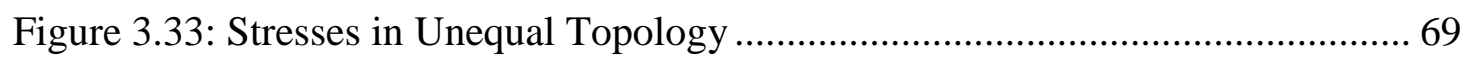

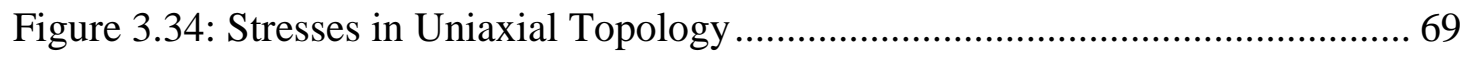

Figure 3.35: Stress Frequency Distribution of the Five Topologies ............................ 70

Figure 3.36: Coarse, Medium and Fine Topology of Uniaxial Loaded Plate ............... 73

Figure 3.37: Random Topologies for Uniaxial Loaded Plate ……………………...... 73

Figure 3.38: Abaqus Stresses in Coarse Topology ………….................................... 74 
Figure 3.39: Abaqus Stresses in Medium Topology ............................................. 74

Figure 3.40: Abaqus Stresses in Fine Topology ................................................... 74

Figure 3.41: Stress Distribution in Coarse Topology ............................................ 75

Figure 3.42: Stress Distribution in Medium Topology ....................................... 75

Figure 3.43: Stress Distribution in Fine Topology ............................................ 75

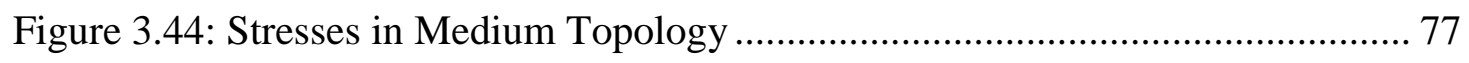

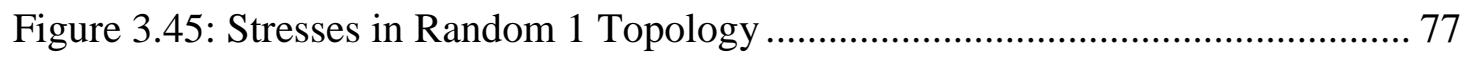

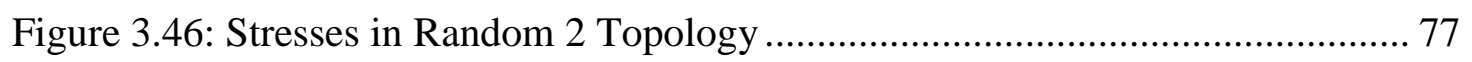

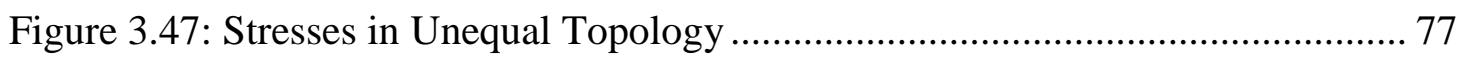

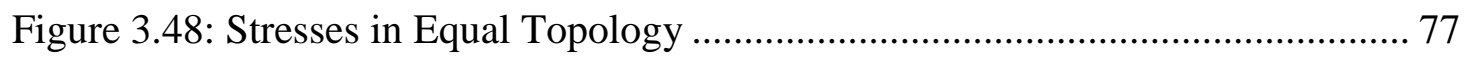

Figure 3.49: Stress Frequency Distribution of the Five Topologies ......................... 78

Figure 3.50: Coarse, Medium and Fine Topology of Unequal Biaxial Loaded Plate.. 80

Figure 3.51: Random Topologies for Unequal Biaxial Loaded Plate ........................ 81

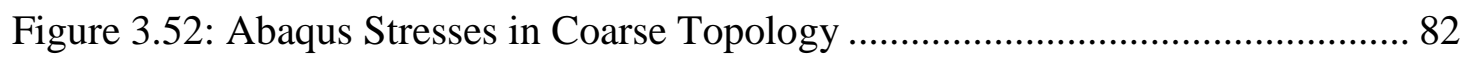

Figure 3.53: Abaqus Stresses in Medium Topology ............................................ 82

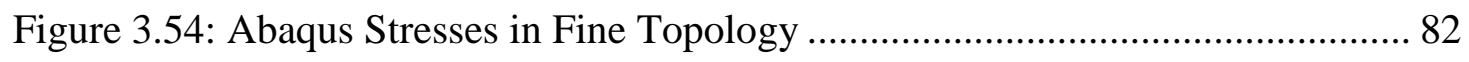

Figure 3.55: Stress Distribution in Coarse Topology ........................................... 83

Figure 3.56: Stress Distribution in Medium Topology ......................................... 83

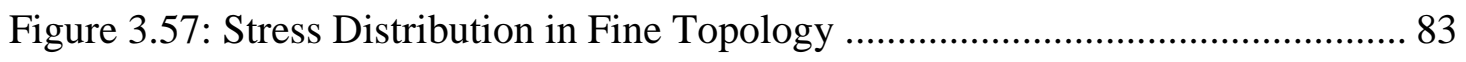

Figure 3.58: Stresses in Medium Topology ...................................................... 85

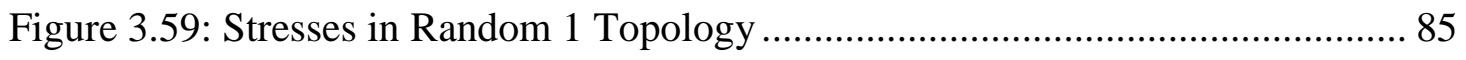

Figure 3.60: Stresses in Random 2 Topology ................................................. 85

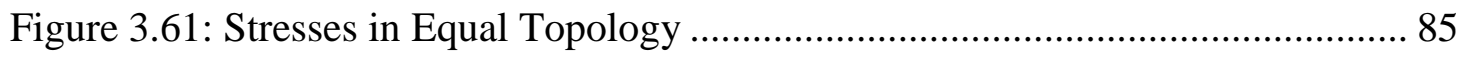


Figure 3.62: Stresses in Uniaxial Topology ....................................................... 85

Figure 3.63: Stress Frequency Distribution of the Five Topologies .......................... 86 


\section{Introduction}

\subsection{Cellular Materials}

A cellular solid is one made up of an interconnected network of solid struts or plates which form the edges and faces of cells (Gibson \& Ashby, 1997). Cellular materials are found throughout nature in wood, cork, bone, coral structures and many other places. There are also many man-made cellular materials comprised of polymers, metals, ceramics and more.

The applications of cellular material are numerous due to their many desirable physical properties. Due to their low thermal conductivity, cellular materials are used from simple applications such as a Styrofoam coffee cup to more elaborate applications such as insulator of the booster rockets for the space shuttle. Their low thermal conductivity also allows them to be used in more common applications like insulating a modern building, keeping refrigerated trucks and rail cars cold and keeping ships carrying natural gas at proper temperatures. The second major use for man-made cellular solids is in packaging (Kiessling, 1961). The cellular foams are able to absorb energy without damaging the contents while keeping the package light. The cellular foams have a low cost per unit volume which helps in the packaging application. Other applications of cellular material include the structure of the deHavilland Mosquito, a World War II bomber, (Hoff, 1951) and the F-15 aircraft which uses carbon-fiber composite skins separated by a aluminum or paper-resin honeycombs.

Research in the field of cellular materials is fairly new with literature beginning to significantly accumulate after 1960. This is different from other 
materials, like metals and concrete, which have been studied continuously for centuries. Cellular materials with repeating geometries are the most studied cellular structures and were the starting point for this analysis. Material properties and damage characteristics of cellular materials have been investigated more recently by engineers. Scientists are learning how to exploit the unique characteristics available in cellular materials. In recent years, more random cellular topologies have been studied including Voronoi topologies and some naturally occurring topologies.

\subsubsection{Geometries of Cellular Materials}

The cells of cellular materials can be broken down into two types of geometry, periodic and non-periodic structures. Periodic structures include honeycombs (like those made by bees), square, and triangular topologies. Examples of these can be seen in Figure 1.1 and are mainly man-made. These structures are used in sandwich panels and for supports of catalysts and components of heat exchangers. Since these geometries are repetitive, one cell can be analyzed and the equations derived can be inferred to the rest of the cells.

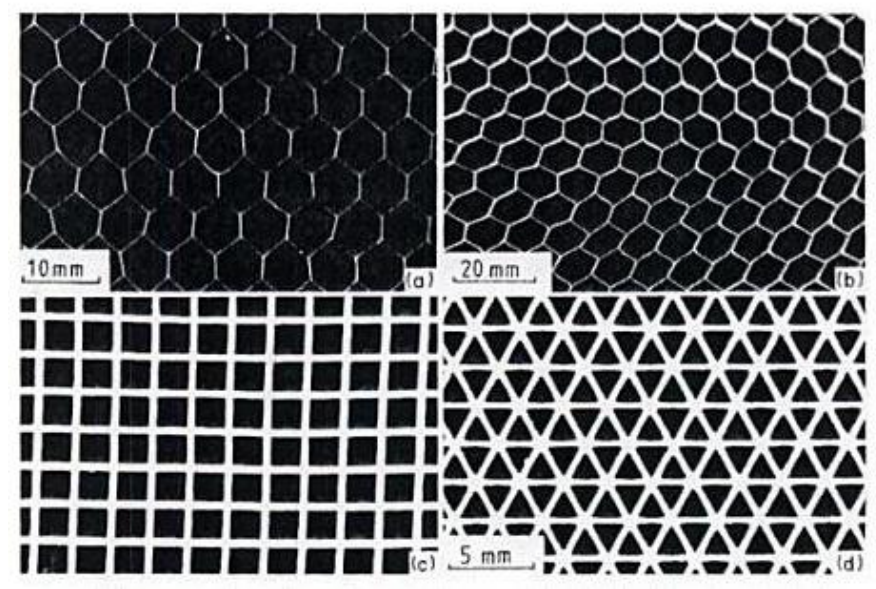

Figure 1.1: Periodic Structures of Cellular Materials 
Non-periodic cellular solids lack the repetitiveness of the periodic structures and are much more varying in size. Many of these types of geometries are found in nature as seen in Figure 1.2. These geometries are found in cork, balsa wood, sponges, bone, coral and more. The shape and size of these structures are determined by the space available when formed. The shape and size can also be dictated by the stress they experience. Man-made non-periodic structures are common also. Metal, ceramic and polymer open and closed face foams are created from bubbles in liquefied material (Gibson \& Ashby, 1997).

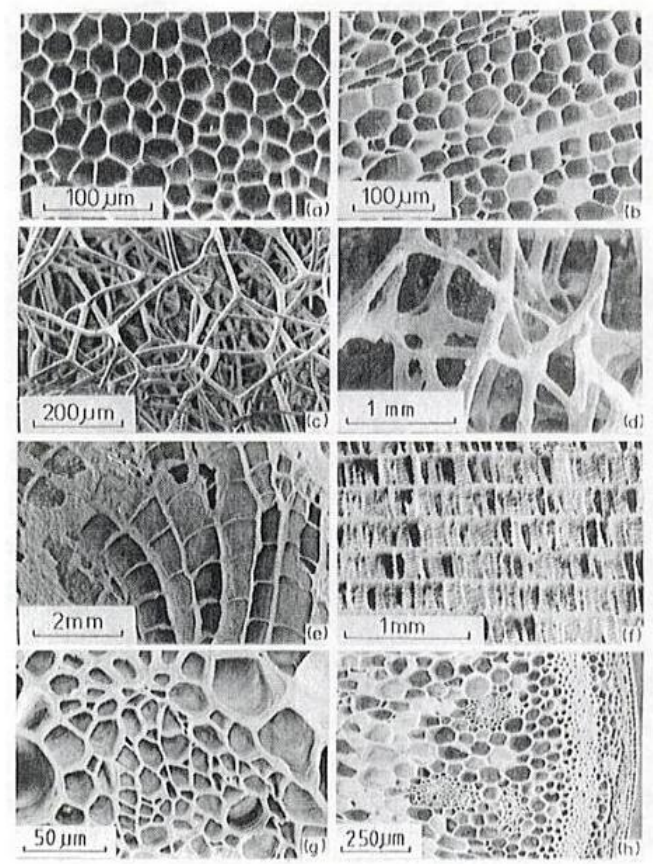

Figure 1.2: (a) Cork, (b) Balsa, (c) Sponge, (d) Cancellous Bone, (e) Coral, (f) Cuttlefish Bone, (g) Iris Leaf, (h) Stalk of a Plant

In both periodic and non-periodic geometries the cells themselves can be quite different. The cells can be can be open or closed as seen in the first row of Figure 1.3. The cells can also be more thin walled like the second row of Figure 1.3 or have wider 
walls like the third row. Finally, the cells can be a mixture of open and closed cells as seen in the bottom two pictures of Figure 1.3.

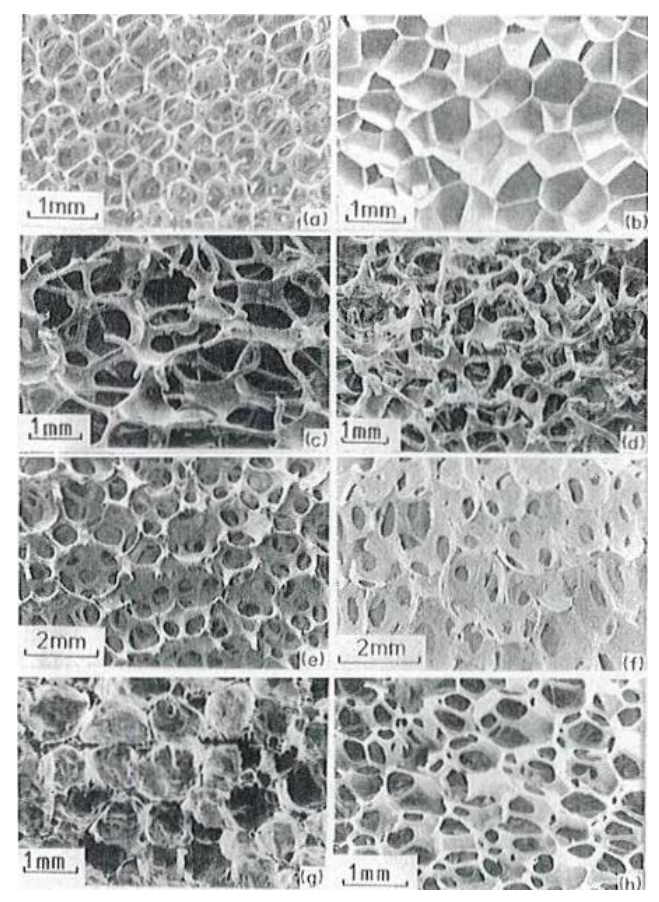

Figure 1.3: (a) Open Cells, (b) Closed Cells, (c) \& (d) Thin Walled Cells, (e) \& (f) Wider Walled Cells, (g) \& (h) Open and Closed Cells Mixed

\subsubsection{Properties of Cellular Materials}

One of the most fundamental properties of cellular topologies or foams is the relative density. The relative density of a cellular material is the density of the cellular material divided by the density of the solid material it is created from. This can be seen in equation (1.1), and typical relative densities range from 0.3 to 0.02 . Commercially available Aluminum foams like Duocel and Alporas have relative densities in the 0.12-0.08 range [(Gibson \& Ashby, 1997), (Silva \& Gibson, 1997), 
(Schaffner G. , Guo, Silva, \& Gibson, 2000), (Alkhader \& Vural, 2008) and (Onch, 2003)].

$$
\text { relative density }=\frac{\rho^{*}}{\rho_{s}}
$$

When looking at cellular materials the size of the individual cell is not as important as the ratio of cells per component dimension. A component with ten or more cells along each dimension has properties that are only weakly dependant on the cell size (Alkhader \& Vural, 2008). This is due to the homogenizing effect that more cells have on the entire structure. In the work of Kumar and McDowell, 2004, they were able to compare cellular models with as few as 10 cells across the component dimensions. In their analyses, it was found that models with more cells were far superior in modeling complex domain geometries for multiple cell structure types. Aluminum foams can be varied based on part per inch (PPI) as shown in Figure 1.4.

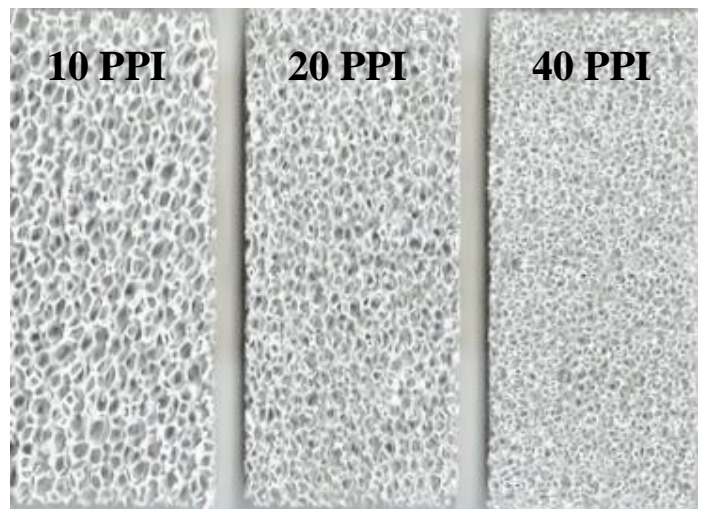

Figure 1.4: Example of Duocel ${ }^{\circledR}$ Aluminum Foam of Various Densities

There are some definitions that aid in the characterization of cellular structures. For a two dimensional cellular structure, the vertices are the point where the edges meet, the edges enclose the cell and the edge-connectivity is the amount of edges that 
meet at a vertex. For two dimensions with a large amount of cells, Euler's Law gives a relationship between several of these microstructural properties

$$
F-E+V=1
$$

where $\mathrm{F}$ is the number of faces of cells, $\mathrm{E}$ is the edges and $\mathrm{V}$ is the vertices. This is a geometric constraint placed on periodic repeating cells (Gibson \& Ashby, 1997). For periodic cellular material with edge lengths $l$, and thickness $t$ and a large difference in their magnitude, that is $t<<l$, the relative density is related to the thickness ratio by

$$
\frac{\rho^{*}}{\rho_{s}}=C_{1} \frac{t}{l}
$$

where $\mathrm{C}_{1}$ is a constant near 1 . Higher order approximations can be used when the relative density is greater than 0.2. Equation (1.4) represents the higher order approximation with an addition constant $\mathrm{D}_{1}$. Equation (1.5) represents the higher order relationship between the relative density and the thickness ratio for a square honeycomb periodic topology.

$$
\begin{gathered}
\frac{\rho^{*}}{\rho_{s}}=C_{1} \frac{t}{l}\left(1-D_{1} \frac{t}{l}\right) \\
\frac{\rho^{*}}{\rho_{s}}=2 \frac{t}{l}\left(1-\frac{1}{2} \frac{t}{l}\right)
\end{gathered}
$$

Cellular solids have physical, mechanical and thermal properties like ordinary continuum solids. The range of these properties can be compared to those of continuum or true solids as shown in Figure 1.5. Many of the properties of cellular materials are close to true solids. With the low densities of cellular materials, they permit the design of light, stiff components. 

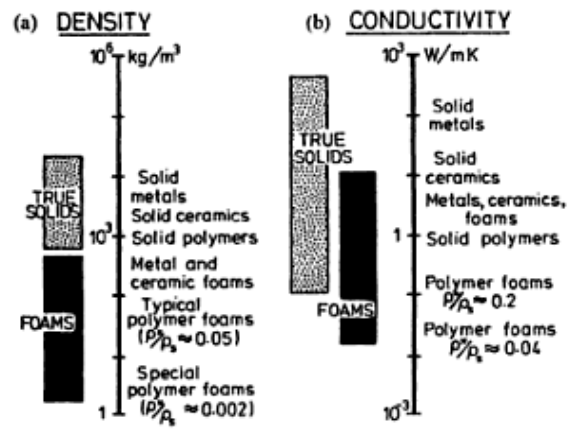

(c) YOUNGS MODULUS

(d) STRENGTH
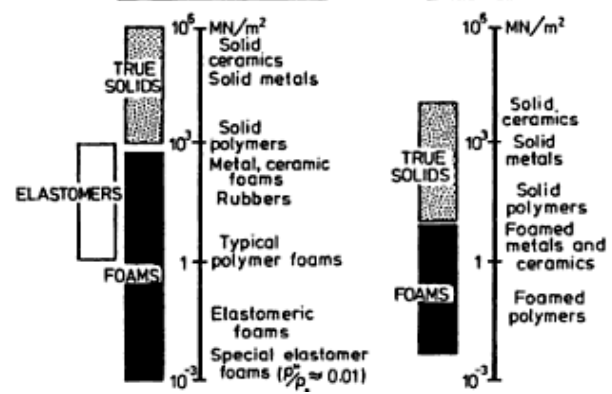

Figure 1.5: Property Comparison of Cellular Materials and True Solids

The mechanical response of cellular structures is highly dependent on the material from which it is made. For example, metal foam when loaded in tension will exhibit linear elastic deformation followed by localized yielding of edges in areas that exceed the yield stress of the metal. The foam will continue to deform until the fracture strength, $\sigma_{\mathrm{fs}}$, is reached. This will cause a process of defects to form within the structure as edges are broken and load is redistributed to the neighboring cells. This continues until the entire structure fails and breaks in two or more pieces.

Ceramic foams would behave differently. After the short period of linear elastic deformation, rapid fracture would take place and the entire foam would fail. Polymer based foams have deformations that are heavily dependent on the temperature of the foam itself. Relatively low temperatures produce brittle type fractures while temperatures approaching the melting temperature of the foam produce large viscous deformations. 
For two dimensional cellular structures like honeycombs, the stiffness and strength is separated into in and out of plane stiffness and strength. Stiffness and strength is much lower in-plane than out-of-plane due to the fact that the edges bend and buckle when a sufficiently large load is place on them. Out-of-plane deformation requires the edges to deform axially and thus is much harder. This is the reason honeycomb is placed in sandwich panels so that their out-of-plane stiffness can augment the material it is bonded to like shown in Figure 1.6 (Composite Honeycombs, 2010).

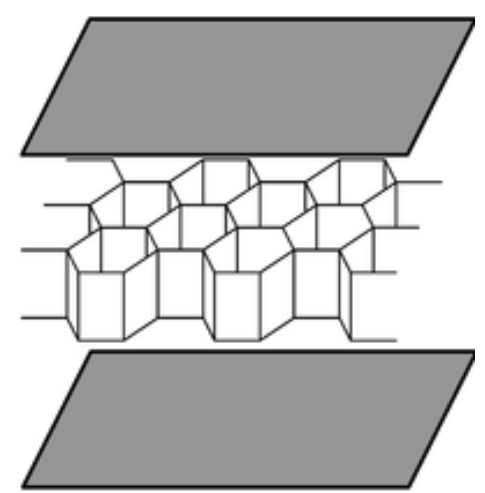

Figure 1.6: Schematic of Honeycomb between Two Panels

\subsection{Finite Element Analysis of Cellular Materials}

Most of the previous work done on cellular topologies has been done using grids, honeycomb periodic and Voronoi topologies. The field of mesh generation is in continual progression as researchers look for ways to improve their effectiveness. This is accomplished by developing algorithms that are autonomous, fast, allow larger amount of user control and create better quality geometric grids. Grids and honeycomb topologies lend them to be used in finite difference methods in heat 
transfer and solid mechanics. Problems with grids and honeycomb mesh occur around non standard geometries such as holes and curved surfaces (Ramamurthy \& Farouki, 1999). Voronoi and other irregular topologies are able to conform to irregular geometries and give the user a greater level of freedom when meshing options are predetermined. Voronoi topologies are also seen in real materials like those shown in Figure 1.2. Examples of irregular topologies can be seen in Figure 1.7.

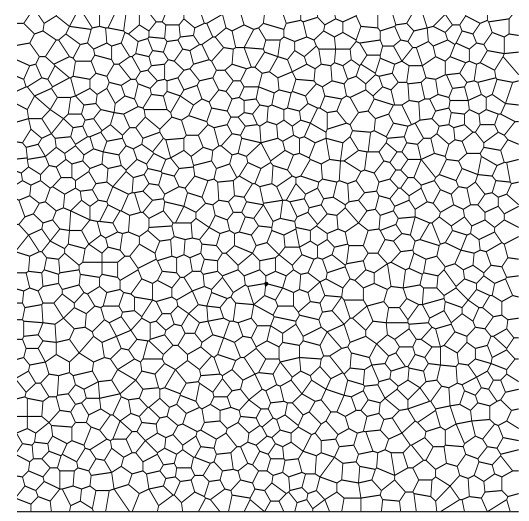

Figure 1.7: Voronoi or Irregular Polygon Topologies

To analyze the effect of the loading on an individual cell or edge the load must be broken into respective components. This is particularly useful when analyzing stress concentrations near imperfections and moving defects like a crack. Edge loading of a regular honeycomb can be described with their components of stress (Chen \& Ozaki, 2009) in Figure 1.8. Each member is broken into components of force in the 1 and 2 directions, $\mathrm{P}_{1}, \mathrm{P}_{2}$, and moments. This is done using statics and strength of materials quite readily but for non regular cellular structures like those normally found in nature resolving the components is not so trivial. For such cases, continuum and finite element numerical methods must be used to determine stress in individual areas or edges. 


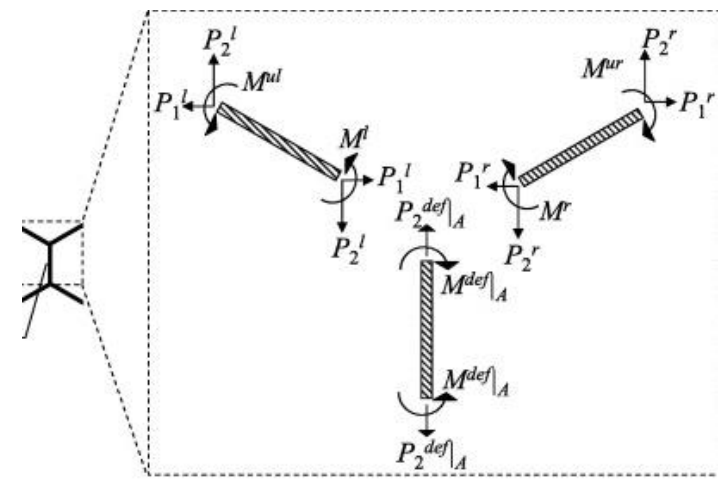

Figure 1.8: Stress Components for Edges of Regular Honeycomb.

Silva and Gibson,1997, analyzed non-periodic cellular structures under compression using finite element methods. They concluded that for the same relative cell size, non-periodic cell structures were $30 \%$ weaker and less stiff than regular honeycomb structures of the same relative density. They performed this study using generated Voronoi meshes then used finite element analysis to simulate its mechanical behavior of each cellular side wall under compressive stress.

Compressive test were performed by Alkhader and Vural, 2008, on regular hexagonal and triangle honeycomb along with non-periodic Voronoi honeycombs. Their study aimed at understanding the effect topology and cell irregularity had on compressive response. The general honeycomb topologies that were studied are shown in Figure 1.9.
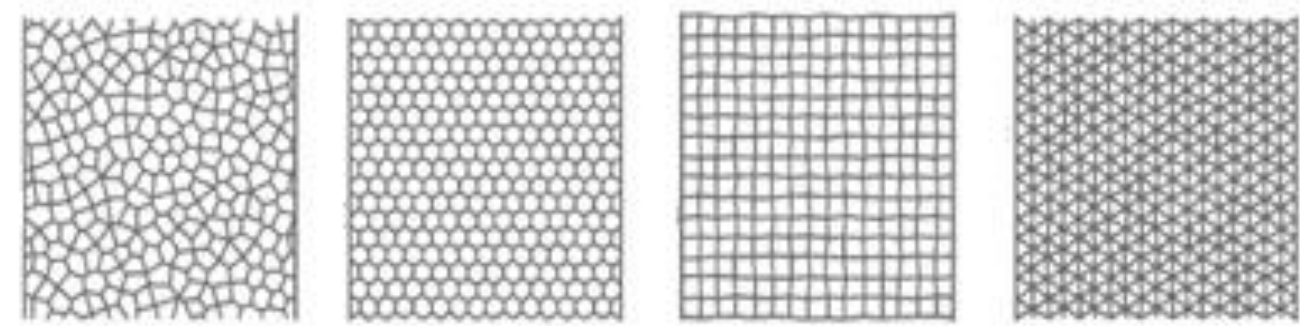

Figure 1.9: Cell Topologies Studied in Compression 
They found that by using a minimum of 10 cells across the specimens they were able to represent bulk foam behavior. An investigation was done into the boundary of each specimen to examine the effects of the boundary morphology and the influence of boundary conditions such as displacement and rotation fixation. They concluded that the boundary morphology was not influential but that the type of boundary condition imposed had a large affect. A connection was made with the type of deformation mode, stretch or bending dominated, and the overall strength of the specimen. Regular honeycombs relied on equally spaced members sharing compressive and tensile loads while irregular honeycombs had a tendency to have higher moment stresses in the edges causing them to fail sooner.

Many other computational studies have explored other aspects of cellular material behavior, such as the work done by (Ramamurthy \& Farouki, 1999) and (Schaffner G. , Guo, Silva, \& Gibson, 2000). These studies continue find better ways to simulate and study cellular materials. With the increasing knowledge obtained from these studies, companies are able to produce cellular components and structures that are better suited for a particular application.

It has been found that a beam using a cellular topology does not always carry the stress in the same manner as the continuum. In the case of uniformly loaded simply supported beams the maximum stress was located along the edges of the beam where they are supported due to compression. For the continuum problem the maximum stresses are found in the center of the beam and decrease towards the supports. 
Voronoi topologies have been studied more in recent years, including by (Jones, 2011) in his Master's Thesis last year. He investigated two dimensional Voronoi topologies around a central stress free hole. He also investigated the effect of grading the cellular material in the topology. He found some elevation of the stresses around the hole and established a gradation effect to reduce the local stress concentration.

Although considerable work has been done on studying the effect of cellular topology, only some preliminary research has explored the use of a topology based on stress trajectories. Most of this work has looked at stress trajectories around earthquakes, tectonic plate collisions and cancellous bone. We wish to explore in detail this concept for two-dimensional behavior of cellular solids and will now discuss these ideas in detail.

\subsection{Stress Trajectories, Michell Structures and Applications to Cellular Materials}

\subsubsection{Stress Trajectories}

Stress trajectories are lines whose direction at each point gives the direction of one of the principal stresses in a continuum material subject to load. For the twodimensional case, stress trajectories consist of two families, principal and secondary, and at each point they insect, orthogonally to each other (Molleda et al, 2005). 


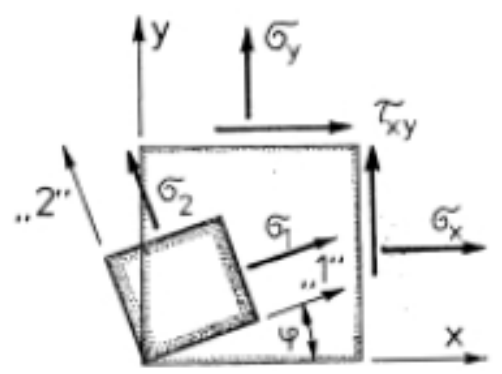

Figure 1.10: Notations Used for the Stress State (Thamm, 2000)

To generate the stress trajectories in two-dimensions, first begin with the set of in plane stresses:

$$
\begin{gathered}
\sigma_{x}=\sigma_{x}(x, y) \\
\sigma_{y}=\sigma_{y}(x, y) \\
\tau_{x y}=\tau_{x y}(x, y)
\end{gathered}
$$

Using standard transformation laws the orientation of the principal stress normal to the stress trajectory is given by:

$$
\tan 2 \varphi=\frac{2 \tau_{x y}}{\sigma_{x}-\sigma_{y}}
$$

where $\varphi$ is the angle between the stress normal to the stress trajectories at a point and the $\mathrm{x}$-axis as seen in Figure 1.10. As $\tan \varphi$ is the slope of the stress under considerations:

$$
\tan \varphi=\frac{d y}{d x}
$$

Using a standard trigonometric identity and combining with equations (1.7) and (1.8) leads to

$$
\tan 2 \varphi=\frac{2 \tan \varphi}{1-\tan ^{2} \varphi}=\frac{2 \frac{d y}{d x}}{1-\left(\frac{d y}{d x}\right)^{2}}=\frac{2 \tau_{x y}}{\sigma_{x}-\sigma_{y}}
$$

Solving for $\frac{d y}{d x}$ leads to the equations of the two families (Molleda et al, 2005) 


$$
\begin{aligned}
& \frac{d y}{d x}=-\frac{\sigma_{x}-\sigma_{y}}{2 \tau_{x y}}+\sqrt{1+\left(\frac{\sigma_{x}-\sigma_{y}}{2 \tau_{x y}}\right)^{2}} \\
& \frac{d y}{d x}=-\frac{\sigma_{x}-\sigma_{y}}{2 \tau_{x y}}-\sqrt{1+\left(\frac{\sigma_{x}-\sigma_{y}}{2 \tau_{x y}}\right)^{2}}
\end{aligned}
$$

Generating stress trajectories can be done in some different ways. Almeida Pereira and Moitinho de Almeida, 1994 developed a method that started at a point and then generated the change in $\mathrm{x}$ and $\mathrm{y}$ at each point along the line until the boundary was reached. Once outside the boundary the process was repeated. At each point equation they calculated the principal stresses $\sigma_{1}$ and $\sigma_{2}$ and the angle at that point. They then calculated dy, $\mathrm{ds} \sin \theta$, and $\mathrm{dx}, \mathrm{ds} \cos \theta$, and moved to the next point and repeated the process (Almeida Perira \& Moitinho de Almeida, 1994). This process was also done and improved upon by Petrucci and Restivo in 2007. They used a grid over the mesh and found the points of the line from boundary to boundary. A different way of generating stress trajectories other than numerical integration is using photoelasticity. This is done by using photoelastic fringes to get the information about the stress field at a given point. Still another way of generating stress trajectories can be done by using load paths as Kelly and Tosh, 1999. They developed stress trajectories using load paths and principal directions and vice versa. In this study, stress trajectories are generated by integrating the ordinary differential equations, (1.10) and (1.11), using MATLAB. A full description of this process can be seen in section 2.1 .

Stress trajectories are different from principal stress contours. Such contours are lines of constant principal stress while stress trajectories are lines normal and 
tangent to principal stress. As shown in Figure 1.11 (large plate with stress free hole under far field horizontal loading 0.5 and vertical loading 1.0 ), primary stress contours and primary stress trajectories are not the same. The stress contours are on the right side of the figure and the stress trajectories are on the left. The stress trajectories form a grid while principal stress contours do not.

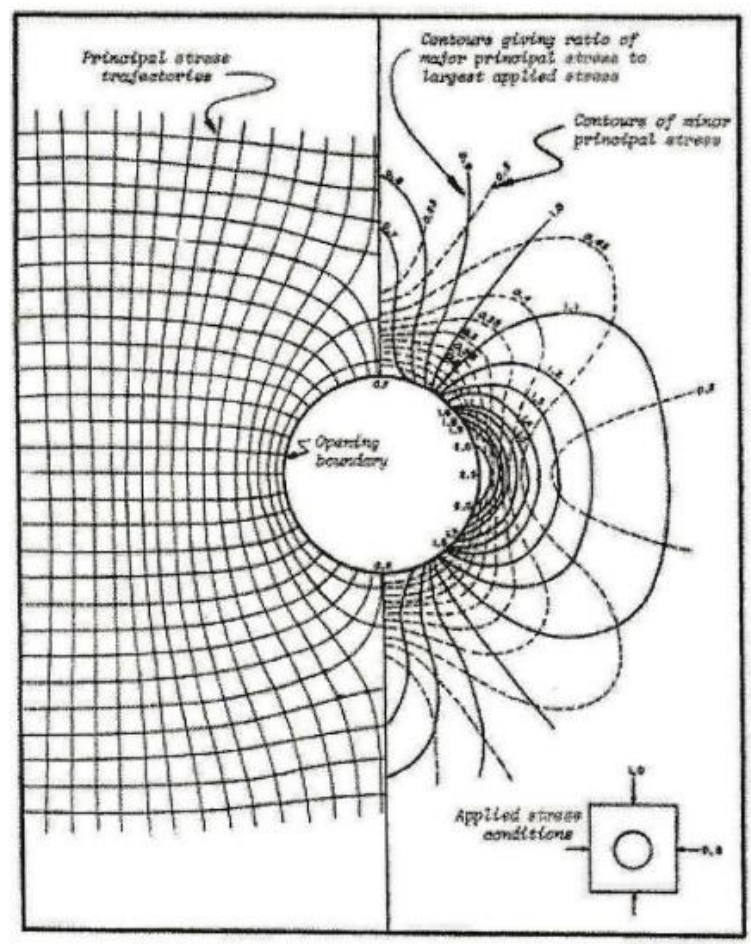

Figure 1.11: Stress Trajectories and Stress Contours

One of the areas in cellular mechanics research that is related to stress trajectories is in human bones. As seen in Figure 1.12, the head of the femur is a cancellous, or cellular bone. Current research has been looking into the principal stress trajectories, shown in Figure 1.13, in the femur head and the cancellous topology of the head. Future research could include the use of cellular materials in hip replacements to mimic the human femur more accurately. 


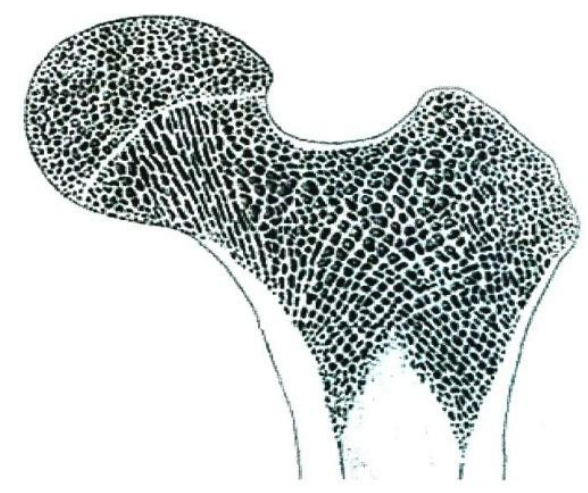

Figure 1.12: Femur Head

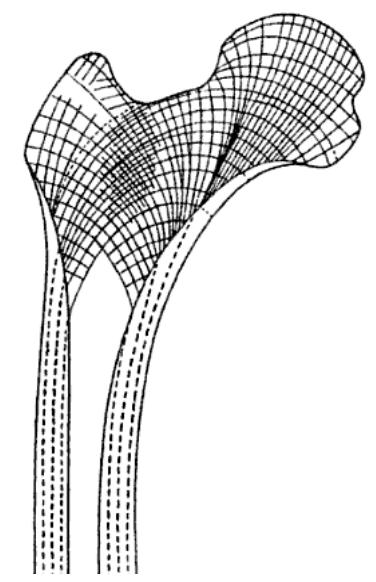

Figure 1.13: Principal Stress Trajectories in the Femur Head (Thompson, 1961)

Other research currently being done that pertains to stress trajectories includes ways to determine the stress field from discrete data. Irsa and Galybin, 2010, were able to do this using subdivision of the domain and piecewise polynomial approximations. This will help the generation of stress trajectories by allowing the stress field of irregular shapes to be determined.

\subsubsection{Michell Structures}

Related to the issue of principal stress trajectories are the Michell structures. These are minimum weight structures optmized to carry a specific load using the least 
amount of material. A. G. M. Michell, 1904 derived the dual form of the problem and exhibited its essential role in determining the optimal design (Whittle, 2007). Michell structures are used for simple structures, such as beams and bars, and more complicated structures in aeronautics and space technology. There are many such optimization methods that are used for various structures, including methods being developed here at the University of Rhode Island (Taggart et al, 2010), (Taggart et al, 2008),(Taggart \& Dewhurst, 2010).

Michell structures are similar to stress trajectories but also quite different. Michell structures look to minimize the material but stress trajectories are lines along principal stress. The case in which the two are the most similar is the cantilever beam. For example the Michell structure of a Chan Cantilever, seen in Figure 1.14, is very similar to the stress trajectories of a cantilever beam. The differences come from the set up of the problems. In the Chan cantilever, it is fixed at two points along the left boundary while a general cantilever beam is normally fixed over that entire boundary. In many cases the Michell structure of the problem would be a grid of straight lines.

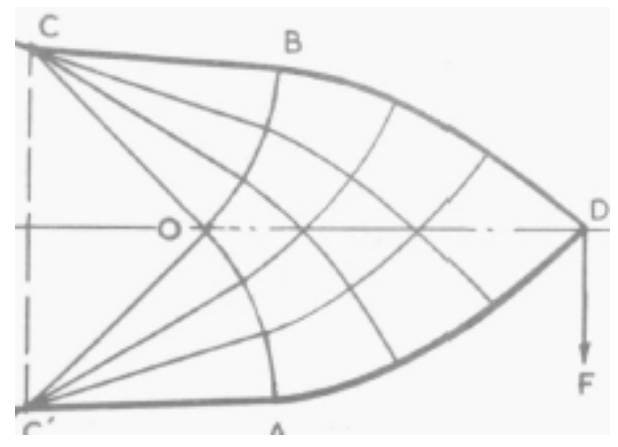

Figure 1.14: Michell Structure for Chan Cantilever (Chan, 1960) 


\subsubsection{Application to Cellular Materials}

As mentioned in section 1.3.1 one potential use of stress trajectories in cellular materials is as a topology for artificial hips in hip replacements. With the improving technology in making cellular materials there is potential to be able to make cellular materials with cell walls that align with the stress trajectories. It is anticipated that stress trajectory topologies would better distribute and lower maximum stress when compared with a more random topology. This would then lead to a lighter weight, higher strength material. It is believed that as more sophisticated manufacturing techniques are developed, future cellular solids could be made in this topology. In principle, stress trajectories could also be applied to three dimensional materials.

\subsection{Purpose of this Study}

The purpose of this study is to determine if a cellular topology aligned with the stress trajectories is a better topology than a more random one for a given problem. It is believed that the topology aligned with the stress trajectories will carry and distribute the stress better than a random topology, which often has localized areas of high stress. The study will be done by examining six different problems and comparing the stress trajectory topology to a control or random topology. The problems include a cantilever beam with shear end loading, simply supported beam under uniform loading, a disk under diametric compression, and a plate with a central hole under equal biaxial, uniaxial and unequal biaxial loadings. In the cantilever beam, simply supported beam and disk problems, the stress trajectory topology will be compared against a uniform control topology. The equal biaxial loaded plate, uniaxial 
loaded plate and unequal biaxial loaded plate problems will compare the stress trajectory topology to two random topologies and also to the medium ST topologies of the other two plate problems not being currently studied. In the three plate problems, the size of the elements in the topology will also be examined. This will be done by comparing a coarse, medium and fine topology to see if the stresses change. Using the methods discussed in this thesis, one should be able to take a continuum problem, find the stresses of the problem, generate the stress trajectories and create an overlay wire frame to be simulated using finite element analysis. The scheme is illustrated in the flow chart shown in Figure 1.15.

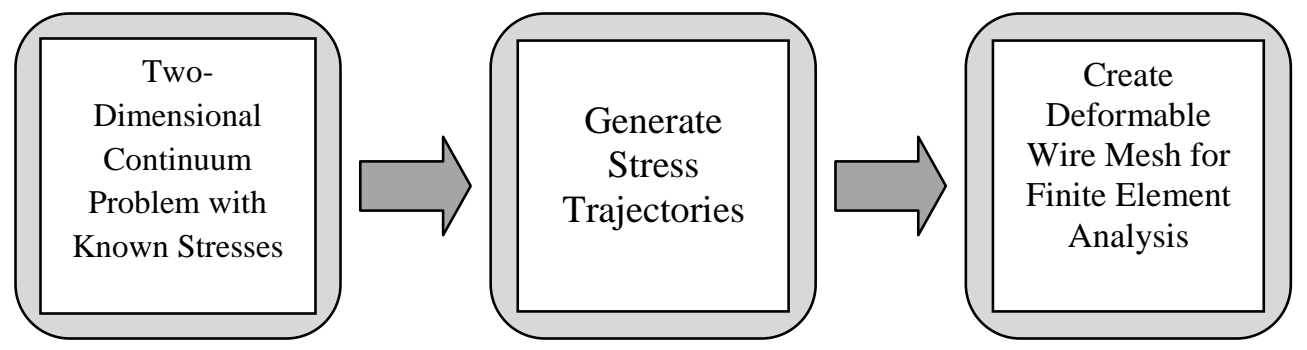

Figure 1.15: Flowchart of Procedure for Given Problems

\subsubsection{Problems Studied}

The seven problems studied in this thesis, all are two-dimensional continuum problems having an analytical solution for the in-plane stress field. This is important for calculating the stress trajectories as discussed in section 1.3.1. The stresses for each problem were taken from Sadd, 2009.

\section{Cantilever Problem}

The first problem examined was a cantilever beam with end shear load. The sketch and stresses can be seen in Figure 1.16. In the problem $\mathrm{L}=5 \mathrm{~cm}, \mathrm{c}=1 \mathrm{~cm}, \mathrm{P}=1$ $\mathrm{N} / \mathrm{m}$ and $\mathrm{N}=0$. The cantilever is fixed along the left boundary. 


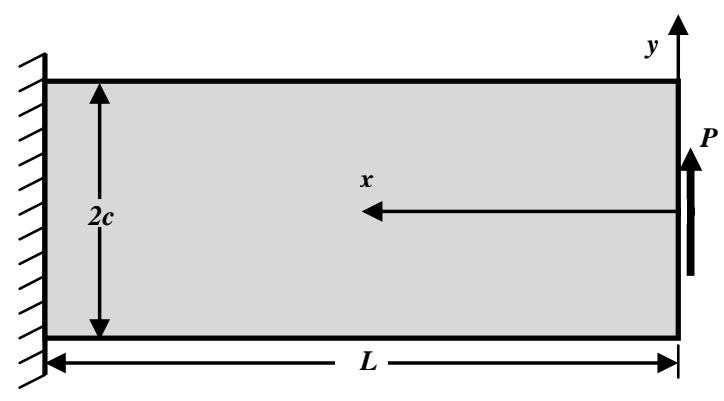

$$
\begin{aligned}
\sigma_{x} & =-\frac{3 P x y}{2 c^{3}} \\
\sigma_{y} & =0 \\
\tau_{x y} & =-\frac{3 P}{4 c}\left(1-\frac{y^{2}}{c^{2}}\right)
\end{aligned}
$$

Figure 1.16: Cantilever Beam Sketch and Stresses

\section{$\underline{\text { Simply Supported Beam Problem }}$}

The second problem studied is a simply supported beam under uniform load.

Two such problems were examined in this thesis. The first beam, shown in Figure

1.17, uses the stresses from elasticity while the second beam, shown in Figure 1.18, uses the stresses from Strength of Materials. In both beams $\mathrm{w}=1 \mathrm{~N} / \mathrm{m}, \mathrm{c}=1 \mathrm{~cm}$, and $l=5$ $\mathrm{cm}$. Both beams are pinned at the bottom left corner of the beam and have a roller at the bottom right corner of the beam.

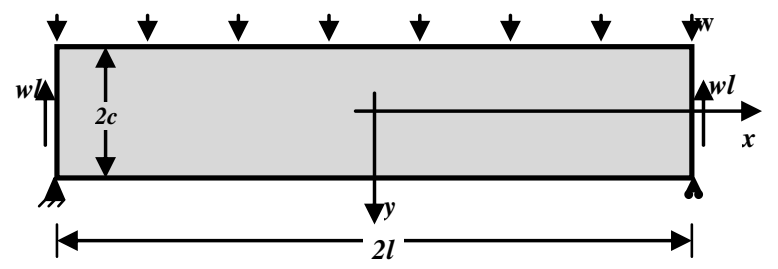

$$
\begin{aligned}
\sigma_{x} & =\frac{3 w}{4 c}\left(\frac{l^{2}}{c^{2}}-\frac{2}{5}\right) y-\frac{3 w}{4 c^{2}}\left(x^{2} y-\frac{2}{3} y^{3}\right) \\
\sigma_{y} & =-\frac{w}{2}+\frac{3 w}{4 c} y-\frac{w}{4 c^{3}} y^{3} \\
\tau_{x y} & =-\frac{3 w}{4 c} x+\frac{3 w}{4 c^{3}} x y^{2}
\end{aligned}
$$

Figure 1.17: Simply Supported Beam using Elasticity Stresses

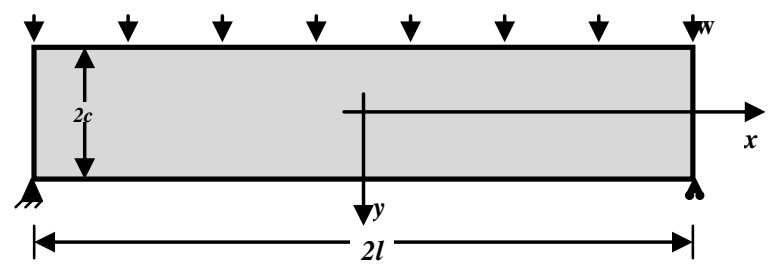

$$
\begin{aligned}
\sigma_{x} & =\frac{3 w}{4 c}\left(\frac{l^{2}}{c^{2}}-\frac{2}{5}\right) y \\
\sigma_{y} & =0 \\
\tau_{x y} & =-\frac{3 w}{4 c} x+\frac{3 w}{4 c^{3}} x y^{2}
\end{aligned}
$$

Figure 1.18: Simply Supported Beam using Strength of Materials Stresses 


\section{$\underline{\text { Disk Problem }}$}

The next problem studied was a disk under diametric compression. The stresses in the disk, seen in Figure 1.19. Equation (1.12) defines $r_{1}$ and $r_{2}$ where the $r_{1}$ uses the minus sign and $\mathrm{r}_{2}$ uses the plus sign. Problem loading and geometry are, $\mathrm{P}=1$ $\mathrm{N}, \mathrm{R}=2 \mathrm{~cm}$, and $\mathrm{D}=4 \mathrm{~cm}$.

$$
r_{1,2}=\sqrt{x^{2}+(R \mp y)}
$$

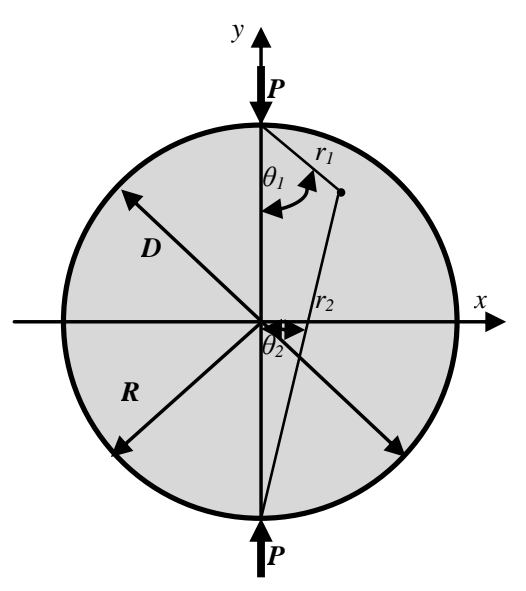

$$
\begin{aligned}
& \sigma_{x}=-\frac{2 P}{\pi}\left[\frac{(R-y) x^{2}}{r_{1}^{4}}+\frac{(R+y) x^{2}}{r_{2}^{4}}-\frac{1}{D}\right] \\
& \sigma_{y}=-\frac{2 P}{\pi}\left[\frac{(R-y)^{3}}{r_{1}^{4}}+\frac{(R+y)^{3}}{r_{2}^{4}}-\frac{1}{D}\right] \\
& \tau_{x y}=\frac{2 P}{\pi}\left[\frac{(R-y)^{2} x}{r_{1}^{4}}+\frac{(R+y)^{2} x}{r_{2}^{4}}\right]
\end{aligned}
$$

Figure 1.19: Disk Sketch and Stresses

\section{$\underline{\text { Plate Problems }}$}

The final three problems are plates with central stress free holes under different loading conditions. The first problem is a plate under equal biaxial loading, shown in Figure 1.20. In the stress equations, $\mathrm{T}=1 \mathrm{~N} / \mathrm{m}, \mathrm{a}=1 \mathrm{~cm}$ and $\mathrm{r}=\sqrt{\mathrm{x}^{2}+\mathrm{y}^{2}}$. Theses parameters were also used for the other plate problems as well. Figure 1.21 illustrates the uniaxial loading case and Figure 1.22 shows an unequal biaxial loading problem. All of these problems have standard elasticity solutions that can be generated from the uniaxial solution, (Sadd, 2009). Each solution is shown in the respective figures. The stresses for the plate problem are for the infinite domain. To minimize the effect of 
the loading on the hole a large ratio for the width of the plate to diameter of the hole was chosen. A ratio of 5:1 was used in the examples.

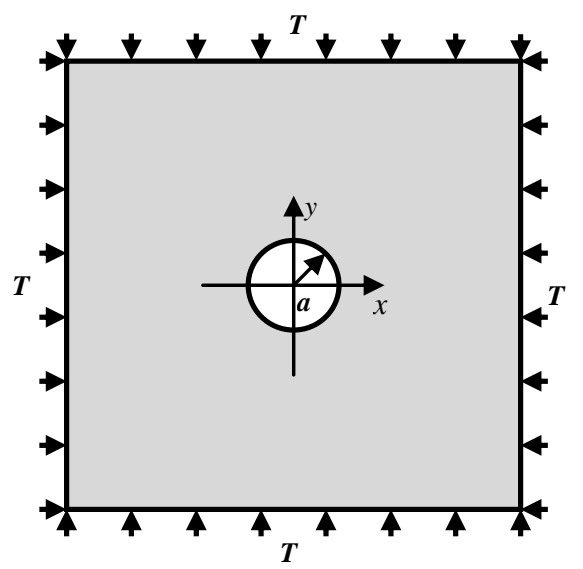

$$
\begin{aligned}
\sigma_{r} & =T\left(1-\frac{a^{2}}{r^{2}}\right) \\
\sigma_{\theta} & =T\left(1+\frac{a^{2}}{r^{2}}\right) \\
\tau_{r \theta} & =0
\end{aligned}
$$

Figure 1.20: Equal Biaxial Loaded Plate Sketch and Stresses

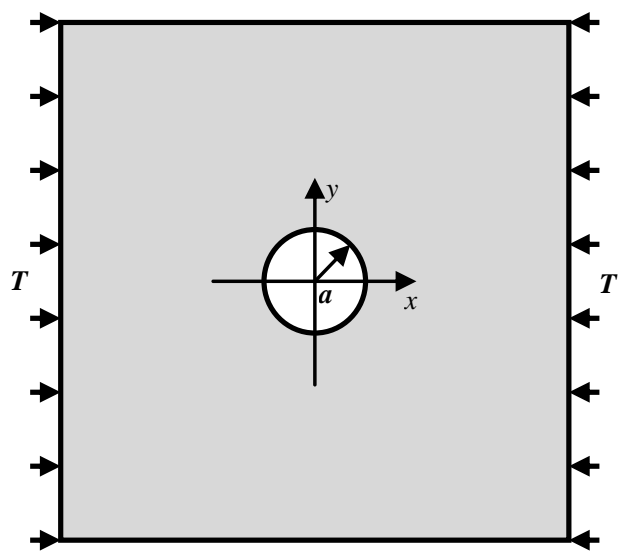

$$
\begin{aligned}
\sigma_{r} & =\frac{T}{2}\left(1-\frac{a^{2}}{r^{2}}\right)+\frac{T}{2}\left(1+\frac{3 a^{4}}{r^{4}}-\frac{4 a^{2}}{r^{2}}\right) \cos 2 \theta \\
\sigma_{\theta} & =\frac{T}{2}\left(1+\frac{a^{2}}{r^{2}}\right)-\frac{T}{2}\left(1+\frac{3 a^{4}}{r^{4}}\right) \cos 2 \theta \\
\tau_{r \theta} & =-\frac{T}{2}\left(1-\frac{3 a^{4}}{r^{4}}+\frac{4 a^{2}}{r^{2}}\right) \sin 2 \theta
\end{aligned}
$$

Figure 1.21: Uniaxial Load Plate Sketch and Stresses

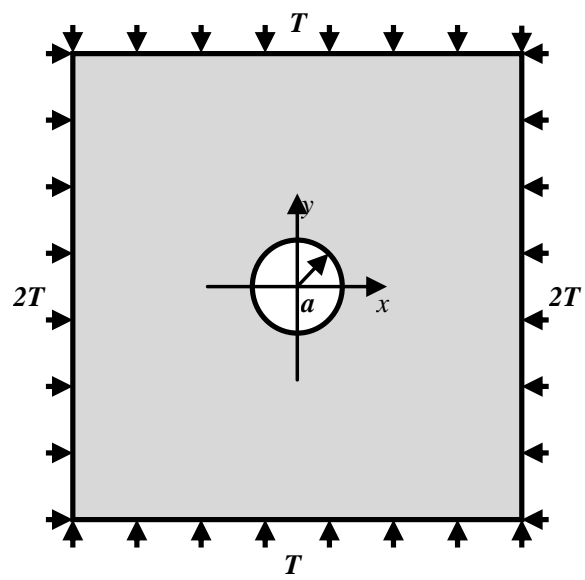

$$
\begin{aligned}
& \sigma_{r}=\frac{3 T}{2}\left(1-\frac{a^{2}}{r^{2}}\right)+\frac{T}{2}\left(1+\frac{3 a^{4}}{r^{4}}-\frac{4 a^{2}}{r^{2}}\right) \cos 2 \theta \\
& \sigma_{\theta}=\frac{3 T}{2}\left(1+\frac{a^{2}}{r^{2}}\right)-\frac{T}{2}\left(1+\frac{3 a^{4}}{r^{4}}\right) \cos 2 \theta \\
& \tau_{r \theta}=-\frac{T}{2}\left(1-\frac{3 a^{4}}{r^{4}}+\frac{4 a^{2}}{r^{2}}\right) \sin 2 \theta
\end{aligned}
$$

Figure 1.22: Unequal Biaxial Loaded Plate Sketch and Stresses 


\section{Modeling Approach}

\subsection{Determining Stress Trajectories in MATLAB}

The first step in determining if stress trajectories are a better topology than a random array is to generate the stress trajectories using MATLAB, a numerical computing program by Math Works. The stress trajectories were generated for the problems discussed in section 1.4.1 to a desired density. Along the way there were some problems that arose but were able to be solved. Once the stress trajectories were generated an overlay mesh can be generated and simulated using Abaqus.

\subsubsection{Setting up the M-file}

The stress trajectories were generated using equations (1.10) and (1.11) in section 1.3.1 and it is necessary to use an ordinary differential equation (ODE) solver. MATLAB has eight different ODE solvers built into the program that cover from basic equations to implicit equations. After examining all the ODE functions available it was determined that ode 45 was the function that would be used. Ode 45 is a nonstiff differential equations solver that has a medium order of accuracy (Gilat, 2008). It solves differential equations with the equation $\mathrm{y}^{\prime}=\mathrm{f}(\mathrm{x}, \mathrm{y})$ with initial state of $\mathrm{y}\left(\mathrm{x}_{0}\right)=\mathrm{y}_{0}$. The function $f(x, y)$ is given by equations (1.10) and (1.11). The syntax for ode45 is [xout,yout $]=$ ode45(odefun, $\mathrm{xspan}, \mathrm{y}_{0}$ ) where [xout,yout $]$ is an output matrix of coordinates, xspan is the span in which the ODE will be solved and odefun is a function that is called to calculate the right hand side, $\mathrm{f}(\mathrm{x}, \mathrm{y})$. 
The ODE equations for stress trajectories are a function of $\sigma_{\mathrm{x}}, \sigma_{\mathrm{y}}$, and $\tau_{\mathrm{xy}}$ and require an imbedded function to be called upon by ode45. There are two imbedded functions that were created, one for equation (1.10) and one for equation (1.11). In these functions are the stresses given in the problem, dimensions that affect the stresses and either equation (1.10) or (1.11). In the simply supported example, defined in Figure 1.18, the imbedded function can be seen below where sx is $\sigma_{\mathrm{x}}$, sy is $\sigma_{\mathrm{y}}$, and txy is $\tau_{\mathrm{xy}}$ :

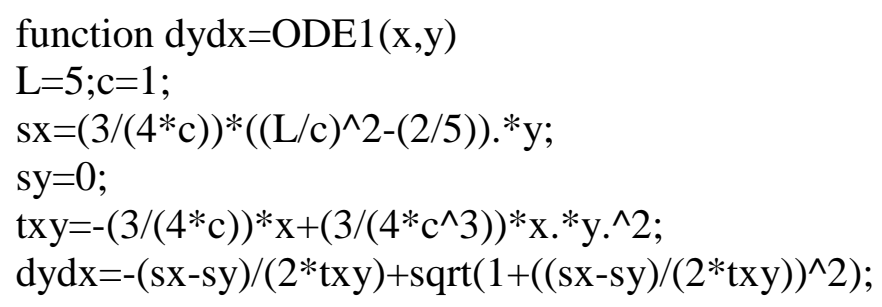

The second imbedded function would be the same as the one above except in the dydx equation before sqrt would be a minus sign. In the plate problems discussed in sections 3.5 to 3.7 the stresses given were $\sigma_{\mathrm{r}}, \sigma_{\theta}$, and $\tau_{\mathrm{r} \theta}$ and therefore had to be transformed to $\sigma_{\mathrm{x}}, \sigma_{\mathrm{y}}$, and $\tau_{\mathrm{xy}}$. Using stress transformation $\sigma_{\mathrm{x}}, \sigma_{\mathrm{y}}$, and $\tau_{\mathrm{xy}}$ become:

$$
\begin{gathered}
\sigma_{\mathrm{x}}=\sigma_{\mathrm{r}} \cos ^{2} \theta+\sigma_{\theta} \sin ^{2} \theta-2 \tau_{\mathrm{r} \theta} \sin \theta \cos \theta \\
\sigma_{\mathrm{y}}=\sigma_{\mathrm{r}} \sin ^{2} \theta+\sigma_{\theta} \cos ^{2} \theta+2 \tau_{\mathrm{r} \theta} \sin \theta \cos \theta \\
\tau_{\mathrm{xy}}=\sigma_{\mathrm{r}} \sin \theta \cos \theta-\sigma_{\theta} \sin \theta \cos \theta+\tau_{\mathrm{r} \theta} \cos ^{2} \theta-\tau_{\mathrm{r} \theta} \sin ^{2} \theta
\end{gathered}
$$

Other small changes were necessary for different problems that arose and will be discussed further in section 2.1.3. 


\subsubsection{Generating the Stress Trajectories}

To be able to use each output stress trajectory matrix in future calculations each line was generated individually and saved as a unique variable. For all the problems the xspan was taken from 0 to the boundary and was broken down into increments three orders of magnitude smaller. Symmetry was used whenever possible for example, for the plate problems, $1 / 4$ of plate was generated and mirrored to fill in the rest of the plate. This was also true for the disk and the simply supported beams that were mirrored over y axis. There were cases in the plate problems in sections 3.5 to 3.7 that called for the xspan to be changed and will be discussed in those sections. Each line generated had a unique $\mathrm{y}_{0}$ with the increments varying from 0.05 to 0.3 depending upon the problem. Each line was called twice to produce a primary stress trajectory and a secondary stress trajectory. An example of the syntax to generate the lines is below:

[x31,y31]=ode45(@ODE1,[0:0.001:5.000],0.50); [x44,y44]=ode45(@ODE2,[0:0.001:5.000],0.50);

In the first line @ODE1 is the imbedded function that generates the primary stress trajectories and @ODE2 generates the secondary stress trajectories. Both lines are generated over the span of 0 to 5 by increments of 0.001 and had an initial value of $y=$ 0.5 at $x=0$. The first column of the output matrix in the first line is saved as $x 31$ with the second column saved as $\mathrm{y} 31$. This allows each line to be plotted individually and simplifies the finding of the intersecting points discussed in section 2.2.1. After each line is generated it is then plotted. For the cantilever beam problem the primary and secondary stress trajectories appear in Figure 2.1 where the black lines are the primary stress trajectories and the blue lines are the secondary stress trajectories. 


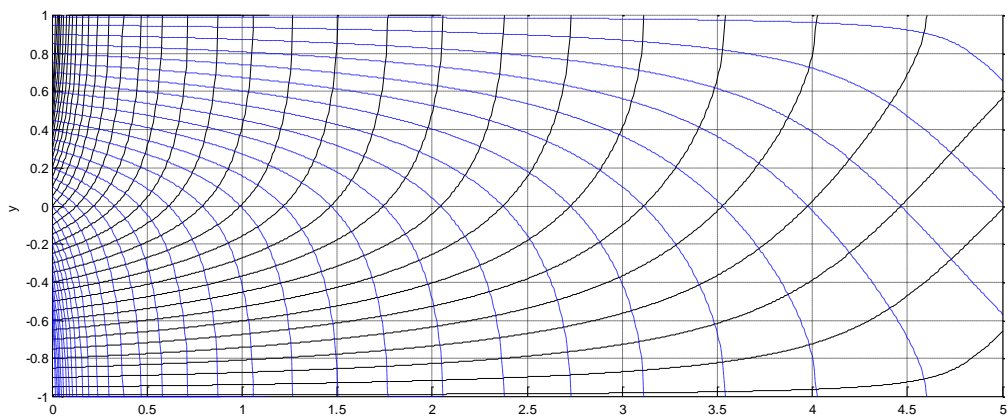

Figure 2.1: Primary and Secondary Stress Trajectories in Cantilever Beam

\subsubsection{Solutions to Problems That Arose}

One of the first problems that arose in the setting up of the stress trajectories were how the stresses were derived. In the case of the cantilever beam the stresses were given from the free end to the fixed end instead of from the fixed end to the free end. This is a problem because the stress trajectories are generated from an initial point with the slope generated at each increment. In the case with the stresses starting on the free end, the stress trajectories began every 0.05 and the slope was generated to the next point. This resulted in the generation of similar stress trajectories which quickly converge and overlap, as seen in Figure 2.2. This does not fill the beam adequately. To solve this problem the stresses were translated to the left boundary. In the case with the stresses starting along the fixed end, the stress trajectories began every 0.05 and again the slope was generated from increment to increment. This scheme successfully filled the domain. To show that the stress trajectories are identical in both cases the top blue dashed line in Figure 2.1 and the top solid black line in Figure 2.2 were compared. When both lines were plotted on the same axis the line overlapped, showing that they were in fact the same lines. The original stresses were translated from $x=5$ to $x=0$ meaning the $x$ in the original stresses became $(5-x)$ in the 
new stresses. This produced the stress trajectories seen in Figure 2.1 that fill in the entire beam. This situation only occurred for the cantilever beam problem.

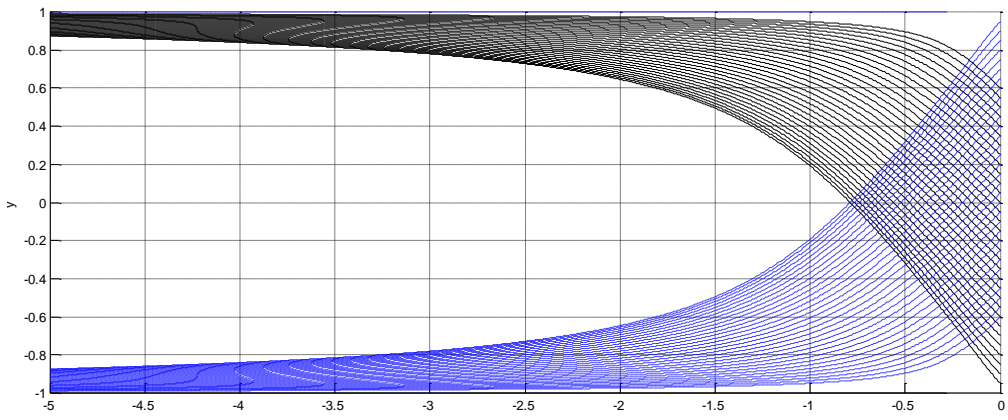

Figure 2.2: Stress Trajectories in a Cantilever Beam with Original Stresses

The next problem that first arose in the disk problem and again later in the plate problems was the secondary stress trajectories being a function of y rather than a function of $\mathrm{x}$. When looking at the left side of Figure 2.3, the primary stress trajectories are horizontal while the secondary stress trajectories are vertical. This poses the problem in the ode45 function in MATLAB since these stress trajectories vary as $y$ changes rather than as $x$ changes. In using ode45 you cannot change the xspan and $\mathrm{y}_{0}$ to get lines that vary as y changes. In order to solve this problem it was determined that the secondary stress trajectories in Figure 2.3 are the same as the primary stress trajectories if the problem is rotated by 90 degrees. The embedded ODE functions were thus edited by switching the loads in the plate examples and switching $\mathrm{x}$ and $\mathrm{y}$ in the disk problem. These changes produced the desired trajectory after properly swapping the output data. 


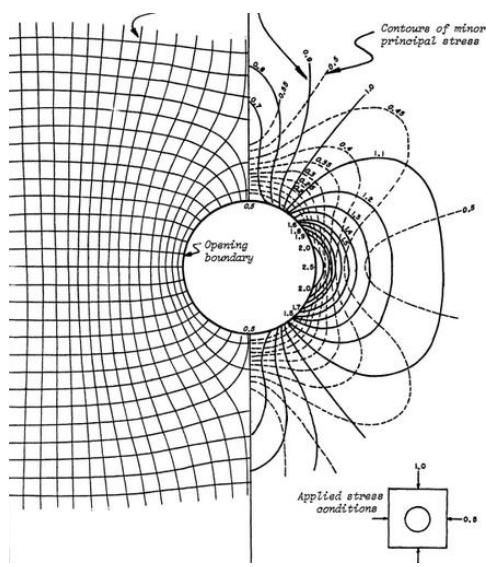

Figure 2.3: Stress Trajectories and Stress Contours in Plate with Unequal Loading

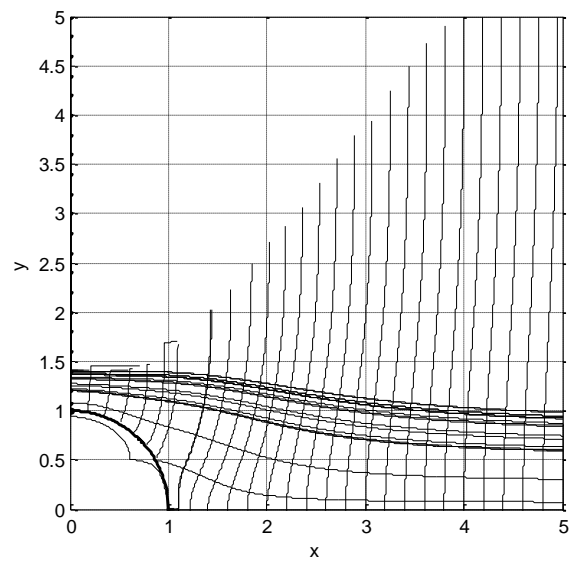

Figure 2.4: Original Stress Trajectories of Uniaxial Loaded Case

The final problem that occurred when trying to generate stress trajectories at points in the generation where the $\tau_{\mathrm{xy}}$ term would go to zero. From equation (1.10), the trajectory slope becomes unbounded and will cause the line to either drop to a much lower value or stop all together. This occurred in the uniaxial and unequal loaded plate problems. As seen in Figure 2.4 the primary stress trajectories, which start every 0.2 along the y axis, have all dropped to below 1.5 and then were generated. The secondary stress trajectories are generated until they reach a point where they stop and become undefined. To solve this problem a MATLAB if conditional statement was placed in the imbedded ODE function that checked the slope and modified it if 
needed. To solve the primary stress trajectories from initially dropping the if condition looked at the dydx value and if the absolute value was greater than 10 then the dydx term was then set to zero. For the case of the secondary trajectories it was determined that the $\tau_{\mathrm{xy}}$ term went to zero as the stress trajectory stopped and the dydx went to infinity. Similar to the primary trajectories an if condition limited the slope and set it to zero if the slope was larger than a set value. With this included in the imbedded ODE function the stress trajectories for the uniaxial loaded case can be seen in Figure 2.5.

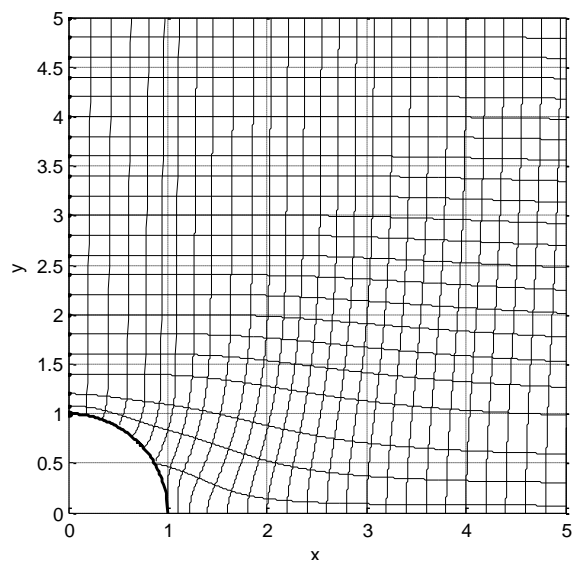

Figure 2.5: Stress Trajectories in Uniaxial Loaded Case

\subsection{Establishing Finite Element Topologies Based on Stress Trajectories}

Once a sufficiently dense collection of stress trajectories has been generated, finite element wire-frame mesh is to be created as an overlay. This requires determination of all intersection points of the stress trajectories. Once these points have been found, the finite element mesh can be easily generated for Abaqus analysis. 


\subsubsection{Curve Intersect}

Finding the intersecting points of each line was done using a shared file from MATLAB Central called Curve Intersect. Curve Intersect finds the intersection points of the two curves described by the vector data pairs x1,y1 and x2,y2 (Holz, 2005). The syntax in MATLAB is $[\mathrm{x}, \mathrm{y}]=\operatorname{curveintersect}(\mathrm{x} 1, \mathrm{y} 1, \mathrm{x} 2, \mathrm{y} 2)$ where $[\mathrm{x}, \mathrm{y}]$ are the coordinates of the intersecting points and $\mathrm{x} 1, \mathrm{y} 1$ and $\mathrm{x} 2, \mathrm{y} 2$ are the two sets of curves. To verify that the function works $\mathrm{x} 1, \mathrm{y} 1, \mathrm{x} 2$, and $\mathrm{y} 2$ were set to $\operatorname{rand}(10,1)$ which generates an array of 10 random points between 0 and 1. Using Curve Intersect and plotting $(\mathrm{x} 1, \mathrm{y} 1)$ as a black line, $(\mathrm{x} 2, \mathrm{y} 2)$ as a blue line and the intersecting points as red stars, it can be seen in Figure 2.6 that the function works. It was able to identify all the locations that the two lines intersected. To verify that the function is accurate, a simple example using two linear lines that intersect at a known point was completed.

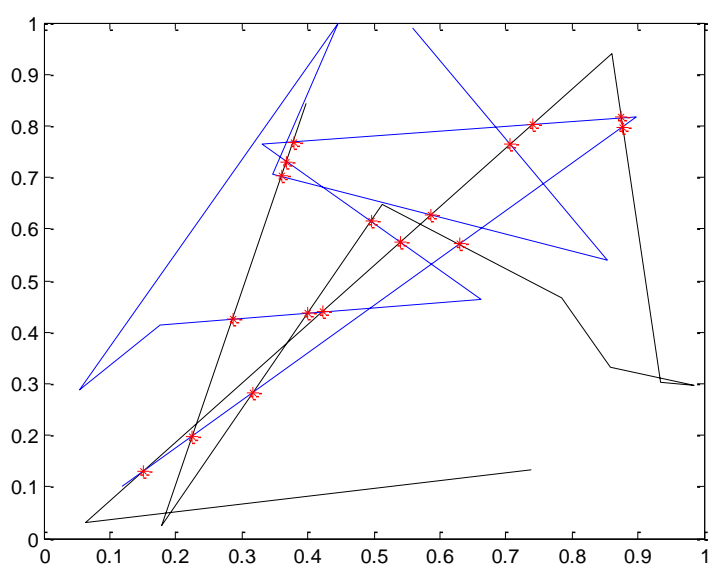

Figure 2.6: Curve Intersect Verification

This was done by setting $\mathrm{y} 1=\mathrm{x} 1$ and $\mathrm{y} 2=-\mathrm{x} 2+0.85$, where the known solution is $\mathrm{x}=$ 0.425 and $y=0.425$ and when ran in MATLAB, the following is returned:

$$
\begin{aligned}
& >>[x, y]=\text { curveintersect }(x 1, y 1, x 2, y 2) \\
& x=0.4250 \\
& y=0.4250
\end{aligned}
$$


This verifies that curve intersect can be used to find the intersecting points of the stress trajectories with confidence.

\subsubsection{Finding the Intersecting points}

Curve Intersect was used find the intersecting points of the stress trajectories. Saving the output matrix of each generated stress trajectory helps to simplify the process. Each primary stress trajectory intersects each secondary stress trajectory. Instead of finding each point individually, all the intersecting points along one trajectory were found using a MATLAB for loop. The for loop used a different secondary trajectory each time through the loop and found the intersecting points with the primary trajectories. The for loop to find the intersecting point between the bottom line in Figure 2.5 and the secondary stress trajectories is given by:

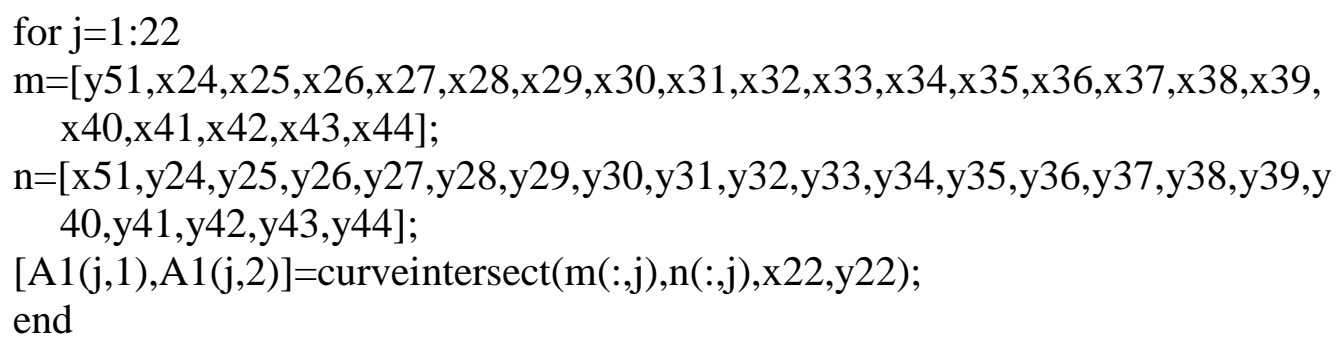

This code found and saved the 22 intersecting points in a 22 by 2 matrix called A1. The other intersecting points were generated and saved in the same manner. The intersecting points of Figure 2.5 can be seen in Figure 2.7 where the intersecting points are represented by magenta asterisks. Once all the points had been found, results were placed into a single matrix. Since the plates with holes are symmetric about the $\mathrm{x}$ and $\mathrm{y}$ axis the results matrix for the quarter plate can be easily used to determine intersection points for the entire plate 


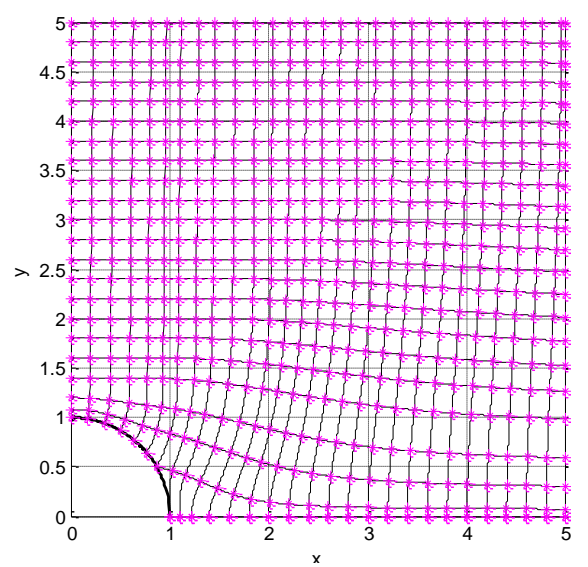

Figure 2.7: Intersecting Points of Uniaxially Loaded Plate

\subsection{Finite Element Analysis}

After all the stress trajectories were generated and the intersecting points were found, the next step was to create a corresponding wire-frame mesh in Abaqus. Abaqus is a Finite Element Analyzer for computer aided engineering. It will allow the cellular mechanics problem to be simulated and report the stresses in each cellular side-wall member.

\subsubsection{Setting up the Problems}

Once the matrices that included all the intersecting point data were generated the next step was to import them into Abaqus. Each $\mathrm{x}$ and $\mathrm{y}$ coordinate was placed into Abaqus and connected with a straight line to form the wire-frame mesh of the 


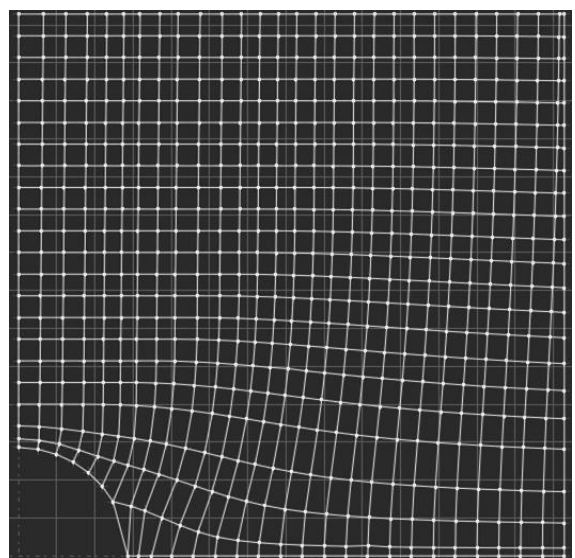

Figure 2.8: Abaqus Graphic of Uniaxially Loaded Cellular Plate

cellular material model. This lead to some errors in the element orientation, thus losing the orthogonality at the intersecting points. The uniaxially loaded plate is shown in Figure 2.8 and is a close match to the stress trajectories in Figure 2.5. Once the mesh generation was completed the entire problem could be created using the mirror feature as shown in Figure 2.9. This established a two-dimensional model, made up of frame elements.

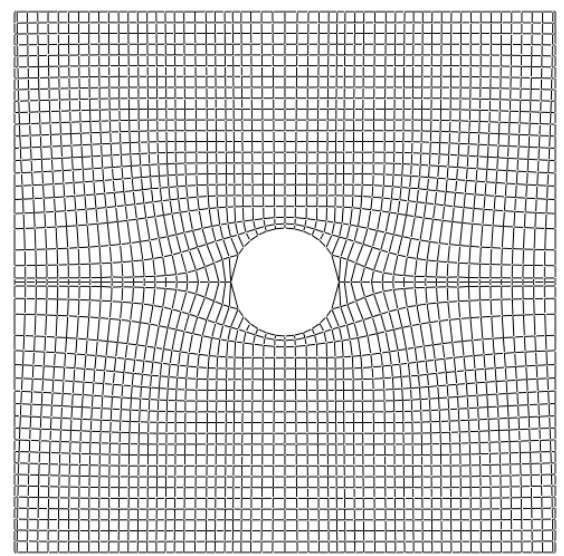

Figure 2.9: Full Uniaxially Loaded Plate

Each problem is made of Aluminum (modulus of Elasticity $=69 \mathrm{GPa}$, Shear Strength $=207 \mathrm{MPa}$ and Poisson's ratio $=0.33$ ) and each element has a square cross section. The section size depends on the problem being run. In order to do proper comparisons 
each topology in a given problem will have the same volume of material. The list of all problems and the topologies is given in Table 2.1 .

Table 2.1: List of Problems and Topologies

\begin{tabular}{|c|c|}
\hline Problem & Topology \\
\hline \multirow{2}{*}{ Cantilever Beam with Shear End Loading } & Along Stress Trajectories \\
\hline & Control \\
\hline \multirow{2}{*}{$\begin{array}{c}\text { Simply Supported Beam with Uniform } \\
\text { Loading }\end{array}$} & Along Stress Trajectories using Elasticity Sol. \\
\hline & Control \\
\hline \multirow{2}{*}{$\begin{array}{c}\text { Simply Supported Beam with Uniform } \\
\text { Loading }\end{array}$} & Along Stress Trajectories using Strength of Mat'ls Sol. \\
\hline & Control \\
\hline \multirow{2}{*}{ Disk with Diametric Compression Loading } & Along Stress Trajectories \\
\hline & Control \\
\hline \multirow{7}{*}{$\begin{array}{l}\text { Plate with Hole under Equal Biaxial } \\
\text { Compression }\end{array}$} & Along Stress Trajectories (coarse) \\
\hline & Along Stress Trajectories (medium) \\
\hline & Along Stress Trajectories (fine) \\
\hline & Random 1 (medium) \\
\hline & Random 2 (medium) \\
\hline & Uniaxial Medium \\
\hline & Unequal Medium \\
\hline \multirow{7}{*}{$\begin{array}{l}\text { Plate with Hole under Uniaxial } \\
\text { Compression }\end{array}$} & Along Stress Trajectories (coarse) \\
\hline & Along Stress Trajectories (medium) \\
\hline & Along Stress Trajectories (fine) \\
\hline & Random 1 (medium) \\
\hline & Random 2 (medium) \\
\hline & Equal Medium \\
\hline & Unequal Medium \\
\hline \multirow{7}{*}{$\begin{array}{l}\text { Plate with Hole under Unequal Biaxial } \\
\text { Compression }\end{array}$} & Along Stress Trajectories (coarse) \\
\hline & Along Stress Trajectories (medium) \\
\hline & Along Stress Trajectories (fine) \\
\hline & Random 1 (medium) \\
\hline & Random 2 (medium) \\
\hline & Equal Medium \\
\hline & Uniaxial Medium \\
\hline
\end{tabular}

The average length of a given element in each problem was calculated and the cross section was determined such that the ratio of the length of the element $(l)$ to section width of the element ( $a$ ) was between five and ten. The cross sectional widths and the average lengths of the elements can be seen in Table 2.2. Once the rectangular profiles had been added, it was made sure that the beams were orientated in the correct manner. This properly establishes section properties like moment of inertia. 
Table 2.2: Properties of Each Problem

\begin{tabular}{|c|c|c|c|c|c|}
\hline Problem & Topology & $\begin{array}{l}\text { Number of } \\
\text { Elements }\end{array}$ & $\begin{array}{c}\text { Avg. } \\
\text { Length }(l)\end{array}$ & $\begin{array}{c}\text { Cross } \\
\text { Section }(a)\end{array}$ & $l / a$ \\
\hline \multirow{2}{*}{ Cantilever } & Along S.T. & 1576 & $0.0944 \mathrm{~cm}$ & $0.0189 \mathrm{~cm}$ & 5.00 \\
\hline & Control & 2070 & $0.1000 \mathrm{~cm}$ & $0.0160 \mathrm{~cm}$ & 6.25 \\
\hline \multirow{2}{*}{$\begin{array}{c}\text { Simply Supported } \\
\text { Elasticity }\end{array}$} & Along S.T. & 1764 & $0.1780 \mathrm{~cm}$ & $0.0237 \mathrm{~cm}$ & 7.50 \\
\hline & Control & 1070 & $0.2000 \mathrm{~cm}$ & $0.0287 \mathrm{~cm}$ & 6.96 \\
\hline \multirow{2}{*}{$\begin{array}{c}\text { Simply Supported } \\
\text { SM }\end{array}$} & Along S.T. & 1596 & $0.1961 \mathrm{~cm}$ & $0.0261 \mathrm{~cm}$ & 7.50 \\
\hline & Control & 1070 & $0.2000 \mathrm{~cm}$ & $0.0316 \mathrm{~cm}$ & 6.33 \\
\hline \multirow{2}{*}{ Disk } & Along S.T. & 544 & $0.2415 \mathrm{~cm}$ & $0.0322 \mathrm{~cm}$ & 7.50 \\
\hline & Control & 416 & $0.2702 \mathrm{~cm}$ & $0.0348 \mathrm{~cm}$ & 7.76 \\
\hline \multirow{7}{*}{$\begin{array}{l}\text { Equal Biaxial } \\
\text { Compression }\end{array}$} & Along S.T. (coarse) & 880 & $0.5623 \mathrm{~cm}$ & $0.0625 \mathrm{~cm}$ & 9.00 \\
\hline & Along S.T. (medium) & 1892 & $0.3723 \mathrm{~cm}$ & $0.0524 \mathrm{~cm}$ & 7.11 \\
\hline & Along S.T. (fine) & 7028 & $0.1908 \mathrm{~cm}$ & $0.0379 \mathrm{~cm}$ & 5.03 \\
\hline & Random 1 & 1892 & $0.3775 \mathrm{~cm}$ & $0.0520 \mathrm{~cm}$ & 7.26 \\
\hline & Random 2 & 1892 & $0.3784 \mathrm{~cm}$ & $0.0519 \mathrm{~cm}$ & 7.28 \\
\hline & Uniaxial Medium & 5072 & $0.2006 \mathrm{~cm}$ & $0.0436 \mathrm{~cm}$ & 4.60 \\
\hline & Unequal Medium & 4076 & $0.2247 \mathrm{~cm}$ & $0.0459 \mathrm{~cm}$ & 4.90 \\
\hline \multirow{7}{*}{$\begin{array}{c}\text { Uniaxial } \\
\text { Compression }\end{array}$} & Along S.T. (coarse) & 2260 & $0.3030 \mathrm{~cm}$ & $0.0337 \mathrm{~cm}$ & 9.00 \\
\hline & Along S.T. (medium) & 5072 & $0.2006 \mathrm{~cm}$ & $0.0276 \mathrm{~cm}$ & 7.26 \\
\hline & Along S.T. (fine) & 18888 & $0.1032 \mathrm{~cm}$ & $0.0200 \mathrm{~cm}$ & 5.17 \\
\hline & Random 1 & 5072 & $0.2050 \mathrm{~cm}$ & $0.0273 \mathrm{~cm}$ & 7.50 \\
\hline & Random 2 & 5072 & $0.2044 \mathrm{~cm}$ & $0.0274 \mathrm{~cm}$ & 7.47 \\
\hline & Equal Medium & 1892 & $0.3723 \mathrm{~cm}$ & $0.0332 \mathrm{~cm}$ & 11.20 \\
\hline & Unequal Medium & 4076 & $0.2247 \mathrm{~cm}$ & $0.0291 \mathrm{~cm}$ & 7.72 \\
\hline \multirow{7}{*}{$\begin{array}{l}\text { Unequal Biaxial } \\
\text { Compression }\end{array}$} & Along S.T. (coarse) & 1800 & $0.3422 \mathrm{~cm}$ & $0.0380 \mathrm{~cm}$ & 9.00 \\
\hline & Along S.T. (medium) & 4076 & $0.2247 \mathrm{~cm}$ & $0.0312 \mathrm{~cm}$ & 7.20 \\
\hline & Along S.T. (fine) & 14736 & $0.1169 \mathrm{~cm}$ & $0.0227 \mathrm{~cm}$ & 5.14 \\
\hline & Random 1 & 4076 & $0.2310 \mathrm{~cm}$ & $0.0308 \mathrm{~cm}$ & 7.51 \\
\hline & Random 2 & 4076 & $0.2285 \mathrm{~cm}$ & $0.0309 \mathrm{~cm}$ & 7.39 \\
\hline & Equal Medium & 1892 & $0.3723 \mathrm{~cm}$ & $0.0356 \mathrm{~cm}$ & 10.47 \\
\hline & Uniaxial Medium & 5072 & $0.2006 \mathrm{~cm}$ & $0.0296 \mathrm{~cm}$ & 6.78 \\
\hline
\end{tabular}

\subsubsection{Element Type}

Each problem was simulated using frame elements to approximate the solution.

Frame elements are straight beams with any cross-section and have three degrees of

freedom per node. They can deform in both the axial and transverse direction.

Moment, axial and transverse loads are incorporated, which make them ideal for

modeling many structural problems (Reddy, 2006). Frame elements differ from

simpler truss elements by incorporating all three loads instead of just axial loads.

They are capable of being used in 2 and 3 dimensional skeletal structures, which is an 
appropriate model of a cellular material and has been widely used in such modeling. Figure 2.10 shows how each cell side wall or strut is modeled using a frame element with combined axial and bending stress.

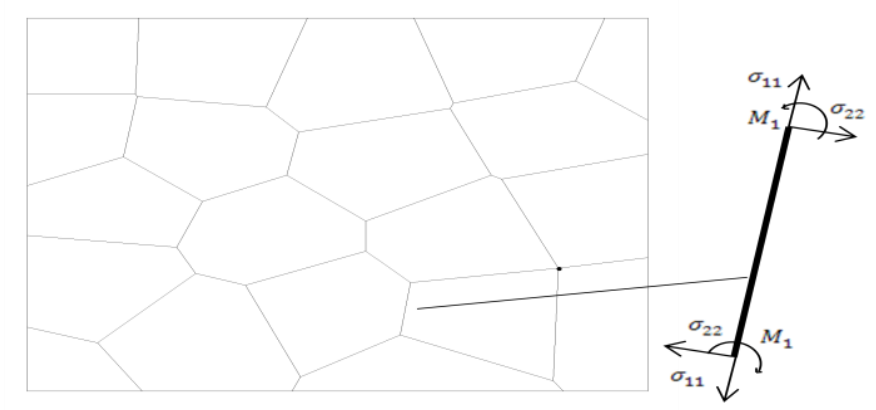

Figure 2.10: Diagram Demonstrating Frame Elements

The most commonly used beam element is the Euler-Bernoulli beam. This is a simplified class of beams that incorporate small deflections of beams in the direction perpendicular to the beam's axis only. Euler-Bernoulli beam theory makes the assumption that plane cross-sections remain plane and normal to the longitudinal axis after bending. This simplification excludes the deformation caused by transverse shear (Reddy, 2006). Deflection of an Euler Bernoulli beam is given by the fourth order equation

$$
\frac{d^{2}}{d x^{2}}\left(E I \frac{d^{2} w}{d x^{2}}\right)=q(x)
$$

where $\mathrm{E}$ is the modulus of elasticity, $\mathrm{I}$ is the second moment of area about the $\mathrm{y}$ axis, $\mathrm{q}$ is the distributed transverse load and $\mathrm{w}$ is the transverse deflection of the beam. Euler Bernoulli beams are acceptable for beams with lengths that are much greater than their cross-section dimension. When this is not the case a more generalized beam theory must be used. 
Timoshenko beam theory models the effect of transverse shear and includes an additional angle of rotation due to shear stress. Plane cross-sections remain plane but not normal to the longitudinal axis. An additional angle of rotation is included and the total angle is given by $\Psi=\gamma_{\mathrm{xy}}-\frac{d w}{d x}$, where $\gamma_{\mathrm{xy}}$ is shear strain and $\frac{d w}{d x}$ is rotation due to bending stress. Figures 2.11 and 2.12 show these differences in the two beam theories.

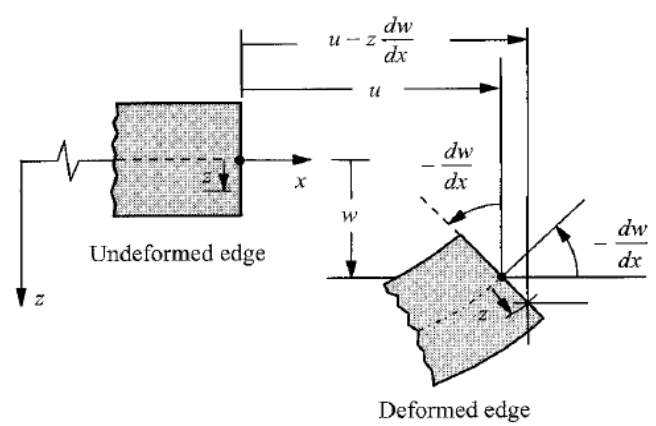

Figure 2.11: Cross-section after deformation Euler Bernoulli Beam Theory (Reddy 2006)

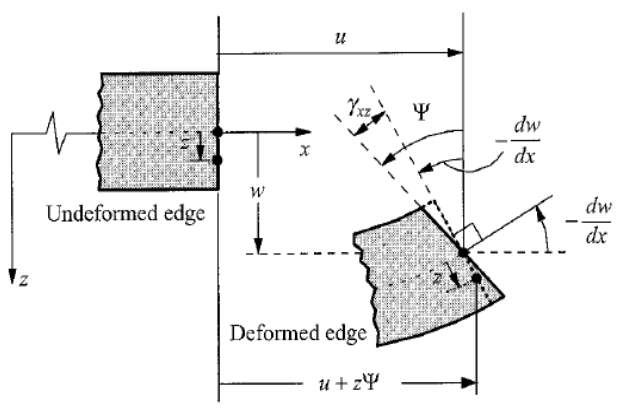

Figure 2.12:Cross-section after deformation Timoshenko Beam Theory (Reddy 2006).

Timoshenko beam theory is governed by equations (2.5) and (2.6). If there is no shear displacement and the angle $\Psi$ is set to $-\frac{d w}{d x}$, equation (2.5) and (2.6) collapse to equation (2.4) Using Timoshenko beams the aspect ratio $l / a$ can be reduced to 1.5 (Reddy, 2006) while still obtaining accurate results.

$$
\begin{gathered}
-\frac{d}{d x}\left[G A K_{S}\left(\Psi+\frac{d w}{d x}\right)\right]=q \\
-\frac{d}{d x}\left(E I \frac{d \Psi}{d x}\right)+G A K_{S}\left(\Psi+\frac{d w}{d x}\right)=0
\end{gathered}
$$

Although, the present study kept $5</ / a<10$, Timoshenko beam elements were used since previous studies have used them. 


\subsubsection{Stress Reporting}

All stresses reported from Abaqus are axial stress, labeled S11 in Abaqus. This component of stress will include bending and axial stress but will not include shear stress. This is acceptable because cellular topologies commonly have large $l / a$ ratios and thus are dominated by bending stress (Ashby \& Gibson, 1997). To verify that axial stress is sufficient to capture the stresses in cellular topologies, a comparison of the reported stress is made between axial stress and a more inclusive von Mises stress. Von Mises stress $\left(\sigma_{v}\right)$ is a combination of axial and shear stress and is given for two dimensions by

$$
\sigma_{v}=\sqrt{\frac{\left(\sigma_{11}-\sigma_{22}\right)^{2}}{2}+\frac{\sigma_{11^{2}}}{2}+\frac{\sigma_{22}^{2}}{2}+6 \frac{\sigma_{12}^{2}}{2}}
$$

where $\sigma_{11}$ and $\sigma_{22}$ is stress in the 11 and 22 direction respectively and $\sigma_{12}$ is shear stress in the 12 direction. If shear stress is negligible in comparison to axial and bending stress, then $\sigma_{11}$ and $\sigma_{\mathrm{v}}$ stress contours should be very similar or identical. To show this the disk problem was used as a comparison. The stress contours are shown below for both axial stress and von Mises stress in Figure 2.13 and 2.14. The stress contours are identical and thus shear stress appears to be negligible.
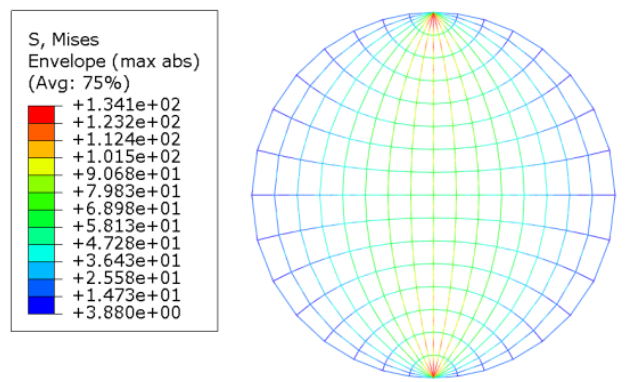

Figure 2.13: Von Mises Stress Output
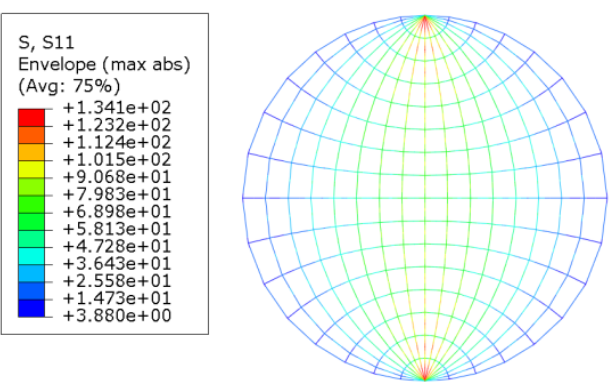

Figure 2.14: S11 Stress Output 
Each edge of a cell will be modeled as an element with 2 nodes, one on each end and also a central read out. The element will not be able to pivot at the central node and will remain continuous. The nodes at the ends of the elements will be shared between elements while the central node will be unique to the element. The stresses reported from Abaqus will be given at each node. The number of nodes is related to the number elements by two-thirds. That is if you take the number of nodes and multiply it by two-thirds you get the number of elements.

\subsection{Verification Examples}

It is important to analyze and compare the stress reported from simpler frame problems to gain confidence in modeling cellular structures. The first verification example is a simple frame structure, and the second example is a more complicated portal frame. A third example used many more elements in a square mesh wire frame.

\subsubsection{Frame Example}

The frame shown in Figure 2.15 has been simulated in Abaqus and compared with the analytical solution. This example was chosen for its simplicity. 


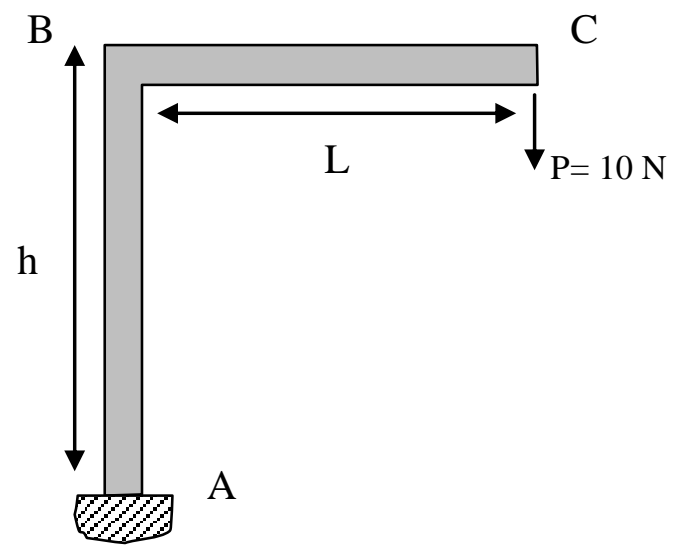

Figure 2.15: Simple Frame Example

The frame has a unit square cross-section and $\mathrm{L}$ and $\mathrm{h}$ both equal $10 \mathrm{~m}$. The axial stress in each member can easily be determined from statics and strength of materials. The load placed on the end of the frame creates a moment equal to PL. The maximum stress of member $\mathrm{BC}$ is calculated from equation (2.8) and member $\mathrm{AB}$ is calculated using equation (2.9)

$$
\sigma_{m}=\frac{M c}{I}
$$

where $\sigma_{m}$ is stress due to moment loading, c half the section thickness, (for maximum stress) and $\mathrm{I}$ is the area moment of inertia.

$$
\sigma_{c}=\left|\frac{P}{A}\right|+\left|\frac{M c}{I}\right|
$$

equation two is the stress due to both axial loads and moments. Table 2.3 compares the exact solution for stress with the results obtained from Abaqus for both members.

Table 2.3: Stresses in Each Member

\begin{tabular}{|c|c|c|}
\hline Member & Calculated Stress $(\mathrm{Pa})$ & Abaqus Stress (Pa) \\
\hline $\mathrm{AB}$ & 610 & 610 \\
\hline $\mathrm{BC}$ & 600 & 600 \\
\hline
\end{tabular}


The stresses listed in Table 2.3 are identical and allow for the continuation to more complex frame examples with confidence.

\subsubsection{Portal Example}

A portal frame has been used to further validate the analysis of framed structures. The solution of this problem is taken from (Hibbeler, 2009). The frame geometry and boundary conditions are shown in Figure 2.24.

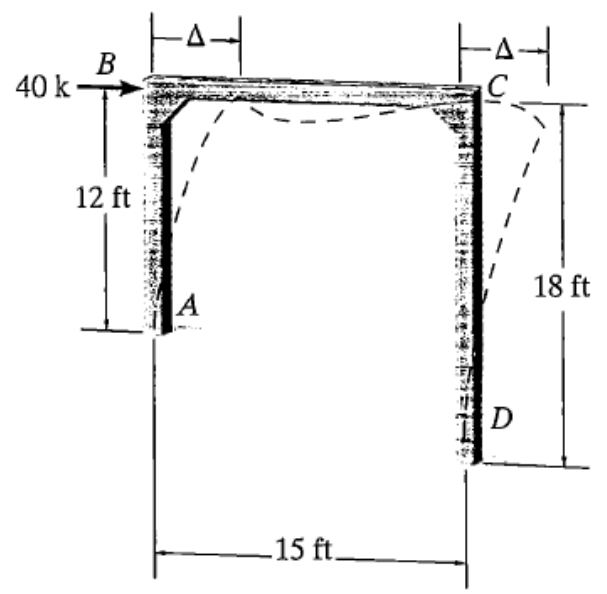

Figure 2.16: Portal Frame Geometry and Boundary Conditions

The problem has been solved analytically using slope-deflection equations.

The solution is given in the form of moments at each joint. The transverse load is also given as a function of these moments. These given values are shown in Table 2.4.

The notation used for the moments corresponds with Figure 2.17.

Table 2.4: Given Moments and Transverse Load

\begin{tabular}{|c|c|c|c|c|c|c|c|}
\hline $\begin{array}{c}\mathrm{M}_{\mathrm{AB}} \\
(\mathrm{k} \mathrm{ft})\end{array}$ & $\begin{array}{c}\mathrm{M}_{\mathrm{BA}} \\
(\mathrm{k} \mathrm{ft})\end{array}$ & $\begin{array}{c}\mathrm{M}_{\mathrm{BC}} \\
(\mathrm{kft})\end{array}$ & $\begin{array}{c}\mathrm{M}_{\mathrm{CB}} \\
(\mathrm{k} \mathrm{ft})\end{array}$ & $\begin{array}{c}\mathrm{M}_{\mathrm{CD}} \\
(\mathrm{k} \mathrm{ft})\end{array}$ & $\begin{array}{c}\mathrm{M}_{\mathrm{DC}} \\
(\mathrm{kft})\end{array}$ & $\begin{array}{c}\mathrm{V}_{\mathrm{A}} \\
(\mathrm{K})\end{array}$ & $\begin{array}{c}\mathrm{V}_{\mathrm{D}} \\
(\mathrm{K})\end{array}$ \\
\hline-208 & -135 & 135 & 94.8 & -94.8 & -110 & 11.33 & 28.67 \\
\hline
\end{tabular}



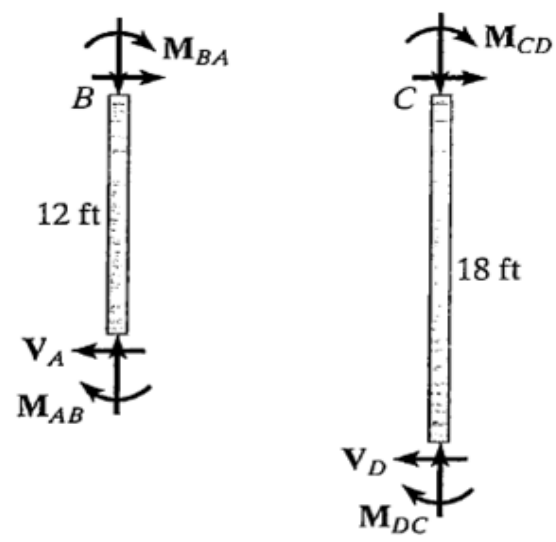

Figure 2.17: Free Body Diagrams of Side Members

Using the moments and forces from Table 2.4 the stress in the frame can be solved for using equation (2.9). The portal frame model simulated in Abaqus uses members with a unit square cross-section. This gives an area (A) of $1 \mathrm{ft}^{2}$, a half thickness (c) of $0.5 \mathrm{ft}$ and an area moment of inertia (I) of $0.0833 \mathrm{ft}^{4}$. Table 2.5 compares the calculated stresses and Abaqus outputted stresses. The locations for the stress comparison correspond with the locations of the moments from which they were calculated. Position AB is the bottom of left member, BA is the top of the left member, $\mathrm{BC}$ is the left of the top member, $\mathrm{CB}$ is the right of the top member, $\mathrm{CD}$ is the top of the right member and DC is the bottom of the right member.

Table 2.5: Analytical vs Abaqus Stresses

\begin{tabular}{|c|c|c|c|c|c|}
\hline $\begin{array}{c}\text { Stress } \\
\text { Location }\end{array}$ & $\begin{array}{c}\text { Axial Load } \\
(\mathrm{K})\end{array}$ & $\begin{array}{c}\text { Moment Load } \\
(\mathrm{K} \mathrm{ft})\end{array}$ & $\begin{array}{c}\text { Analytical Stress } \\
(\mathrm{Kpsi})\end{array}$ & $\begin{array}{c}\text { Abaqus Stress } \\
(\mathrm{Kpsi})\end{array}$ & \% Error \\
\hline $\mathrm{AB}$ & 57.33 & 208.00 & 1305.83 & 1267.60 & $2.9 \%$ \\
\hline $\mathrm{BA}$ & 57.33 & 135.00 & 867.65 & 823.70 & $5.1 \%$ \\
\hline $\mathrm{BC}$ & 11.33 & 135.00 & 821.66 & 819.87 & $0.2 \%$ \\
\hline $\mathrm{CB}$ & 11.33 & 94.80 & 580.36 & 578.32 & $0.4 \%$ \\
\hline $\mathrm{CD}$ & 57.33 & 94.80 & 626.36 & 582.20 & $7.0 \%$ \\
\hline $\mathrm{DC}$ & 57.73 & 110.00 & 717.99 & 676.60 & $5.8 \%$ \\
\hline
\end{tabular}


Also compared were the displacements of nodes B and C. Beam theory solution gives a lateral displacement at point $\mathrm{B}$ and $\mathrm{C}$ of $.002703 \mathrm{ft}$. These points were queried in Abaqus for their $\mathrm{x}$ displacement. Table 2.6 shows a comparison between the two.

Table 2.6: Analytical vs Abaqus Displacement

\begin{tabular}{|c|c|c|c|}
\hline Point & Calculated $\Delta$ & Abaqus $\Delta$ & $\%$ Error \\
\hline B & 0.002703 & 0.002749 & $1.7 \%$ \\
\hline C & 0.002703 & 0.002744 & $1.5 \%$ \\
\hline
\end{tabular}

The difference between the calculated and Abaqus reported displacements are within $2 \%$, and thus Abaqus has been shown to competently analyze frame problems.

\subsubsection{Square Topology Simulation}

The previous frame examples only contained two and three elements, and so as a final verification example we choose a simple square mesh with many elements. A square mesh was used because it can be solved analytically and is easy to model. Since there are no inclined struts in the square topologies the stresses will be almost entirely due to tension or compression (Ashby \& Gibson, 1997). Three different topologies were chosen, a coarse, medium and fine topology. All three topologies are $10 \mathrm{~cm}$ by $10 \mathrm{~cm}$ and will have identical loads, boundary conditions and volumes. The topologies were fixed vertically along the bottom edge and were loaded with a uniform load of $1 \mathrm{~N} / \mathrm{m}$ along the top edge. The coarse topology consisted of 10 cells across, the medium topology had 20 cells across and the fine topology had 40 cells across. The three topologies can be seen in Figures 2.18-20. All topologies were simulated using one Timoshenko beam element per strut as done by (Andrews \& 
Gibson, 2001). All three topologies had uniform stress across the elements with the highest concentration of stress being in the top corners.

The stresses in the vertical elements are equal to the net load divided by the total cross-sectional area of the elements. This is shown in equation (2.10) where the total cross-sectional area is equal to the number of elements $(\mathrm{N})$ multiplied by the cross-sectional area (A) and the stresses for all three topologies can be seen in Table 2.7 .

$$
\sigma=\frac{P_{t}}{N A}
$$

Table 2.7: Calculated Stresses in Vertical Elements

\begin{tabular}{|c|c|c|c|c|c|}
\hline Topology Type & $\mathbf{P t}(\mathbf{N})$ & $\mathbf{N}$ & $\mathbf{A}\left(\mathbf{m}^{\mathbf{2}}\right)$ & Volume $\left.\mathbf{( m}^{\mathbf{3}}\right)$ & Stresses $(\mathbf{P a})$ \\
\hline Coarse & 0.1 & 11 & 1 E -06 & 2.2 E -06 & 9090.91 \\
\hline Medium & 0.1 & 21 & 5.24 E -07 & 2.2 E -06 & 9084.55 \\
\hline Fine & 0.1 & 41 & 2.68 E -08 & 2.2 E -06 & 9089.85 \\
\hline
\end{tabular}

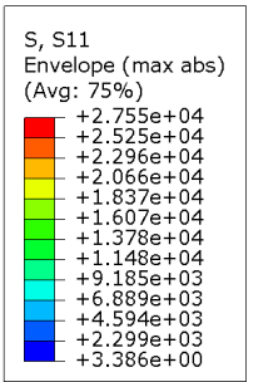

Figure 2.18: S11 Stress in Coarse Topology

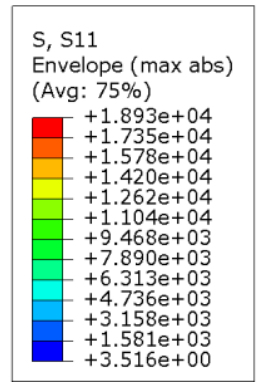

Figure 2.19: S11 Stress in Medium Topology
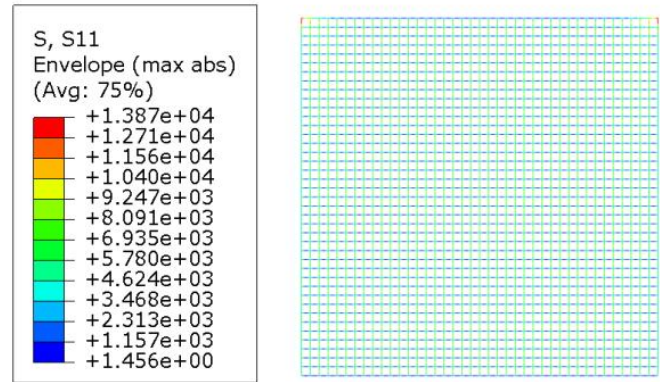

Figure 2.20: S11 Stress in Fine Topology 
The stresses were taken along a path through the middle of the topology similar to that shown in Figure 2.21. The reported stresses from Abaqus were plotted with the values from Table 2.7 and can be seen in Figures 2.22-2.24. In all three figures it can be seen that the stresses reported reasonably match the analytical stresses. Keeping the masses of topologies similar allowed the expected stress to be about the same.

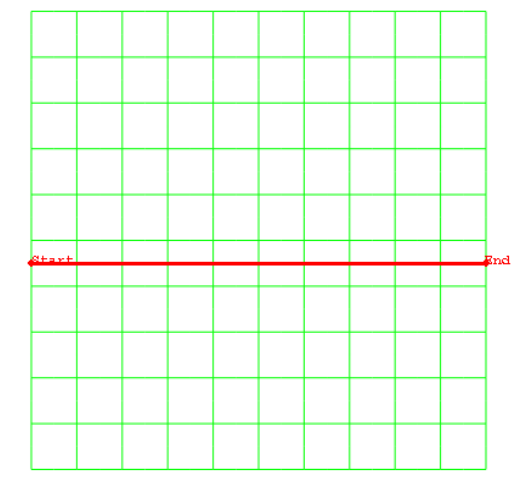

Figure 2.21: Path Where the Stresses Were Reported

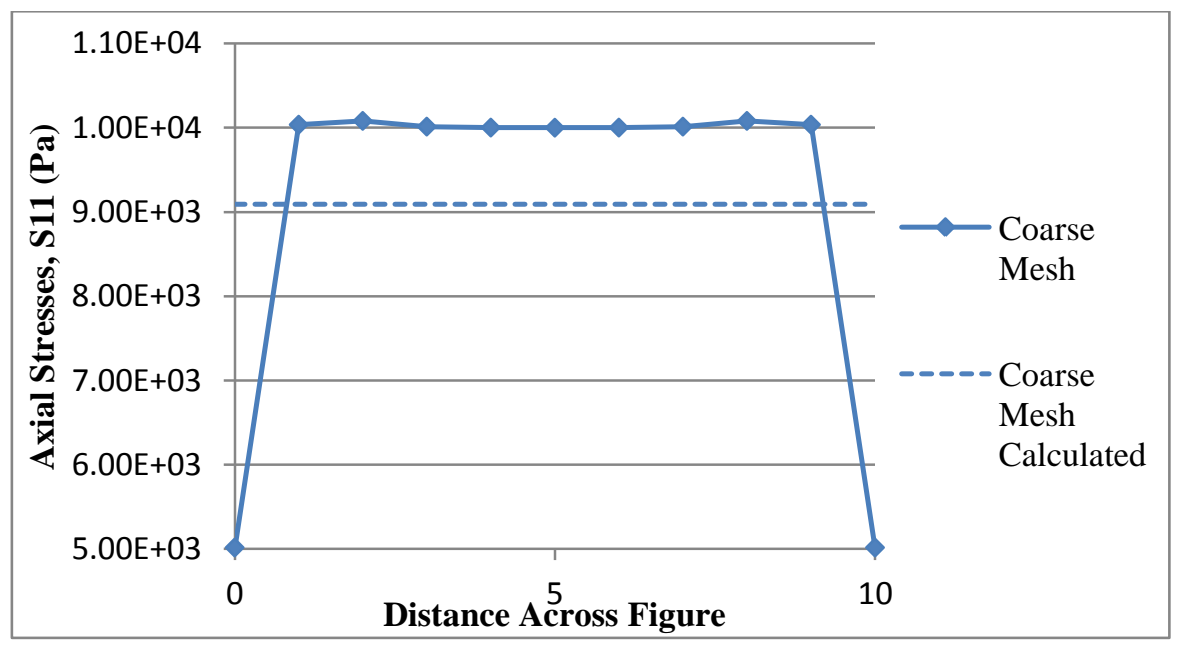

Figure 2.22: Numerical vs Abaqus Stresses in Coarse Topology 


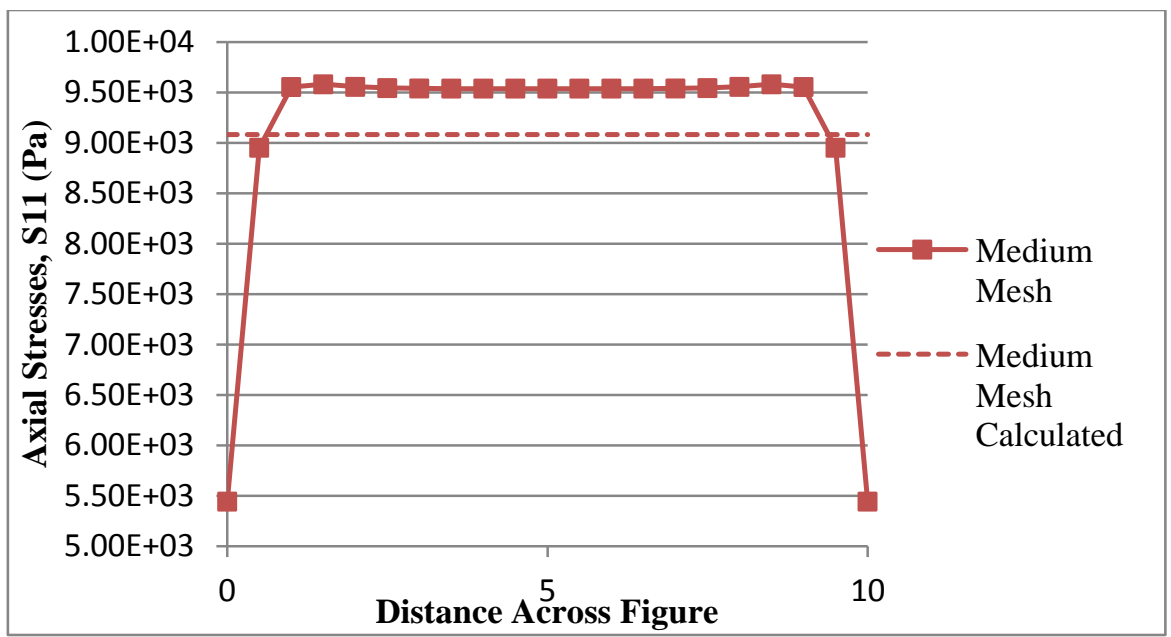

Figure 2.23: Numerical vs Abaqus Stresses in Medium Topology

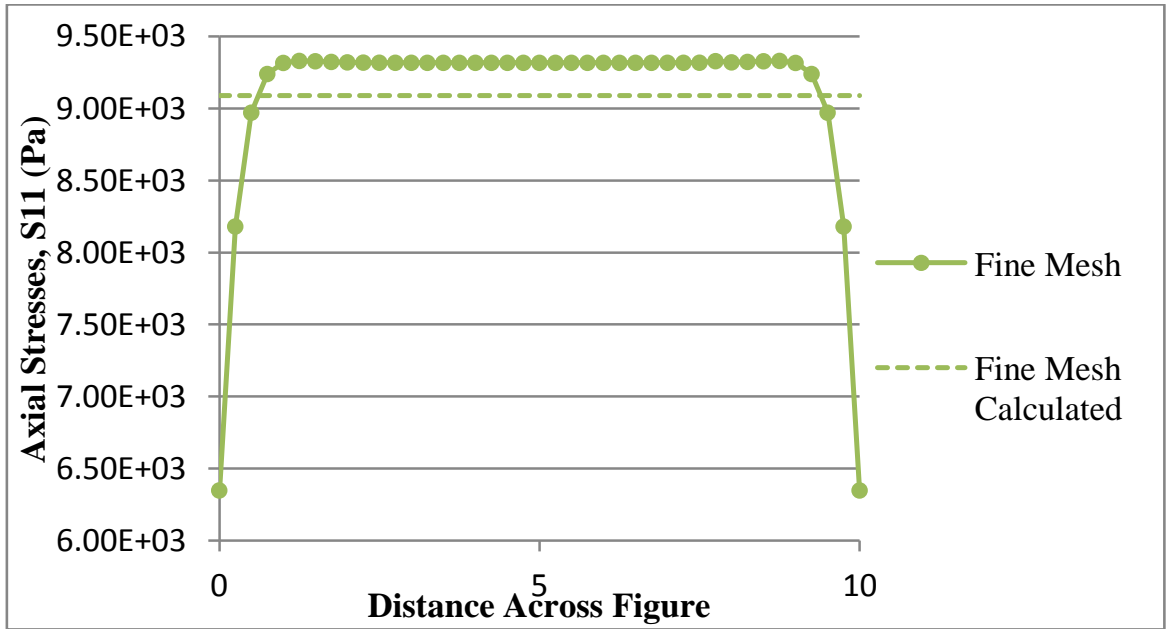

Figure 2.24: Numerical vs Abaqus Stresses in Fine Topology

These verification examples show that Abaqus is adequate in evaluating the stresses in our cellular mechanics problems. 


\section{Simulation Examples and Results}

\subsection{Examples Problems Studied}

As discussed in Section 1.4.1, there are six problems to be examined in this study. The first three problems, the cantilever beam, the simply supported beams, and the disk problem, are used for understanding the stress trajectories and seeing how the stresses are carried along them. The last three problems are plates with holes located in the center under equal biaxial, uniaxial and unequal biaxial loadings. The main focus of this study is to see if following stress trajectories is a better cellular topology for a plate with a hole than other alternative topology choices. For each problem, stresses from stress trajectory (ST) topologies will be compared with other cellular microstructures referred to as uniform, control or alternate topologies

\subsection{Cantilever Beam Problem}

\subsubsection{The Problem}

The cantilever beam is $5 \mathrm{~cm}$ long and $2 \mathrm{~cm}$ in height and will be loaded along its free end, as shown in Figure 1.16. The stress trajectories were generated from the fixed end at $\mathrm{x}=0$ to the free end and started every $0.05 \mathrm{~cm}$ along the $\mathrm{y}$ axis. The stress trajectories can be seen in Figure 3.1. The beam was loaded with a uniform line of 1 $\mathrm{N} / \mathrm{m}$. The stress trajectories for the cantilever beam can also be found in (Gere \& Goodno, 2009). 


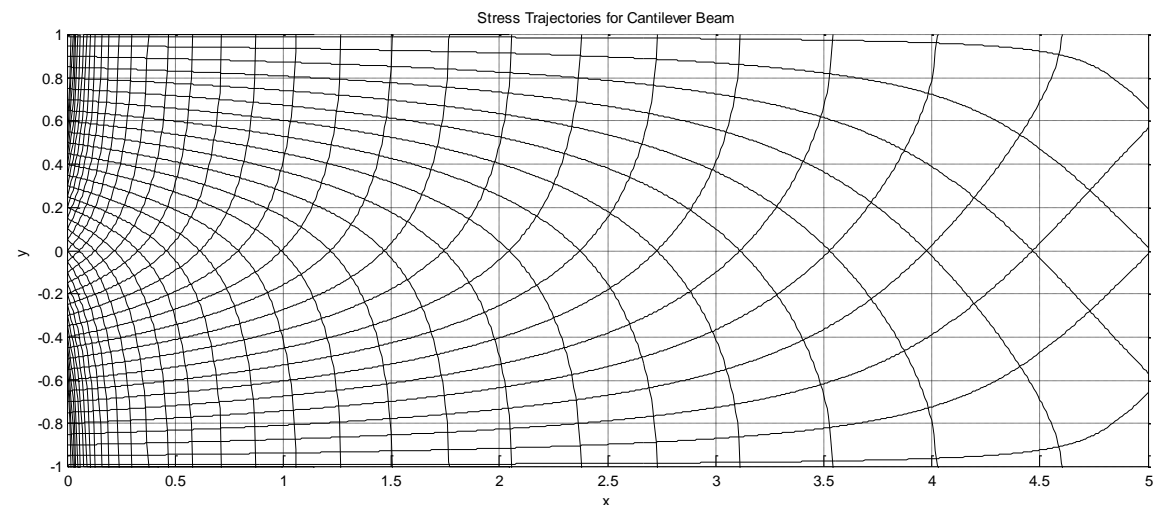

Figure 3.1: Stress Trajectories for Cantilever Beam

Some of the intersecting points along the top and bottom were shifted to the boundary when imported into Abaqus since they were close to the proximity of the boundary. The ST cantilever problem is shown in Figure 3.2. The comparison control problem for the cantilever beam incorporates a uniform rectangular mesh with vertical and horizontal elements spaced every $0.1 \mathrm{~cm}$, see Figure 3.3. The control problem has identical boundary loading as applied to Figure 3.2. The ST cantilever beam and the control problem have the same total material volume, $0.05302 \mathrm{~cm}^{3}$, and cross sectional area adjusted to keep constant volume. This assures that the same amount of material is present in both problems so that proper comparison can be made.

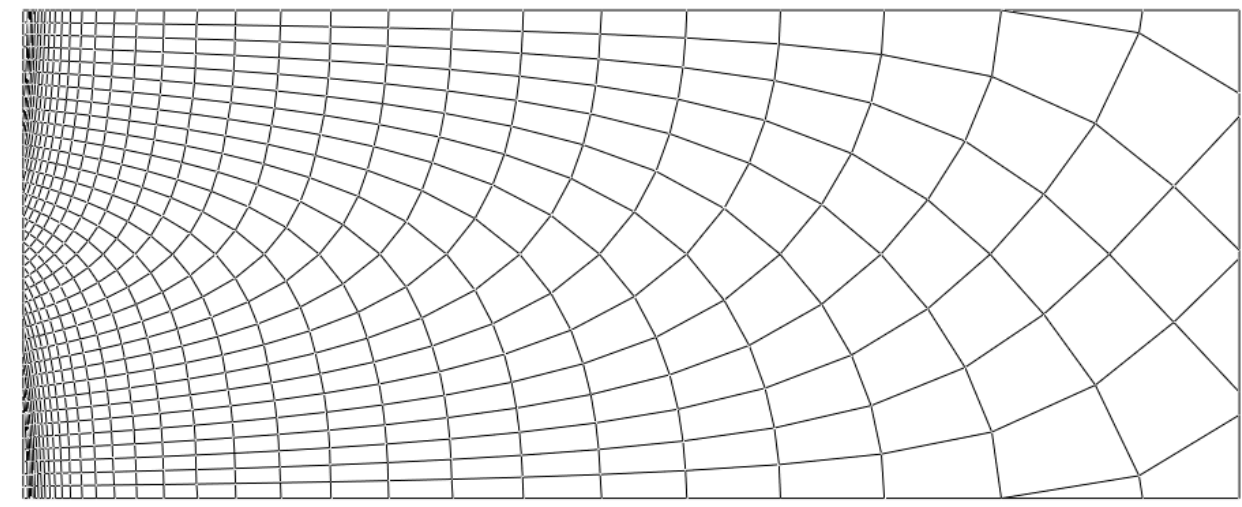

Figure 3.2: Abaqus ST Topology of Cantilever Beam 


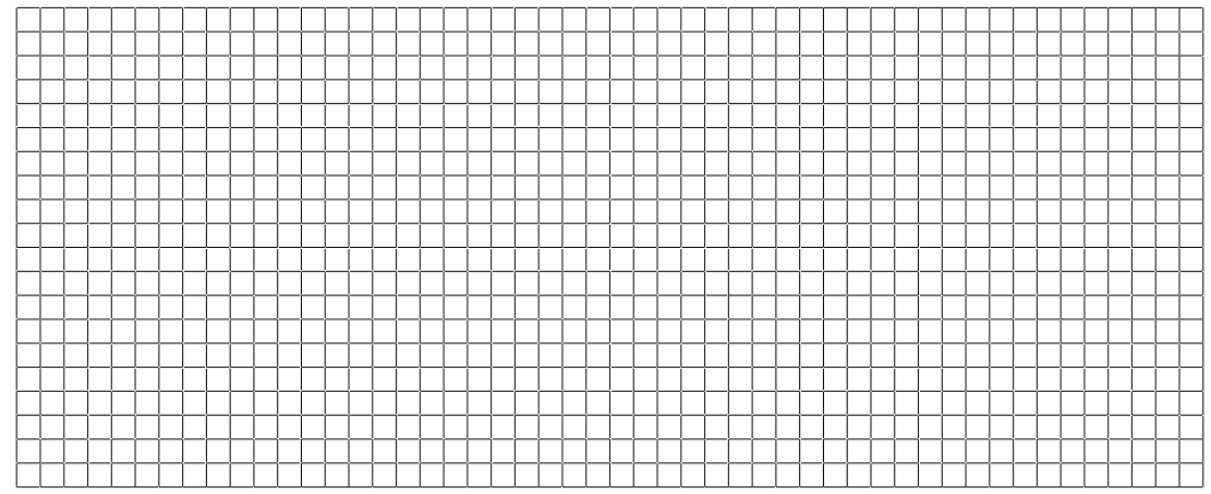

Figure 3.3: Abaqus Control Topology for the Cantilever

\subsubsection{Abaqus Simulations and Results}

The ST cantilever beam and the control beam will be compared using the stress in the topologies, frequency of the stress and the top ten percent of the stresses. Considering the location of the maximum stress in the cantilever problem, the topology using the stress trajectories have the maximum stresses in the center of the beam, as seen in Figure 3.4. The maximum stress in the control topology occurred

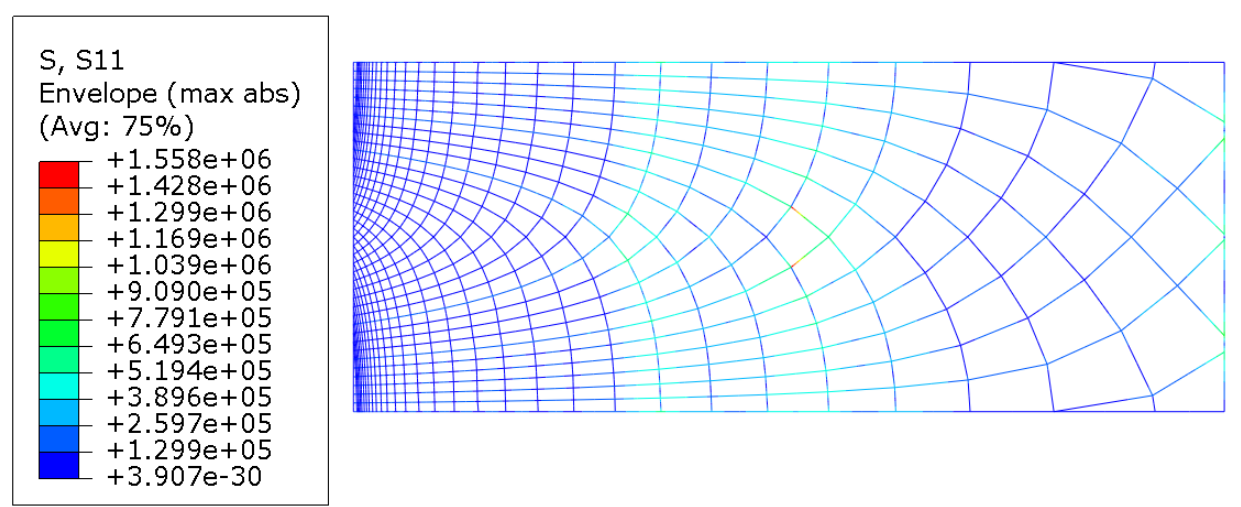

Figure 3.4: Stresses in Cantilever Beam with Stress Trajectory Topology

at the top and bottom corners along the left edge of the beam, similar to continuum theory. The maximum stress at the nodes in the ST topology was 772 kilopascals (kPa.) and 2,377 $\mathrm{kPa}$. in the control topology. It should be pointed out that the 
maximum stress at the nodes is different from Figure 3.4. Abaqus interpolates the stress in increments along the element while the nodes are located at the ends and the center of the elements. In the stress trajectory topology the majority of the beam has low stress while the control topology has generally high stress as seen in Figure 3.5. The average stress in the stress trajectory topology was $97 \mathrm{kPa}$. while the average of the control topology was $312 \mathrm{kPa}$. A summary of the statistics is given in Table 3.1 and it can be seen that the stress trajectory topology have values much lower than that of the control topology.

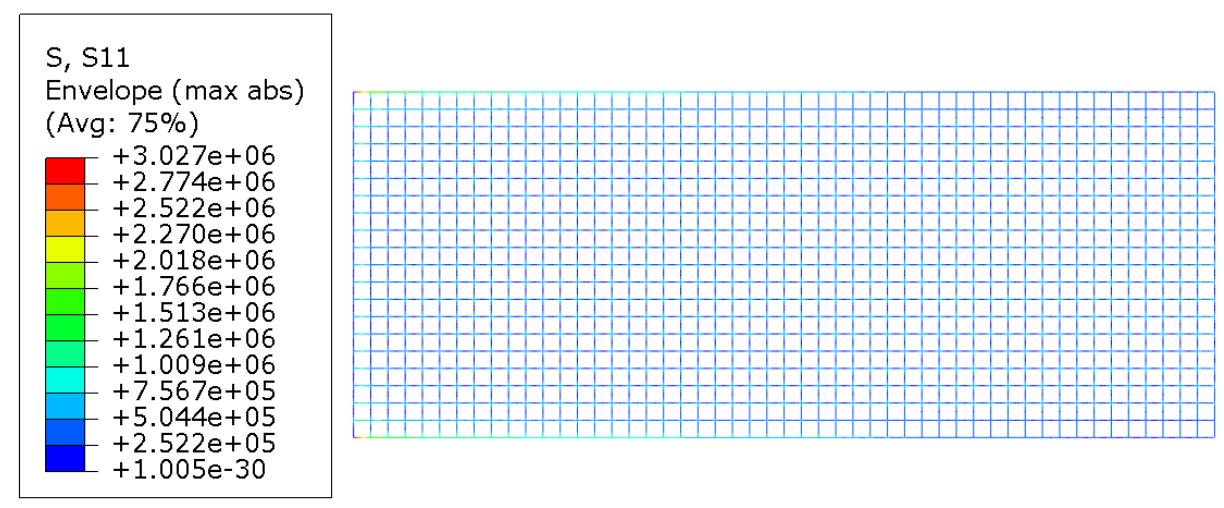

Figure 3.5: Stresses in Cantilever Beam with Control Topology

Table 3.1: Stress Trajectory Topology vs Control Topology

\begin{tabular}{|c|c|c|}
\hline & Stress Trajectory Topology & Control Topology \\
\hline Number of Nodes & 2,387 & 3,141 \\
\hline Maximum Stress & $772 \mathrm{kPa}$. & $2,377 \mathrm{kPa}$. \\
\hline Minimum Stress & $3.9 \mathrm{E}-33 \mathrm{kPa}$. & $1 \mathrm{E}-33 \mathrm{kPa}$. \\
\hline Average Stress & $97 \mathrm{kPa}$. & $312 \mathrm{kPa}$. \\
\hline
\end{tabular}

To examine the distribution, stress frequency plots were constructed as shown in Figure 3.6. These plots show the frequency of nodal stress values over the total range. When comparing the two histograms, it can be seen that the control topology shows a higher frequency at the higher stress levels when compared to the ST 
topology. The peak of the stress trajectory topology is at $200 \mathrm{kPa}$. while the peak of control topology is at the $800 \mathrm{kPa}$. In the stress trajectory topology the majority of the stress is between 100-300 kPa. while the majority of the stress in the control topology is between $200-800 \mathrm{kPa}$.

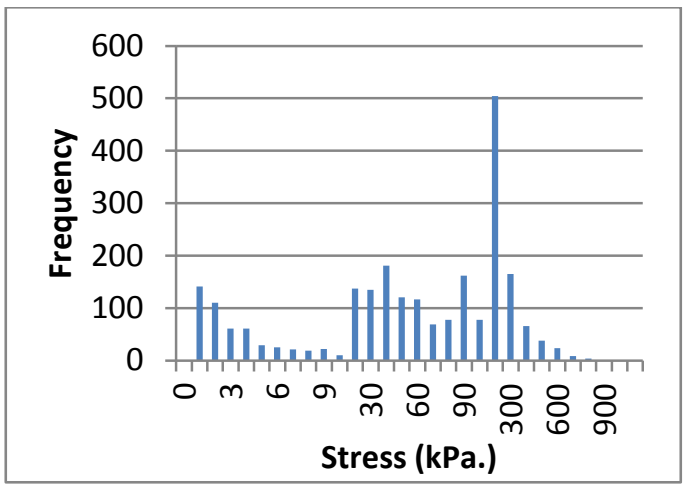

a) Stress Trajectory Topology

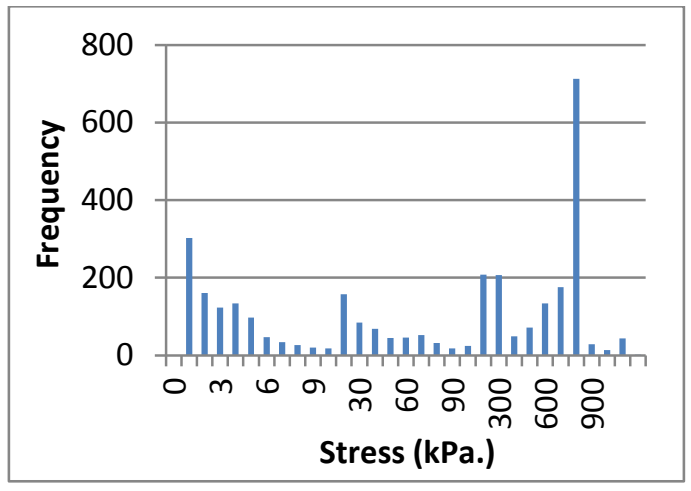

b) Control Topology

Figure 3.6: Histograms of Stress for Cantilever Beam Problem

Besides looking at the stresses found in every node, the nodes that had stress in the top ten percent were examined as well. This was done to look at a smaller sample of nodes and to look more closely at the higher stresses experienced in the topologies. The range of the top ten percent of stress trajectory topology is $772-220 \mathrm{kPa}$. while the top ten percent of the control topology range from 2,377-869 kPa. In fact the control topology has 343 nodes, $11 \%$ of the total number of nodes, with higher stress than the maximum stress in the stress trajectory topology.

Looking at the stresses in both topologies it is apparent that the ST topology is much better than the control topology. The concentration of stress is at lower value of stress in the stress trajectory topology than the control topology. The maximum stress and average stress were much higher in the control topology than the stress trajectory 
model. Many of the nodes in the control topology had higher stress than those in the stress trajectories.

\subsection{Simply Supported Beam Problems}

\subsubsection{The Problem}

The simply supported beam problems are $10 \mathrm{~cm}$ long and $2 \mathrm{~cm}$ wide, as shown in Figures 1.17 and 1.18. They will be supported at the bottom corners of the beam. There are two different simply supported beams being studied. The first problem uses the stresses from the elasticity solution to the problem see (Sadd, 2009), whereas the other problem will use the stresses from strength of materials (SM). These two beam models had slightly different stress distributions as shown in Figures 1.17 and 1.18, and thus generated slightly different stress trajectories. The other way that the two problems differ is the way they are loaded as seen in Figures 1.17 and 1.18. The generated stress trajectories for these two problems can be seen in Figures 3.7 and 3.8. Both beams will be fixed with displacement at the bottom left corner and vertically fixed at the bottom right corner. The main difference between the two sets of stress trajectories is that the stresses from strength of materials problem are symmetrical about the $\mathrm{x}$ and $\mathrm{y}$ axis whereas the theory of elasticity solution is symmetric about the

$\mathrm{y}$ axis only. The control topologies for both problems are the same, a $10 \mathrm{~cm}$ by $2 \mathrm{~cm}$ beam with horizontal and vertical elements spaced every $0.2 \mathrm{~cm}$ similar to the cantilever beam. The control topology will be used with both loading conditions and can be seen in Figure 3.9. Table 3.2 shows the topological data for all cases. 


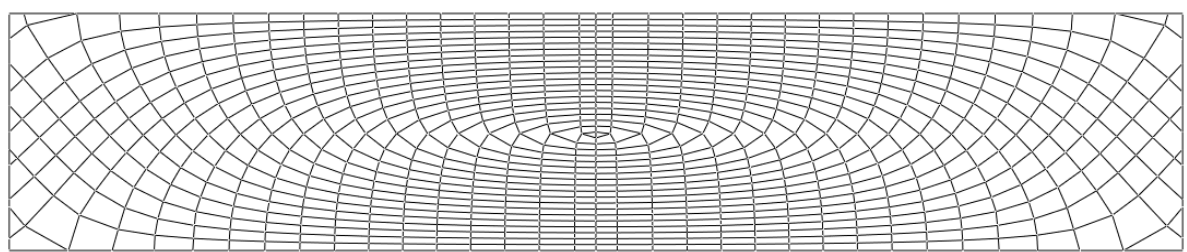

Figure 3.7: Abaqus Topology of Simply Supported Beam using Elasticity Stresses

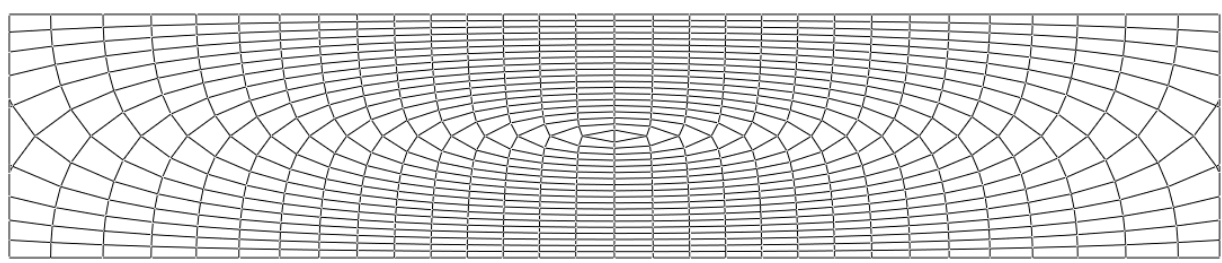

Figure 3.8: Abaqus Topology of Simply Supported Beam Using SM Stresses

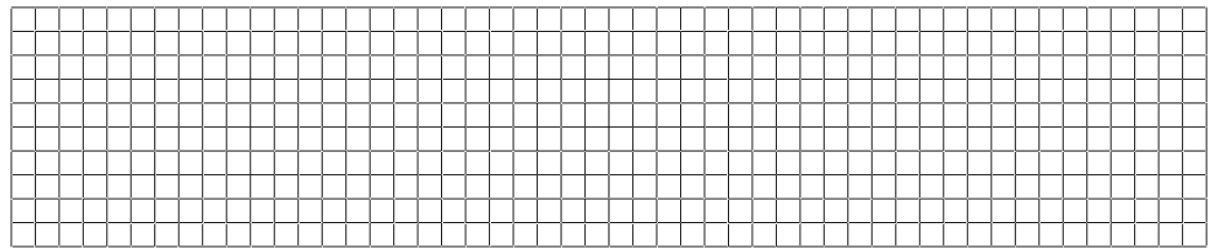

Figure 3.9: Control Topology for Simply Supported Beam

Table 3.2: Topological Data for Simply Supported Beams Examples

\begin{tabular}{|c|c|c|c|c|}
\hline Topology & $\begin{array}{c}\text { Number of } \\
\text { Elements }\end{array}$ & $\begin{array}{c}\text { Avg Length } \\
(\mathbf{c m})\end{array}$ & $\begin{array}{c}\text { Cross Sectional Width } \\
(\mathbf{c m})\end{array}$ & $\begin{array}{c}\text { Volume } \\
\left(\mathbf{c m}^{\mathbf{3}}\right)\end{array}$ \\
\hline SS Elasticity & 1764 & 0.1780 & 0.0237 & 0.1768 \\
\hline Elas. Control & 1070 & 0.2000 & 0.0287 & 0.1768 \\
\hline SS SM & 1596 & 0.1961 & 0.0261 & 0.2138 \\
\hline SM Control & 1070 & 0.2000 & 0.0316 & 0.2138 \\
\hline
\end{tabular}

\subsubsection{Abaqus Simulations and Results}

As shown in Figures 3.10 and 3.11, the highest stresses in the SM and control beam are along the left and right boundaries for both topologies. In the topology using stress trajectories the next highest stress is in a ring around the center of the beam as seen in Figure 3.10. In the control topology the next highest stress level is located 
along most of the beam except for the center as seen in Figure 3.11. The majority of the beam using the stress trajectory topology is under low stress. The maximum stress in the stress trajectory topology is $1,024 \mathrm{kPa}$. and the average stress is $81 \mathrm{kPa}$, meaning that most of the stress in the topology is low. In the control topology the maximum stress is $1,163 \mathrm{kPa}$. and the average stress is $249 \mathrm{kPa}$., both values are higher than the stress trajectory topology.
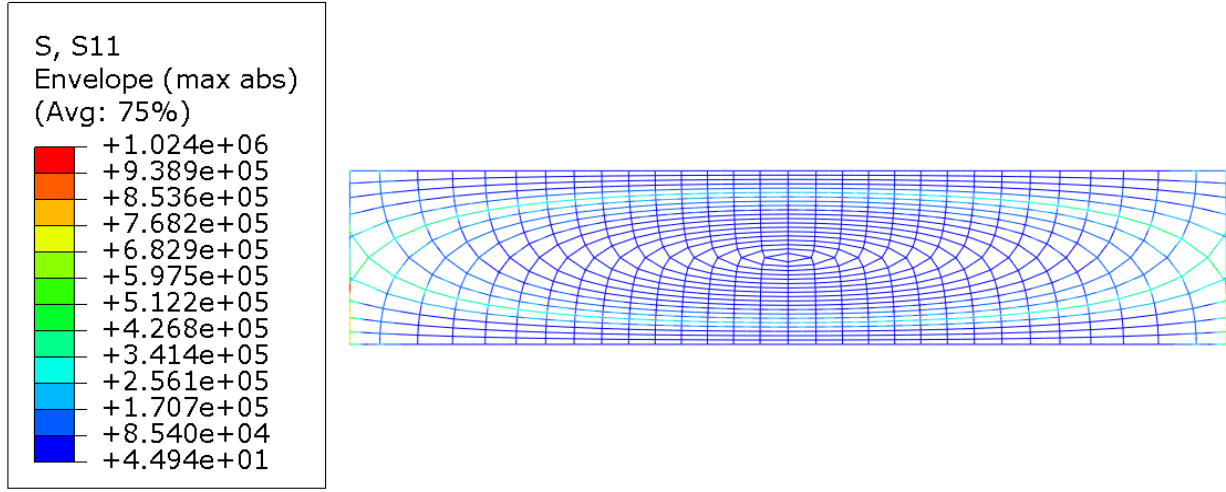

Figure 3.10: Stresses in Simply Supported Beam with Stress Trajectory Topology using SM Stresses
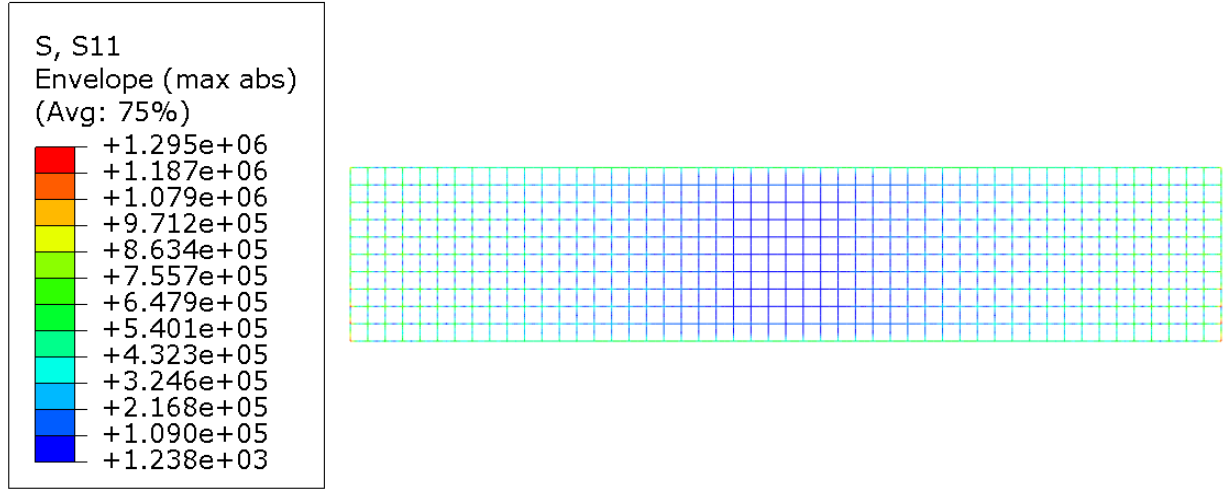

Figure 3.11: Stresses in Simply Supported Beam with Control Topology Using SM Loading

From Figures 3.12 and 3.13, in the beam using the stresses from elasticity, the highest stresses in the stress trajectory topology are along the left and right boundary but only on the very small elements. In the control topology the highest stresses are 
also located along the left and right boundary. While most of the nodes in the control topology have a middle level of stress, the stress trajectory topology has most of its nodes with lower level stress. The maximum and average stresses, seen in Figure 3.12 , in the stress trajectory topology are 1,023 and $91.3 \mathrm{kPa}$. respectively. In the control topology, seen in Figure 3.13, the maximum stress is 1,423 $\mathrm{kPa}$. and the average is $316 \mathrm{kPa}$. Similar to the beam using SM stresses the maximum and average stresses are lower in the stress trajectory topology than the control topology.

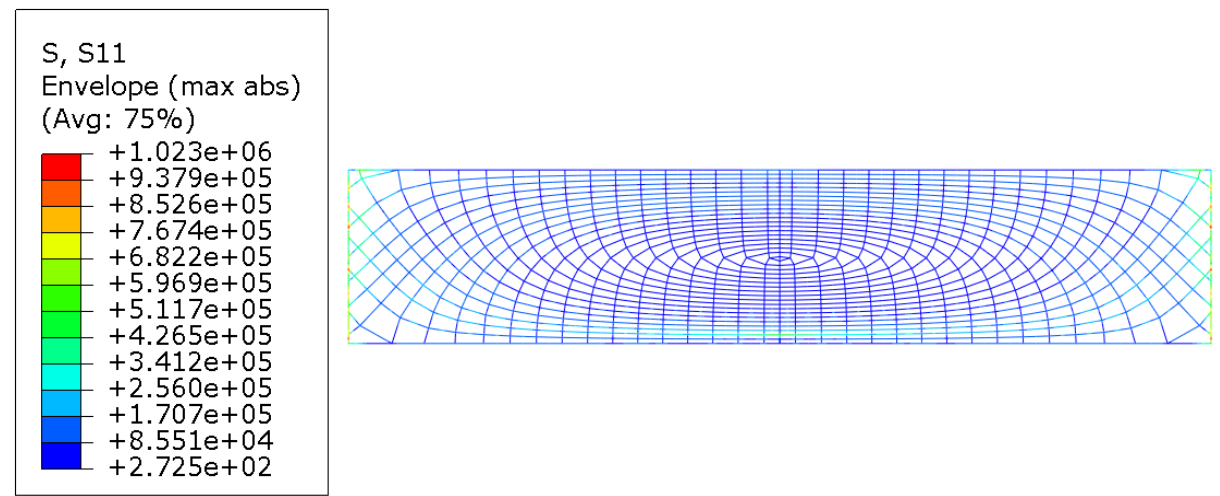

Figure 3.12: Stresses in Simply Supported Beam with Stress Trajectory Topology using Elasticity Stresses

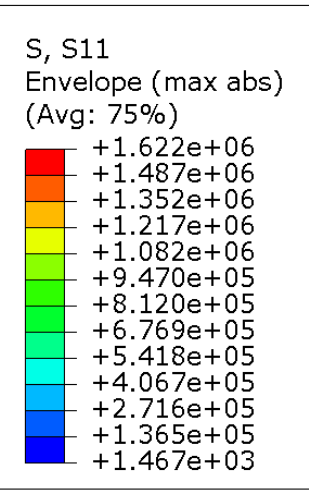

Figure 3.13: Stresses in Simply Supported Beam with Control Topology Using Elasticity Loading

When the stress trajectory and control topologies were run for the strength of materials problem the results were again quite different. There are a few more nodes in the stress trajectory topology than in the control topology, so the shape of the 
histograms will be looked at rather than the node frequency count. When looking at the frequency plots in Figure 3.14, the topology using the stress trajectories indicates a majority of the stress is concentrated at a lower stress value than that of the control topology. The control topology also has many more nodes greater than $400 \mathrm{kPa}$. compared to that of the ST topology despite having less nodes. The majority of the stress in the stress trajectory topology is between $20-200 \mathrm{kPa}$. while the majority of the control topology is between 70-900 $\mathrm{kPa}$.

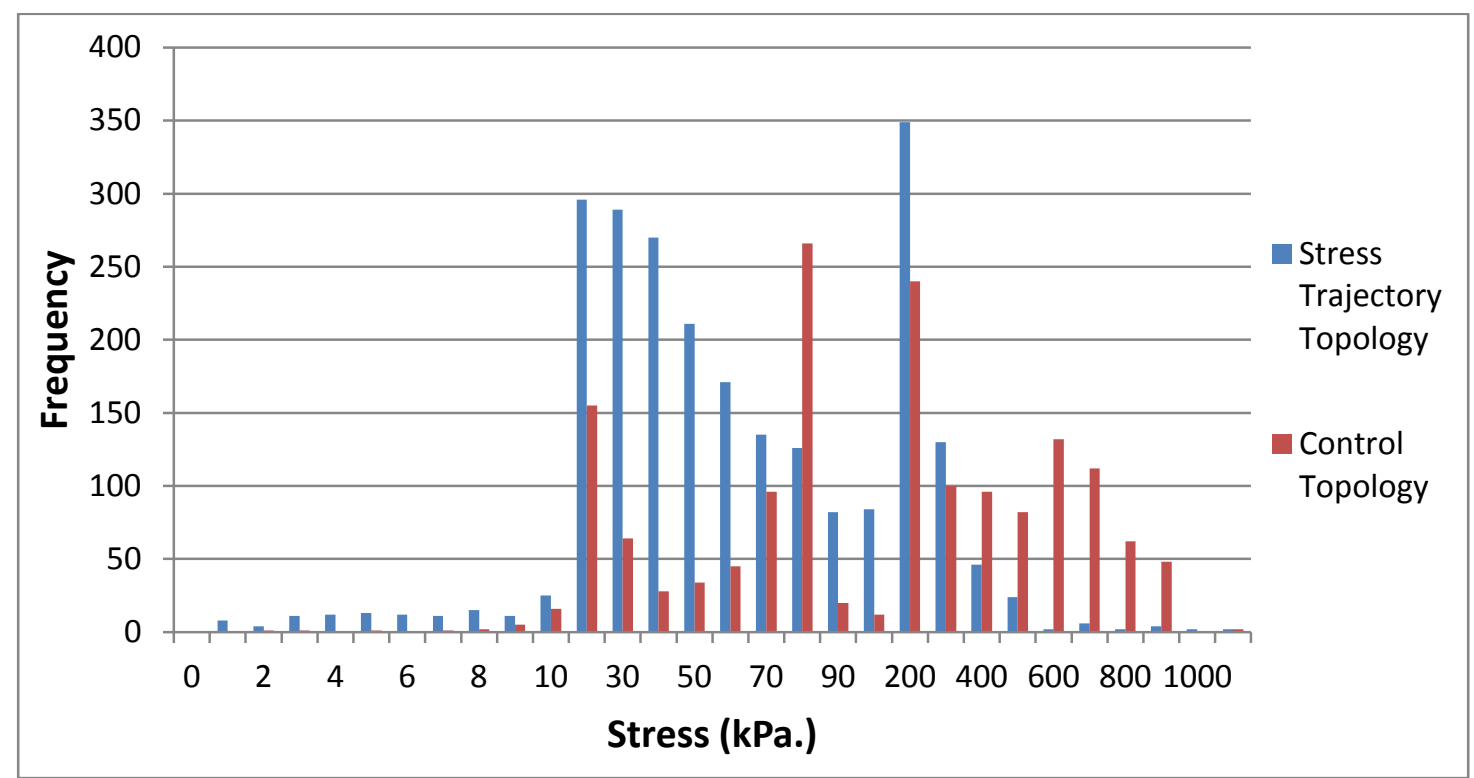

Figure 3.14: Stresses in Simply Supported Beam using SM Stresses

When looking at the histograms of the simply supported beam using the stresses from elasticity, in Figure 3.15, a similar result is seen as in the strength of materials problem. The concentration of stress using stress trajectory topology is much lower compared to that of the control topology problem. For the ST topology the majority of the stress is between $20-200 \mathrm{kPa}$. while majority of stress of the control topology is between $80-2,000 \mathrm{kPa}$. Similar to the SM case, the control topology has many nodes above $400 \mathrm{kPa}$. despite having less nodes. 


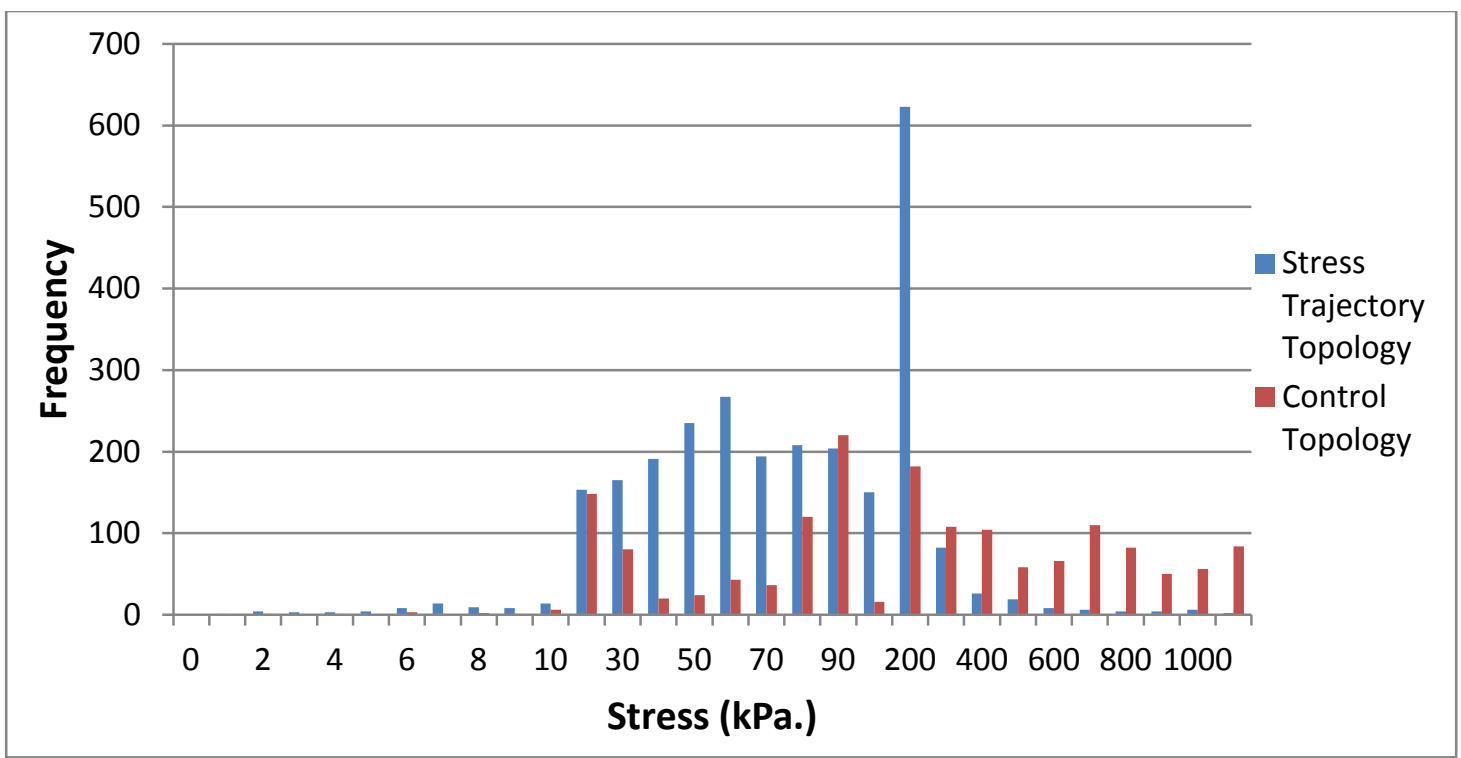

Figure 3.15: Frequency Plots for Simply Supported Beam using Elasticity Stresses

Taking a closer look at the stresses in the topology, the top ten percent of all stresses shows whether there are a lot higher stressed nodes or mostly lower stressed nodes in the topology. Looking at the beam using strength of materials stresses, the top ten percent of the stresses tells a lot about the topologies. In the stress trajectory topology the top ten percent range was $1,000-194 \mathrm{kPa}$. with an average value of $313 \mathrm{kPa}$. This shows that the majority of the stresses are lower, confirming the beliefs from looking at the stress distribution in the topology. In the control topology the top ten percent range was 1,163-653 $\mathrm{kPa}$., a much higher range than the stress trajectory topology. Taking into account the distribution of the stress, and the top ten percent of the stresses it is clear that the stress trajectory topology is better for reducing stresses in the problem. The maximum and average stresses are lower and the stresses are distributed over a lower level.

Likewise, looking at the beam using the elasticity stresses and the top ten percent of all stresses it is apparent that the stress trajectory topology has less nodes 
with higher stress than the control topology. The range of the top ten percent of the stress trajectory topology was from $1,023-157 \mathrm{kPa}$. versus $1,423-872 \mathrm{kPa}$. for the control topology. The control topology has a much higher concentration of high stress nodes than the stress trajectory topology. While looking at the stress distribution of all the nodes and the top ten percent of the stresses it is apparent that the stress trajectory topology is a better topology than the control topology.

\subsection{Disk Problem}

\subsubsection{The Problem}

The disk problem, shown in Figure 1.19, has a $2 \mathrm{~cm}$ radius and will be loaded in diametric compression of $1 \mathrm{~N}$ along the $\mathrm{y}$ axis. The elasticity stresses for the disk were taken from (Sadd, 2009). Stress trajectories were determined in the usual manner previously discussed and are shown in Figure 3.16. These compared perfectly with those shown in (Budynas, 1999). The primary stress trajectories are the more vertical lines and the secondary stress trajectories are the horizontal lines. The stress trajectories were spaced out every $0.25 \mathrm{~cm}$ along the $\mathrm{x}$ and $\mathrm{y}$ axes and were symmetrically distributed over the disk. To get the primary stress trajectories, the $\mathrm{x}$ and y coordinates and related stresses were switched (i.e. rotated the problem) so that we could use our standard numerical integration scheme. The stress trajectories for 


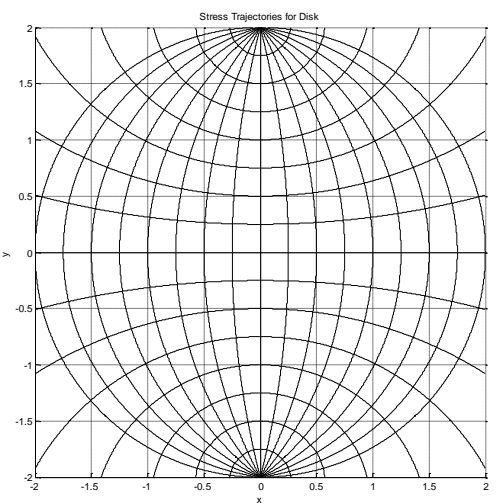

Figure 3.16: Stress Trajectories of Disk in MATLAB

the disk were generated over the span 0 to 2 which means that they went outside the boundary of the disk as shown in Figure 3.16. They were all generated over the same span to make it possible to be placed in a matrix to find all the intersecting points. When creating the Abaqus mesh, stress trajectories outside of the disk boundary were dropped and the final result is shown in Figure 3.17. The control topology for the

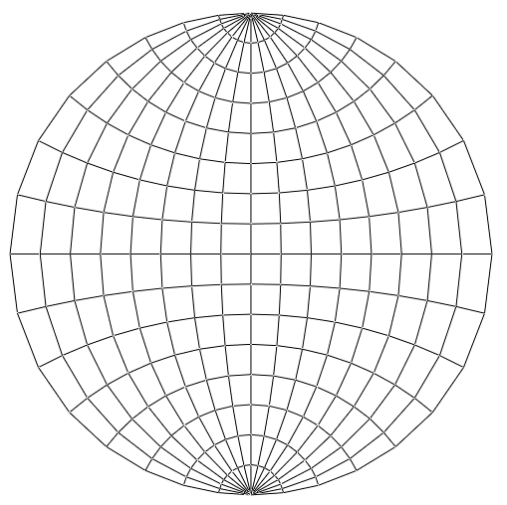

Figure 3.17: Abaqus Topology of Disk Problem

disk problem has the same radius of $2 \mathrm{~cm}$ and consists of radial lines and circles. The radial lines are every 15 degrees and the circles have radii from $0.25 \mathrm{~cm}$ to $2 \mathrm{~cm}$. The control topology can be seen in Figure 3.18. In theory the disk will just compress vertically, however, to make sure that all rigid body movements are removed the 
problems will be fixed in displacement at the bottom and will be horizontally fixed at the top.

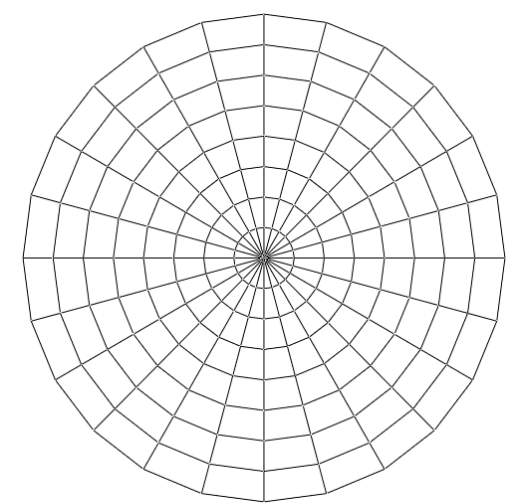

Figure 3.18: Abaqus Control Topology for Disk Problem

\subsubsection{Abaqus Simulations and Results}

When looking at the distribution of the stress in the topologies, see Figures 3.19 and 3.20, it appears that the stress trajectory topology distributes the stress throughout the topology while the control topology has the majority of the stress along the vertical diameter line. The highest stresses in the stress trajectory topology is at the loading points and quickly drops just $0.5 \mathrm{~cm}$ away from the loading point, while the control topology has high stress throughout the vertical line connecting the load points. The majority of nodal stresses in the control topology, as seen in Figure 3.20, is around $354 \mathrm{kPa}$. In the stress trajectory topology, seen in Figure 3.19, the stress is more distributed with the highest stress in the central regions and decreasing stress moving towards the outer edge. 

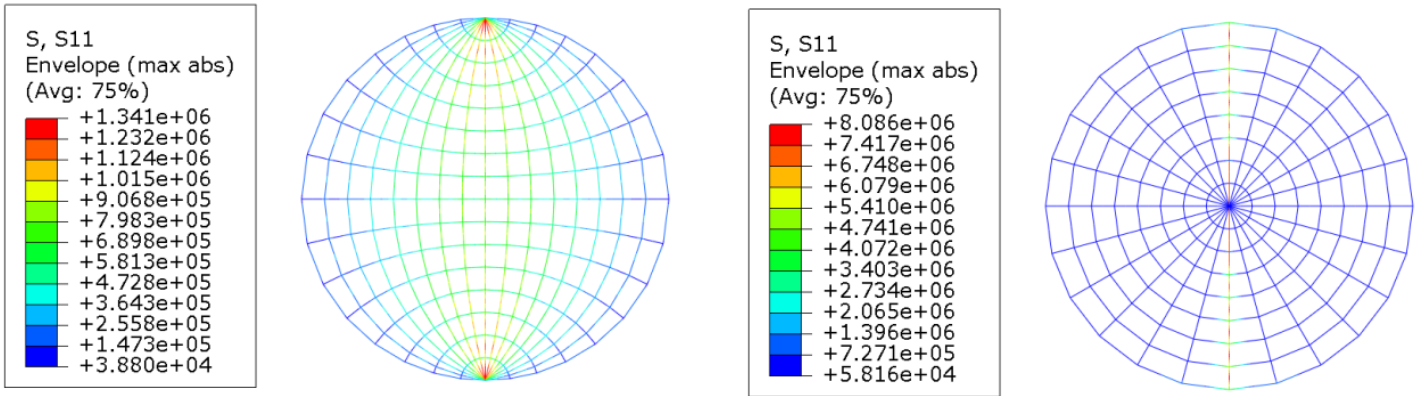

Figure 3.19: Stresses in Stress Trajectory Topology

Figure 3.20: Stresses in Control Topology

When the stress trajectory and control topologies were run for the disk problem, the frequency plots have some similarities with the highest stress occurring near or on the loaded diameter. The control topology had more concentrated stresses along the vertical loading line, as seen in Figure 3.20. The stresses are concentrated between 200 and 2,000 $\mathrm{kPa}$. The only difference between the two topologies is the stress trajectory topology has some nodes with less stress than the concentration whereas the control topology has nodes with higher stress than the concentration. The maximum stress in the stress trajectory topology is $1,341 \mathrm{kPa}$. with an average of $469.5 \mathrm{kPa}$. The maximum stress of the control topology is $8,086 \mathrm{kPa}$. with an average stress of $653.5 \mathrm{kPa}$. The maximum stress in the control topology is about six times greater and the average stress is about $40 \%$ higher when compared with the ST topology. 


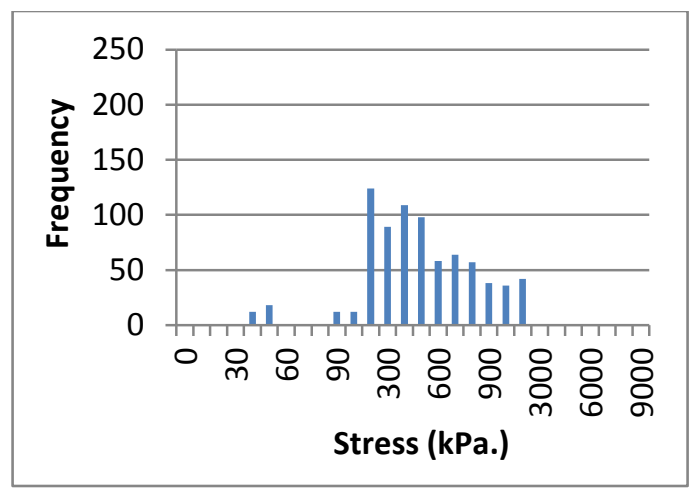

a) Stress Trajectory Topology

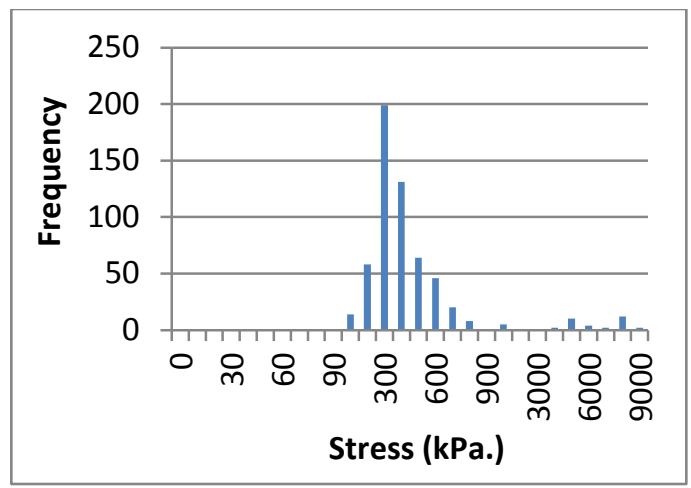

b) Control Topology

Figure 3.21: Stress Distribution in Disk Topologies

To take a different look at the stresses in the topology the top ten percent of the stresses were again examined. The top ten percent of the stress trajectory topology ranges from 1,341-907 $\mathrm{kPa}$. with an average stress of about 1,000 $\mathrm{kPa}$. In the control topology the top ten percent of the stress ranges from 8,086-620 $\mathrm{kPa}$. with an average of 3,647 $\mathrm{kPa}$. This shows that the stress trajectory topology has more nodes closer to the minimum value of the $10 \%$ group than the control topology. Although both topologies have some similarities, there are some key differences. The major differences between the two topologies are the maximum stress and the distribution of the stresses in the topologies. In both of these differences, the stress trajectory topology performed better than the control topology. Overall the stress trajectory topology had much lower stresses. 


\subsection{Equal Biaxial Loaded Plate Problem}

\subsubsection{The Problem}

We now explore the first of the three plate problems containing a single stress free interior circular hole. The current problem considers the case of equal biaxial loading as shown in Figure 1.20. The plate is $10 \mathrm{~cm}$ by $10 \mathrm{~cm}$ with a hole of $2 \mathrm{~cm}$ diameter located in the center of the plate. The plate will be loaded on all 4 sides in compression at $1 \mathrm{~N} / \mathrm{m}$ and the hole will be stress free. Using the infinite domain stress field solution, in Figure 1.20, the stress trajectories for this problem were found to be radial lines and circles, see Figure 3.22. Since the lines are radial and the center of the plate is outside the domain the MATLAB generation of the stress trajectories had to be modified. This was done by adjusting the xspan and the $y_{0}$ such that the stress trajectory started on the hole itself. The stress trajectories were spaced out by predesignated degrees depending on which topology was being generated.

There are seven different topologies for the plate with equal biaxial compression. They all have the same total volume of material, $1.93 \mathrm{~cm}^{3}$, and element cross sectional widths were adjusted to maintain constant material content. Data on the seven topologies can be seen in Table 3.3. FEA results will be analyzed and compared in different ways than done in the previous examples. First, coarse, medium and fine topologies, seen in Figure 3.22, will be compared to determine if element size affects the stress. The coarse topology has radial lines every 15 degrees along the hole and primary stress trajectories every 0.3 along the y axis. The medium topology has 


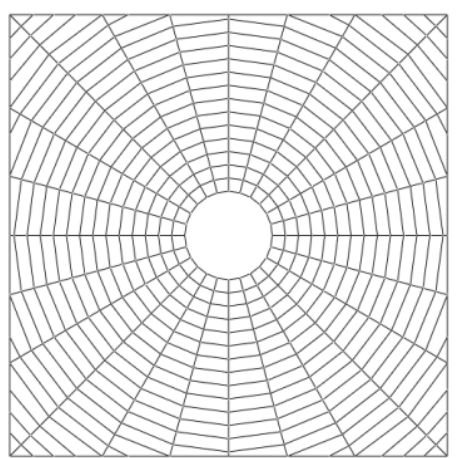

a) Coarse Topology

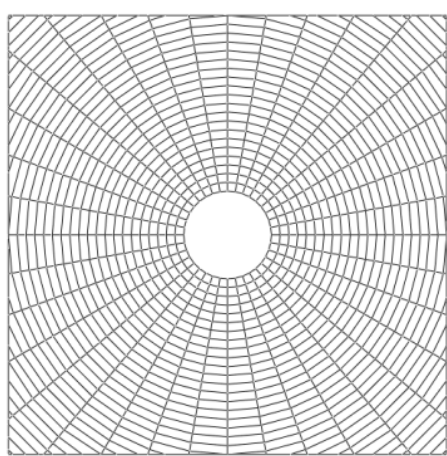

b) Medium Topology

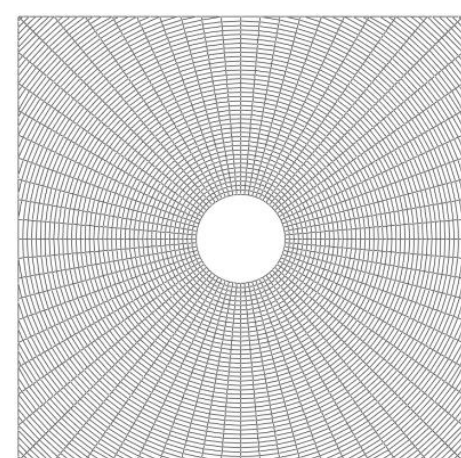

c) Fine Topology

Figure 3.22: Coarse, Medium and Fine Topology of Equal Biaxial Loaded Plate

radial lines every 10 degrees and primary trajectories every 0.2 along the y axis.

Finally, the fine topology has radial lines every 5 degrees along the hole and primary trajectories every 0.1 along the y axis. The two random topologies, shown in Figure 3.23, will be compared to the medium topology of all three plate problems to determine if a stress trajectory topology is better than a random or totally different topology. The medium topologies will be adjusted so that the volumes will all be the same. To eliminate all rigid body movements, the plate will be fixed in vertical displacement along the bottom edge and the center point of the top edge will be horizontally fixed.

The random topologies are generated in a controlled manner such that they would have the same number of elements as the medium topology. This was done by taking the matrix consisting of all the intersection points of the stress trajectory topology and putting it through a random displacement MATLAB generator. Using a for loop consisting of the following:

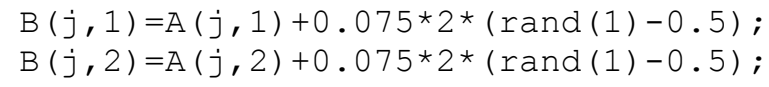


A new location matrix $B$ is generated from the original A matrix. The first line of the code moves the $\mathrm{x}$ coordinate and the second line moves the $\mathrm{y}$ coordinate. To assure that the dimensions will be the same the elements along the edge were allowed to move along the edge but not off of it. The elements around the interior hole were kept from the original matrix so that the circular hole would be preserved. The uniaxial and unequal biaxial medium topologies that will also be run using equal biaxial loading can be seen in sections 3.6 and 3.7 respectively.

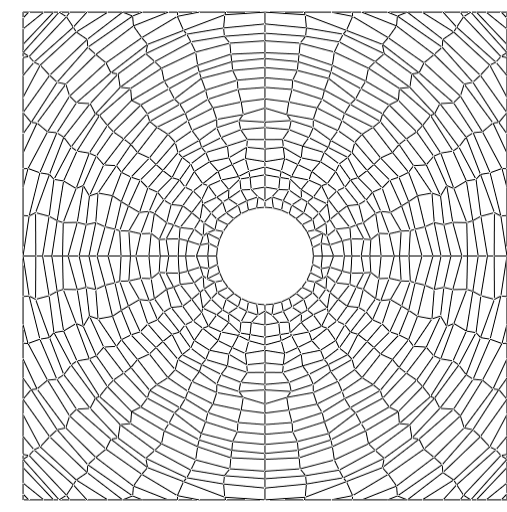

a) Random 1

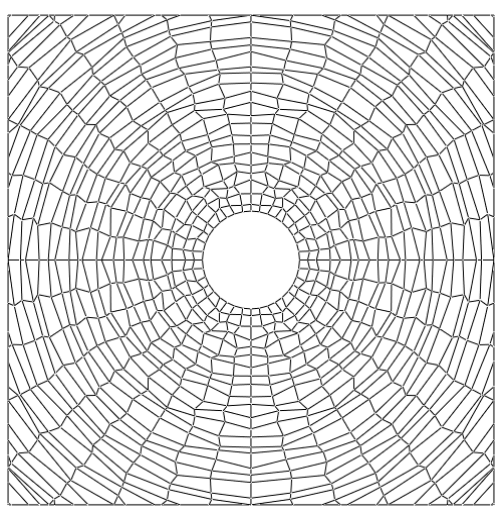

b) Random 2

Figure 3.23: Random Topologies for Equal Biaxial Loaded Plate

Table 3.3: Topological Data for Equal Biaxial Loaded Plate Examples

\begin{tabular}{|c|c|c|c|c|}
\hline Topology & Number of Elements & Avg. Length (L) & Cross Section (A) & L/A \\
\hline Coarse & 880 & 0.5623 & 0.0625 & 9.00 \\
\hline Medium & 1892 & 0.3723 & 0.0524 & 7.11 \\
\hline Fine & 7028 & 0.1908 & 0.0379 & 5.02 \\
\hline Random 1 & 1892 & 0.3775 & 0.0520 & 7.26 \\
\hline Random 2 & 1892 & 0.3784 & 0.0519 & 7.28 \\
\hline Uniaxial Medium & 5072 & 0.2006 & 0.0436 & 4.60 \\
\hline Unequal Medium & 4076 & 0.2247 & 0.0459 & 4.89 \\
\hline
\end{tabular}




\subsubsection{Abaqus Simulations and Results}

The coarse, medium and fine topologies were examined by looking at the stress distributions in each topology and the top ten percent of the stresses. Taking a look at the distribution of the stresses in the ST topologies allows for a visual representation of where the highest stresses are located. In Figures 3.24-26, the stresses are color mapped using Abaqus. It can be seen that in all three topologies the majority of the stress is about the same and the higher stresses are located around the edge of the plate. In the coarse topology there are more higher level stressed nodes than the medium and fine topologies. Overall all three topologies are very similar.
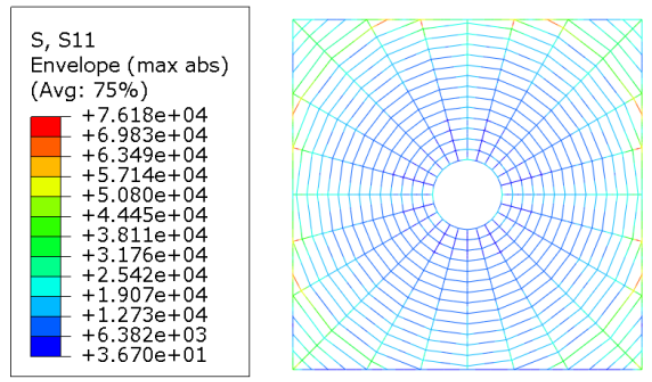

Figure 3.24: Abaqus Stresses in Coarse Topology
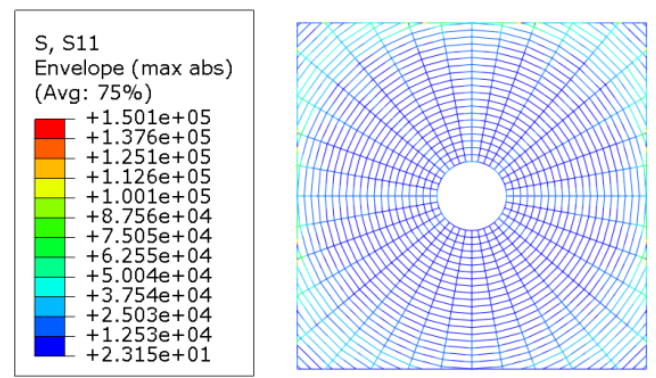

Figure 3.25: Abaqus Stresses in Medium Topology

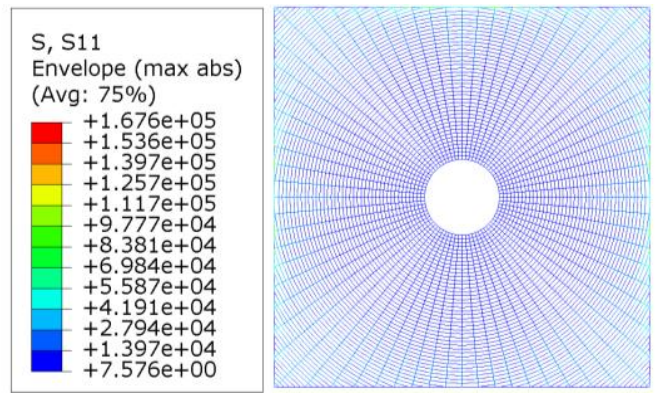

Figure 3.26: Abaqus Stresses in Fine Topology

Comparing the frequency plots of the three topologies in Figures 3.27-29 it can be seen that the general shape of the stress distribution is about the same in all the 
topologies. They all build up slowly to the peak concentration at $20 \mathrm{kPa}$. then rapidly decrease to a maximum stress value around $100 \mathrm{kPa}$. Table 3.4 lists the maximum, minimum and average stresses, and indicates that the three topologies give very similar results.

Table 3.4: Maximum, Minimum and Average Stresses for the Three Topologies

\begin{tabular}{|c|c|c|c|}
\hline Topology & Max. Stress (kPa.) & Min Stress (kPa.) & Avg. Stress (kPa.) \\
\hline Coarse & 76 & .036 & 16 \\
\hline Medium & 95 & .023 & 16 \\
\hline Fine & 116 & .007 & 13 \\
\hline
\end{tabular}

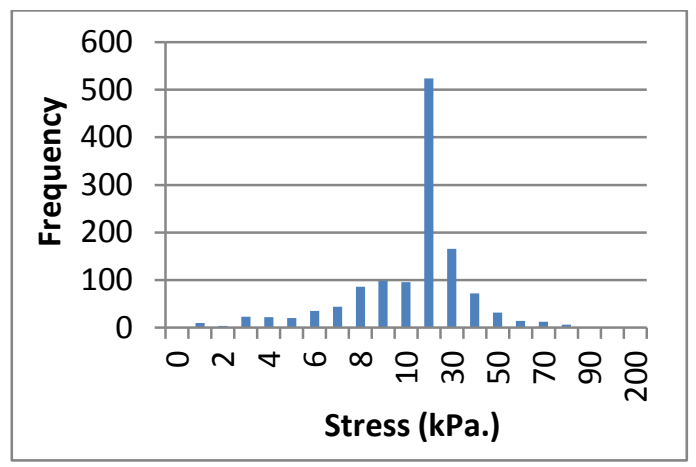

Figure 3.27: Stress Distribution in Coarse Topology

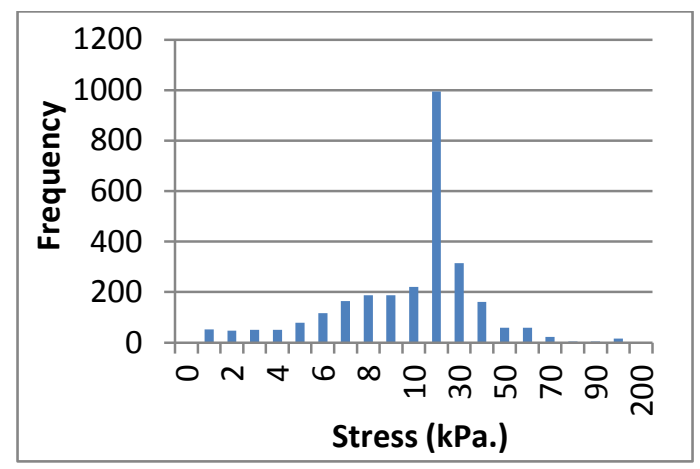

Figure 3.28: Stress Distribution in Medium Topology

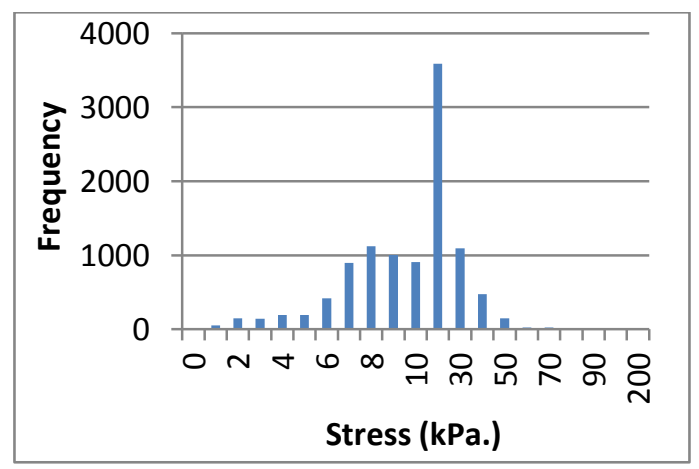

Figure 3.29: Stress Distribution in Fine Topology 
Taking a look at the top ten percent of the stresses allows a different examination of the stress to be done. In the three topologies the top ten percent of the stresses are similar like the overall stress. The average stresses in the top ten percent are 44, 47 and $40 \mathrm{kPa}$. for the coarse, medium, and fine topologies respectively. This shows that the three topologies are relatively the same and the size of the elements does not affect the topology. The fine topology is slightly better than other two topologies but the medium and coarse topologies are very close and are acceptable.

Besides looking at the size of the element in the topology the actual topologies were tested. This was done by comparing the medium topology to the two random topologies and to topologies of two other plate problems. Looking at the stresses in the topologies, seen in Figures 3.30-34, shows there is quite a difference between the medium topology and the rest of them. In the random topologies the highest stress is in the same location as the medium topology, along the left, right and top boundary in a small area. The unequal and uniaxial topologies show the maximum stress is a much larger area located on the holes. The unequal topology has most of its nodes around $27 \mathrm{kPa}$. and the uniaxial topology has most of its nodes around $14 \mathrm{kPa}$. In the medium topology most the nodes are around $12 \mathrm{kPa}$., the random 1 topology is mostly around $22 \mathrm{kPa}$. and the random 2 topology is about $36 \mathrm{kPa}$. 

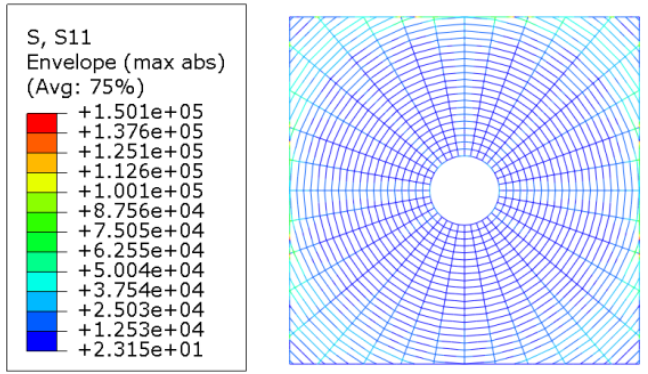

Figure 3.30: Stresses in Medium

Topology
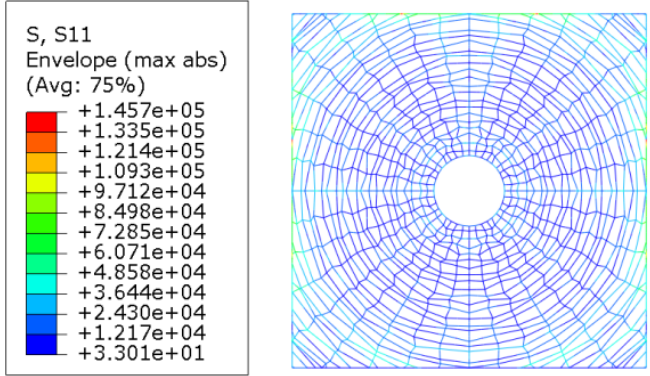

Figure 3.32: Stresses in Random 2 Topology
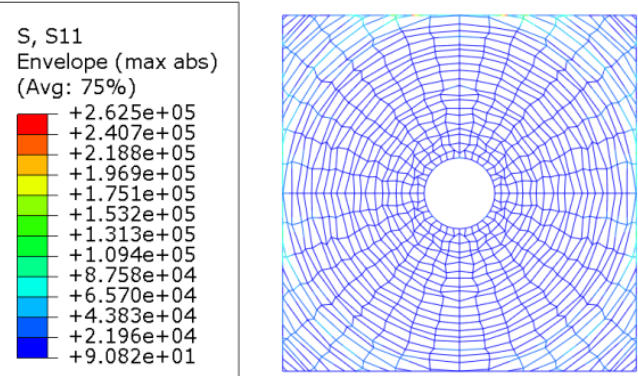

Figure 3.31: Stresses in Random 1

Topology
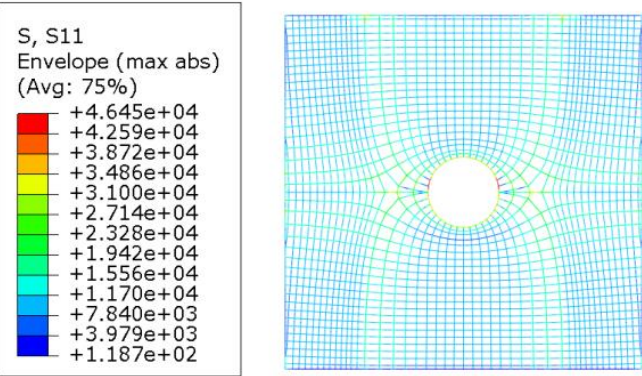

Figure 3.33: Stresses in Unequal Topology
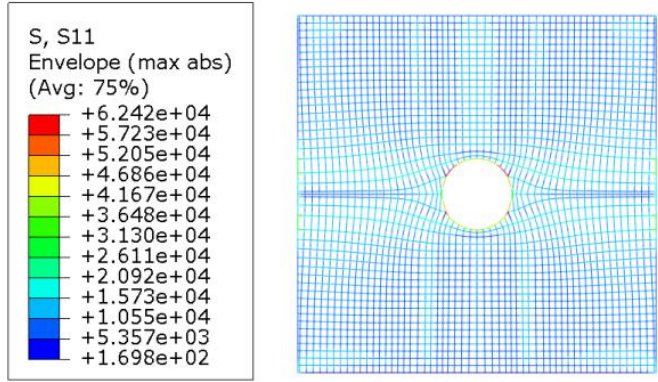

Figure 3.34: Stresses in Uniaxial Topology 


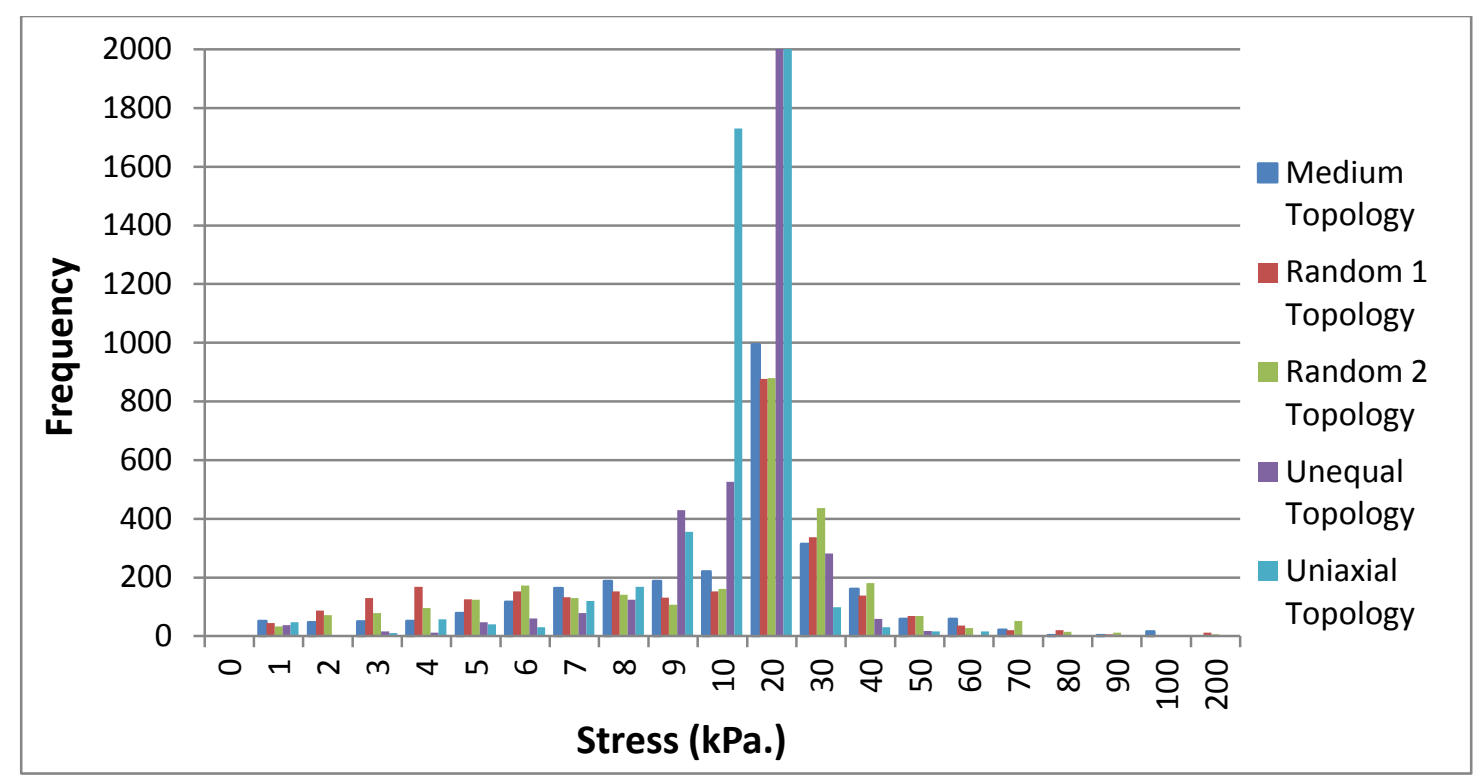

Figure 3.35: Stress Frequency Distribution of the Five Topologies

Histogram plots seen in Figure 3.35, show that frequencies of the stress of the five topologies are similar with some slight differences. The unequal and uniaxial topologies have many more nodes with stress between 9 and $20 \mathrm{kPa}$. compared to the other 3 topologies. The random topologies are close to medium topologies with the only difference being that the random topologies have more nodes at a higher stress. The maximum, minimum and average stresses can be seen in Table 3.5. The random topologies have a higher maximum stress than the medium topology while the unequal and uniaxial topologies have a lower maximum stress. The average stresses are all similar and are within $4.5 \mathrm{kPa}$. of the medium topology. Looking at this only, it appears that the unequal and uniaxial topologies are better than the medium topology, however, the aspect of the location of the higher stresses is important as well. The location of the maximum stress is the most likely spot of the topology to fail. If the maximum stress is on or around the hole and the element fails, a zipper effect could 
take place. If the maximum is on a small element near the boundary and the element fails the stresses are more like to be able to be handled by the surrounding elements.

Table 3.5: Maximum, Minimum and Average Stresses for the Five Topologies

\begin{tabular}{|c|c|c|c|}
\hline Topology & Maximum Stress (kPa.) & Minimum Stress (kPa.) & Average Stress (kPa.) \\
\hline Medium & 95 & .023 & 15.5 \\
\hline Random 1 & 140 & .090 & 15 \\
\hline Random 2 & 144 & .033 & 17 \\
\hline Unequal & 48 & .125 & 12.5 \\
\hline Uniaxial & 60 & .217 & 11 \\
\hline
\end{tabular}

Looking at the stress distribution and the stresses in the topologies it appears that the medium ST topology is indeed the best topology. While the random topologies are similar in average stress and the stresses in the topology when compared to the medium topology they also have maximum stresses $50 \mathrm{kPa}$. greater than the medium topology. The unequal and uniaxial topologies have lower maximum stresses when compared to the medium topology, however, neither topology distribute the stress through the topology like the equal medium topologies does. The unequal and uniaxial topologies also have much greater stresses on the hole, which should have fairly low stress, than the other three topologies.

\subsection{Uniaxial Loaded Plate Problem}

\subsubsection{The Problem}

The uniaxial loaded plate has the same dimensions as the biaxial loaded plate discussed in the previous section and is horizontally loaded in compression of $1 \mathrm{~N} / \mathrm{m}$ as shown in Figure 1.21. The primary stress trajectories run from the $\mathrm{x}=0$ to $\mathrm{x}=5$ and 
starting at 30 degrees from the $\mathrm{x}$ axis on the hole to $\mathrm{x}=5$. The secondary stress trajectories run from $y=0$ to $y=5$, starting from the hole to the plate boundary. This plate problem will also have seven different models and their properties are given in Table 3.6. All seven problems have a total volume of $0.77583 \mathrm{~cm}^{3}$ and cross sectional widths based on that. The topologies will be compared the same way as reported in section 3.5. The coarse topology has primary stress trajectories every 0.3 along the $y$ axis and secondary stress trajectories every 0.3 along the $\mathrm{x}$ axis and every 15 degrees along the hole starting at 30 degrees. The medium topology has primary trajectories

Table 3.6: Topological Data for Uniaxial Loaded Plate Examples

\begin{tabular}{|c|c|c|c|c|}
\hline Topology & Number of Elements & Avg. Length (L) & Cross Section (A) & L/A \\
\hline Coarse & 2260 & 0.3030 & 0.0337 & 9.0000 \\
\hline Medium & 5072 & 0.2006 & 0.0276 & 7.2622 \\
\hline Fine & 18888 & 0.1032 & 0.0200 & 5.1724 \\
\hline Random 1 & 5072 & 0.2050 & 0.0273 & 7.5037 \\
\hline Random 2 & 5072 & 0.2044 & 0.0274 & 7.4717 \\
\hline Equal Medium & 1892 & 0.3723 & 0.0332 & 11.2180 \\
\hline Unequal Medium & 4076 & 0.2247 & 0.0291 & 7.7204 \\
\hline
\end{tabular}

every 0.2 along the $\mathrm{y}$ axis and secondary trajectories every 0.2 along the $\mathrm{x}$ axis and every 10 degrees starting at 30 degrees. The fine trajectories have primary trajectories every 0.1 along the $\mathrm{y}$ axis and secondary trajectories every 0.1 along the $\mathrm{x}$ axis and every 5 degrees along the hole starting at 25 degrees. The coarse, medium and fine topologies can be seen in Figure 3.36. The same boundary conditions were again used to eliminate all rigid body movements. 


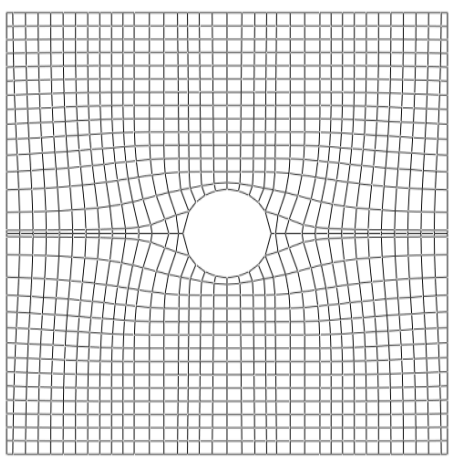

a) Coarse Topology

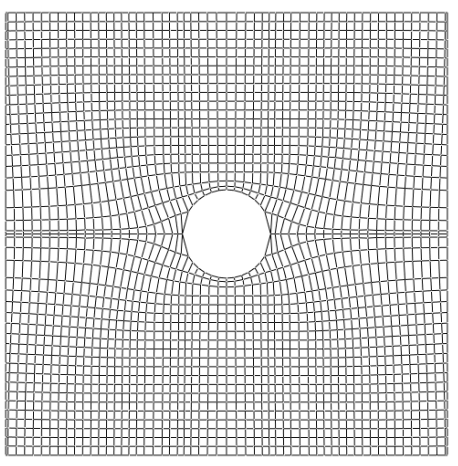

b) Medium Topology

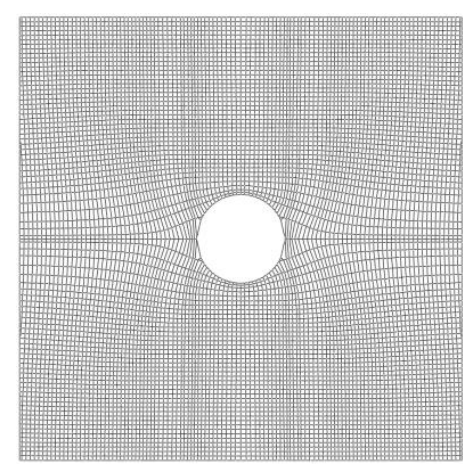

c) Fine Topology

Figure 3.36: Coarse, Medium and Fine Topology of Uniaxial Loaded Plate

The random topologies were generated the same way as the equal biaxial loaded plate except the intersecting points were only allowed to move up to $0.05 \mathrm{~cm}$ in any direction. As before boundary points of the problem were held at constant locations. The random topologies are shown in Figure 3.37 and will be compared to all the medium topologies.

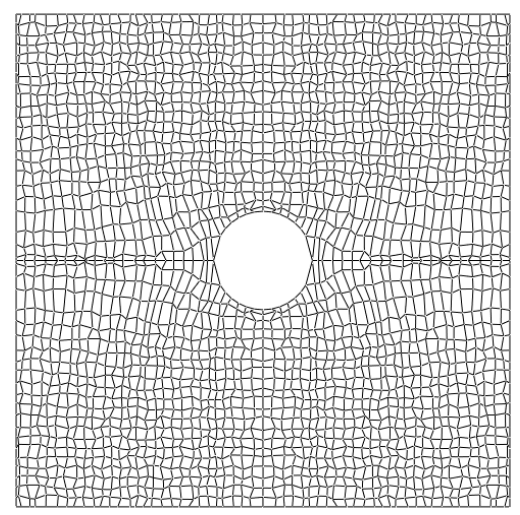

a) Random 1

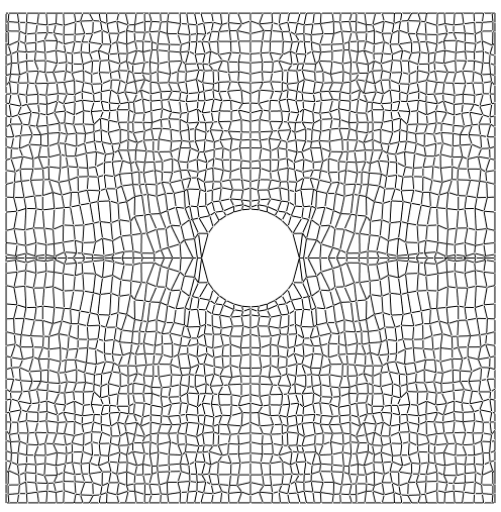

b) Random 2

Figure 3.37: Random Topologies for Uniaxial Loaded Plate

\subsubsection{Abaqus Simulations and Results}

The coarse, medium and fine topologies were again examined by looking at the stress distributions in each topology and the top ten percent of the stresses. Taking a 
look at the distribution of the stresses in the actual topology allows for a visual representation of where the highest stresses are located. In Figures 3.38-40, the stresses are color mapped using Abaqus. It can be seen that in all three topologies the majority of the stress is about the same and the higher stresses are located on the left boundary in small elements. In the fine topology there are more higher level stressed nodes than the medium and coarse topologies. Overall all three topologies are very similar.
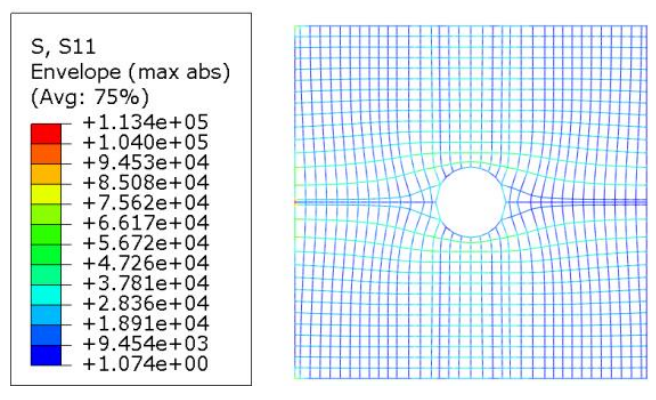

Figure 3.38: Abaqus Stresses in Coarse Topology
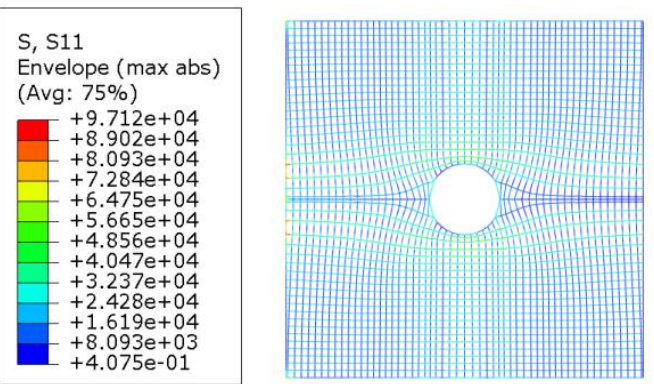

Figure 3.39: Abaqus Stresses in Medium Topology
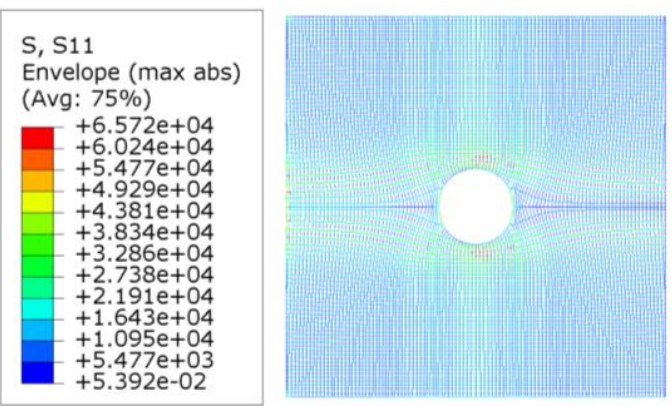

Figure 3.40: Abaqus Stresses in Fine Topology

Comparing the frequency plots of the three topologies in Figures 3.41-43 it can be seen that the general shape of the stress distribution is about the same in all the topologies. They all build up slowly to the peak concentration at 20-30 kPa. then rapidly decrease to a maximum stress value around $75 \mathrm{kPa}$. The only difference is the fine topology decreases quicker than the others. Table 3.7 lists the maximum, 
minimum and average stresses, and indicates that the three topologies give very similar results.

Table 3.7: Maximum, Minimum and Average Stresses for the Three Topologies

\begin{tabular}{|c|c|c|c|}
\hline Topology & Max. Stress (kPa.) & Min Stress (kPa.) & Avg. Stress (kPa.) \\
\hline Coarse & 76 & 0 & 18 \\
\hline Medium & 97 & 0 & 17.7 \\
\hline Fine & 62 & 0 & 13.2 \\
\hline
\end{tabular}

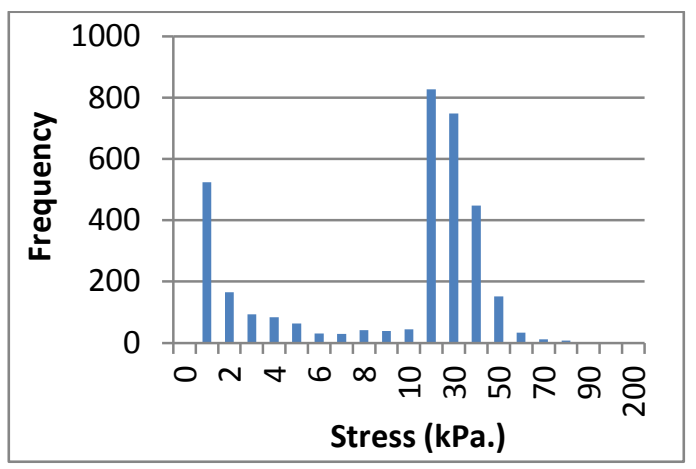

Figure 3.41: Stress Distribution in Coarse Topology

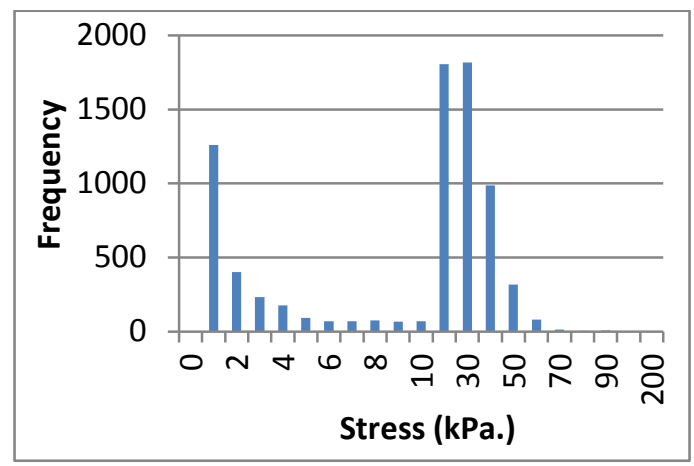

Figure 3.42: Stress Distribution in Medium Topology

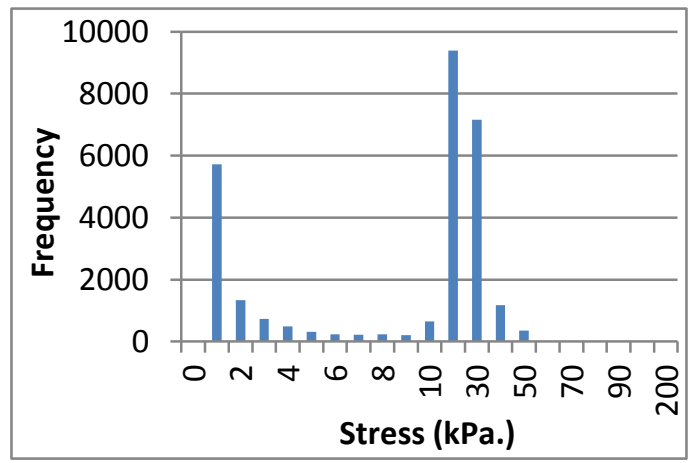

Figure 3.43: Stress Distribution in Fine Topology

Taking a look at the top ten percent of the stresses allows a different examination of the stress to be done. In the three topologies the top ten percent of the stresses are similar like the overall stress. The average stresses in the top ten percent are $35.8,35.2$ and $29.6 \mathrm{kPa}$. for the coarse, medium, and fine topologies respectively. 
This shows that the top ten percent stress of the three topologies is relatively the same and the size of the elements does not affect the stress results.

Apart from looking at the size of the element in the topology, the actual topology was examined. The medium ST topology was compared to the two random topologies along with the ST medium topologies of the equal biaxial and the unequal biaxial problems. It is important to look at the where the maximum stress is located and how the stresses are distributed throughout the topology. Looking at the stress in the topologies, seen in Figures 3.44-48, there are differences between the five topologies. In the medium topology the highest stress is located along the left boundary in a few elements and most of the elements are around $25 \mathrm{kPa}$. In the random topologies, the highest stresses are located in the same area of the topology as the medium. In the first random topology most of the stress is around $50 \mathrm{kPa}$., and in the second random topology most of the elements are around $45 \mathrm{kPa}$. with some small areas with stress around $100 \mathrm{kPa}$. In the unequal topology most of the elements are around $25 \mathrm{kPa}$. like the medium topology. However, unlike the medium topology, the hole experiences its highest stress. The hole experiences stresses that range from 80 to $122.6 \mathrm{kPa}$. compared to $10 \mathrm{kPa}$. in the medium topology. In the equal topology most of the stress is around $125 \mathrm{kPa}$. with the highest stress located on the hole like the unequal topology. 


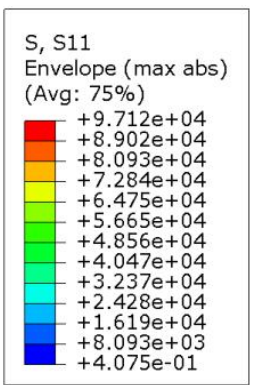

Figure 3.44: Stresses in Medium

Topology
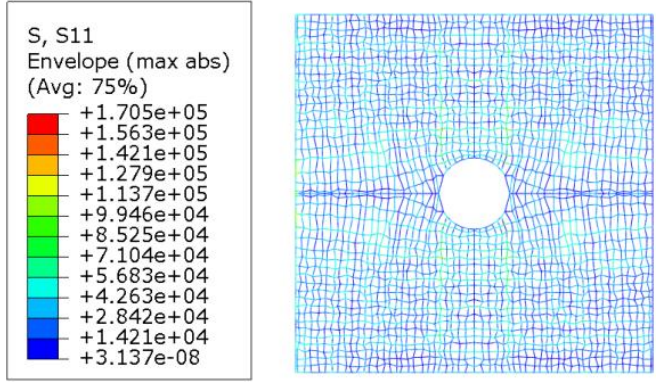

Figure 3.46: Stresses in Random 2 Topology
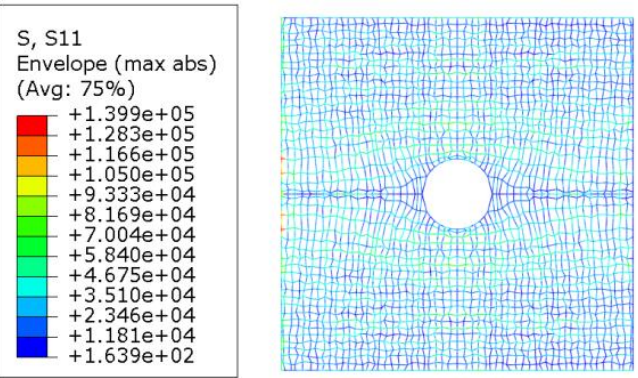

Figure 3.45: Stresses in Random 1

Topology
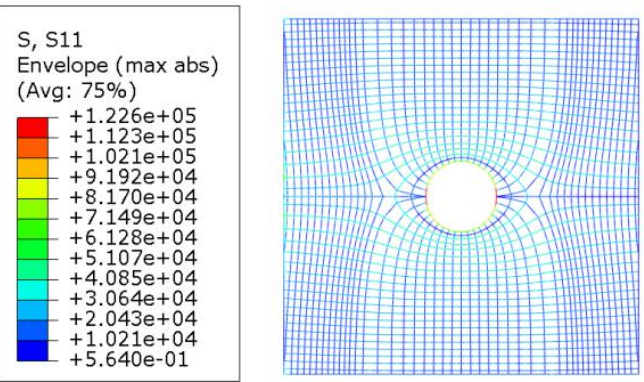

Figure 3.47: Stresses in Unequal Topology
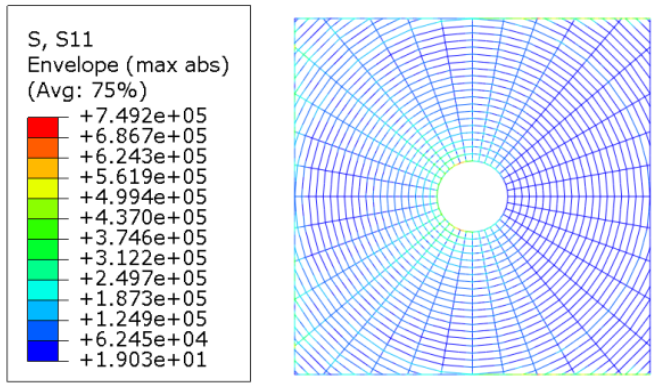

Figure 3.48: Stresses in Equal Topology

Looking at the frequency plots in Figure 3.49, it can be seen that the equal topology has many nodes with higher stress and the medium topology has many nodes with lower stress. All of the topologies appear to peak around $20 \mathrm{kPa}$. and then decrease after that with the medium and unequal topology decreasing more rapidly. The medium and unequal topologies have most of their stress before the common peak while the two random topologies have most of their stresses after the peak. There is also a difference when looking at the maximum, minimum and average stress in Table 
3.8. The medium topology has the lowest maximum stress while the unequal topology has the lowest average stress.

Table 3.8: Maximum, Minimum and Average Stresses for the Five Topologies

\begin{tabular}{|c|c|c|c|}
\hline Topology & Maximum Stress (kPa.) & Minimum Stress (kPa.) & Average Stress (kPa.) \\
\hline Medium & 97 & 0 & 17.7 \\
\hline Random 1 & 140 & .163 & 28 \\
\hline Random 2 & 108 & 0 & 28 \\
\hline Unequal & 122.6 & .118 & 11 \\
\hline Equal & 572 & .019 & 85 \\
\hline
\end{tabular}

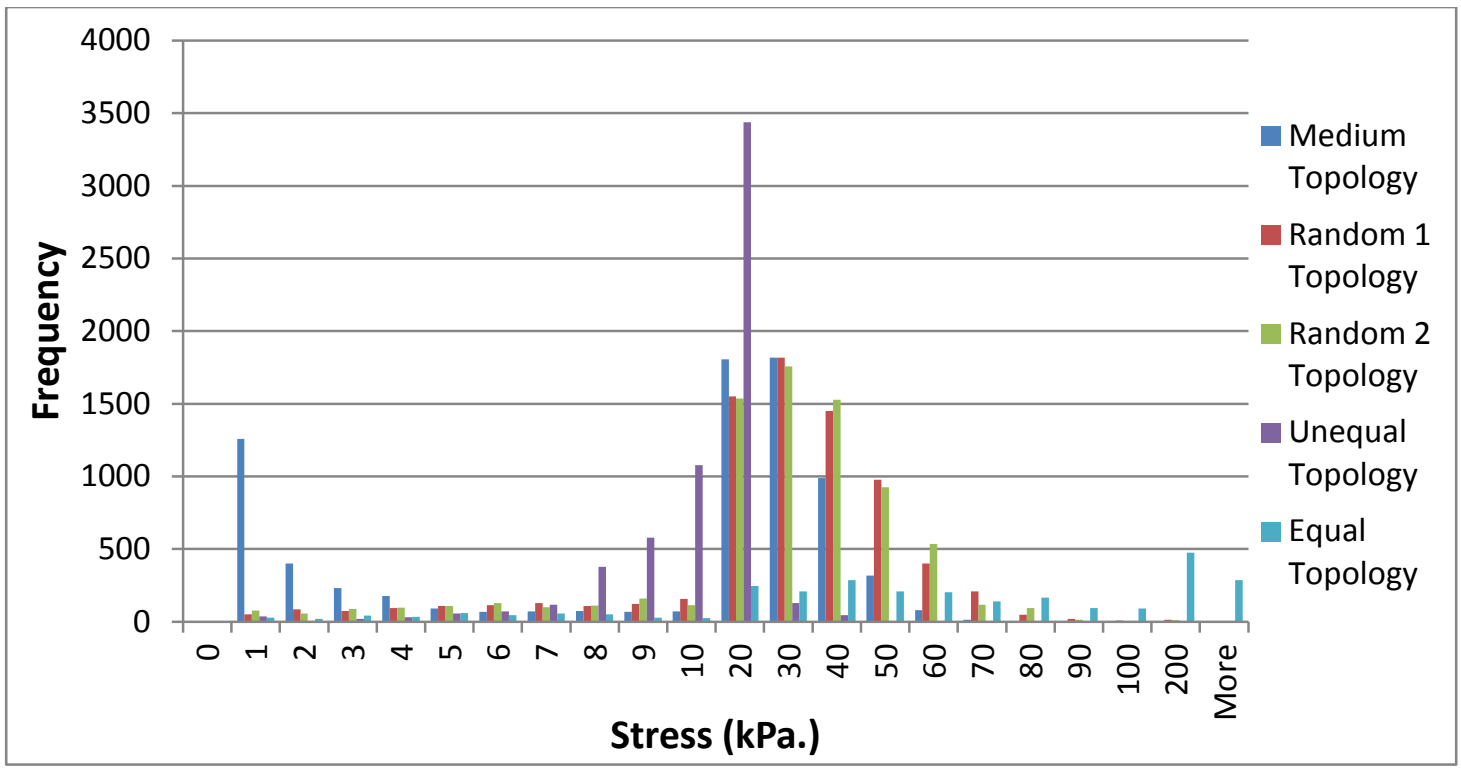

Figure 3.49: Stress Frequency Distribution of the Five Topologies

Taking into account the frequency of the stresses and the distribution of the stresses in the topologies, it is apparent that the medium topology is the best of the five topologies. The unequal topology had the lowest average stress but the medium topology had the lowest maximum stress. When looking at the distribution of the stresses in the topologies the medium topology outperforms the rest of the topologies. Both the ST medium and unequal topologies had the majority of their elements 
experiencing around $25 \mathrm{kPa}$. but the hole in the unequal topology experiences eight to twelve times the stress than that in the medium topology.

\subsection{Unequal Biaxial Loaded Plate Problem}

\subsubsection{The Problem}

Like the previous plate problems the unequal biaxial loaded plate is $10 \mathrm{~cm}$ by $10 \mathrm{~cm}$ with a $2 \mathrm{~cm}$ hole located in the center. The plate will be loaded in compression along all four edges with a value of $1 \mathrm{~N} / \mathrm{m}$ vertically and $2 \mathrm{~N} / \mathrm{m}$ horizontally as shown in Figure 1.15 in section 1.4.1. Also like the previous plate problems, there will be seven example topologies to be analyzed and compared. Their properties can be seen in Table 3.9. All topologies have the same volume of material, $0.89070 \mathrm{~cm}^{3}$. The same boundary conditions as the previous two sections were used to eliminate rigid

Table 3.9: Topological Data for Unequal Biaxial Loaded Plate Examples

\begin{tabular}{|c|c|c|c|c|}
\hline Topology & Number of Elements & Avg. Length (L) & Cross Section (A) & L/A \\
\hline Coarse & 1800 & 0.3422 & 0.0380 & 9.0000 \\
\hline Medium & 4076 & 0.2247 & 0.0312 & 7.2046 \\
\hline Fine & 14736 & 0.1169 & 0.0227 & 5.1384 \\
\hline Random 1 & 4076 & 0.2310 & 0.0308 & 7.5116 \\
\hline Random 2 & 4076 & 0.2285 & 0.0309 & 7.3870 \\
\hline Equal Medium & 1892 & 0.3723 & 0.0356 & 10.4697 \\
\hline Uniaxial Medium & 5072 & 0.2006 & 0.0296 & 6.7799 \\
\hline
\end{tabular}

body motion. The coarse, medium and fine topologies, shown in Figure 3.50, will be compared to see if the size of the element has any affect and the random topologies will be compared to all the medium topologies to see if the stress trajectory microstructure produces the lowest stresses. The coarse topology has primary stress 
trajectories every 0.3 along the y axis and secondary stress trajectories every 0.3 along the $\mathrm{x}$ axis and every 15 degrees around the hole. The medium topology has primary trajectories every 0.2 along the $\mathrm{y}$ axis and secondary trajectories every 0.2 along the $\mathrm{x}$ axis and every 10 degrees around the hole. The fine topology has primary trajectories every 0.1 along the $\mathrm{y}$ axis and secondary trajectories every 0.1 along the $\mathrm{x}$ axis and every 5 degrees along the hole.

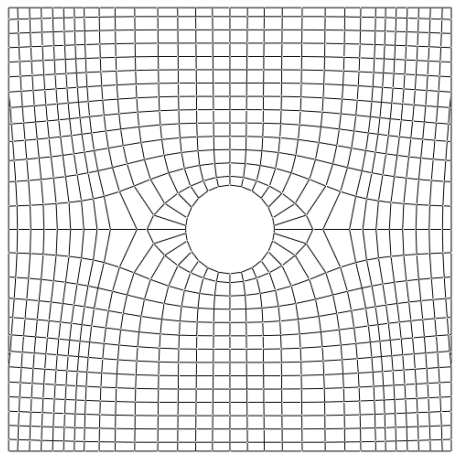

a) Coarse Topology

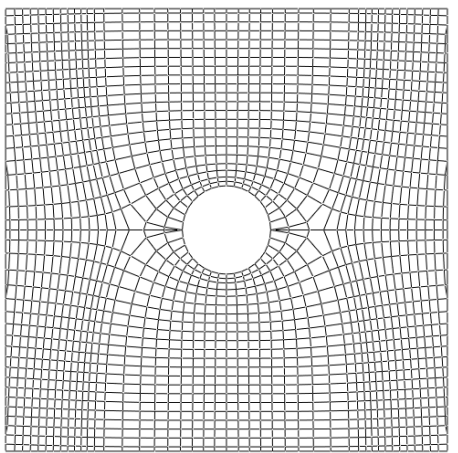

b) Medium Topology

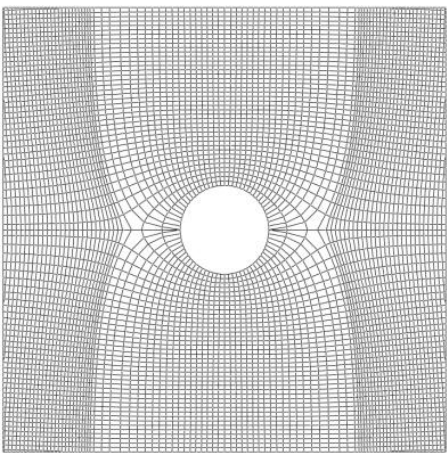

c) Fine Topology

Figure 3.50: Coarse, Medium and Fine Topology of Unequal Biaxial Loaded Plate

To generate the random topologies, the intersecting points of the medium topology were allowed to move $0.05 \mathrm{~cm}$ in any direction. The boundary points were only allowed to move along the boundary and the points around the hole were held in place. The random topologies can be seen in Figure 3.51 and will be compared to medium topologies of each problem. 


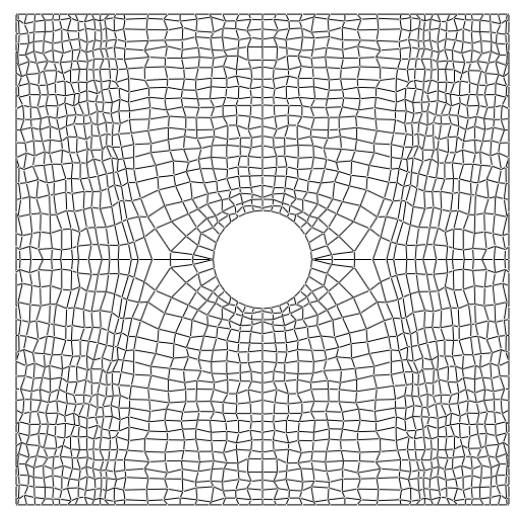

a) Random 1

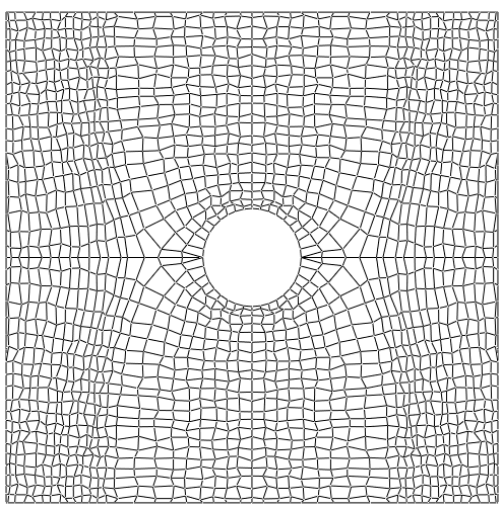

b) Random 2

Figure 3.51: Random Topologies for Unequal Biaxial Loaded Plate

\subsubsection{Abaqus Simulations and Results}

The coarse, medium and fine topologies were examined by looking at the stress distributions in each topology and the top ten percent of the stresses. Taking a look at the distribution of the stresses in the actual topology allows for a visual representation of where the highest stresses are located. In Figures 3.52-54, the stresses are color mapped using Abaqus. It can be seen that the in the all three topologies the majority of the stress is about the same, $55 \mathrm{kPa}$. In the coarse and fine topologies the maximum stress is near the hole on the elliptical stress trajectory surrounding the hole. The highest stress in the medium topology is on the left and right boundary in small elements. This location of higher stress is also found in the fine topology. In the fine topology there are more higher level stressed nodes than the medium and coarse topologies. Overall all three topologies are similar with the medium topology slightly better than the others. 

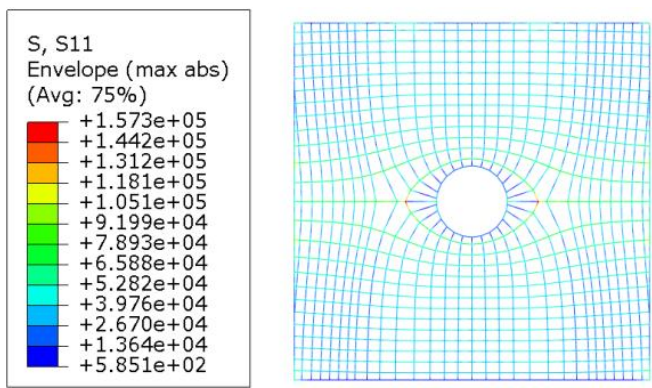

Figure 3.52: Abaqus Stresses in Coarse Topology
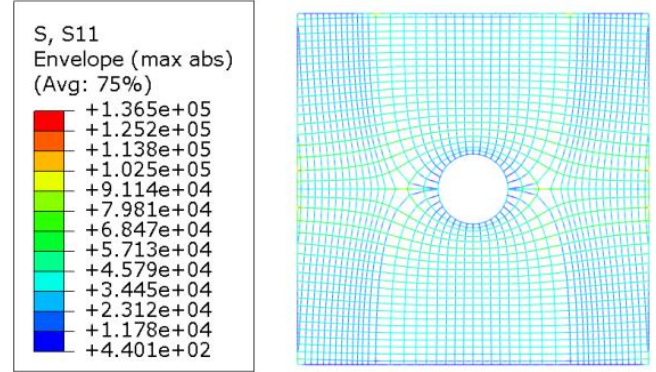

Figure 3.53: Abaqus Stresses in Medium Topology

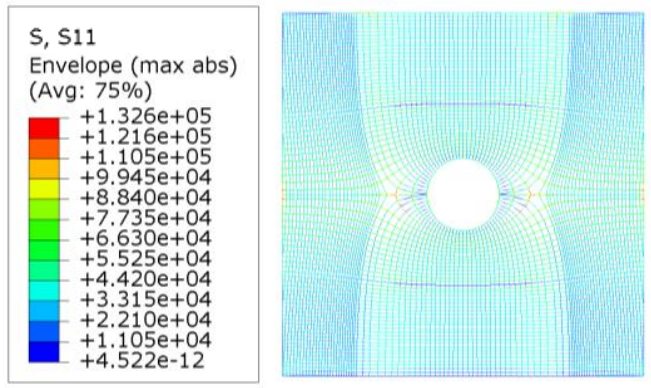

Figure 3.54: Abaqus Stresses in Fine Topology

Comparing the frequency plots of the three topologies in Figures 3.55-57 it can be seen that the general shape of the stress distribution is the same in all the topologies. They all build up slowly to the peak concentration at about $50 \mathrm{kPa}$. then rapidly decrease to a maximum stress value around $125 \mathrm{kPa}$. Table 3.10 lists the maximum, minimum and average stresses, and indicates that the three topologies give very similar results.

Table 3.10: Maximum, Minimum and Average Stresses for the Three Topologies

\begin{tabular}{|c|c|c|c|}
\hline Topology & Max. Stress (kPa.) & Min Stress (kPa.) & Avg. Stress (kPa.) \\
\hline Coarse & 120.1 & .585 & 37.7 \\
\hline Medium & 136.5 & .440 & 36.4 \\
\hline Fine & 126.7 & 0 & 36.3 \\
\hline
\end{tabular}




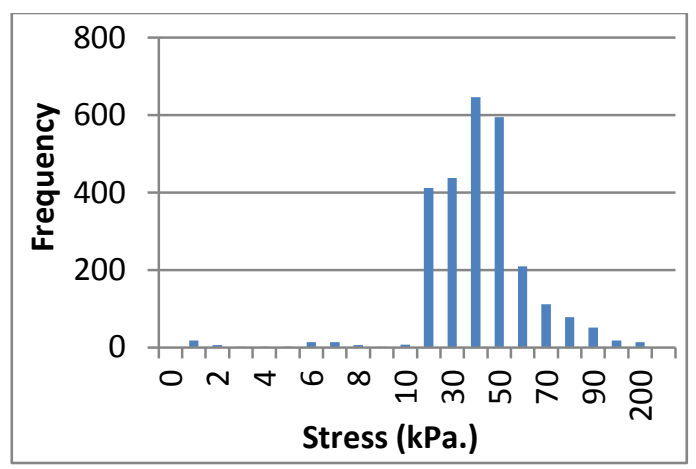

Figure 3.55: Stress Distribution in Coarse Topology

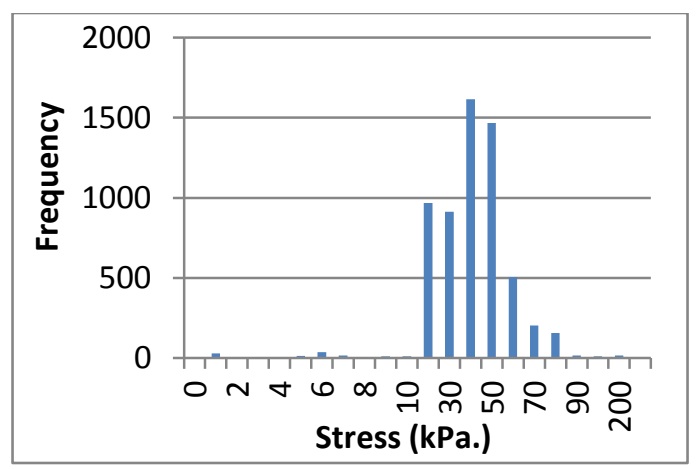

Figure 3.56: Stress Distribution in Medium Topology

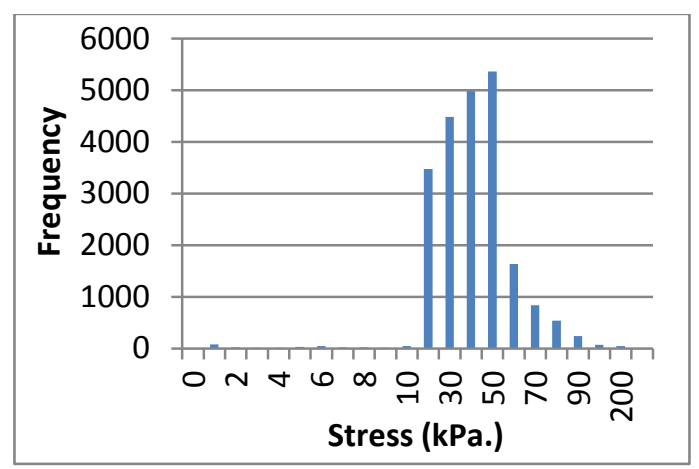

Figure 3.57: Stress Distribution in Fine Topology

Looking at the top ten percent of the stresses allows a different examination of the stress. In the three topologies the top ten percent of the stresses are similar like the overall stress. The average stresses in the top ten percent are 61,56 and $56 \mathrm{kPa}$. for the coarse, medium, and fine topologies respectively. This shows that the three topologies are relatively the same and the size of the elements does not significantly affect the stress distribution. The medium topology is slightly better than other two.

In addition to looking at the size of the element, the topology itself was examined. This was done by comparing the medium ST topology to the random, equal and uniaxial topologies. It is important to look at the distribution of the stress in the topologies in addition to just the frequency plots themselves. When looking at the 
five topologies in Figures 3.58-62, they have significant difference between them but also appear to have some similarities. The five topologies all have the highest stresses in small areas along the left and right boundaries, however, the random 2 and uniaxial topologies also have areas of high stress near or around the hole. There is also a difference in the level of stress the majority of the nodes. The equal topology has the majority of its stress around $210 \mathrm{kPa}$., the random topologies are around $75 \mathrm{kPa}$. and the medium and uniaxial topologies are mostly around $50 \mathrm{kPa}$. The major difference between the two topologies is the stresses found on the hole in the center of the plate. Looking at the medium topology the stresses around the hole are around $10 \mathrm{kPa}$. while in the uniaxial topology the stresses around the hole are between 100 and $150 \mathrm{kPa}$. 

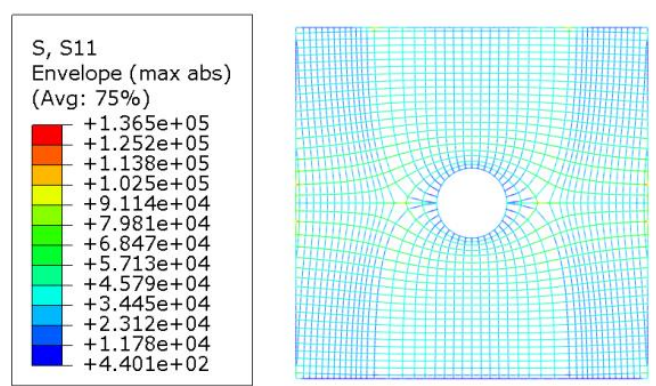

Figure 3.58: Stresses in Medium

Topology
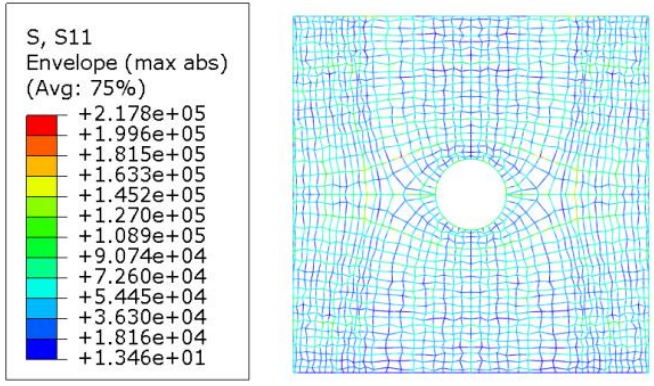

Figure 3.60: Stresses in Random 2 Topology
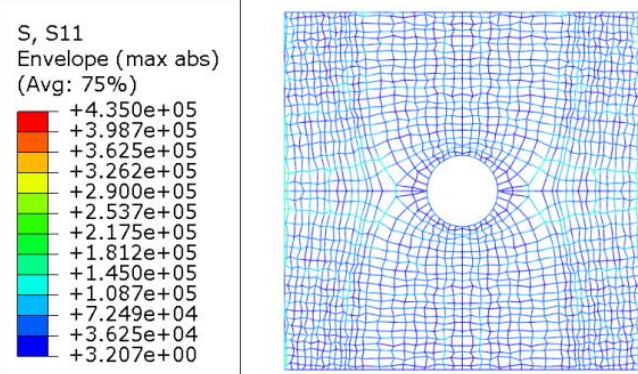

Figure 3.59: Stresses in Random 1

Topology
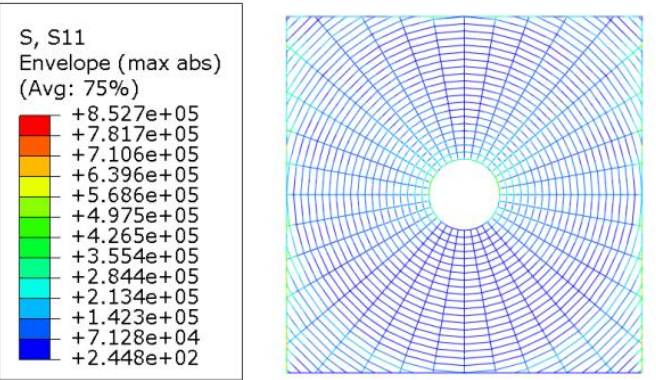

Figure 3.61: Stresses in Equal Topology
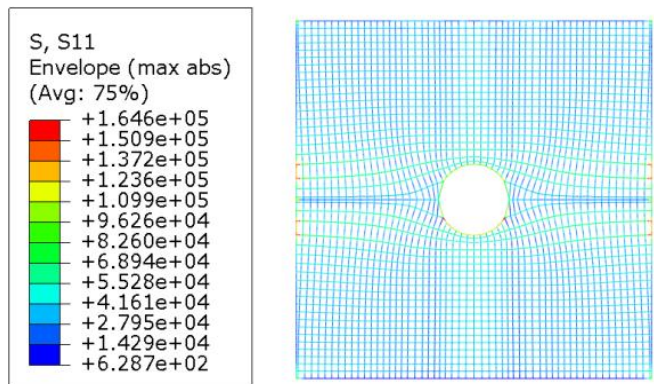

Figure 3.62: Stresses in Uniaxial Topology

When looking at the frequency plots, seen in Figure 3.63, the topologies are somewhat similar except for the equal topology. They all rise to a peak concentration around $40 \mathrm{kPa}$. and then quickly decrease. The random topologies decrease less rapidly as the medium and uniaxial topologies. The equal topology has the greatest maximum stress at $749 \mathrm{kPa}$. while the medium topology has the lowest at $136.5 \mathrm{kPa}$. The medium topology has the lowest average stress of $36 \mathrm{kPa}$. and the uniaxial topology is close at $37 \mathrm{kPa}$. The rest of the statistics of the topologies can be seen in 
Table 3.11. Looking at these stress results indicates that the medium topology is the best at reducing overall stresses. It is also clear that the equal topology is the worst of five topologies.

Table 3.11: Maximum, Minimum and Average Stresses for the Five Topologies

\begin{tabular}{|c|c|c|c|}
\hline Topology & Maximum Stress (kPa.) & Minimum Stress (kPa.) & Average Stress (kPa.) \\
\hline Medium & 136.5 & .440 & 36 \\
\hline Random 1 & 270 & .003 & 52 \\
\hline Random 2 & 174 & .013 & 49.6 \\
\hline Equal & 749 & .244 & 102 \\
\hline Uniaxial & 162 & .628 & 37 \\
\hline
\end{tabular}

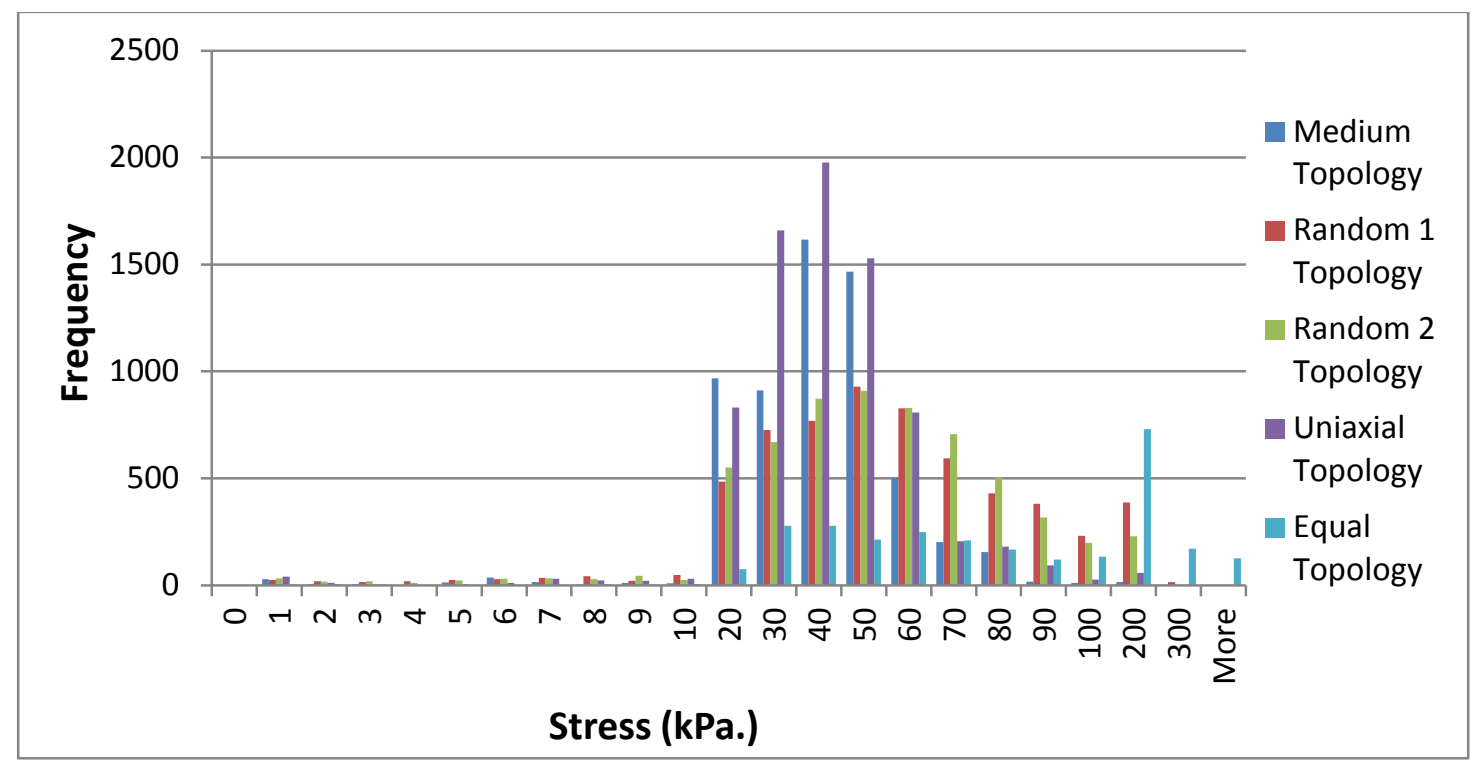

Figure 3.63: Stress Frequency Distribution of the Five Topologies

Overall, like the other two plate problems, it is apparent that the medium topology is the best of the five at handling the applied load. It has the lowest maximum stress and average stress. The uniaxial topology is similar to the medium topology except the hole in the uniaxial case experienced ten times the stress to that in the medium topology. 


\section{Summary, Conclusions and Recommendations}

\subsection{Summary}

A basic computational study of stresses in cellular topologies using stress trajectories (ST) for different problems has been presented. The advantages of cellular solid's mechanical properties like strength to weight ratio and high energy absorption has led them to be a desired material for applications in aerospace industries. The most common cellular material is aluminum foams such as Alporas and Duocel. These foams are commercially available but their expense compared to standard engineering material limits their general use. In recent years the price has been dropping due to improved techniques in manufacturing. An advantage of cellular material is the ability to customize cell size, cell wall thicknesses and the relative density to obtain a customer's desired attributes.

In order to study highly complex and irregular cellular materials, numerical methods have been developed to generate the material model and to conduct finite element analysis. In this study, the stress trajectory topologies were generated using a specially developed MATLAB routine. The stress trajectories were generated by integrating the ordinary differential equations (1.10) and (1.11) to form a wire frame mesh. The intersecting points were then found and inputted into Abaqus to be recreated for simulation. In principle these cellular topologies are applicable to two and three dimensions, but in this study only two dimensional topologies were studied. The topologies used the properties of aluminum in simulation and each element had a square cross section. To be able to compare the topologies for each problem the total material volume was held constant. 
MATLAB software and a commercially available finite element package Abaqus were used to generate and simulate all cellular topologies in this study. There were seven problems, seen in Figures 1.16-22, investigated in this study. In the three beam problems and the disk problem the effect of the topology was investigated. To investigate this the ST topology was compared to a uniform topology for each problem. In the three plate problems, the effects of topology and the size of the elements were investigated. The investigation into the effect of cell size of the material was done by creating three ST topologies for each problem, a coarse, medium and fine topology. To investigate the effect of the topologies, the medium ST topology was compared to two random meshes, and two other ST medium topologies under the same loading conditions. The topologies were compared by looking at the nodal stresses across the topologies, the frequency of the stresses, and the top ten percent of the stress distribution. Although the stress trajectories topologies were only compared to a limited number of alternate controlled topologies it can be assumed that the same results will occur when comparing to other control topologies.

\subsection{Conclusions}

In the investigation into the effect of the topologies in the beam and disk problems it was found the ST topology outperformed the uniform control topologies. In all four problems the maximum stress was less in the ST topology than the uniform topology. The ST topology also had a lower average stress and the concentration of stress was at a lower value. The disk problem was the only case where the majority of the stress was at a lower level in the control topology. However, the maximum stress 
was about six times greater and the average of the top ten percent was three times greater than the ST topology. Overall in these four problems, the stress trajectory topology was better than the control topology.

For the plate problems, it was found that the size of the elements in the ST topologies does not affect the performance of the topology. In the equal biaxial loaded plate, the maximum and average of the three topologies (coarse, medium and fine) were all very close. All three topologies also distributed the stress the same with the majority of the elements around $20 \mathrm{kPa}$. When looking at the top ten percent of the stresses the results are even more similar. The averages of the top ten percent for the coarse, medium and fine topologies were 44,47 and $40 \mathrm{kPa}$. respectively. In the case of the uniaxial loaded plate it was found that all three topologies (coarse, medium and fine) the majority of the nodes experiences stress between 10 and $30 \mathrm{kPa}$. The average stress for the entire topology and the top ten percent of the stresses was relatively the same for each topology. For the unequal loaded plate it was found that the coarse, medium and fine topologies were similar. The average stress of the three topologies was within $1.4 \mathrm{kPa}$. of one another. When looking at the top ten percent of the stresses the same trend was found.

In looking into the effect of the topologies in the plate problems it was found that the medium ST topologies for the given problem were better than the random and medium topologies of the other alternate problems. A summary of the maximum and average stresses for each topology can be seen in Table 4.1. In the case of the equal biaxial loaded plate the unequal and uniaxial medium topologies had a lower maximum stress than the rest but the maximum stress was located on the holes 
compared to the very low stress on the hole in the medium ST mesh. The random topologies were similar to the medium ST topology except with values $10-30 \mathrm{kPa}$. higher. In the uniaxial loaded plate the medium ST topology had a lower maximum stress than the other topologies while the unequal medium topology had the lowest average stress. Like in the equal biaxial case, the unequal and equal topologies had the highest stresses on the hole while the maximum stress in the medium and random topologies were in small elements along the left boundary. In the case of the unequal biaxial loaded plate, the medium ST topology had a lower maximum and average stress than the other topologies. The uniaxial topology had the second lowest maximum and average stresses with the average stress just $1 \mathrm{kPa}$ less than the medium ST topology. As seen in the other cases, the equal and uniaxial topologies experienced much higher stresses on the hole than the medium ST topology. The hole in the medium ST topology had stresses around $10 \mathrm{kPa}$, while the uniaxial topology experienced stresses ranging from $100-150 \mathrm{kPa}$.

Overall in every problem the stress trajectory topologies had more desirable stress distributions than the alternate control topologies. They lowered the maximum and average stresses while carrying the stresses in a more distributed way. It was also found that the size of the element in the stress trajectory topologies does not play a significant role in the stresses values. One will also be able to use finite element analysis to get the stress field from a continuum problem and then generate the stress trajectories for that given problem. 
Table 4.1: Maximum and Average Stress for the Each Problem

\begin{tabular}{|c|c|c|c|}
\hline Problem & Topology & Maximum Stress (kPa.) & Average Stress (kPa.) \\
\hline \multirow{2}{*}{ Cantilever } & Along S.T. & 772 & 97.2 \\
\hline & Control & 2,377 & 312.4 \\
\hline \multirow{2}{*}{$\begin{array}{c}\text { Simply Supported } \\
\text { Elasticity }\end{array}$} & Along S.T. & 1,023 & 91.3 \\
\hline & Control & 1,423 & 316.2 \\
\hline \multirow{2}{*}{$\begin{array}{c}\text { Simply Supported } \\
\text { SM } \\
\end{array}$} & Along S.T. & 1,024 & 81.3 \\
\hline & Control & 1,163 & 248.7 \\
\hline \multirow{2}{*}{ Disk } & Along S.T. & 1,341 & 469.5 \\
\hline & Control & 8,086 & 653.5 \\
\hline \multirow{7}{*}{$\begin{array}{l}\text { Equal Biaxial } \\
\text { Compression }\end{array}$} & Along S.T. (coarse) & 76 & 15.9 \\
\hline & Along S.T. (medium) & 95 & 15.5 \\
\hline & Along S.T. (fine) & 115 & 13.3 \\
\hline & Random 1 & 140 & 15.1 \\
\hline & Random 2 & 144 & 17.0 \\
\hline & Uniaxial Medium & 60 & 11.0 \\
\hline & Unequal Medium & 48 & 12.6 \\
\hline \multirow{7}{*}{ Uniaxial Compression } & Along S.T. (coarse) & 113 & 18.1 \\
\hline & Along S.T. (medium) & 97 & 17.7 \\
\hline & Along S.T. (fine) & 62 & 13.2 \\
\hline & Random 1 & 140 & 28.0 \\
\hline & Random 2 & 108 & 28.2 \\
\hline & Equal Medium & 572 & 84.8 \\
\hline & Unequal Medium & 42.8 & 11.3 \\
\hline \multirow{7}{*}{$\begin{array}{l}\text { Unequal Biaxial } \\
\text { Compression }\end{array}$} & Along S.T. (coarse) & 120 & 37.7 \\
\hline & Along S.T. (medium) & 136 & 36.4 \\
\hline & Along S.T. (fine) & 127 & 36.3 \\
\hline & Random 1 & 270 & 52.3 \\
\hline & Random 2 & 174 & 49.6 \\
\hline & Equal Medium & 749 & 101.9 \\
\hline & Uniaxial Medium & 162 & 37.3 \\
\hline
\end{tabular}

\subsection{Recommendations}

One weakness of this study is large variation of the lengths of each cell wall in the ST topologies. The topologies included some elements with lengths around $0.5 \mathrm{~cm}$ and some elements of length around 0.05 and smaller. To simplify the simulation each cell wall was given the same cross sectional width. This resulted in high stresses in the small elements. This was seen in Figure 3.12 where all of the highest stresses in the topology were in the smallest elements along the left and right boundaries. A 
future study could be done using a way to automate the cell wall thicknesses to keep the $l / a$ ratio consistent throughout the cellular system.

Another recommendation that would benefit this field is the addition of including buckling behavior of the cell side walls. Often in cellular materials cell walls are very thin, and so it is certainly reasonable to expect buckling when loaded in compression. It has been observed that buckling is the major failure mode in cellular materials when loaded in compression (Ashby \& Gibson 1997). Allowing for inelastic behavior of cell side walls would also more accurately predict failure and illustrate additional mechanisms in cellular materials better than the current study. Either using perfectly plastic or a hardening rule could be applied to the localized strain in each cell sidewall. This would require a significantly larger capacity computer to simulate loaded models in feasible way. This could be accomplished in Abaqus using a time dependent loading and more complex finite elements.

Although the stress trajectories appear to be accurate when compared to known stress trajectories, a more accurate generation of stress trajectories can be studied. In generating the stress trajectories using ode45 in MATLAB there were some problems. One problem was the stress trajectories would jump if the spatial increment was too small. Another problem that arose and was later resolved was that stress trajectories would rapidly drop at the beginning of the generation when they had zero slope. More accurate stress trajectories could be generated if the slope limitation condition in section 2.1.3 did not need to be included to fix the stress trajectories. Also a way to keep the orthogonality at the intersecting points would produce more accurate results with less errors. 
Additional studies can be done to compare stress trajectory topologies to other cellular topologies such as Voronoi and triangular topologies. Also additional studies could investigate other boundary conditions and compare the results. 


\section{APPENDIX}

\section{A. MATLAB Codes}

\section{Cantilever Beam:}

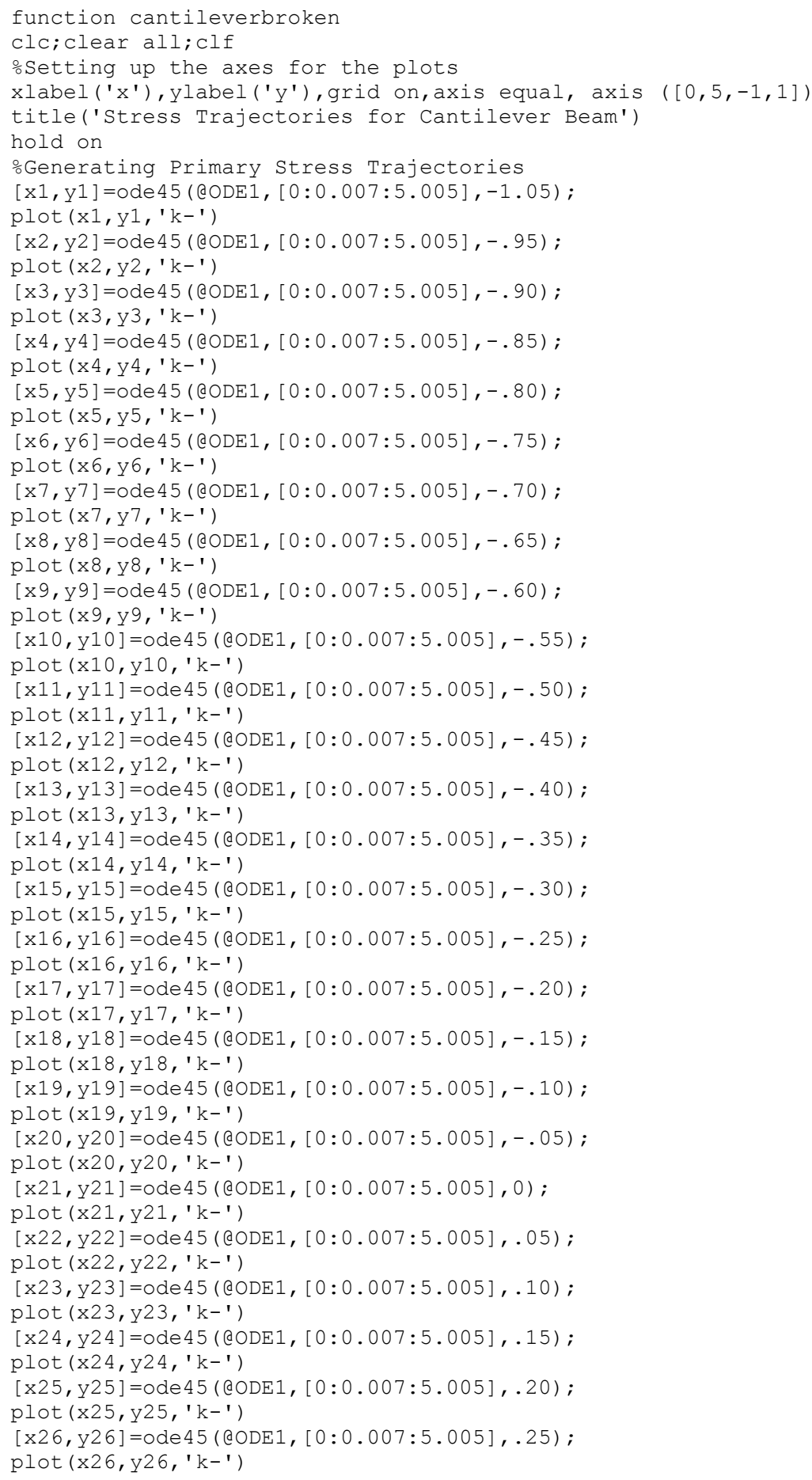


$[\mathrm{x} 27, \mathrm{y} 27]=0 \mathrm{de} 45(@ \operatorname{ODE} 1,[0: 0.007: 5.005], .30) ;$ plot $(x 27, y 27, ' k-')$

$[\mathrm{x} 28, \mathrm{y} 28]=0 \mathrm{de} 45($ @ODE1 $,[0: 0.007: 5.005], .35)$; plot $(x 28, y 28, ' k-')$

$[\mathrm{x} 29, \mathrm{y} 29]=0 \mathrm{de} 45($ @ODE1, $[0: 0.007: 5.005], .40)$; plot $(x 29, y 29, ' k-')$

$[\mathrm{x} 30, \mathrm{y} 30]=0 \mathrm{de} 45(\mathrm{QODE} 1,[0: 0.007: 5.005], .45)$;

plot (x30, y30, ' $k-1)$

$[\mathrm{x} 31, \mathrm{y} 31]=0 \mathrm{de} 45(\varrho \mathrm{ODE} 1,[0: 0.007: 5.005], .50)$;

plot $(x 31, y 31, ' k-')$

$[\mathrm{x} 32, \mathrm{y} 32]=0 \mathrm{de} 45($ @ODE1 $,[0: 0.007: 5.005], .55)$;

plot $\left(x 32, y 32, ' \mathrm{k}-{ }^{\prime}\right)$

$[\mathrm{x} 33, \mathrm{y} 33]=0 \mathrm{de} 45(\mathrm{\varrho ODE} 1,[0: 0.007: 5.005], .60)$;

plot (x33,y33, 'k-')

\%Generating Secondary Stress Trajectories

$[\mathrm{x} 34, \mathrm{y} 34]=0 \mathrm{de} 45(\mathrm{QODE} 2,[0: 0.007: 5.005], 1.05)$;

plot $\left(\mathrm{x} 34, \mathrm{y} 34, \mathrm{~b}--^{\prime}\right)$

$[x 35, y 35]=0$ de4 $5(\operatorname{QODE} 2,[0: 0.007: 5.005], .95)$;

plot $\left(\mathrm{x} 35, \mathrm{y} 35, \mathrm{~b}--^{\prime}\right)$

$[x 36, y 36]=0$ de $45(@ O D E 2,[0: 0.007: 5.005], .90)$;

plot $\left(x 36, y 36, ' b--^{\prime}\right)$

$[\mathrm{x} 37, \mathrm{y} 37]=0 \mathrm{de} 45(\mathrm{CODE} 2,[0: 0.007: 5.005], .85)$;

plot $\left(x 37, y 37,{ }^{\prime} b--^{\prime}\right)$

$[\mathrm{x} 38, \mathrm{y} 38]=0 \mathrm{de} 45(\varrho \mathrm{ODE} 2,[0: 0.007: 5.005], .80)$;

plot $\left(x 38, y 38, ' b--^{\prime}\right)$

$[\mathrm{x} 39, \mathrm{y} 39]=0 \mathrm{de} 45(\varrho \mathrm{ODE} 2,[0: 0.007: 5.005], .75)$;

plot $\left(\mathrm{x} 39, \mathrm{y} 39, \mathrm{\prime}^{\prime} \mathrm{b--}\right)$

$[\mathrm{x} 40, \mathrm{y} 40]=0 \mathrm{de} 45(\mathrm{QODE} 2,[0: 0.007: 5.005], .70)$;

plot $\left(\mathrm{x} 40, \mathrm{y} 40, \mathrm{~b}-\mathrm{-}^{\prime}\right)$

$[\mathrm{x} 41, \mathrm{y} 41]=0 \mathrm{de} 45(@ \mathrm{ODE} 2,[0: 0.007: 5.005], .65)$;

plot $\left(\mathrm{x} 41, \mathrm{y} 41, \mathrm{~b}--^{\prime}\right)$

$[\mathrm{x} 42, \mathrm{y} 42]=0 \mathrm{de} 45(\mathrm{\varrho ODE} 2,[0: 0.007: 5.005], .60)$;

plot $\left(\mathrm{x} 42, \mathrm{y} 42, \mathrm{~b}-\mathrm{-}^{\prime}\right)$

$[\mathrm{x} 43, \mathrm{y} 43]=0 \mathrm{de} 45($ @ODE2 $,[0: 0.007: 5.005], .55)$;

plot $\left(\mathrm{x} 43, \mathrm{y} 43, \mathrm{~b}-\mathrm{-}^{\prime}\right)$

$[\mathrm{x} 44, \mathrm{y} 44]=0 \mathrm{de} 45($ @ODE2 $,[0: 0.007: 5.005], .50)$;

plot $\left(\mathrm{x} 44, \mathrm{y} 44, \mathrm{~b}^{\left.\mathrm{b}-\mathrm{-}^{\prime}\right)}\right.$

$[\mathrm{x} 45, \mathrm{y} 45]=0 \mathrm{de} 45(@ \mathrm{ODE} 2,[0: 0.007: 5.005], .45)$;

plot $\left(\mathrm{x} 45, \mathrm{y} 45, \mathrm{~b}--^{\prime}\right)$

$[\mathrm{x} 46, \mathrm{y} 46]=0 \mathrm{de} 45(\operatorname{eOdE} 2,[0: 0.007: 5.005], .40)$;

plot $(\mathrm{x} 46, \mathrm{y} 46, \mathrm{~b} \mathrm{b--1})$

$[\mathrm{x} 47, \mathrm{y} 47]=0 \mathrm{de} 45(\mathrm{\varrho ODE} 2,[0: 0.007: 5.005], .35)$;

plot $(x 47, y 47, ' b--')$

$[\mathrm{x} 48, \mathrm{y} 48]=0 \mathrm{de} 45(\mathrm{\varrho ODE} 2,[0: 0.007: 5.005], .30)$;

plot $(x 48, y 48, ' b--')$

$[\mathrm{x} 49, \mathrm{y} 49]=0 \mathrm{de} 45(\mathrm{QODE} 2,[0: 0.007: 5.005], .25)$;

plot $\left(x 49, y 49, ' b--^{\prime}\right)$

$[\mathrm{x} 50, \mathrm{y} 50]=0 \mathrm{de} 45(\mathrm{QODE} 2,[0: 0.007: 5.005], .20)$;

plot $\left(\mathrm{x} 50, \mathrm{y} 50, \mathrm{~b}^{\left.\mathrm{b}-\mathrm{-}^{\prime}\right)}\right.$

$[\mathrm{x} 51, \mathrm{y} 51]=0 \mathrm{de} 45(\operatorname{QODE} 2,[0: 0.007: 5.005], .15)$;

plot (x51, y51, 'b--')

$[\mathrm{x} 52, \mathrm{y} 52]=0 \mathrm{de} 45(\mathrm{QODE} 2,[0: 0.007: 5.005], .10)$;

plot $\left(x 52, y 52,{ }^{\prime} b--^{\prime}\right)$

$[\mathrm{x} 53, \mathrm{y} 53]=0 \mathrm{de} 45(\mathrm{QODE} 2,[0: 0.007: 5.005], .05)$;

plot (x53,y53, 'b--')

$[\mathrm{x} 54, \mathrm{y} 54]=0 \mathrm{de} 45(@ \mathrm{ODE} 2,[0: 0.007: 5.005], 0)$;

plot $\left(\mathrm{x} 54, \mathrm{y} 54, \mathrm{~b}^{\left.\mathrm{b}--^{\prime}\right)}\right.$

$[\mathrm{x} 55, \mathrm{y} 55]=0 \mathrm{de} 45(\mathrm{@ODE} 2,[0: 0.007: 5.005],-.05)$;

plot $\left(\mathrm{x} 55, \mathrm{y} 55, \mathrm{~b}-\mathrm{-}^{\prime}\right)$

$[\mathrm{x} 56, \mathrm{y} 56]=0 \mathrm{de} 45(@ \mathrm{ODE} 2,[0: 0.007: 5.005],-.10)$;

plot $\left(x 56, y 56, ' b--^{\prime}\right)$

$[\mathrm{x} 57, \mathrm{y} 57]=0 \mathrm{de} 45(\mathrm{\varrho ODE} 2,[0: 0.007: 5.005],-.15)$;

plot $\left(\mathrm{x} 57, \mathrm{y} 57, \mathrm{~b}--^{\prime}\right)$

$[\mathrm{x} 58, \mathrm{y} 58]=0 \mathrm{de} 45(\mathrm{QODE} 2,[0: 0.007: 5.005],-.20)$;

plot $\left(\mathrm{x} 58, \mathrm{y} 58, \mathrm{~b}--^{\prime}\right)$

$[\mathrm{x} 59, \mathrm{y} 59]=0 \mathrm{de} 45(\mathrm{\varrho ODE} 2,[0: 0.007: 5.005],-.25)$;

plot $(x 59, y 59, ' b--')$

$[\mathrm{x} 60, \mathrm{y} 60]=0 \mathrm{de} 45(\mathrm{QODE} 2,[0: 0.007: 5.005],-.30)$;

plot $(x 60, y 60, ' b--1)$

$[\mathrm{x} 61, \mathrm{y} 61]=0 \mathrm{de} 45(\mathrm{QODE} 2,[0: 0.007: 5.005],-.35)$;

plot $\left.\left(x 61, y 61, b^{--}\right)^{\prime}\right)$ 
$[\mathrm{x} 62, \mathrm{y} 62]=0 \mathrm{de} 45(\mathrm{QODE} 2,[0: 0.007: 5.005],-.40) ;$

plot $(x 62, y 62, ' b--')$

$[\mathrm{x} 63, \mathrm{y} 63]=0 \mathrm{de} 45(\mathrm{QODE} 2,[0: 0.007: 5.005],-.45)$;

plot $\left(x 63, y 63,{ }^{\prime} b--^{\prime}\right)$

$[\mathrm{x} 64, \mathrm{y} 64]=0 \mathrm{de} 45(\mathrm{eODE} 2,[0: 0.007: 5.005],-.50)$;

plot $\left(x 64, y 64, ' b--^{\prime}\right)$

$[\mathrm{x} 65, \mathrm{y} 65]=0 \mathrm{de} 45(@ \mathrm{ODE} 2,[0: 0.007: 5.005],-.55)$;

plot $\left(x 65, y 65, ' b--^{\prime}\right)$

$[\mathrm{x} 66, \mathrm{y} 66]=0 \mathrm{de} 45(\mathrm{CODE} 2,[0: 0.007: 5.005],-.60)$;

plot $\left(x 66, y 66, ' b--^{\prime}\right)$

$\times 67=[0: .007: 5.005]^{\prime}$;

$\mathrm{y} 67=\mathrm{x} 67 . * 0+5$;

plot $\left(\mathrm{y} 67, \mathrm{x} 67, \mathrm{k}^{\prime} \mathrm{-}^{\prime}\right)$

plot $\left(y 67,-x 67,{ }^{\prime}-^{\prime}\right)$

oautomatically finding intersecting points

for $j=1: 30$

$m=\left[y_{4}, y 5, y 6, y 7, y 8, y 9, y 10, y 11, y 12, y 13, y 14, y 15, y 16, y 17, y 18, y 19, y 20, y 21, y 22, y 23, y 24, y 2\right.$

$5, y 26, y 27, y 28, y 29, y 30, y 31, y 32, y 33]$

$[A 1(j, 1), A 1(j, 2)]=$ curveintersect $(x 2, m(:, j), x 34, y 34)$

end

for $j=1: 31$

$m=\left[y^{3}, y^{4}, y 5, y 6, y 7, y 8, y 9, y 10, y 11, y 12, y 13, y 14, y 15, y 16, y 17, y 18, y 19, y 20, y 21, y 22, y 23, y 24\right.$

$, \mathrm{y} 25, \mathrm{y} 26, \mathrm{y} 27, \mathrm{y} 28, \mathrm{y} 29, \mathrm{y} 30, \mathrm{y} 31, \mathrm{y} 32, \mathrm{y} 33]$

$[A 2(j, 1), A 2(j, 2)]=$ curveintersect $(x 2, m(:, j), x 35, y 35)$;

end

for $j=1: 32$

$m=\left[y_{2}, y^{3}, y^{4}, y^{5}, y 6, y 7, y 8, y 9, y 10, y 11, y 12, y 13, y 14, y 15, y 16, y 17, y 18, y 19, y 20, y 21, y 22, y 23\right.$, $\mathrm{y} 24, \mathrm{y} 25, \mathrm{y} 26, \mathrm{y} 27, \mathrm{y} 28, \mathrm{y} 29, \mathrm{y} 30, \mathrm{y} 31, \mathrm{y} 32, \mathrm{y} 33]$

end

$[A 3(j, 1), A 3(j, 2)]=$ curveintersect $(x 2, m(:, j), x 36, y 36)$;

for $j=1: 33$

$m=[y 1, y 2, y 3, y 4, y 5, y 6, y 7, y 8, y 9, y 10, y 11, y 12, y 13, y 14, y 15, y 16, y 17, y 18, y 19, y 20, y 21, y 22, y$ $23, \mathrm{y} 24, \mathrm{y} 25, \mathrm{y} 26, \mathrm{y} 27, \mathrm{y} 28, \mathrm{y} 29, \mathrm{y} 30, \mathrm{y} 31, \mathrm{y} 32, \mathrm{y} 33]$

end

$[A 4(j, 1), A 4(j, 2)]=$ curveintersect $(x 2, m(:, j), x 37, y 37)$;

for $j=1: 33$

$m=[y 1, y 2, y 3, y 4, y 5, y 6, y 7, y 8, y 9, y 10, y 11, y 12, y 13, y 14, y 15, y 16, y 17, y 18, y 19, y 20, y 21, y 22, y$ $23, y 24, y 25, y 26, y 27, y 28, y 29, y 30, y 31, y 32, y 33] ;$

$[A 5(j, 1), A 5(j, 2)]=$ curveintersect $(x 2, m(:, j), x 38, y 38)$;

end

for $j=1: 33$

$m=[y 1, y 2, y 3, y 4, y 5, y 6, y 7, y 8, y 9, y 10, y 11, y 12, y 13, y 14, y 15, y 16, y 17, y 18, y 19, y 20, y 21, y 22, y$ $23, y 24, y 25, y 26, y 27, y 28, y 29, y 30, y 31, y 32, y 33]$

end $[A 6(j, 1), A 6(j, 2)]=$ curveintersect $(x 2, m(:, j), x 39, y 39)$;

for $j=1: 33$

$m=\left[y_{1}, y^{2}, y^{3}, y 4, y 5, y 6, y 7, y 8, y 9, y 10, y 11, y 12, y 13, y 14, y 15, y 16, y 17, y 18, y 19, y 20, y 21, y 22, y\right.$ $23, y 24, y 25, y 26, y 27, y 28, y 29, y 30, y 31, y 32, y 33] ;$

end $[A 7(j, 1), A 7(j, 2)]=$ curveintersect $(x 2, m(:, j), x 40, y 40)$;

for $j=1: 33$

$m=[y 1, y 2, y 3, y 4, y 5, y 6, y 7, y 8, y 9, y 10, y 11, y 12, y 13, y 14, y 15, y 16, y 17, y 18, y 19, y 20, y 21, y 22, y$ $23, \mathrm{y} 24, \mathrm{y} 25, \mathrm{y} 26, \mathrm{y} 27, \mathrm{y} 28, \mathrm{y} 29, \mathrm{y} 30, \mathrm{y} 31, \mathrm{y} 32, \mathrm{y} 33]$

end $[\mathrm{A} 8(j, 1), \mathrm{A} 8(j, 2)]=$ curveintersect $(\mathrm{x} 2, \mathrm{~m}(:, j), \mathrm{x} 41, \mathrm{y} 41)$;

for $j=1: 33$

$m=[y 1, y 2, y 3, y 4, y 5, y 6, y 7, y 8, y 9, y 10, y 11, y 12, y 13, y 14, y 15, y 16, y 17, y 18, y 19, y 20, y 21, y 22, y$ $23, \mathrm{y} 24, \mathrm{y} 25, \mathrm{y} 26, \mathrm{y} 27, \mathrm{y} 28, \mathrm{y} 29, \mathrm{y} 30, \mathrm{y} 31, \mathrm{y} 32, \mathrm{y} 33] ;$

end $[A 9(j, 1), A 9(j, 2)]=$ curveintersect $(x 2, m(:, j), x 42, y 42)$;

for $j=1: 32$

$m=[y 1, y 2, y 3, y 4, y 5, y 6, y 7, y 8, y 9, y 10, y 11, y 12, y 13, y 14, y 15, y 16, y 17, y 18, y 19, y 20, y 21, y 22, y$ $23, \mathrm{y} 24, \mathrm{y} 25, \mathrm{y} 26, \mathrm{y} 27, \mathrm{y} 28, \mathrm{y} 29, \mathrm{y} 30, \mathrm{y} 31, \mathrm{y} 32]$

end

$[A 10(j, 1), A 10(j, 2)]=$ curveintersect $(x 2, m(:, j), x 43, y 43)$;

for $j=1: 31$

$m=\left[y_{1}, y^{2}, y^{3}, y 4, y 5, y 6, y 7, y 8, y 9, y 10, y 11, y 12, y 13, y 14, y 15, y 16, y 17, y 18, y 19, y 20, y 21, y 22, y\right.$ $23, y 24, y 25, y 26, y 27, y 28, y 29, y 30, y 31]$

$[\mathrm{A} 11(j, 1), \mathrm{A} 11(j, 2)]=$ Curveintersect $(\mathrm{x} 2, \mathrm{~m}(:, j), \mathrm{x} 44, \mathrm{y} 44) ;$

end

for $j=1: 30$ 
$m=[y 1, y 2, y 3, y 4, y 5, y 6, y 7, y 8, y 9, y 10, y 11, y 12, y 13, y 14, y 15, y 16, y 17, y 18, y 19, y 20, y 21, y 22, y$ $23, \mathrm{y} 24, \mathrm{y} 25, \mathrm{y} 26, \mathrm{y} 27, \mathrm{y} 28, \mathrm{y} 29, \mathrm{y} 30]$

end

$[A 12(j, 1), A 12(j, 2)]=$ Curveintersect $(x 2, m(:, j), x 45, y 45)$;

for $j=1: 29$

$m=[y 1, y 2, y 3, y 4, y 5, y 6, y 7, y 8, y 9, y 10, y 11, y 12, y 13, y 14, y 15, y 16, y 17, y 18, y 19, y 20, y 21, y 22, y$ $23, \mathrm{y} 24, \mathrm{y} 25, \mathrm{y} 26, \mathrm{y} 27, \mathrm{y} 28, \mathrm{y} 29] ;$

end

$[\mathrm{A} 13(j, 1), \mathrm{A} 13(j, 2)]=$ curveintersect $(\mathrm{x} 2, \mathrm{~m}(:, j), \mathrm{x} 46, \mathrm{y} 46)$;

for $j=1: 28$

$m=\left[y_{1}, y^{2}, y^{3}, y^{4}, y 5, y 6, y 7, y 8, y 9, y 10, y 11, y 12, y 13, y 14, y 15, y 16, y 17, y 18, y 19, y 20, y 21, y 22, y\right.$ $23, \mathrm{y} 24, \mathrm{y} 25, \mathrm{y} 26, \mathrm{y} 27, \mathrm{y} 28]$

end

$[A 14(j, 1), A 14(j, 2)]=$ curveintersect $(x 2, m(:, j), x 47, y 47)$;

for $j=1: 27$

$m=[y 1, y 2, y 3, y 4, y 5, y 6, y 7, y 8, y 9, y 10, y 11, y 12, y 13, y 14, y 15, y 16, y 17, y 18, y 19, y 20, y 21, y 22, y$ $23, \mathrm{y} 24, \mathrm{y} 25, \mathrm{y} 26, \mathrm{y} 27]$

end

$[\mathrm{A} 15(j, 1), \mathrm{A} 15(j, 2)]=$ curveintersect $(\mathrm{x} 2, \mathrm{~m}(:, j), \mathrm{x} 48, \mathrm{y} 48)$;

for $j=1: 26$

$m=[y 1, y 2, y 3, y 4, y 5, y 6, y 7, y 8, y 9, y 10, y 11, y 12, y 13, y 14, y 15, y 16, y 17, y 18, y 19, y 20, y 21, y 22, y$ $23, \mathrm{y} 24, \mathrm{y} 25, \mathrm{y} 26] ;$

end

$[A 16(j, 1), A 16(j, 2)]=$ curveintersect $(x 2, m(:, j), x 49, y 49)$;

for $j=1: 25$

$m=[y 1, y 2, y 3, y 4, y 5, y 6, y 7, y 8, y 9, y 10, y 11, y 12, y 13, y 14, y 15, y 16, y 17, y 18, y 19, y 20, y 21, y 22, y$ $23, \mathrm{y} 24, \mathrm{y} 25]$;

end

$[\mathrm{A} 17(j, 1), \mathrm{A} 17(j, 2)]=$ curveintersect $(\mathrm{x} 2, \mathrm{~m}(:, j), \mathrm{x} 50, \mathrm{y} 50)$;

for $j=1: 24$

$m=[y 1, y 2, y 3, y 4, y 5, y 6, y 7, y 8, y 9, y 10, y 11, y 12, y 13, y 14, y 15, y 16, y 17, y 18, y 19, y 20, y 21, y 22, y$ $23, \mathrm{y} 24]$

end

$[A 18(j, 1), A 18(j, 2)]=$ curveintersect $(x 2, m(:, j), x 51, y 51) ;$

for $j=1: 23$

$m=\left[y_{1}, y^{2}, y^{3}, y^{4}, y^{5}, y 6, y 7, y 8, y 9, y 10, y 11, y 12, y 13, y 14, y 15, y 16, y 17, y 18, y 19, y 20, y 21, y 22, y\right.$ 23] ;

end

$[A 19(j, 1), A 19(j, 2)]=$ curveintersect $(x 2, m(:, j), x 52, y 52)$;

for $j=1: 22$

$m=[y 1, y 2, y 3, y 4, y 5, y 6, y 7, y 8, y 9, y 10, y 11, y 12, y 13, y 14, y 15, y 16, y 17, y 18, y 19, y 20, y 21, y 22, y$ $23, y 24, y 25, y 26, y 27, y 28, y 29, y 30, y 31, y 32, y 33] ;$

end

$[\mathrm{A} 20(j, 1), \mathrm{A} 20(j, 2)]=$ curveintersect $(\mathrm{x} 2, \mathrm{~m}(:, j), \mathrm{x} 53, \mathrm{y} 53)$;

for $j=1: 21$

$m=[y 1, y 2, y 3, y 4, y 5, y 6, y 7, y 8, y 9, y 10, y 11, y 12, y 13, y 14, y 15, y 16, y 17, y 18, y 19, y 20, y 21, y 22, y$

$23, \mathrm{y} 24, \mathrm{y} 25, \mathrm{y} 26, \mathrm{y} 27, \mathrm{y} 28, \mathrm{y} 29, \mathrm{y} 30, \mathrm{y} 31, \mathrm{y} 32, \mathrm{y} 33] ;$

$[A 21(j, 1), A 21(j, 2)]=$ curveintersect $(x 2, m(:, j), x 54, y 54) ;$

end

for $j=1: 20$

$m=[y 1, y 2, y 3, y 4, y 5, y 6, y 7, y 8, y 9, y 10, y 11, y 12, y 13, y 14, y 15, y 16, y 17, y 18, y 19, y 20, y 21, y 22, y$

$23, y 24, y 25, y 26, y 27, y 28, y 29, y 30, y 31, y 32, y 33]$;

end

$[\mathrm{A} 22(j, 1), \mathrm{A} 22(j, 2)]=$ curveintersect $(\mathrm{x} 2, \mathrm{~m}(:, j), \mathrm{x} 55, \mathrm{y} 55)$;

for $j=1: 19$

$m=\left[y_{1}, y^{2}, y^{3}, y^{4}, y^{5}, y^{6}, y 7, y 8, y 9, y 10, y 11, y 12, y 13, y 14, y 15, y 16, y 17, y 18, y 19, y 20, y 21, y 22, y\right.$

$23, y 24, y 25, y 26, y 27, y 28, y 29, y 30, y 31, y 32, y 33] ;$

end

$[\mathrm{A} 23(j, 1), \mathrm{A} 23(j, 2)]=$ curveintersect $(x 2, \mathrm{~m}(:, j), \mathrm{x} 56, \mathrm{y} 56)$;

for $j=1: 18$

$m=\left[y_{1}, y^{2}, y^{3}, y^{4}, y_{5}, y 6, y 7, y 8, y 9, y 10, y 11, y 12, y 13, y 14, y 15, y 16, y 17, y 18, y 19, y 20, y 21, y 22, y\right.$

$23, y 24, y 25, y 26, y 27, y 28, y 29, y 30, y 31, y 32, y 33] ;$

end

$[\mathrm{A} 24(j, 1), \mathrm{A} 24(j, 2)]=$ Curveintersect $(\mathrm{x} 2, \mathrm{~m}(:, j), \mathrm{x} 57, \mathrm{y} 57)$;

for $j=1: 17$

$m=[y 1, y 2, y 3, y 4, y 5, y 6, y 7, y 8, y 9, y 10, y 11, y 12, y 13, y 14, y 15, y 16, y 17, y 18, y 19, y 20, y 21, y 22, y$

$23, y 24, y 25, y 26, y 27, y 28, y 29, y 30, y 31, y 32, y 33] ;$

end

$[A 25(j, 1), A 25(j, 2)]=$ curveintersect $(x 2, m(:, j), x 58, y 58)$;

for $j=1: 16$ 


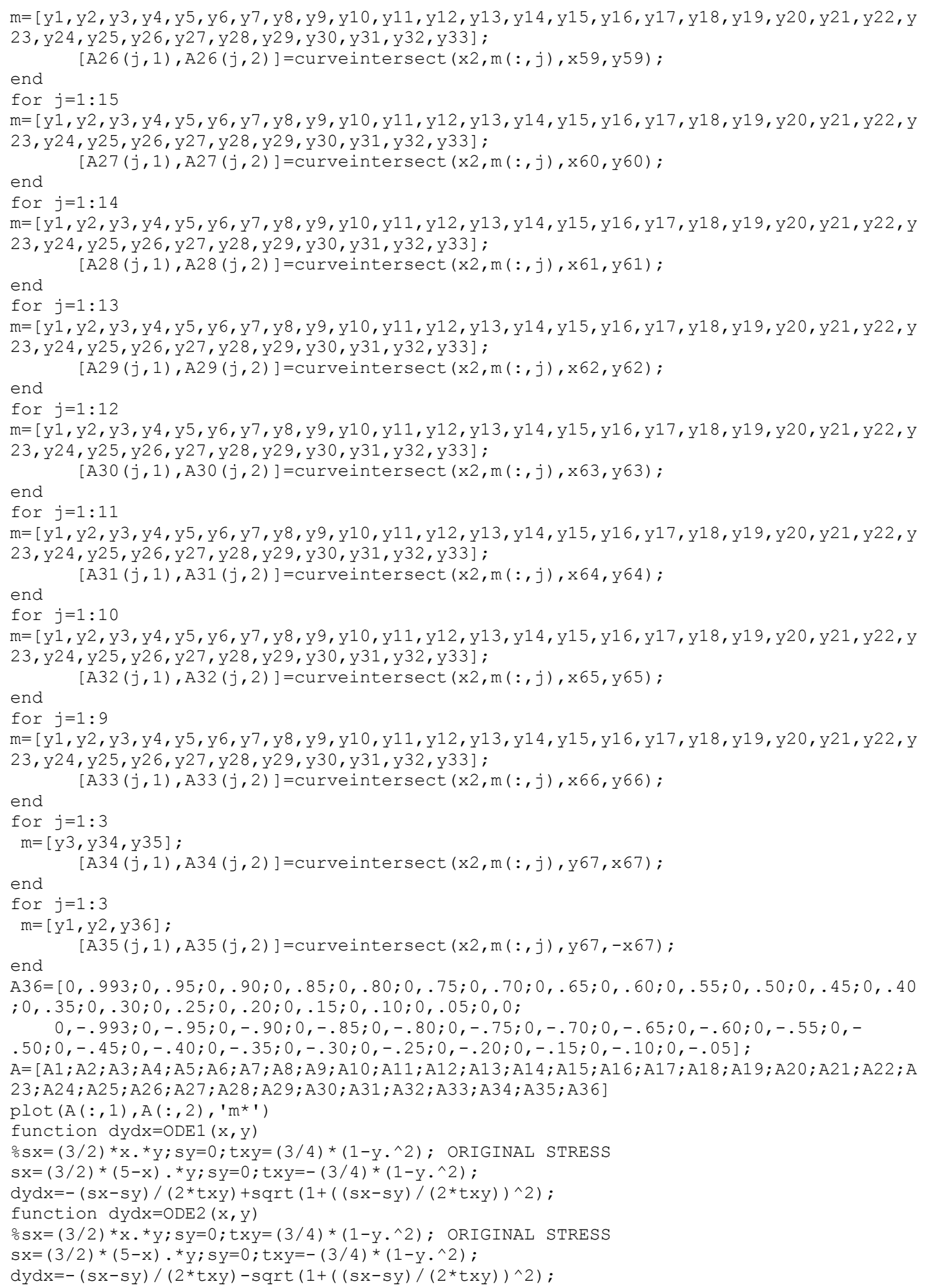

\section{Simply Supported Beam using Elasticity Stresses}

function ELASTICITYSOL

clc;clear all;clf

hold on

xlabel ('x'), ylabel('y'), grid on, axis equal, axis $([0,5,-1,1])$

title('Stress Trajectories Simply Supported Beam Under Uniform Load')

oGenerating the Primary Stress Trajectories 
$[\mathrm{x} 1, \mathrm{y} 1]=0 \mathrm{de} 45(@ 0 D E 1,[0.001: 0.001: 5.001],-1.05) ;$

plot $\left([\mathrm{x} 1,-\mathrm{x} 1],[\mathrm{y} 1, \mathrm{y} 1], \mathrm{k}^{-}\right)$

$[\mathrm{x} 2, \mathrm{y} 2]=0 \operatorname{de} 45(\operatorname{OODE} 1,[0.001: 0.001: 5.001],-0.95)$;

plot $\left([\mathrm{x} 2,-\mathrm{x} 2],[\mathrm{y} 2, \mathrm{y} 2], \mathrm{k}^{-1}\right)$

$[\mathrm{x} 3, \mathrm{y} 3]=0 \mathrm{de} 45(\mathrm{QODE} 1,[0.001: 0.001: 5.001],-0.90)$;

plot $\left([\mathrm{x} 3,-\mathrm{x} 3],[\mathrm{y} 3, \mathrm{y} 3], \mathrm{k}-\mathrm{-}^{\prime}\right)$

$[\mathrm{x} 4, \mathrm{y} 4]=0 \mathrm{de} 45($ @ODE1, $[0.001: 0.001: 5.001],-0.85)$;

plot $([x 4,-x 4],[y 4, y 4], ' k-1)$

$[\mathrm{x} 5, \mathrm{y} 5]=0 \mathrm{de} 45($ @ODE1, $[0.001: 0.001: 5.001],-0.80)$;

plot $([x 5,-x 5],[y 5, y 5], ' k-')$

$[\mathrm{x} 6, \mathrm{y} 6]=0 \mathrm{de} 45($ @ODE1 $,[0.001: 0.001: 5.001],-0.75)$;

plot $\left([x 6,-x 6],[y 6, y 6], ' k^{-1}\right)$

$[\mathrm{x} 7, \mathrm{y} 7]=0 \mathrm{de} 45($ @ODE1, $[0.001: 0.001: 5.001],-0.70)$;

$\operatorname{plot}([x 7,-x 7],[y 7, y 7], ' k-1)$

$[\mathrm{x} 8, \mathrm{y} 8]=0 \mathrm{de} 45(\operatorname{CODE} 1,[0.001: 0.001: 5.001],-0.65) ;$

plot $\left([\mathrm{x} 8,-\mathrm{x} 8],[\mathrm{y} 8, \mathrm{y} 8], \mathrm{k}^{\prime}\right)$

$[\mathrm{x} 9, \mathrm{y} 9]=0 \mathrm{de} 45(\mathrm{QODE} 1,[0.001: 0.001: 5.001],-0.60)$;

plot $([x 9,-x 9],[y 9, y 9], ' k-')$

$[\mathrm{x} 10, \mathrm{y} 10]=0 \mathrm{de} 45(\mathrm{QODE} 1,[0.001: 0.001: 5.001],-0.55)$;

plot $\left([\mathrm{x} 10,-\mathrm{x} 10],[\mathrm{y} 10, \mathrm{y} 10], \mathrm{k}^{-1}\right)$

$[\mathrm{x} 11, \mathrm{y} 11]=0 \mathrm{de} 45(\mathrm{QODE} 1,[0.001: 0.001: 5.001],-0.50)$;

plot $\left([\mathrm{x} 11,-\mathrm{x} 11],[\mathrm{y} 11, \mathrm{y} 11], \mathrm{k}^{-}\right)$

$[\mathrm{x} 12, \mathrm{y} 12]=0 \mathrm{de} 45(\mathrm{QODE} 1,[0.001: 0.001: 5.001],-0.45)$;

plot $\left([\mathrm{x} 12,-\mathrm{x} 12],[\mathrm{y} 12, \mathrm{y} 12], \mathrm{k}^{-\prime}\right)$

$[\mathrm{x} 13, \mathrm{y} 13]=0 \mathrm{de} 45(\operatorname{CODE} 1,[0.001: 0.001: 5.001],-0.40)$;

plot $([x 13,-x 13],[y 13, y 13], ' k-')$

$[\mathrm{x} 14, \mathrm{y} 14]=0 \operatorname{de} 45($ @ODE1, $[0.001: 0.001: 5.001],-0.35)$;

plot $\left([\mathrm{x} 14,-\mathrm{x} 14],[\mathrm{y} 14, \mathrm{y} 14], \mathrm{k}^{-1}\right)$

$[\mathrm{x} 15, \mathrm{y} 15]=0 \mathrm{de} 45(\mathrm{QODE} 1,[0.001: 0.001: 5.001],-0.30)$;

plot $([\mathrm{x} 15,-\mathrm{x} 15],[y 15, \mathrm{y} 15], ' \mathrm{k}-\mathrm{\prime})$

$[\mathrm{x} 16, \mathrm{y} 16]=0 \mathrm{de} 45($ @ODE $1,[0.001: 0.001: 5.001],-0.25)$;

plot $([\mathrm{x} 16,-\mathrm{x} 16],[\mathrm{y} 16, \mathrm{y} 16], 1 \mathrm{k}-1)$

$[\mathrm{x} 17, \mathrm{y} 17]=0 \mathrm{de} 45(\mathrm{QODE} 1,[0.001: 0.001: 5.001],-0.20)$;

plot $\left([\mathrm{x} 17,-\mathrm{x} 17],[y 17, \mathrm{y} 17], \mathrm{k}^{\prime}\right)$

$[\mathrm{x} 18, \mathrm{y} 18]=0 \mathrm{de} 45(\operatorname{CODE} 1,[0.001: 0.001: 5.001],-0.15)$;

plot $\left([\mathrm{x} 18,-\mathrm{x} 18],[\mathrm{y} 18, \mathrm{y} 18], \mathrm{k}^{\prime} \mathrm{-}^{\prime}\right)$

$[\mathrm{x} 19, \mathrm{y} 19]=0 \mathrm{de} 45($ @ODE1, $[0.001: 0.001: 5.001],-0.10)$;

$\operatorname{plot}\left([\mathrm{x} 19,-\mathrm{x} 19],[\mathrm{y} 19, \mathrm{y} 19], \mathrm{k}^{-1}\right)$

$[\mathrm{x} 20, \mathrm{y} 20]=0 \mathrm{de} 45(\mathrm{QODE} 1,[0.001: 0.001: 5.001],-0.05)$;

plot $\left([\mathrm{x} 20,-\mathrm{x} 20],[y 20, y 20], \mathrm{y}^{-\prime}\right)$

oGenerating the Secondary Stress Trajectories

$[\mathrm{x} 21, \mathrm{y} 21]=0 \mathrm{de} 45(@ \mathrm{ODE} 2,[0.001: 0.001: 5.001], 0.00)$;

plot $\left([\mathrm{x} 21,-\mathrm{x} 21],[\mathrm{y} 21, \mathrm{y} 21], \mathrm{k}^{\prime} \mathrm{\prime}\right)$

$[\mathrm{x} 22, \mathrm{y} 22]=0 \mathrm{de} 45(@ \mathrm{ODE} 2,[0.001: 0.001: 5.001], 0.05)$;

plot $\left([\mathrm{x} 22,-\mathrm{x} 22],[\mathrm{y} 22, \mathrm{y} 22], \mathrm{k}^{-\prime}\right)$

$[\mathrm{x} 23, \mathrm{y} 23]=0 \mathrm{de} 45(\mathrm{QODE} 2,[0.001: 0.001: 5.001], 0.10)$;

plot $\left([x 23,-x 23],[y 23, y 23], k^{\prime}{ }^{\prime}\right)$

$[\mathrm{x} 24, \mathrm{y} 24]=0 \operatorname{de} 45(@ \mathrm{ODE} 2,[0.001: 0.001: 5.001], 0.15)$;

$\operatorname{plot}\left([\mathrm{x} 24,-\mathrm{x} 24],[\mathrm{y} 24, \mathrm{y} 24], \mathrm{k}^{-1}\right)$

$[\mathrm{x} 25, \mathrm{y} 25]=0 \mathrm{de} 45(\mathrm{QODE} 2,[0.001: 0.001: 5.001], 0.20)$;

plot $([x 25,-x 25],[y 25, y 25], ' k-')$

$[\mathrm{x} 26, \mathrm{y} 26]=0 \mathrm{de} 45(\mathrm{QODE} 2,[0.001: 0.001: 5.001], 0.25)$;

plot $\left([\mathrm{x} 26,-\mathrm{x} 26],[\mathrm{y} 26, \mathrm{y} 26], \mathrm{k}^{-1}\right)$

$[\mathrm{x} 27, \mathrm{y} 27]=0 \mathrm{de} 45(\mathrm{OODE} 2,[0.001: 0.001: 5.001], 0.30)$;

plot $\left([\mathrm{x} 27,-\mathrm{x} 27],[\mathrm{y} 27, \mathrm{y} 27], ' \mathrm{k}-\mathrm{\prime}^{\prime}\right)$

$[\mathrm{x} 28, \mathrm{y} 28]=0 \mathrm{de} 45(\varrho \mathrm{ODE} 2,[0.001: 0.001: 5.001], 0.35)$;

plot $([x 28,-x 28],[y 28, y 28], ' k-')$

$[\mathrm{x} 29, \mathrm{y} 29]=0 \mathrm{de} 45(\mathrm{QODE} 2,[0.001: 0.001: 5.001], 0.40)$;

plot $([x 29,-x 29],[y 29, y 29], ' k-1)$

$[\mathrm{x} 30, \mathrm{y} 30]=0 \mathrm{de} 45(\mathrm{QODE} 2,[0.001: 0.001: 5.001], 0.45)$;

plot $\left([\mathrm{x} 30,-\mathrm{x} 30],[\mathrm{y} 30, \mathrm{y} 30], \mathrm{k}^{\prime}\right.$ ')

$[\mathrm{x} 31, \mathrm{y} 31]=0 \mathrm{de} 45(\mathrm{QODE} 2,[0.001: 0.001: 5.001], 0.50)$;

plot $\left([\mathrm{x} 31,-\mathrm{x} 31],[\mathrm{y} 31, \mathrm{y} 31], \mathrm{k}^{\prime}\right)$

$[\mathrm{x} 32, \mathrm{y} 32]=0 \mathrm{de} 45(\mathrm{@ODE} 2,[0.001: 0.001: 5.001], 0.55)$;

plot $\left([\mathrm{x} 32,-\mathrm{x} 32],[\mathrm{y} 32, \mathrm{y} 32], \mathrm{k}^{-\prime}\right)$

$[\mathrm{x} 33, \mathrm{y} 33]=0 \mathrm{de} 45(\mathrm{QODE} 2,[0.001: 0.001: 5.001], 0.60)$;

plot $\left([\mathrm{x} 33,-\mathrm{x} 33],[\mathrm{y} 33, \mathrm{y} 33], \mathrm{k}^{-1}\right)$

$[\mathrm{x} 34, \mathrm{y} 34]=0 \mathrm{de} 45(@ \mathrm{ODE} 2,[0.001: 0.001: 5.001], 0.65)$;

plot $\left([\mathrm{x} 34,-\mathrm{x} 34],[\mathrm{y} 34, \mathrm{y} 34], \mathrm{k}^{\prime}\right)$

$[\mathrm{x} 35, \mathrm{y} 35]=0 \mathrm{de} 45(\mathrm{QODE} 2,[0.001: 0.001: 5.001], 0.70)$;

plot $([x 35,-x 35],[y 35, y 35], ' k-')$ 
$[\mathrm{x} 36, \mathrm{y} 36]=0 \mathrm{de} 45(\mathrm{QODE} 2,[0.001: 0.001: 5.001], 0.75) ;$

plot $\left([\mathrm{x} 36,-\mathrm{x} 36],[\mathrm{y} 36, \mathrm{y} 36], \mathrm{k}^{\prime}\right)$

$[\mathrm{x} 37, \mathrm{y} 37]=0 \mathrm{de} 45(\mathrm{QODE} 2,[0.001: 0.001: 5.001], 0.80)$;

plot $\left([\mathrm{x} 37,-\mathrm{x} 37],[\mathrm{y} 37, \mathrm{y} 37], \mathrm{k}^{\prime}\right.$ ')

$[\mathrm{x} 38, \mathrm{y} 38]=0 \mathrm{de} 45(\mathrm{QODE} 2,[0.001: 0.001: 5.001], 0.85)$;

plot $\left([\mathrm{x} 38,-\mathrm{x} 38],[\mathrm{y} 38, \mathrm{y} 38], \mathrm{k}^{\prime}\right)$

$[\mathrm{x} 39, \mathrm{y} 39]=0 \mathrm{de} 45(@ \mathrm{ODE} 2,[0.001: 0.001: 5.001], 0.90)$;

plot $([x 39,-x 39],[y 39, y 39], ' k-1)$

$[\mathrm{x} 40, \mathrm{y} 40]=0 \mathrm{de} 45(\mathrm{QODE} 2,[0.001: 0.001: 5.001], 0.95)$;

plot $\left([\mathrm{x} 40,-\mathrm{x} 40],[\mathrm{y} 40, \mathrm{y} 40], \mathrm{k}^{\prime} \mathrm{\prime}\right)$

$[\mathrm{x} 41, \mathrm{y} 41]=0 \mathrm{de} 45(@ \mathrm{ODE} 2,[0.001: 0.001: 5.001], 1.05)$;

plot $\left([\mathrm{x} 41,-\mathrm{x} 41],[\mathrm{y} 41, \mathrm{y} 41], \mathrm{k}^{-}\right)$

$\mathrm{x} 42=[0: .001: 5]^{\prime}$;

$\mathrm{y} 42=\mathrm{x} 42 . * 0+5$

for $j=1: 16$

$m=[y 21, y 22, y 23, y 24, y 25, y 26, y 27, y 28, y 29, y 30, y 31, y 32, y 33, y 34, y 35, y 36, y 37, y 38, y 39, y 40$, $\mathrm{Y} 41]$;

$[\mathrm{A} 1(j, 1), \mathrm{A} 1(j, 2)]=\mathrm{curveintersect}(\mathrm{x} 1, \mathrm{y} 1, \mathrm{x} 21, \mathrm{~m}(:, j))$;

end

for $j=1: 18$

$m=[y 21, y 22, y 23, y 24, y 25, y 26, y 27, y 28, y 29, y 30, y 31, y 32, y 33, y 34, y 35, y 36, y 37, y 38, y 39, y 40$, $\mathrm{Y} 41]$

$[A 2(j, 1), A 2(j, 2)]=$ curveintersect $(x 2, y 2, x 21, m(:, j))$;

end

for $j=1: 19$

$m=[y 21, y 22, y 23, y 24, y 25, y 26, y 27, y 28, y 29, y 30, y 31, y 32, y 33, y 34, y 35, y 36, y 37, y 38, y 39, y 40$, $\mathrm{y} 41]$

$[A 3(j, 1), A 3(j, 2)]=$ curveintersect $(x 3, y 3, x 21, m(:, j))$;

end

for $j=1: 20$

$m=[y 21, y 22, y 23, y 24, y 25, y 26, y 27, y 28, y 29, y 30, y 31, y 32, y 33, y 34, y 35, y 36, y 37, y 38, y 39, y 40$, $\mathrm{y} 41]$;

$[A 4(j, 1), A 4(j, 2)]=$ curveintersect $(x 4, y 4, x 21, m(:, j))$;

end

for $j=1: 21$

$m=[y 21, y 22, y 23, y 24, y 25, y 26, y 27, y 28, y 29, y 30, y 31, y 32, y 33, y 34, y 35, y 36, y 37, y 38, y 39, y 40$, $\mathrm{y} 41]$

$[A 5(j, 1), A 5(j, 2)]=$ curveintersect $(x 5, y 5, x 21, m(:, j))$;

end

for $j=1: 21$

$m=[y 21, y 22, y 23, y 24, y 25, y 26, y 27, y 28, y 29, y 30, y 31, y 32, y 33, y 34, y 35, y 36, y 37, y 38, y 39, y 40$, $\mathrm{y} 41]$

$[A 6(j, 1), A 6(j, 2)]=$ curveintersect $(x 6, y 6, x 21, m(:, j)) ;$

end

for $j=1: 21$

$m=[y 21, y 22, y 23, y 24, y 25, y 26, y 27, y 28, y 29, y 30, y 31, y 32, y 33, y 34, y 35, y 36, y 37, y 38, y 39, y 40$, $\mathrm{y} 41]$

$[A 7(j, 1), A 7(j, 2)]=$ curveintersect $(x 7, y 7, x 21, m(:, j))$;

end

for $j=1: 21$

$m=[y 21, y 22, y 23, y 24, y 25, y 26, y 27, y 28, y 29, y 30, y 31, y 32, y 33, y 34, y 35, y 36, y 37, y 38, y 39, y 40$, $\mathrm{Y} 41]$;

$[A 8(j, 1), A 8(j, 2)]=$ curveintersect $(x 8, y 8, x 21, m(:, j))$;

end

for $j=1: 21$

$m=[y 21, y 22, y 23, y 24, y 25, y 26, y 27, y 28, y 29, y 30, y 31, y 32, y 33, y 34, y 35, y 36, y 37, y 38, y 39, y 40$, $\mathrm{y} 41]$

$[A 9(j, 1), A 9(j, 2)]=$ curveintersect $(x 9, y 9, x 21, m(:, j)) ;$

end

for $j=1: 21$ 


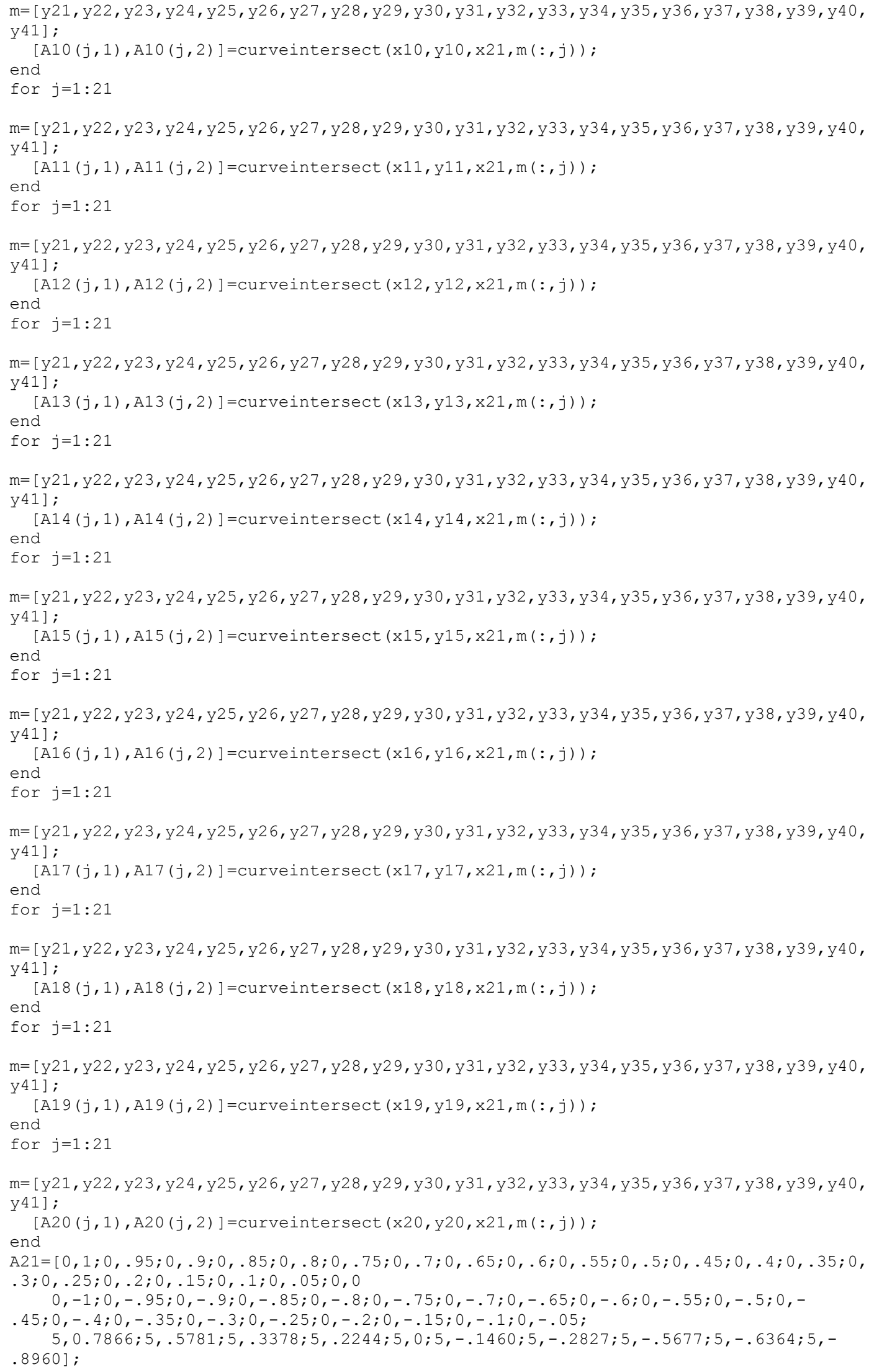




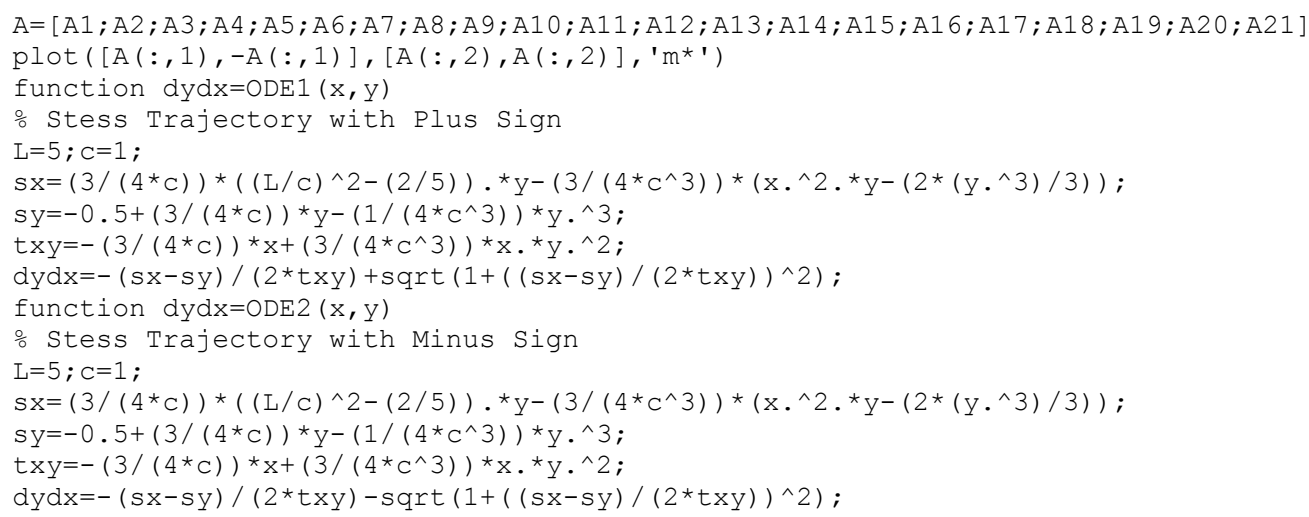

\section{Simply Supported Beam using Strength of Materials Stresses}

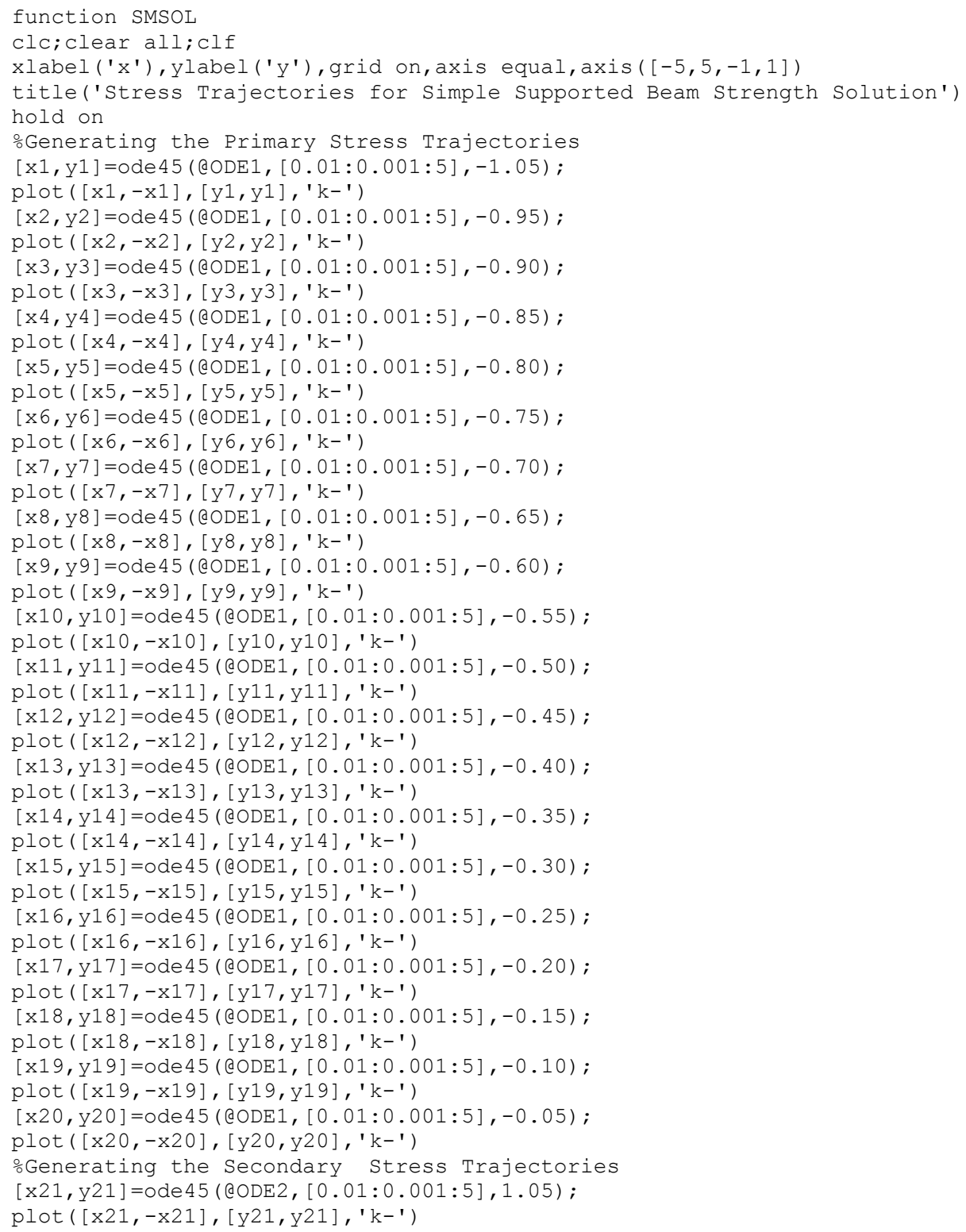




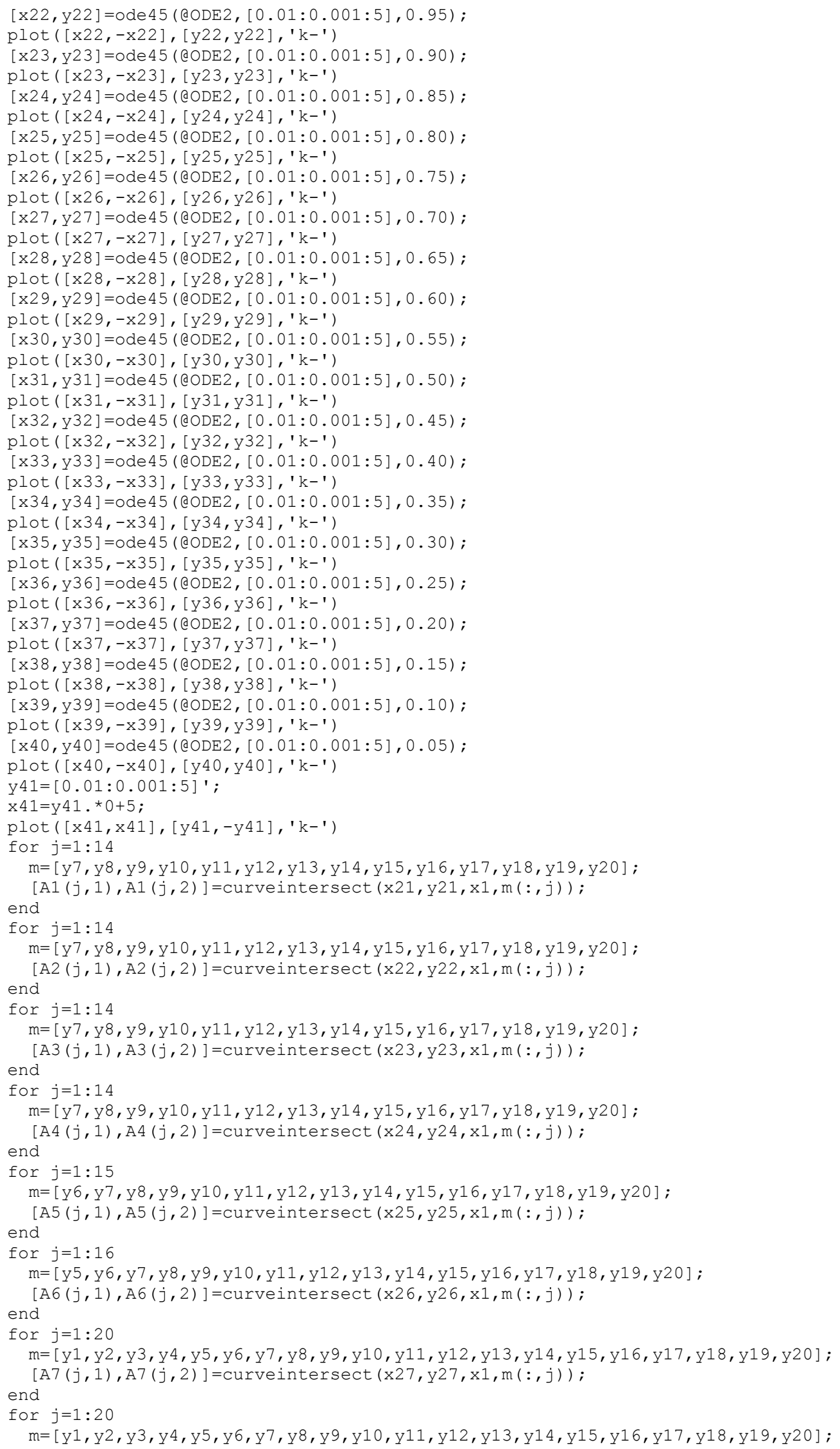




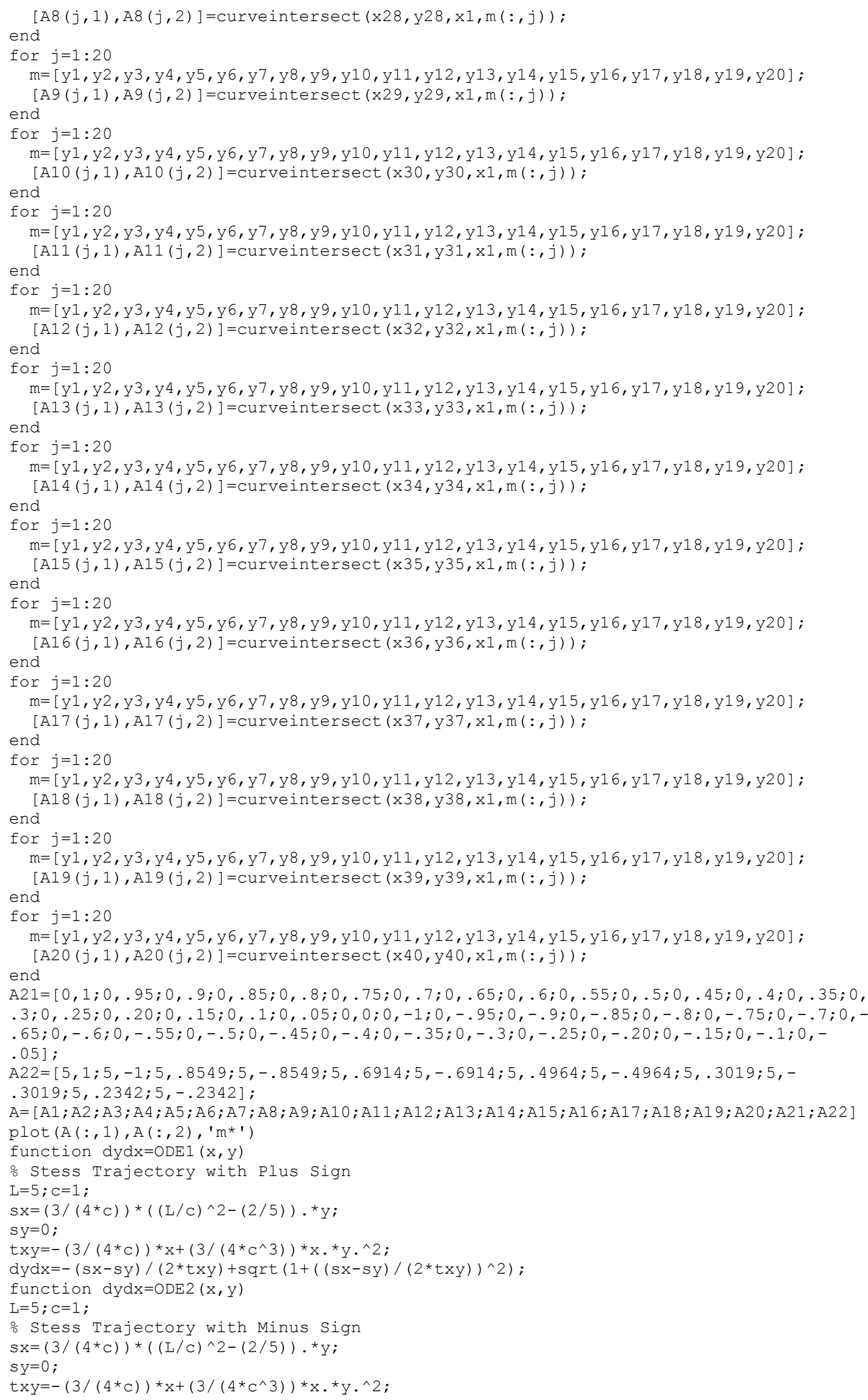


$d y d x=-(s x-s y) /(2 * t x y)-\operatorname{sqrt}\left(1+((s x-s y) /(2 * t x y))^{\wedge} 2\right)$

\section{Disk}

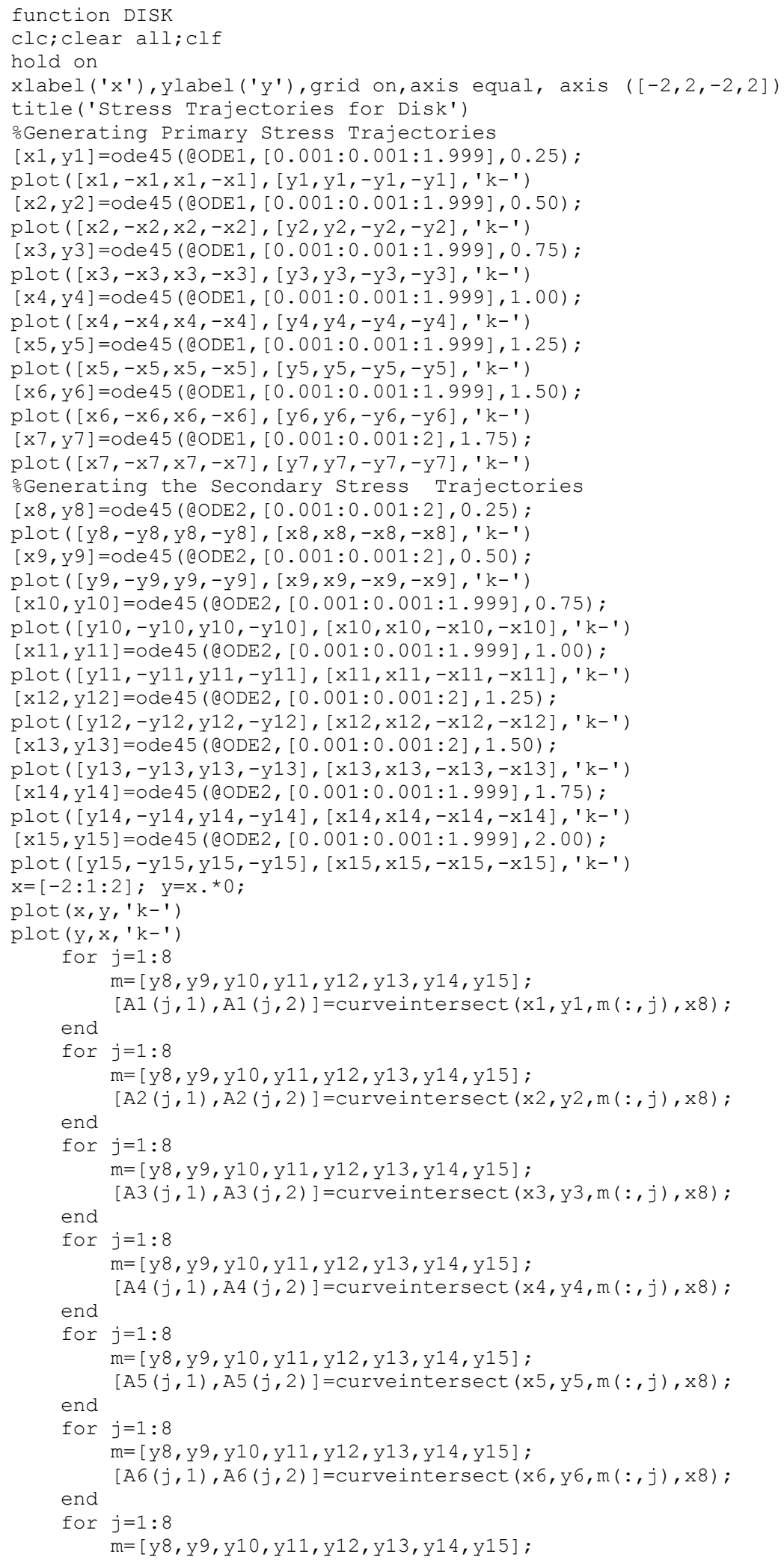


[A7 $(j, 1), A 7(j, 2)]=$ curveintersect $(x 7, y 7, m(:, j), x 8)$;

$\mathrm{A} 8=[0,2 ; 0,1.75 ; 0,1.5 ; 0,1.25 ; 0,1 ; 0, .75 ; 0, .5 ; 0, .25 ; 0,0 ; .25,0 ; .5,0 ; .75,0 ; 1,0 ; 1.25,0 ; 1$. $5,0 ; 1.75,0 ; 2,0] ;$

$\mathrm{A}=[\mathrm{A} 1 ; \mathrm{A} 2 ; \mathrm{A} 3 ; \mathrm{A} 4 ; \mathrm{A} 5 ; \mathrm{A} 6 ; \mathrm{A} 7 ; \mathrm{A} 8]$

$\operatorname{plot}\left([A(:, 1),-A(:, 1), A(:, 1),-A(:, 1)],[A(:, 2),-A(:, 2),-A(:, 2), A(:, 2)], ' r^{*}\right)$, axis $([-2$

$2-2$ 2 $]$ )

$\operatorname{plot}\left(A(:, 1), A(:, 2),{ }^{\prime} k \cdot{ }^{\prime}\right)$, axis $([0,2,0,2])$

function $d y d x=O D E 1(x, y)$

$\mathrm{R}=2 ; \mathrm{D}=4$;

$r 1=\operatorname{sqrt}\left(\left(\left(x \cdot{ }^{\wedge} 2\right)+\left((R-y) \cdot{ }^{\wedge} 2\right)\right)\right) ;$

$r 2=\operatorname{sqrt}\left(\left(\left(x \cdot{ }^{\wedge} 2\right)+\left((\mathrm{R}+\mathrm{y}) \cdot{ }^{\wedge} 2\right)\right)\right) ;$

$\mathrm{sx}=(-2 / \mathrm{pi}) *\left(\left(\left((\mathrm{R}-\mathrm{y}) \cdot{ }^{\star}\left(\mathrm{x} \cdot{ }^{\wedge} 2\right)\right) \cdot /\left(\mathrm{r} 1 \cdot{ }^{\wedge} 4\right)\right)+\left(\left((\mathrm{R}+\mathrm{y}) \cdot{ }^{\star}\left(\mathrm{x} \cdot{ }^{\wedge} 2\right)\right) \cdot /\left(\mathrm{r} 2 \cdot{ }^{\wedge} 4\right)\right)-(1 / \mathrm{D})\right)$;

$\mathrm{sy}=(-2 / \mathrm{pi}) *\left(\left(\left((\mathrm{R}-\mathrm{y}) \cdot{ }^{\wedge} 3\right) \cdot /\left(\mathrm{r} 1 \cdot{ }^{\wedge} 4\right)\right)+\left(\left((\mathrm{R}+\mathrm{y}) \cdot{ }^{\wedge} 3\right) \cdot /\left(\mathrm{r} 2 \cdot{ }^{\wedge} 4\right)\right)-(1 / \mathrm{D})\right)$;

$\operatorname{txy}=(2 / \mathrm{pi}){ }^{\star}\left(\left(\left(\left((\mathrm{R}-\mathrm{y}) \cdot \cdot^{\wedge} 2\right) \cdot{ }^{\star} \mathrm{x}\right) \cdot /\left(\mathrm{r} 1 \cdot \cdot^{\wedge} 4\right)\right)-\left(\left(\left((\mathrm{R}+\mathrm{y}) \cdot{ }^{\wedge} 2\right) \cdot{ }^{\star} \mathrm{x}\right) \cdot /\left(\mathrm{r} 2 \cdot{ }^{\wedge} 4\right)\right)\right) ;$

$d y d x=-(s x-s y) /(2 * t x y)+\operatorname{sqrt}\left(1+((s x-s y) /(2 * t x y))^{\wedge} 2\right)$;

function $d y d x=\operatorname{ODE} 2(x, y)$

$\mathrm{R}=2 ; \mathrm{D}=4$;

$\mathrm{r} 1=\operatorname{sqrt}\left(\left(\left(\mathrm{y} \cdot{ }^{\wedge} 2\right)+\left((\mathrm{R}-\mathrm{x}) \cdot \cdot^{\wedge} 2\right)\right)\right)$;

$\mathrm{r} 2=\operatorname{sqrt}\left(\left(\left(\mathrm{y} \cdot{ }^{\wedge} 2\right)+\left((\mathrm{R}+\mathrm{x}) \cdot \cdot^{\wedge} 2\right)\right)\right)$;

$\mathrm{sx}=(-2 / \mathrm{pi}) *\left(\left(\left((\mathrm{R}-\mathrm{x}) \cdot *^{\star}\left(\mathrm{y} \cdot \wedge^{\wedge} 2\right)\right) \cdot /\left(\mathrm{r} 1 \cdot{ }^{\wedge} 4\right)\right)+\left(\left((\mathrm{R}+\mathrm{x}) \cdot *^{*}\left(\mathrm{y} \cdot{ }^{\wedge} 2\right)\right) \cdot /\left(\mathrm{r} 2 \cdot \cdot^{\wedge} 4\right)\right)-(1 / \mathrm{D})\right)$;

$\mathrm{sy}=(-2 / \mathrm{pi}) *\left(\left(\left((\mathrm{R}-\mathrm{x}) \cdot{ }^{\wedge} 3\right) \cdot /\left(\mathrm{r} 1 \cdot{ }^{\wedge} 4\right)\right)+\left(\left((\mathrm{R}+\mathrm{x}) \cdot{ }^{\wedge} 3\right) \cdot /\left(\mathrm{r} 2 \cdot{ }^{\wedge} 4\right)\right)-(1 / \mathrm{D})\right) ;$

$\operatorname{txy}=(2 / \mathrm{pi}){ }^{\star}\left(\left(\left(\left((\mathrm{R}-\mathrm{x}) \cdot \cdot^{\wedge} 2\right) \cdot{ }^{\star} \mathrm{y}\right) \cdot /\left(\mathrm{r} 1 \cdot \cdot^{\wedge} 4\right)\right)-\left(\left(\left((\mathrm{R}+\mathrm{x}) \cdot \cdot^{\wedge} 2\right) \cdot{ }^{\star} \mathrm{y}\right) \cdot /\left(\mathrm{r} 2 \cdot{ }^{\wedge} 4\right)\right)\right)$;

$d y d x=-(\operatorname{sy}-s x) /(2 \star t x y)-\operatorname{sqrt}\left(1+\left((\operatorname{sy}-s x) /\left(2^{\star} \operatorname{txy}\right)\right)^{\wedge} 2\right)$;

\section{Equal Biaxial Compression Coarse Topology}

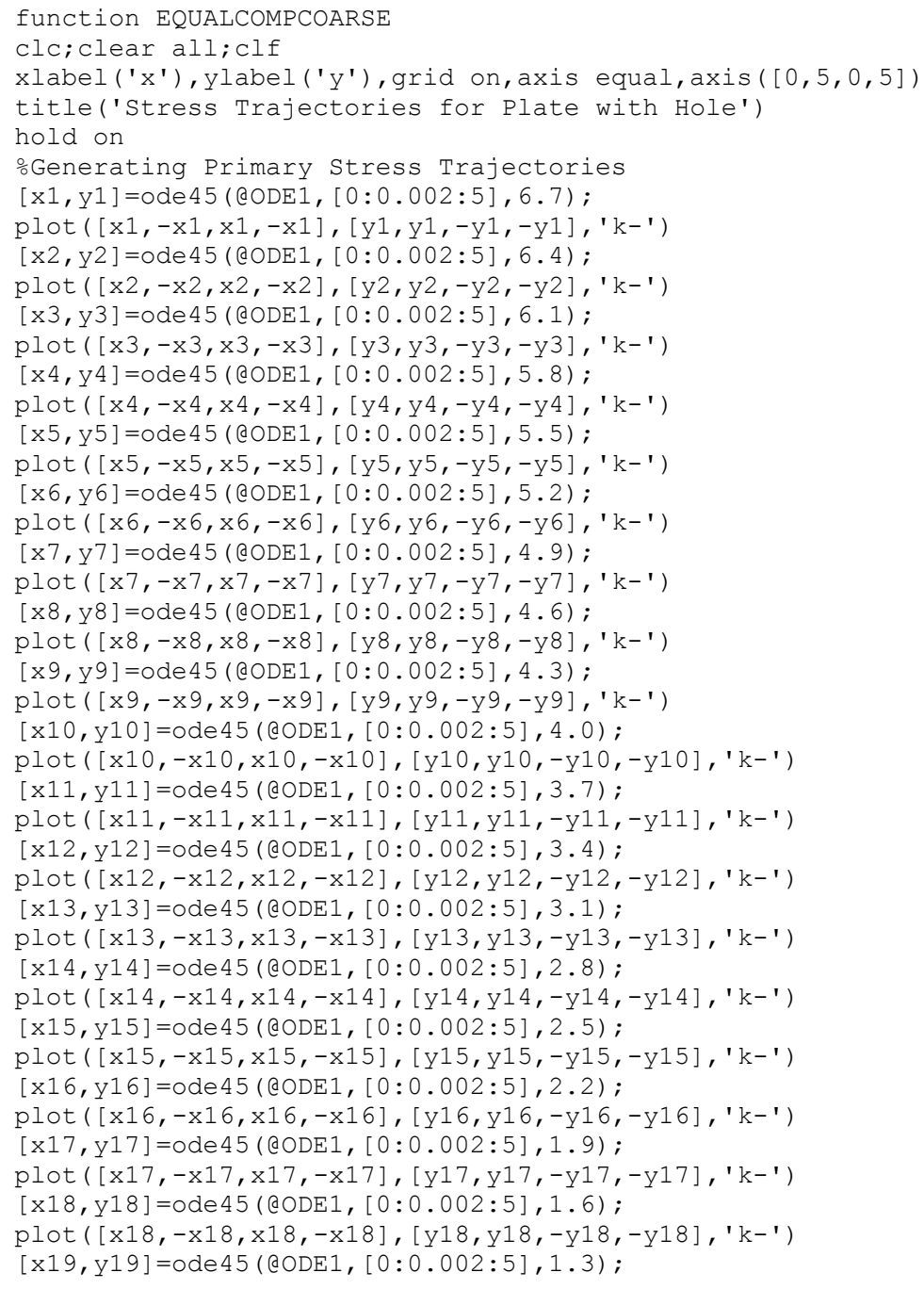




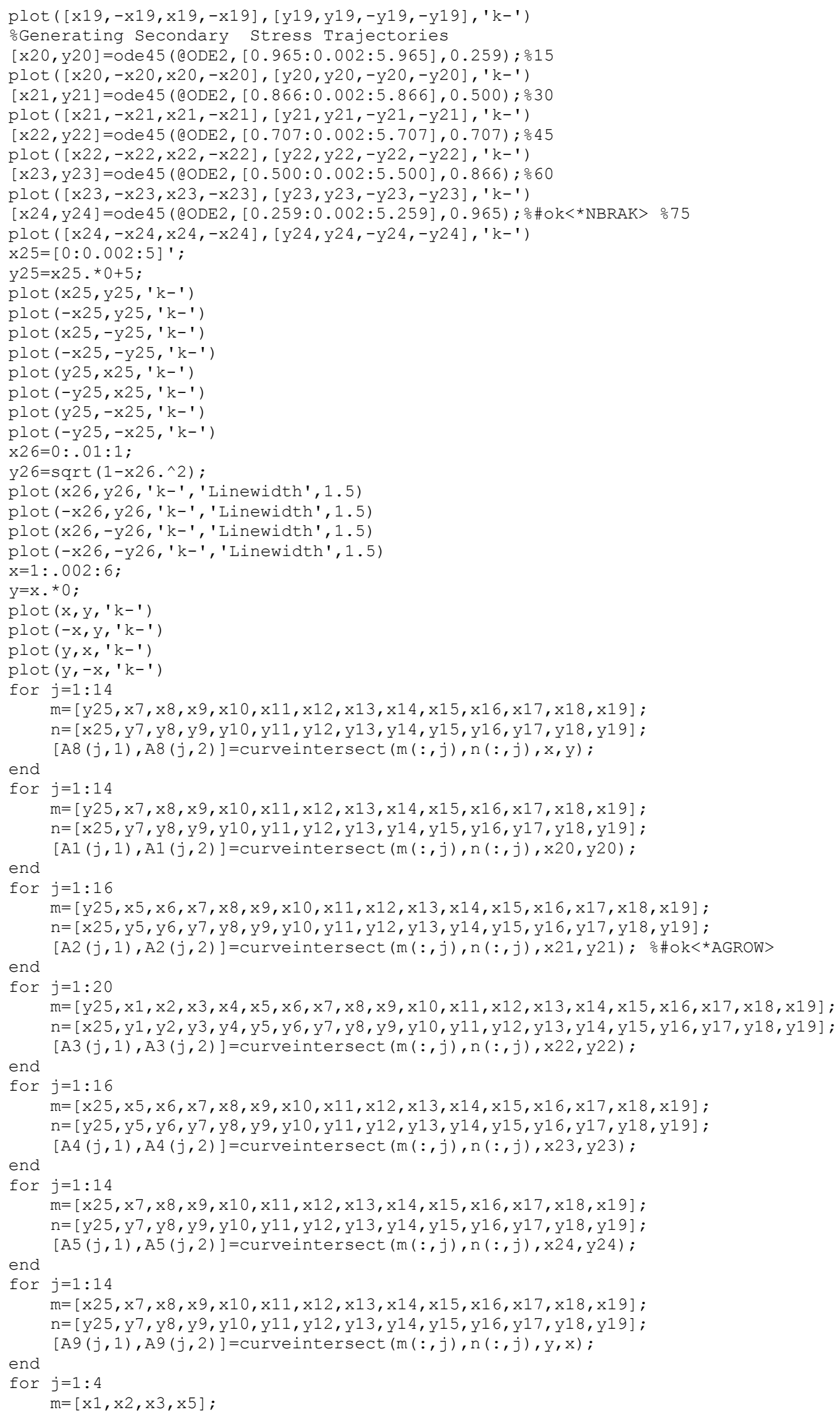




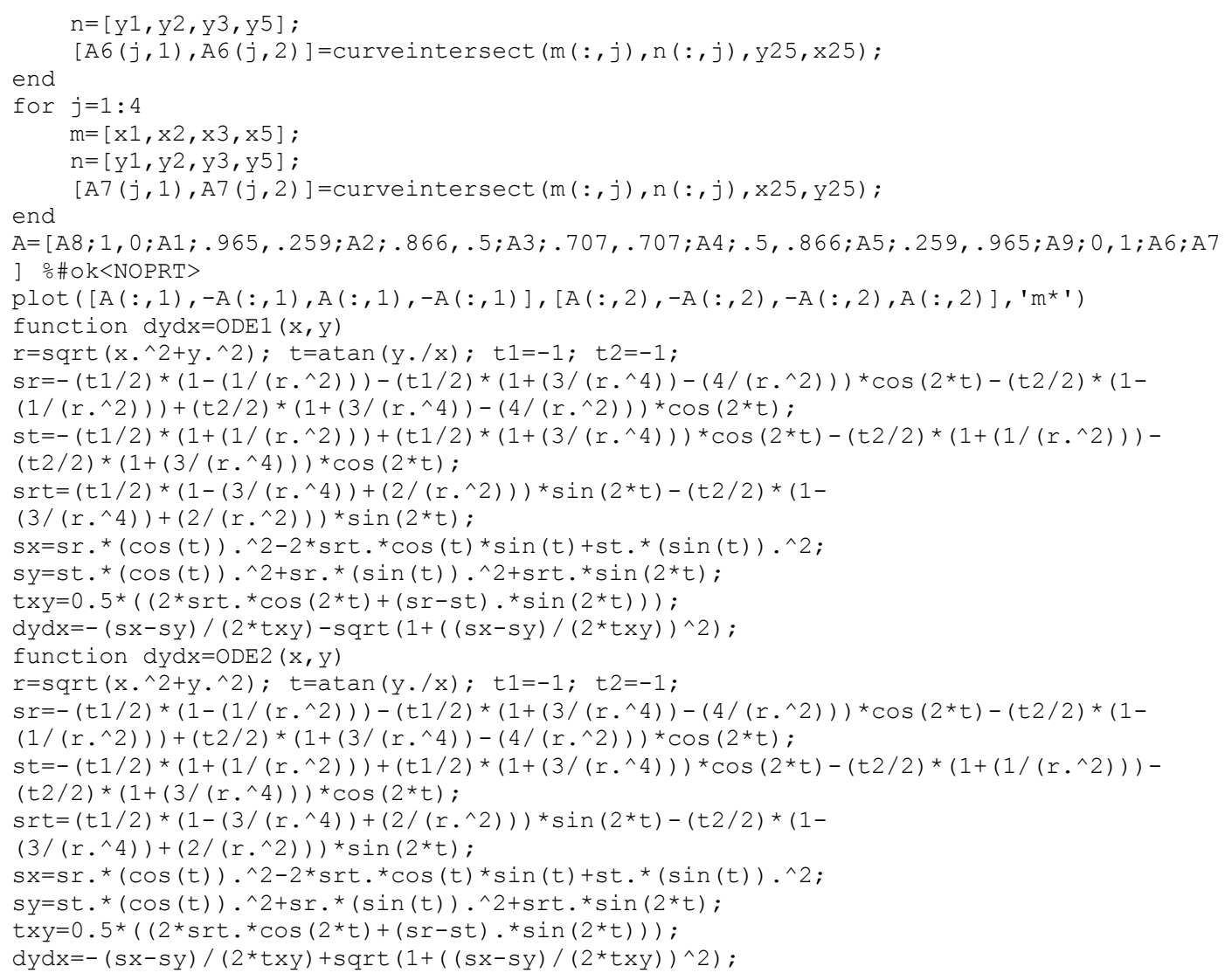

\section{Equal Biaxial Compression Medium Topology}

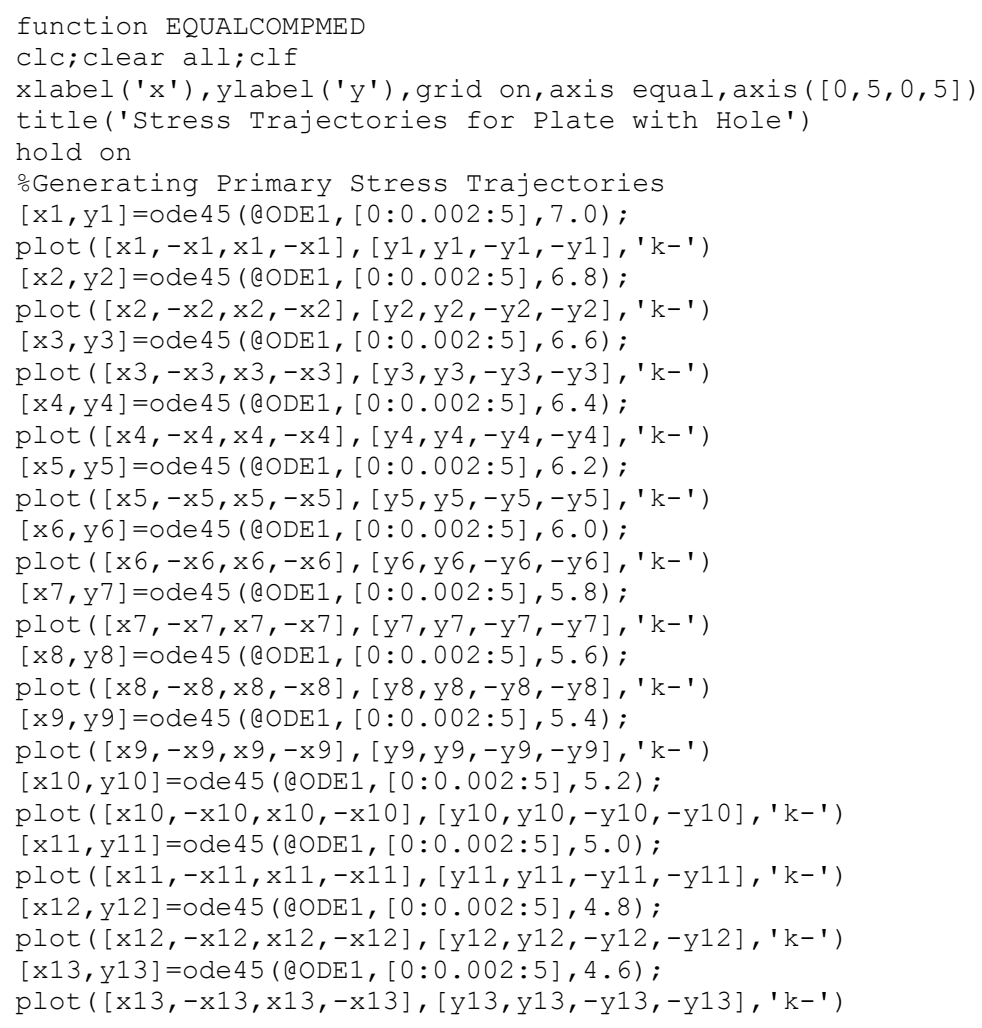


[x14,y14] =ode45 (@ODE1, [0:0.002:5],4.4);

plot $\left([\mathrm{x} 14,-\mathrm{x} 14, \mathrm{x} 14,-\mathrm{x} 14],[y 14, y 14,-y 14,-y 14], \mathrm{k}^{-\prime}\right)$

$[\mathrm{x} 15, \mathrm{y} 15]=0 \mathrm{de} 45(\mathrm{QODE} 1,[0: 0.002: 5], 4.2)$;

plot $([x 15,-x 15, x 15,-x 15],[y 15, y 15,-y 15,-y 15], ' k-')$

$[\mathrm{x} 16, \mathrm{y} 16]=0 \mathrm{de} 45(\mathrm{QODE} 1,[0: 0.002: 5], 4.0)$;

plot $\left([\mathrm{x} 16,-\mathrm{x} 16, \mathrm{x} 16,-\mathrm{x} 16],[\mathrm{y} 16, \mathrm{y} 16,-\mathrm{y} 16,-\mathrm{y} 16], \mathrm{k}^{\prime} \mathrm{\prime}^{\prime}\right)$

$[\mathrm{x} 17, \mathrm{y} 17]=0 \mathrm{de} 45($ @ODE $1,[0: 0.002: 5], 3.8)$;

plot $\left([x 17,-x 17, x 17,-x 17],[y 17, y 17,-y 17,-y 17], ' k^{\prime}\right)$

$[\mathrm{x} 18, \mathrm{y} 18]=0 \mathrm{de} 45(\mathrm{\varrho ODE} 1,[0: 0.002: 5], 3.6)$;

plot $\left([x 18,-x 18, x 18,-x 18],[y 18, y 18,-y 18,-y 18], ' k^{\prime}\right)$

$[\mathrm{x} 19, \mathrm{y} 19]=0 \mathrm{de} 45($ @ODE $1,[0: 0.002: 5], 3.4)$;

plot $\left([x 19,-x 19, x 19,-x 19],[y 19, y 19,-y 19,-y 19], k^{\prime}-^{\prime}\right)$

$[\mathrm{x} 20, \mathrm{y} 20]=0 \mathrm{de} 45(\mathrm{\varrho ODE} 1,[0: 0.002: 5], 3.2)$;

plot $\left([x 20,-x 20, x 20,-x 20],[y 20, y 20,-y 20,-y 20], k^{\prime}\right.$ ')

$[\mathrm{x} 21, \mathrm{y} 21]=0 \mathrm{de} 45(\mathrm{QODE} 1,[0: 0.002: 5], 3.0)$;

plot $\left([x 21,-x 21, x 21,-x 21],[y 21, y 21,-y 21,-y 21], k^{\prime}{ }^{\prime}\right)$

$[\mathrm{x} 22, \mathrm{y} 22]=0 \mathrm{de} 45(\mathrm{\varrho ODE} 1,[0: 0.002: 5], 2.8)$;

plot $([x 22,-x 22, x 22,-x 22],[y 22, y 22,-y 22,-y 22], ' k-')$

$[\mathrm{x} 23, \mathrm{y} 23]=0 \mathrm{de} 45(\mathrm{OODE} 1,[0: 0.002: 5], 2.6)$;

plot $\left([x 23,-x 23, x 23,-x 23],[y 23, y 23,-y 23,-y 23],{ }^{\prime} k-^{\prime}\right)$

$[\mathrm{x} 24, \mathrm{y} 24]=0 \mathrm{de} 45(\mathrm{@ODE} 1,[0: 0.002: 5], 2.4)$; \%\# Ok<*NBRAK>

plot $\left([x 24,-x 24, x 24,-x 24],[y 24, y 24,-y 24,-y 24], k^{-\prime}\right)$

$[\mathrm{x} 25, \mathrm{y} 25]=0 \mathrm{de} 45(\mathrm{QODE} 1,[0: 0.002: 5], 2.2)$;

plot $\left([x 25,-x 25, x 25,-x 25],[y 25, y 25,-y 25,-y 25], '-^{\prime}\right)$

$[\mathrm{x} 26, \mathrm{y} 26]=0 \mathrm{de} 45(\mathrm{eODE} 1,[0: 0.002: 5], 2.0)$;

plot $\left([x 26,-x 26, x 26,-x 26],[y 26, y 26,-y 26,-y 26], ' k^{-'}\right)$

$[\mathrm{x} 27, \mathrm{y} 27]=0 \mathrm{de} 45(\mathrm{QODE} 1,[0: 0.002: 5], 1.8)$;

plot $\left([x 27,-x 27, x 27,-x 27],[y 27, y 27,-y 27,-y 27], ' k^{-'}\right)$

$[\mathrm{x} 28, \mathrm{y} 28]=0 \mathrm{de} 45(\mathrm{eODE} 1,[0: 0.002: 5], 1.6)$;

plot $\left([x 28,-x 28, x 28,-x 28],[y 28, y 28,-y 28,-y 28], ' k^{\prime}\right.$ ')

$[\mathrm{x} 29, \mathrm{y} 29]=0 \mathrm{de} 45($ @ODE1, $[0: 0.002: 5], 1.4)$;

plot $([x 29,-x 29, x 29,-x 29],[y 29, y 29,-y 29,-y 29], ' k-')$

$[\mathrm{x} 30, \mathrm{y} 30]=0 \mathrm{de} 45(\mathrm{\varrho ODE} 1,[0: 0.002: 5], 1.2)$;

plot $\left([x 30,-x 30, x 30,-x 30],[y 30, y 30,-y 30,-y 30],{ }^{\prime}-^{\prime}\right)$

oGenerating Secondary Stress Trajectories

$[\mathrm{x} 31, \mathrm{y} 31]=0 \mathrm{de} 45(\mathrm{QODE} 2,[0.984: 0.002: 5.984], 0.174) ; \circ 10$

plot $\left([x 31,-x 31, x 31,-x 31],[y 31, y 31,-y 31,-y 31],{ }^{\prime}-^{\prime}\right)$

$[\mathrm{x} 32, \mathrm{y} 32]=0 \mathrm{de} 45(\mathrm{QODE} 2,[0.939: 0.002: 5.939], 0.342) ; \circ 20$

plot $\left([x 32,-x 32, x 32,-x 32],[y 32, y 32,-y 32,-y 32], '{ }^{\prime}-^{\prime}\right)$

$[\mathrm{x} 33, \mathrm{y} 33]=0 \mathrm{de} 45(\mathrm{QODE} 2,[0.866: 0.002: 5.866], 0.500) ; \circ 30$

plot $\left([x 33,-x 33, x 33,-x 33],[y 33, y 33,-y 33,-y 33],{ }^{\prime}-^{\prime}\right)$

$[\mathrm{x} 34, \mathrm{y} 34]=0 \mathrm{de} 45(\mathrm{QODE} 2,[0.7659: 0.002: 5.7659], 0.6429) ; \% 40$

plot $\left([x 34,-x 34, x 34,-x 34],[y 34, y 34,-y 34,-y 34],{ }^{\prime}-^{\prime}\right)$

$[\mathrm{x} 35, \mathrm{y} 35]=0 \mathrm{de} 45(\mathrm{OODE} 2,[0.6428: 0.002: 5.6428], 0.7658) ; \% 50$

plot $([x 35,-x 35, x 35,-x 35],[y 35, y 35,-y 35,-y 35], ' k-1)$

$[\mathrm{x} 36, \mathrm{y} 36]=0 \mathrm{de} 45(\mathrm{QODE} 2,[0.500: 0.002: 5.500], 0.866) ; \circ 60$

plot $\left([x 36,-x 36, x 36,-x 36],[y 36, y 36,-y 36,-y 36],{ }^{\prime} k-^{\prime}\right)$

$[\mathrm{x} 37, \mathrm{y} 37]=0 \mathrm{de} 45(\mathrm{QODE} 2,[0.342: 0.002: 5.342], 0.939) ; \circ 70$

plot $\left([x 37,-x 37, x 37,-x 37],[y 37, y 37,-y 37,-y 37], k^{-\prime}\right)$

$[\mathrm{x} 38, \mathrm{y} 38]=0 \mathrm{de} 45(\mathrm{\varrho ODE} 2,[0.174: 0.002: 5.174], 0.984) ; \circ 80$

plot $([x 38,-x 38, x 38,-x 38],[y 38, y 38,-y 38,-y 38], ' k-')$

$\times 39=[0: 0.002: 5] '$;

$\mathrm{y} 39=\mathrm{x} 39 \cdot * 0+5$

plot $\left(x 39, \mathrm{y} 39, \mathrm{\prime}^{\prime} \mathrm{-} \cdot\right)$

plot $\left(-\mathrm{x} 39, \mathrm{y} 39, \mathrm{\prime}^{\mathrm{k}} \mathrm{-}^{\prime}\right)$

plot $\left(x 39,-y 39,{ }^{\prime}-{ }^{\prime}\right)$

plot $\left(-x 39,-y 39,{ }^{\prime} k-'\right)$

plot $(y 39, x 39, ' k-')$

plot $\left(-y 39, x 39,{ }^{\prime} k-'\right)$

plot $\left(y 39,-x 39,{ }^{\prime} k-'\right)$

plot $\left(-y 39,-x 39,{ }^{\prime} k^{\prime}\right)$

$\mathrm{x}=1: .002: 5$;

$\mathrm{y}=\mathrm{x} \cdot{ }^{*} 0$;

plot $\left(x, y,{ }^{\prime}-'\right)$

plot $\left(-x, y,{ }^{\prime}-^{\prime}\right)$

$\operatorname{plot}\left(y, x,{ }^{\prime}-^{\prime}\right)$

plot $\left(y,-x, k^{\prime}\right)$

$\mathrm{x}=0: .01: 1$;

$\mathrm{y}=\operatorname{sqrt}\left(1-\mathrm{x} \cdot{ }^{\wedge} 2\right)$;

plot $(x, y, ' k-'$ ' 'Linewidth', 1.5)

plot $(-x, y, ' k-'$, 'Linewidth', 1.5) 
plot $\left(x,-y,{ }^{\prime}-{ }^{\prime}\right.$ ' ' Linewidth', 1.5)

plot $\left(-x,-y,{ }^{\prime}-^{\prime}, '\right.$ 'Linewidth', 1.5)

for $j=1: 21$

$m=[y 39, x 11, x 12, x 13, x 14, x 15, x 16, x 17, x 18, x 19, x 20, x 21, x 22, x 23, x 24, x 25, x 26, x 27, x 28, x 29$, $\mathrm{x} 30]$;

$n=[x 39, y 11, y 12, y 13, y 14, y 15, y 16, y 17, y 18, y 19, y 20, y 21, y 22, y 23, y 24, y 25, y 26, y 27, y 28, y 29$, y30];

end

$[A 1(j, 1), A 1(j, 2)]=$ curveintersect $(m(:, j), n(:, j), x 31, y 31) ;$ o\#०k<*AGROW>

for $j=1: 22$

$m=[y 39, x 10, x 11, x 12, x 13, x 14, x 15, x 16, x 17, x 18, x 19, x 20, x 21, x 22, x 23, x 24, x 25, x 26, x 27, x 28$, $\times 29, \times 30]$

$\mathrm{n}=[\mathrm{x} 39, \mathrm{y} 10, \mathrm{y} 11, \mathrm{y} 12, \mathrm{y} 13, \mathrm{y} 14, \mathrm{y} 15, \mathrm{y} 16, \mathrm{y} 17, \mathrm{y} 18, \mathrm{y} 19, \mathrm{y} 20, \mathrm{y} 21, \mathrm{y} 22, \mathrm{y} 23, \mathrm{y} 24, \mathrm{y} 25, \mathrm{y} 26, \mathrm{y} 27, \mathrm{y} 28$, y29, y30];

end

$[A 2(j, 1), A 2(j, 2)]=$ curveintersect $(m(:, j), n(:, j), x 32, y 32) ;$

for $j=1: 24$

$m=[y 39, x 8, x 9, x 10, x 11, x 12, x 13, x 14, x 15, x 16, x 17, x 18, x 19, x 20, x 21, x 22, x 23, x 24, x 25, x 26, x 2$ $7, \times 28, \times 29, \times 30]$

$n=[x 39, y 8, y 9, y 10, y 11, y 12, y 13, y 14, y 15, y 16, y 17, y 18, y 19, y 20, y 21, y 22, y 23, y 24, y 25, y 26, y 2$ $7, \mathrm{y} 28, \mathrm{y} 29, \mathrm{y} 30]$

end

$[A 3(j, 1), A 3(j, 2)]=$ curveintersect $(m(:, j), n(:, j), x 33, y 33)$;

for $j=1: 28$

$m=[y 39, x 4, x 5, x 6, x 7, x 8, x 9, x 10, x 11, x 12, x 13, x 14, x 15, x 16, x 17, x 18, x 19, x 20, x 21, x 22, x 23, x 2$ $4, x 25, x 26, x 27, x 28, x 29, x 30]$

$n=[x 39, y 4, y 5, y 6, y 7, y 8, y 9, y 10, y 11, y 12, y 13, y 14, y 15, y 16, y 17, y 18, y 19, y 20, y 21, y 22, y 23, y 2$ $4, \mathrm{y} 25, \mathrm{y} 26, \mathrm{y} 27, \mathrm{y} 28, \mathrm{y} 29, \mathrm{y} 30]$ end

$[A 4(j, 1), A 4(j, 2)]=$ curveintersect $(m(:, j), n(:, j), x 34, y 34) ;$

for $j=1: 28$

$\mathrm{m}=[\mathrm{x} 39, \mathrm{x} 4, \mathrm{x} 5, \mathrm{x} 6, \mathrm{x} 7, \mathrm{x} 8, \mathrm{x} 9, \mathrm{x} 10, \mathrm{x} 11, \mathrm{x} 12, \mathrm{x} 13, \mathrm{x} 14, \mathrm{x} 15, \mathrm{x} 16, \mathrm{x} 17, \mathrm{x} 18, \mathrm{x} 19, \mathrm{x} 20, \mathrm{x} 21, \mathrm{x} 22, \mathrm{x} 23, \mathrm{x} 2$ $4, x 25, x 26, x 27, x 28, x 29, x 30]$

$n=[y 39, y 4, y 5, y 6, y 7, y 8, y 9, y 10, y 11, y 12, y 13, y 14, y 15, y 16, y 17, y 18, y 19, y 20, y 21, y 22, y 23, y 2$ $4, \mathrm{y} 25, \mathrm{y} 26, \mathrm{y} 27, \mathrm{y} 28, \mathrm{y} 29, \mathrm{y} 30]$

end

$[A 5(j, 1), A 5(j, 2)]=$ curveintersect $(m(:, j), n(:, j), x 35, y 35)$;

for $j=1: 24$

$m=[x 39, x 8, x 9, x 10, x 11, x 12, x 13, x 14, x 15, x 16, x 17, x 18, x 19, x 20, x 21, x 22, x 23, x 24, x 25, x 26, x 2$ $7, \times 28, \times 29, \times 30]$

$\mathrm{n}=[\mathrm{y} 39, \mathrm{y} 8, \mathrm{y} 9, \mathrm{y} 10, \mathrm{y} 11, \mathrm{y} 12, \mathrm{y} 13, \mathrm{y} 14, \mathrm{y} 15, \mathrm{y} 16, \mathrm{y} 17, \mathrm{y} 18, \mathrm{y} 19, \mathrm{y} 20, \mathrm{y} 21, \mathrm{y} 22, \mathrm{y} 23, \mathrm{y} 24, \mathrm{y} 25, \mathrm{y} 26, \mathrm{y} 2$ $7, \mathrm{y} 28, \mathrm{y} 29, \mathrm{y} 30]$ end

$[A 6(j, 1), A 6(j, 2)]=$ curveintersect $(m(:, j), n(:, j), x 36, y 36)$;

for $j=1: 22$

$m=[x 39, x 10, x 11, x 12, x 13, x 14, x 15, x 16, x 17, x 18, x 19, x 20, x 21, x 22, x 23, x 24, x 25, x 26, x 27, x 28$, $\times 29, \times 30]$

$n=[y 39, y 10, y 11, y 12, y 13, y 14, y 15, y 16, y 17, y 18, y 19, y 20, y 21, y 22, y 23, y 24, y 25, y 26, y 27, y 28$, $\mathrm{y} 29, \mathrm{y} 30]$

$m=[x 39, x 11, x 12, x 13, x 14, x 15, x 16, x 17, x 18, x 19, x 20, x 21, x 22, x 23, x 24, x 25, x 26, x 27, x 28, x 29$, $\times 30]$;

$n=[y 39, y 11, y 12, y 13, y 14, y 15, y 16, y 17, y 18, y 19, y 20, y 21, y 22, y 23, y 24, y 25, y 26, y 27, y 28, y 29$, y30] ;

end

$[A 8(j, 1), A 8(j, 2)]=$ curveintersect $(m(:, j), n(:, j), x 38, y 38)$;

for $j=1: 9$

$\mathrm{m}=[\mathrm{x} 1, \mathrm{x} 2, \mathrm{x} 3, \mathrm{x} 4, \mathrm{x} 5, \mathrm{x} 6, \mathrm{x} 8, \mathrm{x} 9, \mathrm{x} 10]$;

$\mathrm{n}=[\mathrm{y} 1, \mathrm{y} 2, \mathrm{y} 3, \mathrm{y} 4, \mathrm{y} 5, \mathrm{y} 6, \mathrm{y} 8, \mathrm{y} 9, \mathrm{y} 10] ;$

end

$[A 9(j, 1), A 9(j, 2)]=$ curveintersect $(m(:, j), n(:, j), \times 39, y 39)$;

for $j=1: 9$

$\mathrm{m}=[\mathrm{x} 1, \mathrm{x} 2, \mathrm{x} 3, \mathrm{x} 4, \mathrm{x} 5, \mathrm{x} 6, \mathrm{x} 8, \mathrm{x} 9, \mathrm{x} 10]$;

$\mathrm{n}=[\mathrm{y} 1, \mathrm{y} 2, \mathrm{y} 3, \mathrm{y} 4, \mathrm{y} 5, \mathrm{y} 6, \mathrm{y} 8, \mathrm{y} 9, \mathrm{y} 10]$

$[A 10(j, 1), A 10(j, 2)]=$ curveintersect $(m(:, j), n(:, j), y 39, x 39)$; 
end

$\mathrm{A} 11=[5,0 ; 0,5 ; 4.8,0 ; 0,4.8 ; 4.6,0 ; 0,4.6 ; 4.4,0 ; 0,4.4 ; 4.2,0 ; 0,4.2 ; 4,0 ; 0,4 ; 3.8,0 ; 0,3.8 ; 3$. 6,$0 ; 0,3.6 ; 3.4,0 ; 0,3.4 ; 3.2,0 ; 0,3.2 ; 3,0 ; 0,3 ; 2.8,0 ; 0,2.8 ; 2.6,0 ; 0,2.6 ; 2.4,0 ; 0,2.4 ; 2.2,0$ $; 0,2.2 ; 2,0 ; 0,2 ; 1.8,0 ; 0,1.8 ; 1.6,0 ; 0,1.6 ; 1.4,0 ; 0,1.4 ; 1.2,0 ; 0,1.2 ; 1,0 ; 0,1 ; .984, .174 ; .1$ $74, .984 ; .939, .342 ; .342, .939 ; .866, .5 ; .5, .866 ; .766, .643 ; .643, .766] ;$ $\mathrm{A}=[\mathrm{A} 1 ; \mathrm{A} 2 ; \mathrm{A} 3 ; \mathrm{A} 4 ; \mathrm{A} 5 ; \mathrm{A} 6 ; \mathrm{A} 7 ; \mathrm{A} 8 ; \mathrm{A} 9 ; \mathrm{A} 10 ; \mathrm{A} 11]$ 읏NOPRT $>$

plot $([A(:, 1),-A(:, 1), A(:, 1),-A(:, 1)],[A(:, 2),-A(:, 2),-A(:, 2), A(:, 2)], ' m \star ')$

function dydx=ODE1 $(x, y)$

$r=\operatorname{sqrt}(x \cdot \wedge 2+y \cdot \wedge 2) ; t=\operatorname{atan}(y \cdot / x) ; t 1=-1 ; t 2=-1 ;$

$\mathrm{sr}=-(t 1 / 2) *\left(1-\left(1 /\left(r \cdot{ }^{\wedge} 2\right)\right)\right)-(t 1 / 2) *(1+(3 /(r \cdot \wedge 4))-(4 /(r . \wedge 2))) * \cos (2 * t)-(t 2 / 2) *(1-$ $\left.\left(1 /\left(r \cdot{ }^{\wedge} 2\right)\right)\right)+(t 2 / 2) *(1+(3 /(r \cdot \wedge 4))-(4 /(r \cdot \wedge 2))){ }^{*} \cos (2 * t) ;$ $s t=-(t 1 / 2) *\left(1+\left(1 /\left(r \wedge^{\wedge} 2\right)\right)\right)+(t 1 / 2) *(1+(3 /(r \cdot \wedge 4))) * \cos (2 * t)-(t 2 / 2) *(1+(1 /(r . \wedge 2)))-$ $(t 2 / 2) *\left(1+\left(3 /\left(r \cdot{ }^{\wedge} 4\right)\right)\right) * \cos (2 * t) ;$

$\operatorname{srt}=(t 1 / 2) *(1-(3 /(r \cdot \wedge 4))+(2 /(r \cdot \wedge 2))) * \sin (2 * t)-(t 2 / 2) *(1-$ $(3 /(r \cdot \wedge 4))+(2 /(r \cdot \wedge 2))) * \sin (2 * t) ;$

$\operatorname{sx}=\operatorname{sr} \cdot{ }^{*}(\cos (t)) \cdot{ }^{\wedge} 2-2 * \operatorname{srt} \cdot{ }^{*} \cos (t) * \sin (t)+\operatorname{st} \cdot{ }^{*}(\sin (t)) \cdot{ }^{\wedge} 2 ;$

$\operatorname{sy}=s t \cdot{ }^{*}(\cos (t)) \cdot{ }^{\wedge} 2+\operatorname{sr} \cdot{ }^{*}(\sin (t)) \cdot{ }^{\wedge} 2+\operatorname{srt} \cdot{ }^{*} \sin (2 * t) \cdot$

txy $=0.5 *\left(\left(2 * \operatorname{srt} .{ }^{*} \cos (2 * t)+(\operatorname{sr}-\operatorname{st}) \cdot{ }^{*} \sin (2 * t)\right)\right) ;$

$d y d x=-($ sx - sy $) /(2 * t x y)-\operatorname{sqrt}\left(1+((\text { sx }- \text { sy }) /(2 * t x y))^{\wedge} 2\right)$;

function $d y d x=\operatorname{ODE} 2(x, y)$

$r=\operatorname{sqrt}(x \cdot \wedge 2+y \cdot \wedge 2) ; t=\operatorname{atan}(y \cdot / x) ; t 1=-1 ; t 2=-1 ;$

$\mathrm{sr}=-(t 1 / 2) *\left(1-\left(1 /\left(r \cdot{ }^{\wedge} 2\right)\right)\right)-(t 1 / 2) *(1+(3 /(r \cdot \wedge 4))-(4 /(r . \wedge 2))) * \cos (2 * t)-(t 2 / 2) *(1-$

$(1 /(r \cdot \wedge 2)))+(t 2 / 2) *(1+(3 /(r \cdot \wedge 4))-(4 /(r \cdot \wedge 2))) * \cos (2 * t)$;

$s t=-(t 1 / 2) *\left(1+\left(1 /\left(r \cdot{ }^{\wedge} 2\right)\right)\right)+(t 1 / 2) *\left(1+\left(3 /\left(r \cdot{ }^{\wedge} 4\right)\right)\right){ }^{*} \cos (2 * t)-(t 2 / 2) *\left(1+\left(1 /\left(r \cdot{ }^{\wedge} 2\right)\right)\right)-$

$(t 2 / 2) *(1+(3 /(r \cdot \wedge 4))) * \cos (2 * t) ;$

$\operatorname{srt}=(t 1 / 2) *\left(1-\left(3 /\left(r \cdot{ }^{\wedge} 4\right)\right)+\left(2 /\left(r \cdot{ }^{\wedge} 2\right)\right)\right) * \sin (2 * t)-(t 2 / 2) *(1-$

$(3 /(r \cdot \wedge 4))+(2 /(r \cdot \wedge 2))) * \sin (2 * t) ;$

$\operatorname{sx}=\operatorname{sr} \cdot{ }^{*}(\cos (t)) \cdot{ }^{\wedge} 2-2 * \operatorname{srt} .{ }^{*} \cos (t) * \sin (t)+\operatorname{st} \cdot{ }^{*}(\sin (t)) \cdot{ }^{\wedge} 2$;

$\operatorname{sy}=s t \cdot{ }^{*}(\cos (t)) \cdot{ }^{\wedge} 2+\operatorname{sr} \cdot{ }^{*}(\sin (t)) \cdot{ }^{\wedge} 2+\operatorname{srt} \cdot{ }^{*} \sin (2 * t) ;$

$t x y=0.5 *\left(\left(2 * \operatorname{srt} .{ }^{*} \cos (2 * t)+(\operatorname{sr}-s t) \cdot{ }^{*} \sin (2 * t)\right)\right) ;$

$d y d x=-(s x-s y) /(2 * \operatorname{txy})+\operatorname{sqrt}\left(1+((s x-s y) /(2 * \operatorname{txy}))^{\wedge} 2\right)$;

\section{Equal Biaxial Compression Fine Topology}

function EQUALCOMPFINE

clc;clear all;clf

xlabel ('x'), ylabel ('y'), grid on, axis equal, axis $([0,5,0,5])$

title('Stress Trajectories for Plate with Hole')

hold on

oGenerating Primary Stress Trajctories

$[\mathrm{x} 1, \mathrm{y} 1]=0 \mathrm{de} 45(@ \mathrm{ODE} 1,[0: 0.002: 5], 7.0)$;

plot $\left([\mathrm{x} 1,-\mathrm{x} 1, \mathrm{x} 1,-\mathrm{x} 1],[\mathrm{y} 1, \mathrm{y} 1,-\mathrm{y} 1,-\mathrm{y} 1], \mathrm{\prime}^{\prime}-^{\prime}\right)$

$[\mathrm{x} 2, \mathrm{y} 2]=0 \mathrm{de} 45(@ \mathrm{ODE} 1,[0: 0.002: 5], 6.9)$;

plot $([x 2,-x 2, x 2,-x 2],[y 2, y 2,-y 2,-y 2], ' k-1)$

$[\mathrm{x} 3, \mathrm{y} 3]=0 \mathrm{de} 45(@ \mathrm{ODE} 1,[0: 0.002: 5], 6.8)$;

plot $\left([x 3,-x 3, x 3,-x 3],[y 3, y 3,-y 3,-y 3], k^{\prime}\right)$

$[\mathrm{x} 4, \mathrm{y} 4]=0 \mathrm{de} 45(@ \mathrm{ODE} 1,[0: 0.002: 5], 6.7)$;

plot $\left([\mathrm{x} 4,-\mathrm{x} 4, \mathrm{x} 4,-\mathrm{x} 4],\left[\mathrm{y}^{4}, \mathrm{y} 4,-\mathrm{y} 4,-\mathrm{y} 4\right], \mathrm{k}^{-1}\right)$

$[\mathrm{x} 5, \mathrm{y} 5]=0 \mathrm{de} 45(\mathrm{QODE} 1,[0: 0.002: 5], 6.6)$;

plot $([x 5,-x 5, x 5,-x 5],[y 5, y 5,-y 5,-y 5], ' k-1)$

$[\mathrm{x} 6, \mathrm{y} 6]=0 \mathrm{de} 45(@ \mathrm{ODE} 1,[0: 0.002: 5], 6.5)$;

plot $\left([x 6,-x 6, x 6,-x 6],\left[y 6, y^{6},-y 6,-y 6\right], ' k-1\right)$

$[\mathrm{x} 7, \mathrm{y} 7]=0 \mathrm{de} 45(@ \mathrm{ODE} 1,[0: 0.002: 5], 6.4)$;

plot $([x 7,-x 7, x 7,-x 7],[y 7, y 7,-y 7,-y 7], ' k-1)$

$[\mathrm{x} 8, \mathrm{y} 8]=0 \mathrm{de} 45(\mathrm{@ODE} 1,[0: 0.002: 5], 6.3)$;

plot $\left([\mathrm{x} 8,-\mathrm{x} 8, \mathrm{x} 8,-\mathrm{x} 8],[\mathrm{y} 8, \mathrm{y} 8,-\mathrm{y} 8,-\mathrm{y} 8], \mathrm{k}^{\prime} \mathrm{\prime}^{\prime}\right)$

$[\mathrm{x} 9, \mathrm{y} 9]=0 \mathrm{de} 45($ @ODE $1,[0: 0.002: 5], 6.2)$;

plot $\left([x 9,-x 9, x 9,-x 9],[y 9, y 9,-y 9,-y 9], k^{\prime}\right)$

$[\mathrm{x} 10, \mathrm{y} 10]=0 \mathrm{de} 45(\mathrm{QODE} 1,[0: 0.002: 5], 6.1)$;

plot $\left([x 10,-x 10, x 10,-x 10],[y 10, y 10,-y 10,-y 10], ' k^{-'}\right)$

$[\mathrm{x} 11, \mathrm{y} 11]=0 \mathrm{de} 45(\mathrm{QODE} 1,[0: 0.002: 5], 6.0)$;

plot $\left([x 11,-x 11, x 11,-x 11],[y 11, y 11,-y 11,-y 11], k^{-\prime}\right)$

$[\mathrm{x} 12, \mathrm{y} 12]=0 \mathrm{de} 45(\mathrm{\varrho ODE} 1,[0: 0.002: 5], 5.9)$;

plot $([x 12,-x 12, x 12,-x 12],[y 12, y 12,-y 12,-y 12], ' k-')$

$[\mathrm{x} 13, \mathrm{y} 13]=0 \mathrm{de} 45(\mathrm{\varrho ODE} 1,[0: 0.002: 5], 5.8)$;

plot $([x 13,-x 13, x 13,-x 13],[y 13, y 13,-y 13,-y 13], ' k-')$

$[\mathrm{x} 14, \mathrm{y} 14]=0 \mathrm{de} 45(\mathrm{\varrho ODE} 1,[0: 0.002: 5], 5.7)$;

plot $([x 14,-x 14, x 14,-x 14],[y 14, y 14,-y 14,-y 14], ' k-')$

$[\mathrm{x} 15, \mathrm{y} 15]=0 \mathrm{de} 45($ @ODE1, $[0: 0.002: 5], 5.6)$;

plot $\left([x 15,-x 15, x 15,-x 15],[y 15, y 15,-y 15,-y 15], k^{\prime}-^{\prime}\right)$ 
$[\mathrm{x} 16, \mathrm{y} 16]=0 \mathrm{de} 45(@ O D E 1,[0: 0.002: 5], 5.5)$;

plot $\left([\mathrm{x} 16,-\mathrm{x} 16, \mathrm{x} 16,-\mathrm{x} 16],[\mathrm{y} 16, \mathrm{y} 16,-\mathrm{y} 16,-\mathrm{y} 16], \mathrm{k}^{\prime}\right)$

$[\mathrm{x} 17, \mathrm{y} 17]=0 \mathrm{de} 45(\mathrm{QODE} 1,[0: 0.002: 5], 5.4)$;

plot $\left([x 17,-x 17, x 17,-x 17],[y 17, y 17,-y 17,-y 17], k^{-\prime}\right)$

$[\mathrm{x} 18, \mathrm{y} 18]=0 \mathrm{de} 45(@ O D E 1,[0: 0.002: 5], 5.3)$;

plot $\left([x 18,-x 18, x 18,-x 18],[y 18, y 18,-y 18,-y 18], k^{-\prime}\right)$

$[\mathrm{x} 19, \mathrm{y} 19]=0 \mathrm{de} 45(\mathrm{@ODE} 1,[0: 0.002: 5], 5.2)$;

plot $([x 19,-x 19, x 19,-x 19],[y 19, y 19,-y 19,-y 19], ' k-')$

$[\mathrm{x} 20, \mathrm{y} 20]=0 \mathrm{de} 45(\mathrm{QODE} 1,[0: 0.002: 5], 5.1)$;

plot $\left([x 20,-x 20, x 20,-x 20],[y 20, y 20,-y 20,-y 20], ' k^{\prime}\right.$ ')

$[\mathrm{x} 21, \mathrm{y} 21]=0 \mathrm{de} 45(\mathrm{QODE} 1,[0: 0.002: 5], 5.0)$;

plot $\left([x 21,-x 21, x 21,-x 21],[y 21, y 21,-y 21,-y 21], k^{-\prime}\right)$

$[\mathrm{x} 22, \mathrm{y} 22]=0 \mathrm{de} 45(\mathrm{\varrho ODE} 1,[0: 0.002: 5], 4.9)$;

plot $\left([x 22,-x 22, x 22,-x 22],[y 22, y 22,-y 22,-y 22], k^{\prime}-^{\prime}\right)$

$[\mathrm{x} 23, \mathrm{y} 23]=0 \mathrm{de} 45(\mathrm{QODE} 1,[0: 0.002: 5], 4.8)$;

plot $([x 23,-x 23, x 23,-x 23],[y 23, y 23,-y 23,-y 23], ' k-1)$

$[\mathrm{x} 24, \mathrm{y} 24]=0 \mathrm{de} 45(@ \mathrm{QDE} 1,[0: 0.002: 5], 4.7)$;

plot $([x 24,-x 24, x 24,-x 24],[y 24, y 24,-y 24,-y 24], ' k-')$

$[\mathrm{x} 25, \mathrm{y} 25]=0 \mathrm{de} 45(\mathrm{QODE} 1,[0: 0.002: 5], 4.6)$;

plot $\left([x 25,-x 25, x 25,-x 25],[y 25, y 25,-y 25,-y 25], ' k^{-\prime}\right)$

$[\mathrm{x} 26, \mathrm{y} 26]=0 \mathrm{de} 45(\mathrm{QODE} 1,[0: 0.002: 5], 4.5)$;

plot $([x 26,-x 26, x 26,-x 26],[y 26, y 26,-y 26,-y 26], ' k-')$

$[\mathrm{x} 27, \mathrm{y} 27]=0 \mathrm{de} 45(\mathrm{@ODE} 1,[0: 0.002: 5], 4.4)$;

plot $([x 27,-x 27, x 27,-x 27],[y 27, y 27,-y 27,-y 27], ' k-')$

$[\mathrm{x} 28, \mathrm{y} 28]=0 \mathrm{de} 45(\mathrm{QODE} 1,[0: 0.002: 5], 4.3)$;

plot $\left([x 28,-x 28, x 28,-x 28],[y 28, y 28,-y 28,-y 28], ' k^{-\prime}\right)$

$[\mathrm{x} 29, \mathrm{y} 29]=0 \mathrm{de} 45(\mathrm{@ODE} 1,[0: 0.002: 5], 4.2)$;

plot $\left([x 29,-x 29, x 29,-x 29],[y 29, y 29,-y 29,-y 29], ' k^{\prime}\right)$

$[\mathrm{x} 30, \mathrm{y} 30]=0 \mathrm{de} 45(\mathrm{QODE} 1,[0: 0.002: 5], 4.1)$;

plot $\left([x 30,-x 30, x 30,-x 30],[y 30, y 30,-y 30,-y 30],{ }^{\prime} k-^{\prime}\right)$

$[\mathrm{x} 31, \mathrm{y} 31]=0 \mathrm{de} 45(@ O D E 1,[0: 0.002: 5], 4.0)$;

plot $\left([x 31,-x 31, x 31,-x 31],[y 31, y 31,-y 31,-y 31], k^{-\prime}\right)$

$[\mathrm{x} 32, \mathrm{y} 32]=0 \mathrm{de} 45(\mathrm{\varrho ODE} 1,[0: 0.002: 5], 3.9)$;

plot $\left([x 32,-x 32, x 32,-x 32],[y 32, y 32,-y 32,-y 32],{ }^{\prime}-^{\prime}\right)$

$[\mathrm{x} 33, \mathrm{y} 33]=0 \mathrm{de} 45(\mathrm{QODE} 1,[0: 0.002: 5], 3.8)$;

plot $\left([x 33,-x 33, x 33,-x 33],[y 33, y 33,-y 33,-y 33], ' k^{\prime}\right)$

$[\mathrm{x} 34, \mathrm{y} 34]=0 \mathrm{de} 45(\mathrm{QODE} 1,[0: 0.002: 5], 3.7)$;

plot $\left([x 34,-x 34, x 34,-x 34],[y 34, y 34,-y 34,-y 34], k^{\prime}{ }^{\prime}\right)$

$[\mathrm{x} 35, \mathrm{y} 35]=0 \mathrm{de} 45(\mathrm{QODE} 1,[0: 0.002: 5], 3.6)$;

plot $\left([x 35,-x 35, x 35,-x 35],[y 35, y 35,-y 35,-y 35],{ }^{\prime}-^{\prime}\right)$

$[\mathrm{x} 36, \mathrm{y} 36]=0 \mathrm{de} 45(\mathrm{QODE} 1,[0: 0.002: 5], 3.5)$;

plot $\left([x 36,-x 36, x 36,-x 36],[y 36, y 36,-y 36,-y 36],{ }^{\prime}-^{\prime}\right)$

$[\mathrm{x} 37, \mathrm{y} 37]=0 \mathrm{de} 45($ @ODE1, $[0: 0.002: 5], 3.4)$;

plot $([x 37,-x 37, x 37,-x 37],[y 37, y 37,-y 37,-y 37], ' k-')$

$[\mathrm{x} 38, \mathrm{y} 38]=0 \mathrm{de} 45(\mathrm{QODE} 1,[0: 0.002: 5], 3.3)$;

plot $([x 38,-x 38, x 38,-x 38],[y 38, y 38,-y 38,-y 38], ' k-')$

$[\mathrm{x} 39, \mathrm{y} 39]=0 \mathrm{de} 45(@ \mathrm{ODE} 1,[0: 0.002: 5], 3.2)$;

plot $\left([x 39,-x 39, x 39,-x 39],[y 39, y 39,-y 39,-y 39],{ }^{\prime}-^{\prime}\right)$

$[\mathrm{x} 40, \mathrm{y} 40]=0 \mathrm{de} 45(\mathrm{QODE} 1,[0: 0.002: 5], 3.1)$;

plot $\left([x 40,-x 40, x 40,-x 40],[y 40, y 40,-y 40,-y 40], k^{\prime}-^{\prime}\right)$

$[\mathrm{x} 41, \mathrm{y} 41]=0 \mathrm{de} 45(\mathrm{\varrho ODE} 1,[0: 0.002: 5], 3.0)$;

plot $\left([x 41,-x 41, x 41,-x 41],[y 41, y 41,-y 41,-y 41], k^{-\prime}\right)$

$[\mathrm{x} 42, \mathrm{y} 42]=0 \mathrm{de} 45(\mathrm{\varrho ODE} 1,[0: 0.002: 5], 2.9)$;

plot $\left([x 42,-x 42, x 42,-x 42],[y 42, y 42,-y 42,-y 42], k^{\prime}-^{\prime}\right)$

$[\mathrm{x} 43, \mathrm{y} 43]=0 \mathrm{de} 45(\mathrm{\varrho ODE} 1,[0: 0.002: 5], 2.8)$;

plot $\left([x 43,-x 43, x 43,-x 43],[y 43, y 43,-y 43,-y 43], k^{\prime}-^{\prime}\right)$

$[\mathrm{x} 44, \mathrm{y} 44]=0 \mathrm{de} 45(\mathrm{@ODE} 1,[0: 0.002: 5], 2.7)$; \%\# Ok<*NBRAK>

plot $\left([x 44,-x 44, x 44,-x 44],[y 44, y 44,-y 44,-y 44], k^{-\prime}\right)$

$[\mathrm{x} 45, \mathrm{y} 45]=0 \mathrm{de} 45(\mathrm{QODE} 1,[0: 0.002: 5], 2.6)$;

$\operatorname{plot}\left([x 45,-x 45, x 45,-x 45],[y 45, y 45,-y 45,-y 45], k^{\prime}-^{\prime}\right)$

$[\mathrm{x} 46, \mathrm{y} 46]=0 \mathrm{de} 45(\mathrm{QODE} 1,[0: 0.002: 5], 2.5)$;

plot $([x 46,-x 46, x 46,-x 46],[y 46, y 46,-y 46,-y 46], ' k-')$

$[\mathrm{x} 47, \mathrm{y} 47]=0 \mathrm{de} 45(\mathrm{\varrho ODE} 1,[0: 0.002: 5], 2.4)$;

plot $([x 47,-x 47, x 47,-x 47],[y 47, y 47,-y 47,-y 47], ' k-')$

$[\mathrm{x} 48, \mathrm{y} 48]=0 \mathrm{de} 45(\mathrm{eODE} 1,[0: 0.002: 5], 2.3)$;

plot $\left([x 48,-x 48, x 48,-x 48],[y 48, y 48,-y 48,-y 48], k^{-\prime}\right)$

$[\mathrm{x} 49, \mathrm{y} 49]=0 \mathrm{de} 45(\mathrm{QODE} 1,[0: 0.002: 5], 2.2)$;

plot $\left([x 49,-x 49, x 49,-x 49],[y 49, y 49,-y 49,-y 49], k^{-\prime}\right)$

$[\mathrm{x} 50, \mathrm{y} 50]=0 \mathrm{de} 45(\mathrm{QODE} 1,[0: 0.002: 5], 2.1)$;

plot $\left([x 50,-x 50, x 50,-x 50],[y 50, y 50,-y 50,-y 50], k^{\prime}-^{\prime}\right)$

$[\mathrm{x} 51, \mathrm{y} 51]=0 \mathrm{de} 45(@ O D E 1,[0: 0.002: 5], 2.0)$; 
plot $\left([x 51,-x 51, x 51,-x 51],[y 51, y 51,-y 51,-y 51],{ }^{\prime}-^{\prime}\right)$ $[\mathrm{x} 52, \mathrm{y} 52]=0 \mathrm{de} 45(\mathrm{\varrho ODE} 1,[0: 0.002: 5], 1.9)$;

plot $\left([x 52,-x 52, x 52,-x 52],[y 52, y 52,-y 52,-y 52],{ }^{\prime}-^{\prime}\right)$ $[\mathrm{x} 53, \mathrm{y} 53]=0 \mathrm{de} 45(\mathrm{QODE} 1,[0: 0.002: 5], 1.8)$;

plot $\left([x 53,-x 53, x 53,-x 53],[y 53, y 53,-y 53,-y 53],{ }^{\prime}-^{\prime}\right)$ $[\mathrm{x} 54, \mathrm{y} 54]=0 \mathrm{de} 45(\mathrm{QODE} 1,[0: 0.002: 5], 1.7)$;

plot $\left([x 54,-x 54, x 54,-x 54],[y 54, y 54,-y 54,-y 54], k^{-\prime}\right)$ $[\mathrm{x} 55, \mathrm{y} 55]=0 \mathrm{de} 45(\mathrm{QODE} 1,[0: 0.002: 5], 1.6)$;

plot $\left([x 55,-x 55, x 55,-x 55],[y 55, y 55,-y 55,-y 55], ' k^{\prime}\right)$ $[\mathrm{x} 56, \mathrm{y} 56]=0 \mathrm{de} 45(\mathrm{QODE} 1,[0: 0.002: 5], 1.5)$.

plot $\left([x 56,-x 56, x 56,-x 56],[y 56, y 56,-y 56,-y 56], k^{\prime}-^{\prime}\right)$ $[\mathrm{x} 57, \mathrm{y} 57]=0 \mathrm{de} 45($ @ODE $1,[0: 0.002: 5], 1.4)$;

plot $\left([x 57,-x 57, x 57,-x 57],[y 57, y 57,-y 57,-y 57], k^{-\prime}\right)$ $[\mathrm{x} 58, \mathrm{y} 58]=0 \mathrm{de} 45(@ \mathrm{ODE} 1,[0: 0.002: 5], 1.3)$;

plot $\left([x 58,-x 58, x 58,-x 58],[y 58, y 58,-y 58,-y 58], ' k^{\prime} '\right)$

$[\mathrm{x} 59, \mathrm{y} 59]=0 \mathrm{de} 45(\mathrm{QODE} 1,[0: 0.002: 5], 1.2)$;

plot $([x 59,-x 59, x 59,-x 59],[y 59, y 59,-y 59,-y 59], ' k-')$

$[\mathrm{x} 60, \mathrm{y} 60]=0 \mathrm{de} 45(\mathrm{eODE} 1,[0: 0.002: 5], 1.1)$;

plot $\left([\mathrm{x} 60,-\mathrm{x} 60, \mathrm{x} 60,-\mathrm{x} 60],[\mathrm{y} 60, \mathrm{y} 60,-\mathrm{y} 60,-\mathrm{y} 60], \mathrm{\prime}^{\prime} \mathrm{-}^{\prime}\right)$

oGenerating Secondary Stress Trajectories

$[\mathrm{x} 61, \mathrm{y} 61]=0 \mathrm{de} 45(\mathrm{QODE} 2,[0.996: 0.002: 5.996], 0.087) ; \circ 5$

plot $\left([x 61,-x 61, x 61,-x 61],[y 61, y 61,-y 61,-y 61], '-^{\prime}\right)$

$[\mathrm{x} 62, \mathrm{y} 62]=0 \mathrm{de} 45(@ \mathrm{ODE} 2,[0.984: 0.002: 5.984], 0.174) ; \circ 10$

plot $\left([x 62,-x 62, x 62,-x 62],[y 62, y 62,-y 62,-y 62], ' k^{\prime} '\right)$

$[\mathrm{x} 63, \mathrm{y} 63]=0 \mathrm{de} 45(\mathrm{eODE} 2,[0.965: 0.002: 5.965], 0.259) ; \circ 15$

plot $\left([x 63,-x 63, x 63,-x 63],[y 63, y 63,-y 63,-y 63],{ }^{\prime}-^{\prime}\right)$

$[\mathrm{x} 64, \mathrm{y} 64]=0 \mathrm{de} 45(@$ ODE $2,[0.939: 0.002: 5.939], 0.342) ; \circ 20$

plot $\left([x 64,-x 64, x 64,-x 64],[y 64, y 64,-y 64,-y 64], k^{-\prime}\right)$

$[\mathrm{x} 65, \mathrm{y} 65]=0 \mathrm{de} 45(@ \mathrm{ODE} 2,[0.906: 0.002: 5.906], 0.423) ; \circ 25$

plot $\left([x 65,-x 65, x 65,-x 65],[y 65, y 65,-y 65,-y 65], ' k^{\prime}\right.$ ')

$[\mathrm{x} 66, \mathrm{y} 66]=0 \mathrm{de} 45(\mathrm{QDEE} 2,[0.866: 0.002: 5.866], 0.500) ; \div 30$

plot $\left([x 66,-x 66, x 66,-x 66],[y 66, y 66,-y 66,-y 66], \prime^{\prime-\prime}\right)$

$[\mathrm{x} 67, \mathrm{y} 67]=0 \mathrm{de} 45(\mathrm{QODE} 2,[0.819: 0.002: 5.819], 0.573) ; \circ 35$

$\operatorname{plot}\left([x 67,-x 67, x 67,-x 67],[y 67, y 67,-y 67,-y 67], k^{\prime}-^{\prime}\right)$

$[\mathrm{x} 68, \mathrm{y} 68]=0 \mathrm{de} 45(\mathrm{QODE} 2,[0.766: 0.002: 5.766], 0.643) ; \circ 40$

plot $\left([x 68,-x 68, x 68,-x 68],[y 68, y 68,-y 68,-y 68],{ }^{\prime} k-^{\prime}\right)$

$[\mathrm{x} 69, \mathrm{y} 69]=0 \mathrm{de} 45(\mathrm{QDEE} 2,[0.707: 0.002: 5.707], 0.707) ; \circ 45$

plot $\left([x 69,-x 69, x 69,-x 69],[y 69, y 69,-y 69,-y 69], k^{-\prime}\right)$

$[\mathrm{x} 70, \mathrm{y} 70]=0 \mathrm{de} 45(\mathrm{QODE} 2,[0.643: 0.002: 5.643], 0.766) ; \circ 50$

$\operatorname{plot}\left([x 70,-x 70, x 70,-x 70],[y 70, y 70,-y 70,-y 70], k^{-\prime}\right)$

$[\mathrm{x} 71, \mathrm{y} 71]=0 \mathrm{de} 45(\mathrm{QODE} 2,[0.573: 0.002: 5.573], 0.819) ; \circ 55$

plot $\left([x 71,-x 71, x 71,-x 71],[y 71, y 71,-y 71,-y 71], ' k-^{\prime}\right)$

$[\mathrm{x} 72, \mathrm{y} 72]=0 \mathrm{de} 45(@ \mathrm{ODE} 2,[0.500: 0.002: 5.500], 0.866) ; \circ 60$

plot $([x 72,-x 72, x 72,-x 72],[y 72, y 72,-y 72,-y 72], ' k-')$

$[\mathrm{x} 73, \mathrm{y} 73]=0 \mathrm{de} 45(\mathrm{QODE} 2,[0.423: 0.002: 5.423], 0.906) ; \circ 65$

plot $\left([x 73,-x 73, x 73,-x 73],[y 73, y 73,-y 73,-y 73], ' k-^{\prime}\right)$

$[\mathrm{x} 74, \mathrm{y} 74]=0 \mathrm{de} 45(\mathrm{QODE} 2,[0.342: 0.002: 5.342], 0.939) ; \circ 70$

plot $\left([x 74,-x 74, x 74,-x 74],[y 74, y 74,-y 74,-y 74],{ }^{\prime}-^{\prime}\right)$

$[\mathrm{x} 75, \mathrm{y} 75]=0 \mathrm{de} 45(\mathrm{QODE} 2,[0.259: 0.002: 5.259], 0.965) ; \circ 75$

plot $\left([x 75,-x 75, x 75,-x 75],[y 75, y 75,-y 75,-y 75],{ }^{\prime}-^{\prime}\right.$ ')

$[\mathrm{x} 76, \mathrm{y} 76]=0 \mathrm{de} 45(@ \mathrm{ODE} 2,[0.174: 0.002: 5.174], 0.984) ; \circ 80$

plot $\left([x 76,-x 76, x 76,-x 76],[y 76, y 76,-y 76,-y 76], k^{-1}\right)$

$[\mathrm{x} 77, \mathrm{y} 77]=0 \mathrm{de} 45(@ \mathrm{ODE} 2,[0.087: 0.002: 5.087], 0.996) ; \circ 85$

plot $\left([x 77,-x 77, x 77,-x 77],[y 77, y 77,-y 77,-y 77], k^{\prime}\right)$

$\mathrm{x} 78=[0: 0.002: 5]$ ';

$\mathrm{y} 78=\mathrm{x} 78 . * 0+5$;

plot $(x 78, y 78, ' k-')$

plot $\left(-x 78, y 78, k^{\prime}-'\right)$

plot $\left(x 78,-y 78,{ }^{\prime}-^{\prime}\right)$

plot $\left(-x 78,-y 78, \mathrm{k}^{-}\right.$')

$\operatorname{plot}\left(\mathrm{y} 78, \mathrm{x} 78, \mathrm{\prime}^{\left.\mathrm{k}-\mathrm{\prime}^{\prime}\right)}\right.$

plot $\left(-y 78, x 78,{ }^{\prime} k-^{\prime}\right)$

plot $\left(y 78,-x 78,{ }^{\prime} \mathrm{k}-{ }^{\prime}\right)$

plot $\left(-y 78,-x 78,{ }^{\prime} k-'\right)$

$\mathrm{x}=[1: .002: 6]^{\prime}$;

$\mathrm{y}=\mathrm{x} \cdot * 0$;

plot $\left(x, y,{ }^{\prime}-^{\prime}\right)$

plot $\left(-x, y,{ }^{\prime} k-'\right)$

plot $(y, x, ' k-')$

plot $\left(y,-x, k^{\prime}-{ }^{\prime}\right)$

$\mathrm{x}=0: .01: 1$; 
$\mathrm{y}=\operatorname{squt}\left(1-\mathrm{x} \cdot{ }^{\wedge} 2\right) ;$

plot $(x, y, ' k-'$ ' 'Linewidth', 1.5)

plot $(-x, y, ' k-', '$ Linewidth', 1.5$)$

plot $\left(x,-y,{ }^{\prime}-{ }^{\prime}, '\right.$ 'Linewidth', 1.5)

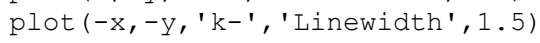

for $j=1: 40$

$m=[y 78, x 22, x 23, x 24, x 25, x 26, x 27, x 28, x 29, x 30, x 31, x 32, x 33, x 34, x 35, x 36, x 37, x 38, x 39, x 40$, $x 41, x 42, x 43, x 44, x 45, x 46, x 47, x 48, x 49, x 50, x 51, x 52, x 53, x 54, x 55, x 56, x 57, x 58, x 59, x 60] ;$ $\mathrm{n}=[\mathrm{x} 78, \mathrm{y} 22, \mathrm{y} 23, \mathrm{y} 24, \mathrm{y} 25, \mathrm{y} 26, \mathrm{y} 27, \mathrm{y} 28, \mathrm{y} 29, \mathrm{y} 30, \mathrm{y} 31, \mathrm{y} 32, \mathrm{y} 33, \mathrm{y} 34, \mathrm{y} 35, \mathrm{y} 36, \mathrm{y} 37, \mathrm{y} 38, \mathrm{y} 39, \mathrm{y} 40$, $\mathrm{y} 41, \mathrm{y} 42, \mathrm{y} 43, \mathrm{y} 44, \mathrm{y} 45, \mathrm{y} 46, \mathrm{y} 47, \mathrm{y} 48, \mathrm{y} 49, \mathrm{y} 50, \mathrm{y} 51, \mathrm{y} 52, \mathrm{y} 53, \mathrm{y} 54, \mathrm{y} 55, \mathrm{y} 56, \mathrm{y} 57, \mathrm{y} 58, \mathrm{y} 59, \mathrm{y} 60]$; end $[A 1(j, 1), A 1(j, 2)]=$ curveintersect $(m(:, j), \mathrm{n}(:, j), \mathrm{x} 61, \mathrm{y} 61) ;$ \% $\mathrm{ok}<\star A G R O W>$

for $j=1: 41$

$\mathrm{m}=[\mathrm{y} 78, \mathrm{x} 21, \mathrm{x} 22, \mathrm{x} 23, \mathrm{x} 24, \mathrm{x} 25, \mathrm{x} 26, \mathrm{x} 27, \mathrm{x} 28, \mathrm{x} 29, \mathrm{x} 30, \mathrm{x} 31, \mathrm{x} 32, \mathrm{x} 33, \mathrm{x} 34, \mathrm{x} 35, \mathrm{x} 36, \mathrm{x} 37, \mathrm{x} 38, \mathrm{x} 39$, $x 40, x 41, x 42, x 43, x 44, x 45, x 46, x 47, x 48, x 49, x 50, x 51, x 52, x 53, x 54, x 55, x 56, x 57, x 58, x 59, x 60$ ] ;

$n=[x 78, y 21, y 22, y 23, y 24, y 25, y 26, y 27, y 28, y 29, y 30, y 31, y 32, y 33, y 34, y 35, y 36, y 37, y 38, y 39$, $\mathrm{y} 40, \mathrm{y} 41, \mathrm{y} 42, \mathrm{y} 43, \mathrm{y} 44, \mathrm{y} 45, \mathrm{y} 46, \mathrm{y} 47, \mathrm{y} 48, \mathrm{y} 49, \mathrm{y} 50, \mathrm{y} 51, \mathrm{y} 52, \mathrm{y} 53, \mathrm{y} 54, \mathrm{y} 55, \mathrm{y} 56, \mathrm{y} 57, \mathrm{y} 58, \mathrm{y} 59, \mathrm{y} 60$ ] ; end

$[A 2(j, 1), A 2(j, 2)]=$ curveintersect $(m(:, j), n(:, j), x 62, y 62) ; \% \#$ ok<*AGROW>

for $j=1: 42$

$\mathrm{m}=[\mathrm{y} 78, \mathrm{x} 20, \mathrm{x} 21, \mathrm{x} 22, \mathrm{x} 23, \mathrm{x} 24, \mathrm{x} 25, \mathrm{x} 26, \mathrm{x} 27, \mathrm{x} 28, \mathrm{x} 29, \mathrm{x} 30, \mathrm{x} 31, \mathrm{x} 32, \mathrm{x} 33, \mathrm{x} 34, \mathrm{x} 35, \mathrm{x} 36, \mathrm{x} 37, \mathrm{x} 38$, $x 39, x 40, x 41, x 42, x 43, x 44, x 45, x 46, x 47, x 48, x 49, x 50, x 51, x 52, x 53, x 54, x 55, x 56, x 57, x 58, x 59$ $, \times 60]$;

$n=[x 78, y 20, y 21, y 22, y 23, y 24, y 25, y 26, y 27, y 28, y 29, y 30, y 31, y 32, y 33, y 34, y 35, y 36, y 37, y 38$, $\mathrm{y} 39, \mathrm{y} 40, \mathrm{y} 41, \mathrm{y} 42, \mathrm{y} 43, \mathrm{y} 44, \mathrm{y} 45, \mathrm{y} 46, \mathrm{y} 47, \mathrm{y} 48, \mathrm{y} 49, \mathrm{y} 50, \mathrm{y} 51, \mathrm{y} 52, \mathrm{y} 53, \mathrm{y} 54, \mathrm{y} 55, \mathrm{y} 56, \mathrm{y} 57, \mathrm{y} 58, \mathrm{y} 59$ $, \mathrm{y} 60]$; end

$[A 3(j, 1), A 3(j, 2)]=$ curveintersect $(m(:, j), n(:, j), x 63, y 63) ;$ o\# ok<*AGROW>

for $j=1: 43$

$\mathrm{m}=[\mathrm{y} 78, \mathrm{x} 19, \mathrm{x} 20, \mathrm{x} 21, \mathrm{x} 22, \mathrm{x} 23, \mathrm{x} 24, \mathrm{x} 25, \mathrm{x} 26, \mathrm{x} 27, \mathrm{x} 28, \mathrm{x} 29, \mathrm{x} 30, \mathrm{x} 31, \mathrm{x} 32, \mathrm{x} 33, \mathrm{x} 34, \mathrm{x} 35, \mathrm{x} 36, \mathrm{x} 37$, $x 38, x 39, x 40, x 41, x 42, x 43, x 44, x 45, x 46, x 47, x 48, x 49, x 50, x 51, x 52, x 53, x 54, x 55, x 56, x 57, x 58$ $, \times 59, \times 60] ;$

$n=[x 78, y 19, y 20, y 21, y 22, y 23, y 24, y 25, y 26, y 27, y 28, y 29, y 30, y 31, y 32, y 33, y 34, y 35, y 36, y 37$, $\mathrm{y} 38, \mathrm{y} 39, \mathrm{y} 40, \mathrm{y} 41, \mathrm{y} 42, \mathrm{y} 43, \mathrm{y} 44, \mathrm{y} 45, \mathrm{y} 46, \mathrm{y} 47, \mathrm{y} 48, \mathrm{y} 49, \mathrm{y} 50, \mathrm{y} 51, \mathrm{y} 52, \mathrm{y} 53, \mathrm{y} 54, \mathrm{y} 55, \mathrm{y} 56, \mathrm{y} 57, \mathrm{y} 58$ $, \mathrm{y} 59, \mathrm{y} 60]$

end

$[A 4(j, 1), A 4(j, 2)]=$ curveintersect $(m(:, j), n(:, j), x 64, y 64) ;$ o\# ok<*AGROW>

for $j=1: 45$

$m=[y 78, x 17, x 18, x 19, x 20, x 21, x 22, x 23, x 24, x 25, x 26, x 27, x 28, x 29, x 30, x 31, x 32, x 33, x 34, x 35$, $x 36, x 37, x 38, x 39, x 40, x 41, x 42, x 43, x 44, x 45, x 46, x 47, x 48, x 49, x 50, x 51, x 52, x 53, x 54, x 55, x 56$ $, \times 57, x 58, \times 59, \times 60]$;

$n=[x 78, y 17, y 18, y 19, y 20, y 21, y 22, y 23, y 24, y 25, y 26, y 27, y 28, y 29, y 30, y 31, y 32, y 33, y 34, y 35$, $\mathrm{y} 36, \mathrm{y} 37, \mathrm{y} 38, \mathrm{y} 39, \mathrm{y} 40, \mathrm{y} 41, \mathrm{y} 42, \mathrm{y} 43, \mathrm{y} 44, \mathrm{y} 45, \mathrm{y} 46, \mathrm{y} 47, \mathrm{y} 48, \mathrm{y} 49, \mathrm{y} 50, \mathrm{y} 51, \mathrm{y} 52, \mathrm{y} 53, \mathrm{y} 54, \mathrm{y} 55, \mathrm{y} 56$ $, \mathrm{y} 57, \mathrm{y} 58, \mathrm{y} 59, \mathrm{y} 60]$

end

$[A 5(j, 1), A 5(j, 2)]=$ curveintersect $(m(:, j), n(:, j), x 65, y 65) ;$ o\#०k<*AGROW>

for $j=1: 48$

$\mathrm{m}=[\mathrm{y} 78, \mathrm{x} 14, \mathrm{x} 15, \mathrm{x} 16, \mathrm{x} 17, \mathrm{x} 18, \mathrm{x} 19, \mathrm{x} 20, \mathrm{x} 21, \mathrm{x} 22, \mathrm{x} 23, \mathrm{x} 24, \mathrm{x} 25, \mathrm{x} 26, \mathrm{x} 27, \mathrm{x} 28, \mathrm{x} 29, \mathrm{x} 30, \mathrm{x} 31, \mathrm{x} 32$, $x 33, x 34, x 35, x 36, x 37, x 38, x 39, x 40, x 41, x 42, x 43, x 44, x 45, x 46, x 47, x 48, x 49, x 50, x 51, x 52, x 53$ $, x 54, x 55, x 56, x 57, x 58, x 59, x 60]$;

$\mathrm{n}=[\mathrm{x} 78, \mathrm{y} 14, \mathrm{y} 15, \mathrm{y} 16, \mathrm{y} 17, \mathrm{y} 18, \mathrm{y} 19, \mathrm{y} 20, \mathrm{y} 21, \mathrm{y} 22, \mathrm{y} 23, \mathrm{y} 24, \mathrm{y} 25, \mathrm{y} 26, \mathrm{y} 27, \mathrm{y} 28, \mathrm{y} 29, \mathrm{y} 30, \mathrm{y} 31, \mathrm{y} 32$, $\mathrm{y} 33, \mathrm{y} 34, \mathrm{y} 35, \mathrm{y} 36, \mathrm{y} 37, \mathrm{y} 38, \mathrm{y} 39, \mathrm{y} 40, \mathrm{y} 41, \mathrm{y} 42, \mathrm{y} 43, \mathrm{y} 44, \mathrm{y} 45, \mathrm{y} 46, \mathrm{y} 47, \mathrm{y} 48, \mathrm{y} 49, \mathrm{y} 50, \mathrm{y} 51, \mathrm{y} 52, \mathrm{y} 53$ $, \mathrm{y} 54, \mathrm{y} 55, \mathrm{y} 56, \mathrm{y} 57, \mathrm{y} 58, \mathrm{y} 59, \mathrm{y} 60]$ end

$[A 6(j, 1), A 6(j, 2)]=$ curveintersect $(m(:, j), n(:, j), x 66, y 66) ;$ o\#ok<*AGROW>

for $j=1: 51$

$\mathrm{m}=[\mathrm{y} 78, \mathrm{x} 11, \mathrm{x} 12, \mathrm{x} 13, \mathrm{x} 14, \mathrm{x} 15, \mathrm{x} 16, \mathrm{x} 17, \mathrm{x} 18, \mathrm{x} 19, \mathrm{x} 20, \mathrm{x} 21, \mathrm{x} 22, \mathrm{x} 23, \mathrm{x} 24, \mathrm{x} 25, \mathrm{x} 26, \mathrm{x} 27, \mathrm{x} 28, \mathrm{x} 29$, $x 30, x 31, x 32, x 33, x 34, x 35, x 36, x 37, x 38, x 39, x 40, x 41, x 42, x 43, x 44, x 45, x 46, x 47, x 48, x 49, x 50$ $, x 51, x 52, x 53, x 54, x 55, x 56, x 57, x 58, x 59, x 60]$

$n=[x 78, y 11, y 12, y 13, y 14, y 15, y 16, y 17, y 18, y 19, y 20, y 21, y 22, y 23, y 24, y 25, y 26, y 27, y 28, y 29$, y30,y31,y32,y33,y34, y35, y36,y37,y38,y39, y40,y41,y42,y43,y44,y45, y46, y47, y48, y49, y50 $, \mathrm{y} 51, \mathrm{y} 52, \mathrm{y} 53, \mathrm{y} 54, \mathrm{y} 55, \mathrm{y} 56, \mathrm{y} 57, \mathrm{y} 58, \mathrm{y} 59, \mathrm{y} 60] ;$ end

$[A 7(j, 1), A 7(j, 2)]=$ curveintersect $(m(:, j), n(:, j), x 67, y 67) ;$ o\#०k<*AGROW>

for $j=1: 55$

$\mathrm{m}=[\mathrm{y} 78, \mathrm{x} 7, \mathrm{x} 8, \mathrm{x} 9, \mathrm{x} 10, \mathrm{x} 11, \mathrm{x} 12, \mathrm{x} 13, \mathrm{x} 14, \mathrm{x} 15, \mathrm{x} 16, \mathrm{x} 17, \mathrm{x} 18, \mathrm{x} 19, \mathrm{x} 20, \mathrm{x} 21, \mathrm{x} 22, \mathrm{x} 23, \mathrm{x} 24, \mathrm{x} 25, \mathrm{x} 26$ $, x 27, x 28, x 29, x 30, x 31, x 32, x 33, x 34, x 35, x 36, x 37, x 38, x 39, x 40, x 41, x 42, x 43, x 44, x 45, x 46, x 4$ $7, x 48, x 49, x 50, x 51, x 52, x 53, x 54, x 55, x 56, x 57, x 58, x 59, x 60]$; 
$\mathrm{n}=[\mathrm{x} 78, \mathrm{y} 7, \mathrm{y} 8, \mathrm{y} 9, \mathrm{y} 10, \mathrm{y} 11, \mathrm{y} 12, \mathrm{y} 13, \mathrm{y} 14, \mathrm{y} 15, \mathrm{y} 16, \mathrm{y} 17, \mathrm{y} 18, \mathrm{y} 19, \mathrm{y} 20, \mathrm{y} 21, \mathrm{y} 22, \mathrm{y} 23, \mathrm{y} 24, \mathrm{y} 25, \mathrm{y} 26$ $, \mathrm{y} 27, \mathrm{y} 28, \mathrm{y} 29, \mathrm{y} 30, \mathrm{y} 31, \mathrm{y} 32, \mathrm{y} 33, \mathrm{y} 34, \mathrm{y} 35, \mathrm{y} 36, \mathrm{y} 37, \mathrm{y} 38, \mathrm{y} 39, \mathrm{y} 40, \mathrm{y} 41, \mathrm{y} 42, \mathrm{y} 43, \mathrm{y} 44, \mathrm{y} 45, \mathrm{y} 46, \mathrm{y} 4$ $7, y 48, y 49, y 50, y 51, y 52, y 53, y 54, y 55, y 56, y 57, y 58, y 59, y 60]$

end

$[A 8(j, 1), A 8(j, 2)]=$ curveintersect $(m(:, j), n(:, j), x 68, y 68) ;$ \%\#ok<*AGROW>

for $j=1: 61$

$\mathrm{m}=[\mathrm{y} 78, \mathrm{x} 1, \mathrm{x} 2, \mathrm{x} 3, \mathrm{x} 4, \mathrm{x} 5, \mathrm{x} 6, \mathrm{x} 7, \mathrm{x} 8, \mathrm{x} 9, \mathrm{x} 10, \mathrm{x} 11, \mathrm{x} 12, \mathrm{x} 13, \mathrm{x} 14, \mathrm{x} 15, \mathrm{x} 16, \mathrm{x} 17, \mathrm{x} 18, \mathrm{x} 19, \mathrm{x} 20, \mathrm{x} 21, \mathrm{x}$ $22, x 23, x 24, x 25, x 26, x 27, x 28, x 29, x 30, x 31, x 32, x 33, x 34, x 35, x 36, x 37, x 38, x 39, x 40, x 41, x 42$, $x 43, x 44, x 45, x 46, x 47, x 48, x 49, x 50, x 51, x 52, x 53, x 54, x 55, x 56, x 57, x 58, x 59, x 60]$;

$n=[x 78, y 1, y 2, y 3, y 4, y 5, y 6, y 7, y 8, y 9, y 10, y 11, y 12, y 13, y 14, y 15, y 16, y 17, y 18, y 19, y 20, y 21, y$ $22, y 23, y 24, y 25, y 26, y 27, y 28, y 29, y 30, y 31, y 32, y 33, y 34, y 35, y 36, y 37, y 38, y 39, y 40, y 41, y 42$, $\mathrm{y} 43, \mathrm{y} 44, \mathrm{y} 45, \mathrm{y} 46, \mathrm{y} 47, \mathrm{y} 48, \mathrm{y} 49, \mathrm{y} 50, \mathrm{y} 51, \mathrm{y} 52, \mathrm{y} 53, \mathrm{y} 54, \mathrm{y} 55, \mathrm{y} 56, \mathrm{y} 57, \mathrm{y} 58, \mathrm{y} 59, \mathrm{y} 60] ;$

end

$[A 9(j, 1), A 9(j, 2)]=$ curveintersect $(m(:, j), n(:, j), x 69, y 69) ;$ \%\#०k<*AGROW>

for $j=1: 55$

$m=[x 78, x 7, x 8, x 9, x 10, x 11, x 12, x 13, x 14, x 15, x 16, x 17, x 18, x 19, x 20, x 21, x 22, x 23, x 24, x 25, x 26$ $, x 27, x 28, x 29, x 30, x 31, x 32, x 33, x 34, x 35, x 36, x 37, x 38, x 39, x 40, x 41, x 42, x 43, x 44, x 45, x 46, x 4$ $7, x 48, x 49, x 50, x 51, x 52, x 53, x 54, x 55, x 56, x 57, x 58, x 59, x 60]$;

$\mathrm{n}=[\mathrm{y} 78, \mathrm{y} 7, \mathrm{y} 8, \mathrm{y} 9, \mathrm{y} 10, \mathrm{y} 11, \mathrm{y} 12, \mathrm{y} 13, \mathrm{y} 14, \mathrm{y} 15, \mathrm{y} 16, \mathrm{y} 17, \mathrm{y} 18, \mathrm{y} 19, \mathrm{y} 20, \mathrm{y} 21, \mathrm{y} 22, \mathrm{y} 23, \mathrm{y} 24, \mathrm{y} 25, \mathrm{y} 26$ $, \mathrm{y} 27, \mathrm{y} 28, \mathrm{y} 29, \mathrm{y} 30, \mathrm{y} 31, \mathrm{y} 32, \mathrm{y} 33, \mathrm{y} 34, \mathrm{y} 35, \mathrm{y} 36, \mathrm{y} 37, \mathrm{y} 38, \mathrm{y} 39, \mathrm{y} 40, \mathrm{y} 41, \mathrm{y} 42, \mathrm{y} 43, \mathrm{y} 44, \mathrm{y} 45, \mathrm{y} 46, \mathrm{y} 4$ $7, \mathrm{y} 48, \mathrm{y} 49, \mathrm{y} 50, \mathrm{y} 51, \mathrm{y} 52, \mathrm{y} 53, \mathrm{y} 54, \mathrm{y} 55, \mathrm{y} 56, \mathrm{y} 57, \mathrm{y} 58, \mathrm{y} 59, \mathrm{y} 60]$ end $[A 10(j, 1), A 10(j, 2)]=$ curveintersect $(m(:, j), n(:, j), x 70, y 70) ;$ o\#०k<*AGROW>

for $j=1: 51$

$\mathrm{m}=[\mathrm{x} 78, \mathrm{x} 11, \mathrm{x} 12, \mathrm{x} 13, \mathrm{x} 14, \mathrm{x} 15, \mathrm{x} 16, \mathrm{x} 17, \mathrm{x} 18, \mathrm{x} 19, \mathrm{x} 20, \mathrm{x} 21, \mathrm{x} 22, \mathrm{x} 23, \mathrm{x} 24, \mathrm{x} 25, \mathrm{x} 26, \mathrm{x} 27, \mathrm{x} 28, \mathrm{x} 29$, $x 30, x 31, x 32, x 33, x 34, x 35, x 36, x 37, x 38, x 39, x 40, x 41, x 42, x 43, x 44, x 45, x 46, x 47, x 48, x 49, x 50$ $, x 51, x 52, x 53, x 54, x 55, x 56, x 57, x 58, x 59, x 60]$

$\mathrm{n}=[\mathrm{y} 78, \mathrm{y} 11, \mathrm{y} 12, \mathrm{y} 13, \mathrm{y} 14, \mathrm{y} 15, \mathrm{y} 16, \mathrm{y} 17, \mathrm{y} 18, \mathrm{y} 19, \mathrm{y} 20, \mathrm{y} 21, \mathrm{y} 22, \mathrm{y} 23, \mathrm{y} 24, \mathrm{y} 25, \mathrm{y} 26, \mathrm{y} 27, \mathrm{y} 28, \mathrm{y} 29$, $\mathrm{y} 30, \mathrm{y} 31, \mathrm{y} 32, \mathrm{y} 33, \mathrm{y} 34, \mathrm{y} 35, \mathrm{y} 36, \mathrm{y} 37, \mathrm{y} 38, \mathrm{y} 39, \mathrm{y} 40, \mathrm{y} 41, \mathrm{y} 42, \mathrm{y} 43, \mathrm{y} 44, \mathrm{y} 45, \mathrm{y} 46, \mathrm{y} 47, \mathrm{y} 48, \mathrm{y} 49, \mathrm{y} 50$ $, \mathrm{y} 51, \mathrm{y} 52, \mathrm{y} 53, \mathrm{y} 54, \mathrm{y} 55, \mathrm{y} 56, \mathrm{y} 57, \mathrm{y} 58, \mathrm{y} 59, \mathrm{y} 60] ;$ end $[A 11(j, 1), A 11(j, 2)]=$ curveintersect $(m(:, j), n(:, j), x 71, y 71) ;$ o\# ok<*AGROW>

for $j=1: 48$

$\mathrm{m}=[\mathrm{x} 78, \mathrm{x} 14, \mathrm{x} 15, \mathrm{x} 16, \mathrm{x} 17, \mathrm{x} 18, \mathrm{x} 19, \mathrm{x} 20, \mathrm{x} 21, \mathrm{x} 22, \mathrm{x} 23, \mathrm{x} 24, \mathrm{x} 25, \mathrm{x} 26, \mathrm{x} 27, \mathrm{x} 28, \mathrm{x} 29, \mathrm{x} 30, \mathrm{x} 31, \mathrm{x} 32$, $x 33, x 34, x 35, x 36, x 37, x 38, x 39, x 40, x 41, x 42, x 43, x 44, x 45, x 46, x 47, x 48, x 49, x 50, x 51, x 52, x 53$ $, \times 54, x 55, x 56, x 57, x 58, x 59, \times 60]$;

$\mathrm{n}=[\mathrm{y} 78, \mathrm{y} 14, \mathrm{y} 15, \mathrm{y} 16, \mathrm{y} 17, \mathrm{y} 18, \mathrm{y} 19, \mathrm{y} 20, \mathrm{y} 21, \mathrm{y} 22, \mathrm{y} 23, \mathrm{y} 24, \mathrm{y} 25, \mathrm{y} 26, \mathrm{y} 27, \mathrm{y} 28, \mathrm{y} 29, \mathrm{y} 30, \mathrm{y} 31, \mathrm{y} 32$, $\mathrm{y} 33, \mathrm{y} 34, \mathrm{y} 35, \mathrm{y} 36, \mathrm{y} 37, \mathrm{y} 38, \mathrm{y} 39, \mathrm{y} 40, \mathrm{y} 41, \mathrm{y} 42, \mathrm{y} 43, \mathrm{y} 44, \mathrm{y} 45, \mathrm{y} 46, \mathrm{y} 47, \mathrm{y} 48, \mathrm{y} 49, \mathrm{y} 50, \mathrm{y} 51, \mathrm{y} 52, \mathrm{y} 53$ $, \mathrm{y} 54, \mathrm{y} 55, \mathrm{y} 56, \mathrm{y} 57, \mathrm{y} 58, \mathrm{y} 59, \mathrm{y} 60]$ end $[A 12(j, 1), A 12(j, 2)]=$ curveintersect $(m(:, j), n(:, j), x 72, y 72) ;$ o\# ok<*AGROW>

for $j=1: 45$

$\mathrm{m}=[\mathrm{x} 78, \mathrm{x} 17, \mathrm{x} 18, \mathrm{x} 19, \mathrm{x} 20, \mathrm{x} 21, \mathrm{x} 22, \mathrm{x} 23, \mathrm{x} 24, \mathrm{x} 25, \mathrm{x} 26, \mathrm{2} 27, \mathrm{x} 28, \mathrm{x} 29, \mathrm{x} 30, \mathrm{x} 31, \mathrm{x} 32, \mathrm{x} 33, \mathrm{x} 34, \mathrm{x} 35$, $x 36, x 37, x 38, x 39, x 40, x 41, x 42, x 43, x 44, x 45, x 46, x 47, x 48, x 49, x 50, x 51, x 52, x 53, x 54, x 55, x 56$ $, x 57, x 58, x 59, x 60]$;

$n=[y 78, y 17, y 18, y 19, y 20, y 21, y 22, y 23, y 24, y 25, y 26, y 27, y 28, y 29, y 30, y 31, y 32, y 33, y 34, y 35$, $\mathrm{y} 36, \mathrm{y} 37, \mathrm{y} 38, \mathrm{y} 39, \mathrm{y} 40, \mathrm{y} 41, \mathrm{y} 42, \mathrm{y} 43, \mathrm{y} 44, \mathrm{y} 45, \mathrm{y} 46, \mathrm{y} 47, \mathrm{y} 48, \mathrm{y} 49, \mathrm{y} 50, \mathrm{y} 51, \mathrm{y} 52, \mathrm{y} 53, \mathrm{y} 54, \mathrm{y} 55, \mathrm{y} 56$ $, \mathrm{y} 57, \mathrm{y} 58, \mathrm{y} 59, \mathrm{y} 60]$ end $[A 13(j, 1), A 13(j, 2)]=$ curveintersect $(m(:, j), n(:, j), x 73, y 73) ;$ o\#ok<*AGROW>

for $j=1: 43$

$m=[x 78, x 19, x 20, x 21, x 22, x 23, x 24, x 25, x 26, x 27, x 28, x 29, x 30, x 31, x 32, x 33, x 34, x 35, x 36, x 37$, $x 38, x 39, x 40, x 41, x 42, x 43, x 44, x 45, x 46, x 47, x 48, x 49, x 50, x 51, x 52, x 53, x 54, x 55, x 56, x 57, x 58$ $, \times 59, x 60]$

$n=[y 78, y 19, y 20, y 21, y 22, y 23, y 24, y 25, y 26, y 27, y 28, y 29, y 30, y 31, y 32, y 33, y 34, y 35, y 36, y 37$, $\mathrm{y} 38, y 39, y 40, y 41, y 42, y 43, y 44, y 45, y 46, y 47, y 48, y 49, y 50, y 51, y 52, y 53, y 54, y 55, y 56, y 57, y 58$ $, \mathrm{y} 59, \mathrm{y} 60]$

$[\mathrm{A} 14(j, 1), \mathrm{Al} 4(j, 2)]=$ curveintersect $(\mathrm{m}(:, j), \mathrm{n}(:, j), \mathrm{x} 74, \mathrm{y} 74) ;$ o\# ok<*AGROW>

end

for $j=1: 42$

$m=[x 78, x 20, x 21, x 22, x 23, x 24, x 25, x 26, x 27, x 28, x 29, x 30, x 31, x 32, x 33, x 34, x 35, x 36, x 37, x 38$, $x 39, x 40, x 41, x 42, x 43, x 44, x 45, x 46, x 47, x 48, x 49, x 50, x 51, x 52, x 53, x 54, x 55, x 56, x 57, x 58, x 59$ $, \times 60]$;

$n=[y 78, y 20, y 21, y 22, y 23, y 24, y 25, y 26, y 27, y 28, y 29, y 30, y 31, y 32, y 33, y 34, y 35, y 36, y 37, y 38$, $\mathrm{y} 39, \mathrm{y} 40, \mathrm{y} 41, \mathrm{y} 42, \mathrm{y} 43, \mathrm{y} 44, \mathrm{y} 45, \mathrm{y} 46, \mathrm{y} 47, \mathrm{y} 48, \mathrm{y} 49, \mathrm{y} 50, \mathrm{y} 51, \mathrm{y} 52, \mathrm{y} 53, \mathrm{y} 54, \mathrm{y} 55, \mathrm{y} 56, \mathrm{y} 57, \mathrm{y} 58, \mathrm{y} 59$ $, \mathrm{y} 60]$

$[A 15(j, 1), A 15(j, 2)]=$ curveintersect $(m(:, j), n(:, j), x 75, y 75) ;$ o\# ok<*AGROW> end

for $j=1: 41$ 
$m=[x 78, x 21, x 22, x 23, x 24, x 25, x 26, x 27, x 28, x 29, x 30, x 31, x 32, x 33, x 34, x 35, x 36, x 37, x 38, x 39$, $x 40, x 41, x 42, x 43, x 44, x 45, x 46, x 47, x 48, x 49, x 50, x 51, x 52, x 53, x 54, x 55, x 56, x 57, x 58, x 59, x 60$ ] ;

$\mathrm{n}=[\mathrm{y} 78, \mathrm{y} 21, \mathrm{y} 22, \mathrm{y} 23, \mathrm{y} 24, \mathrm{y} 25, \mathrm{y} 26, \mathrm{y} 27, \mathrm{y} 28, \mathrm{y} 29, \mathrm{y} 30, \mathrm{y} 31, \mathrm{y} 32, \mathrm{y} 33, \mathrm{y} 34, \mathrm{y} 35, \mathrm{y} 36, \mathrm{y} 37, \mathrm{y} 38, \mathrm{y} 39$, $\mathrm{y} 40, \mathrm{y} 41, \mathrm{y} 42, \mathrm{y} 43, \mathrm{y} 44, \mathrm{y} 45, \mathrm{y} 46, \mathrm{y} 47, \mathrm{y} 48, \mathrm{y} 49, \mathrm{y} 50, \mathrm{y} 51, \mathrm{y} 52, \mathrm{y} 53, \mathrm{y} 54, \mathrm{y} 55, \mathrm{y} 56, \mathrm{y} 57, \mathrm{y} 58, \mathrm{y} 59, \mathrm{y} 60$ ] ;

end

$[A 16(j, 1), A 16(j, 2)]=$ curveintersect $(m(:, j), n(:, j), x 76, y 76) ; \% \# \circ k<A G R O W>$

for $j=1: 40$

$m=[x 78, x 22, x 23, x 24, x 25, x 26, x 27, x 28, x 29, x 30, x 31, x 32, x 33, x 34, x 35, x 36, x 37, x 38, x 39, x 40$, $x 41, x 42, x 43, x 44, x 45, x 46, x 47, x 48, x 49, x 50, x 51, x 52, x 53, x 54, x 55, x 56, x 57, x 58, x 59, x 60] ;$ $\mathrm{n}=[\mathrm{y} 78, \mathrm{y} 22, \mathrm{y} 23, \mathrm{y} 24, \mathrm{y} 25, \mathrm{y} 26, \mathrm{y} 27, \mathrm{y} 28, \mathrm{y} 29, \mathrm{y} 30, \mathrm{y} 31, \mathrm{y} 32, \mathrm{y} 33, \mathrm{y} 34, \mathrm{y} 35, \mathrm{y} 36, \mathrm{y} 37, \mathrm{y} 38, \mathrm{y} 39, \mathrm{y} 40$, $\mathrm{y} 41, \mathrm{y} 42, \mathrm{y} 43, \mathrm{y} 44, \mathrm{y} 45, \mathrm{y} 46, \mathrm{y} 47, \mathrm{y} 48, \mathrm{y} 49, \mathrm{y} 50, \mathrm{y} 51, \mathrm{y} 52, \mathrm{y} 53, \mathrm{y} 54, \mathrm{y} 55, \mathrm{y} 56, \mathrm{y} 57, \mathrm{y} 58, \mathrm{y} 59, \mathrm{y} 60] ;$ end $[A 17(j, 1), A 17(j, 2)]=$ curveintersect $(m(:, j), n(:, j), x 77, y 77) ; \quad \circ \# \circ k<{ }^{*} A G R O W>$

for $j=1: 15$ $m=[x 1, x 2, x 3, x 4, x 5, x 7, x 8, x 9, x 11, x 12, x 14, x 15, x 17, x 19, x 20]$; $\mathrm{n}=\left[\mathrm{y} 1, \mathrm{y} 2, \mathrm{y}^{3}, \mathrm{y}^{4}, \mathrm{y} 5, \mathrm{y} 7, \mathrm{y} 8, \mathrm{y}^{9}, \mathrm{y} 11, \mathrm{y} 12, \mathrm{y} 14, \mathrm{y} 15, \mathrm{y} 17, \mathrm{y} 19, \mathrm{y} 20\right]$ end $[A 18(j, 1), A 18(j, 2)]=$ curveintersect $(m(:, j), n(:, j), y 78, x 78) ; \% \# \circ k<A G R O W>$

for $j=1: 15$ $m=[x 1, x 2, x 3, x 4, x 5, x 7, x 8, x 9, x 11, x 12, x 14, x 15, x 17, x 19, x 20]$; $\mathrm{n}=[\mathrm{y} 1, \mathrm{y} 2, \mathrm{y} 3, \mathrm{y} 4, \mathrm{y} 5, \mathrm{y} 7, \mathrm{y} 8, \mathrm{y} 9, \mathrm{y} 11, \mathrm{y} 12, \mathrm{y} 14, \mathrm{y} 15, \mathrm{y} 17, \mathrm{y} 19, \mathrm{y} 20] ;$ end $[A 19(j, 1), A 19(j, 2)]=$ curveintersect $(m(:, j), n(:, j), x 78, y 78) ; \% \#$ ok $<\star A G R O W>$

$\mathrm{A} 20=[5,0 ; 0,5 ; 4.9,0 ; 0,4.9 ; 4.8,0 ; 0,4.8 ; 4.7,0 ; 0,4.7 ; 4.6,0 ; 0,4.6 ; 4.5,0 ; 0,4.5 ; 4.4,0 ; 0,4$. $4 ; 4.3,0 ; 0,4.3 ; 4.2,0 ; 0,4.2 ; 4.1,0 ; 0,4.1 ; 4,0 ; 0,4 ; 3.9,0 ; 0,3.9 ; 3.8,0 ; 0,3.8 ; 3.7,0 ; 0,3.7 ; 3$ $.6,0 ; 0,3.6 ; 3.5,0 ; 0,3.5 ; 3.4,0 ; 0,3.4 ; 3.3,0 ; 0,3.3 ; 3.2,0 ; 0,3.2 ; 3.1,0 ; 0,3.1 ; 3,0 ; 0,3 ; 2.9$, $0 ; 0,2.9 ; 2.8,0 ; 0,2.8 ; 2.7,0 ; 0,2.7 ; 2.6,0 ; 0,2.6 ; 2.5,0 ; 0,2.5 ; 2.4,0 ; 0,2.4 ; 2.3,0 ; 0,2.3 ; 2.2$ , $0 ; 0,2.2 ; 2.1,0 ; 0,2.1 ; 2,0 ; 0,2 ; 1.9,0 ; 0,1.9 ; 1.8,0 ; 0,1.8 ; 1.7,0 ; 0,1.7 ; 1.6,0 ; 0,1.6 ; 1.5,0 ;$ $0,1.5 ; 1.4,0 ; 0,1.4 ; 1.3,0 ; 0,1.3 ; 1.2,0 ; 0,1.2 ; 1.1,0 ; 0,1.1 ; 0,1 ; 1,0 ; .984, .174 ; .174, .984 ;$. $965, .259 ; .259, .965 ; .939, .342 ; .342, .939 ; .906, .423 ; .423, .906 ; .866, .5 ; .5, .866 ; .819, .57$ $3 ; .573, .819 ; .766, .643 ; .643, .766 ; .707, .707 ; .996, .087 ; .087, .996]$; $\mathrm{A}=[\mathrm{A} 1 ; \mathrm{A} 2 ; \mathrm{A} 3 ; \mathrm{A} 4 ; \mathrm{A} 5 ; \mathrm{A} 6 ; \mathrm{A} 7 ; \mathrm{A} 8 ; \mathrm{A} 9 ; \mathrm{A} 10 ; \mathrm{A} 11 ; \mathrm{A} 12 ; \mathrm{A} 13 ; \mathrm{A} 14 ; \mathrm{A} 15 ; \mathrm{A} 16 ; \mathrm{A} 17 ; \mathrm{A} 18 ; \mathrm{A} 19 ; \mathrm{A} 20]$ $\circ \# \circ \mathrm{k}<\mathrm{NOPRT}>$

plot $\left([A(:, 1),-A(:, 1), A(:, 1),-A(:, 1)],[A(:, 2),-A(:, 2),-A(:, 2), A(:, 2)], ' m^{\star} '\right)$ function dydx=ODE1 $(x, y)$ $r=\operatorname{sqrt}(x \cdot \wedge 2+y \cdot \wedge 2) ; t=\operatorname{atan}(y \cdot / x) ; t 1=-1 ; t 2=-1 ;$ $\mathrm{sr}=-(t 1 / 2) *(1-(1 /(r \cdot \wedge 2)))-(t 1 / 2) *\left(1+(3 /(r \cdot \wedge 4))-\left(4 /\left(r \cdot{ }^{\wedge} 2\right)\right)\right) * \cos (2 * t)-(t 2 / 2) *(1-$ $(1 /(r \cdot \wedge 2)))+(t 2 / 2) *(1+(3 /(r \cdot \wedge 4))-(4 /(r \cdot \wedge 2))){ }^{*} \cos (2 * t) ;$ $s t=-(t 1 / 2) *(1+(1 /(r \cdot \wedge 2)))+(t 1 / 2) *(1+(3 /(r \cdot \wedge 4))) * \cos (2 * t)-(t 2 / 2) *(1+(1 /(r . \wedge 2)))-$ $(t 2 / 2) *(1+(3 /(r \cdot \wedge 4))) * \cos (2 * t)$ $\operatorname{srt}=(t 1 / 2) *\left(1-\left(3 /\left(r \cdot{ }^{\wedge} 4\right)\right)+\left(2 /\left(r \cdot{ }^{\wedge} 2\right)\right)\right) * \sin (2 * t)-(t 2 / 2) *(1-$ $\left.(3 /(r \cdot \wedge 4))+\left(2 /\left(r \cdot{ }^{\wedge} 2\right)\right)\right) * \sin (2 * t) ;$

$\mathrm{sx}=\operatorname{sr} \cdot{ }^{*}(\cos (t)) \cdot{ }^{\wedge} 2-2{ }^{*} \operatorname{srt} \cdot{ }^{*} \cos (t){ }^{\star} \sin (t)+\operatorname{st} \cdot{ }^{*}(\sin (t)) \cdot{ }^{\wedge} 2 ;$

$\operatorname{sy}=\operatorname{st} \cdot{ }^{*}(\cos (t)) \cdot{ }^{\wedge} 2+\operatorname{sr} \cdot{ }^{*}(\sin (t)) \cdot{ }^{\wedge} 2+\operatorname{srt} \cdot{ }^{*} \sin (2 * t) ;$

$\operatorname{txy}=0.5 *\left(\left(2 * \operatorname{srt} .{ }^{*} \cos (2 * t)+(\operatorname{sr}-\mathrm{st}) \cdot{ }^{\star} \sin (2 * t)\right)\right)$;

$d y d x=-(s x-s y) /(2 \star t x y)-\operatorname{sqrt}(1+((s x-s y) /(2 \star t x y)) \wedge 2)$

function $d y d x=\operatorname{ODE} 2(x, y)$

$r=\operatorname{sqrt}(x \cdot \wedge 2+y \cdot \wedge 2) ; t=\operatorname{atan}(y \cdot / x) ; t 1=-1 ; t 2=-1 ;$

$\operatorname{sr}=-(t 1 / 2) *(1-(1 /(r \cdot \wedge 2)))-(t 1 / 2) *(1+(3 /(r \cdot \wedge 4))-(4 /(r \cdot \wedge 2))) * \cos (2 * t)-(t 2 / 2) *(1-$ $(1 /(r \cdot \wedge 2)))+(t 2 / 2) *(1+(3 /(r \cdot \wedge 4))-(4 /(r \cdot \wedge 2))) * \cos (2 * t)$;

$s t=-(t 1 / 2) *\left(1+\left(1 /\left(r \cdot^{\wedge} 2\right)\right)\right)+(t 1 / 2) *\left(1+\left(3 /\left(r \wedge^{\wedge} 4\right)\right)\right){ }^{\star} \cos (2 * t)-(t 2 / 2) *\left(1+\left(1 /\left(r \cdot{ }^{\wedge} 2\right)\right)\right)-$ $(t 2 / 2) *(1+(3 /(r \cdot \wedge 4))) * \cos (2 * t) ;$

$\operatorname{srt}=(t 1 / 2) *\left(1-\left(3 /\left(r \cdot{ }^{\wedge} 4\right)\right)+\left(2 /\left(r \cdot{ }^{\wedge} 2\right)\right)\right) * \sin (2 * t)-(t 2 / 2) *(1-$

$(3 /(r \cdot \wedge 4))+(2 /(r \cdot \wedge 2))) * \sin (2 * t) ;$

$\operatorname{sx}=\operatorname{sr} \cdot{ }^{*}(\cos (t)) \cdot{ }^{\wedge} 2-2{ }^{*} \operatorname{srt} .{ }^{*} \cos (t){ }^{*} \sin (t)+\operatorname{st} \cdot{ }^{*}(\sin (t)) \cdot{ }^{\wedge} 2$;

$\operatorname{sy}=\operatorname{st} \cdot{ }^{*}(\cos (t)) \cdot{ }^{\wedge} 2+\operatorname{sr} \cdot{ }^{*}(\sin (t)) \cdot{ }^{\wedge} 2+\operatorname{srt} \cdot{ }^{\star} \sin (2 * t)$;

txy $=0.5^{\star}\left(\left(2 * \operatorname{srt} .{ }^{\star} \cos (2 * t)+(\operatorname{sr}-s t) \cdot{ }^{\star} \sin (2 * t)\right)\right) ;$

$d y d x=-(s x-s y) /(2 \star t x y)+\operatorname{sqrt}\left(1+((s x-s y) /(2 \star t x y))^{\wedge} 2\right)$;

\section{Uniaxial Compression Coarse Topology}

function UNIAXIALCOARSE

clc;clear all; clf

xlabel ('x'), ylabel ('y'), grid on, axis equal, axis $([0,5,0,5])$

title('Stress Trajectories for Plate with Hole')

hold on

\%Generating Primary Stress Trajectories

$[\mathrm{x} 1, \mathrm{y} 1]=0 \mathrm{de} 45(@ O D E 1,[0: 0.002: 5], 4.7) ;$ o\#०k<*NBRAK$>$ 
plot $\left([x 1,-x 1, x 1,-x 1],[y 1, y 1,-y 1,-y 1], k^{\prime}\right)$

$[\mathrm{x} 2, \mathrm{y} 2]=0 \mathrm{de} 45(\mathrm{QDEE} 1,[0: 0.002: 5], 4.4)$;

plot $([x 2,-x 2, x 2,-x 2],[y 2, y 2,-y 2,-y 2], ' k-1)$

$[\mathrm{x} 3, \mathrm{y} 3]=0 \mathrm{de} 45(\mathrm{@ODE} 1,[0: 0.002: 5], 4.1)$;

plot $\left([x 3,-x 3, x 3,-x 3],[y 3, y 3,-y 3,-y 3], k^{\prime}\right)$

$[\mathrm{x} 4, \mathrm{y} 4]=0 \operatorname{de} 45(@ O D E 1,[0: 0.002: 5], 3.8)$;

$\operatorname{plot}\left([\mathrm{x} 4,-\mathrm{x} 4, \mathrm{x} 4,-\mathrm{x} 4],[\mathrm{y} 4, \mathrm{y} 4,-\mathrm{y} 4,-\mathrm{y} 4], \mathrm{k}^{-1}\right)$

$[\mathrm{x} 5, \mathrm{y} 5]=0 \mathrm{de} 45(@ \mathrm{ODE} 1,[0: 0.002: 5], 3.5)$;

plot $\left([x 5,-x 5, x 5,-x 5],[y 5, y 5,-y 5,-y 5], k^{\prime}\right)$

$[\mathrm{x} 6, \mathrm{y} 6]=0 \mathrm{de} 45$ (@ODE1, [0:0.002:5],3.2);

$\operatorname{plot}\left([\mathrm{x} 6,-\mathrm{x} 6, \mathrm{x} 6,-\mathrm{x} 6],\left[\mathrm{y}^{6}, \mathrm{y} 6,-\mathrm{y} 6,-\mathrm{y} 6\right], \mathrm{k}^{\prime} \mathrm{-}^{\prime}\right)$

$[\mathrm{x} 7, \mathrm{y} 7]=0 \mathrm{de} 45($ @ODE $1,[0: 0.002: 5], 2.9)$;

$\operatorname{plot}([x 7,-x 7, x 7,-x 7],[y 7, y 7,-y 7,-y 7], ' k-1)$

$[\mathrm{x} 8, \mathrm{y} 8]=0 \mathrm{de} 45(\mathrm{QODE} 1,[0: 0.002: 5], 2.6)$;

plot $\left([\mathrm{x} 8,-\mathrm{x} 8, \mathrm{x} 8,-\mathrm{x} 8],[\mathrm{y} 8, \mathrm{y} 8,-\mathrm{y} 8,-\mathrm{y} 8], \mathrm{k}^{\prime}\right.$ ')

$[\mathrm{x} 9, \mathrm{y} 9]=0 \mathrm{de} 45($ @ODE $1,[0: 0.002: 5], 2.3)$;

plot $([x 9,-x 9, x 9,-x 9],[y 9, y 9,-y 9,-y 9], ' k-1)$

$[\mathrm{x} 10, \mathrm{y} 10]=0 \mathrm{de} 45(\mathrm{\varrho ODE} 1,[0: 0.002: 5], 2.0)$;

plot $\left([\mathrm{x} 10,-\mathrm{x} 10, \mathrm{x} 10,-\mathrm{x} 10],[\mathrm{y} 10, \mathrm{y} 10,-\mathrm{y} 10,-\mathrm{y} 10], \mathrm{\prime}^{\prime}-^{\prime}\right)$

$[\mathrm{x} 11, \mathrm{y} 11]=0 \mathrm{de} 45(\mathrm{QODE} 1,[0: 0.002: 5], 1.7)$;

plot $\left([x 11,-x 11, x 11,-x 11],[y 11, y 11,-y 11,-y 11], k^{-\prime}\right)$

$[\mathrm{x} 12, \mathrm{y} 12]=0 \mathrm{de} 45(\mathrm{QODE} 1,[0: 0.002: 5], 1.4)$;

plot $\left([x 12,-x 12, x 12,-x 12],[y 12, y 12,-y 12,-y 12], k^{\prime}-^{\prime}\right)$

$[\mathrm{x} 13, \mathrm{y} 13]=0 \mathrm{de} 45($ @ODE1, $[0: 0.002: 5], 1.15)$;

plot $\left([x 13,-x 13, x 13,-x 13],[y 13, y 13,-y 13,-y 13],{ }^{\prime}-^{\prime}\right)$

$[\mathrm{x} 14, \mathrm{y} 14]=0 \mathrm{de} 45(@ O D E 1,[0.866: 0.001: 5.866], 0.500)$;

plot $\left([\mathrm{x} 14,-\mathrm{x} 14, \mathrm{x} 14,-\mathrm{x} 14],[y 14, y 14,-y 14,-y 14], \mathrm{k}^{\prime}\right.$ ')

\%Generating Secondary Stress Trajectories

$[y 15, x 15]=0 d e 45(\operatorname{eODE} 2,[0: 0.002: 5], 4.7)$;

plot $\left([x 15,-x 15, x 15,-x 15],[y 15, y 15,-y 15,-y 15], '{ }^{-\prime}\right)$

$[y 16, x 16]=0 d e 45(@ O D E 2,[0: 0.002: 5], 4.4)$;

plot $\left([\mathrm{x} 16,-\mathrm{x} 16, \mathrm{x} 16,-\mathrm{x} 16],[\mathrm{y} 16, \mathrm{y} 16,-\mathrm{y} 16,-\mathrm{y} 16], \mathrm{k}^{\prime}\right.$ ')

$[y 17, x 17]=0$ de4 $5(@ O D E 2,[0: 0.002: 5], 4.1)$;

plot $\left([x 17,-x 17, x 17,-x 17],[y 17, y 17,-y 17,-y 17], k^{-\prime}\right)$

$[y 18, x 18]=0$ de4 $5($ @ODE $2,[0: 0.002: 5], 3.8)$;

plot $\left([x 18,-x 18, x 18,-x 18],[y 18, y 18,-y 18,-y 18], k^{\prime}-^{\prime}\right)$ [y19, x19] =ode45 (@ODE2, [0:0.002:5],3.5);

plot $\left([x 19,-x 19, x 19,-x 19],[y 19, y 19,-y 19,-y 19], k^{-\prime}\right)$

$[y 20, x 20]=0$ de4 $5(@ O D E 2,[0: 0.002: 5], 3.2)$;

$\operatorname{plot}\left([\mathrm{x} 20,-\mathrm{x} 20, \mathrm{x} 20,-\mathrm{x} 20],[y 20, y 20,-y 20,-y 20], \mathrm{k}^{\prime}{ }^{\prime}\right)$

$[y 21, x 21]=0$ de45 (@ODE2, [0:0.002:5],2.9):

plot $([x 21,-x 21, x 21,-x 21],[y 21, y 21,-y 21,-y 21], ' k-')$

$[y 22, x 22]=0$ de $45($ @ODE $2,[0: 0.002: 5], 2.6)$;

plot $([x 22,-x 22, x 22,-x 22],[y 22, y 22,-y 22,-y 22], ' k-')$

$[y 23, x 23]=0$ de $45($ @ODE2, $[0: 0.002: 5], 2.3)$;

plot $([x 23,-x 23, x 23,-x 23],[y 23, y 23,-y 23,-y 23], ' k-')$

$[y 24, x 24]=0$ de $45($ @ODE $2,[0: 0.002: 5], 2.0)$;

plot $([x 24,-x 24, x 24,-x 24],[y 24, y 24,-y 24,-y 24], ' k-')$

$[y 25, x 25]=0$ de $45(@ O D E 2,[0: 0.002: 5], 1.7)$;

plot $\left([x 25,-x 25, x 25,-x 25],[y 25, y 25,-y 25,-y 25], '{ }^{-\prime}\right)$

$[y 26, \mathrm{x} 26]=0 \mathrm{de} 45(\mathrm{QODE} 2,[0: 0.002: 5], 1.32)$;

plot $\left([x 26,-x 26, x 26,-x 26],[y 26, y 26,-y 26,-y 26],{ }^{\prime}-^{\prime}\right)$

[y27, x27] =ode45 (@ODE2, [0:0.002:5],1.1);

plot $\left([x 27,-x 27, x 27,-x 27],[y 27, y 27,-y 27,-y 27], k^{-1}\right)$

$[\mathrm{x} 28, \mathrm{y} 28]=0 \mathrm{de} 45(\mathrm{QODE} 3,[0.875: 0.002: 5.875], 0.485) ; \circ 30$

plot $\left([x 28,-x 28, x 28,-x 28],[y 28, y 28,-y 28,-y 28],{ }^{\prime} k-^{\prime}\right)$

$[\mathrm{x} 29, \mathrm{y} 29]=0 \mathrm{de} 45(\mathrm{eODE} 3,[0.707: 0.002: 5.707], 0.707) ; \circ 45$

plot $\left.\left([x 29,-x 29, x 29,-x 29],[y 29, y 29,-y 29,-y 29], k^{\prime}\right)^{\prime}\right)$

$[\mathrm{x} 30, \mathrm{y} 30]=0 \mathrm{de} 45(\mathrm{QODE} 3,[0.500: 0.002: 5.500], 0.866) ; \circ 60$

plot $\left([x 30,-x 30, x 30,-x 30],[y 30, y 30,-y 30,-y 30], k^{\prime}\right)$

$[\mathrm{x} 31, \mathrm{y} 31]=0 \mathrm{de} 45(\mathrm{@ODE} 3,[0.259: 0.002: 5.259], 0.965) ; \circ 75$

plot $([x 31,-x 31, x 31,-x 31],[y 31, y 31,-y 31,-y 31], ' k-')$

$\times 32=[0: 0.002: 5]^{\prime}$;

$\mathrm{y} 32=\times 32 \cdot{ }^{*} 0+5$

plot (x32, y32, 'k-')

plot $\left(-\mathrm{x} 32, \mathrm{y} 32, \mathrm{\prime}^{\mathrm{k}} \mathrm{-}^{\prime}\right)$

plot $\left(x 32,-y 32,{ }^{\prime}-{ }^{\prime}\right)$

plot $(-x 32,-y 32, ' k-')$

plot $(y 32, x 32, ' \mathrm{k}-')$

plot $(-y 32, x 32, ' k-')$

plot $\left(y 32,-x 32, k^{-\prime}\right)$ 


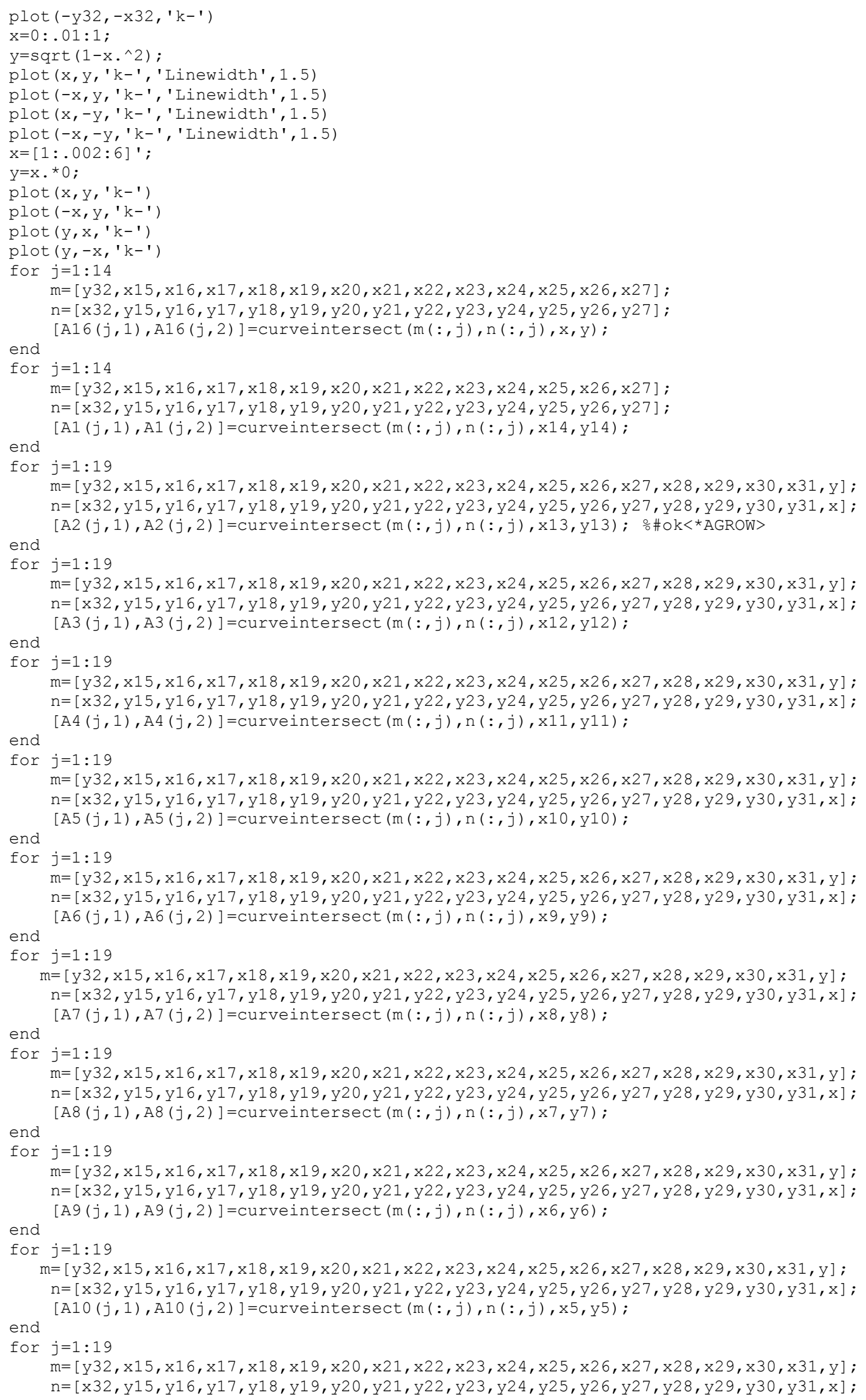


$[\mathrm{A} 11(j, 1), \mathrm{A} 11(j, 2)]$ =curveintersect $\left(\mathrm{m}(:, j), \mathrm{n}(:, j), \mathrm{x} 4, \mathrm{y}^{4}\right)$;

end

for $j=1: 19$

$m=[y 32, x 15, x 16, x 17, x 18, x 19, x 20, x 21, x 22, x 23, x 24, x 25, x 26, x 27, x 28, x 29, x 30, x 31, y] ;$ $\mathrm{n}=[\mathrm{x} 32, \mathrm{y} 15, \mathrm{y} 16, \mathrm{y} 17, \mathrm{y} 18, \mathrm{y} 19, \mathrm{y} 20, \mathrm{y} 21, \mathrm{y} 22, \mathrm{y} 23, \mathrm{y} 24, \mathrm{y} 25, \mathrm{y} 26, \mathrm{y} 27, \mathrm{y} 28, \mathrm{y} 29, \mathrm{y} 30, \mathrm{y} 31, \mathrm{x}] ;$ end $[A 12(j, 1), A 12(j, 2)]=$ curveintersect $(m(:, j), n(:, j), x 3, y 3)$;

for $j=1: 19$

$m=[y 32, x 15, x 16, x 17, x 18, x 19, x 20, x 21, x 22, x 23, x 24, x 25, x 26, x 27, x 28, x 29, x 30, x 31, y] ;$ $\mathrm{n}=[\mathrm{x} 32, \mathrm{y} 15, \mathrm{y} 16, \mathrm{y} 17, \mathrm{y} 18, \mathrm{y} 19, \mathrm{y} 20, \mathrm{y} 21, \mathrm{y} 22, \mathrm{y} 23, \mathrm{y} 24, \mathrm{y} 25, \mathrm{y} 26, \mathrm{y} 27, \mathrm{y} 28, \mathrm{y} 29, \mathrm{y} 30, \mathrm{y} 31, \mathrm{x}] ;$ end $[A 13(j, 1), A 13(j, 2)]=$ curveintersect $(m(:, j), n(:, j), x 2, y 2)$;

for $j=1: 19$

$m=[y 32, x 15, x 16, x 17, x 18, x 19, x 20, x 21, x 22, x 23, x 24, x 25, x 26, x 27, x 28, x 29, x 30, x 31, y] ;$ $\mathrm{n}=[\mathrm{x} 32, \mathrm{y} 15, \mathrm{y} 16, \mathrm{y} 17, \mathrm{y} 18, \mathrm{y} 19, \mathrm{y} 20, \mathrm{y} 21, \mathrm{y} 22, \mathrm{y} 23, \mathrm{y} 24, \mathrm{y} 25, \mathrm{y} 26, \mathrm{y} 27, \mathrm{y} 28, \mathrm{y} 29, \mathrm{y} 30, \mathrm{y} 31, \mathrm{x}] ;$ end $[\mathrm{A} 14(j, 1), \mathrm{A} 14(j, 2)]=$ curveintersect $(\mathrm{m}(:, j), \mathrm{n}(:, j), \mathrm{x} 1, \mathrm{y} 1)$;

for $j=1: 19$ $m=[y 32, x 15, x 16, x 17, x 18, x 19, x 20, x 21, x 22, x 23, x 24, x 25, x 26, x 27, x 28, x 29, x 30, x 31, y]$ $\mathrm{n}=[\mathrm{x} 32, \mathrm{y} 15, \mathrm{y} 16, \mathrm{y} 17, \mathrm{y} 18, \mathrm{y} 19, \mathrm{y} 20, \mathrm{y} 21, \mathrm{y} 22, \mathrm{y} 23, \mathrm{y} 24, \mathrm{y} 25, \mathrm{y} 26, \mathrm{y} 27, \mathrm{y} 28, \mathrm{y} 29, \mathrm{y} 30, \mathrm{y} 31, \mathrm{x}]$; end $[A 15(j, 1), A 15(j, 2)]=$ curveintersect $(m(:, j), n(:, j), x 32, y 32)$;

$\mathrm{A} 17=[0,1 ; 1,0 ; .866, .5 ; .5, .866 ; .707, .707 ; .259, .965]$;

$\mathrm{A}=[\mathrm{A} 16 ; \mathrm{A} 1 ; \mathrm{A} 2 ; \mathrm{A} 3 ; \mathrm{A} 4 ; \mathrm{A} 5 ; \mathrm{A} 6 ; \mathrm{A} 7 ; \mathrm{A} 8 ; \mathrm{A} 9 ; \mathrm{A} 10 ; \mathrm{A} 11 ; \mathrm{A} 12 ; \mathrm{A} 13 ; \mathrm{A} 14 ; \mathrm{A} 15 ; \mathrm{A} 17]$

$\operatorname{plot}\left([A(:, 1),-A(:, 1), A(:, 1),-A(:, 1)],[A(:, 2),-A(:, 2),-A(:, 2), A(:, 2)], ' m^{*} '\right)$

function $d y d x=\operatorname{ODE} 1(\mathrm{x}, \mathrm{y})$

$r=\operatorname{sqrt}(x \cdot \wedge 2+y \cdot \wedge 2) ; t=\operatorname{atan}(y \cdot / x) ;$

$\operatorname{sr}=0.5 *(1-(r \cdot \wedge-2))+0.5 *(1+3 *(r \cdot \wedge-4)-4 *(r \cdot \wedge-2)) * \cos (2 * t)$

$\mathrm{st}=0.5 *\left(1+\left(r \cdot{ }^{\wedge}-2\right)\right)-0.5 *\left(1+3 *\left(r \cdot{ }^{\wedge}-4\right)\right) * \cos (2 * t)$;

$\operatorname{srt}=-0.5^{*}\left(1-3 *\left(r \cdot{ }^{\wedge}-4\right)+2 *\left(r \cdot{ }^{\wedge}-2\right)\right) * \sin (2 * t) ;$

$\mathrm{sx}=\operatorname{sr}{ }^{*} \cos (t) \cdot{ }^{\wedge} 2+\operatorname{st}{ }^{*} \sin (t) \cdot{ }^{\wedge} 2-2{ }^{*} \operatorname{srt}{ }^{*} \sin (t){ }^{*} \cos (t)$;

$\operatorname{sy}=\operatorname{sr}{ }^{*} \sin (t) \cdot{ }^{\wedge} 2+\operatorname{st}{ }^{*} \cos (t) \cdot{ }^{\wedge} 2+2{ }^{*} \operatorname{srt}{ }^{\star} \sin (t){ }^{*} \cos (t)$;

$t x y=\operatorname{sr}{ }^{*} \sin (t){ }^{*} \cos (t)-\operatorname{st}{ }^{*} \cos (t){ }^{*} \sin (t)+\operatorname{srt}{ }^{*} \cos (t) \cdot{ }^{\wedge} 2-\operatorname{srt}{ }^{*} \sin (t) \cdot{ }^{\wedge} 2 ;$

$d y d x=-(s x-s y) . /(2 * t x y)-\operatorname{sqrt}(1+((s x-s y) . /(2 * t x y)) . \wedge 2)$;

\% Introduce slope limiter for case where tau goes to zero

if $\operatorname{abs}(d y d x)>10$

$d y d x=0$;

end

function $d y d x=\operatorname{ODE} 2(x, y)$

$r=\operatorname{sqrt}\left(x \cdot{ }^{\wedge} 2+y \cdot{ }^{\wedge} 2\right) ; t=\operatorname{atan}(y \cdot / x)$;

$\mathrm{sr}=0.5 *\left(1-\left(r .^{\wedge}-2\right)\right)-0.5 *\left(1+3 *\left(r \wedge^{\wedge}-4\right)-4 *\left(r \cdot{ }^{\wedge}-2\right)\right){ }^{*} \cos (2 * t)$

st $=0.5^{\star}\left(1+\left(r \cdot{ }^{\wedge}-2\right)\right)+0.5^{\star}\left(1+3 *\left(r \cdot^{\wedge}-4\right)\right){ }^{*} \cos (2 * t)$;

$\operatorname{srt}=0.5 *\left(1-3 *\left(r \cdot^{\wedge}-4\right)+2 *\left(r \cdot^{\wedge}-2\right)\right) * \sin (2 * t) ;$

$\operatorname{sx}=\operatorname{sr}^{*} \cos (t) \cdot{ }^{\wedge} 2+\operatorname{st}{ }^{*} \sin (t) \cdot{ }^{\wedge} 2-2{ }^{*} \operatorname{srt}{ }^{*} \sin (t){ }^{*} \cos (t)$

$\operatorname{sy}=\operatorname{sr}{ }^{*} \sin (t) \cdot{ }^{\wedge} 2+\operatorname{st}{ }^{*} \cos (t) \cdot{ }^{\wedge} 2+2 * \operatorname{srt}{ }^{*} \sin (t) * \cos (t)$;

$t x y=\operatorname{sr}{ }^{*} \sin (t){ }^{*} \cos (t)-\operatorname{st}{ }^{*} \cos (t){ }^{*} \sin (t)+\operatorname{srt}{ }^{*} \cos (t) \cdot{ }^{\wedge} 2-\operatorname{srt}{ }^{*} \sin (t) \cdot{ }^{\wedge} 2$

$d y d x=-(s x-s y) . /(2 * t x y)+\operatorname{sqrt}\left(1+((s x-s y) . /(2 * t x y)) \cdot{ }^{\wedge} 2\right)$;

\% Introduce slope limiter for case where tau goes to zero

if $\operatorname{abs}(d y d x)>4.1$

end $d y d x=0$;

function $d y d x=\operatorname{ODE} 3(x, y)$

$r=\operatorname{sqrt}\left(x \cdot \wedge 2+y \cdot{ }^{\wedge} 2\right) ; t=\operatorname{atan}(y \cdot / x)$;

$\mathrm{sr}=0.5 *\left(1-\left(r \cdot{ }^{\wedge}-2\right)\right)+0.5 *\left(1+3 *\left(r \cdot^{\wedge}-4\right)-4 *(r \cdot \wedge-2)\right){ }^{\star} \cos (2 * t)$

st $=0.5 *\left(1+\left(r \cdot{ }^{\wedge}-2\right)\right)-0.5 *\left(1+3 *\left(r \cdot{ }^{\wedge}-4\right)\right) * \cos (2 * t)$;

$\operatorname{srt}=-0.5 *\left(1-3^{*}\left(r \cdot{ }^{\wedge}-4\right)+2 *\left(r \cdot{ }^{\wedge}-2\right)\right) * \sin (2 * t) ;$

$\operatorname{sx}=\operatorname{sr}{ }^{*} \cos (t) \cdot{ }^{\wedge} 2+\operatorname{st}{ }^{*} \sin (t) \cdot{ }^{\wedge} 2-2{ }^{*} \operatorname{srt}{ }^{*} \sin (t){ }^{*} \cos (t) ;$

$\operatorname{sy}=\operatorname{sr}{ }^{*} \sin (t) \cdot{ }^{\wedge} 2+\operatorname{st}{ }^{*} \cos (t) \cdot{ }^{\wedge} 2+2{ }^{*} \operatorname{srt}{ }^{*} \sin (t) * \cos (t) ;$

$\operatorname{txy}=\operatorname{sr}{ }^{\star} \sin (t){ }^{*} \cos (t)-\operatorname{st}^{*} \cos (t){ }^{*} \sin (t)+\operatorname{srt}{ }^{*} \cos (t) \cdot{ }^{\wedge} 2-\operatorname{srt}{ }^{*} \sin (t) \cdot{ }^{\wedge} 2$;

$d y d x=-(s x-s y) \cdot /(2 * t x y)+\operatorname{sqr} t\left(1+((s x-s y) \cdot /(2 * t x y)) \cdot{ }^{\wedge} 2\right)$;

\% Introduce slope limiter for case where tau goes to zero

if $\operatorname{abs}(d y d x)>10$ $d y d x=100$

elseif abs $(d y d x)<0.1$ $d y d x=100$

end

\section{Uniaxial Compression Medium Topology}

function UNIAXIALMED 
clc;clear all; clf

xlabel ('x'), ylabel ('y'), grid on, axis equal, axis $([0,5,0,5])$

title('Stress Trajectories for Plate with Hole')

hold on

oGenerating Primary Stress Trajectories

$[\mathrm{x} 1, \mathrm{y} 1]=0 \mathrm{de} 45(@ O D E 1,[0: 0.002: 5], 5.0)$;

plot $([x 1,-x 1, x 1,-x 1],[y 1, y 1,-y 1,-y 1], ' k-1)$

$[\mathrm{x} 2, \mathrm{y} 2]=0 \mathrm{de} 45(@ \mathrm{ODE} 1,[0: 0.002: 5], 4.8)$;

$\operatorname{plot}\left([\mathrm{x} 2,-\mathrm{x} 2, \mathrm{x} 2,-\mathrm{x} 2],[\mathrm{y} 2, \mathrm{y} 2,-\mathrm{y} 2,-\mathrm{y} 2], \mathrm{k}^{\prime}\right)$

$[\mathrm{x} 3, \mathrm{y} 3]=0 \mathrm{de} 45(\mathrm{QODE} 1,[0: 0.002: 5], 4.6)$;

$\operatorname{plot}\left([\mathrm{x} 3,-\mathrm{x} 3, \mathrm{x} 3,-\mathrm{x} 3],[y 3, \mathrm{y} 3,-\mathrm{y} 3,-\mathrm{y} 3], \mathrm{y}^{\prime} \mathrm{-}\right)$

$[\mathrm{x} 4, \mathrm{y} 4]=0 \mathrm{de} 45(\mathrm{QODE} 1,[0: 0.002: 5], 4.4)$;

plot $([x 4,-x 4, x 4,-x 4],[y 4, y 4,-y 4,-y 4], ' k-1)$

$[\mathrm{x} 5, \mathrm{y} 5]=0 \mathrm{de} 45(\mathrm{QODE} 1,[0: 0.002: 5], 4.2)$;

plot $([x 5,-x 5, x 5,-x 5],[y 5, y 5,-y 5,-y 5], ' k-1)$

$[\mathrm{x} 6, \mathrm{y} 6]=0 \mathrm{de} 45(@ \mathrm{ODE} 1,[0: 0.002: 5], 4.0)$;

plot $\left(\left[x 6,-x 6, x^{6},-x 6\right],\left[y 6, y^{6},-y 6,-y 6\right], ' k-1\right)$

$[\mathrm{x} 7, \mathrm{y} 7]=0 \operatorname{de} 45(@ \mathrm{ODE} 1,[0: 0.002: 5], 3.8)$;

plot $([x 7,-x 7, x 7,-x 7],[y 7, y 7,-y 7,-y 7], ' k-1)$

$[\mathrm{x} 8, \mathrm{y} 8]=0 \mathrm{de} 45($ @ODE $1,[0: 0.002: 5], 3.6)$;

plot $\left([\mathrm{x} 8,-\mathrm{x} 8, \mathrm{x} 8,-\mathrm{x} 8],[\mathrm{y} 8, \mathrm{y} 8,-\mathrm{y} 8,-\mathrm{y} 8], \mathrm{k}^{\prime}\right)$

$[\mathrm{x} 9, \mathrm{y} 9]=$ ode $45(@ O D E 1,[0: 0.002: 5], 3.4)$;

plot $\left([x 9,-x 9, x 9,-x 9],[y 9, y 9,-y 9,-y 9], k^{\prime}\right)$

$[\mathrm{x} 10, \mathrm{y} 10]=0 \mathrm{de} 45($ @ODE1, $[0: 0.002: 5], 3.2)$;

plot $\left([x 10,-x 10, x 10,-x 10],[y 10, y 10,-y 10,-y 10], k^{-\prime}\right)$

$[\mathrm{x} 11, \mathrm{y} 11]=0 \mathrm{de} 45(\mathrm{QODE} 1,[0: 0.002: 5], 3.0)$;

plot $\left([x 11,-x 11, x 11,-x 11],[y 11, y 11,-y 11,-y 11], k^{-\prime}\right)$

$[\mathrm{x} 12, \mathrm{y} 12]=0 \mathrm{de} 45(\mathrm{CODE} 1,[0: 0.002: 5], 2.8)$;

$\operatorname{plot}\left([\mathrm{x} 12,-\mathrm{x} 12, \mathrm{x} 12,-\mathrm{x} 12],[\mathrm{y} 12, \mathrm{y} 12,-\mathrm{y} 12,-\mathrm{y} 12], \mathrm{k}^{\prime} \mathbf{\prime}^{\prime}\right)$

$[\mathrm{x} 13, \mathrm{y} 13]=0 \mathrm{de} 45($ @ODE1, $[0: 0.002: 5], 2.6)$;

plot $\left([x 13,-x 13, x 13,-x 13],[y 13, y 13,-y 13,-y 13], k^{\prime}-^{\prime}\right)$

$[\mathrm{x} 14, \mathrm{y} 14]=0 \mathrm{de} 45($ @ODE1, $[0: 0.002: 5], 2.4)$; #ㅡㄴ <*NBRAK>

plot $\left([\mathrm{x} 14,-\mathrm{x} 14, \mathrm{x} 14,-\mathrm{x} 14],[y 14, y 14,-y 14,-y 14], \mathrm{k}^{\prime}\right)$

$[\mathrm{x} 15, \mathrm{y} 15]=0 \mathrm{de} 45($ @ODE1, $[0: 0.002: 5], 2.2)$;

plot $\left([x 15,-x 15, x 15,-x 15],[y 15, y 15,-y 15,-y 15], ' k^{\prime}\right)$

$[\mathrm{x} 16, \mathrm{y} 16]=0 \mathrm{de} 45(\mathrm{QODE} 1,[0: 0.002: 5], 2.0)$;

plot $\left([\mathrm{x} 16,-\mathrm{x} 16, \mathrm{x} 16,-\mathrm{x} 16],[\mathrm{y} 16, \mathrm{y} 16,-\mathrm{y} 16,-\mathrm{y} 16], \mathrm{k}^{-\prime}\right)$

$[\mathrm{x} 17, \mathrm{y} 17]=0 \mathrm{de} 45(\mathrm{QODE} 1,[0: 0.002: 5], 1.8)$;

plot $\left([x 17,-x 17, x 17,-x 17],[y 17, y 17,-y 17,-y 17], k^{-\prime}\right)$

$[\mathrm{x} 18, \mathrm{y} 18]=0 \mathrm{de} 45(\mathrm{\varrho ODE} 1,[0: 0.002: 5], 1.6)$;

plot $\left([x 18,-x 18, x 18,-x 18],[y 18, y 18,-y 18,-y 18], k^{-\prime}\right)$

$[\mathrm{x} 19, \mathrm{y} 19]=0 \mathrm{de} 45($ @ODE1, $[0: 0.002: 5], 1.4)$;

plot $\left([x 19,-x 19, x 19,-x 19],[y 19, y 19,-y 19,-y 19], k^{\prime}-^{\prime}\right)$

$[\mathrm{x} 20, \mathrm{y} 20]=0 \mathrm{de} 45(\mathrm{QODE} 1,[0: 0.002: 5], 1.2)$;

plot $\left([x 20,-x 20, x 20,-x 20],[y 20, y 20,-y 20,-y 20], k^{\prime}-^{\prime}\right)$

$[\mathrm{x} 21, \mathrm{y} 21]=0 \mathrm{de} 45(\mathrm{QODE} 1,[0: 0.002: 5], 1.08)$;

plot $\left([x 21,-x 21, x 21,-x 21],[y 21, y 21,-y 21,-y 21], ' k-{ }^{\prime}\right)$

$[\mathrm{x} 22, \mathrm{y} 22]=0 \mathrm{de} 45(\mathrm{QODE} 1,[0.866: 0.001: 5.866], 0.500)$;

plot $\left([x 22,-x 22, x 22,-x 22],[y 22, y 22,-y 22,-y 22], k^{-\prime}\right)$

oGenerating the Secondary Stress Trajectories

$[y 23, x 23]=0$ de $45($ @ODE $2,[0: 0.002: 5], 5.0)$;

plot $\left([x 23,-x 23, x 23,-x 23],[y 23, y 23,-y 23,-y 23],{ }^{\prime}-^{\prime}\right)$

$[y 24, x 24]=0 d e 45(@ O D E 2,[0: 0.002: 5], 4.8)$;

plot $\left([x 24,-x 24, x 24,-x 24],[y 24, y 24,-y 24,-y 24], k^{\prime}-^{\prime}\right)$

$[y 25, x 25]=0$ de $45($ @ODE2, $[0: 0.002: 5], 4.6)$;

plot $\left([x 25,-x 25, x 25,-x 25],[y 25, y 25,-y 25,-y 25], ' k^{\prime}\right.$ ')

$[y 26, x 26]=0$ de $45(@ O D E 2,[0: 0.002: 5], 4.4)$;

plot $\left([x 26,-x 26, x 26,-x 26],[y 26, y 26,-y 26,-y 26], ' k^{\prime}\right.$ ' $)$

$[y 27, x 27]=0$ de $45($ @ODE2, $[0: 0.002: 5], 4.2)$;

$\operatorname{plot}\left([x 27,-x 27, x 27,-x 27],[y 27, y 27,-y 27,-y 27], k^{\prime}{ }^{\prime}\right)$

$[y 28, x 28]=0$ de $45($ @ODE $2,[0: 0.002: 5], 4.0)$;

plot $([x 28,-x 28, x 28,-x 28],[y 28, y 28,-y 28,-y 28], ' k-1)$

$[y 29, \times 29]=0$ de $45($ @ODE $2,[0: 0.002: 5], 3.8)$;

plot $([x 29,-x 29, x 29,-x 29],[y 29, y 29,-y 29,-y 29], ' k-')$

$[y 30, x 30]=0$ de $45($ @ODE $2,[0: 0.002: 5], 3.6)$;

plot $\left([x 30,-x 30, x 30,-x 30],[y 30, y 30,-y 30,-y 30], k^{\prime}-^{\prime}\right)$

$[y 31, x 31]=0$ de $45($ @ODE2, $[0: 0.002: 5], 3.4)$;

plot $\left([x 31,-x 31, x 31,-x 31],[y 31, y 31,-y 31,-y 31],{ }^{\prime}-^{\prime}\right)$

$[y 32, x 32]=0 d e 45($ @ODE $2,[0: 0.002: 5], 3.2)$;

plot $\left([x 32,-x 32, x 32,-x 32],[y 32, y 32,-y 32,-y 32], k^{\prime}-^{\prime}\right)$

$[y 33, x 33]=0$ de $45(@ O D E 2,[0: 0.002: 5], 3.0)$; 
plot $([x 33,-x 33, x 33,-x 33],[y 33, y 33,-y 33,-y 33], ' k-')$

$[y 34, x 34]=0 d e 45(@ O D E 2,[0: 0.002: 5], 2.8)$;

plot $\left([x 34,-x 34, x 34,-x 34],[y 34, y 34,-y 34,-y 34],{ }^{\prime}-^{\prime}\right)$

$[y 35, x 35]=0$ de $45($ @ODE2, $[0: 0.002: 5], 2.6)$;

plot $\left([x 35,-x 35, x 35,-x 35],[y 35, y 35,-y 35,-y 35],{ }^{\prime} k-^{\prime}\right)$

$[y 36, x 36]=0 d e 45(@ O D E 2,[0: 0.002: 5], 2.4)$; \%\#ok<*NBRAK>

plot $([x 36,-x 36, x 36,-x 36],[y 36, y 36,-y 36,-y 36], ' k-')$

$[y 37, x 37]=0 d e 45(@ O D E 2,[0: 0.002: 5], 2.2)$;

plot $\left([x 37,-x 37, x 37,-x 37],[y 37, y 37,-y 37,-y 37], k^{\prime}{ }^{\prime}\right)$

$[y 38, x 38]=0$ de $45($ @ODE $2,[0: 0.002: 5], 2.0)$;

plot $\left([x 38,-x 38, x 38,-x 38],[y 38, y 38,-y 38,-y 38], k^{\prime}-^{\prime}\right)$

$[y 39, \times 39]=0$ de $45($ @ODE2, $[0: 0.002: 5], 1.8)$;

plot $\left([x 39,-x 39, x 39,-x 39],[y 39, y 39,-y 39,-y 39],{ }^{\prime}-^{-\prime}\right)$

$[\mathrm{y} 40, \mathrm{x} 40]=0 \mathrm{de} 45(\mathrm{QODE} 2,[0: 0.002: 5], 1.6)$;

plot $\left([x 40,-x 40, x 40,-x 40],[y 40, y 40,-y 40,-y 40], k^{-\prime}\right)$

$[y 41, x 41]=0$ de $45(@ O D E 2,[0: 0.002: 5], 1.4)$;

plot $([x 41,-x 41, x 41,-x 41],[y 41, y 41,-y 41,-y 41], ' k-')$

$[y 42, x 42]=0 d e 45(@ O D E 2,[0: 0.002: 5], 1.22)$;

plot $\left([x 42,-x 42, x 42,-x 42],[y 42, y 42,-y 42,-y 42],{ }^{\prime}-^{\prime}\right)$

$[y 43, x 43]=0 d e 45(@ O D E 2,[0: 0.002: 5], 1.1)$;

plot $\left([x 43,-x 43, x 43,-x 43],[y 43, y 43,-y 43,-y 43], ' k-^{\prime}\right)$

$[y 44, x 44]=0 d e 45(@ O D E 2,[0: 0.002: 5], 1.0)$;

plot $\left([x 44,-x 44, x 44,-x 44],[y 44, y 44,-y 44,-y 44], k^{-\prime}\right)$

$[\mathrm{x} 45, \mathrm{y} 45]=0 \mathrm{de} 45(\mathrm{QODE} 3,[0.866: 0.002: 5.866], 0.500) ; \circ 30$

plot $([x 45,-x 45, x 45,-x 45],[y 45, y 45,-y 45,-y 45], ' k-1)$

$[\mathrm{x} 46, \mathrm{y} 46]=0 \mathrm{de} 45(\mathrm{QODE} 3,[0.7659: 0.002: 5.7659], 0.6429) ; \% 40$

$\operatorname{plot}\left([\mathrm{x} 46,-\mathrm{x} 46, \mathrm{x} 46,-\mathrm{x} 46],[\mathrm{y} 46, \mathrm{y} 46,-\mathrm{y} 46,-\mathrm{y} 46], \mathrm{\prime}^{\prime} \mathbf{-}^{\prime}\right)$

$[\mathrm{x} 47, \mathrm{y} 47]=0 \mathrm{de} 45(\mathrm{\varrho ODE} 3,[0.6428: 0.002: 5.6428], 0.7658) ; \% 50$

$\operatorname{plot}\left([x 47,-x 47, x 47,-x 47],[y 47, y 47,-y 47,-y 47], ' k-{ }^{\prime}\right)$

$[\mathrm{x} 48, \mathrm{y} 48]=0 \mathrm{de} 45(\mathrm{QODE} 3,[0.500: 0.002: 5.500], 0.866) ; \circ 60$

plot $([x 48,-x 48, x 48,-x 48],[y 48, y 48,-y 48,-y 48], ' k-')$

$[\mathrm{x} 49, \mathrm{y} 49]=0 \mathrm{de} 45(\mathrm{eODE} 3,[0.342: 0.002: 5.342], 0.939) ; \circ 70$

plot $\left([x 49,-x 49, x 49,-x 49],[y 49, y 49,-y 49,-y 49], ' k-^{\prime}\right)$

$[\mathrm{x} 50, \mathrm{y} 50]=0 \mathrm{de} 45(\mathrm{QODE} 3,[0.174: 0.002: 5.174], 0.984) ; \circ 80$

plot $\left([x 50,-x 50, x 50,-x 50],[y 50, y 50,-y 50,-y 50], k^{-\prime}\right)$

$x 51=[0: 0.002: 5]^{\prime}$;

$\mathrm{y} 51=\mathrm{x} 51 \cdot * 0+5$

plot $(x 51, y 51, ' k-')$

plot $\left(-\mathrm{x} 51, \mathrm{y} 51, \mathrm{k}^{-1}\right)$

plot $\left(x 51,-\mathrm{y} 51, \mathrm{k}^{-1}\right)$

plot $(-x 51,-y 51, ' k-')$

plot (y51, x51,' k-')

plot $\left(-\mathrm{y} 51, \mathrm{x} 51, \mathrm{\prime}^{\mathrm{k}} \mathrm{-}^{\prime}\right)$

plot $\left(y 51,-x 51, k^{-1}\right)$

plot $\left(-\mathrm{y} 51,-\mathrm{x} 51, \mathrm{k}-\mathrm{-}^{\prime}\right)$

$\mathrm{x} 52=0: .01: 1$;

y52=sqrt $(1-x 52 \cdot \wedge 2)$;

plot (x52, y52, 'k-', 'Linewidth' , 1.5)

plot $(-x 52, y 52, '$ 'k-', 'Linewidth' ', 1.5)

plot (x52,-y52, 'k-', 'Linewidth', 1.5)

plot $\left(-x 52,-y 52\right.$, 'k-' $^{-}$'Linewidth' , 1.5)

$\mathrm{x}=[1: .002: 6] '$;

$\mathrm{y}=\mathrm{x} \cdot{ }^{*} 0$;

plot $(x, y, ' k-')$

plot $\left(-x, y,{ }^{\prime}-^{\prime}\right)$

plot $\left(y, x, x^{\prime}-{ }^{\prime}\right)$

plot $\left(y,-x, k^{\prime}-'\right)$

for $j=1: 22$

$\mathrm{m}=[\mathrm{y} 51, \mathrm{x} 24, \mathrm{x} 25, \mathrm{x} 26, \mathrm{x} 27, \mathrm{x} 28, \mathrm{x} 29, \mathrm{x} 30, \mathrm{x} 31, \mathrm{x} 32, \mathrm{x} 33, \mathrm{x} 34, \mathrm{x} 35, \mathrm{x} 36, \mathrm{x} 37, \mathrm{x} 38, \mathrm{x} 39, \mathrm{x} 40, \mathrm{x} 41, \mathrm{x} 42$, $\mathrm{x} 43, \mathrm{x} 44]$;

$n=[x 51, y 24, y 25, y 26, y 27, y 28, y 29, y 30, y 31, y 32, y 33, y 34, y 35, y 36, y 37, y 38, y 39, y 40, y 41, y 42$, $\mathrm{y} 43, \mathrm{y} 44]$

end

$[A 1(j, 1), A 1(j, 2)]=$ curveintersect $(m(:, j), n(:, j), x 22, y 22)$;

for $j=1: 29$

$m=[y, y 51, x 24, x 25, x 26, x 27, x 28, x 29, x 30, x 31, x 32, x 33, x 34, x 35, x 36, x 37, x 38, x 39, x 40, x 41, x 4$

$2, x 43, x 44, x 45, x 46, x 47, x 48, x 49, x 50]$

$n=[x, x 51, y 24, y 25, y 26, y 27, y 28, y 29, y 30, y 31, y 32, y 33, y 34, y 35, y 36, y 37, y 38, y 39, y 40, y 41, y 4$

$2, \mathrm{y} 43, \mathrm{y} 44, \mathrm{y} 45, \mathrm{y} 46, \mathrm{y} 47, \mathrm{y} 48, \mathrm{y} 49, \mathrm{y} 50]$ end

$[A 2(j, 1), A 2(j, 2)]=$ curveintersect $(m(:, j), n(:, j), x 21, y 21)$; 
for $j=1: 29$

$\mathrm{m}=[\mathrm{y}, \mathrm{y} 51, \mathrm{x} 24, \mathrm{x} 25, \mathrm{x} 26, \mathrm{x} 27, \mathrm{x} 28, \mathrm{x} 29, \mathrm{x} 30, \mathrm{x} 31, \mathrm{x} 32, \mathrm{x} 33, \mathrm{x} 34, \mathrm{x} 35, \mathrm{x} 36, \mathrm{x} 37, \mathrm{x} 38, \mathrm{x} 39, \mathrm{x} 40, \mathrm{x} 41, \mathrm{x} 4$ $2, x 43, x 44, x 45, x 46, x 47, x 48, x 49, x 50]$

$n=[x, x 51, y 24, y 25, y 26, y 27, y 28, y 29, y 30, y 31, y 32, y 33, y 34, y 35, y 36, y 37, y 38, y 39, y 40, y 41, y 4$ $2, \mathrm{y} 43, \mathrm{y} 44, \mathrm{y} 45, \mathrm{y} 46, \mathrm{y} 47, \mathrm{y} 48, \mathrm{y} 49, \mathrm{y} 50]$

end

$[A 3(j, 1), A 3(j, 2)]=$ curveintersect $(m(:, j), n(:, j), x 20, y 20) ;$

for $j=1: 29$

$m=[y, y 51, x 24, x 25, x 26, x 27, x 28, x 29, x 30, x 31, x 32, x 33, x 34, x 35, x 36, x 37, x 38, x 39, x 40, x 41, x 4$ $2, x 43, x 44, x 45, x 46, x 47, x 48, x 49, x 50]$

$n=[x, x 51, y 24, y 25, y 26, y 27, y 28, y 29, y 30, y 31, y 32, y 33, y 34, y 35, y 36, y 37, y 38, y 39, y 40, y 41, y 4$ $2, \mathrm{y} 43, \mathrm{y} 44, \mathrm{y} 45, \mathrm{y} 46, \mathrm{y} 47, \mathrm{y} 48, \mathrm{y} 49, \mathrm{y} 50]$

end

$[A 4(j, 1), A 4(j, 2)]=$ curveintersect $(m(:, j), n(:, j), x 19, y 19)$;

for $j=1: 29$

$m=[y, y 51, x 24, x 25, x 26, x 27, x 28, x 29, x 30, x 31, x 32, x 33, x 34, x 35, x 36, x 37, x 38, x 39, x 40, x 41, x 4$ $2, x 43, x 44, x 45, x 46, x 47, x 48, x 49, x 50]$

$n=[x, x 51, y 24, y 25, y 26, y 27, y 28, y 29, y 30, y 31, y 32, y 33, y 34, y 35, y 36, y 37, y 38, y 39, y 40, y 41, y 4$ $2, \mathrm{y} 43, \mathrm{y} 44, \mathrm{y} 45, \mathrm{y} 46, \mathrm{y} 47, \mathrm{y} 48, \mathrm{y} 49, \mathrm{y} 50]$ end $[A 5(j, 1), A 5(j, 2)]=$ curveintersect $(m(:, j), n(:, j), x 18, y 18) ;$

for $j=1: 29$

$m=[y, y 51, x 24, x 25, x 26, x 27, x 28, x 29, x 30, x 31, x 32, x 33, x 34, x 35, x 36, x 37, x 38, x 39, x 40, x 41, x 4$ $2, x 43, x 44, x 45, x 46, x 47, x 48, x 49, x 50]$

$n=[x, x 51, y 24, y 25, y 26, y 27, y 28, y 29, y 30, y 31, y 32, y 33, y 34, y 35, y 36, y 37, y 38, y 39, y 40, y 41, y 4$ $2, \mathrm{y} 43, \mathrm{y} 44, \mathrm{y} 45, \mathrm{y} 46, \mathrm{y} 47, \mathrm{y} 48, \mathrm{y} 49, \mathrm{y} 50]$

$[A 6(j, 1), A 6(j, 2)]=$ curveintersect $(m(:, j), n(:, j), x 17, y 17) ;$

end

for $j=1: 29$

$m=[y, y 51, x 24, x 25, x 26, x 27, x 28, x 29, x 30, x 31, x 32, x 33, x 34, x 35, x 36, x 37, x 38, x 39, x 40, x 41, x 4$ $2, x 43, x 44, x 45, x 46, x 47, x 48, x 49, x 50]$

$n=[x, x 51, y 24, y 25, y 26, y 27, y 28, y 29, y 30, y 31, y 32, y 33, y 34, y 35, y 36, y 37, y 38, y 39, y 40, y 41, y 4$ $2, \mathrm{y} 43, \mathrm{y} 44, \mathrm{y} 45, \mathrm{y} 46, \mathrm{y} 47, \mathrm{y} 48, \mathrm{y} 49, \mathrm{y} 50]$ end $[A 7(j, 1), A 7(j, 2)]=$ curveintersect $(m(:, j), n(:, j), x 16, y 16)$;

for $j=1: 29$

$m=[y, y 51, x 24, x 25, x 26, x 27, x 28, x 29, x 30, x 31, x 32, x 33, x 34, x 35, x 36, x 37, x 38, x 39, x 40, x 41, x 4$ $2, x 43, x 44, x 45, x 46, x 47, x 48, x 49, x 50]$

$n=[x, x 51, y 24, y 25, y 26, y 27, y 28, y 29, y 30, y 31, y 32, y 33, y 34, y 35, y 36, y 37, y 38, y 39, y 40, y 41, y 4$ $2, \mathrm{y} 43, \mathrm{y} 44, \mathrm{y} 45, \mathrm{y} 46, \mathrm{y} 47, \mathrm{y} 48, \mathrm{y} 49, \mathrm{y} 50]$ end $[A 8(j, 1), A 8(j, 2)]=$ curveintersect $(m(:, j), n(:, j), x 15, y 15)$

for $j=1: 29$

$m=[y, y 51, x 24, x 25, x 26, x 27, x 28, x 29, x 30, x 31, x 32, x 33, x 34, x 35, x 36, x 37, x 38, x 39, x 40, x 41, x 4$ $2, x 43, x 44, x 45, x 46, x 47, x 48, x 49, x 50]$

$n=[x, x 51, y 24, y 25, y 26, y 27, y 28, y 29, y 30, y 31, y 32, y 33, y 34, y 35, y 36, y 37, y 38, y 39, y 40, y 41, y 4$ $2, \mathrm{y} 43, \mathrm{y} 44, \mathrm{y} 45, \mathrm{y} 46, \mathrm{y} 47, \mathrm{y} 48, \mathrm{y} 49, \mathrm{y} 50]$

$[A 9(j, 1), A 9(j, 2)]=$ curveintersect $(m(:, j), n(:, j), x 14, y 14) ;$

end

for $j=1: 29$

$m=[y, y 51, x 24, x 25, x 26, x 27, x 28, x 29, x 30, x 31, x 32, x 33, x 34, x 35, x 36, x 37, x 38, x 39, x 40, x 41, x 4$ $2, x 43, x 44, x 45, x 46, x 47, x 48, x 49, x 50]$

$n=[x, x 51, y 24, y 25, y 26, y 27, y 28, y 29, y 30, y 31, y 32, y 33, y 34, y 35, y 36, y 37, y 38, y 39, y 40, y 41, y 4$ $2, \mathrm{y} 43, \mathrm{y} 44, \mathrm{y} 45, \mathrm{y} 46, \mathrm{y} 47, \mathrm{y} 48, \mathrm{y} 49, \mathrm{y} 50]$

end

$[A 10(j, 1), A 10(j, 2)]=$ curveintersect $(m(:, j), n(:, j), x 13, y 13)$;

for $j=1: 29$

$m=[y, y 51, x 24, x 25, x 26, x 27, x 28, x 29, x 30, x 31, x 32, x 33, x 34, x 35, x 36, x 37, x 38, x 39, x 40, x 41, x 4$ $2, x 43, x 44, x 45, x 46, x 47, x 48, x 49, x 50]$

$n=[x, x 51, y 24, y 25, y 26, y 27, y 28, y 29, y 30, y 31, y 32, y 33, y 34, y 35, y 36, y 37, y 38, y 39, y 40, y 41, y 4$ $2, \mathrm{y} 43, \mathrm{y} 44, \mathrm{y} 45, \mathrm{y} 46, \mathrm{y} 47, \mathrm{y} 48, \mathrm{y} 49, \mathrm{y} 50]$ end

$[A 11(j, 1), \operatorname{Al1}(j, 2)]=$ curveintersect $(m(:, j), n(:, j), x 12, y 12)$;

for $j=1: 29$

$\mathrm{m}=[\mathrm{y}, \mathrm{y} 51, \mathrm{x} 24, \mathrm{x} 25, \mathrm{x} 26, \mathrm{x} 27, \mathrm{x} 28, \mathrm{x} 29, \mathrm{x} 30, \mathrm{x} 31, \mathrm{x} 32, \mathrm{x} 33, \mathrm{x} 34, \mathrm{x} 35, \mathrm{x} 36, \mathrm{x} 37, \mathrm{x} 38, \mathrm{x} 39, \mathrm{x} 40, \mathrm{x} 41, \mathrm{x} 4$ $2, x 43, x 44, x 45, x 46, x 47, x 48, x 49, x 50]$

$n=[x, x 51, y 24, y 25, y 26, y 27, y 28, y 29, y 30, y 31, y 32, y 33, y 34, y 35, y 36, y 37, y 38, y 39, y 40, y 41, y 4$ $2, \mathrm{y} 43, \mathrm{y} 44, \mathrm{y} 45, \mathrm{y} 46, \mathrm{y} 47, \mathrm{y} 48, \mathrm{y} 49, \mathrm{y} 50]$

$[A 12(j, 1), A 12(j, 2)]=$ curveintersect $(m(:, j), n(:, j), x 11, y 11)$;

end

for $j=1: 29$ 
$m=[y, y 51, x 24, x 25, x 26, x 27, x 28, x 29, x 30, x 31, x 32, x 33, x 34, x 35, x 36, x 37, x 38, x 39, x 40, x 41, x 4$ $2, x 43, x 44, x 45, x 46, x 47, x 48, x 49, x 50]$

$\mathrm{n}=[\mathrm{x}, \mathrm{x} 51, \mathrm{y} 24, \mathrm{y} 25, \mathrm{y} 26, \mathrm{y} 27, \mathrm{y} 28, \mathrm{y} 29, \mathrm{y} 30, \mathrm{y} 31, \mathrm{y} 32, \mathrm{y} 33, \mathrm{y} 34, \mathrm{y} 35, \mathrm{y} 36, \mathrm{y} 37, \mathrm{y} 38, \mathrm{y} 39, \mathrm{y} 40, \mathrm{y} 41, \mathrm{y} 4$ $2, \mathrm{y} 43, \mathrm{y} 44, \mathrm{y} 45, \mathrm{y} 46, \mathrm{y} 47, \mathrm{y} 48, \mathrm{y} 49, \mathrm{y} 50]$

$[A 13(j, 1), A 13(j, 2)]=$ curveintersect $(m(:, j), n(:, j), x 10, y 10)$;

end

for $j=1: 29$

$m=[y, y 51, x 24, x 25, x 26, x 27, x 28, x 29, x 30, x 31, x 32, x 33, x 34, x 35, x 36, x 37, x 38, x 39, x 40, x 41, x 4$ $2, x 43, x 44, x 45, x 46, x 47, x 48, x 49, x 50]$

$\mathrm{n}=[\mathrm{x}, \mathrm{x} 51, \mathrm{y} 24, \mathrm{y} 25, \mathrm{y} 26, \mathrm{y} 27, \mathrm{y} 28, \mathrm{y} 29, \mathrm{y} 30, \mathrm{y} 31, \mathrm{y} 32, \mathrm{y} 33, \mathrm{y} 34, \mathrm{y} 35, \mathrm{y} 36, \mathrm{y} 37, \mathrm{y} 38, \mathrm{y} 39, \mathrm{y} 40, \mathrm{y} 41, \mathrm{y} 4$ $2, \mathrm{y} 43, \mathrm{y} 44, \mathrm{y} 45, \mathrm{y} 46, \mathrm{y} 47, \mathrm{y} 48, \mathrm{y} 49, \mathrm{y} 50]$ end

$[A 14(j, 1), A 14(j, 2)]=$ curveintersect $(m(:, j), n(:, j), x 9, y 9)$;

for $j=1: 29$

$m=[y, y 51, x 24, x 25, x 26, x 27, x 28, x 29, x 30, x 31, x 32, x 33, x 34, x 35, x 36, x 37, x 38, x 39, x 40, x 41, x 4$ $2, x 43, x 44, x 45, x 46, x 47, x 48, x 49, x 50]$

$n=[x, x 51, y 24, y 25, y 26, y 27, y 28, y 29, y 30, y 31, y 32, y 33, y 34, y 35, y 36, y 37, y 38, y 39, y 40, y 41, y 4$ $2, \mathrm{y} 43, \mathrm{y} 44, \mathrm{y} 45, \mathrm{y} 46, \mathrm{y} 47, \mathrm{y} 48, \mathrm{y} 49, \mathrm{y} 50]$

end

$[A 15(j, 1), A 15(j, 2)]=$ curveintersect $(m(:, j), n(:, j), x 8, y 8)$;

for $j=1: 29$

$m=[y, y 51, x 24, x 25, x 26, x 27, x 28, x 29, x 30, x 31, x 32, x 33, x 34, x 35, x 36, x 37, x 38, x 39, x 40, x 41, x 4$

$2, x 43, x 44, x 45, x 46, x 47, x 48, x 49, x 50]$

$\mathrm{n}=[\mathrm{x}, \mathrm{x} 51, \mathrm{y} 24, \mathrm{y} 25, \mathrm{y} 26, \mathrm{y} 27, \mathrm{y} 28, \mathrm{y} 29, \mathrm{y} 30, \mathrm{y} 31, \mathrm{y} 32, \mathrm{y} 33, \mathrm{y} 34, \mathrm{y} 35, \mathrm{y} 36, \mathrm{y} 37, \mathrm{y} 38, \mathrm{y} 39, \mathrm{y} 40, \mathrm{y} 41, \mathrm{y} 4$ $2, \mathrm{y} 43, \mathrm{y} 44, \mathrm{y} 45, \mathrm{y} 46, \mathrm{y} 47, \mathrm{y} 48, \mathrm{y} 49, \mathrm{y} 50]$

end

$[A 16(j, 1), A 16(j, 2)]=$ curveintersect $(m(:, j), n(:, j), x 7, y 7)$;

for $j=1: 29$

$m=[y, y 51, x 24, x 25, x 26, x 27, x 28, x 29, x 30, x 31, x 32, x 33, x 34, x 35, x 36, x 37, x 38, x 39, x 40, x 41, x 4$ $2, x 43, x 44, x 45, x 46, x 47, x 48, x 49, x 50]$

$n=[x, x 51, y 24, y 25, y 26, y 27, y 28, y 29, y 30, y 31, y 32, y 33, y 34, y 35, y 36, y 37, y 38, y 39, y 40, y 41, y 4$ $2, \mathrm{y} 43, \mathrm{y} 44, \mathrm{y} 45, \mathrm{y} 46, \mathrm{y} 47, \mathrm{y} 48, \mathrm{y} 49, \mathrm{y} 50]$

end

$[A 17(j, 1), A 17(j, 2)]=$ curveintersect $(m(:, j), n(:, j), x 6, y 6) ;$

for $j=1: 29$

$m=[y, y 51, x 24, x 25, x 26, x 27, x 28, x 29, x 30, x 31, x 32, x 33, x 34, x 35, x 36, x 37, x 38, x 39, x 40, x 41, x 4$ $2, x 43, x 44, x 45, x 46, x 47, x 48, x 49, x 50]$

$n=[x, x 51, y 24, y 25, y 26, y 27, y 28, y 29, y 30, y 31, y 32, y 33, y 34, y 35, y 36, y 37, y 38, y 39, y 40, y 41, y 4$ $2, \mathrm{y} 43, \mathrm{y} 44, \mathrm{y} 45, \mathrm{y} 46, \mathrm{y} 47, \mathrm{y} 48, \mathrm{y} 49, \mathrm{y} 50]$ end

$[A 18(j, 1), A 18(j, 2)]=$ curveintersect $(m(:, j), n(:, j), x 5, y 5)$;

for $j=1: 29$

$m=[y, y 51, x 24, x 25, x 26, x 27, x 28, x 29, x 30, x 31, x 32, x 33, x 34, x 35, x 36, x 37, x 38, x 39, x 40, x 41, x 4$ $2, x 43, x 44, x 45, x 46, x 47, x 48, x 49, x 50]$

$\mathrm{n}=[\mathrm{x}, \mathrm{x} 51, \mathrm{y} 24, \mathrm{y} 25, \mathrm{y} 26, \mathrm{y} 27, \mathrm{y} 28, \mathrm{y} 29, \mathrm{y} 30, \mathrm{y} 31, \mathrm{y} 32, \mathrm{y} 33, \mathrm{y} 34, \mathrm{y} 35, \mathrm{y} 36, \mathrm{y} 37, \mathrm{y} 38, \mathrm{y} 39, \mathrm{y} 40, \mathrm{y} 41, \mathrm{y} 4$ $2, \mathrm{y} 43, \mathrm{y} 44, \mathrm{y} 45, \mathrm{y} 46, \mathrm{y} 47, \mathrm{y} 48, \mathrm{y} 49, \mathrm{y} 50]$

$[A 19(j, 1), A 19(j, 2)]=$ curveintersect $\left(m(:, j), n(:, j), x 4, y^{4}\right)$;

end

for $j=1: 29$

$m=[y, y 51, x 24, x 25, x 26, x 27, x 28, x 29, x 30, x 31, x 32, x 33, x 34, x 35, x 36, x 37, x 38, x 39, x 40, x 41, x 4$ $2, x 43, x 44, x 45, x 46, x 47, x 48, x 49, x 50]$

$\mathrm{n}=[\mathrm{x}, \mathrm{x} 51, \mathrm{y} 24, \mathrm{y} 25, \mathrm{y} 26, \mathrm{y} 27, \mathrm{y} 28, \mathrm{y} 29, \mathrm{y} 30, \mathrm{y} 31, \mathrm{y} 32, \mathrm{y} 33, \mathrm{y} 34, \mathrm{y} 35, \mathrm{y} 36, \mathrm{y} 37, \mathrm{y} 38, \mathrm{y} 39, \mathrm{y} 40, \mathrm{y} 41, \mathrm{y} 4$ $2, \mathrm{y} 43, \mathrm{y} 44, \mathrm{y} 45, \mathrm{y} 46, \mathrm{y} 47, \mathrm{y} 48, \mathrm{y} 49, \mathrm{y} 50]$ end $[A 20(j, 1), A 20(j, 2)]=$ curveintersect $(m(:, j), n(:, j), x 3, y 3)$;

for $j=1: 29$

$m=[y, y 51, x 24, x 25, x 26, x 27, x 28, x 29, x 30, x 31, x 32, x 33, x 34, x 35, x 36, x 37, x 38, x 39, x 40, x 41, x 4$ $2, x 43, x 44, x 45, x 46, x 47, x 48, x 49, x 50]$

$n=[x, x 51, y 24, y 25, y 26, y 27, y 28, y 29, y 30, y 31, y 32, y 33, y 34, y 35, y 36, y 37, y 38, y 39, y 40, y 41, y 4$ $2, \mathrm{y} 43, \mathrm{y} 44, \mathrm{y} 45, \mathrm{y} 46, \mathrm{y} 47, \mathrm{y} 48, \mathrm{y} 49, \mathrm{y} 50]$ end $[A 21(j, 1), A 21(j, 2)]=$ curveintersect $(m(:, j), n(:, j), x 2, y 2)$

for $j=1: 29$

$\mathrm{m}=[\mathrm{y}, \mathrm{y} 51, \mathrm{x} 24, \mathrm{x} 25, \mathrm{x} 26, \mathrm{x} 27, \mathrm{x} 28, \mathrm{x} 29, \mathrm{x} 30, \mathrm{x} 31, \mathrm{x} 32, \mathrm{x} 33, \mathrm{x} 34, \mathrm{x} 35, \mathrm{x} 36, \mathrm{x} 37, \mathrm{x} 38, \mathrm{x} 39, \mathrm{x} 40, \mathrm{x} 41, \mathrm{x} 4$ $2, x 43, x 44, x 45, x 46, x 47, x 48, x 49, x 50]$

$\mathrm{n}=[\mathrm{x}, \mathrm{x} 51, \mathrm{y} 24, \mathrm{y} 25, \mathrm{y} 26, \mathrm{y} 27, \mathrm{y} 28, \mathrm{y} 29, \mathrm{y} 30, \mathrm{y} 31, \mathrm{y} 32, \mathrm{y} 33, \mathrm{y} 34, \mathrm{y} 35, \mathrm{y} 36, \mathrm{y} 37, \mathrm{y} 38, \mathrm{y} 39, \mathrm{y} 40, \mathrm{y} 41, \mathrm{y} 4$

$2, \mathrm{y} 43, \mathrm{y} 44, \mathrm{y} 45, \mathrm{y} 46, \mathrm{y} 47, \mathrm{y} 48, \mathrm{y} 49, \mathrm{y} 50]$

end

$[A 22(j, 1), A 22(j, 2)]=$ curveintersect $(m(:, j), n(:, j), x 51, y 51)$;

for $j=1: 21$ 


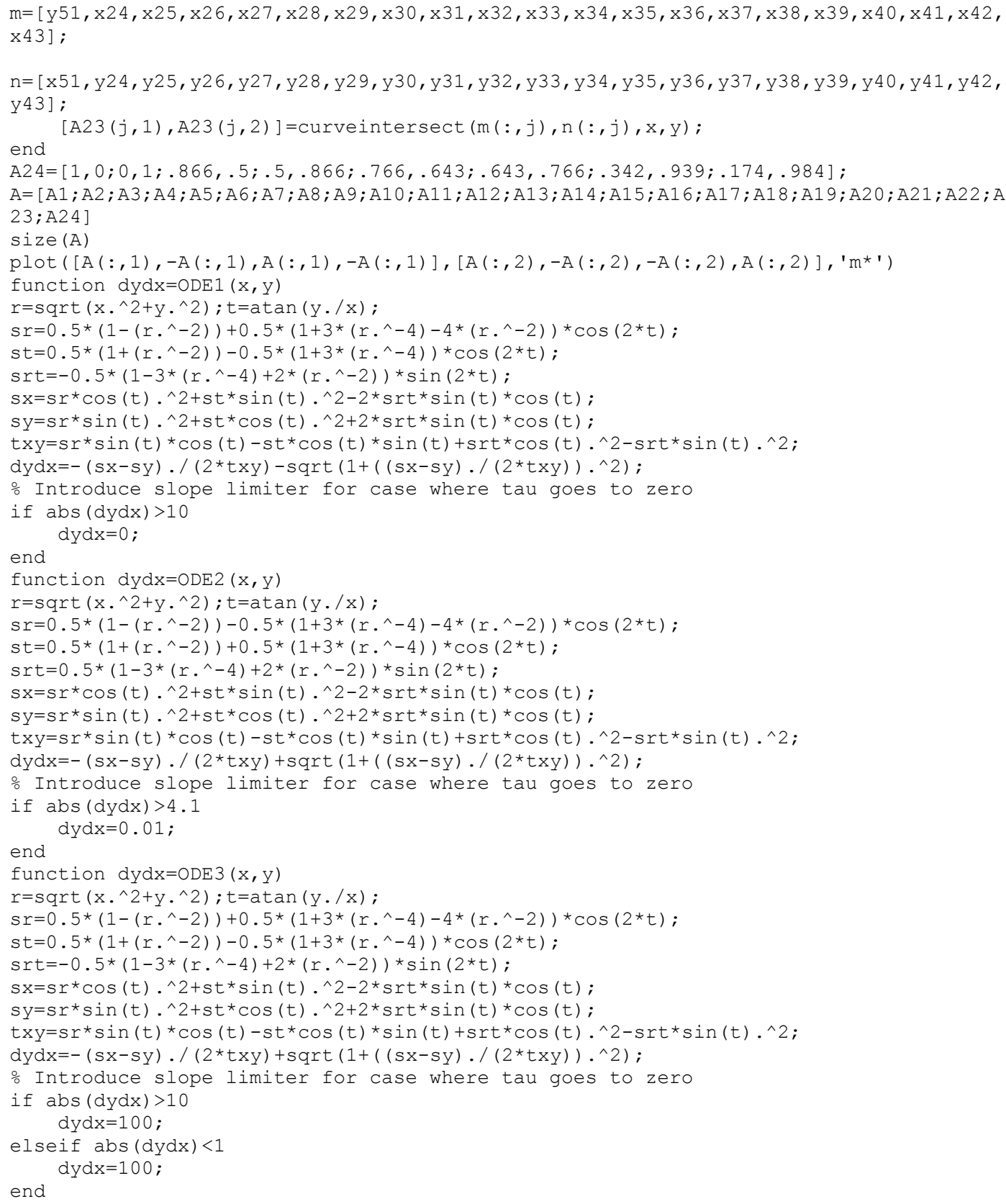

\section{Uniaxial Compression Fine Topology}

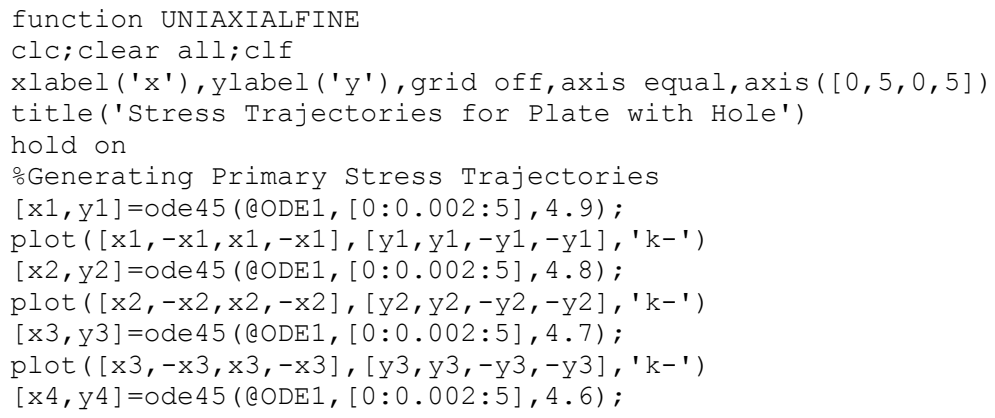


plot $\left([\mathrm{x} 4,-\mathrm{x} 4, \mathrm{x} 4,-\mathrm{x} 4],[\mathrm{y} 4, \mathrm{y} 4,-\mathrm{y} 4,-\mathrm{y} 4], \mathrm{k}^{\prime}\right)$

$[\mathrm{x} 5, \mathrm{y} 5]=0 \mathrm{de} 45(\mathrm{QODE} 1,[0: 0.002: 5], 4.5)$;

plot $\left([\mathrm{x} 5,-\mathrm{x} 5, \mathrm{x} 5,-\mathrm{x} 5],[\mathrm{y} 5, \mathrm{y} 5,-\mathrm{y} 5,-\mathrm{y} 5], \mathrm{k}^{\mathrm{k}}\right.$ ')

$[\mathrm{x} 6, \mathrm{y} 6]=0 \mathrm{de} 45(@ \mathrm{ODE} 1,[0: 0.002: 5], 4.4)$;

plot $([x 6,-x 6, x 6,-x 6],[y 6, y 6,-y 6,-y 6], ' k-1)$

$[\mathrm{x} 7, \mathrm{y} 7]=0 \mathrm{de} 45(@ \mathrm{ODE} 1,[0: 0.002: 5], 4.3)$;

$\operatorname{plot}([x 7,-x 7, x 7,-x 7],[y 7, y 7,-y 7,-y 7], ' k-1)$

$[\mathrm{x} 8, \mathrm{y} 8]=0 \mathrm{de} 45(@ \mathrm{ODE} 1,[0: 0.002: 5], 4.2)$;

$\operatorname{plot}\left([\mathrm{x} 8,-\mathrm{x} 8, \mathrm{x} 8,-\mathrm{x} 8],[\mathrm{y} 8, \mathrm{y} 8,-\mathrm{y} 8,-\mathrm{y} 8], \mathrm{k}^{\prime}\right)$

$[\mathrm{x} 9, \mathrm{y} 9]=0 \mathrm{de} 45($ @ODE $1,[0: 0.002: 5], 4.1)$;

plot $([x 9,-x 9, x 9,-x 9],[y 9, y 9,-y 9,-y 9], ' k-')$

$[\mathrm{x} 10, \mathrm{y} 10]=0 \mathrm{de} 45($ @ODE $1,[0: 0.002: 5], 4.0)$;

plot $([x 10,-x 10, x 10,-x 10],[y 10, y 10,-y 10,-y 10], ' k-')$

$[\mathrm{x} 11, \mathrm{y} 11]=0 \mathrm{de} 45(\mathrm{QODE} 1,[0: 0.002: 5], 3.9)$;

plot $\left([x 11,-x 11, x 11,-x 11],[y 11, y 11,-y 11,-y 11], ' k-^{\prime}\right)$

$[\mathrm{x} 12, \mathrm{y} 12]=0 \mathrm{de} 45(\mathrm{QODE} 1,[0: 0.002: 5], 3.8)$;

plot $\left([\mathrm{x} 12,-\mathrm{x} 12, \mathrm{x} 12,-\mathrm{x} 12],[\mathrm{y} 12, \mathrm{y} 12,-\mathrm{y} 12,-\mathrm{y} 12], \mathrm{\prime}^{\prime} \mathbf{-}^{\prime}\right)$

$[\mathrm{x} 13, \mathrm{y} 13]=0 \mathrm{de} 45(\operatorname{eODE} 1,[0: 0.002: 5], 3.7)$;

plot $\left([x 13,-x 13, x 13,-x 13],[y 13, y 13,-y 13,-y 13],{ }^{\prime}-^{\prime}\right)$

$[\mathrm{x} 14, \mathrm{y} 14]=0 \mathrm{de} 45($ @ODE $1,[0: 0.002: 5], 3.6)$;

plot $([x 14,-x 14, x 14,-x 14],[y 14, y 14,-y 14,-y 14], ' k-')$

$[\mathrm{x} 15, \mathrm{y} 15]=0 \mathrm{de} 45($ @ODE1, $[0: 0.002: 5], 3.5)$;

plot $\left([x 15,-x 15, x 15,-x 15],[y 15, y 15,-y 15,-y 15],{ }^{\prime}-^{\prime}\right)$

$[\mathrm{x} 16, \mathrm{y} 16]=0 \mathrm{de} 45(\mathrm{QODE} 1,[0: 0.002: 5], 3.4)$;

plot $\left([x 16,-x 16, x 16,-x 16],[y 16, y 16,-y 16,-y 16], ' k^{\prime}\right)$

$[\mathrm{x} 17, \mathrm{y} 17]=0 \mathrm{de} 45(\mathrm{eODE} 1,[0: 0.002: 5], 3.3)$;

plot $\left([x 17,-x 17, x 17,-x 17],[y 17, y 17,-y 17,-y 17], k^{-\prime}\right)$

$[\mathrm{x} 18, \mathrm{y} 18]=0 \mathrm{de} 45(\mathrm{QODE} 1,[0: 0.002: 5], 3.2)$.

plot $([x 18,-x 18, x 18,-x 18],[y 18, y 18,-y 18,-y 18], ' k-')$

$[\mathrm{x} 19, \mathrm{y} 19]=0 \mathrm{de} 45($ @ODE1, $[0: 0.002: 5], 3.1)$;

plot $([x 19,-x 19, x 19,-x 19],[y 19, y 19,-y 19,-y 19], ' k-')$

$[\mathrm{x} 20, \mathrm{y} 20]=0 \mathrm{de} 45(\mathrm{\varrho ODE} 1,[0: 0.002: 5], 3.0)$;

plot $\left([x 20,-x 20, x 20,-x 20],[y 20, y 20,-y 20,-y 20],{ }^{\prime}-^{\prime}\right)$

$[\mathrm{x} 21, \mathrm{y} 21]=0 \mathrm{de} 45(\mathrm{QODE} 1,[0: 0.002: 5], 2.9)$;

plot $([x 21,-x 21, x 21,-x 21],[y 21, y 21,-y 21,-y 21], ' k-')$

$[\mathrm{x} 22, \mathrm{y} 22]=0 \mathrm{de} 45(\mathrm{QODE} 1,[0: 0.002: 5], 2.8)$;

plot $\left([x 22,-x 22, x 22,-x 22],[y 22, y 22,-y 22,-y 22], ' k^{\prime} '\right)$

$[\mathrm{x} 23, \mathrm{y} 23]=0 \mathrm{de} 45(\mathrm{QODE} 1,[0: 0.002: 5], 2.7)$; 。\# Ok<*NBRAK>

plot $\left([x 23,-x 23, x 23,-x 23],[y 23, y 23,-y 23,-y 23], ' k^{\prime}\right.$ ')

$[\mathrm{x} 24, \mathrm{y} 24]=0 \mathrm{de} 45(\mathrm{QODE} 1,[0: 0.002: 5], 2.6)$;

plot $\left([x 24,-x 24, x 24,-x 24],[y 24, y 24,-y 24,-y 24], ' k^{\prime}\right.$ ')

$[\mathrm{x} 25, \mathrm{y} 25]=0 \mathrm{de} 45(\mathrm{QODE} 1,[0: 0.002: 5], 2.5)$;

plot $\left([x 25,-x 25, x 25,-x 25],[y 25, y 25,-y 25,-y 25],{ }^{\prime} k-1\right)$

$[\mathrm{x} 26, \mathrm{y} 26]=0 \mathrm{de} 45(\mathrm{QODE} 1,[0: 0.002: 5], 2.4)$;

plot $\left([x 26,-x 26, x 26,-x 26],[y 26, y 26,-y 26,-y 26], ' k^{\prime}\right)$

$[\mathrm{x} 27, \mathrm{y} 27]=0 \mathrm{de} 45(\mathrm{QODE} 1,[0: 0.002: 5], 2.3)$;

plot $\left([x 27,-x 27, x 27,-x 27],[y 27, y 27,-y 27,-y 27], k^{\prime}{ }^{\prime}\right)$

$[\mathrm{x} 28, \mathrm{y} 28]=0 \mathrm{de} 45(\mathrm{eODE} 1,[0: 0.002: 5], 2.2)$;

plot $([x 28,-x 28, x 28,-x 28],[y 28, y 28,-y 28,-y 28], ' k-')$

$[\mathrm{x} 29, \mathrm{y} 29]=0 \mathrm{de} 45(\mathrm{QODE} 1,[0: 0.002: 5], 2.1)$;

plot $\left([x 29,-x 29, x 29,-x 29],[y 29, y 29,-y 29,-y 29], k^{\prime}{ }^{\prime}\right)$

$[\mathrm{x} 30, \mathrm{y} 30]=0 \mathrm{de} 45(\mathrm{QODE} 1,[0: 0.002: 5], 2.0)$;

plot $\left([x 30,-x 30, x 30,-x 30],[y 30, y 30,-y 30,-y 30], ' k-^{\prime}\right)$

$[\mathrm{x} 31, \mathrm{y} 31]=0 \mathrm{de} 45(\mathrm{QODE} 1,[0: 0.002: 5], 1.9)$;

plot $\left([x 31,-x 31, x 31,-x 31],[y 31, y 31,-y 31,-y 31],{ }^{\prime}-^{\prime}\right)$

$[\mathrm{x} 32, \mathrm{y} 32]=0 \mathrm{de} 45(\mathrm{\varrho ODE} 1,[0: 0.002: 5], 1.8)$;

plot $\left([x 32,-x 32, x 32,-x 32],[y 32, y 32,-y 32,-y 32], ' k^{\prime} '\right)$

$[\mathrm{x} 33, \mathrm{y} 33]=0 \mathrm{de} 45(\mathrm{\varrho ODE} 1,[0: 0.002: 5], 1.7)$;

plot $([x 33,-x 33, x 33,-x 33],[y 33, y 33,-y 33,-y 33], ' k-')$

$[\mathrm{x} 34, \mathrm{y} 34]=0 \mathrm{de} 45(\mathrm{QODE} 1,[0: 0.002: 5], 1.6)$;

plot $\left([x 34,-x 34, x 34,-x 34],[y 34, y 34,-y 34,-y 34],{ }^{\prime}-^{\prime}\right)$

$[\mathrm{x} 35, \mathrm{y} 35]=0 \mathrm{de} 45(\mathrm{QODE} 1,[0: 0.002: 5], 1.5)$;

plot $([x 35,-x 35, x 35,-x 35],[y 35, y 35,-y 35,-y 35], ' k-1)$

$[\mathrm{x} 36, \mathrm{y} 36]=0 \mathrm{de} 45(\mathrm{eODE} 1,[0: 0.002: 5], 1.4)$;

plot $\left([x 36,-x 36, x 36,-x 36],[y 36, y 36,-y 36,-y 36],{ }^{\prime}-^{\prime}\right)$ $[\mathrm{x} 37, \mathrm{y} 37]=0 \mathrm{de} 45(\mathrm{\varrho ODE} 1,[0: 0.002: 5], 1.3)$;

plot $\left([x 37,-x 37, x 37,-x 37],[y 37, y 37,-y 37,-y 37], k^{\prime}{ }^{\prime}\right)$ $[\mathrm{x} 38, \mathrm{y} 38]=0 \mathrm{de} 45(\mathrm{QODE} 1,[0: 0.002: 5], 1.2)$;

plot $\left([x 38,-x 38, x 38,-x 38],[y 38, y 38,-y 38,-y 38], ' k^{\prime} '\right)$

$[\mathrm{x} 39, \mathrm{y} 39]=0 \mathrm{de} 45(\mathrm{QODE} 1,[0: 0.002: 5], 1.11)$;

plot $\left([x 39,-x 39, x 39,-x 39],[y 39, y 39,-y 39,-y 39], k^{-\prime}\right)$ 
$[\mathrm{x} 40, \mathrm{y} 40]=0 \mathrm{de} 45(\operatorname{eodE} 1,[0: 0.002: 5], 1.05) ;$

plot $\left([x 40,-x 40, x 40,-x 40],[y 40, y 40,-y 40,-y 40], ' k-^{\prime}\right)$

$[\mathrm{x} 41, \mathrm{y} 41]=0 \mathrm{de} 45(\mathrm{eODE} 1,[0.866: 0.001: 5.866], 0.500)$;

plot $\left([x 41,-x 41, x 41,-x 41],[y 41, y 41,-y 41,-y 41],{ }^{\prime}-^{\prime}\right)$

oGenerating Secondary Stress Trajectories

$[y 42, x 42]=0$ de $45($ @ODE $2,[0: 0.002: 5], 4.9)$ :

plot $\left([x 42,-x 42, x 42,-x 42],[y 42, y 42,-y 42,-y 42], k^{\prime}-^{\prime}\right)$

$[y 43, x 43]=0$ de $45(@ O D E 2,[0: 0.002: 5], 4.8)$;

$\operatorname{plot}\left([x 43,-x 43, x 43,-x 43],[y 43, y 43,-y 43,-y 43], k^{-\prime}\right)$

$[y 44, x 44]=0$ de $45(\operatorname{CODE} 2,[0: 0.002: 5], 4.7)$;

plot $\left([\mathrm{x} 44,-\mathrm{x} 44, \mathrm{x} 44,-\mathrm{x} 44],[\mathrm{y} 44, \mathrm{y} 44,-\mathrm{y} 44,-\mathrm{y} 44], \mathrm{k}^{\prime} \mathbf{\prime}^{\prime}\right)$

$[y 45, x 45]=0$ de $45($ @ODE $2,[0: 0.002: 5], 4.6)$;

plot $([x 45,-x 45, x 45,-x 45],[y 45, y 45,-y 45,-y 45], ' k-')$

$[y 46, x 46]=0$ de $45(@ O D E 2,[0: 0.002: 5], 4.5)$;

plot $\left([\mathrm{x} 46,-\mathrm{x} 46, \mathrm{x} 46,-\mathrm{x} 46],[\mathrm{y} 46, \mathrm{y} 46,-\mathrm{y} 46,-\mathrm{y} 46], \mathrm{l}^{\left.\mathrm{k}-\mathrm{I}^{\prime}\right)}\right.$

$[y 47, x 47]=0$ de4 $5(@ O D E 2,[0: 0.002: 5], 4.4)$;

plot $\left([x 47,-x 47, x 47,-x 47],[y 47, y 47,-y 47,-y 47], ' k^{-1}\right)$

$[y 48, x 48]=0$ de4 $5($ @ODE $2,[0: 0.002: 5], 4.3)$;

plot $\left([x 48,-x 48, x 48,-x 48],[y 48, y 48,-y 48,-y 48],{ }^{\prime}-^{\prime}\right)$

$[y 49, x 49]=0 d e 45($ @ODE $2,[0: 0.002: 5], 4.2)$;

plot $([x 49,-x 49, x 49,-x 49],[y 49, y 49,-y 49,-y 49], ' k-')$

$[y 50, x 50]=0 d e 45(@ O D E 2,[0: 0.002: 5], 4.1)$;

plot $\left([x 50,-x 50, x 50,-x 50],[y 50, y 50,-y 50,-y 50], k^{-\prime}\right)$

$[y 51, x 51]=0$ de4 $5($ @ODE2, $[0: 0.002: 5], 4.0)$;

plot $\left([x 51,-x 51, x 51,-x 51],[y 51, y 51,-y 51,-y 51], k^{\prime}{ }^{\prime}\right)$ $[y 52, x 52]=0$ de $45(@ O D E 2,[0: 0.002: 5], 3.9)$;

plot $\left([x 52,-x 52, x 52,-x 52],[y 52, y 52,-y 52,-y 52], k^{-\prime}\right)$ $[y 53, x 53]=0$ de $45(@ O D E 2,[0: 0.002: 5], 3.8)$;

plot $([x 53,-x 53, x 53,-x 53],[y 53, y 53,-y 53,-y 53], ' k-')$ $[y 54, x 54]=0$ de $45(@ O D E 2,[0: 0.002: 5], 3.7)$.

plot $([x 54,-x 54, x 54,-x 54],[y 54, y 54,-y 54,-y 54], ' k-')$

$[y 55, x 55]=0 d e 45($ @ODE $2,[0: 0.002: 5], 3.6)$;

plot $([x 55,-x 55, x 55,-x 55],[y 55, y 55,-y 55,-y 55], ' k-1)$

$[y 56, x 56]=0$ de $45($ @ODE $2,[0: 0.002: 5], 3.5)$;

plot $\left([x 56,-x 56, x 56,-x 56],[y 56, y 56,-y 56,-y 56], ' k^{-\prime}\right)$

$[y 57, x 57]=0$ de $45($ @ODE $2,[0: 0.002: 5], 3.4)$;

plot $\left([x 57,-x 57, x 57,-x 57],[y 57, y 57,-y 57,-y 57], ' k^{-1}\right)$

$[y 58, x 58]=0$ de $45($ @ODE $2,[0: 0.002: 5], 3.3)$;

plot $\left([x 58,-x 58, x 58,-x 58],[y 58, y 58,-y 58,-y 58], \prime^{\prime}-^{\prime}\right)$

$[y 59, x 59]=0 d e 45($ @ODE $2,[0: 0.002: 5], 3.2)$;

plot $\left([x 59,-x 59, x 59,-x 59],[y 59, y 59,-y 59,-y 59],{ }^{\prime}-^{\prime}\right)$

$[y 60, x 60]=0 d e 45(@ O D E 2,[0: 0.002: 5], 3.1)$;

plot $\left([x 60,-x 60, x 60,-x 60],[y 60, y 60,-y 60,-y 60],{ }^{\prime} k-1\right)$

$[y 61, x 61]=0$ de4 $5(@ O D E 2,[0: 0.002: 5], 3.0)$;

plot $([x 61,-x 61, x 61,-x 61],[y 61, y 61,-y 61,-y 61], ' k-1)$ $[y 62, x 62]=0$ de $45(@ O D E 2,[0: 0.002: 5], 2.9)$;

plot $\left([x 62,-x 62, x 62,-x 62],[y 62, y 62,-y 62,-y 62],{ }^{\prime} k-^{\prime}\right)$ $[y 63, x 63]=0 d e 45(@ O D E 2,[0: 0.002: 5], 2.8)$.

plot $\left([x 63,-x 63, x 63,-x 63],[y 63, y 63,-y 63,-y 63], ' k-^{\prime}\right)$

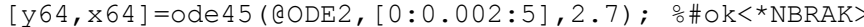
plot $\left([x 64,-x 64, x 64,-x 64],[y 64, y 64,-y 64,-y 64], ' k^{-\prime}\right)$ $[y 65, x 65]=0 d e 45(@ O D E 2,[0: 0.002: 5], 2.6)$;

plot $\left([x 65,-x 65, x 65,-x 65],[y 65, y 65,-y 65,-y 65], ' k^{-1}\right)$ $[y 66, x 66]=0 \operatorname{de} 45($ @ODE $2,[0: 0.002: 5], 2.5)$;

plot $\left([x 66,-x 66, x 66,-x 66],[y 66, y 66,-y 66,-y 66],{ }^{\prime}-^{\prime}\right)$

$[y 67, x 67]=0$ de $45(@ O D E 2,[0: 0.002: 5], 2.4)$;

plot $\left([x 67,-x 67, x 67,-x 67],[y 67, y 67,-y 67,-y 67], ' k^{\prime}{ }^{\prime}\right)$

$[y 68, x 68]=0$ de $45($ @ODE $2,[0: 0.002: 5], 2.3)$;

plot $\left([x 68,-x 68, x 68,-x 68],[y 68, y 68,-y 68,-y 68], k^{\prime-1}\right)$

$[y 69, x 69]=0 d e 45(@ O D E 2,[0: 0.002: 5], 2.2)$;

plot $([x 69,-x 69, x 69,-x 69],[y 69, y 69,-y 69,-y 69], ' k-1)$

$[y 70, x 70]=0 d e 45(@ O D E 2,[0: 0.002: 5], 2.1)$;

plot $\left([x 70,-x 70, x 70,-x 70],[y 70, y 70,-y 70,-y 70], ' k-^{\prime}\right)$

$[y 71, x 71]=0 d e 45(@ O D E 2,[0: 0.002: 5], 2.0)$;

plot $\left([x 71,-x 71, x 71,-x 71],[y 71, y 71,-y 71,-y 71], k^{\prime}{ }^{\prime}\right)$

$[y 72, x 72]=0$ de4 $5($ @ODE $2,[0: 0.002: 5], 1.9)$;

plot $([x 72,-x 72, x 72,-x 72],[y 72, y 72,-y 72,-y 72], ' k-')$ $[y 73, x 73]=0$ de $45($ @ODE $2,[0: 0.002: 5], 1.8)$;

plot $\left([x 73,-x 73, x 73,-x 73],[y 73, y 73,-y 73,-y 73], k^{-1}\right)$

$[y 74, x 74]=0$ de $45(@ O D E 2,[0: 0.002: 5], 1.7)$;

plot $\left([x 74,-x 74, x 74,-x 74],[y 74, y 74,-y 74,-y 74], k^{-'}\right)$ 
$[y 75, x 75]=0$ de $45($ @ODE $2,[0: 0.002: 5], 1.6)$;

plot $([x 75,-x 75, x 75,-x 75],[y 75, y 75,-y 75,-y 75], ' k-')$

$[y 76, x 76]=0 d e 45(@ O D E 2,[0: 0.002: 5], 1.5)$;

plot $\left([x 76,-x 76, x 76,-x 76],[y 76, y 76,-y 76,-y 76],{ }^{\prime}-^{\prime}\right)$

$[y 77, x 77]=0$ de $45(@ O D E 2,[0: 0.002: 5], 1.4)$;

plot $\left([x 77,-x 77, x 77,-x 77],[y 77, y 77,-y 77,-y 77], k^{\prime}{ }^{\prime}\right)$

$[y 78, x 78]=0$ de $45($ @ODE $2,[0: 0.002: 5], 1.3)$;

plot $\left([x 78,-x 78, x 78,-x 78],[y 78, y 78,-y 78,-y 78], ' k^{\prime}\right.$ ')

$[y 79, x 79]=0 d e 45(@ O D E 2,[0: 0.002: 5], 1.22)$;

plot $\left([x 79,-x 79, x 79,-x 79],[y 79, y 79,-y 79,-y 79], k^{\prime-1}\right)$

$[y 80, x 80]=0$ de $45($ @ODE2, $[0: 0.002: 5], 1.15)$;

plot $\left([\mathrm{x} 80,-\mathrm{x} 80, \mathrm{x} 80,-\mathrm{x} 80],[\mathrm{y} 80, \mathrm{y} 80,-\mathrm{y} 80,-\mathrm{y} 80], \mathrm{k}^{\left.\mathrm{k}-\mathrm{\prime}^{\prime}\right)}\right.$

$[y 81, x 81]=0 d e 45($ @ODE $2,[0: 0.002: 5], 1.1)$;

plot $\left([x 81,-x 81, x 81,-x 81],[y 81, y 81,-y 81,-y 81], ' k^{\prime}\right)$

$[y 82, x 82]=0$ de $45(@ O D E 2,[0: 0.002: 5], 1.05)$ :

plot $\left([x 82,-x 82, x 82,-x 82],[y 82, y 82,-y 82,-y 82], k^{\prime}-^{\prime}\right)$

$[y 83, x 83]=0$ de $45($ @ODE $2,[0: 0.002: 5], 1.0)$.

plot $([x 83,-x 83, x 83,-x 83],[y 83, y 83,-y 83,-y 83], ' k-')$

$[\mathrm{x} 84, \mathrm{y} 84]=0 \mathrm{de} 45(\mathrm{OODE} 3,[0.921: 0.002: 5.921], 0.391) ; \% 25$

plot $\left([x 84,-x 84, x 84,-x 84],[y 84, y 84,-y 84,-y 84], ' k^{-\prime}\right)$

$[\mathrm{x} 85, \mathrm{y} 85]=0 \mathrm{de} 45(\mathrm{QODE} 3,[0.875: 0.002: 5.875], 0.485) ; \div 30$

plot $\left([x 85,-x 85, x 85,-x 85],[y 85, y 85,-y 85,-y 85], k^{-\prime}\right)$

$[\mathrm{x} 86, \mathrm{y} 86]=0 \mathrm{de} 45(@ \mathrm{ODE} 3,[0.819: 0.002: 5.819], 0.573) ; \circ 35$

plot $\left([\mathrm{x} 86,-\mathrm{x} 86, \mathrm{x} 86,-\mathrm{x} 86],[\mathrm{y} 86, \mathrm{y} 86,-\mathrm{y} 86,-\mathrm{y} 86], \mathrm{k}^{\prime} \mathrm{-}^{\prime}\right)$

$[\mathrm{x} 87, \mathrm{y} 87]=0 \mathrm{de} 45(\mathrm{eODE} 3,[0.766: 0.002: 5.766], 0.643) ; \circ 40$

plot $\left([x 87,-x 87, x 87,-x 87],[y 87, y 87,-y 87,-y 87], k^{\prime}-^{\prime}\right)$

$[\mathrm{x} 88, \mathrm{y} 88]=0 \mathrm{de} 45(\mathrm{QODE} 3,[0.707: 0.002: 5.707], 0.707) ; \% 45$

plot $\left([x 88,-x 88, x 88,-x 88],[y 88, y 88,-y 88,-y 88],{ }^{\prime}-^{\prime}\right.$ ')

$[\mathrm{x} 89, \mathrm{y} 89]=0 \mathrm{de} 45(@ \mathrm{ODE} 3,[0.643: 0.002: 5.643], 0.766) ; \circ 50$

plot $\left([x 89,-x 89, x 89,-x 89],[y 89, y 89,-y 89,-y 89], k^{\prime}-^{\prime}\right)$

$[\mathrm{x} 90, \mathrm{y} 90]=0 \mathrm{de} 45(\mathrm{QODE} 3,[0.573: 0.002: 5.573], 0.819) ; \% 55$

plot $\left([x 90,-x 90, x 90,-x 90],[y 90, y 90,-y 90,-y 90], k^{\prime}-^{\prime}\right)$

$[\mathrm{x} 91, \mathrm{y} 91]=0 \mathrm{de} 45(\mathrm{@ODE} 3,[0.500: 0.002: 5.500], 0.866) ; \circ 60$

plot $\left([x 91,-x 91, x 91,-x 91],[y 91, y 91,-y 91,-y 91], k^{-\prime}\right)$

$[\mathrm{x} 92, \mathrm{y} 92]=0 \mathrm{de} 45(\mathrm{QODE} 3,[0.423: 0.002: 5.423], 0.906) ; \circ 65$

plot $\left([x 92,-x 92, x 92,-x 92],[y 92, y 92,-y 92,-y 92],{ }^{\prime} k-^{\prime}\right)$

[x93,y93] =ode45 (@ODE3, [0.342:0.002:5.342], 0.939); 구

plot $\left([x 93,-x 93, x 93,-x 93],[y 93, y 93,-y 93,-y 93], k^{-\prime}\right)$

[x94,y94 ] =ode45 (@ODE3, [0.259:0.002:5.259], 0.965); 은

plot $([x 94,-x 94, x 94,-x 94],[y 94, y 94,-y 94,-y 94], ' k-')$

$[\mathrm{x} 95, \mathrm{y} 95]=0 \mathrm{de} 45(\mathrm{QODE} 3,[0.174: 0.002: 5.174], 0.984) ; \circ 80$

plot ( [x95,-x95, x95,-x95], [y95,y95,-y95,-y95], 'k-')

$[\mathrm{x} 96, \mathrm{y} 96]=0 \mathrm{de} 45($ @ODE3, $[0.087: 0.002: 5.087], 0.996) ; \circ 85$

plot $([x 96,-x 96, x 96,-x 96],[y 96, y 96,-y 96,-y 96], ' k-')$

$\times 97=[0: 0.002: 5]$ ';

$\mathrm{y} 97=\mathrm{x} 97 . * 0+5$

plot $(x 97, y 97, ' k-')$

plot $\left(-x 97, y 97,{ }^{\prime} k-'\right)$

plot (x97,-y97, 'k-')

plot $\left(-x 97,-y 97,{ }^{\prime} k-'\right)$

plot (y97, x97, 'k-')

plot $(-y 97, x 97, ' k-')$

plot $(y 97,-x 97, ' k-')$

plot $(-y 97,-x 97, ' k-1)$

$\mathrm{x}=0: .01: 1$;

$\mathrm{y}=\operatorname{squt}\left(1-\mathrm{x} \cdot{ }^{\wedge} 2\right)$;

plot ( $x, y,{ }^{\prime}-^{\prime}$ ' ' 'Linewidth' , 1.5)

plot $\left(-x, y,{ }^{\prime}-^{\prime}\right.$ ', 'Linewidth', 1.5$)$

plot $\left(x,-y,{ }^{\prime}{ }^{-}\right.$', 'Linewidth', 1.5)

plot $\left(-x,-y,{ }^{\prime}-'\right.$ ' ' Linewidth' , 1.5)

$\mathrm{x}=[1: .002: 6]^{\prime}$;

$\mathrm{y}=\mathrm{x} \cdot{ }^{*} 0$;

$\operatorname{plot}\left(\mathrm{x}, \mathrm{y}, \mathrm{k}^{-\prime}\right)$

$\operatorname{plot}\left(-x, y,{ }^{\prime}-{ }^{\prime}\right)$

$\operatorname{plot}\left(y, x,{ }^{\prime}-^{\prime}\right)$

plot $\left(y,-x,{ }^{\prime}-^{\prime}\right)$

for $j=1: 43$

$\mathrm{m}=[\mathrm{y} 97, \mathrm{x} 42, \mathrm{x} 43, \mathrm{x} 44, \mathrm{x} 45, \mathrm{x} 46, \mathrm{x} 47, \mathrm{x} 48, \mathrm{x} 49, \mathrm{x} 50, \mathrm{x} 51, \mathrm{x} 52, \mathrm{x} 53, \mathrm{x} 54, \mathrm{x} 55, \mathrm{x} 56, \mathrm{x} 57, \mathrm{x} 58, \mathrm{x} 59, \mathrm{x} 60$, $x 61, x 62, x 63, x 64, x 65, x 66, x 67, x 68, x 69, x 70, x 71, x 72, x 73, x 74, x 75, x 76, x 77, x 78, x 79, x 80, x 81$ $, \times 82, \times 83]$; 
$n=[x 97, y 42, y 43, y 44, y 45, y 46, y 47, y 48, y 49, y 50, y 51, y 52, y 53, y 54, y 55, y 56, y 57, y 58, y 59, y 60$, $y 61, y 62, y 63, y 64, y 65, y 66, y 67, y 68, y 69, y 70, y 71, y 72, y 73, y 74, y 75, y 76, y 77, y 78, y 79, y 80, y 81$ $, \mathrm{y} 82, \mathrm{y} 83]$

end

$[A 1(j, 1), A 1(j, 2)]=$ curveintersect $(m(:, j), n(:, j), x, y)$; #ㅐ ${ }^{*}$ AGROW>

for $j=1: 44$

$m=[y 97, x 42, x 43, x 44, x 45, x 46, x 47, x 48, x 49, x 50, x 51, x 52, x 53, x 54, x 55, x 56, x 57, x 58, x 59, x 60$, $x 61, x 62, x 63, x 64, x 65, x 66, x 67, x 68, x 69, x 70, x 71, x 72, x 73, x 74, x 75, x 76, x 77, x 78, x 79, x 80, x 81$ $, x 82, x 83, x 84]$

$\mathrm{n}=[\mathrm{x} 97, \mathrm{y} 42, \mathrm{y} 43, \mathrm{y} 44, \mathrm{y} 45, \mathrm{y} 46, \mathrm{y} 47, \mathrm{y} 48, \mathrm{y} 49, \mathrm{y} 50, \mathrm{y} 51, \mathrm{y} 52, \mathrm{y} 53, \mathrm{y} 54, \mathrm{y} 55, \mathrm{y} 56, \mathrm{y} 57, \mathrm{y} 58, \mathrm{y} 59, \mathrm{y} 60$, $\mathrm{y} 61, y 62, y 63, y 64, y 65, y 66, y 67, y 68, y 69, y 70, y 71, y 72, y 73, y 74, y 75, y 76, y 77, y 78, y 79, y 80, y 81$ $, \mathrm{y} 82, \mathrm{y} 83, \mathrm{y} 84]$

end

$[A 2(j, 1), A 2(j, 2)]=$ curveintersect $(m(:, j), n(:, j), x 41, y 41) ;$

for $j=1: 57$

$m=[y 97, x 42, x 43, x 44, x 45, x 46, x 47, x 48, x 49, x 50, x 51, x 52, x 53, x 54, x 55, x 56, x 57, x 58, x 59, x 60$, $x 61, x 62, x 63, x 64, x 65, x 66, x 67, x 68, x 69, x 70, x 71, x 72, x 73, x 74, x 75, x 76, x 77, x 78, x 79, x 80, x 81$ $, \mathrm{x} 82, \mathrm{x} 83, \mathrm{x} 84, \mathrm{x} 85, \mathrm{x} 86, \mathrm{x} 87, \mathrm{x} 88, \mathrm{x} 89, \mathrm{x} 90, \mathrm{x} 91, \mathrm{x} 92, \mathrm{x} 93, \mathrm{x} 94, \mathrm{x} 95, \mathrm{x} 96, \mathrm{y}]$;

$\mathrm{n}=[\mathrm{x} 97, \mathrm{y} 42, \mathrm{y} 43, \mathrm{y} 44, \mathrm{y} 45, \mathrm{y} 46, \mathrm{y} 47, \mathrm{y} 48, \mathrm{y} 49, \mathrm{y} 50, \mathrm{y} 51, \mathrm{y} 52, \mathrm{y} 53, \mathrm{y} 54, \mathrm{y} 55, \mathrm{y} 56, \mathrm{y} 57, \mathrm{y} 58, \mathrm{y} 59, \mathrm{y} 60$, $\mathrm{y} 61, \mathrm{y} 62, \mathrm{y} 63, \mathrm{y} 64, \mathrm{y} 65, \mathrm{y} 66, \mathrm{y} 67, \mathrm{y} 68, \mathrm{y} 69, \mathrm{y} 70, \mathrm{y} 71, \mathrm{y} 72, \mathrm{y} 73, \mathrm{y} 74, \mathrm{y} 75, \mathrm{y} 76, \mathrm{y} 77, \mathrm{y} 78, \mathrm{y} 79, \mathrm{y} 80, \mathrm{y} 81$ $, \mathrm{y} 82, \mathrm{y} 83, \mathrm{y} 84, \mathrm{y} 85, \mathrm{y} 86, \mathrm{y} 87, \mathrm{y} 88, \mathrm{y} 89, \mathrm{y} 90, \mathrm{y} 91, \mathrm{y} 92, \mathrm{y} 93, \mathrm{y} 94, \mathrm{y} 95, \mathrm{y} 96, \mathrm{x}] ;$

end

$[A 3(j, 1), A 3(j, 2)]=$ curveintersect $(m(:, j), n(:, j), x 40, y 40)$;

for $j=1: 57$

$\mathrm{m}=[\mathrm{y} 97, \mathrm{x} 42, \mathrm{x} 43, \mathrm{x} 44, \mathrm{x} 45, \mathrm{x} 46, \mathrm{x} 47, \mathrm{x} 48, \mathrm{x} 49, \mathrm{x} 50, \mathrm{x} 51, \mathrm{x} 52, \mathrm{x} 53, \mathrm{x} 54, \mathrm{x} 55, \mathrm{x} 56, \mathrm{x} 57, \mathrm{x} 58, \mathrm{x} 59, \mathrm{x} 60$, $x 61, x 62, x 63, x 64, x 65, x 66, x 67, x 68, x 69, x 70, x 71, x 72, x 73, x 74, x 75, x 76, x 77, x 78, x 79, x 80, x 81$ $, x 82, x 83, x 84, x 85, x 86, x 87, x 88, x 89, x 90, x 91, x 92, x 93, x 94, x 95, x 96, y] ;$

$n=[x 97, y 42, y 43, y 44, y 45, y 46, y 47, y 48, y 49, y 50, y 51, y 52, y 53, y 54, y 55, y 56, y 57, y 58, y 59, y 60$, $\mathrm{y} 61, \mathrm{y} 62, \mathrm{y} 63, \mathrm{y} 64, \mathrm{y} 65, \mathrm{y} 66, \mathrm{y} 67, \mathrm{y} 68, \mathrm{y} 69, \mathrm{y} 70, \mathrm{y} 71, \mathrm{y} 72, \mathrm{y} 73, \mathrm{y} 74, \mathrm{y} 75, \mathrm{y} 76, \mathrm{y} 77, \mathrm{y} 78, \mathrm{y} 79, \mathrm{y} 80, \mathrm{y} 81$ , y82,y83,y84,y85,y86,y87,y88,y89,y90,y91,y92,y93,y94,y95,y96, x] ;

end

$[A 4(j, 1), A 4(j, 2)]=$ curveintersect $(m(:, j), n(:, j), x 39, y 39)$;

for $j=1: 57$

$\mathrm{m}=[\mathrm{y} 97, \mathrm{x} 42, \mathrm{x} 43, \mathrm{x} 44, \mathrm{x} 45, \mathrm{x} 46, \mathrm{x} 47, \mathrm{x} 48, \mathrm{x} 49, \mathrm{x} 50, \mathrm{x} 51, \mathrm{x} 52, \mathrm{x} 53, \mathrm{x} 54, \mathrm{x} 55, \mathrm{x} 56, \mathrm{x} 57, \mathrm{x} 58, \mathrm{x} 59, \mathrm{x} 60$, $x 61, x 62, x 63, x 64, x 65, x 66, x 67, x 68, x 69, x 70, x 71, x 72, x 73, x 74, x 75, x 76, x 77, x 78, x 79, x 80, x 81$ $, x 82, x 83, x 84, x 85, x 86, x 87, x 88, x 89, x 90, x 91, x 92, x 93, x 94, x 95, x 96, y] ;$

$\mathrm{n}=[\mathrm{x} 97, \mathrm{y} 42, \mathrm{y} 43, \mathrm{y} 44, \mathrm{y} 45, \mathrm{y} 46, \mathrm{y} 47, \mathrm{y} 48, \mathrm{y} 49, \mathrm{y} 50, \mathrm{y} 51, \mathrm{y} 52, \mathrm{y} 53, \mathrm{y} 54, \mathrm{y} 55, \mathrm{y} 56, \mathrm{y} 57, \mathrm{y} 58, \mathrm{y} 59, \mathrm{y} 60$, $\mathrm{y} 61, y 62, y 63, y 64, y 65, y 66, y 67, y 68, y 69, y 70, y 71, y 72, y 73, y 74, y 75, y 76, y 77, y 78, y 79, y 80, y 81$ $, \mathrm{y} 82, \mathrm{y} 83, \mathrm{y} 84, \mathrm{y} 85, \mathrm{y} 86, \mathrm{y} 87, \mathrm{y} 88, \mathrm{y} 89, \mathrm{y} 90, \mathrm{y} 91, \mathrm{y} 92, \mathrm{y} 93, \mathrm{y} 94, \mathrm{y} 95, \mathrm{y} 96, \mathrm{x}] ;$

end

$[A 5(j, 1), A 5(j, 2)]=$ curveintersect $(m(:, j), n(:, j), x 38, y 38) ;$

for $j=1: 57$

$\mathrm{m}=[\mathrm{y} 97, \mathrm{x} 42, \mathrm{x} 43, \mathrm{x} 44, \mathrm{x} 45, \mathrm{x} 46, \mathrm{x} 47, \mathrm{x} 48, \mathrm{x} 49, \mathrm{x} 50, \mathrm{x} 51, \mathrm{x} 52, \mathrm{x} 53, \mathrm{x} 54, \mathrm{x} 55, \mathrm{x} 56, \mathrm{x} 57, \mathrm{x} 58, \mathrm{x} 59, \mathrm{x} 60$, $x 61, x 62, x 63, x 64, x 65, x 66, x 67, x 68, x 69, x 70, x 71, x 72, x 73, x 74, x 75, x 76, x 77, x 78, x 79, x 80, x 81$ $, x 82, x 83, x 84, x 85, x 86, x 87, x 88, x 89, x 90, x 91, x 92, x 93, x 94, x 95, x 96, y]$;

$n=[x 97, y 42, y 43, y 44, y 45, y 46, y 47, y 48, y 49, y 50, y 51, y 52, y 53, y 54, y 55, y 56, y 57, y 58, y 59, y 60$, $y 61, y 62, y 63, y 64, y 65, y 66, y 67, y 68, y 69, y 70, y 71, y 72, y 73, y 74, y 75, y 76, y 77, y 78, y 79, y 80, y 81$ $, \mathrm{y} 82, \mathrm{y} 83, \mathrm{y} 84, \mathrm{y} 85, \mathrm{y} 86, \mathrm{y} 87, \mathrm{y} 88, \mathrm{y} 89, \mathrm{y} 90, \mathrm{y} 91, \mathrm{y} 92, \mathrm{y} 93, \mathrm{y} 94, \mathrm{y} 95, \mathrm{y} 96, \mathrm{x}] ;$

end

$[A 6(j, 1), A 6(j, 2)]=$ curveintersect $(m(:, j), n(:, j), x 37, y 37)$;

for $j=1: 57$

$m=[y 97, x 42, x 43, x 44, x 45, x 46, x 47, x 48, x 49, x 50, x 51, x 52, x 53, x 54, x 55, x 56, x 57, x 58, x 59, x 60$, $x 61, x 62, x 63, x 64, x 65, x 66, x 67, x 68, x 69, x 70, x 71, x 72, x 73, x 74, x 75, x 76, x 77, x 78, x 79, x 80, x 81$ $, x 82, x 83, x 84, x 85, x 86, x 87, x 88, x 89, x 90, x 91, x 92, x 93, x 94, x 95, x 96, y]$

$\mathrm{n}=[\mathrm{x} 97, \mathrm{y} 42, \mathrm{y} 43, \mathrm{y} 44, \mathrm{y} 45, \mathrm{y} 46, \mathrm{y} 47, \mathrm{y} 48, \mathrm{y} 49, \mathrm{y} 50, \mathrm{y} 51, \mathrm{y} 52, \mathrm{y} 53, \mathrm{y} 54, \mathrm{y} 55, \mathrm{y} 56, \mathrm{y} 57, \mathrm{y} 58, \mathrm{y} 59, \mathrm{y} 60$, $\mathrm{y} 61, y 62, y 63, y 64, y 65, y 66, y 67, y 68, y 69, y 70, y 71, y 72, y 73, y 74, y 75, y 76, y 77, y 78, y 79, y 80, y 81$ $, \mathrm{y} 82, \mathrm{y} 83, \mathrm{y} 84, \mathrm{y} 85, \mathrm{y} 86, \mathrm{y} 87, \mathrm{y} 88, \mathrm{y} 89, \mathrm{y} 90, \mathrm{y} 91, \mathrm{y} 92, \mathrm{y} 93, \mathrm{y} 94, \mathrm{y} 95, \mathrm{y} 96, \mathrm{x}] ;$

end

$[A 7(j, 1), A 7(j, 2)]=$ curveintersect $(m(:, j), n(:, j), x 36, y 36)$;

for $j=1: 57$

$m=[y 97, x 42, x 43, x 44, x 45, x 46, x 47, x 48, x 49, x 50, x 51, x 52, x 53, x 54, x 55, x 56, x 57, x 58, x 59, x 60$, $x 61, x 62, x 63, x 64, x 65, x 66, x 67, x 68, x 69, x 70, x 71, x 72, x 73, x 74, x 75, x 76, x 77, x 78, x 79, x 80, x 81$ $, x 82, x 83, x 84, x 85, x 86, x 87, x 88, x 89, x 90, x 91, x 92, x 93, x 94, x 95, x 96, y] ;$

$n=[x 97, y 42, y 43, y 44, y 45, y 46, y 47, y 48, y 49, y 50, y 51, y 52, y 53, y 54, y 55, y 56, y 57, y 58, y 59, y 60$, $y 61, y 62, y 63, y 64, y 65, y 66, y 67, y 68, y 69, y 70, y 71, y 72, y 73, y 74, y 75, y 76, y 77, y 78, y 79, y 80, y 81$ $, \mathrm{y} 82, \mathrm{y} 83, \mathrm{y} 84, \mathrm{y} 85, \mathrm{y} 86, \mathrm{y} 87, \mathrm{y} 88, \mathrm{y} 89, \mathrm{y} 90, \mathrm{y} 91, \mathrm{y} 92, \mathrm{y} 93, \mathrm{y} 94, \mathrm{y} 95, \mathrm{y} 96, \mathrm{x}] ;$

end

$[A 8(j, 1), A 8(j, 2)]=$ curveintersect $(m(:, j), n(:, j), x 35, y 35)$;

for $j=1: 57$ 
$m=[y 97, x 42, x 43, x 44, x 45, x 46, x 47, x 48, x 49, x 50, x 51, x 52, x 53, x 54, x 55, x 56, x 57, x 58, x 59, x 60$, $x 61, x 62, x 63, x 64, x 65, x 66, x 67, x 68, x 69, x 70, x 71, x 72, x 73, x 74, x 75, x 76, x 77, x 78, x 79, x 80, x 81$ $, x 82, x 83, x 84, x 85, x 86, x 87, x 88, x 89, x 90, x 91, x 92, x 93, x 94, x 95, x 96, y]$;

$\mathrm{n}=[\mathrm{x} 97, \mathrm{y} 42, \mathrm{y} 43, \mathrm{y} 44, \mathrm{y} 45, \mathrm{y} 46, \mathrm{y} 47, \mathrm{y} 48, \mathrm{y} 49, \mathrm{y} 50, \mathrm{y} 51, \mathrm{y} 52, \mathrm{y} 53, \mathrm{y} 54, \mathrm{y} 55, \mathrm{y} 56, \mathrm{y} 57, \mathrm{y} 58, \mathrm{y} 59, \mathrm{y} 60$, $\mathrm{y} 61, \mathrm{y} 62, \mathrm{y} 63, \mathrm{y} 64, \mathrm{y} 65, \mathrm{y} 66, \mathrm{y} 67, \mathrm{y} 68, \mathrm{y} 69, \mathrm{y} 70, \mathrm{y} 71, \mathrm{y} 72, \mathrm{y} 73, \mathrm{y} 74, \mathrm{y} 75, \mathrm{y} 76, \mathrm{y} 77, \mathrm{y} 78, \mathrm{y} 79, \mathrm{y} 80, \mathrm{y} 81$ $, \mathrm{y} 82, \mathrm{y} 83, \mathrm{y} 84, \mathrm{y} 85, \mathrm{y} 86, \mathrm{y} 87, \mathrm{y} 88, \mathrm{y} 89, \mathrm{y} 90, \mathrm{y} 91, \mathrm{y} 92, \mathrm{y} 93, \mathrm{y} 94, \mathrm{y} 95, \mathrm{y} 96, \mathrm{x}] ;$

end $[A 9(j, 1), A 9(j, 2)]=$ curveintersect $(m(:, j), n(:, j), x 34, y 34)$;

for $j=1: 57$

$m=[y 97, x 42, x 43, x 44, x 45, x 46, x 47, x 48, x 49, x 50, x 51, x 52, x 53, x 54, x 55, x 56, x 57, x 58, x 59, x 60$, $x 61, x 62, x 63, x 64, x 65, x 66, x 67, x 68, x 69, x 70, x 71, x 72, x 73, x 74, x 75, x 76, x 77, x 78, x 79, x 80, x 81$ $, x 82, x 83, x 84, x 85, x 86, x 87, x 88, x 89, x 90, x 91, x 92, x 93, x 94, x 95, x 96, y]$;

$\mathrm{n}=[\mathrm{x} 97, \mathrm{y} 42, \mathrm{y} 43, \mathrm{y} 44, \mathrm{y} 45, \mathrm{y} 46, \mathrm{y} 47, \mathrm{y} 48, \mathrm{y} 49, \mathrm{y} 50, \mathrm{y} 51, \mathrm{y} 52, \mathrm{y} 53, \mathrm{y} 54, \mathrm{y} 55, \mathrm{y} 56, \mathrm{y} 57, \mathrm{y} 58, \mathrm{y} 59, \mathrm{y} 60$, $\mathrm{y} 61, \mathrm{y} 62, \mathrm{y} 63, \mathrm{y} 64, \mathrm{y} 65, \mathrm{y} 66, \mathrm{y} 67, \mathrm{y} 68, \mathrm{y} 69, \mathrm{y} 70, \mathrm{y} 71, \mathrm{y} 72, \mathrm{y} 73, \mathrm{y} 74, \mathrm{y} 75, \mathrm{y} 76, \mathrm{y} 77, \mathrm{y} 78, \mathrm{y} 79, \mathrm{y} 80, \mathrm{y} 81$ $, \mathrm{y} 82, \mathrm{y} 83, \mathrm{y} 84, \mathrm{y} 85, \mathrm{y} 86, \mathrm{y} 87, \mathrm{y} 88, \mathrm{y} 89, \mathrm{y} 90, \mathrm{y} 91, \mathrm{y} 92, \mathrm{y} 93, \mathrm{y} 94, \mathrm{y} 95, \mathrm{y} 96, \mathrm{x}] ;$

end

$[A 10(j, 1), A 10(j, 2)]=$ curveintersect $(m(:, j), n(:, j), x 33, y 33)$;

for $j=1: 57$

$m=[y 97, x 42, x 43, x 44, x 45, x 46, x 47, x 48, x 49, x 50, x 51, x 52, x 53, x 54, x 55, x 56, x 57, x 58, x 59, x 60$, $x 61, x 62, x 63, x 64, x 65, x 66, x 67, x 68, x 69, x 70, x 71, x 72, x 73, x 74, x 75, x 76, x 77, x 78, x 79, x 80, x 81$ $, x 82, x 83, x 84, x 85, x 86, x 87, x 88, x 89, x 90, x 91, x 92, x 93, x 94, x 95, x 96, y]$;

$\mathrm{n}=[\mathrm{x} 97, \mathrm{y} 42, \mathrm{y} 43, \mathrm{y} 44, \mathrm{y} 45, \mathrm{y} 46, \mathrm{y} 47, \mathrm{y} 48, \mathrm{y} 49, \mathrm{y} 50, \mathrm{y} 51, \mathrm{y} 52, \mathrm{y} 53, \mathrm{y} 54, \mathrm{y} 55, \mathrm{y} 56, \mathrm{y} 57, \mathrm{y} 58, \mathrm{y} 59, \mathrm{y} 60$, $\mathrm{y} 61, \mathrm{y} 62, \mathrm{y} 63, \mathrm{y} 64, \mathrm{y} 65, \mathrm{y} 66, \mathrm{y} 67, \mathrm{y} 68, \mathrm{y} 69, \mathrm{y} 70, \mathrm{y} 71, \mathrm{y} 72, \mathrm{y} 73, \mathrm{y} 74, \mathrm{y} 75, \mathrm{y} 76, \mathrm{y} 77, \mathrm{y} 78, \mathrm{y} 79, \mathrm{y} 80, \mathrm{y} 81$ $, \mathrm{y} 82, \mathrm{y} 83, \mathrm{y} 84, \mathrm{y} 85, \mathrm{y} 86, \mathrm{y} 87, \mathrm{y} 88, \mathrm{y} 89, \mathrm{y} 90, \mathrm{y} 91, \mathrm{y} 92, \mathrm{y} 93, \mathrm{y} 94, \mathrm{y} 95, \mathrm{y} 96, \mathrm{x}] ;$

end

$[\mathrm{A} 11(j, 1), \mathrm{A} 11(j, 2)]=$ curveintersect $(\mathrm{m}(:, j), \mathrm{n}(:, j), \mathrm{x} 32, \mathrm{y} 32)$;

for $j=1: 57$

$m=[y 97, x 42, x 43, x 44, x 45, x 46, x 47, x 48, x 49, x 50, x 51, x 52, x 53, x 54, x 55, x 56, x 57, x 58, x 59, x 60$, $x 61, x 62, x 63, x 64, x 65, x 66, x 67, x 68, x 69, x 70, x 71, x 72, x 73, x 74, x 75, x 76, x 77, x 78, x 79, x 80, x 81$ $, x 82, x 83, x 84, x 85, x 86, x 87, x 88, x 89, x 90, x 91, x 92, x 93, x 94, x 95, x 96, y]$;

$\mathrm{n}=[\mathrm{x} 97, \mathrm{y} 42, \mathrm{y} 43, \mathrm{y} 44, \mathrm{y} 45, \mathrm{y} 46, \mathrm{y} 47, \mathrm{y} 48, \mathrm{y} 49, \mathrm{y} 50, \mathrm{y} 51, \mathrm{y} 52, \mathrm{y} 53, \mathrm{y} 54, \mathrm{y} 55, \mathrm{y} 56, \mathrm{y} 57, \mathrm{y} 58, \mathrm{y} 59, \mathrm{y} 60$, $\mathrm{y} 61, \mathrm{y} 62, \mathrm{y} 63, \mathrm{y} 64, \mathrm{y} 65, \mathrm{y} 66, \mathrm{y} 67, \mathrm{y} 68, \mathrm{y} 69, \mathrm{y} 70, \mathrm{y} 71, \mathrm{y} 72, \mathrm{y} 73, \mathrm{y} 74, \mathrm{y} 75, \mathrm{y} 76, \mathrm{y} 77, \mathrm{y} 78, \mathrm{y} 79, \mathrm{y} 80, \mathrm{y} 81$ , $\mathrm{y} 82, \mathrm{y} 83, \mathrm{y} 84, \mathrm{y} 85, \mathrm{y} 86, \mathrm{y} 87, \mathrm{y} 88, \mathrm{y} 89, \mathrm{y} 90, \mathrm{y} 91, \mathrm{y} 92, \mathrm{y} 93, \mathrm{y} 94, \mathrm{y} 95, \mathrm{y} 96, \mathrm{x}]$;

end

$[A 12(j, 1), A 12(j, 2)]=$ curveintersect $(m(:, j), n(:, j), x 31, y 31)$;

for $j=1: 57$

$m=[y 97, x 42, x 43, x 44, x 45, x 46, x 47, x 48, x 49, x 50, x 51, x 52, x 53, x 54, x 55, x 56, x 57, x 58, x 59, x 60$, $x 61, x 62, x 63, x 64, x 65, x 66, x 67, x 68, x 69, x 70, x 71, x 72, x 73, x 74, x 75, x 76, x 77, x 78, x 79, x 80, x 81$ $, x 82, x 83, x 84, x 85, x 86, x 87, x 88, x 89, x 90, x 91, x 92, x 93, x 94, x 95, x 96, y]$;

$\mathrm{n}=[\mathrm{x} 97, \mathrm{y} 42, \mathrm{y} 43, \mathrm{y} 44, \mathrm{y} 45, \mathrm{y} 46, \mathrm{y} 47, \mathrm{y} 48, \mathrm{y} 49, \mathrm{y} 50, \mathrm{y} 51, \mathrm{y} 52, \mathrm{y} 53, \mathrm{y} 54, \mathrm{y} 55, \mathrm{y} 56, \mathrm{y} 57, \mathrm{y} 58, \mathrm{y} 59, \mathrm{y} 60$, $\mathrm{y} 61, \mathrm{y} 62, \mathrm{y} 63, \mathrm{y} 64, \mathrm{y} 65, \mathrm{y} 66, \mathrm{y} 67, \mathrm{y} 68, \mathrm{y} 69, \mathrm{y} 70, \mathrm{y} 71, \mathrm{y} 72, \mathrm{y} 73, \mathrm{y} 74, \mathrm{y} 75, \mathrm{y} 76, \mathrm{y} 77, \mathrm{y} 78, \mathrm{y} 79, \mathrm{y} 80, \mathrm{y} 81$ , y82, y83,y84, y85, y86,y87,y88,y89,y90,y91,y92,y93,y94,y95,y96, x ;

end

$[A 13(j, 1), A 13(j, 2)]=$ curveintersect $(m(:, j), n(:, j), x 30, y 30)$;

for $j=1: 57$

$\mathrm{m}=[\mathrm{y} 97, \mathrm{x} 42, \mathrm{x} 43, \mathrm{x} 44, \mathrm{x} 45, \mathrm{x} 46, \mathrm{x} 47, \mathrm{x} 48, \mathrm{x} 49, \mathrm{x} 50, \mathrm{x} 51, \mathrm{x} 52, \mathrm{x} 53, \mathrm{x} 54, \mathrm{x} 55, \mathrm{x} 56, \mathrm{x} 57, \mathrm{x} 58, \mathrm{x} 59, \mathrm{x} 60$, $x 61, x 62, x 63, x 64, x 65, x 66, x 67, x 68, x 69, x 70, x 71, x 72, x 73, x 74, x 75, x 76, x 77, x 78, x 79, x 80, x 81$ $, x 82, x 83, x 84, x 85, x 86, x 87, x 88, x 89, x 90, x 91, x 92, x 93, x 94, x 95, x 96, y]$;

$\mathrm{n}=[\mathrm{x} 97, \mathrm{y} 42, \mathrm{y} 43, \mathrm{y} 44, \mathrm{y} 45, \mathrm{y} 46, \mathrm{y} 47, \mathrm{y} 48, \mathrm{y} 49, \mathrm{y} 50, \mathrm{y} 51, \mathrm{y} 52, \mathrm{y} 53, \mathrm{y} 54, \mathrm{y} 55, \mathrm{y} 56, \mathrm{y} 57, \mathrm{y} 58, \mathrm{y} 59, \mathrm{y} 60$, $\mathrm{y} 61, \mathrm{y} 62, \mathrm{y} 63, \mathrm{y} 64, \mathrm{y} 65, \mathrm{y} 66, \mathrm{y} 67, \mathrm{y} 68, \mathrm{y} 69, \mathrm{y} 70, \mathrm{y} 71, \mathrm{y} 72, \mathrm{y} 73, \mathrm{y} 74, \mathrm{y} 75, \mathrm{y} 76, \mathrm{y} 77, \mathrm{y} 78, \mathrm{y} 79, \mathrm{y} 80, \mathrm{y} 81$ , $\mathrm{y} 82, \mathrm{y} 83, \mathrm{y} 84, \mathrm{y} 85, \mathrm{y} 86, \mathrm{y} 87, \mathrm{y} 88, \mathrm{y} 89, \mathrm{y} 90, \mathrm{y} 91, \mathrm{y} 92, \mathrm{y} 93, \mathrm{y} 94, \mathrm{y} 95, \mathrm{y} 96, \mathrm{x}]$;

end $[A 14(j, 1), A 14(j, 2)]=$ curveintersect $(m(:, j), n(:, j), x 29, y 29)$;

for $j=1: 57$

$m=[y 97, x 42, x 43, x 44, x 45, x 46, x 47, x 48, x 49, x 50, x 51, x 52, x 53, x 54, x 55, x 56, x 57, x 58, x 59, x 60$, $x 61, x 62, x 63, x 64, x 65, x 66, x 67, x 68, x 69, x 70, x 71, x 72, x 73, x 74, x 75, x 76, x 77, x 78, x 79, x 80, x 81$ $, x 82, x 83, x 84, x 85, x 86, x 87, x 88, x 89, x 90, x 91, x 92, x 93, x 94, x 95, x 96, y] ;$

$\mathrm{n}=[\mathrm{x} 97, \mathrm{y} 42, \mathrm{y} 43, \mathrm{y} 44, \mathrm{y} 45, \mathrm{y} 46, \mathrm{y} 47, \mathrm{y} 48, \mathrm{y} 49, \mathrm{y} 50, \mathrm{y} 51, \mathrm{y} 52, \mathrm{y} 53, \mathrm{y} 54, \mathrm{y} 55, \mathrm{y} 56, \mathrm{y} 57, \mathrm{y} 58, \mathrm{y} 59, \mathrm{y} 60$, $y 61, y 62, y 63, y 64, y 65, y 66, y 67, y 68, y 69, y 70, y 71, y 72, y 73, y 74, y 75, y 76, y 77, y 78, y 79, y 80, y 81$ $, \mathrm{y} 82, \mathrm{y} 83, \mathrm{y} 84, \mathrm{y} 85, \mathrm{y} 86, \mathrm{y} 87, \mathrm{y} 88, \mathrm{y} 89, \mathrm{y} 90, \mathrm{y} 91, \mathrm{y} 92, \mathrm{y} 93, \mathrm{y} 94, \mathrm{y} 95, \mathrm{y} 96, \mathrm{x}] ;$

end $[\mathrm{A} 15(j, 1), \mathrm{A} 15(j, 2)]=$ curveintersect $(\mathrm{m}(:, j), \mathrm{n}(:, j), \mathrm{x} 28, \mathrm{y} 28)$;

for $j=1: 57$

$m=[y 97, x 42, x 43, x 44, x 45, x 46, x 47, x 48, x 49, x 50, x 51, x 52, x 53, x 54, x 55, x 56, x 57, x 58, x 59, x 60$, $x 61, x 62, x 63, x 64, x 65, x 66, x 67, x 68, x 69, x 70, x 71, x 72, x 73, x 74, x 75, x 76, x 77, x 78, x 79, x 80, x 81$ $, x 82, x 83, x 84, x 85, x 86, x 87, x 88, x 89, x 90, x 91, x 92, x 93, x 94, x 95, x 96, y] ;$

$\mathrm{n}=[\mathrm{x} 97, \mathrm{y} 42, \mathrm{y} 43, \mathrm{y} 44, \mathrm{y} 45, \mathrm{y} 46, \mathrm{y} 47, \mathrm{y} 48, \mathrm{y} 49, \mathrm{y} 50, \mathrm{y} 51, \mathrm{y} 52, \mathrm{y} 53, \mathrm{y} 54, \mathrm{y} 55, \mathrm{y} 56, \mathrm{y} 57, \mathrm{y} 58, \mathrm{y} 59, \mathrm{y} 60$, $\mathrm{y} 61, \mathrm{y} 62, \mathrm{y} 63, \mathrm{y} 64, \mathrm{y} 65, \mathrm{y} 66, \mathrm{y} 67, \mathrm{y} 68, \mathrm{y} 69, \mathrm{y} 70, \mathrm{y} 71, \mathrm{y} 72, \mathrm{y} 73, \mathrm{y} 74, \mathrm{y} 75, \mathrm{y} 76, \mathrm{y} 77, \mathrm{y} 78, \mathrm{y} 79, \mathrm{y} 80, \mathrm{y} 81$ , $\mathrm{y} 82, \mathrm{y} 83, \mathrm{y} 84, \mathrm{y} 85, \mathrm{y} 86, \mathrm{y} 87, \mathrm{y} 88, \mathrm{y} 89, \mathrm{y} 90, \mathrm{y} 91, \mathrm{y} 92, \mathrm{y} 93, \mathrm{y} 94, \mathrm{y} 95, \mathrm{y} 96, \mathrm{x}]$; end

$[A 16(j, 1), A 16(j, 2)]=$ curveintersect $(m(:, j), n(:, j), x 27, y 27)$; 
for $j=1: 56$

$\mathrm{m}=[\mathrm{y} 97, \mathrm{x} 43, \mathrm{x} 44, \mathrm{x} 45, \mathrm{x} 46, \mathrm{x} 47, \mathrm{x} 48, x 49, x 50, x 51, x 52, x 53, x 54, x 55, x 56, x 57, x 58, x 59, x 60, x 61$, $x 62, x 63, x 64, x 65, x 66, x 67, x 68, x 69, x 70, x 71, x 72, x 73, x 74, x 75, x 76, x 77, x 78, x 79, x 80, x 81, x 82$ $, \mathrm{x} 83, \mathrm{x} 84, \mathrm{x} 85, \mathrm{x} 86, \mathrm{x} 87, \mathrm{x} 88, \mathrm{x} 89, \mathrm{x} 90, \mathrm{x} 91, \mathrm{x} 92, \mathrm{x} 93, \mathrm{x} 94, \mathrm{x} 95, \mathrm{x} 96, \mathrm{y}]$;

$\mathrm{n}=[\mathrm{x} 97, \mathrm{y} 43, \mathrm{y} 44, \mathrm{y} 45, \mathrm{y} 46, \mathrm{y} 47, \mathrm{y} 48, \mathrm{y} 49, \mathrm{y} 50, \mathrm{y} 51, \mathrm{y} 52, \mathrm{y} 53, \mathrm{y} 54, \mathrm{y} 55, \mathrm{y} 56, \mathrm{y} 57, \mathrm{y} 58, \mathrm{y} 59, \mathrm{y} 60, \mathrm{y} 61$, $\mathrm{y} 62, \mathrm{y} 63, \mathrm{y} 64, \mathrm{y} 65, \mathrm{y} 66, \mathrm{y} 67, \mathrm{y} 68, \mathrm{y} 69, \mathrm{y} 70, \mathrm{y} 71, \mathrm{y} 72, \mathrm{y} 73, \mathrm{y} 74, \mathrm{y} 75, \mathrm{y} 76, \mathrm{y} 77, \mathrm{y} 78, \mathrm{y} 79, \mathrm{y} 80, \mathrm{y} 81, \mathrm{y} 82$ , y83,y84,y85,y86,y87,y88,y89,y90,y91,y92,y93,y94,y95,y96,x];

end

$[A 17(j, 1), A 17(j, 2)]=$ curveintersect $(m(:, j), n(:, j), x 26, y 26) ;$

for $j=1: 56$

$m=[y 97, x 43, x 44, x 45, x 46, x 47, x 48, x 49, x 50, x 51, x 52, x 53, x 54, x 55, x 56, x 57, x 58, x 59, x 60, x 61$, $x 62, x 63, x 64, x 65, x 66, x 67, x 68, x 69, x 70, x 71, x 72, x 73, x 74, x 75, x 76, x 77, x 78, x 79, x 80, x 81, x 82$ $, x 83, x 84, x 85, x 86, x 87, x 88, x 89, x 90, x 91, x 92, x 93, x 94, x 95, x 96, y]$

$n=[x 97, y 43, y 44, y 45, y 46, y 47, y 48, y 49, y 50, y 51, y 52, y 53, y 54, y 55, y 56, y 57, y 58, y 59, y 60, y 61$, $\mathrm{y} 62, \mathrm{y} 63, \mathrm{y} 64, \mathrm{y} 65, \mathrm{y} 66, \mathrm{y} 67, \mathrm{y} 68, \mathrm{y} 69, \mathrm{y} 70, \mathrm{y} 71, \mathrm{y} 72, \mathrm{y} 73, \mathrm{y} 74, \mathrm{y} 75, \mathrm{y} 76, \mathrm{y} 77, \mathrm{y} 78, \mathrm{y} 79, \mathrm{y} 80, \mathrm{y} 81, \mathrm{y} 82$ , y83,y84,y85,y86,y87,y88,y89,y90,y91,y92,y93,y94,y95,y96,x];

end

$[A 18(j, 1), A 18(j, 2)]=$ curveintersect $(m(:, j), n(:, j), x 25, y 25)$;

for $j=1: 56$

$m=[y 97, x 43, x 44, x 45, x 46, x 47, x 48, x 49, x 50, x 51, x 52, x 53, x 54, x 55, x 56, x 57, x 58, x 59, x 60, x 61$, $x 62, x 63, x 64, x 65, x 66, x 67, x 68, x 69, x 70, x 71, x 72, x 73, x 74, x 75, x 76, x 77, x 78, x 79, x 80, x 81, x 82$ $, x 83, x 84, x 85, x 86, x 87, x 88, x 89, x 90, x 91, x 92, x 93, x 94, x 95, x 96, y]$;

$\mathrm{n}=[\mathrm{x} 97, \mathrm{y} 43, \mathrm{y} 44, \mathrm{y} 45, \mathrm{y} 46, \mathrm{y} 47, \mathrm{y} 48, \mathrm{y} 49, \mathrm{y} 50, \mathrm{y} 51, \mathrm{y} 52, \mathrm{y} 53, \mathrm{y} 54, \mathrm{y} 55, \mathrm{y} 56, \mathrm{y} 57, \mathrm{y} 58, \mathrm{y} 59, \mathrm{y} 60, \mathrm{y} 61$, $\mathrm{y} 62, \mathrm{y} 63, \mathrm{y} 64, \mathrm{y} 65, \mathrm{y} 66, \mathrm{y} 67, \mathrm{y} 68, \mathrm{y} 69, \mathrm{y} 70, \mathrm{y} 71, \mathrm{y} 72, \mathrm{y} 73, \mathrm{y} 74, \mathrm{y} 75, \mathrm{y} 76, \mathrm{y} 77, \mathrm{y} 78, \mathrm{y} 79, \mathrm{y} 80, \mathrm{y} 81, \mathrm{y} 82$ , y83,y84,y85,y86,y87,y88,y89,y90,y91,y92,y93,y94,y95,y96,x];

end

$[\mathrm{A} 19(j, 1), \mathrm{A} 19(j, 2)]=$ curveintersect $(\mathrm{m}(:, j), \mathrm{n}(:, j), \mathrm{x} 24, \mathrm{y} 24) ;$

for $j=1: 56$

$\mathrm{m}=[\mathrm{y} 97, \mathrm{x} 43, \mathrm{x} 44, \mathrm{x} 45, \mathrm{x} 46, \mathrm{x} 47, \mathrm{x} 48, \mathrm{x} 49, \mathrm{x} 50, \mathrm{x} 51, \mathrm{x} 52, \mathrm{x} 53, \mathrm{x} 54, \mathrm{x} 55, \mathrm{x} 56, \mathrm{x} 57, \mathrm{x} 58, \mathrm{x} 59, \mathrm{x} 60, \mathrm{x} 61$, $x 62, x 63, x 64, x 65, x 66, x 67, x 68, x 69, x 70, x 71, x 72, x 73, x 74, x 75, x 76, x 77, x 78, x 79, x 80, x 81, x 82$ $, x 83, x 84, x 85, x 86, x 87, x 88, x 89, x 90, x 91, x 92, x 93, x 94, x 95, x 96, y] ;$

$\mathrm{n}=[\mathrm{x} 97, \mathrm{y} 43, \mathrm{y} 44, \mathrm{y} 45, \mathrm{y} 46, \mathrm{y} 47, \mathrm{y} 48, \mathrm{y} 49, \mathrm{y} 50, \mathrm{y} 51, \mathrm{y} 52, \mathrm{y} 53, \mathrm{y} 54, \mathrm{y} 55, \mathrm{y} 56, \mathrm{y} 57, \mathrm{y} 58, \mathrm{y} 59, \mathrm{y} 60, \mathrm{y} 61$, $\mathrm{y} 62, \mathrm{y} 63, \mathrm{y} 64, \mathrm{y} 65, \mathrm{y} 66, \mathrm{y} 67, \mathrm{y} 68, \mathrm{y} 69, \mathrm{y} 70, \mathrm{y} 71, \mathrm{y} 72, \mathrm{y} 73, \mathrm{y} 74, \mathrm{y} 75, \mathrm{y} 76, \mathrm{y} 77, \mathrm{y} 78, \mathrm{y} 79, \mathrm{y} 80, \mathrm{y} 81, \mathrm{y} 82$ $, \mathrm{y} 83, \mathrm{y} 84, \mathrm{y} 85, \mathrm{y} 86, \mathrm{y} 87, \mathrm{y} 88, \mathrm{y} 89, \mathrm{y} 90, \mathrm{y} 91, \mathrm{y} 92, \mathrm{y} 93, \mathrm{y} 94, \mathrm{y} 95, \mathrm{y} 96, \mathrm{x}]$;

end

$[\mathrm{A} 20(j, 1), \mathrm{A} 20(j, 2)]=$ curveintersect $(\mathrm{m}(:, j), \mathrm{n}(:, j), \mathrm{x} 23, \mathrm{y} 23)$;

for $j=1: 56$

$m=[y 97, x 43, x 44, x 45, x 46, x 47, x 48, x 49, x 50, x 51, x 52, x 53, x 54, x 55, x 56, x 57, x 58, x 59, x 60, x 61$, $x 62, x 63, x 64, x 65, x 66, x 67, x 68, x 69, x 70, x 71, x 72, x 73, x 74, x 75, x 76, x 77, x 78, x 79, x 80, x 81, x 82$ $, x 83, x 84, x 85, x 86, x 87, x 88, x 89, x 90, x 91, x 92, x 93, x 94, x 95, x 96, y] ;$

$\mathrm{n}=[\mathrm{x} 97, \mathrm{y} 43, \mathrm{y} 44, \mathrm{y} 45, \mathrm{y} 46, \mathrm{y} 47, \mathrm{y} 48, \mathrm{y} 49, \mathrm{y} 50, \mathrm{y} 51, \mathrm{y} 52, \mathrm{y} 53, \mathrm{y} 54, \mathrm{y} 55, \mathrm{y} 56, \mathrm{y} 57, \mathrm{y} 58, \mathrm{y} 59, \mathrm{y} 60, \mathrm{y} 61$, $\mathrm{y} 62, \mathrm{y} 63, \mathrm{y} 64, \mathrm{y} 65, \mathrm{y} 66, \mathrm{y} 67, \mathrm{y} 68, \mathrm{y} 69, \mathrm{y} 70, \mathrm{y} 71, \mathrm{y} 72, \mathrm{y} 73, \mathrm{y} 74, \mathrm{y} 75, \mathrm{y} 76, \mathrm{y} 77, \mathrm{y} 78, \mathrm{y} 79, \mathrm{y} 80, \mathrm{y} 81, \mathrm{y} 82$ , y83,y84,y85,y86,y87,y88,y89,y90,y91,y92,y93,y94,y95,y96, x];

$[A 21(j, 1), A 21(j, 2)]=$ curveintersect $(m(:, j), n(:, j), x 22, y 22) ;$

end

for $j=1: 56$

$\mathrm{m}=[\mathrm{y} 97, \mathrm{x} 43, \mathrm{x} 44, \mathrm{x} 45, \mathrm{x} 46, \mathrm{x} 47, \mathrm{x} 48, \mathrm{x} 49, \mathrm{x} 50, \mathrm{x} 51, \mathrm{x} 52, \mathrm{x} 53, \mathrm{x} 54, \mathrm{x} 55, \mathrm{x} 56, x 57, x 58, x 59, x 60, x 61$, $x 62, x 63, x 64, x 65, x 66, x 67, x 68, x 69, x 70, x 71, x 72, x 73, x 74, x 75, x 76, x 77, x 78, x 79, x 80, x 81, x 82$ $, x 83, x 84, x 85, x 86, x 87, x 88, x 89, x 90, x 91, x 92, x 93, x 94, x 95, x 96, y]$;

$\mathrm{n}=[\mathrm{x} 97, \mathrm{y} 43, \mathrm{y} 44, \mathrm{y} 45, \mathrm{y} 46, \mathrm{y} 47, \mathrm{y} 48, \mathrm{y} 49, \mathrm{y} 50, \mathrm{y} 51, \mathrm{y} 52, \mathrm{y} 53, \mathrm{y} 54, \mathrm{y} 55, \mathrm{y} 56, \mathrm{y} 57, \mathrm{y} 58, \mathrm{y} 59, \mathrm{y} 60, \mathrm{y} 61$, $\mathrm{y} 62, \mathrm{y} 63, \mathrm{y} 64, \mathrm{y} 65, \mathrm{y} 66, \mathrm{y} 67, \mathrm{y} 68, \mathrm{y} 69, \mathrm{y} 70, \mathrm{y} 71, \mathrm{y} 72, \mathrm{y} 73, \mathrm{y} 74, \mathrm{y} 75, \mathrm{y} 76, \mathrm{y} 77, \mathrm{y} 78, \mathrm{y} 79, \mathrm{y} 80, \mathrm{y} 81, \mathrm{y} 82$ , y83,y84,y85, y86,y87,y88,y89,y90,y91,y92,y93,y94,y95,y96,x];

end

$[\mathrm{A} 22(j, 1), \mathrm{A} 22(j, 2)]=$ curveintersect $(\mathrm{m}(:, j), \mathrm{n}(:, j), \mathrm{x} 21, \mathrm{y} 21) ;$

for $j=1: 56$

$\mathrm{m}=[\mathrm{y} 97, \mathrm{x} 43, \mathrm{x} 44, \mathrm{x} 45, \mathrm{x} 46, \mathrm{x} 47, \mathrm{x} 48, \mathrm{x} 49, \mathrm{x} 50, \mathrm{x} 51, \mathrm{x} 52, \mathrm{x} 53, \mathrm{x} 54, \mathrm{x} 55, \mathrm{x} 56, \mathrm{x} 57, \mathrm{x} 58, \mathrm{x} 59, \mathrm{x} 60, \mathrm{x} 61$, $x 62, x 63, x 64, x 65, x 66, x 67, x 68, x 69, x 70, x 71, x 72, x 73, x 74, x 75, x 76, x 77, x 78, x 79, x 80, x 81, x 82$ $, x 83, x 84, x 85, x 86, x 87, x 88, x 89, x 90, x 91, x 92, x 93, x 94, x 95, x 96, y] ;$

$\mathrm{n}=[\mathrm{x} 97, \mathrm{y} 43, \mathrm{y} 44, \mathrm{y} 45, \mathrm{y} 46, \mathrm{y} 47, \mathrm{y} 48, \mathrm{y} 49, \mathrm{y} 50, \mathrm{y} 51, \mathrm{y} 52, \mathrm{y} 53, \mathrm{y} 54, \mathrm{y} 55, \mathrm{y} 56, \mathrm{y} 57, \mathrm{y} 58, \mathrm{y} 59, \mathrm{y} 60, \mathrm{y} 61$, $\mathrm{y} 62, \mathrm{y} 63, \mathrm{y} 64, \mathrm{y} 65, \mathrm{y} 66, \mathrm{y} 67, \mathrm{y} 68, \mathrm{y} 69, \mathrm{y} 70, \mathrm{y} 71, \mathrm{y} 72, \mathrm{y} 73, \mathrm{y} 74, \mathrm{y} 75, \mathrm{y} 76, \mathrm{y} 77, \mathrm{y} 78, \mathrm{y} 79, \mathrm{y} 80, \mathrm{y} 81, \mathrm{y} 82$ , y83,y84,y85,y86,y87,y88,y89,y90,y91,y92,y93,y94,y95,y96,x]; end $[\mathrm{A} 23(j, 1), \mathrm{A} 23(j, 2)]=$ curveintersect $(\mathrm{m}(:, j), \mathrm{n}(:, j), \mathrm{x} 20, \mathrm{y} 20)$;

for $j=1: 56$

$\mathrm{m}=[\mathrm{y} 97, \mathrm{x} 43, \mathrm{x} 44, \mathrm{x} 45, \mathrm{x} 46, \mathrm{x} 47, \mathrm{x} 48, \mathrm{x} 49, \mathrm{x} 50, \mathrm{x} 51, \mathrm{x} 52, \mathrm{x} 53, \mathrm{x} 54, \mathrm{x} 55, \mathrm{x} 56, \mathrm{x} 57, \mathrm{x} 58, \mathrm{x} 59, \mathrm{x} 60, \mathrm{x} 61$, $x 62, x 63, x 64, x 65, x 66, x 67, x 68, x 69, x 70, x 71, x 72, x 73, x 74, x 75, x 76, x 77, x 78, x 79, x 80, x 81, x 82$ $, \mathrm{x} 83, \mathrm{x} 84, \mathrm{x} 85, \mathrm{x} 86, \mathrm{x} 87, \mathrm{x} 88, \mathrm{x} 89, \mathrm{x} 90, \mathrm{x} 91, \mathrm{x} 92, \mathrm{x} 93, \mathrm{x} 94, \mathrm{x} 95, \mathrm{x} 96, \mathrm{y}]$

$\mathrm{n}=[\mathrm{x} 97, \mathrm{y} 43, \mathrm{y} 44, \mathrm{y} 45, \mathrm{y} 46, \mathrm{y} 47, \mathrm{y} 48, \mathrm{y} 49, \mathrm{y} 50, \mathrm{y} 51, \mathrm{y} 52, \mathrm{y} 53, \mathrm{y} 54, \mathrm{y} 55, \mathrm{y} 56, \mathrm{y} 57, \mathrm{y} 58, \mathrm{y} 59, \mathrm{y} 60, \mathrm{y} 61$, $\mathrm{y} 62, \mathrm{y} 63, \mathrm{y} 64, \mathrm{y} 65, \mathrm{y} 66, \mathrm{y} 67, \mathrm{y} 68, \mathrm{y} 69, \mathrm{y} 70, \mathrm{y} 71, \mathrm{y} 72, \mathrm{y} 73, \mathrm{y} 74, \mathrm{y} 75, \mathrm{y} 76, \mathrm{y} 77, \mathrm{y} 78, \mathrm{y} 79, \mathrm{y} 80, \mathrm{y} 81, \mathrm{y} 82$ , y83,y84,y85,y86,y87,y88,y89,y90,y91,y92,y93,y94,y95,y96,x];

$[A 24(j, 1), A 24(j, 2)]=$ curveintersect $(m(:, j), n(:, j), x 19, y 19) ;$ 
end

for $j=1: 56$

$m=[y 97, x 43, x 44, x 45, x 46, x 47, x 48, x 49, x 50, x 51, x 52, x 53, x 54, x 55, x 56, x 57, x 58, x 59, x 60, x 61$, $x 62, x 63, x 64, x 65, x 66, x 67, x 68, x 69, x 70, x 71, x 72, x 73, x 74, x 75, x 76, x 77, x 78, x 79, x 80, x 81, x 82$ $, x 83, x 84, x 85, x 86, x 87, x 88, x 89, x 90, x 91, x 92, x 93, x 94, x 95, x 96, y]$;

$\mathrm{n}=[\mathrm{x} 97, \mathrm{y} 43, \mathrm{y} 44, \mathrm{y} 45, \mathrm{y} 46, \mathrm{y} 47, \mathrm{y} 48, \mathrm{y} 49, \mathrm{y} 50, \mathrm{y} 51, \mathrm{y} 52, \mathrm{y} 53, \mathrm{y} 54, \mathrm{y} 55, \mathrm{y} 56, \mathrm{y} 57, \mathrm{y} 58, \mathrm{y} 59, \mathrm{y} 60, \mathrm{y} 61$, $\mathrm{y} 62, \mathrm{y} 63, \mathrm{y} 64, \mathrm{y} 65, \mathrm{y} 66, \mathrm{y} 67, \mathrm{y} 68, \mathrm{y} 69, \mathrm{y} 70, \mathrm{y} 71, \mathrm{y} 72, \mathrm{y} 73, \mathrm{y} 74, \mathrm{y} 75, \mathrm{y} 76, \mathrm{y} 77, \mathrm{y} 78, \mathrm{y} 79, \mathrm{y} 80, \mathrm{y} 81, \mathrm{y} 82$ $, \mathrm{y} 83, \mathrm{y} 84, \mathrm{y} 85, \mathrm{y} 86, \mathrm{y} 87, \mathrm{y} 88, \mathrm{y} 89, \mathrm{y} 90, \mathrm{y} 91, \mathrm{y} 92, \mathrm{y} 93, \mathrm{y} 94, \mathrm{y} 95, \mathrm{y} 96, \mathrm{x}] ;$

end $[A 25(j, 1), A 25(j, 2)]=$ curveintersect $(m(:, j), n(:, j), x 18, y 18)$;

for $j=1: 56$

$\mathrm{m}=[\mathrm{y} 97, \mathrm{x} 43, \mathrm{x} 44, \mathrm{x} 45, \mathrm{x} 46, \mathrm{x} 47, \mathrm{x} 48, \mathrm{x} 49, \mathrm{x} 50, \mathrm{x} 51, x 52, x 53, x 54, x 55, x 56, x 57, x 58, x 59, x 60, x 61$, $x 62, x 63, x 64, x 65, x 66, x 67, x 68, x 69, x 70, x 71, x 72, x 73, x 74, x 75, x 76, x 77, x 78, x 79, x 80, x 81, x 82$ $, x 83, x 84, x 85, x 86, x 87, x 88, x 89, x 90, x 91, x 92, x 93, x 94, x 95, x 96, y]$;

$\mathrm{n}=[\mathrm{x} 97, \mathrm{y} 43, \mathrm{y} 44, \mathrm{y} 45, \mathrm{y} 46, \mathrm{y} 47, \mathrm{y} 48, \mathrm{y} 49, \mathrm{y} 50, \mathrm{y} 51, \mathrm{y} 52, \mathrm{y} 53, \mathrm{y} 54, \mathrm{y} 55, \mathrm{y} 56, \mathrm{y} 57, \mathrm{y} 58, \mathrm{y} 59, \mathrm{y} 60, \mathrm{y} 61$, $y 62, y 63, y 64, y 65, y 66, y 67, y 68, y 69, y 70, y 71, y 72, y 73, y 74, y 75, y 76, y 77, y 78, y 79, y 80, y 81, y 82$ $, \mathrm{y} 83, \mathrm{y} 84, \mathrm{y} 85, \mathrm{y} 86, \mathrm{y} 87, \mathrm{y} 88, \mathrm{y} 89, \mathrm{y} 90, \mathrm{y} 91, \mathrm{y} 92, \mathrm{y} 93, \mathrm{y} 94, \mathrm{y} 95, \mathrm{y} 96, \mathrm{x}] ;$ end $[A 26(j, 1), A 26(j, 2)]=$ curveintersect $(m(:, j), n(:, j), x 17, y 17)$;

for $j=1: 56$

$\mathrm{m}=[\mathrm{y} 97, \mathrm{x} 43, \mathrm{x} 44, \mathrm{x} 45, \mathrm{x} 46, \mathrm{x} 47, \mathrm{x} 48, \mathrm{x} 49, \mathrm{x} 50, \mathrm{x} 51, x 52, x 53, x 54, x 55, x 56, x 57, x 58, x 59, x 60, x 61$, $x 62, x 63, x 64, x 65, x 66, x 67, x 68, x 69, x 70, x 71, x 72, x 73, x 74, x 75, x 76, x 77, x 78, x 79, x 80, x 81, x 82$ $, x 83, x 84, x 85, x 86, x 87, x 88, x 89, x 90, x 91, x 92, x 93, x 94, x 95, x 96, y] ;$

$\mathrm{n}=[\mathrm{x} 97, \mathrm{y} 43, \mathrm{y} 44, \mathrm{y} 45, \mathrm{y} 46, \mathrm{y} 47, \mathrm{y} 48, \mathrm{y} 49, \mathrm{y} 50, \mathrm{y} 51, \mathrm{y} 52, \mathrm{y} 53, \mathrm{y} 54, \mathrm{y} 55, \mathrm{y} 56, \mathrm{y} 57, \mathrm{y} 58, \mathrm{y} 59, \mathrm{y} 60, \mathrm{y} 61$, $\mathrm{y} 62, \mathrm{y} 63, \mathrm{y} 64, \mathrm{y} 65, \mathrm{y} 66, \mathrm{y} 67, \mathrm{y} 68, \mathrm{y} 69, \mathrm{y} 70, \mathrm{y} 71, \mathrm{y} 72, \mathrm{y} 73, \mathrm{y} 74, \mathrm{y} 75, \mathrm{y} 76, \mathrm{y} 77, \mathrm{y} 78, \mathrm{y} 79, \mathrm{y} 80, \mathrm{y} 81, \mathrm{y} 82$ $, \mathrm{y} 83, \mathrm{y} 84, \mathrm{y} 85, \mathrm{y} 86, \mathrm{y} 87, \mathrm{y} 88, \mathrm{y} 89, \mathrm{y} 90, \mathrm{y} 91, \mathrm{y} 92, \mathrm{y} 93, \mathrm{y} 94, \mathrm{y} 95, \mathrm{y} 96, \mathrm{x}] ;$

end $[A 27(j, 1), A 27(j, 2)]=$ curveintersect $(m(:, j), n(:, j), x 16, y 16) ;$

for $j=1: 56$

$\mathrm{m}=[\mathrm{y} 97, \mathrm{x} 43, \mathrm{x} 44, \mathrm{x} 45, \mathrm{x} 46, \mathrm{x} 47, \mathrm{x} 48, \mathrm{x} 49, \mathrm{x} 50, \mathrm{x} 51, \mathrm{x} 52, \mathrm{x} 53, \mathrm{x} 54, \mathrm{x} 55, \mathrm{x} 56, \mathrm{x} 57, \mathrm{x} 58, \mathrm{x} 59, \mathrm{x} 60, \mathrm{x} 61$, $x 62, x 63, x 64, x 65, x 66, x 67, x 68, x 69, x 70, x 71, x 72, x 73, x 74, x 75, x 76, x 77, x 78, x 79, x 80, x 81, x 82$ $, x 83, x 84, x 85, x 86, x 87, x 88, x 89, x 90, x 91, x 92, x 93, x 94, x 95, x 96, y] ;$

$\mathrm{n}=[\mathrm{x} 97, \mathrm{y} 43, \mathrm{y} 44, \mathrm{y} 45, \mathrm{y} 46, \mathrm{y} 47, \mathrm{y} 48, \mathrm{y} 49, \mathrm{y} 50, \mathrm{y} 51, \mathrm{y} 52, \mathrm{y} 53, \mathrm{y} 54, \mathrm{y} 55, \mathrm{y} 56, \mathrm{y} 57, \mathrm{y} 58, \mathrm{y} 59, \mathrm{y} 60, \mathrm{y} 61$, $\mathrm{y} 62, \mathrm{y} 63, \mathrm{y} 64, \mathrm{y} 65, \mathrm{y} 66, \mathrm{y} 67, \mathrm{y} 68, \mathrm{y} 69, \mathrm{y} 70, \mathrm{y} 71, \mathrm{y} 72, \mathrm{y} 73, \mathrm{y} 74, \mathrm{y} 75, \mathrm{y} 76, \mathrm{y} 77, \mathrm{y} 78, \mathrm{y} 79, \mathrm{y} 80, \mathrm{y} 81, \mathrm{y} 82$ $, \mathrm{y} 83, \mathrm{y} 84, \mathrm{y} 85, \mathrm{y} 86, \mathrm{y} 87, \mathrm{y} 88, \mathrm{y} 89, \mathrm{y} 90, \mathrm{y} 91, \mathrm{y} 92, \mathrm{y} 93, \mathrm{y} 94, \mathrm{y} 95, \mathrm{y} 96, \mathrm{x}] ;$

end $[A 28(j, 1), A 28(j, 2)]=$ curveintersect $(m(:, j), n(:, j), x 15, y 15) ;$

for $j=1: 56$

$\mathrm{m}=[\mathrm{y} 97, \mathrm{x} 43, \mathrm{x} 44, \mathrm{x} 45, \mathrm{x} 46, \mathrm{x} 47, \mathrm{x} 48, \mathrm{x} 49, \mathrm{x} 50, \mathrm{x} 51, \mathrm{x} 52, \mathrm{x} 53, \mathrm{x} 54, \mathrm{x} 55, \mathrm{x} 56, \mathrm{x} 57, \mathrm{x} 58, \mathrm{x} 59, \mathrm{x} 60, \mathrm{x} 61$, $x 62, x 63, x 64, x 65, x 66, x 67, x 68, x 69, x 70, x 71, x 72, x 73, x 74, x 75, x 76, x 77, x 78, x 79, x 80, x 81, x 82$ $, x 83, x 84, x 85, x 86, x 87, x 88, x 89, x 90, x 91, x 92, x 93, x 94, x 95, x 96, y] ;$

$\mathrm{n}=[\mathrm{x} 97, \mathrm{y} 43, \mathrm{y} 44, \mathrm{y} 45, \mathrm{y} 46, \mathrm{y} 47, \mathrm{y} 48, \mathrm{y} 49, \mathrm{y} 50, \mathrm{y} 51, \mathrm{y} 52, \mathrm{y} 53, \mathrm{y} 54, \mathrm{y} 55, \mathrm{y} 56, \mathrm{y} 57, \mathrm{y} 58, \mathrm{y} 59, \mathrm{y} 60, \mathrm{y} 61$, $\mathrm{y} 62, \mathrm{y} 63, \mathrm{y} 64, \mathrm{y} 65, \mathrm{y} 66, \mathrm{y} 67, \mathrm{y} 68, \mathrm{y} 69, \mathrm{y} 70, \mathrm{y} 71, \mathrm{y} 72, \mathrm{y} 73, \mathrm{y} 74, \mathrm{y} 75, \mathrm{y} 76, \mathrm{y} 77, \mathrm{y} 78, \mathrm{y} 79, \mathrm{y} 80, \mathrm{y} 81, \mathrm{y} 82$ $, \mathrm{y} 83, \mathrm{y} 84, \mathrm{y} 85, \mathrm{y} 86, \mathrm{y} 87, \mathrm{y} 88, \mathrm{y} 89, \mathrm{y} 90, \mathrm{y} 91, \mathrm{y} 92, \mathrm{y} 93, \mathrm{y} 94, \mathrm{y} 95, \mathrm{y} 96, \mathrm{x}] ;$

end $[\mathrm{A} 29(j, 1), \mathrm{A} 29(j, 2)]=$ curveintersect $(\mathrm{m}(:, j), \mathrm{n}(:, j), \mathrm{x} 14, \mathrm{y} 14)$;

for $j=1: 56$

$\mathrm{m}=[\mathrm{y} 97, \mathrm{x} 43, \mathrm{x} 44, \mathrm{x} 45, \mathrm{x} 46, \mathrm{x} 47, \mathrm{x} 48, \mathrm{x} 49, \mathrm{x} 50, \mathrm{x} 51, \mathrm{x} 52, \mathrm{x} 53, \mathrm{x} 54, \mathrm{x} 55, \mathrm{x} 56, \mathrm{x} 57, \mathrm{x} 58, \mathrm{x} 59, \mathrm{x} 60, \mathrm{x} 61$, $x 62, x 63, x 64, x 65, x 66, x 67, x 68, x 69, x 70, x 71, x 72, x 73, x 74, x 75, x 76, x 77, x 78, x 79, x 80, x 81, x 82$ $, x 83, x 84, x 85, x 86, x 87, x 88, x 89, x 90, x 91, x 92, x 93, x 94, x 95, x 96, y] ;$

$\mathrm{n}=[\mathrm{x} 97, \mathrm{y} 43, \mathrm{y} 44, \mathrm{y} 45, \mathrm{y} 46, \mathrm{y} 47, \mathrm{y} 48, \mathrm{y} 49, \mathrm{y} 50, \mathrm{y} 51, \mathrm{y} 52, \mathrm{y} 53, \mathrm{y} 54, \mathrm{y} 55, \mathrm{y} 56, \mathrm{y} 57, \mathrm{y} 58, \mathrm{y} 59, \mathrm{y} 60, \mathrm{y} 61$, $\mathrm{y} 62, \mathrm{y} 63, \mathrm{y} 64, \mathrm{y} 65, \mathrm{y} 66, \mathrm{y} 67, \mathrm{y} 68, \mathrm{y} 69, \mathrm{y} 70, \mathrm{y} 71, \mathrm{y} 72, \mathrm{y} 73, \mathrm{y} 74, \mathrm{y} 75, \mathrm{y} 76, \mathrm{y} 77, \mathrm{y} 78, \mathrm{y} 79, \mathrm{y} 80, \mathrm{y} 81, \mathrm{y} 82$ , y83, y84, y85, y86, y87, y88,y89,y90,y91,y92,y93,y94,y95, y96, x];

end $[A 30(j, 1), A 30(j, 2)]=$ curveintersect $(m(:, j), n(:, j), x 13, y 13)$;

for $j=1: 56$

$\mathrm{m}=[\mathrm{y} 97, \mathrm{x} 43, \mathrm{x} 44, \mathrm{x} 45, \mathrm{x} 46, \mathrm{x} 47, \mathrm{x} 48, \mathrm{x} 49, \mathrm{x} 50, \mathrm{x} 51, \mathrm{x} 52, \mathrm{x} 53, \mathrm{x} 54, \mathrm{x} 55, \mathrm{x} 56, \mathrm{x} 57, \mathrm{x} 58, \mathrm{x} 59, \mathrm{x} 60, \mathrm{x} 61$, $x 62, x 63, x 64, x 65, x 66, x 67, x 68, x 69, x 70, x 71, x 72, x 73, x 74, x 75, x 76, x 77, x 78, x 79, x 80, x 81, x 82$ $, x 83, x 84, x 85, x 86, x 87, x 88, x 89, x 90, x 91, x 92, x 93, x 94, x 95, x 96, y]$

$\mathrm{n}=[\mathrm{x} 97, \mathrm{y} 43, \mathrm{y} 44, \mathrm{y} 45, \mathrm{y} 46, \mathrm{y} 47, \mathrm{y} 48, \mathrm{y} 49, \mathrm{y} 50, \mathrm{y} 51, \mathrm{y} 52, \mathrm{y} 53, \mathrm{y} 54, \mathrm{y} 55, \mathrm{y} 56, \mathrm{y} 57, \mathrm{y} 58, \mathrm{y} 59, \mathrm{y} 60, \mathrm{y} 61$, $\mathrm{y} 62, \mathrm{y} 63, \mathrm{y} 64, \mathrm{y} 65, \mathrm{y} 66, \mathrm{y} 67, \mathrm{y} 68, \mathrm{y} 69, \mathrm{y} 70, \mathrm{y} 71, \mathrm{y} 72, \mathrm{y} 73, \mathrm{y} 74, \mathrm{y} 75, \mathrm{y} 76, \mathrm{y} 77, \mathrm{y} 78, \mathrm{y} 79, \mathrm{y} 80, \mathrm{y} 81, \mathrm{y} 82$ , y83, y84, y85, y86, y87, y88, y89, y90,y91,y92,y93,y94,y95, y96, x];

end $[A 31(j, 1), A 31(j, 2)]=$ curveintersect $(m(:, j), n(:, j), x 12, y 12)$;

for $j=1: 56$

$m=[y 97, x 43, x 44, x 45, x 46, x 47, x 48, x 49, x 50, x 51, x 52, x 53, x 54, x 55, x 56, x 57, x 58, x 59, x 60, x 61$, $x 62, x 63, x 64, x 65, x 66, x 67, x 68, x 69, x 70, x 71, x 72, x 73, x 74, x 75, x 76, x 77, x 78, x 79, x 80, x 81, x 82$ $, x 83, x 84, x 85, x 86, x 87, x 88, x 89, x 90, x 91, x 92, x 93, x 94, x 95, x 96, y] ;$

$\mathrm{n}=[\mathrm{x} 97, \mathrm{y} 43, \mathrm{y} 44, \mathrm{y} 45, \mathrm{y} 46, \mathrm{y} 47, \mathrm{y} 48, \mathrm{y} 49, \mathrm{y} 50, \mathrm{y} 51, \mathrm{y} 52, \mathrm{y} 53, \mathrm{y} 54, \mathrm{y} 55, \mathrm{y} 56, \mathrm{y} 57, \mathrm{y} 58, \mathrm{y} 59, \mathrm{y} 60, \mathrm{y} 61$, $\mathrm{y} 62, \mathrm{y} 63, \mathrm{y} 64, \mathrm{y} 65, \mathrm{y} 66, \mathrm{y} 67, \mathrm{y} 68, \mathrm{y} 69, \mathrm{y} 70, \mathrm{y} 71, \mathrm{y} 72, \mathrm{y} 73, \mathrm{y} 74, \mathrm{y} 75, \mathrm{y} 76, \mathrm{y} 77, \mathrm{y} 78, \mathrm{y} 79, \mathrm{y} 80, \mathrm{y} 81, \mathrm{y} 82$ $, \mathrm{y} 83, \mathrm{y} 84, \mathrm{y} 85, \mathrm{y} 86, \mathrm{y} 87, \mathrm{y} 88, \mathrm{y} 89, \mathrm{y} 90, \mathrm{y} 91, \mathrm{y} 92, \mathrm{y} 93, \mathrm{y} 94, \mathrm{y} 95, \mathrm{y} 96, \mathrm{x}] ;$ 
$[A 32(j, 1), A 32(j, 2)]=$ curveintersect $(m(:, j), n(:, j), x 11, y 11)$;

end

for $j=1: 56$

$m=[y 97, x 43, x 44, x 45, x 46, x 47, x 48, x 49, x 50, x 51, x 52, x 53, x 54, x 55, x 56, x 57, x 58, x 59, x 60, x 61$, $x 62, x 63, x 64, x 65, x 66, x 67, x 68, x 69, x 70, x 71, x 72, x 73, x 74, x 75, x 76, x 77, x 78, x 79, x 80, x 81, x 82$ $, x 83, x 84, x 85, x 86, x 87, x 88, x 89, x 90, x 91, x 92, x 93, x 94, x 95, x 96, y] ;$

$\mathrm{n}=[\mathrm{x} 97, \mathrm{y} 43, \mathrm{y} 44, \mathrm{y} 45, \mathrm{y} 46, \mathrm{y} 47, \mathrm{y} 48, \mathrm{y} 49, \mathrm{y} 50, \mathrm{y} 51, \mathrm{y} 52, \mathrm{y} 53, \mathrm{y} 54, \mathrm{y} 55, \mathrm{y} 56, \mathrm{y} 57, \mathrm{y} 58, \mathrm{y} 59, \mathrm{y} 60, \mathrm{y} 61$, $\mathrm{y} 62, \mathrm{y} 63, \mathrm{y} 64, \mathrm{y} 65, \mathrm{y} 66, \mathrm{y} 67, \mathrm{y} 68, \mathrm{y} 69, \mathrm{y} 70, \mathrm{y} 71, \mathrm{y} 72, \mathrm{y} 73, \mathrm{y} 74, \mathrm{y} 75, \mathrm{y} 76, \mathrm{y} 77, \mathrm{y} 78, \mathrm{y} 79, \mathrm{y} 80, \mathrm{y} 81, \mathrm{y} 82$ , y83,y84,y85,y86,y87,y88,y89,y90,y91,y92,y93,y94,y95,y96,x];

end $[\mathrm{A} 33(j, 1), \mathrm{A} 33(j, 2)]=$ curveintersect $(\mathrm{m}(:, j), \mathrm{n}(:, j), \mathrm{x} 10, \mathrm{y} 10)$;

for $j=1: 56$

$m=[y 97, x 43, x 44, x 45, x 46, x 47, x 48, x 49, x 50, x 51, x 52, x 53, x 54, x 55, x 56, x 57, x 58, x 59, x 60, x 61$, $x 62, x 63, x 64, x 65, x 66, x 67, x 68, x 69, x 70, x 71, x 72, x 73, x 74, x 75, x 76, x 77, x 78, x 79, x 80, x 81, x 82$ $, x 83, x 84, x 85, x 86, x 87, x 88, x 89, x 90, x 91, x 92, x 93, x 94, x 95, x 96, y] ;$

$\mathrm{n}=[\mathrm{x} 97, \mathrm{y} 43, \mathrm{y} 44, \mathrm{y} 45, \mathrm{y} 46, \mathrm{y} 47, \mathrm{y} 48, \mathrm{y} 49, \mathrm{y} 50, \mathrm{y} 51, \mathrm{y} 52, \mathrm{y} 53, \mathrm{y} 54, \mathrm{y} 55, \mathrm{y} 56, \mathrm{y} 57, \mathrm{y} 58, \mathrm{y} 59, \mathrm{y} 60, \mathrm{y} 61$, $\mathrm{y} 62, \mathrm{y} 63, \mathrm{y} 64, \mathrm{y} 65, \mathrm{y} 66, \mathrm{y} 67, \mathrm{y} 68, \mathrm{y} 69, \mathrm{y} 70, \mathrm{y} 71, \mathrm{y} 72, \mathrm{y} 73, \mathrm{y} 74, \mathrm{y} 75, \mathrm{y} 76, \mathrm{y} 77, \mathrm{y} 78, \mathrm{y} 79, \mathrm{y} 80, \mathrm{y} 81, \mathrm{y} 82$ , y83,y84,y85,y86,y87,y88,y89,y90,y91,y92,y93,y94,y95,y96,x];

end

$[\mathrm{A} 34(j, 1), \mathrm{A} 34(j, 2)]=$ curveintersect $(\mathrm{m}(:, j), \mathrm{n}(:, j), \mathrm{x} 9, \mathrm{y} 9)$;

for $j=1: 56$

$m=[y 97, x 43, x 44, x 45, x 46, x 47, x 48, x 49, x 50, x 51, x 52, x 53, x 54, x 55, x 56, x 57, x 58, x 59, x 60, x 61$, $x 62, x 63, x 64, x 65, x 66, x 67, x 68, x 69, x 70, x 71, x 72, x 73, x 74, x 75, x 76, x 77, x 78, x 79, x 80, x 81, x 82$ $, x 83, x 84, x 85, x 86, x 87, x 88, x 89, x 90, x 91, x 92, x 93, x 94, x 95, x 96, y] ;$

$\mathrm{n}=[\mathrm{x} 97, \mathrm{y} 43, \mathrm{y} 44, \mathrm{y} 45, \mathrm{y} 46, \mathrm{y} 47, \mathrm{y} 48, \mathrm{y} 49, \mathrm{y} 50, \mathrm{y} 51, \mathrm{y} 52, \mathrm{y} 53, \mathrm{y} 54, \mathrm{y} 55, \mathrm{y} 56, \mathrm{y} 57, \mathrm{y} 58, \mathrm{y} 59, \mathrm{y} 60, \mathrm{y} 61$, $\mathrm{y} 62, \mathrm{y} 63, \mathrm{y} 64, \mathrm{y} 65, \mathrm{y} 66, \mathrm{y} 67, \mathrm{y} 68, \mathrm{y} 69, \mathrm{y} 70, \mathrm{y} 71, \mathrm{y} 72, \mathrm{y} 73, \mathrm{y} 74, \mathrm{y} 75, \mathrm{y} 76, \mathrm{y} 77, \mathrm{y} 78, \mathrm{y} 79, \mathrm{y} 80, \mathrm{y} 81, \mathrm{y} 82$ $, \mathrm{y} 83, \mathrm{y} 84, \mathrm{y} 85, \mathrm{y} 86, \mathrm{y} 87, \mathrm{y} 88, \mathrm{y} 89, \mathrm{y} 90, \mathrm{y} 91, \mathrm{y} 92, \mathrm{y} 93, \mathrm{y} 94, \mathrm{y} 95, \mathrm{y} 96, \mathrm{x}]$;

end

$[\mathrm{A} 35(j, 1), \mathrm{A} 35(j, 2)]=$ curveintersect $(\mathrm{m}(:, j), \mathrm{n}(:, j), \mathrm{x} 8, \mathrm{y} 8)$;

for $j=1: 56$

$m=[y 97, x 43, x 44, x 45, x 46, x 47, x 48, x 49, x 50, x 51, x 52, x 53, x 54, x 55, x 56, x 57, x 58, x 59, x 60, x 61$, $x 62, x 63, x 64, x 65, x 66, x 67, x 68, x 69, x 70, x 71, x 72, x 73, x 74, x 75, x 76, x 77, x 78, x 79, x 80, x 81, x 82$ $, x 83, x 84, x 85, x 86, x 87, x 88, x 89, x 90, x 91, x 92, x 93, x 94, x 95, x 96, y]$;

$\mathrm{n}=[\mathrm{x} 97, \mathrm{y} 43, \mathrm{y} 44, \mathrm{y} 45, \mathrm{y} 46, \mathrm{y} 47, \mathrm{y} 48, \mathrm{y} 49, \mathrm{y} 50, \mathrm{y} 51, \mathrm{y} 52, \mathrm{y} 53, \mathrm{y} 54, \mathrm{y} 55, \mathrm{y} 56, \mathrm{y} 57, \mathrm{y} 58, \mathrm{y} 59, \mathrm{y} 60, \mathrm{y} 61$, $\mathrm{y} 62, \mathrm{y} 63, \mathrm{y} 64, \mathrm{y} 65, \mathrm{y} 66, \mathrm{y} 67, \mathrm{y} 68, \mathrm{y} 69, \mathrm{y} 70, \mathrm{y} 71, \mathrm{y} 72, \mathrm{y} 73, \mathrm{y} 74, \mathrm{y} 75, \mathrm{y} 76, \mathrm{y} 77, \mathrm{y} 78, \mathrm{y} 79, \mathrm{y} 80, \mathrm{y} 81, \mathrm{y} 82$ $, \mathrm{y} 83, \mathrm{y} 84, \mathrm{y} 85, \mathrm{y} 86, \mathrm{y} 87, \mathrm{y} 88, \mathrm{y} 89, \mathrm{y} 90, \mathrm{y} 91, \mathrm{y} 92, \mathrm{y} 93, \mathrm{y} 94, \mathrm{y} 95, \mathrm{y} 96, \mathrm{x}]$;

end

$[A 36(j, 1), A 36(j, 2)]=$ curveintersect $(m(:, j), n(:, j), x 7, y 7)$;

for $j=1: 56$

$m=[y 97, x 43, x 44, x 45, x 46, x 47, x 48, x 49, x 50, x 51, x 52, x 53, x 54, x 55, x 56, x 57, x 58, x 59, x 60, x 61$, $x 62, x 63, x 64, x 65, x 66, x 67, x 68, x 69, x 70, x 71, x 72, x 73, x 74, x 75, x 76, x 77, x 78, x 79, x 80, x 81, x 82$ $, \mathrm{x} 83, \mathrm{x} 84, \mathrm{x} 85, \mathrm{x} 86, \mathrm{x} 87, \mathrm{x} 88, \mathrm{x} 89, \mathrm{x} 90, \mathrm{x} 91, \mathrm{x} 92, \mathrm{x} 93, \mathrm{x} 94, \mathrm{x} 95, \mathrm{x} 96, \mathrm{y}]$;

$\mathrm{n}=[\mathrm{x} 97, \mathrm{y} 43, \mathrm{y} 44, \mathrm{y} 45, \mathrm{y} 46, \mathrm{y} 47, \mathrm{y} 48, \mathrm{y} 49, \mathrm{y} 50, \mathrm{y} 51, \mathrm{y} 52, \mathrm{y} 53, \mathrm{y} 54, \mathrm{y} 55, \mathrm{y} 56, \mathrm{y} 57, \mathrm{y} 58, \mathrm{y} 59, \mathrm{y} 60, \mathrm{y} 61$, $\mathrm{y} 62, \mathrm{y} 63, \mathrm{y} 64, \mathrm{y} 65, \mathrm{y} 66, \mathrm{y} 67, \mathrm{y} 68, \mathrm{y} 69, \mathrm{y} 70, \mathrm{y} 71, \mathrm{y} 72, \mathrm{y} 73, \mathrm{y} 74, \mathrm{y} 75, \mathrm{y} 76, \mathrm{y} 77, \mathrm{y} 78, \mathrm{y} 79, \mathrm{y} 80, \mathrm{y} 81, \mathrm{y} 82$ , y83,y84,y85,y86,y87,y88,y89,y90,y91,y92,y93,y94,y95,y96, x];

end

$[A 37(j, 1), A 37(j, 2)]=$ curveintersect $(m(:, j), n(:, j), x 6, y 6)$;

for $j=1: 56$

$\mathrm{m}=[\mathrm{y} 97, \mathrm{x} 43, \mathrm{x} 44, \mathrm{x} 45, \mathrm{x} 46, \mathrm{x} 47, \mathrm{x} 48, \mathrm{x} 49, \mathrm{x} 50, \mathrm{x} 51, \mathrm{x} 52, \mathrm{x} 53, \mathrm{x} 54, \mathrm{x} 55, \mathrm{x} 56, \mathrm{x} 57, \mathrm{x} 58, \mathrm{x} 59, \mathrm{x} 60, \mathrm{x} 61$, $x 62, x 63, x 64, x 65, x 66, x 67, x 68, x 69, x 70, x 71, x 72, x 73, x 74, x 75, x 76, x 77, x 78, x 79, x 80, x 81, x 82$ $, x 83, x 84, x 85, x 86, x 87, x 88, x 89, x 90, x 91, x 92, x 93, x 94, x 95, x 96, y] ;$

$\mathrm{n}=[\mathrm{x} 97, \mathrm{y} 43, \mathrm{y} 44, \mathrm{y} 45, \mathrm{y} 46, \mathrm{y} 47, \mathrm{y} 48, \mathrm{y} 49, \mathrm{y} 50, \mathrm{y} 51, \mathrm{y} 52, \mathrm{y} 53, \mathrm{y} 54, \mathrm{y} 55, \mathrm{y} 56, \mathrm{y} 57, \mathrm{y} 58, \mathrm{y} 59, \mathrm{y} 60, \mathrm{y} 61$, $y 62, y 63, y 64, y 65, y 66, y 67, y 68, y 69, y 70, y 71, y 72, y 73, y 74, y 75, y 76, y 77, y 78, y 79, y 80, y 81, y 82$ , y83,y84,y85,y86,y87,y88,y89,y90,y91,y92,y93,y94,y95,y96,x];

end

$[\mathrm{A} 38(j, 1), \mathrm{A} 38(j, 2)]=$ curveintersect $(\mathrm{m}(:, j), \mathrm{n}(:, j), \mathrm{x} 5, \mathrm{y} 5)$;

for $j=1: 56$

$m=[y 97, x 43, x 44, x 45, x 46, x 47, x 48, x 49, x 50, x 51, x 52, x 53, x 54, x 55, x 56, x 57, x 58, x 59, x 60, x 61$, $x 62, x 63, x 64, x 65, x 66, x 67, x 68, x 69, x 70, x 71, x 72, x 73, x 74, x 75, x 76, x 77, x 78, x 79, x 80, x 81, x 82$ $, x 83, x 84, x 85, x 86, x 87, x 88, x 89, x 90, x 91, x 92, x 93, x 94, x 95, x 96, y]$

$\mathrm{n}=[\mathrm{x} 97, \mathrm{y} 43, \mathrm{y} 44, \mathrm{y} 45, \mathrm{y} 46, \mathrm{y} 47, \mathrm{y} 48, \mathrm{y} 49, \mathrm{y} 50, \mathrm{y} 51, \mathrm{y} 52, \mathrm{y} 53, \mathrm{y} 54, \mathrm{y} 55, \mathrm{y} 56, \mathrm{y} 57, \mathrm{y} 58, \mathrm{y} 59, \mathrm{y} 60, \mathrm{y} 61$, $y 62, y 63, y 64, y 65, y 66, y 67, y 68, y 69, y 70, y 71, y 72, y 73, y 74, y 75, y 76, y 77, y 78, y 79, y 80, y 81, y 82$ $, \mathrm{y} 83, \mathrm{y} 84, \mathrm{y} 85, \mathrm{y} 86, \mathrm{y} 87, \mathrm{y} 88, \mathrm{y} 89, \mathrm{y} 90, \mathrm{y} 91, \mathrm{y} 92, \mathrm{y} 93, \mathrm{y} 94, \mathrm{y} 95, \mathrm{y} 96, \mathrm{x}]$;

end

$[A 39(j, 1), A 39(j, 2)]=$ curveintersect $(m(:, j), n(:, j), x 4, y 4) ;$

for $j=1: 56$

$\mathrm{m}=[\mathrm{y} 97, \mathrm{x} 43, \mathrm{x} 44, \mathrm{x} 45, \mathrm{x} 46, \mathrm{x} 47, \mathrm{x} 48, \mathrm{x} 49, \mathrm{x} 50, \mathrm{x} 51, \mathrm{x} 52, \mathrm{x} 53, \mathrm{x} 54, \mathrm{x} 55, \mathrm{x} 56, \mathrm{x} 57, \mathrm{x} 58, \mathrm{x} 59, \mathrm{x} 60, \mathrm{x} 61$, $x 62, x 63, x 64, x 65, x 66, x 67, x 68, x 69, x 70, x 71, x 72, x 73, x 74, x 75, x 76, x 77, x 78, x 79, x 80, x 81, x 82$ $, x 83, x 84, x 85, x 86, x 87, x 88, x 89, x 90, x 91, x 92, x 93, x 94, x 95, x 96, y]$ 
$n=[x 97, y 43, y 44, y 45, y 46, y 47, y 48, y 49, y 50, y 51, y 52, y 53, y 54, y 55, y 56, y 57, y 58, y 59, y 60, y 61$, $\mathrm{y} 62, y 63, y 64, y 65, y 66, y 67, y 68, y 69, y 70, y 71, y 72, y 73, y 74, y 75, y 76, y 77, y 78, y 79, y 80, y 81, y 82$ , y83,y84,y85,y86,y87,y88,y89,y90,y91,y92,y93,y94,y95,y96,x];

end $[A 40(j, 1), A 40(j, 2)]=$ curveintersect $(m(:, j), n(:, j), x 3, y 3)$;

for $j=1: 56$

$m=[y 97, x 43, x 44, x 45, x 46, x 47, x 48, x 49, x 50, x 51, x 52, x 53, x 54, x 55, x 56, x 57, x 58, x 59, x 60, x 61$, $x 62, x 63, x 64, x 65, x 66, x 67, x 68, x 69, x 70, x 71, x 72, x 73, x 74, x 75, x 76, x 77, x 78, x 79, x 80, x 81, x 82$ $, x 83, x 84, x 85, x 86, x 87, x 88, x 89, x 90, x 91, x 92, x 93, x 94, x 95, x 96, y]$

$n=[x 97, y 43, y 44, y 45, y 46, y 47, y 48, y 49, y 50, y 51, y 52, y 53, y 54, y 55, y 56, y 57, y 58, y 59, y 60, y 61$, $\mathrm{y} 62, \mathrm{y} 63, \mathrm{y} 64, \mathrm{y} 65, \mathrm{y} 66, \mathrm{y} 67, \mathrm{y} 68, \mathrm{y} 69, \mathrm{y} 70, \mathrm{y} 71, \mathrm{y} 72, \mathrm{y} 73, \mathrm{y} 74, \mathrm{y} 75, \mathrm{y} 76, \mathrm{y} 77, \mathrm{y} 78, \mathrm{y} 79, \mathrm{y} 80, \mathrm{y} 81, \mathrm{y} 82$ , y83,y84,y85,y86,y87,y88,y89,y90,y91,y92,y93,y94,y95,y96, x] ;

end

$[A 41(j, 1), A 41(j, 2)]=$ curveintersect $(m(:, j), n(:, j), x 2, y 2) ;$

for $j=1: 56$

$m=[y 97, x 43, x 44, x 45, x 46, x 47, x 48, x 49, x 50, x 51, x 52, x 53, x 54, x 55, x 56, x 57, x 58, x 59, x 60, x 61$, $x 62, x 63, x 64, x 65, x 66, x 67, x 68, x 69, x 70, x 71, x 72, x 73, x 74, x 75, x 76, x 77, x 78, x 79, x 80, x 81, x 82$ $, x 83, x 84, x 85, x 86, x 87, x 88, x 89, x 90, x 91, x 92, x 93, x 94, x 95, x 96, y]$

$\mathrm{n}=[\mathrm{x} 97, \mathrm{y} 43, \mathrm{y} 44, \mathrm{y} 45, \mathrm{y} 46, \mathrm{y} 47, \mathrm{y} 48, \mathrm{y} 49, \mathrm{y} 50, \mathrm{y} 51, \mathrm{y} 52, \mathrm{y} 53, \mathrm{y} 54, \mathrm{y} 55, \mathrm{y} 56, \mathrm{y} 57, \mathrm{y} 58, \mathrm{y} 59, \mathrm{y} 60, \mathrm{y} 61$, $\mathrm{y} 62, \mathrm{y} 63, \mathrm{y} 64, \mathrm{y} 65, \mathrm{y} 66, \mathrm{y} 67, \mathrm{y} 68, \mathrm{y} 69, \mathrm{y} 70, \mathrm{y} 71, \mathrm{y} 72, \mathrm{y} 73, \mathrm{y} 74, \mathrm{y} 75, \mathrm{y} 76, \mathrm{y} 77, \mathrm{y} 78, \mathrm{y} 79, \mathrm{y} 80, \mathrm{y} 81, \mathrm{y} 82$ , y83,y84,y85,y86,y87,y88,y89,y90,y91,y92,y93,y94,y95,y96,x];

end

$[A 42(j, 1), A 42(j, 2)]=$ curveintersect $(m(:, j), n(:, j), x 1, y 1)$;

for $j=1: 56$

$\mathrm{m}=[\mathrm{y} 97, \mathrm{x} 43, \mathrm{x} 44, \mathrm{x} 45, \mathrm{x} 46, \mathrm{x} 47, \mathrm{x} 48, \mathrm{x} 49, \mathrm{x} 50, \mathrm{x} 51, \mathrm{x} 52, \mathrm{x} 53, \mathrm{x} 54, \mathrm{x} 55, \mathrm{x} 56, \mathrm{x} 57, \mathrm{x} 58, \mathrm{x} 59, \mathrm{x} 60, \mathrm{x} 61$, $x 62, x 63, x 64, x 65, x 66, x 67, x 68, x 69, x 70, x 71, x 72, x 73, x 74, x 75, x 76, x 77, x 78, x 79, x 80, x 81, x 82$ $, x 83, x 84, x 85, x 86, x 87, x 88, x 89, x 90, x 91, x 92, x 93, x 94, x 95, x 96, y] ;$

$\mathrm{n}=[\mathrm{x} 97, \mathrm{y} 43, \mathrm{y} 44, \mathrm{y} 45, \mathrm{y} 46, \mathrm{y} 47, \mathrm{y} 48, \mathrm{y} 49, \mathrm{y} 50, \mathrm{y} 51, \mathrm{y} 52, \mathrm{y} 53, \mathrm{y} 54, \mathrm{y} 55, \mathrm{y} 56, \mathrm{y} 57, \mathrm{y} 58, \mathrm{y} 59, \mathrm{y} 60, \mathrm{y} 61$, $\mathrm{y} 62, \mathrm{y} 63, \mathrm{y} 64, \mathrm{y} 65, \mathrm{y} 66, \mathrm{y} 67, \mathrm{y} 68, \mathrm{y} 69, \mathrm{y} 70, \mathrm{y} 71, \mathrm{y} 72, \mathrm{y} 73, \mathrm{y} 74, \mathrm{y} 75, \mathrm{y} 76, \mathrm{y} 77, \mathrm{y} 78, \mathrm{y} 79, \mathrm{y} 80, \mathrm{y} 81, \mathrm{y} 82$ $, \mathrm{y} 83, \mathrm{y} 84, \mathrm{y} 85, \mathrm{y} 86, \mathrm{y} 87, \mathrm{y} 88, \mathrm{y} 89, \mathrm{y} 90, \mathrm{y} 91, \mathrm{y} 92, \mathrm{y} 93, \mathrm{y} 94, \mathrm{y} 95, \mathrm{y} 96, \mathrm{x}]$ end $[A 43(j, 1), A 43(j, 2)]=$ curveintersect $(m(:, j), n(:, j), x 97, y 97)$;

$\mathrm{A} 44=[0,1 ; .087, .996 ; .174, .984 ; .259, .965 ; .342, .939 ; .906, .423 ; .5, .866 ; .573, .819 ; .643,$. $766 ; .707, .707 ; .766, .643 ; .819, .573 ; .866, .5 ; .423, .906] ;$

$\mathrm{A}=[\mathrm{A} 1 ; \mathrm{A} 2 ; \mathrm{A} 3 ; \mathrm{A} 4 ; \mathrm{A} 5 ; \mathrm{A} 6 ; \mathrm{A} 7 ; \mathrm{A} 8 ; \mathrm{A} 9 ; \mathrm{A} 10 ; \mathrm{A} 11 ; \mathrm{A} 12 ; \mathrm{A} 13 ; \mathrm{A} 14 ; \mathrm{A} 15 ; \mathrm{A} 16 ; \mathrm{A} 17 ; \mathrm{A} 18 ; \mathrm{A} 19 ; \mathrm{A} 20 ; \mathrm{A} 21 ; \mathrm{A} 22 ; \mathrm{A}$ 23;A24;A25;A26;A27;A28;A29;A30;A31;A32;A33;A34;A35;A36;A37;A38;A39;A40;A41;A42;A43; A4 4$]$

$\operatorname{plot}([A(:, 1),-A(:, 1), A(:, 1),-A(:, 1)],[A(:, 2),-A(:, 2),-A(:, 2), A(:, 2)], ' m * ')$

function $d y d x=O D E 1(x, y)$

$\mathrm{r}=\operatorname{sqrt}\left(\mathrm{x} \cdot{ }^{\wedge} 2+\mathrm{y} \cdot{ }^{\wedge} 2\right) ; \mathrm{t}=\operatorname{atan}(\mathrm{y} \cdot / \mathrm{x}) ;$

$\operatorname{sr}=0.5 *\left(1-\left(r \cdot{ }^{\wedge}-2\right)\right)+0.5 *\left(1+3 *\left(r \cdot \cdot^{\wedge}-4\right)-4 *\left(r \cdot \cdot^{\wedge}-2\right)\right) * \cos (2 * t) ;$

$s t=0.5 *\left(1+\left(r \cdot{ }^{\wedge}-2\right)\right)-0.5 *\left(1+3 *\left(r \cdot^{\wedge}-4\right)\right) * \cos (2 * t)$;

$\operatorname{srt}=-0.5 *\left(1-3 *\left(r \cdot^{\wedge}-4\right)+2 *\left(r \cdot^{\wedge}-2\right)\right) * \sin (2 * t)$;

$\operatorname{sx}=\operatorname{sr}{ }^{*} \cos (t) \cdot{ }^{\wedge} 2+\operatorname{st}{ }^{*} \sin (t) \cdot{ }^{\wedge} 2-2 * \operatorname{srt}{ }^{*} \sin (t) * \cos (t) ;$

$\operatorname{sy}=\operatorname{sr}{ }^{*} \sin (t) \cdot{ }^{\wedge} 2+\operatorname{st}{ }^{*} \cos (t) \cdot{ }^{\wedge} 2+2 * \operatorname{srt}{ }^{*} \sin (t) * \cos (t) ;$

$t x y=\operatorname{sr}{ }^{*} \sin (t){ }^{*} \cos (t)-s t{ }^{*} \cos (t) * \sin (t)+\operatorname{srt}{ }^{*} \cos (t) .{ }^{\wedge} 2-\operatorname{srt}{ }^{*} \sin (t) \cdot{ }^{\wedge} 2$

$d y d x=-(s x-s y) \cdot /(2 * t x y)-\operatorname{sqrt}\left(1+((s x-s y) \cdot /(2 * t x y)) \cdot{ }^{\wedge} 2\right) ;$

\% Introduce slope limiter for case where tau goes to zero

if $\operatorname{abs}(\mathrm{dydx})>10$

$d y d x=0$;

end

function $d y d x=\operatorname{ODE} 2(x, y)$

$\mathrm{r}=\operatorname{sqrt}\left(\mathrm{x} \cdot{ }^{\wedge} 2+\mathrm{y} \cdot{ }^{\wedge} 2\right) ; \mathrm{t}=\operatorname{atan}(\mathrm{y} \cdot / \mathrm{x})$;

$\mathrm{sr}=0.5^{*}\left(1-\left(r \cdot^{\wedge}-2\right)\right)-0.5^{*}\left(1+3^{*}\left(r \cdot^{\wedge}-4\right)-4 *\left(r \cdot^{\wedge}-2\right)\right) * \cos (2 * t)$

st $=0.5 *\left(1+\left(r \cdot{ }^{\wedge}-2\right)\right)+0 \cdot 5^{*}\left(1+3 *\left(r \cdot{ }^{\wedge}-4\right)\right){ }^{*} \cos (2 * t)$;

$\operatorname{srt}=0.5 *\left(1-3 *\left(r \cdot{ }^{\wedge}-4\right)+2 *\left(r \cdot^{\wedge}-2\right)\right) * \sin (2 * t) ;$

$\operatorname{sx}=\mathrm{sr}^{*} \cos (t) \cdot{ }^{\wedge} 2+\operatorname{st}{ }^{*} \sin (t) \cdot{ }^{\wedge} 2-2 * \operatorname{srt}{ }^{*} \sin (t){ }^{*} \cos (t) ;$

$\operatorname{sy}=\operatorname{sr}{ }^{*} \sin (t) \cdot{ }^{\wedge} 2+\operatorname{st}{ }^{*} \cos (t) \cdot{ }^{\wedge} 2+2{ }^{*} \operatorname{srt}{ }^{*} \sin (t){ }^{*} \cos (t) ;$

$\operatorname{txy}=\operatorname{sr}^{*} \sin (t){ }^{*} \cos (t)-\mathrm{st}^{*} \cos (t){ }^{*} \sin (t)+\operatorname{srt}{ }^{*} \cos (t) \cdot{ }^{\wedge} 2-\operatorname{srt}{ }^{*} \sin (t) \cdot{ }^{\wedge} 2$

$d y d x=-(s x-s y) \cdot /(2 * t x y)+\operatorname{sgrt}\left(1+((s x-s y) \cdot /(2 * t x y)) \cdot{ }^{\wedge} 2\right) ;$

$\circ$ Introduce slope limiter for case where tau goes to zero

if $\operatorname{abs}(d y d x)>4.1$

end $d y d x=0.01$

function $d y d x=\operatorname{ODE} 3(x, y)$

$r=\operatorname{sqrt}\left(\mathrm{x} \cdot{ }^{\wedge} 2+\mathrm{y} \cdot{ }^{\wedge} 2\right) ; \mathrm{t}=\operatorname{atan}(\mathrm{y} \cdot / \mathrm{x})$;

$\operatorname{sr}=0.5^{*}\left(1-\left(r \cdot^{\wedge}-2\right)\right)+0.5^{*}\left(1+3^{*}\left(r \cdot^{\wedge}-4\right)-4 *\left(r \cdot^{\wedge}-2\right)\right){ }^{*} \cos (2 * t) ;$

st $=0.5 *\left(1+\left(r \cdot^{\wedge}-2\right)\right)-0.5 *\left(1+3 *\left(r \cdot^{\wedge}-4\right)\right) * \cos (2 * t)$;

$\operatorname{srt}=-0.5 *\left(1-3 *\left(r \cdot{ }^{\wedge}-4\right)+2 *\left(r \cdot{ }^{\wedge}-2\right)\right) * \sin (2 * t) ;$

$\mathrm{s} x=\mathrm{sr}^{*} \cos (t) \cdot{ }^{\wedge} 2+\mathrm{st}{ }^{\star} \sin (t) \cdot{ }^{\wedge} 2-2^{*} \operatorname{srt} t^{*} \sin (t){ }^{*} \cos (t)$;

$\operatorname{sy}=\operatorname{sr}{ }^{*} \sin (t) \cdot{ }^{\wedge} 2+\operatorname{st}{ }^{*} \cos (t) \cdot{ }^{\wedge} 2+2 * \operatorname{srt}{ }^{*} \sin (t) * \cos (t) ;$ 


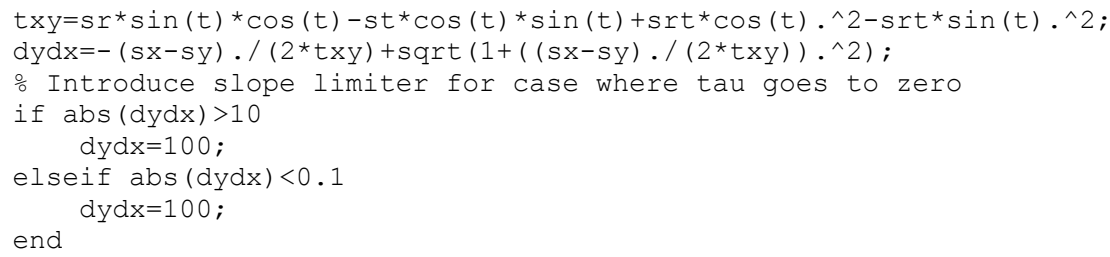

\section{Unequal Biaxial Compression Coarse Topology}

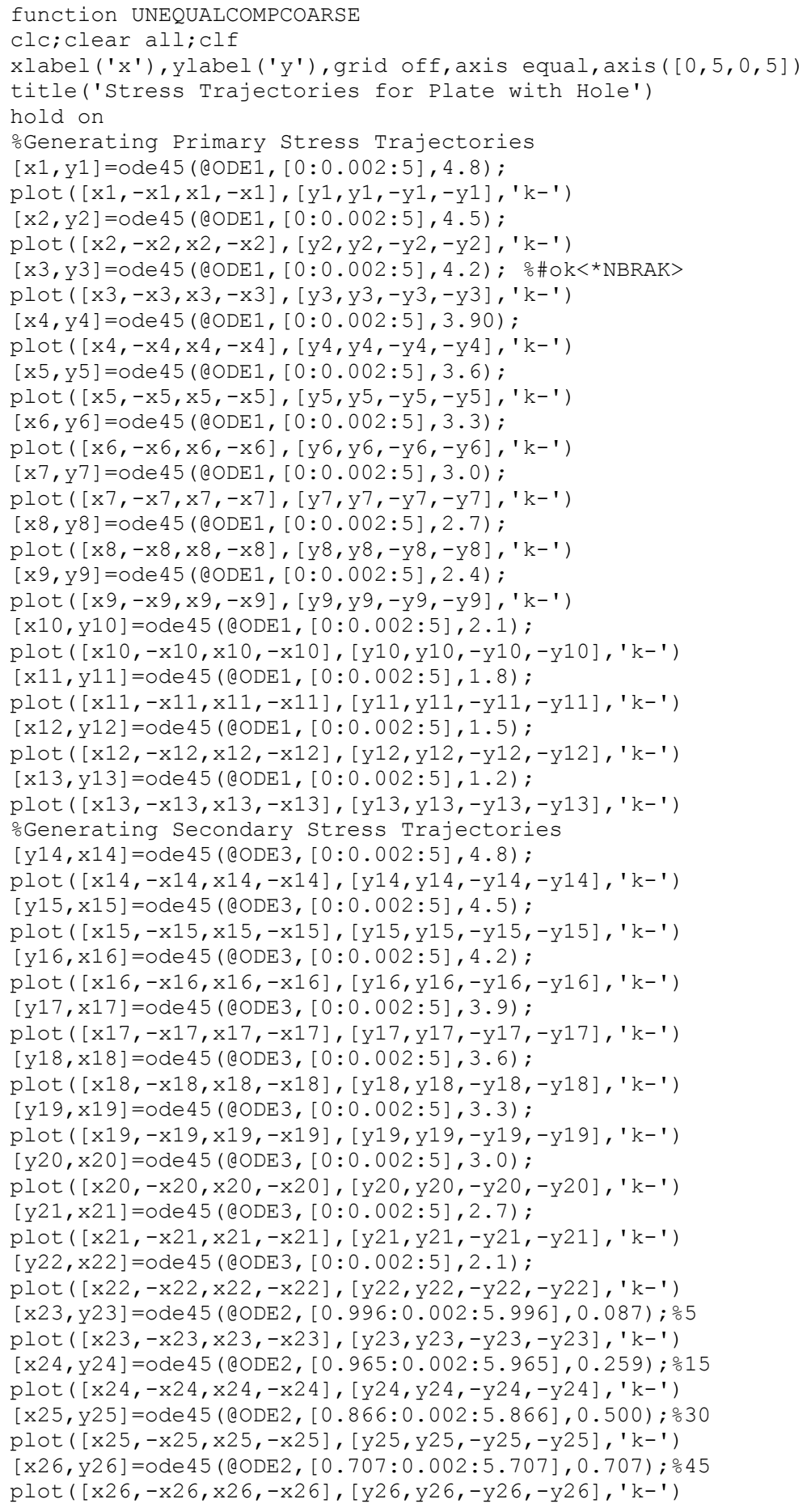


$[\mathrm{x} 27, \mathrm{y} 27]=0 \mathrm{de} 45(\mathrm{QODE} 2,[0.500: 0.002: 5.500], 0.866) ; \% 60$

plot $([x 27,-x 27, x 27,-x 27],[y 27, y 27,-y 27,-y 27], ' k-')$

$[\mathrm{x} 28, \mathrm{y} 28]=0 \mathrm{de} 45(\mathrm{QODE} 2,[0.259: 0.002: 5.259], 0.965) ; \circ 75$

plot $([x 28,-x 28, x 28,-x 28],[y 28, y 28,-y 28,-y 28], ' k-')$

$\mathrm{x} 29=[0: 0.002: 5]^{\prime}$;

$\mathrm{y} 29=\mathrm{x} 29 \cdot * 0+5$

plot $\left(\mathrm{x} 29, \mathrm{y} 29, \mathrm{k}^{-1}\right)$

plot $\left(-\mathrm{x} 29, \mathrm{y} 29, \mathrm{\prime}^{\mathrm{k}} \mathrm{-}^{\prime}\right)$

plot $\left(x 29,-y 29,{ }^{\prime} \mathrm{k}-{ }^{\prime}\right)$

plot $\left(-x 29,-y 29,{ }^{\prime} k-'\right)$

plot $\left(y 29, x 29,{ }^{\prime}-^{\prime}\right)$

plot $\left(-y 29, x 29, k^{\prime}-{ }^{\prime}\right)$

plot $(y 29,-x 29, ' k-')$

plot $(-y 29,-x 29, ' k-')$

$\mathrm{x}=1: .002: 5$;

$\mathrm{y}=\mathrm{x} \cdot * 0$;

plot $(x, y, ' k-')$

plot $\left(-x, y,{ }^{\prime}-^{\prime}\right)$

plot $\left(y, x, x^{\prime}-{ }^{\prime}\right)$

$\operatorname{plot}\left(y,-x,{ }^{\prime}-^{\prime}\right)$

$\mathrm{x}=0: .01: 1$

$\mathrm{y}=\operatorname{sqrt}\left(1-\mathrm{x} \cdot{ }^{\wedge} 2\right)$;

plot $\left(x, y,{ }^{\prime}-', '\right.$ 'Linewidth', 1.5$)$

plot $\left(-x, y,{ }^{\prime}-', '\right.$ Linewidth', 1.5$)$

plot $\left(x,-y,{ }^{\prime}-{ }^{\prime}, '\right.$ 'Linewidth', 1.5)

plot $\left(-x,-y,{ }^{\prime}-^{\prime}\right.$ ', 'Linewidth', 1.5)

for $j=1: 6$

$\mathrm{m}=[\mathrm{x} 23, \mathrm{x} 24, \mathrm{x} 25, \mathrm{x} 26, \mathrm{x} 27, \mathrm{x} 28]$;

$\mathrm{n}=[\mathrm{y} 23, \mathrm{y} 24, \mathrm{y} 25, \mathrm{y} 26, \mathrm{y} 27, \mathrm{y} 28]$;

end

$[A 1(j, 1), A 1(j, 2)]=$ curveintersect $(m(:, j), n(:, j), x 13, y 13)$;

for $j=1: 16$

$m=[y 29, x 14, x 15, x 16, x 17, x 18, x 19, x 20, x 21, x 22, x 23, x 24, x 25, x 26, x 27, x 28]$;

$\mathrm{n}=[\mathrm{x} 29, \mathrm{y} 14, \mathrm{y} 15, \mathrm{y} 16, \mathrm{y} 17, \mathrm{y} 18, \mathrm{y} 19, \mathrm{y} 20, \mathrm{y} 21, \mathrm{y} 22, \mathrm{y} 23, \mathrm{y} 24, \mathrm{y} 25, \mathrm{y} 26, \mathrm{y} 27, \mathrm{y} 28]$;

end

$[A 2(j, 1), A 2(j, 2)]=$ curveintersect $(m(:, j), n(:, j), x 12, y 12)$;

for $j=1: 16$

$\mathrm{m}=[\mathrm{y} 29, \mathrm{x} 14, \mathrm{x} 15, \mathrm{x} 16, \mathrm{x} 17, \mathrm{x} 18, \mathrm{x} 19, \mathrm{x} 20, \mathrm{x} 21, \mathrm{x} 22, \mathrm{x} 23, \mathrm{x} 24, \mathrm{x} 25, \mathrm{x} 26, \mathrm{x} 27, \mathrm{x} 28]$; $\mathrm{n}=[\mathrm{x} 29, \mathrm{y} 14, \mathrm{y} 15, \mathrm{y} 16, \mathrm{y} 17, \mathrm{y} 18, \mathrm{y} 19, \mathrm{y} 20, \mathrm{y} 21, \mathrm{y} 22, \mathrm{y} 23, \mathrm{y} 24, \mathrm{y} 25, \mathrm{y} 26, \mathrm{y} 27, \mathrm{y} 28] ;$

end

$[A 3(j, 1), A 3(j, 2)]=$ curveintersect $(m(:, j), n(:, j), x 11, y 11)$;

for $j=1: 16$

$m=[y 29, x 14, x 15, x 16, x 17, x 18, x 19, x 20, x 21, x 22, x 23, x 24, x 25, x 26, x 27, x 28]$;

$n=[x 29, y 14, y 15, y 16, y 17, y 18, y 19, y 20, y 21, y 22, y 23, y 24, y 25, y 26, y 27, y 28] ;$

end

$[A 4(j, 1), A 4(j, 2)]=$ curveintersect $(m(:, j), n(:, j), x 10, y 10)$;

for $j=1: 16$

$\mathrm{m}=[\mathrm{y} 29, \mathrm{x} 14, \mathrm{x} 15, \mathrm{x} 16, \mathrm{x} 17, \mathrm{x} 18, \mathrm{x} 19, \mathrm{x} 20, \mathrm{x} 21, \mathrm{x} 22, \mathrm{x} 23, \mathrm{x} 24, \mathrm{x} 25, \mathrm{x} 26, \mathrm{x} 27, \mathrm{x} 28]$;

$n=[x 29, y 14, y 15, y 16, y 17, y 18, y 19, y 20, y 21, y 22, y 23, y 24, y 25, y 26, y 27, y 28] ;$

end

$[A 5(j, 1), A 5(j, 2)]=$ curveintersect $(m(:, j), n(:, j), x 9, y 9)$;

for $j=1: 16$

$m=[y 29, x 14, x 15, x 16, x 17, x 18, x 19, x 20, x 21, x 22, x 23, x 24, x 25, x 26, x 27, x 28]$;

$\mathrm{n}=[\mathrm{x} 29, \mathrm{y} 14, \mathrm{y} 15, \mathrm{y} 16, \mathrm{y} 17, \mathrm{y} 18, \mathrm{y} 19, \mathrm{y} 20, \mathrm{y} 21, \mathrm{y} 22, \mathrm{y} 23, \mathrm{y} 24, \mathrm{y} 25, \mathrm{y} 26, \mathrm{y} 27, \mathrm{y} 28]$;

end

$[A 6(j, 1), A 6(j, 2)]=$ curveintersect $(m(:, j), n(:, j), x 8, y 8)$;

for $j=1: 16$

$\mathrm{m}=[\mathrm{y} 29, \mathrm{x} 14, \mathrm{x} 15, \mathrm{x} 16, \mathrm{x} 17, \mathrm{x} 18, \mathrm{x} 19, \mathrm{x} 20, \mathrm{x} 21, \mathrm{x} 22, \mathrm{x} 23, \mathrm{x} 24, \mathrm{x} 25, \mathrm{x} 26, \mathrm{x} 27, \mathrm{x} 28]$; $\mathrm{n}=[\mathrm{x} 29, \mathrm{y} 14, \mathrm{y} 15, \mathrm{y} 16, \mathrm{y} 17, \mathrm{y} 18, \mathrm{y} 19, \mathrm{y} 20, \mathrm{y} 21, \mathrm{y} 22, \mathrm{y} 23, \mathrm{y} 24, \mathrm{y} 25, \mathrm{y} 26, \mathrm{y} 27, \mathrm{y} 28]$;

end $[A 7(j, 1), A 7(j, 2)]=$ curveintersect $(m(:, j), n(:, j), x 7, y 7)$;

for $j=1: 16$

$m=[y 29, x 14, x 15, x 16, x 17, x 18, x 19, x 20, x 21, x 22, x 23, x 24, x 25, x 26, x 27, x 28]$;

$n=[x 29, y 14, y 15, y 16, y 17, y 18, y 19, y 20, y 21, y 22, y 23, y 24, y 25, y 26, y 27, y 28] ;$

end

$[A 8(j, 1), A 8(j, 2)]=$ curveintersect $\left(m(:, j), n(:, j), x 6, y^{6}\right)$;

for $j=1: 15$

$\mathrm{m}=[\mathrm{y} 29, \mathrm{x} 15, \mathrm{x} 16, \mathrm{x} 17, \mathrm{x} 18, \mathrm{x} 19, \mathrm{x} 20, \mathrm{x} 21, \mathrm{x} 22, \mathrm{x} 23, \mathrm{x} 24, \mathrm{x} 25, \mathrm{x} 26, \mathrm{x} 27, \mathrm{x} 28]$;

$\mathrm{n}=[\mathrm{x} 29, \mathrm{y} 15, \mathrm{y} 16, \mathrm{y} 17, \mathrm{y} 18, \mathrm{y} 19, \mathrm{y} 20, \mathrm{y} 21, \mathrm{y} 22, \mathrm{y} 23, \mathrm{y} 24, \mathrm{y} 25, \mathrm{y} 26, \mathrm{y} 27, \mathrm{y} 28] ;$

$[A 9(j, 1), A 9(j, 2)]=$ curveintersect $(m(:, j), n(:, j), x 5, y 5)$;

end 


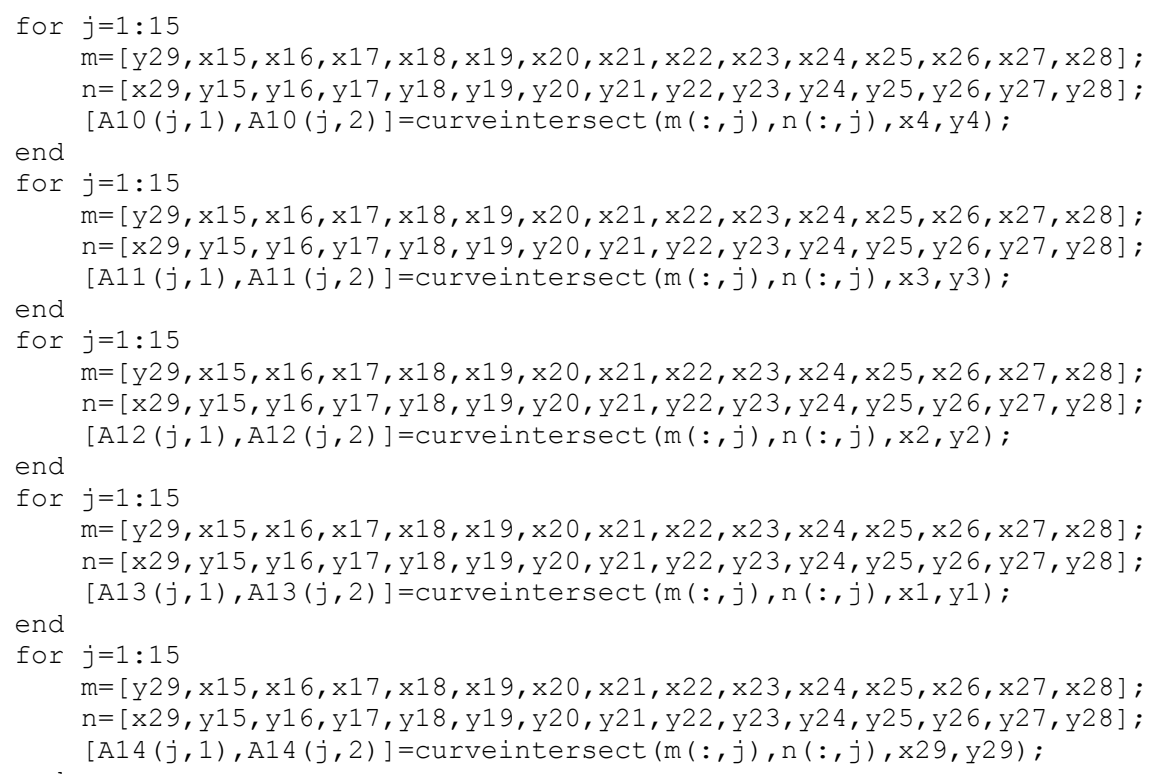


$\operatorname{srt}=-(t 1 / 2) *\left(1-\left(3 /\left(r \cdot^{\wedge} 4\right)\right)+\left(2 /\left(r \cdot^{\wedge} 2\right)\right)\right) * \sin (2 * t)+(t 2 / 2) *(1-$ $(3 /(r \cdot \wedge 4))+(2 /(r \cdot \wedge 2))) * \sin (2 * t) ;$

$\operatorname{sx}=\operatorname{sr} \cdot{ }^{*}(\cos (t)) \cdot{ }^{*} 2-2{ }^{*} \operatorname{srt} .{ }^{*} \cos (t){ }^{*} \sin (t)+\operatorname{st} \cdot{ }^{*}(\sin (t)) \cdot{ }^{\wedge} 2$

$\operatorname{sy}=s t .{ }^{*}(\cos (t)) \cdot{ }^{\wedge} 2+\operatorname{sr} .{ }^{*}(\sin (t)) \cdot{ }^{\wedge} 2+\operatorname{srt} .{ }^{*} \sin (2 * t)$;

txy $=0.5 *\left(\left(2 * \operatorname{srt} .{ }^{*} \cos (2 * t)+(\operatorname{sr}-\operatorname{st}) \cdot{ }^{*} \sin (2 * t)\right)\right) ;$

$d y d x=-(s x-s y) /(2 * t x y)+\operatorname{sqrt}(1+((s x-s y) /(2 * t x y)) \wedge 2)$;

\section{Unequal Biaxial Compression Medium Topology}

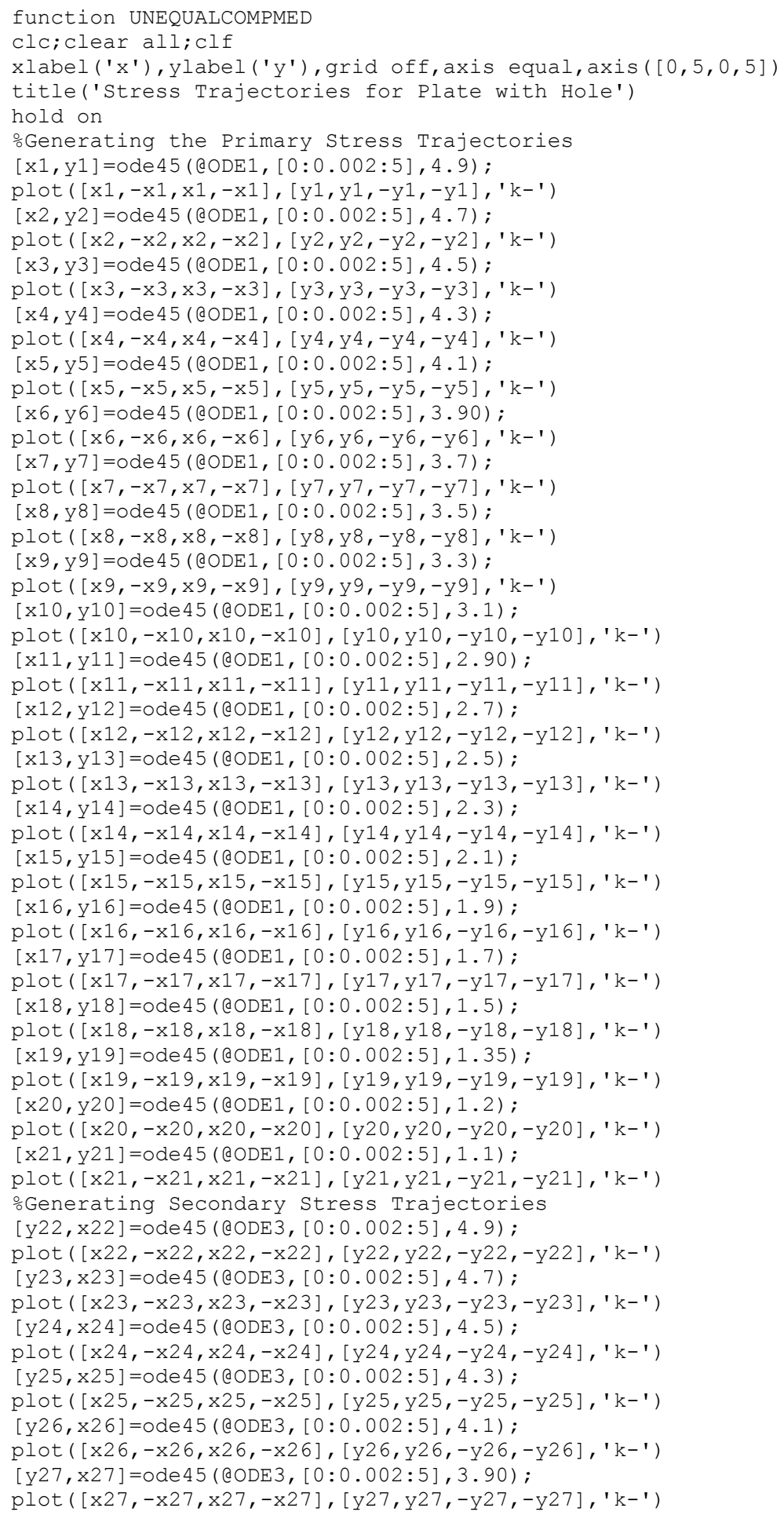


$[y 28, x 28]=0$ de $45($ @ODE $3,[0: 0.002: 5], 3.7)$;

plot $([x 28,-x 28, x 28,-x 28],[y 28, y 28,-y 28,-y 28], ' k-')$

$[y 29, x 29]=0$ de $45($ @ODE $3,[0: 0.002: 5], 3.5)$; o\# ok<*NBRAK>

plot $\left([x 29,-x 29, x 29,-x 29],[y 29, y 29,-y 29,-y 29], k^{\prime}{ }^{\prime}\right)$

$[y 30, x 30]=0 d e 45(\operatorname{QodE} 3,[0: 0.002: 5], 3.3)$;

plot $([x 30,-x 30, x 30,-x 30],[y 30, y 30,-y 30,-y 30], ' k-')$

$[y 31, x 31]=0 d e 45(@ O D E 3,[0: 0.002: 5], 3.1)$;

plot $([x 31,-x 31, x 31,-x 31],[y 31, y 31,-y 31,-y 31], ' k-')$

$[y 32, x 32]=0 d e 45($ @ODE $3,[0: 0.002: 5], 2.90)$;

plot $\left([x 32,-x 32, x 32,-x 32],[y 32, y 32,-y 32,-y 32], '-^{\prime}\right)$

$[y 33, x 33]=0 d e 45(@ O D E 3,[0: 0.002: 5], 2.7)$;

plot $\left([x 33,-x 33, x 33,-x 33],[y 33, y 33,-y 33,-y 33],{ }^{\prime} k-^{\prime}\right)$

[y34,x34] =ode45 (@ODE $3,[0: 0.002: 5], 2.5)$;

plot $\left([x 34,-x 34, x 34,-x 34],[y 34, y 34,-y 34,-y 34], k^{\prime}-^{\prime}\right)$

$[y 35, x 35]=0$ de $45(@ O D E 3,[0: 0.002: 5], 2.2)$;

plot $([x 35,-x 35, x 35,-x 35],[y 35, y 35,-y 35,-y 35], ' k-')$

$[\mathrm{x} 36, \mathrm{y} 36]=0 \mathrm{de} 45(\mathrm{\varrho ODE} 2,[0.9993: 0.002: 5.9993], 0.0348) ; \% 0.5$

plot $([x 36,-x 36, x 36,-x 36],[y 36, y 36,-y 36,-y 36], ' k-')$

$[\mathrm{x} 37, \mathrm{y} 37]=0 \mathrm{de} 45(\mathrm{QODE} 2,[0.984: 0.002: 5.984], 0.173)$;

plot $([x 37,-x 37, x 37,-x 37],[y 37, y 37,-y 37,-y 37], ' k-')$

$[\mathrm{x} 38, \mathrm{y} 38]=0 \mathrm{de} 45(\mathrm{CODE} 2,[0.939: 0.002: 5.939], 0.342)$;

plot $\left([x 38,-x 38, x 38,-x 38],[y 38, y 38,-y 38,-y 38], k^{-\prime}\right)$

$[\mathrm{x} 39, \mathrm{y} 39]=0 \mathrm{de} 45(\mathrm{QODE} 2,[0.866: 0.002: 5.866], 0.500)$;

plot $\left([x 39,-x 39, x 39,-x 39],[y 39, y 39,-y 39,-y 39], k^{-\prime}\right)$

$[\mathrm{x} 40, \mathrm{y} 40]=0 \mathrm{de} 45(\mathrm{QODE} 2,[0.642: 0.002: 5.642], 0.766)$;

plot $\left([\mathrm{x} 40,-\mathrm{x} 40, \mathrm{x} 40,-\mathrm{x} 40],[\mathrm{y} 40, \mathrm{y} 40,-\mathrm{y} 40,-\mathrm{y} 40], \mathrm{k}^{-\prime}\right)$

$[\mathrm{x} 41, \mathrm{y} 41]=0 \mathrm{de} 45(\mathrm{\varrho ODE} 2,[0.766: 0.002: 5.766], 0.642)$;

plot $\left([\mathrm{x} 41,-\mathrm{x} 41, \mathrm{x} 41,-\mathrm{x} 41],[\mathrm{y} 41, \mathrm{y} 41,-\mathrm{y} 41,-\mathrm{y} 41], \mathrm{k}^{-1}\right)$

$[\mathrm{x} 42, \mathrm{y} 42]=0 \mathrm{de} 45(\mathrm{QODE} 2,[0.500: 0.002: 5.500], 0.866)$;

plot $\left([x 42,-x 42, x 42,-x 42],[y 42, y 42,-y 42,-y 42], ' k^{\prime} '\right)$

$[\mathrm{x} 43, \mathrm{y} 43]=0 \mathrm{de} 45(\mathrm{@ODE} 2,[0.342: 0.002: 5.342], 0.939)$;

plot $([x 43,-x 43, x 43,-x 43],[y 43, y 43,-y 43,-y 43], ' k-1)$

$[\mathrm{x} 44, \mathrm{y} 44]=0 \mathrm{de} 45(\mathrm{\varrho ODE} 2,[0.173: 0.002: 5.173], 0.984)$;

plot $\left([x 44,-x 44, x 44,-x 44],[y 44, y 44,-y 44,-y 44], k^{\prime}\right)$

$\mathrm{x} 45=[0: 0.002: 5]^{\prime}$;

$\mathrm{y} 45=\mathrm{x} 45 \cdot * 0+5$

plot $(x 45, y 45, ' k-1)$

plot $\left(-x 45, y 45, k^{\prime}-'\right)$

plot $\left(x 45,-y 45,{ }^{\prime}-^{\prime}\right)$

plot $\left(-x 45,-y 45,{ }^{\prime} k-'\right)$

plot $\left(y 45, x 45,{ }^{\prime} k-'\right)$

plot $(-y 45, x 45, ' k-')$

plot $\left(y 45,-x 45,{ }^{\prime} k-'\right)$

plot $\left(-y 45,-x 45,{ }^{\prime} k-'\right)$

$\mathrm{x}=1: .002: 5$;

$\mathrm{y}=\mathrm{x} \cdot{ }^{*} 0$;

plot $(x, y, ' k-')$

$\operatorname{plot}\left(-x, y,{ }^{\prime} k-1\right)$

$\operatorname{plot}\left(\mathrm{y}, \mathrm{x}, \mathrm{\prime}^{\mathrm{k}-')}\right.$

plot $\left(y,-x, k^{\prime}-^{\prime}\right)$

$\mathrm{x}=0: .01: 1$;

$\mathrm{y}=\operatorname{squt}\left(1-\mathrm{x} \cdot{ }^{\wedge} 2\right) ;$

plot $\left(x, y,{ }^{-}-^{\prime}\right.$ ' 'Linewidth', 1.5)

plot $\left(-x, y,{ }^{\prime}-{ }^{\prime}\right.$ ' 'Linewidth', 1.5$)$

plot $\left(x,-y,{ }^{\prime}-^{\prime}\right.$ ', 'Linewidth', 1.5)

plot $\left(-x,-y,{ }^{\prime}-'\right.$ ' ' Linewidth' , 1.5)

for $j=1: 9$

$m=[x 36, x 37, x 38, x 39, x 40, x 41, x 42, x 43, x 44]$ $\mathrm{n}=[\mathrm{y} 36, \mathrm{y} 37, \mathrm{y} 38, \mathrm{y} 39, \mathrm{y} 40, \mathrm{y} 41, \mathrm{y} 42, \mathrm{y} 43, \mathrm{y} 44]$

$[A 1(j, 1), A 1(j, 2)]=$ curveintersect $(m(:, j), n(:, j), x 21, y 21)$;

end

for $j=1: 9$

$m=[x 36, x 37, x 38, x 39, x 40, x 41, x 42, x 43, x 44]$

$\mathrm{n}=[\mathrm{y} 36, \mathrm{y} 37, \mathrm{y} 38, \mathrm{y} 39, \mathrm{y} 40, \mathrm{y} 41, \mathrm{y} 42, \mathrm{y} 43, \mathrm{y} 44]$

end

$[A 2(j, 1), A 2(j, 2)]=$ curveintersect $(m(:, j), n(:, j), x 20, y 20)$;

for $j=1: 24$

$m=[y 45, x 22, x 23, x 24, x 25, x 26, x 27, x 28, x 29, x 30, x 31, x 32, x 33, x 34, x 35, x 36, x 37, x 38, x 39, x 40$, $\mathrm{x} 41, \mathrm{x} 42, \mathrm{x} 43, \mathrm{x} 44]$;

$n=[x 45, y 22, y 23, y 24, y 25, y 26, y 27, y 28, y 29, y 30, y 31, y 32, y 33, y 34, y 35, y 36, y 37, y 38, y 39, y 40$, $\mathrm{y} 41, \mathrm{y} 42, \mathrm{y} 43, \mathrm{y} 44]$ 
$[A 3(j, 1), A 3(j, 2)]=$ curveintersect $(m(:, j), n(:, j), x 19, y 19) ;$

end

for $j=1: 24$

$m=[y 45, x 22, x 23, x 24, x 25, x 26, x 27, x 28, x 29, x 30, x 31, x 32, x 33, x 34, x 35, x 36, x 37, x 38, x 39, x 40$, $\mathrm{x} 41, \mathrm{x} 42, \mathrm{x} 43, \mathrm{x} 44]$;

$n=[x 45, y 22, y 23, y 24, y 25, y 26, y 27, y 28, y 29, y 30, y 31, y 32, y 33, y 34, y 35, y 36, y 37, y 38, y 39, y 40$, $\mathrm{y} 41, \mathrm{y} 42, \mathrm{y} 43, \mathrm{y} 44]$

end

$[A 4(j, 1), A 4(j, 2)]=$ curveintersect $(m(:, j), n(:, j), x 18, y 18)$;

for $j=1: 24$

$m=[y 45, x 22, x 23, x 24, x 25, x 26, x 27, x 28, x 29, x 30, x 31, x 32, x 33, x 34, x 35, x 36, x 37, x 38, x 39, x 40$, $\mathrm{x} 41, \mathrm{x} 42, \mathrm{x} 43, \mathrm{x} 44]$

$n=[x 45, y 22, y 23, y 24, y 25, y 26, y 27, y 28, y 29, y 30, y 31, y 32, y 33, y 34, y 35, y 36, y 37, y 38, y 39, y 40$, $\mathrm{y} 41, \mathrm{y} 42, \mathrm{y} 43, \mathrm{y} 44]$

end

$[A 5(j, 1), A 5(j, 2)]=$ curveintersect $(m(:, j), n(:, j), x 17, y 17) ; \quad \% \# \circ k<{ }^{*} A G R O W>$

for $j=1: 24$

$m=[y 45, x 22, x 23, x 24, x 25, x 26, x 27, x 28, x 29, x 30, x 31, x 32, x 33, x 34, x 35, x 36, x 37, x 38, x 39, x 40$, $\mathrm{x} 41, \mathrm{x} 42, \mathrm{x} 43, \mathrm{x} 44]$

$n=[x 45, y 22, y 23, y 24, y 25, y 26, y 27, y 28, y 29, y 30, y 31, y 32, y 33, y 34, y 35, y 36, y 37, y 38, y 39, y 40$, $\mathrm{y} 41, \mathrm{y} 42, \mathrm{y} 43, \mathrm{y} 44]$

end

$[A 6(j, 1), A 6(j, 2)]=$ curveintersect $(m(:, j), n(:, j), x 16, y 16) ;$

for $j=1: 24$

$m=[y 45, x 22, x 23, x 24, x 25, x 26, x 27, x 28, x 29, x 30, x 31, x 32, x 33, x 34, x 35, x 36, x 37, x 38, x 39, x 40$, $\times 41, x 42, x 43, x 44]$

$n=[x 45, y 22, y 23, y 24, y 25, y 26, y 27, y 28, y 29, y 30, y 31, y 32, y 33, y 34, y 35, y 36, y 37, y 38, y 39, y 40$, $\mathrm{y} 41, \mathrm{y} 42, \mathrm{y} 43, \mathrm{y} 44]$

end

$[A 7(j, 1), A 7(j, 2)]=$ curveintersect $(m(:, j), n(:, j), x 15, y 15)$;

for $j=1: 24$

$m=[y 45, x 22, x 23, x 24, x 25, x 26, x 27, x 28, x 29, x 30, x 31, x 32, x 33, x 34, x 35, x 36, x 37, x 38, x 39, x 40$, $x 41, x 42, x 43, x 44]$

$\mathrm{n}=[\mathrm{x} 45, \mathrm{y} 22, \mathrm{y} 23, \mathrm{y} 24, \mathrm{y} 25, \mathrm{y} 26, \mathrm{y} 27, \mathrm{y} 28, \mathrm{y} 29, \mathrm{y} 30, \mathrm{y} 31, \mathrm{y} 32, \mathrm{y} 33, \mathrm{y} 34, \mathrm{y} 35, \mathrm{y} 36, \mathrm{y} 37, \mathrm{y} 38, \mathrm{y} 39, \mathrm{y} 40$, $\mathrm{y} 41, \mathrm{y} 42, \mathrm{y} 43, \mathrm{y} 44]$

end

$[A 8(j, 1), A 8(j, 2)]=$ curveintersect $(m(:, j), n(:, j), x 14, y 14)$;

for $j=1: 23$

$m=[y 45, x 23, x 24, x 25, x 26, x 27, x 28, x 29, x 30, x 31, x 32, x 33, x 34, x 35, x 36, x 37, x 38, x 39, x 40, x 41$, $\mathrm{x} 42, \mathrm{x} 43, \mathrm{x} 44]$;

$\mathrm{n}=[\mathrm{x} 45, \mathrm{y} 23, \mathrm{y} 24, \mathrm{y} 25, \mathrm{y} 26, \mathrm{y} 27, \mathrm{y} 28, \mathrm{y} 29, \mathrm{y} 30, \mathrm{y} 31, \mathrm{y} 32, \mathrm{y} 33, \mathrm{y} 34, \mathrm{y} 35, \mathrm{y} 36, \mathrm{y} 37, \mathrm{y} 38, \mathrm{y} 39, \mathrm{y} 40, \mathrm{y} 41$, $\mathrm{y} 42, \mathrm{y} 43, \mathrm{y} 44]$;

end

$[A 9(j, 1), A 9(j, 2)]=$ curveintersect $(m(:, j), n(:, j), x 13, y 13)$;

for $j=1: 23$

$m=[y 45, x 23, x 24, x 25, x 26, x 27, x 28, x 29, x 30, x 31, x 32, x 33, x 34, x 35, x 36, x 37, x 38, x 39, x 40, x 41$, $\mathrm{x} 42, \mathrm{x} 43, \mathrm{x} 44]$;

$n=[x 45, y 23, y 24, y 25, y 26, y 27, y 28, y 29, y 30, y 31, y 32, y 33, y 34, y 35, y 36, y 37, y 38, y 39, y 40, y 41$, $\mathrm{y} 42, \mathrm{y} 43, \mathrm{y} 44] ;$

end

$[A 10(j, 1), A 10(j, 2)]=$ curveintersect $(m(:, j), n(:, j), x 12, y 12)$;

for $j=1: 23$

$m=[y 45, x 23, x 24, x 25, x 26, x 27, x 28, x 29, x 30, x 31, x 32, x 33, x 34, x 35, x 36, x 37, x 38, x 39, x 40, x 41$, $x 42, x 43, x 44]$

$n=[x 45, y 23, y 24, y 25, y 26, y 27, y 28, y 29, y 30, y 31, y 32, y 33, y 34, y 35, y 36, y 37, y 38, y 39, y 40, y 41$, $\mathrm{y} 42, \mathrm{y} 43, \mathrm{y} 44] ;$

end

$[A 11(j, 1), A 11(j, 2)]=$ curveintersect $(m(:, j), n(:, j), x 11, y 11)$;

for $j=1: 23$

$m=[y 45, x 23, x 24, x 25, x 26, x 27, x 28, x 29, x 30, x 31, x 32, x 33, x 34, x 35, x 36, x 37, x 38, x 39, x 40, x 41$, $\mathrm{x} 42, \mathrm{x} 43, \mathrm{x} 44]$;

$\mathrm{n}=[\mathrm{x} 45, \mathrm{y} 23, \mathrm{y} 24, \mathrm{y} 25, \mathrm{y} 26, \mathrm{y} 27, \mathrm{y} 28, \mathrm{y} 29, \mathrm{y} 30, \mathrm{y} 31, \mathrm{y} 32, \mathrm{y} 33, \mathrm{y} 34, \mathrm{y} 35, \mathrm{y} 36, \mathrm{y} 37, \mathrm{y} 38, \mathrm{y} 39, \mathrm{y} 40, \mathrm{y} 41$, $\mathrm{y} 42, \mathrm{Y} 43, \mathrm{y} 44] ;$

end

$[A 12(j, 1), A 12(j, 2)]=$ curveintersect $(m(:, j), n(:, j), x 10, y 10)$;

for $j=1: 23$

$m=[y 45, x 23, x 24, x 25, x 26, x 27, x 28, x 29, x 30, x 31, x 32, x 33, x 34, x 35, x 36, x 37, x 38, x 39, x 40, x 41$, $\mathrm{x} 42, \mathrm{x} 43, \mathrm{x} 44]$

$\mathrm{n}=[\mathrm{x} 45, \mathrm{y} 23, \mathrm{y} 24, \mathrm{y} 25, \mathrm{y} 26, \mathrm{y} 27, \mathrm{y} 28, \mathrm{y} 29, \mathrm{y} 30, \mathrm{y} 31, \mathrm{y} 32, \mathrm{y} 33, \mathrm{y} 34, \mathrm{y} 35, \mathrm{y} 36, \mathrm{y} 37, \mathrm{y} 38, \mathrm{y} 39, \mathrm{y} 40, \mathrm{y} 41$, $\mathrm{y} 42, \mathrm{y} 43, \mathrm{y} 44]$

$[A 13(j, 1), A 13(j, 2)]=$ curveintersect $\left(m(:, j), n(:, j), x 9, y^{9}\right)$; 
end

for $j=1: 23$

$m=[y 45, x 23, x 24, x 25, x 26, x 27, x 28, x 29, x 30, x 31, x 32, x 33, x 34, x 35, x 36, x 37, x 38, x 39, x 40, x 41$, $\mathrm{x} 42, \mathrm{x} 43, \mathrm{x} 44] ;$

$n=[x 45, y 23, y 24, y 25, y 26, y 27, y 28, y 29, y 30, y 31, y 32, y 33, y 34, y 35, y 36, y 37, y 38, y 39, y 40, y 41$, $\mathrm{y} 42, \mathrm{y} 43, \mathrm{y} 44]$

end

$[A 14(j, 1), A 14(j, 2)]=$ curveintersect $(m(:, j), n(:, j), x 8, y 8)$;

for $j=1: 23$

$m=[y 45, x 23, x 24, x 25, x 26, x 27, x 28, x 29, x 30, x 31, x 32, x 33, x 34, x 35, x 36, x 37, x 38, x 39, x 40, x 41$, $x 42, x 43, x 44]$

$\mathrm{n}=[\mathrm{x} 45, \mathrm{y} 23, \mathrm{y} 24, \mathrm{y} 25, \mathrm{y} 26, \mathrm{y} 27, \mathrm{y} 28, \mathrm{y} 29, \mathrm{y} 30, \mathrm{y} 31, \mathrm{y} 32, \mathrm{y} 33, \mathrm{y} 34, \mathrm{y} 35, \mathrm{y} 36, \mathrm{y} 37, \mathrm{y} 38, \mathrm{y} 39, \mathrm{y} 40, \mathrm{y} 41$, $\mathrm{y} 42, \mathrm{y} 43, \mathrm{y} 44]$

end

$[A 15(j, 1), A 15(j, 2)]=$ curveintersect $(m(:, j), n(:, j), x 7, y 7)$;

for $j=1: 23$

$m=[y 45, x 23, x 24, x 25, x 26, x 27, x 28, x 29, x 30, x 31, x 32, x 33, x 34, x 35, x 36, x 37, x 38, x 39, x 40, x 41$, $\times 42, x 43, x 44]$;

$n=[x 45, y 23, y 24, y 25, y 26, y 27, y 28, y 29, y 30, y 31, y 32, y 33, y 34, y 35, y 36, y 37, y 38, y 39, y 40, y 41$, $\mathrm{y} 42, \mathrm{y} 43, \mathrm{y} 44]$;

end

$[A 16(j, 1), A 16(j, 2)]=$ curveintersect $(m(:, j), n(:, j), x 6, y 6)$;

for $j=1: 23$

$m=[y 45, x 23, x 24, x 25, x 26, x 27, x 28, x 29, x 30, x 31, x 32, x 33, x 34, x 35, x 36, x 37, x 38, x 39, x 40, x 41$, $x 42, x 43, x 44]$

$n=[x 45, y 23, y 24, y 25, y 26, y 27, y 28, y 29, y 30, y 31, y 32, y 33, y 34, y 35, y 36, y 37, y 38, y 39, y 40, y 41$, $\mathrm{y} 42, \mathrm{y} 43, \mathrm{y} 44] ;$

end

$[A 17(j, 1), A 17(j, 2)]=$ curveintersect $(m(:, j), n(:, j), x 5, y 5)$

for $j=1: 23$

$m=[y 45, x 23, x 24, x 25, x 26, x 27, x 28, x 29, x 30, x 31, x 32, x 33, x 34, x 35, x 36, x 37, x 38, x 39, x 40, x 41$, $\mathrm{x} 42, \mathrm{x} 43, \mathrm{x} 44]$

$n=[x 45, y 23, y 24, y 25, y 26, y 27, y 28, y 29, y 30, y 31, y 32, y 33, y 34, y 35, y 36, y 37, y 38, y 39, y 40, y 41$, $\mathrm{y} 42, \mathrm{y} 43, \mathrm{y} 44] ;$

end

$[A 18(j, 1), A 18(j, 2)]=$ curveintersect $\left(m(:, j), n(:, j), x 4, y^{4}\right)$;

for $j=1: 23$

$m=[y 45, x 23, x 24, x 25, x 26, x 27, x 28, x 29, x 30, x 31, x 32, x 33, x 34, x 35, x 36, x 37, x 38, x 39, x 40, x 41$, $\mathrm{x} 42, \mathrm{x} 43, \mathrm{x} 44]$

$\mathrm{n}=[\mathrm{x} 45, \mathrm{y} 23, \mathrm{y} 24, \mathrm{y} 25, \mathrm{y} 26, \mathrm{y} 27, \mathrm{y} 28, \mathrm{y} 29, \mathrm{y} 30, \mathrm{y} 31, \mathrm{y} 32, \mathrm{y} 33, \mathrm{y} 34, \mathrm{y} 35, \mathrm{y} 36, \mathrm{y} 37, \mathrm{y} 38, \mathrm{y} 39, \mathrm{y} 40, \mathrm{y} 41$, $\mathrm{y} 42, \mathrm{y} 43, \mathrm{y} 44]$ end

$[A 19(j, 1), A 19(j, 2)]=$ curveintersect $(m(:, j), n(:, j), x 3, y 3)$;

for $j=1: 23$

$\mathrm{m}=[\mathrm{y} 45, \mathrm{x} 23, \mathrm{x} 24, \mathrm{x} 25, \mathrm{x} 26, \mathrm{x} 27, \mathrm{x} 28, \mathrm{x} 29, \mathrm{x} 30, \mathrm{x} 31, \mathrm{x} 32, \mathrm{x} 33, \mathrm{x} 34, \mathrm{x} 35, \mathrm{x} 36, \mathrm{x} 37, \mathrm{x} 38, \mathrm{x} 39, \mathrm{x} 40, \mathrm{x} 41$, $\mathrm{x} 42, \mathrm{x} 43, \mathrm{x} 44]$

$n=[x 45, y 23, y 24, y 25, y 26, y 27, y 28, y 29, y 30, y 31, y 32, y 33, y 34, y 35, y 36, y 37, y 38, y 39, y 40, y 41$, $\mathrm{y} 42, \mathrm{y} 43, \mathrm{y} 44]$

end

$[A 20(j, 1), A 20(j, 2)]=$ curveintersect $(m(:, j), n(:, j), x 2, y 2)$;

for $j=1: 23$

$m=[y 45, x 23, x 24, x 25, x 26, x 27, x 28, x 29, x 30, x 31, x 32, x 33, x 34, x 35, x 36, x 37, x 38, x 39, x 40, x 41$, $\mathrm{x} 42, \mathrm{x} 43, \mathrm{x} 44]$;

$\mathrm{n}=[\mathrm{x} 45, \mathrm{y} 23, \mathrm{y} 24, \mathrm{y} 25, \mathrm{y} 26, \mathrm{y} 27, \mathrm{y} 28, \mathrm{y} 29, \mathrm{y} 30, \mathrm{y} 31, \mathrm{y} 32, \mathrm{y} 33, \mathrm{y} 34, \mathrm{y} 35, \mathrm{y} 36, \mathrm{y} 37, \mathrm{y} 38, \mathrm{y} 39, \mathrm{y} 40, \mathrm{y} 41$, $\mathrm{y} 42, \mathrm{y} 43, \mathrm{y} 44]$

end

$[A 21(j, 1), A 21(j, 2)]=$ curveintersect $(m(:, j), n(:, j), x 1, y 1)$;

for $j=1: 23$

$m=[y 45, x 23, x 24, x 25, x 26, x 27, x 28, x 29, x 30, x 31, x 32, x 33, x 34, x 35, x 36, x 37, x 38, x 39, x 40, x 41$, $\mathrm{x} 42, \mathrm{x} 43, \mathrm{x} 44]$;

$\mathrm{n}=[\mathrm{x} 45, \mathrm{y} 23, \mathrm{y} 24, \mathrm{y} 25, \mathrm{y} 26, \mathrm{y} 27, \mathrm{y} 28, \mathrm{y} 29, \mathrm{y} 30, \mathrm{y} 31, \mathrm{y} 32, \mathrm{y} 33, \mathrm{y} 34, \mathrm{y} 35, \mathrm{y} 36, \mathrm{y} 37, \mathrm{y} 38, \mathrm{y} 39, \mathrm{y} 40, \mathrm{y} 41$, $\mathrm{y} 42, \mathrm{y} 43, \mathrm{y} 44] ;$ end $[A 22(j, 1), A 22(j, 2)]=$ curveintersect $(m(:, j), n(:, j), x 45, y 45)$;

$\mathrm{A} 23=[0,5 ; 0,4.9 ; 0,4.7 ; 0,4.5 ; 0,4.3 ; 0,4.1 ; 0,3.9 ; 0,3.7 ; 0,3.5 ; 0,3.3 ; 0,3.1 ; 0,2.9 ; 0,2.7 ; 0$, $2.5 ; 0,2.3 ; 0,2.1 ; 0,1.9 ; 0,1.7 ; 0,1.5 ; 0,1.3 ; 0,1.2 ; 0,1.1 ; 5,0 ; 4.9,0 ; 4.7,0 ; 4.5,0 ; 4.3,0 ; 4.1$ , $0 ; 3.9,0 ; 3.7,0 ; 3.5,0 ; 3.3,0 ; 3.1,0 ; 2.9,0 ; 2.7,0 ; 2.5,0 ; 2.2,0 ; 1.874,0 ; 1.41,0 ; 1,0 ; 0,1 ; .17$ $3, .984 ; .984, .173 ; .342, .939 ; .939, .342 ; .866, .5 ; .5, .866 ; .766, .642 ; .642, .766] ;$ $\mathrm{A}=[\mathrm{A} 1 ; \mathrm{A} 2 ; \mathrm{A} 3 ; \mathrm{A} 4 ; \mathrm{A} 5 ; \mathrm{A} 6 ; \mathrm{A} 7 ; \mathrm{A} 8 ; \mathrm{A} 9 ; \mathrm{A} 10 ; \mathrm{A} 11 ; \mathrm{A} 12 ; \mathrm{A} 13 ; \mathrm{A} 14 ; \mathrm{A} 15 ; \mathrm{A} 16 ; \mathrm{A} 17 ; \mathrm{A} 18 ; \mathrm{A} 19 ; \mathrm{A} 20 ; \mathrm{A} 21 ; \mathrm{A} 22 ; \mathrm{A}$ 23] $\% \# O k<N O P R T>$

$\operatorname{plot}([A(:, 1),-A(:, 1), A(:, 1),-A(:, 1)],[A(:, 2),-A(:, 2),-A(:, 2), A(:, 2)], ' m * ')$ 


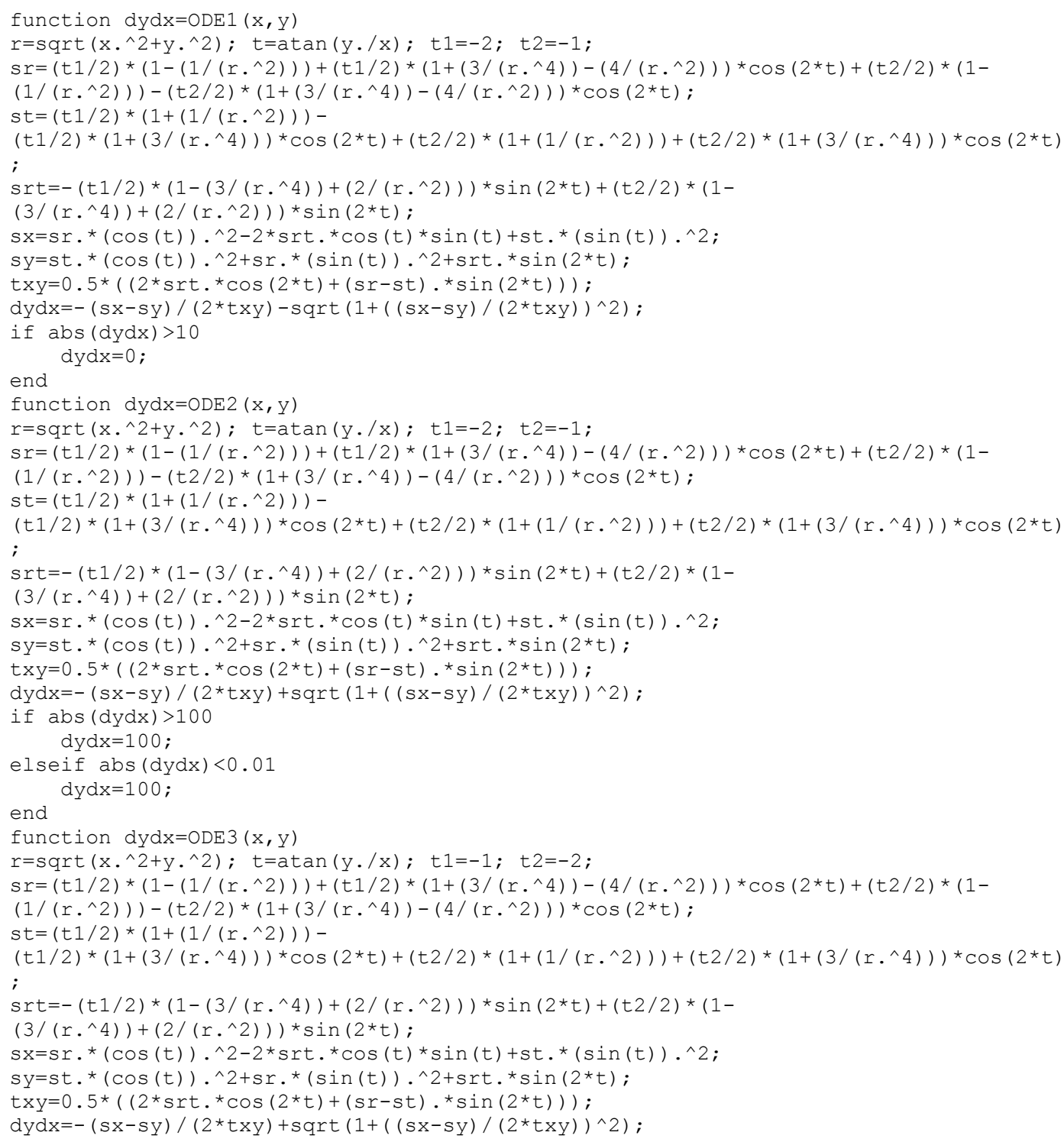

\section{Unequal Biaxial Compression Fine Topology}

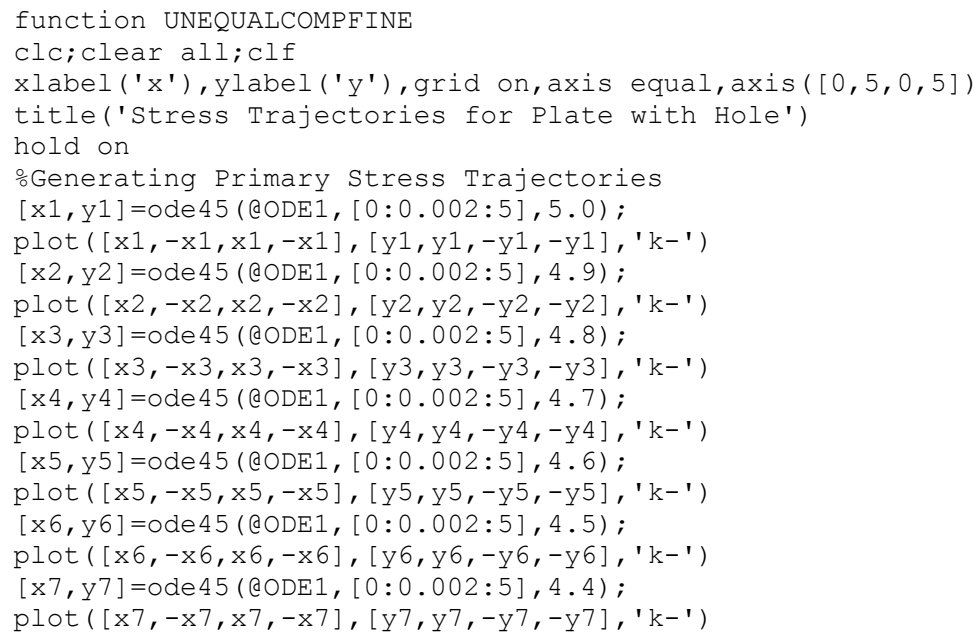


$[\mathrm{x} 8, \mathrm{y} 8]=0 \operatorname{de} 45(\mathrm{QODE} 1,[0: 0.002: 5], 4.3)$;

plot $([x 8,-x 8, x 8,-x 8],[y 8, y 8,-y 8,-y 8], ' k-1)$

$[\mathrm{x} 9, \mathrm{y} 9]=0 \mathrm{de} 45($ @ODE $1,[0: 0.002: 5], 4.2)$;

plot $\left([x 9,-x 9, x 9,-x 9],[y 9, y 9,-y 9,-y 9], k^{\prime}\right)$

$[\mathrm{x} 10, \mathrm{y} 10]=0 \operatorname{de} 45(@ O D E 1,[0: 0.002: 5], 4.1)$;

plot $\left([x 10,-x 10, x 10,-x 10],[y 10, y 10,-y 10,-y 10], k^{-\prime}\right)$

$[\mathrm{x} 11, \mathrm{y} 11]=0 \mathrm{de} 45(\mathrm{QODE} 1,[0: 0.002: 5], 4.0)$;

plot $([x 11,-x 11, x 11,-x 11],[y 11, y 11,-y 11,-y 11], ' k-')$

$[\mathrm{x} 12, \mathrm{y} 12]=0 \mathrm{de} 45($ @ODE1, $[0: 0.002: 5], 3.90)$;

plot $\left([x 12,-x 12, x 12,-x 12],[y 12, y 12,-y 12,-y 12], ' k^{\prime}\right)$

$[\mathrm{x} 13, \mathrm{y} 13]=0 \mathrm{de} 45(\mathrm{QODE} 1,[0: 0.002: 5], 3.8)$;

plot $\left([x 13,-x 13, x 13,-x 13],[y 13, y 13,-y 13,-y 13], ' k^{\prime}\right)$

$[\mathrm{x} 14, \mathrm{y} 14]=0 \mathrm{de} 45($ @ODE1, $[0: 0.002: 5], 3.7)$;

plot $\left([x 14,-x 14, x 14,-x 14],[y 14, y 14,-y 14,-y 14], k^{-\prime}\right)$

$[\mathrm{x} 15, \mathrm{y} 15]=0 \mathrm{de} 45(\mathrm{QODE} 1,[0: 0.002: 5], 3.6)$;

plot $([x 15,-x 15, x 15,-x 15],[y 15, y 15,-y 15,-y 15], ' k-')$

$[\mathrm{x} 16, \mathrm{y} 16]=0 \mathrm{de} 45(\mathrm{QODE} 1,[0: 0.002: 5], 3.5)$;

$\operatorname{plot}\left([\mathrm{x} 16,-\mathrm{x} 16, \mathrm{x} 16,-\mathrm{x} 16],[\mathrm{y} 16, \mathrm{y} 16,-\mathrm{y} 16,-\mathrm{y} 16], \mathrm{k}^{\prime} \mathrm{\prime}^{\prime}\right)$

$[\mathrm{x} 17, \mathrm{y} 17]=0 \mathrm{de} 45(\mathrm{QODE} 1,[0: 0.002: 5], 3.4)$;

plot $\left([x 17,-x 17, x 17,-x 17],[y 17, y 17,-y 17,-y 17], k^{-'}\right)$

$[\mathrm{x} 18, \mathrm{y} 18]=0 \mathrm{de} 45(\mathrm{QODE} 1,[0: 0.002: 5], 3.3)$;

plot $\left([\mathrm{x} 18,-\mathrm{x} 18, \mathrm{x} 18,-\mathrm{x} 18],[y 18, \mathrm{y} 18,-\mathrm{y} 18,-\mathrm{y} 18], \mathrm{k}^{-\prime}\right)$

$[\mathrm{x} 19, \mathrm{y} 19]=0 \mathrm{de} 45($ @ODE1 $,[0: 0.002: 5], 3.2)$;

plot $([x 19,-x 19, x 19,-x 19],[y 19, y 19,-y 19,-y 19], ' k-')$

$[\mathrm{x} 20, \mathrm{y} 20]=0 \mathrm{de} 45(\operatorname{\varrho ODE} 1,[0: 0.002: 5], 3.1)$;

plot $\left([x 20,-x 20, x 20,-x 20],[y 20, y 20,-y 20,-y 20], ' k^{-\prime}\right)$

$[\mathrm{x} 21, \mathrm{y} 21]=0 \mathrm{de} 45(\mathrm{@ODE} 1,[0: 0.002: 5], 3.0)$;

plot $([x 21,-x 21, x 21,-x 21],[y 21, y 21,-y 21,-y 21], ' k-')$

$[\mathrm{x} 22, \mathrm{y} 22]=0 \mathrm{de} 45(\mathrm{QODE} 1,[0: 0.002: 5], 2.90)$;

plot $\left([x 22,-x 22, x 22,-x 22],[y 22, y 22,-y 22,-y 22], ' k^{\prime} '\right)$

$[\mathrm{x} 23, \mathrm{y} 23]=0 \mathrm{de} 45(\mathrm{QODE} 1,[0: 0.002: 5], 2.8)$;

$\operatorname{plot}\left([x 23,-x 23, x 23,-x 23],[y 23, y 23,-y 23,-y 23], k^{\prime}-^{\prime}\right)$

$[\mathrm{x} 24, \mathrm{y} 24]=0 \mathrm{de} 45(\mathrm{QODE} 1,[0: 0.002: 5], 2.7)$;

plot $\left([x 24,-x 24, x 24,-x 24],[y 24, y 24,-y 24,-y 24], k^{\prime}{ }^{\prime}\right)$

$[\mathrm{x} 25, \mathrm{y} 25]=0 \mathrm{de} 45(\mathrm{eODE} 1,[0: 0.002: 5], 2.6)$;

plot $\left([x 25,-x 25, x 25,-x 25],[y 25, y 25,-y 25,-y 25], k^{\prime}-^{\prime}\right)$

$[\mathrm{x} 26, \mathrm{y} 26]=0 \mathrm{de} 45(\mathrm{QODE} 1,[0: 0.002: 5], 2.5)$;

plot $\left([x 26,-x 26, x 26,-x 26],[y 26, y 26,-y 26,-y 26], ' k-^{\prime}\right)$

$[\mathrm{x} 27, \mathrm{y} 27]=0 \mathrm{de} 45(\mathrm{QODE} 1,[0: 0.002: 5], 2.4)$;

plot $([x 27,-x 27, x 27,-x 27],[y 27, y 27,-y 27,-y 27], ' k-')$

$[\mathrm{x} 28, \mathrm{y} 28]=0 \mathrm{de} 45(\mathrm{QODE} 1,[0: 0.002: 5], 2.3)$;

plot $\left([x 28,-x 28, x 28,-x 28],[y 28, y 28,-y 28,-y 28], '-^{\prime}\right.$ )

$[\mathrm{x} 29, \mathrm{y} 29]=0 \mathrm{de} 45($ @ODE1 $,[0: 0.002: 5], 2.2)$;

plot $\left([x 29,-x 29, x 29,-x 29],[y 29, y 29,-y 29,-y 29], ' k-{ }^{\prime}\right)$

$[\mathrm{x} 30, \mathrm{y} 30]=0 \mathrm{de} 45(\mathrm{\varrho ODE} 1,[0: 0.002: 5], 2.1)$;

plot $([x 30,-x 30, x 30,-x 30],[y 30, y 30,-y 30,-y 30], ' k-')$

$[\mathrm{x} 31, \mathrm{y} 31]=0 \mathrm{de} 45(\mathrm{QODE} 1,[0: 0.002: 5], 2.0)$;

plot $([x 31,-x 31, x 31,-x 31],[y 31, y 31,-y 31,-y 31], ' k-')$

$[\mathrm{x} 32, \mathrm{y} 32]=0 \mathrm{de} 45(\mathrm{QODE} 1,[0: 0.002: 5], 1.9)$;

plot $\left([x 32,-x 32, x 32,-x 32],[y 32, y 32,-y 32,-y 32],{ }^{\prime}-^{\prime}\right)$

$[\mathrm{x} 33, \mathrm{y} 33]=0 \mathrm{de} 45(\mathrm{\varrho ODE} 1,[0: 0.002: 5], 1.8)$;

plot $\left([x 33,-x 33, x 33,-x 33],[y 33, y 33,-y 33,-y 33],{ }^{\prime}-^{\prime}\right)$

$[\mathrm{x} 34, \mathrm{y} 34]=0 \mathrm{de} 45(\mathrm{\varrho ODE} 1,[0: 0.002: 5], 1.7)$;

plot $\left([x 34,-x 34, x 34,-x 34],[y 34, y 34,-y 34,-y 34], k^{\prime}{ }^{\prime}\right)$

$[\mathrm{x} 35, \mathrm{y} 35]=0 \mathrm{de} 45(\mathrm{QODE} 1,[0: 0.002: 5], 1.6)$;

plot $\left([x 35,-x 35, x 35,-x 35],[y 35, y 35,-y 35,-y 35],{ }^{\prime}-^{\prime}\right)$

$[\mathrm{x} 36, \mathrm{y} 36]=0 \mathrm{de} 45(\mathrm{QODE} 1,[0: 0.002: 5], 1.5)$;

plot $\left([x 36,-x 36, x 36,-x 36],[y 36, y 36,-y 36,-y 36], k^{\prime}-^{\prime}\right)$

$[\mathrm{x} 37, \mathrm{y} 37]=0 \mathrm{de} 45(\mathrm{QODE} 1,[0: 0.002: 5], 1.4)$;

plot $\left([x 37,-x 37, x 37,-x 37],[y 37, y 37,-y 37,-y 37], k^{\prime}{ }^{\prime}\right)$

$[\mathrm{x} 38, \mathrm{y} 38]=0 \mathrm{de} 45(\mathrm{\varrho ODE} 1,[0: 0.002: 5], 1.3)$;

plot $([x 38,-x 38, x 38,-x 38],[y 38, y 38,-y 38,-y 38], ' k-1)$

$[\mathrm{x} 39, \mathrm{y} 39]=0 \mathrm{de} 45(\mathrm{CODE} 1,[0: 0.002: 5], 1.2)$;

plot $([x 39,-x 39, x 39,-x 39],[y 39, y 39,-y 39,-y 39], ' k-')$

$[\mathrm{x} 40, \mathrm{y} 40]=0 \mathrm{de} 45(\mathrm{QODE} 1,[0: 0.002: 5], 1.1)$;

plot $\left([x 40,-x 40, x 40,-x 40],[y 40, y 40,-y 40,-y 40],{ }^{\prime}-^{\prime}\right)$

○Generating Secondary Stress Trajectories

$[y 41, x 41]=0 d e 45(@ O D E 3,[0: 0.002: 5], 5.0)$;

plot $\left([x 41,-x 41, x 41,-x 41],[y 41, y 41,-y 41,-y 41], k^{\prime}{ }^{\prime}\right)$

$[y 42, x 42]=0 d e 45(@ O D E 3,[0: 0.002: 5], 4.9)$;

plot $([x 42,-x 42, x 42,-x 42],[y 42, y 42,-y 42,-y 42], ' k-')$ 
$[y 43, x 43]=0$ de $45(@ O D E 3,[0: 0.002: 5], 4.8)$;

plot $([x 43,-x 43, x 43,-x 43],[y 43, y 43,-y 43,-y 43], ' k-1)$

$[y 44, x 44]=0 \operatorname{de} 45($ @ODE $3,[0: 0.002: 5], 4.7)$;

plot $\left([\mathrm{x} 44,-\mathrm{x} 44, \mathrm{x} 44,-\mathrm{x} 44],[\mathrm{y} 44, \mathrm{y} 44,-\mathrm{y} 44,-\mathrm{y} 44], \mathrm{k}^{-}{ }^{\prime}\right)$

$[y 45, x 45]=0$ de $45(@ O D E 3,[0: 0.002: 5], 4.6)$;

plot $\left([x 45,-x 45, x 45,-x 45],[y 45, y 45,-y 45,-y 45], ' k^{-\prime}\right)$

$[y 46, x 46]=0 d e 45(@ O D E 3,[0: 0.002: 5], 4.5)$;

plot $\left([\mathrm{x} 46,-\mathrm{x} 46, \mathrm{x} 46,-\mathrm{x} 46],[\mathrm{y} 46, \mathrm{y} 46,-\mathrm{y} 46,-\mathrm{y} 46], \mathrm{k}^{\prime} \mathrm{-}^{\prime}\right)$

$[y 47, x 47]=0$ de4 $5(@ O D E 3,[0: 0.002: 5], 4.4)$;

plot $\left([x 47,-x 47, x 47,-x 47],[y 47, y 47,-y 47,-y 47], ' k^{\prime}\right.$ ')

$[y 48, x 48]=0$ de4 $5($ @ODE $3,[0: 0.002: 5], 4.3)$;

plot $\left([x 48,-x 48, x 48,-x 48],[y 48, y 48,-y 48,-y 48], k^{-\prime}\right)$

$[y 49, x 49]=0$ de $45($ @ODE $3,[0: 0.002: 5], 4.2)$;

plot $\left([x 49,-x 49, x 49,-x 49],[y 49, y 49,-y 49,-y 49], k^{\prime}-^{\prime}\right)$ $[y 50, x 50]=0 d e 45(@ O D E 3,[0: 0.002: 5], 4.1)$;

plot $\left([x 50,-x 50, x 50,-x 50],[y 50, y 50,-y 50,-y 50], k^{\prime}-^{\prime}\right)$ $[y 51, x 51]=0$ de4 $5($ @ODE $3,[0: 0.002: 5], 4.0)$.

plot $\left([x 51,-x 51, x 51,-x 51],[y 51, y 51,-y 51,-y 51], k^{\prime}-^{\prime}\right)$

$[y 52, x 52]=0 d e 45(@ O D E 3,[0: 0.002: 5], 3.9)$;

plot $([x 52,-x 52, x 52,-x 52],[y 52, y 52,-y 52,-y 52], ' k-')$

$[y 53, x 53]=0 d e 45(@ O D E 3,[0: 0.002: 5], 3.8)$;

plot $\left([x 53,-x 53, x 53,-x 53],[y 53, y 53,-y 53,-y 53],{ }^{\prime}-^{\prime}\right)$

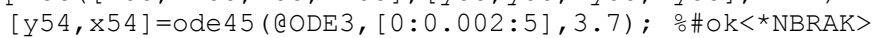

plot $([x 54,-x 54, x 54,-x 54],[y 54, y 54,-y 54,-y 54], ' k-')$

$[y 55, x 55]=0$ de $45($ @ODE $3,[0: 0.002: 5], 3.6)$;

plot $\left([x 55,-x 55, x 55,-x 55],[y 55, y 55,-y 55,-y 55], ' k^{-\prime}\right)$

$[y 56, x 56]=0 d e 45(@ O D E 3,[0: 0.002: 5], 3.5)$;

plot $\left([x 56,-x 56, x 56,-x 56],[y 56, y 56,-y 56,-y 56], ' k^{-\prime}\right)$

$[y 57, x 57]=0 d e 45(@ O D E 3,[0: 0.002: 5], 3.4)$;

plot $\left([x 57,-x 57, x 57,-x 57],[y 57, y 57,-y 57,-y 57], ' k^{\prime}\right)$

$[y 58, x 58]=0 d e 45(@ O D E 3,[0: 0.002: 5], 3.3)$;

plot $\left([x 58,-x 58, x 58,-x 58],[y 58, y 58,-y 58,-y 58], ' k^{\prime}\right)$

[y59, x59] =ode45 (@ODE3, [0:0.002:5],3.2);

plot $\left([x 59,-x 59, x 59,-x 59],[y 59, y 59,-y 59,-y 59],{ }^{\prime}-^{\prime}\right)$

$[y 60, x 60]=0$ de4 $5(@ O D E 3,[0: 0.002: 5], 3.1)$;

plot $\left([x 60,-x 60, x 60,-x 60],[y 60, y 60,-y 60,-y 60],{ }^{\prime} k-^{\prime}\right)$

$[y 61, x 61]=0$ de45 (@ODE $3,[0: 0.002: 5], 3.0)$;

plot $\left([x 61,-x 61, x 61,-x 61],[y 61, y 61,-y 61,-y 61], k^{-\prime}\right)$

$[y 62, x 62]=0$ de4 $5(@ 0 D E 3,[0: 0.002: 5], 2.9)$;

$\operatorname{plot}\left([\mathrm{x} 62,-\mathrm{x} 62, \mathrm{x} 62,-\mathrm{x} 62],[\mathrm{y} 62, \mathrm{y} 62,-\mathrm{y} 62,-\mathrm{y} 62], \mathrm{k}^{\prime} \mathbf{-}^{\prime}\right)$

$[y 63, x 63]=0$ de4 $5(@ 0 D E 3,[0: 0.002: 5], 2.8)$;

plot $\left([x 63,-x 63, x 63,-x 63],[y 63, y 63,-y 63,-y 63],{ }^{\prime}-^{\prime}\right)$

$[y 64, x 64]=0 d e 45(@ O D E 3,[0: 0.002: 5], 2.7)$;

plot $\left([x 64,-x 64, x 64,-x 64],[y 64, y 64,-y 64,-y 64], ' k^{\prime}\right.$ ')

$[y 65, x 65]=0$ de $45($ @ODE $3,[0: 0.002: 5], 2.6)$;

plot $\left([x 65,-x 65, x 65,-x 65],[y 65, y 65,-y 65,-y 65], ' k^{-1}\right)$

$[y 66, x 66]=0$ de $45($ @ODE $3,[0: 0.002: 5], 2.5)$;

plot $([x 66,-x 66, x 66,-x 66],[y 66, y 66,-y 66,-y 66], ' k-1)$

$[y 67, x 67]=0 \operatorname{de} 45(\operatorname{eDE} 3,[0: 0.002: 5], 2.4)$;

plot $\left([x 67,-x 67, x 67,-x 67],[y 67, y 67,-y 67,-y 67], ' k^{-1}\right)$

$[y 68, x 68]=0$ de4 $5($ @ODE $3,[0: 0.002: 5], 2.3)$;

plot $\left([x 68,-x 68, x 68,-x 68],[y 68, y 68,-y 68,-y 68], k^{\prime}-^{\prime}\right)$

$[y 69, x 69]=0 d e 45($ @ODE $3,[0: 0.002: 5], 2.1)$;

plot $\left([x 69,-x 69, x 69,-x 69],[y 69, y 69,-y 69,-y 69], k^{-1}\right)$

$[\mathrm{x} 70, \mathrm{y} 70]=0 \mathrm{de} 45(@ \mathrm{ODE} 2,[0.9993: 0.002: 5.9993], 0.0348) ; \div 0.5$

plot $\left([x 70,-x 70, x 70,-x 70],[y 70, y 70,-y 70,-y 70], k^{-1}\right)$

$[\mathrm{x} 71, \mathrm{y} 71]=0 \mathrm{de} 45(\mathrm{QODE} 2,[0.996: 0.002: 5.996], 0.087) ; \% 5$

plot $\left.\left([x 71,-x 71, x 71,-x 71],[y 71, y 71,-y 71,-y 71], k^{\prime}\right)^{\prime}\right)$

$[\mathrm{x} 72, \mathrm{y} 72]=0 \mathrm{de} 45(\mathrm{eODE} 2,[0.984: 0.002: 5.984], 0.174) ; \% 10$

plot $([x 72,-x 72, x 72,-x 72],[y 72, y 72,-y 72,-y 72], ' k-')$

$[\mathrm{x} 73, \mathrm{y} 73]=0 \mathrm{de} 45(\mathrm{QODE} 2,[0.965: 0.002: 5.965], 0.259) ; \circ 15$

plot $([x 73,-x 73, x 73,-x 73],[y 73, y 73,-y 73,-y 73], ' k-')$

$[\mathrm{x} 74, \mathrm{y} 74]=0 \mathrm{de} 45(\mathrm{\varrho ODE} 2,[0.939: 0.002: 5.939], 0.342) ; \div 20$

plot $\left([x 74,-x 74, x 74,-x 74],[y 74, y 74,-y 74,-y 74], ' k-^{\prime}\right)$

$[\mathrm{x} 75, \mathrm{y} 75]=0 \mathrm{de} 45(\mathrm{QODE} 2,[0.906: 0.002: 5.906], 0.423) ; \circ 25$

plot $\left([x 75,-x 75, x 75,-x 75],[y 75, y 75,-y 75,-y 75],{ }^{\prime}-^{\prime}\right)$

$[\mathrm{x} 76, \mathrm{y} 76]=0 \mathrm{de} 45(\mathrm{QODE} 2,[0.866: 0.002: 5.866], 0.500) ; \circ 30$

plot $\left([x 76,-x 76, x 76,-x 76],[y 76, y 76,-y 76,-y 76],{ }^{\prime}-^{\prime}\right)$

$[\mathrm{x} 77, \mathrm{y} 77]=0 \mathrm{de} 45(\mathrm{@ODE} 2,[0.819: 0.002: 5.819], 0.573) ; \circ 35$

plot $\left([x 77,-x 77, x 77,-x 77],[y 77, y 77,-y 77,-y 77], k^{\prime}{ }^{\prime}\right)$

$[\mathrm{x} 78, \mathrm{y} 78]=0 \mathrm{de} 45(@ \mathrm{ODE} 2,[0.766: 0.002: 5.766], 0.643) ; \% 40$ 
$\operatorname{plot}([x 78,-x 78, x 78,-x 78],[y 78, y 78,-y 78,-y 78], ' k-1)$

$[\mathrm{x} 79, \mathrm{y} 79]=0 \mathrm{de} 45(@ \mathrm{ODE} 2,[0.707: 0.002: 5.707], 0.707) ; \circ 45$

plot $\left([x 79,-x 79, x 79,-x 79],[y 79, y 79,-y 79,-y 79], k^{\prime}{ }^{\prime}\right)$

$[\mathrm{x} 80, \mathrm{y} 80]=0 \mathrm{de} 45(\mathrm{QODE} 2,[0.643: 0.002: 5.643], 0.766) ; \circ 50$

$\operatorname{plot}\left([\mathrm{x} 80,-\mathrm{x} 80, \mathrm{x} 80,-\mathrm{x} 80],[y 80, y 80,-y 80,-y 80], \mathrm{y}^{\prime}-^{\prime}\right)$

$[\mathrm{x} 81, \mathrm{y} 81]=0 \mathrm{de} 45($ @ODE $2,[0.573: 0.002: 5.573], 0.819) ; \circ 55$

plot $\left([x 81,-x 81, x 81,-x 81],[y 81, y 81,-y 81,-y 81], k^{-1}\right)$

$[\mathrm{x} 82, \mathrm{y} 82]=0 \mathrm{de} 45(\mathrm{QODE} 2,[0.500: 0.002: 5.500], 0.866) ; \circ 60$

plot $\left([x 82,-x 82, x 82,-x 82],[y 82, y 82,-y 82,-y 82], ' k^{-\prime}\right)$

$[\mathrm{x} 83, \mathrm{y} 83]=0 \mathrm{de} 45(\mathrm{QODE} 2,[0.423: 0.002: 5.423], 0.906) ; \circ 65$

plot $\left([x 83,-x 83, x 83,-x 83],[y 83, y 83,-y 83,-y 83],{ }^{\prime} k-^{\prime}\right)$

$[\mathrm{x} 84, \mathrm{y} 84]=0 \mathrm{de} 45(\mathrm{eODE} 2,[0.342: 0.002: 5.342], 0.939) ; \circ 70$

plot $\left([x 84,-x 84, x 84,-x 84],[y 84, y 84,-y 84,-y 84], k^{-\prime}\right)$

$[\mathrm{x} 85, \mathrm{y} 85]=0 \mathrm{de} 45($ @ODE $2,[0.259: 0.002: 5.259], 0.965) ; \circ 75$

plot $([x 85,-x 85, x 85,-x 85],[y 85, y 85,-y 85,-y 85], ' k-')$

$[\mathrm{x} 86, \mathrm{y} 86]=0 \mathrm{de} 45(\mathrm{QODE} 2,[0.174: 0.002: 5.174], 0.984) ; \circ 80$

plot $([x 86,-x 86, x 86,-x 86],[y 86, y 86,-y 86,-y 86], ' k-')$

$[\mathrm{x} 87, \mathrm{y} 87]=0 \mathrm{de} 45(\mathrm{QODE} 2,[0.087: 0.002: 5.087], 0.996) ; \circ 85$

plot $\left([x 87,-x 87, x 87,-x 87],[y 87, y 87,-y 87,-y 87], k^{\prime}-^{\prime}\right)$

$\mathrm{x} 88=[0: 0.002: 5]^{\prime}$;

$\mathrm{y} 88=\mathrm{x} 88 \cdot * 0+5$

plot $\left(\mathrm{x} 88, \mathrm{y} 88, \mathrm{\prime}^{\mathrm{k}-1}\right)$

plot $\left(-x 88, y 88,{ }^{\prime} k^{\prime}\right)$

plot $\left(x 88,-y 88,{ }^{\prime} k-^{\prime}\right)$

plot $(-x 88,-y 88, ' k-')$

plot $(y 88, x 88, ' k-')$

plot $\left(-\mathrm{y} 88, \mathrm{x} 88, \mathrm{k}^{\mathrm{k}} \mathrm{-}^{\prime}\right)$

plot $\left(y 88,-x 88,{ }^{\prime} \mathrm{k}^{\prime}\right)$

plot $\left(-y 88,-x 88,{ }^{\prime} k-'\right)$

$\mathrm{x}=1: .002: 5$;

$\mathrm{y}=\mathrm{x} \cdot{ }^{*} 0$;

plot $(x, y, ' k-')$

$\operatorname{plot}\left(-x, y,{ }^{\prime}-^{\prime}\right)$

plot $(y, x, ' k-')$

$\operatorname{plot}\left(y,-x,{ }^{\prime}-{ }^{\prime}\right)$

$\mathrm{x}=0: .01: 1$;

$\mathrm{y}=\operatorname{sqrt}\left(1-\mathrm{x} \cdot{ }^{\wedge} 2\right)$;

plot $(x, y, ' k-'$, 'Linewidth', 1.5)

plot $\left(-x, y,{ }^{\prime}-^{\prime}\right.$ ', 'Linewidth' , 1.5)

plot ( $x,-y,{ }^{\prime}-^{-}$', 'Linewidth', 1.5$)$

plot $\left(-x,-y,{ }^{\prime}-'\right.$ ' ' Linewidth', 1.5$)$

for $j=1: 18$

$\mathrm{m}=[\mathrm{x} 70, \mathrm{x} 71, \mathrm{x} 72, \mathrm{x} 73, \mathrm{x} 74, \mathrm{x} 75, \mathrm{x} 76, \mathrm{x} 77, \mathrm{x} 78, \mathrm{x} 79, \mathrm{x} 80, \mathrm{x} 81, \mathrm{x} 82, \mathrm{x} 83, \mathrm{x} 84, \mathrm{x} 85, \mathrm{x} 86, \mathrm{x} 87]$; $\mathrm{n}=[\mathrm{y} 70, \mathrm{y} 71, \mathrm{y} 72, \mathrm{y} 73, \mathrm{y} 74, \mathrm{y} 75, \mathrm{y} 76, \mathrm{y} 77, \mathrm{y} 78, \mathrm{y} 79, \mathrm{y} 80, \mathrm{y} 81, \mathrm{y} 82, \mathrm{y} 83, \mathrm{y} 84, \mathrm{y} 85, \mathrm{y} 86, \mathrm{y} 87]$;

end

$[A 1(j, 1), A 1(j, 2)]=$ curveintersect $(m(:, j), n(:, j), x 40, y 40)$;

for $j=1: 18$

$\mathrm{m}=[\mathrm{x} 70, \mathrm{x} 71, \mathrm{x} 72, \mathrm{x} 73, \mathrm{x} 74, \mathrm{x} 75, \mathrm{x} 76, \mathrm{x} 77, \mathrm{x} 78, \mathrm{x} 79, \mathrm{x} 80, \mathrm{x} 81, \mathrm{x} 82, \mathrm{x} 83, \mathrm{x} 84, \mathrm{x} 85, \mathrm{x} 86, \mathrm{x} 87]$;

$\mathrm{n}=[\mathrm{y} 70, \mathrm{y} 71, \mathrm{y} 72, \mathrm{y} 73, \mathrm{y} 74, \mathrm{y} 75, \mathrm{y} 76, \mathrm{y} 77, \mathrm{y} 78, \mathrm{y} 79, \mathrm{y} 80, \mathrm{y} 81, \mathrm{y} 82, \mathrm{y} 83, \mathrm{y} 84, \mathrm{y} 85, \mathrm{y} 86, \mathrm{y} 87]$;

end

$[A 2(j, 1), A 2(j, 2)]=$ curveintersect $(m(:, j), n(:, j), x 39, y 39)$

for $j=1: 47$

$m=[y 88, x 42, x 43, x 44, x 45, x 46, x 47, x 48, x 49, x 50, x 51, x 52, x 53, x 54, x 55, x 56, x 57, x 58, x 59, x 60$, $x 61, x 62, x 63, x 64, x 65, x 66, x 67, x 68, x 69, x 70, x 71, x 72, x 73, x 74, x 75, x 76, x 77, x 78, x 79, x 80, x 81$ $, x 82, x 83, x 84, x 85, x 86, x 87]$

$\mathrm{n}=[\mathrm{x} 88, \mathrm{y} 42, \mathrm{y} 43, \mathrm{y} 44, \mathrm{y} 45, \mathrm{y} 46, \mathrm{y} 47, \mathrm{y} 48, \mathrm{y} 49, \mathrm{y} 50, \mathrm{y} 51, \mathrm{y} 52, \mathrm{y} 53, \mathrm{y} 54, \mathrm{y} 55, \mathrm{y} 56, \mathrm{y} 57, \mathrm{y} 58, \mathrm{y} 59, \mathrm{y} 60$, $y 61, y 62, y 63, y 64, y 65, y 66, y 67, y 68, y 69, y 70, y 71, y 72, y 73, y 74, y 75, y 76, y 77, y 78, y 79, y 80, y 81$ $, \mathrm{y} 82, \mathrm{y} 83, \mathrm{y} 84, \mathrm{y} 85, \mathrm{y} 86, \mathrm{y} 87]$

end

$[A 3(j, 1), A 3(j, 2)]=$ curveintersect $(m(:, j), n(:, j), x 38, y 38)$

for $j=1: 47$

$m=[y 88, x 42, x 43, x 44, x 45, x 46, x 47, x 48, x 49, x 50, x 51, x 52, x 53, x 54, x 55, x 56, x 57, x 58, x 59, x 60$, $x 61, x 62, x 63, x 64, x 65, x 66, x 67, x 68, x 69, x 70, x 71, x 72, x 73, x 74, x 75, x 76, x 77, x 78, x 79, x 80, x 81$ $, x 82, x 83, x 84, x 85, x 86, x 87]$

$n=[x 88, y 42, y 43, y 44, y 45, y 46, y 47, y 48, y 49, y 50, y 51, y 52, y 53, y 54, y 55, y 56, y 57, y 58, y 59, y 60$, $\mathrm{y} 61, \mathrm{y} 62, \mathrm{y} 63, \mathrm{y} 64, \mathrm{y} 65, \mathrm{y} 66, \mathrm{y} 67, \mathrm{y} 68, \mathrm{y} 69, \mathrm{y} 70, \mathrm{y} 71, \mathrm{y} 72, \mathrm{y} 73, \mathrm{y} 74, \mathrm{y} 75, \mathrm{y} 76, \mathrm{y} 77, \mathrm{y} 78, \mathrm{y} 79, \mathrm{y} 80, \mathrm{y} 81$ $, \mathrm{y} 82, \mathrm{y} 83, \mathrm{y} 84, \mathrm{y} 85, \mathrm{y} 86, \mathrm{y} 87]$

end

$[A 4(j, 1), A 4(j, 2)]=$ curveintersect $(m(:, j), n(:, j), x 37, y 37)$;

for $j=1: 47$ 
$m=[y 88, x 42, x 43, x 44, x 45, x 46, x 47, x 48, x 49, x 50, x 51, x 52, x 53, x 54, x 55, x 56, x 57, x 58, x 59, x 60$, $x 61, x 62, x 63, x 64, x 65, x 66, x 67, x 68, x 69, x 70, x 71, x 72, x 73, x 74, x 75, x 76, x 77, x 78, x 79, x 80, x 81$ $, x 82, x 83, x 84, x 85, x 86, x 87]$

$\mathrm{n}=[\mathrm{x} 88, \mathrm{y} 42, \mathrm{y} 43, \mathrm{y} 44, \mathrm{y} 45, \mathrm{y} 46, \mathrm{y} 47, \mathrm{y} 48, \mathrm{y} 49, \mathrm{y} 50, \mathrm{y} 51, \mathrm{y} 52, \mathrm{y} 53, \mathrm{y} 54, \mathrm{y} 55, \mathrm{y} 56, \mathrm{y} 57, \mathrm{y} 58, \mathrm{y} 59, \mathrm{y} 60$, $\mathrm{y} 61, \mathrm{y} 62, \mathrm{y} 63, \mathrm{y} 64, \mathrm{y} 65, \mathrm{y} 66, \mathrm{y} 67, \mathrm{y} 68, \mathrm{y} 69, \mathrm{y} 70, \mathrm{y} 71, \mathrm{y} 72, \mathrm{y} 73, \mathrm{y} 74, \mathrm{y} 75, \mathrm{y} 76, \mathrm{y} 77, \mathrm{y} 78, \mathrm{y} 79, \mathrm{y} 80, \mathrm{y} 81$ $, \mathrm{y} 82, \mathrm{y} 83, \mathrm{y} 84, \mathrm{y} 85, \mathrm{y} 86, \mathrm{y} 87]$

end

$[A 5(j, 1), A 5(j, 2)]=$ curveintersect $(m(:, j), n(:, j), x 36, y 36)$;

for $j=1: 47$

$m=[y 88, x 42, x 43, x 44, x 45, x 46, x 47, x 48, x 49, x 50, x 51, x 52, x 53, x 54, x 55, x 56, x 57, x 58, x 59, x 60$, $x 61, x 62, x 63, x 64, x 65, x 66, x 67, x 68, x 69, x 70, x 71, x 72, x 73, x 74, x 75, x 76, x 77, x 78, x 79, x 80, x 81$ $, x 82, x 83, x 84, x 85, x 86, x 87]$

$\mathrm{n}=[\mathrm{x} 88, \mathrm{y} 42, \mathrm{y} 43, \mathrm{y} 44, \mathrm{y} 45, \mathrm{y} 46, \mathrm{y} 47, \mathrm{y} 48, \mathrm{y} 49, \mathrm{y} 50, \mathrm{y} 51, \mathrm{y} 52, \mathrm{y} 53, \mathrm{y} 54, \mathrm{y} 55, \mathrm{y} 56, \mathrm{y} 57, \mathrm{y} 58, \mathrm{y} 59, \mathrm{y} 60$, $\mathrm{y} 61, \mathrm{y} 62, \mathrm{y} 63, \mathrm{y} 64, \mathrm{y} 65, \mathrm{y} 66, \mathrm{y} 67, \mathrm{y} 68, \mathrm{y} 69, \mathrm{y} 70, \mathrm{y} 71, \mathrm{y} 72, \mathrm{y} 73, \mathrm{y} 74, \mathrm{y} 75, \mathrm{y} 76, \mathrm{y} 77, \mathrm{y} 78, \mathrm{y} 79, \mathrm{y} 80, \mathrm{y} 81$ $, \mathrm{y} 82, \mathrm{y} 83, \mathrm{y} 84, \mathrm{y} 85, \mathrm{y} 86, \mathrm{y} 87]$

end

$[A 6(j, 1), A 6(j, 2)]=$ curveintersect $(m(:, j), n(:, j), x 35, y 35)$;

for $j=1: 47$

$m=[y 88, x 42, x 43, x 44, x 45, x 46, x 47, x 48, x 49, x 50, x 51, x 52, x 53, x 54, x 55, x 56, x 57, x 58, x 59, x 60$, $x 61, x 62, x 63, x 64, x 65, x 66, x 67, x 68, x 69, x 70, x 71, x 72, x 73, x 74, x 75, x 76, x 77, x 78, x 79, x 80, x 81$ $, \times 82, x 83, x 84, x 85, x 86, x 87]$

$\mathrm{n}=[\mathrm{x} 88, \mathrm{y} 42, \mathrm{y} 43, \mathrm{y} 44, \mathrm{y} 45, \mathrm{y} 46, \mathrm{y} 47, \mathrm{y} 48, \mathrm{y} 49, \mathrm{y} 50, \mathrm{y} 51, \mathrm{y} 52, \mathrm{y} 53, \mathrm{y} 54, \mathrm{y} 55, \mathrm{y} 56, \mathrm{y} 57, \mathrm{y} 58, \mathrm{y} 59, \mathrm{y} 60$, $\mathrm{y} 61, \mathrm{y} 62, \mathrm{y} 63, \mathrm{y} 64, \mathrm{y} 65, \mathrm{y} 66, \mathrm{y} 67, \mathrm{y} 68, \mathrm{y} 69, \mathrm{y} 70, \mathrm{y} 71, \mathrm{y} 72, \mathrm{y} 73, \mathrm{y} 74, \mathrm{y} 75, \mathrm{y} 76, \mathrm{y} 77, \mathrm{y} 78, \mathrm{y} 79, \mathrm{y} 80, \mathrm{y} 81$ $, \mathrm{y} 82, \mathrm{y} 83, \mathrm{y} 84, \mathrm{y} 85, \mathrm{y} 86, \mathrm{y} 87]$

end

$[A 7(j, 1), A 7(j, 2)]=$ curveintersect $(m(:, j), n(:, j), x 34, y 34)$;

for $j=1: 47$

$m=[y 88, x 42, x 43, x 44, x 45, x 46, x 47, x 48, x 49, x 50, x 51, x 52, x 53, x 54, x 55, x 56, x 57, x 58, x 59, x 60$, $x 61, x 62, x 63, x 64, x 65, x 66, x 67, x 68, x 69, x 70, x 71, x 72, x 73, x 74, x 75, x 76, x 77, x 78, x 79, x 80, x 81$ $, x 82, x 83, x 84, x 85, x 86, x 87] ;$

$\mathrm{n}=[\mathrm{x} 88, \mathrm{y} 42, \mathrm{y} 43, \mathrm{y} 44, \mathrm{y} 45, \mathrm{y} 46, \mathrm{y} 47, \mathrm{y} 48, \mathrm{y} 49, \mathrm{y} 50, \mathrm{y} 51, \mathrm{y} 52, \mathrm{y} 53, \mathrm{y} 54, \mathrm{y} 55, \mathrm{y} 56, \mathrm{y} 57, \mathrm{y} 58, \mathrm{y} 59, \mathrm{y} 60$, $y 61, y 62, y 63, y 64, y 65, y 66, y 67, y 68, y 69, y 70, y 71, y 72, y 73, y 74, y 75, y 76, y 77, y 78, y 79, y 80, y 81$ $, \mathrm{y} 82, \mathrm{y} 83, \mathrm{y} 84, \mathrm{y} 85, \mathrm{y} 86, \mathrm{y} 87]$

end

$[A 8(j, 1), A 8(j, 2)]=$ curveintersect $(m(:, j), n(:, j), x 33, y 33)$;

for $j=1: 47$

$m=[y 88, x 42, x 43, x 44, x 45, x 46, x 47, x 48, x 49, x 50, x 51, x 52, x 53, x 54, x 55, x 56, x 57, x 58, x 59, x 60$, $x 61, x 62, x 63, x 64, x 65, x 66, x 67, x 68, x 69, x 70, x 71, x 72, x 73, x 74, x 75, x 76, x 77, x 78, x 79, x 80, x 81$ $, x 82, x 83, x 84, x 85, x 86, x 87] ;$

$\mathrm{n}=[\mathrm{x} 88, \mathrm{y} 42, \mathrm{y} 43, \mathrm{y} 44, \mathrm{y} 45, \mathrm{y} 46, \mathrm{y} 47, \mathrm{y} 48, \mathrm{y} 49, \mathrm{y} 50, \mathrm{y} 51, \mathrm{y} 52, \mathrm{y} 53, \mathrm{y} 54, \mathrm{y} 55, \mathrm{y} 56, \mathrm{y} 57, \mathrm{y} 58, \mathrm{y} 59, \mathrm{y} 60$, $\mathrm{y} 61, \mathrm{y} 62, \mathrm{y} 63, \mathrm{y} 64, \mathrm{y} 65, \mathrm{y} 66, \mathrm{y} 67, \mathrm{y} 68, \mathrm{y} 69, \mathrm{y} 70, \mathrm{y} 71, \mathrm{y} 72, \mathrm{y} 73, \mathrm{y} 74, \mathrm{y} 75, \mathrm{y} 76, \mathrm{y} 77, \mathrm{y} 78, \mathrm{y} 79, \mathrm{y} 80, \mathrm{y} 81$ $, \mathrm{y} 82, \mathrm{y} 83, \mathrm{y} 84, \mathrm{y} 85, \mathrm{y} 86, \mathrm{y} 87]$

end

$[A 9(j, 1), A 9(j, 2)]=$ curveintersect $(m(:, j), n(:, j), x 32, y 32)$;

for $j=1: 47$

$\mathrm{m}=[\mathrm{y} 88, \mathrm{x} 42, \mathrm{x} 43, \mathrm{x} 44, \mathrm{x} 45, \mathrm{x} 46, \mathrm{x} 47, \mathrm{x} 48, \mathrm{x} 49, \mathrm{x} 50, \mathrm{x} 51, \mathrm{x} 52, \mathrm{x} 53, \mathrm{x} 54, \mathrm{x} 55, \mathrm{x} 56, \mathrm{x} 57, \mathrm{x} 58, \mathrm{x} 59, \mathrm{x} 60$, $x 61, x 62, x 63, x 64, x 65, x 66, x 67, x 68, x 69, x 70, x 71, x 72, x 73, x 74, x 75, x 76, x 77, x 78, x 79, x 80, x 81$ $, \times 82, x 83, x 84, x 85, x 86, x 87]$

$\mathrm{n}=[\mathrm{x} 88, \mathrm{y} 42, \mathrm{y} 43, \mathrm{y} 44, \mathrm{y} 45, \mathrm{y} 46, \mathrm{y} 47, \mathrm{y} 48, \mathrm{y} 49, \mathrm{y} 50, \mathrm{y} 51, \mathrm{y} 52, \mathrm{y} 53, \mathrm{y} 54, \mathrm{y} 55, \mathrm{y} 56, \mathrm{y} 57, \mathrm{y} 58, \mathrm{y} 59, \mathrm{y} 60$, y61, y62, y63, y64, y65, y66, y67,y68,y69, y70,y71,y72,y73,y74, y75, y76, y77,y78, y79, y80, y81 $, \mathrm{y} 82, \mathrm{y} 83, \mathrm{y} 84, \mathrm{y} 85, \mathrm{y} 86, \mathrm{y} 87]$

end

$[A 10(j, 1), A 10(j, 2)]=$ Curveintersect $(m(:, j), n(:, j), x 31, y 31)$;

for $j=1: 47$

$m=[y 88, x 42, x 43, x 44, x 45, x 46, x 47, x 48, x 49, x 50, x 51, x 52, x 53, x 54, x 55, x 56, x 57, x 58, x 59, x 60$, $x 61, x 62, x 63, x 64, x 65, x 66, x 67, x 68, x 69, x 70, x 71, x 72, x 73, x 74, x 75, x 76, x 77, x 78, x 79, x 80, x 81$ $, x 82, x 83, x 84, x 85, x 86, x 87]$

$\mathrm{n}=[\mathrm{x} 88, \mathrm{y} 42, \mathrm{y} 43, \mathrm{y} 44, \mathrm{y} 45, \mathrm{y} 46, \mathrm{y} 47, \mathrm{y} 48, \mathrm{y} 49, \mathrm{y} 50, \mathrm{y} 51, \mathrm{y} 52, \mathrm{y} 53, \mathrm{y} 54, \mathrm{y} 55, \mathrm{y} 56, \mathrm{y} 57, \mathrm{y} 58, \mathrm{y} 59, \mathrm{y} 60$, $\mathrm{y} 61, \mathrm{y} 62, \mathrm{y} 63, \mathrm{y} 64, \mathrm{y} 65, \mathrm{y} 66, \mathrm{y} 67, \mathrm{y} 68, \mathrm{y} 69, \mathrm{y} 70, \mathrm{y} 71, \mathrm{y} 72, \mathrm{y} 73, \mathrm{y} 74, \mathrm{y} 75, \mathrm{y} 76, \mathrm{y} 77, \mathrm{y} 78, \mathrm{y} 79, \mathrm{y} 80, \mathrm{y} 81$ $, \mathrm{y} 82, \mathrm{y} 83, \mathrm{y} 84, \mathrm{y} 85, \mathrm{y} 86, \mathrm{y} 87]$

end

$[A 11(j, 1), A 11(j, 2)]=$ Curveintersect $(m(:, j), n(:, j), x 30, y 30)$;

for $j=1: 47$

$m=[y 88, x 42, x 43, x 44, x 45, x 46, x 47, x 48, x 49, x 50, x 51, x 52, x 53, x 54, x 55, x 56, x 57, x 58, x 59, x 60$, $x 61, x 62, x 63, x 64, x 65, x 66, x 67, x 68, x 69, x 70, x 71, x 72, x 73, x 74, x 75, x 76, x 77, x 78, x 79, x 80, x 81$ $, \times 82, x 83, x 84, x 85, x 86, x 87]$

$\mathrm{n}=[\mathrm{x} 88, \mathrm{y} 42, \mathrm{y} 43, \mathrm{y} 44, \mathrm{y} 45, \mathrm{y} 46, \mathrm{y} 47, \mathrm{y} 48, \mathrm{y} 49, \mathrm{y} 50, \mathrm{y} 51, \mathrm{y} 52, \mathrm{y} 53, \mathrm{y} 54, \mathrm{y} 55, \mathrm{y} 56, \mathrm{y} 57, \mathrm{y} 58, \mathrm{y} 59, \mathrm{y} 60$, $y 61, y 62, y 63, y 64, y 65, y 66, y 67, y 68, y 69, y 70, y 71, y 72, y 73, y 74, y 75, y 76, y 77, y 78, y 79, y 80, y 81$ $, \mathrm{y} 82, \mathrm{y} 83, \mathrm{y} 84, \mathrm{y} 85, \mathrm{y} 86, \mathrm{y} 87]$

end

$[A 12(j, 1), A 12(j, 2)]=$ curveintersect $(m(:, j), n(:, j), x 29, y 29)$; 
for $j=1: 47$

$m=[y 88, x 42, x 43, x 44, x 45, x 46, x 47, x 48, x 49, x 50, x 51, x 52, x 53, x 54, x 55, x 56, x 57, x 58, x 59, x 60$, $x 61, x 62, x 63, x 64, x 65, x 66, x 67, x 68, x 69, x 70, x 71, x 72, x 73, x 74, x 75, x 76, x 77, x 78, x 79, x 80, x 81$ $, x 82, x 83, x 84, x 85, x 86, x 87]$

$\mathrm{n}=[\mathrm{x} 88, \mathrm{y} 42, \mathrm{y} 43, \mathrm{y} 44, \mathrm{y} 45, \mathrm{y} 46, \mathrm{y} 47, \mathrm{y} 48, \mathrm{y} 49, \mathrm{y} 50, \mathrm{y} 51, \mathrm{y} 52, \mathrm{y} 53, \mathrm{y} 54, \mathrm{y} 55, \mathrm{y} 56, \mathrm{y} 57, \mathrm{y} 58, \mathrm{y} 59, \mathrm{y} 60$, $\mathrm{y} 61, \mathrm{y} 62, \mathrm{y} 63, \mathrm{y} 64, \mathrm{y} 65, \mathrm{y} 66, \mathrm{y} 67, \mathrm{y} 68, \mathrm{y} 69, \mathrm{y} 70, \mathrm{y} 71, \mathrm{y} 72, \mathrm{y} 73, \mathrm{y} 74, \mathrm{y} 75, \mathrm{y} 76, \mathrm{y} 77, \mathrm{y} 78, \mathrm{y} 79, \mathrm{y} 80, \mathrm{y} 81$ $, \mathrm{y} 82, \mathrm{y} 83, \mathrm{y} 84, \mathrm{y} 85, \mathrm{y} 86, \mathrm{y} 87]$

end

$[A 13(j, 1), A 13(j, 2)]=$ curveintersect $(m(:, j), n(:, j), x 28, y 28) ;$

for $j=1: 46$

$m=[y 88, x 43, x 44, x 45, x 46, x 47, x 48, x 49, x 50, x 51, x 52, x 53, x 54, x 55, x 56, x 57, x 58, x 59, x 60, x 61$, $x 62, x 63, x 64, x 65, x 66, x 67, x 68, x 69, x 70, x 71, x 72, x 73, x 74, x 75, x 76, x 77, x 78, x 79, x 80, x 81, x 82$ $, x 83, x 84, x 85, x 86, x 87]$

$n=[x 88, y 43, y 44, y 45, y 46, y 47, y 48, y 49, y 50, y 51, y 52, y 53, y 54, y 55, y 56, y 57, y 58, y 59, y 60, y 61$, $\mathrm{y} 62, \mathrm{y} 63, \mathrm{y} 64, \mathrm{y} 65, \mathrm{y} 66, \mathrm{y} 67, \mathrm{y} 68, \mathrm{y} 69, \mathrm{y} 70, \mathrm{y} 71, \mathrm{y} 72, \mathrm{y} 73, \mathrm{y} 74, \mathrm{y} 75, \mathrm{y} 76, \mathrm{y} 77, \mathrm{y} 78, \mathrm{y} 79, \mathrm{y} 80, \mathrm{y} 81, \mathrm{y} 82$ $, \mathrm{y} 83, \mathrm{y} 84, \mathrm{y} 85, \mathrm{y} 86, \mathrm{y} 87]$

end

$[\mathrm{A} 14(j, 1), \mathrm{A} 14(j, 2)]=$ curveintersect $(m(:, j), \mathrm{n}(:, j), \mathrm{x} 27, \mathrm{y} 27) ;$

for $j=1: 46$

$m=[y 88, x 43, x 44, x 45, x 46, x 47, x 48, x 49, x 50, x 51, x 52, x 53, x 54, x 55, x 56, x 57, x 58, x 59, x 60, x 61$, $x 62, x 63, x 64, x 65, x 66, x 67, x 68, x 69, x 70, x 71, x 72, x 73, x 74, x 75, x 76, x 77, x 78, x 79, x 80, x 81, x 82$ $, x 83, x 84, x 85, x 86, x 87]$

$\mathrm{n}=[\mathrm{x} 88, \mathrm{y} 43, \mathrm{y} 44, \mathrm{y} 45, \mathrm{y} 46, \mathrm{y} 47, \mathrm{y} 48, \mathrm{y} 49, \mathrm{y} 50, \mathrm{y} 51, \mathrm{y} 52, \mathrm{y} 53, \mathrm{y} 54, \mathrm{y} 55, \mathrm{y} 56, \mathrm{y} 57, \mathrm{y} 58, \mathrm{y} 59, \mathrm{y} 60, \mathrm{y} 61$, $\mathrm{y} 62, \mathrm{y} 63, \mathrm{y} 64, \mathrm{y} 65, \mathrm{y} 66, \mathrm{y} 67, \mathrm{y} 68, \mathrm{y} 69, \mathrm{y} 70, \mathrm{y} 71, \mathrm{y} 72, \mathrm{y} 73, \mathrm{y} 74, \mathrm{y} 75, \mathrm{y} 76, \mathrm{y} 77, \mathrm{y} 78, \mathrm{y} 79, \mathrm{y} 80, \mathrm{y} 81, \mathrm{y} 82$ $, \mathrm{y} 83, \mathrm{y} 84, \mathrm{y} 85, \mathrm{y} 86, \mathrm{y} 87]$

$[\mathrm{A} 15(j, 1), \mathrm{A} 15(j, 2)]=$ curveintersect $(\mathrm{m}(:, j), \mathrm{n}(:, j), \mathrm{x} 26, \mathrm{y} 26) ;$

end

for $j=1: 46$

$m=[y 88, x 43, x 44, x 45, x 46, x 47, x 48, x 49, x 50, x 51, x 52, x 53, x 54, x 55, x 56, x 57, x 58, x 59, x 60, x 61$, $x 62, x 63, x 64, x 65, x 66, x 67, x 68, x 69, x 70, x 71, x 72, x 73, x 74, x 75, x 76, x 77, x 78, x 79, x 80, x 81, x 82$ $, x 83, x 84, x 85, x 86, x 87]$

$\mathrm{n}=[\mathrm{x} 88, \mathrm{y} 43, \mathrm{y} 44, \mathrm{y} 45, \mathrm{y} 46, \mathrm{y} 47, \mathrm{y} 48, \mathrm{y} 49, \mathrm{y} 50, \mathrm{y} 51, \mathrm{y} 52, \mathrm{y} 53, \mathrm{y} 54, \mathrm{y} 55, \mathrm{y} 56, \mathrm{y} 57, \mathrm{y} 58, \mathrm{y} 59, \mathrm{y} 60, \mathrm{y} 61$, $\mathrm{y} 62, \mathrm{y} 63, \mathrm{y} 64, \mathrm{y} 65, \mathrm{y} 66, \mathrm{y} 67, \mathrm{y} 68, \mathrm{y} 69, \mathrm{y} 70, \mathrm{y} 71, \mathrm{y} 72, \mathrm{y} 73, \mathrm{y} 74, \mathrm{y} 75, \mathrm{y} 76, \mathrm{y} 77, \mathrm{y} 78, \mathrm{y} 79, \mathrm{y} 80, \mathrm{y} 81, \mathrm{y} 82$ $, \mathrm{y} 83, \mathrm{y} 84, \mathrm{y} 85, \mathrm{y} 86, \mathrm{y} 87] ;$

$[\mathrm{A} 16(j, 1), \mathrm{A} 16(j, 2)]=$ curveintersect $(m(:, j), \mathrm{n}(:, j), \mathrm{x} 25, \mathrm{y} 25) ;$

end

for $j=1: 46$

$m=[y 88, x 43, x 44, x 45, x 46, x 47, x 48, x 49, x 50, x 51, x 52, x 53, x 54, x 55, x 56, x 57, x 58, x 59, x 60, x 61$, $x 62, x 63, x 64, x 65, x 66, x 67, x 68, x 69, x 70, x 71, x 72, x 73, x 74, x 75, x 76, x 77, x 78, x 79, x 80, x 81, x 82$ $, x 83, x 84, x 85, x 86, x 87]$

$\mathrm{n}=[\mathrm{x} 88, \mathrm{y} 43, \mathrm{y} 44, \mathrm{y} 45, \mathrm{y} 46, \mathrm{y} 47, \mathrm{y} 48, \mathrm{y} 49, \mathrm{y} 50, \mathrm{y} 51, \mathrm{y} 52, \mathrm{y} 53, \mathrm{y} 54, \mathrm{y} 55, \mathrm{y} 56, \mathrm{y} 57, \mathrm{y} 58, \mathrm{y} 59, \mathrm{y} 60, \mathrm{y} 61$, $\mathrm{y} 62, \mathrm{y} 63, \mathrm{y} 64, \mathrm{y} 65, \mathrm{y} 66, \mathrm{y} 67, \mathrm{y} 68, \mathrm{y} 69, \mathrm{y} 70, \mathrm{y} 71, \mathrm{y} 72, \mathrm{y} 73, \mathrm{y} 74, \mathrm{y} 75, \mathrm{y} 76, \mathrm{y} 77, \mathrm{y} 78, \mathrm{y} 79, \mathrm{y} 80, \mathrm{y} 81, \mathrm{y} 82$ $, \mathrm{y} 83, \mathrm{y} 84, \mathrm{y} 85, \mathrm{y} 86, \mathrm{y} 87] ;$

$[A 17(j, 1), A 17(j, 2)]=$ curveintersect $(m(:, j), n(:, j), x 24, y 24) ;$

end

for $j=1: 46$

$m=[y 88, x 43, x 44, x 45, x 46, x 47, x 48, x 49, x 50, x 51, x 52, x 53, x 54, x 55, x 56, x 57, x 58, x 59, x 60, x 61$, $x 62, x 63, x 64, x 65, x 66, x 67, x 68, x 69, x 70, x 71, x 72, x 73, x 74, x 75, x 76, x 77, x 78, x 79, x 80, x 81, x 82$ $, \times 83, x 84, x 85, x 86, x 87]$

$\mathrm{n}=[\mathrm{x} 88, \mathrm{y} 43, \mathrm{y} 44, \mathrm{y} 45, \mathrm{y} 46, \mathrm{y} 47, \mathrm{y} 48, \mathrm{y} 49, \mathrm{y} 50, \mathrm{y} 51, \mathrm{y} 52, \mathrm{y} 53, \mathrm{y} 54, \mathrm{y} 55, \mathrm{y} 56, \mathrm{y} 57, \mathrm{y} 58, \mathrm{y} 59, \mathrm{y} 60, \mathrm{y} 61$, $y 62, y 63, y 64, y 65, y 66, y 67, y 68, y 69, y 70, y 71, y 72, y 73, y 74, y 75, y 76, y 77, y 78, y 79, y 80, y 81, y 82$ $, \mathrm{y} 83, \mathrm{y} 84, \mathrm{y} 85, \mathrm{y} 86, \mathrm{y} 87] ;$

$[A 18(j, 1), A 18(j, 2)]=$ curveintersect $(m(:, j), n(:, j), x 23, y 23) ;$

end

for $j=1: 46$

$\mathrm{m}=[\mathrm{y} 88, \mathrm{x} 43, \mathrm{x} 44, \mathrm{x} 45, \mathrm{x} 46, \mathrm{x} 47, \mathrm{x} 48, \mathrm{x} 49, \mathrm{x} 50, \mathrm{x} 51, \mathrm{x} 52, \mathrm{x} 53, \mathrm{x} 54, \mathrm{x} 55, \mathrm{x} 56, \mathrm{x} 57, \mathrm{x} 58, \mathrm{x} 59, \mathrm{x} 60, \mathrm{x} 61$, $x 62, x 63, x 64, x 65, x 66, x 67, x 68, x 69, x 70, x 71, x 72, x 73, x 74, x 75, x 76, x 77, x 78, x 79, x 80, x 81, x 82$ $, x 83, x 84, x 85, x 86, x 87]$

$\mathrm{n}=[\mathrm{x} 88, \mathrm{y} 43, \mathrm{y} 44, \mathrm{y} 45, \mathrm{y} 46, \mathrm{y} 47, \mathrm{y} 48, \mathrm{y} 49, \mathrm{y} 50, \mathrm{y} 51, \mathrm{y} 52, \mathrm{y} 53, \mathrm{y} 54, \mathrm{y} 55, \mathrm{y} 56, \mathrm{y} 57, \mathrm{y} 58, \mathrm{y} 59, \mathrm{y} 60, \mathrm{y} 61$, $\mathrm{y} 62, \mathrm{y} 63, \mathrm{y} 64, \mathrm{y} 65, \mathrm{y} 66, \mathrm{y} 67, \mathrm{y} 68, \mathrm{y} 69, \mathrm{y} 70, \mathrm{y} 71, \mathrm{y} 72, \mathrm{y} 73, \mathrm{y} 74, \mathrm{y} 75, \mathrm{y} 76, \mathrm{y} 77, \mathrm{y} 78, \mathrm{y} 79, \mathrm{y} 80, \mathrm{y} 81, \mathrm{y} 82$ $, \mathrm{y} 83, \mathrm{y} 84, \mathrm{y} 85, \mathrm{y} 86, \mathrm{y} 87]$

end

$[A 19(j, 1), A 19(j, 2)]=$ curveintersect $(m(:, j), n(:, j), x 22, y 22)$;

for $j=1: 46$

$\mathrm{m}=[\mathrm{y} 88, \mathrm{x} 43, \mathrm{x} 44, \mathrm{x} 45, \mathrm{x} 46, \mathrm{x} 47, \mathrm{x} 48, \mathrm{x} 49, \mathrm{x} 50, \mathrm{x} 51, \mathrm{x} 52, \mathrm{x} 53, \mathrm{x} 54, \mathrm{x} 55, \mathrm{x} 56, \mathrm{x} 57, \mathrm{x} 58, \mathrm{x} 59, \mathrm{x} 60, \mathrm{x} 61$, $x 62, x 63, x 64, x 65, x 66, x 67, x 68, x 69, x 70, x 71, x 72, x 73, x 74, x 75, x 76, x 77, x 78, x 79, x 80, x 81, x 82$ $, \times 83, x 84, x 85, \times 86, x 87]$

$\mathrm{n}=[\mathrm{x} 88, \mathrm{y} 43, \mathrm{y} 44, \mathrm{y} 45, \mathrm{y} 46, \mathrm{y} 47, \mathrm{y} 48, \mathrm{y} 49, \mathrm{y} 50, \mathrm{y} 51, \mathrm{y} 52, \mathrm{y} 53, \mathrm{y} 54, \mathrm{y} 55, \mathrm{y} 56, \mathrm{y} 57, \mathrm{y} 58, \mathrm{y} 59, \mathrm{y} 60, \mathrm{y} 61$, $y 62, y 63, y 64, y 65, y 66, y 67, y 68, y 69, y 70, y 71, y 72, y 73, y 74, y 75, y 76, y 77, y 78, y 79, y 80, y 81, y 82$ $, \mathrm{y} 83, \mathrm{y} 84, \mathrm{y} 85, \mathrm{y} 86, \mathrm{y} 87]$

$[\mathrm{A} 20(j, 1), \mathrm{A} 20(j, 2)]=$ curveintersect $(\mathrm{m}(:, j), \mathrm{n}(:, j), \mathrm{x} 21, \mathrm{y} 21) ;$ 
end

for $j=1: 46$

$m=[y 88, x 43, x 44, x 45, x 46, x 47, x 48, x 49, x 50, x 51, x 52, x 53, x 54, x 55, x 56, x 57, x 58, x 59, x 60, x 61$, $x 62, x 63, x 64, x 65, x 66, x 67, x 68, x 69, x 70, x 71, x 72, x 73, x 74, x 75, x 76, x 77, x 78, x 79, x 80, x 81, x 82$ $, \times 83, x 84, x 85, x 86, x 87] ;$

$\mathrm{n}=[\mathrm{x} 88, \mathrm{y} 43, \mathrm{y} 44, \mathrm{y} 45, \mathrm{y} 46, \mathrm{y} 47, \mathrm{y} 48, \mathrm{y} 49, \mathrm{y} 50, \mathrm{y} 51, \mathrm{y} 52, \mathrm{y} 53, \mathrm{y} 54, \mathrm{y} 55, \mathrm{y} 56, \mathrm{y} 57, \mathrm{y} 58, \mathrm{y} 59, \mathrm{y} 60, \mathrm{y} 61$, $\mathrm{y} 62, \mathrm{y} 63, \mathrm{y} 64, \mathrm{y} 65, \mathrm{y} 66, \mathrm{y} 67, \mathrm{y} 68, \mathrm{y} 69, \mathrm{y} 70, \mathrm{y} 71, \mathrm{y} 72, \mathrm{y} 73, \mathrm{y} 74, \mathrm{y} 75, \mathrm{y} 76, \mathrm{y} 77, \mathrm{y} 78, \mathrm{y} 79, \mathrm{y} 80, \mathrm{y} 81, \mathrm{y} 82$ $, \mathrm{y} 83, \mathrm{y} 84, \mathrm{y} 85, \mathrm{y} 86, \mathrm{y} 87]$

end

$[A 21(j, 1), A 21(j, 2)]=$ curveintersect $(m(:, j), n(:, j), x 20, y 20) ;$

for $j=1: 46$

$m=[y 88, x 43, x 44, x 45, x 46, x 47, x 48, x 49, x 50, x 51, x 52, x 53, x 54, x 55, x 56, x 57, x 58, x 59, x 60, x 61$, $x 62, x 63, x 64, x 65, x 66, x 67, x 68, x 69, x 70, x 71, x 72, x 73, x 74, x 75, x 76, x 77, x 78, x 79, x 80, x 81, x 82$ $, x 83, x 84, x 85, x 86, x 87]$;

$\mathrm{n}=[\mathrm{x} 88, \mathrm{y} 43, \mathrm{y} 44, \mathrm{y} 45, \mathrm{y} 46, \mathrm{y} 47, \mathrm{y} 48, \mathrm{y} 49, \mathrm{y} 50, \mathrm{y} 51, \mathrm{y} 52, \mathrm{y} 53, \mathrm{y} 54, \mathrm{y} 55, \mathrm{y} 56, \mathrm{y} 57, \mathrm{y} 58, \mathrm{y} 59, \mathrm{y} 60, \mathrm{y} 61$, $y 62, y 63, y 64, y 65, y 66, y 67, y 68, y 69, y 70, y 71, y 72, y 73, y 74, y 75, y 76, y 77, y 78, y 79, y 80, y 81, y 82$ $, \mathrm{y} 83, \mathrm{y} 84, \mathrm{y} 85, \mathrm{y} 86, \mathrm{y} 87]$

end

$[A 22(j, 1), A 22(j, 2)]=$ curveintersect $(m(:, j), n(:, j), x 19, y 19)$;

for $j=1: 46$

$m=[y 88, x 43, x 44, x 45, x 46, x 47, x 48, x 49, x 50, x 51, x 52, x 53, x 54, x 55, x 56, x 57, x 58, x 59, x 60, x 61$, $x 62, x 63, x 64, x 65, x 66, x 67, x 68, x 69, x 70, x 71, x 72, x 73, x 74, x 75, x 76, x 77, x 78, x 79, x 80, x 81, x 82$ $, x 83, x 84, x 85, x 86, x 87] ;$

$\mathrm{n}=[\mathrm{x} 88, \mathrm{y} 43, \mathrm{y} 44, \mathrm{y} 45, \mathrm{y} 46, \mathrm{y} 47, \mathrm{y} 48, \mathrm{y} 49, \mathrm{y} 50, \mathrm{y} 51, \mathrm{y} 52, \mathrm{y} 53, \mathrm{y} 54, \mathrm{y} 55, \mathrm{y} 56, \mathrm{y} 57, \mathrm{y} 58, \mathrm{y} 59, \mathrm{y} 60, \mathrm{y} 61$, $\mathrm{y} 62, \mathrm{y} 63, \mathrm{y} 64, \mathrm{y} 65, \mathrm{y} 66, \mathrm{y} 67, \mathrm{y} 68, \mathrm{y} 69, \mathrm{y} 70, \mathrm{y} 71, \mathrm{y} 72, \mathrm{y} 73, \mathrm{y} 74, \mathrm{y} 75, \mathrm{y} 76, \mathrm{y} 77, \mathrm{y} 78, \mathrm{y} 79, \mathrm{y} 80, \mathrm{y} 81, \mathrm{y} 82$ $, \mathrm{y} 83, \mathrm{y} 84, \mathrm{y} 85, \mathrm{y} 86, \mathrm{y} 87]$

end

$[A 23(j, 1), A 23(j, 2)]=$ curveintersect $(m(:, j), n(:, j), x 18, y 18)$;

for $j=1: 46$

$\mathrm{m}=[\mathrm{y} 88, \mathrm{x} 43, \mathrm{x} 44, \mathrm{x} 45, \mathrm{x} 46, \mathrm{x} 47, \mathrm{x} 48, \mathrm{x} 49, \mathrm{x} 50, \mathrm{x} 51, \mathrm{x} 52, \mathrm{x} 53, \mathrm{x} 54, \mathrm{x} 55, \mathrm{x} 56, \mathrm{x} 57, \mathrm{x} 58, \mathrm{x} 59, \mathrm{x} 60, \mathrm{x} 61$, $x 62, x 63, x 64, x 65, x 66, x 67, x 68, x 69, x 70, x 71, x 72, x 73, x 74, x 75, x 76, x 77, x 78, x 79, x 80, x 81, x 82$ $, x 83, x 84, x 85, x 86, x 87]$;

$\mathrm{n}=[\mathrm{x} 88, \mathrm{y} 43, \mathrm{y} 44, \mathrm{y} 45, \mathrm{y} 46, \mathrm{y} 47, \mathrm{y} 48, \mathrm{y} 49, \mathrm{y} 50, \mathrm{y} 51, \mathrm{y} 52, \mathrm{y} 53, \mathrm{y} 54, \mathrm{y} 55, \mathrm{y} 56, \mathrm{y} 57, \mathrm{y} 58, \mathrm{y} 59, \mathrm{y} 60, \mathrm{y} 61$, $\mathrm{y} 62, \mathrm{y} 63, \mathrm{y} 64, \mathrm{y} 65, \mathrm{y} 66, \mathrm{y} 67, \mathrm{y} 68, \mathrm{y} 69, \mathrm{y} 70, \mathrm{y} 71, \mathrm{y} 72, \mathrm{y} 73, \mathrm{y} 74, \mathrm{y} 75, \mathrm{y} 76, \mathrm{y} 77, \mathrm{y} 78, \mathrm{y} 79, \mathrm{y} 80, \mathrm{y} 81, \mathrm{y} 82$ $, \mathrm{y} 83, \mathrm{y} 84, \mathrm{y} 85, \mathrm{y} 86, \mathrm{y} 87] ;$

end $[A 24(j, 1), A 24(j, 2)]=$ curveintersect $(m(:, j), n(:, j), x 17, y 17)$;

for $j=1: 46$

$m=[y 88, x 43, x 44, x 45, x 46, x 47, x 48, x 49, x 50, x 51, x 52, x 53, x 54, x 55, x 56, x 57, x 58, x 59, x 60, x 61$, $x 62, x 63, x 64, x 65, x 66, x 67, x 68, x 69, x 70, x 71, x 72, x 73, x 74, x 75, x 76, x 77, x 78, x 79, x 80, x 81, x 82$ $, \times 83, x 84, x 85, x 86, x 87]$

$\mathrm{n}=[\mathrm{x} 88, \mathrm{y} 43, \mathrm{y} 44, \mathrm{y} 45, \mathrm{y} 46, \mathrm{y} 47, \mathrm{y} 48, \mathrm{y} 49, \mathrm{y} 50, \mathrm{y} 51, \mathrm{y} 52, \mathrm{y} 53, \mathrm{y} 54, \mathrm{y} 55, \mathrm{y} 56, \mathrm{y} 57, \mathrm{y} 58, \mathrm{y} 59, \mathrm{y} 60, \mathrm{y} 61$, $\mathrm{y} 62, \mathrm{y} 63, \mathrm{y} 64, \mathrm{y} 65, \mathrm{y} 66, \mathrm{y} 67, \mathrm{y} 68, \mathrm{y} 69, \mathrm{y} 70, \mathrm{y} 71, \mathrm{y} 72, \mathrm{y} 73, \mathrm{y} 74, \mathrm{y} 75, \mathrm{y} 76, \mathrm{y} 77, \mathrm{y} 78, \mathrm{y} 79, \mathrm{y} 80, \mathrm{y} 81, \mathrm{y} 82$ $, \mathrm{y} 83, \mathrm{y} 84, \mathrm{y} 85, \mathrm{y} 86, \mathrm{y} 87] ;$

end

$[A 25(j, 1), A 25(j, 2)]=$ curveintersect $(m(:, j), n(:, j), x 16, y 16)$;

for $j=1: 45$

$m=[y 88, x 44, x 45, x 46, x 47, x 48, x 49, x 50, x 51, x 52, x 53, x 54, x 55, x 56, x 57, x 58, x 59, x 60, x 61, x 62$, $x 63, x 64, x 65, x 66, x 67, x 68, x 69, x 70, x 71, x 72, x 73, x 74, x 75, x 76, x 77, x 78, x 79, x 80, x 81, x 82, x 83$ $, \times 84, x 85, x 86, x 87]$

$\mathrm{n}=[\mathrm{x} 88, \mathrm{y} 44, \mathrm{y} 45, \mathrm{y} 46, \mathrm{y} 47, \mathrm{y} 48, \mathrm{y} 49, \mathrm{y} 50, \mathrm{y} 51, \mathrm{y} 52, \mathrm{y} 53, \mathrm{y} 54, \mathrm{y} 55, \mathrm{y} 56, \mathrm{y} 57, \mathrm{y} 58, \mathrm{y} 59, \mathrm{y} 60, \mathrm{y} 61, \mathrm{y} 62$, $\mathrm{y} 63, \mathrm{y} 64, \mathrm{y} 65, \mathrm{y} 66, \mathrm{y} 67, \mathrm{y} 68, \mathrm{y} 69, \mathrm{y} 70, \mathrm{y} 71, \mathrm{y} 72, \mathrm{y} 73, \mathrm{y} 74, \mathrm{y} 75, \mathrm{y} 76, \mathrm{y} 77, \mathrm{y} 78, \mathrm{y} 79, \mathrm{y} 80, \mathrm{y} 81, \mathrm{y} 82, \mathrm{y} 83$ $, \mathrm{y} 84, \mathrm{y} 85, \mathrm{y} 86, \mathrm{y} 87]$

$[A 26(j, 1), A 26(j, 2)]=$ curveintersect $(m(:, j), n(:, j), x 15, y 15)$;

end

for $j=1: 45$

$\mathrm{m}=[\mathrm{y} 88, \mathrm{x} 44, \mathrm{x} 45, \mathrm{x} 46, \mathrm{x} 47, \mathrm{x} 48, \mathrm{x} 49, \mathrm{x} 50, \mathrm{x} 51, \mathrm{x} 52, \mathrm{x} 53, \mathrm{x} 54, \mathrm{x} 55, \mathrm{x} 56, \mathrm{x} 57, \mathrm{x} 58, \mathrm{x} 59, \mathrm{x} 60, \mathrm{x} 61, \mathrm{x} 62$, $x 63, x 64, x 65, x 66, x 67, x 68, x 69, x 70, x 71, x 72, x 73, x 74, x 75, x 76, x 77, x 78, x 79, x 80, x 81, x 82, x 83$ $, \times 84, \times 85, \times 86, \times 87]$

$\mathrm{n}=\left[\mathrm{x} 88, \mathrm{y} 44, \mathrm{y} 45, \mathrm{y} 46, \mathrm{y} 47, \mathrm{y} 48, \mathrm{y} 49, \mathrm{y} 50, \mathrm{y} 51, \mathrm{y} 52, \mathrm{y} 53, \mathrm{y} 54, \mathrm{y} 55, \mathrm{y} 56, \mathrm{y} 57, \mathrm{y}^{58}, \mathrm{y} 59, \mathrm{y} 60, \mathrm{y} 61, \mathrm{y} 62\right.$, $\mathrm{y} 63, \mathrm{y} 64, \mathrm{y} 65, \mathrm{y} 66, \mathrm{y} 67, \mathrm{y} 68, \mathrm{y} 69, \mathrm{y} 70, \mathrm{y} 71, \mathrm{y} 72, \mathrm{y} 73, \mathrm{y} 74, \mathrm{y} 75, \mathrm{y} 76, \mathrm{y} 77, \mathrm{y} 78, \mathrm{y} 79, \mathrm{y} 80, \mathrm{y} 81, \mathrm{y} 82, \mathrm{y} 83$ $, \mathrm{y} 84, \mathrm{y} 85, \mathrm{y} 86, \mathrm{y} 87] ;$

$[A 27(j, 1), A 27(j, 2)]=$ curveintersect $(m(:, j), n(:, j), x 14, y 14)$;

end

for $j=1: 45$

$m=[y 88, x 44, x 45, x 46, x 47, x 48, x 49, x 50, x 51, x 52, x 53, x 54, x 55, x 56, x 57, x 58, x 59, x 60, x 61, x 62$, $x 63, x 64, x 65, x 66, x 67, x 68, x 69, x 70, x 71, x 72, x 73, x 74, x 75, x 76, x 77, x 78, x 79, x 80, x 81, x 82, x 83$ $, \times 84, \times 85, \times 86, \times 87]$

$\mathrm{n}=[\mathrm{x} 88, \mathrm{y} 44, \mathrm{y} 45, \mathrm{y} 46, \mathrm{y} 47, \mathrm{y} 48, \mathrm{y} 49, \mathrm{y} 50, \mathrm{y} 51, \mathrm{y} 52, \mathrm{y} 53, \mathrm{y} 54, \mathrm{y} 55, \mathrm{y} 56, \mathrm{y} 57, \mathrm{y} 58, \mathrm{y} 59, \mathrm{y} 60, \mathrm{y} 61, \mathrm{y} 62$, $\mathrm{y} 63, \mathrm{y} 64, \mathrm{y} 65, \mathrm{y} 66, \mathrm{y} 67, \mathrm{y} 68, \mathrm{y} 69, \mathrm{y} 70, \mathrm{y} 71, \mathrm{y} 72, \mathrm{y} 73, \mathrm{y} 74, \mathrm{y} 75, \mathrm{y} 76, \mathrm{y} 77, \mathrm{y} 78, \mathrm{y} 79, \mathrm{y} 80, \mathrm{y} 81, \mathrm{y} 82, \mathrm{y} 83$ $, \mathrm{y} 84, \mathrm{y} 85, \mathrm{y} 86, \mathrm{y} 87]$; 
$[A 28(j, 1), A 28(j, 2)]=$ curveintersect $(m(:, j), n(:, j), x 13, y 13)$;

end

for $j=1: 45$

$m=[y 88, x 44, x 45, x 46, x 47, x 48, x 49, x 50, x 51, x 52, x 53, x 54, x 55, x 56, x 57, x 58, x 59, x 60, x 61, x 62$, $x 63, x 64, x 65, x 66, x 67, x 68, x 69, x 70, x 71, x 72, x 73, x 74, x 75, x 76, x 77, x 78, x 79, x 80, x 81, x 82, x 83$ $, \times 84, x 85, x 86, x 87]$

$\mathrm{n}=[\mathrm{x} 88, \mathrm{y} 44, \mathrm{y} 45, \mathrm{y} 46, \mathrm{y} 47, \mathrm{y} 48, \mathrm{y} 49, \mathrm{y} 50, \mathrm{y} 51, \mathrm{y} 52, \mathrm{y} 53, \mathrm{y} 54, \mathrm{y} 55, \mathrm{y} 56, \mathrm{y} 57, \mathrm{y} 58, \mathrm{y} 59, \mathrm{y} 60, \mathrm{y} 61, \mathrm{y} 62$, $\mathrm{y} 63, \mathrm{y} 64, \mathrm{y} 65, \mathrm{y} 66, \mathrm{y} 67, \mathrm{y} 68, \mathrm{y} 69, \mathrm{y} 70, \mathrm{y} 71, \mathrm{y} 72, \mathrm{y} 73, \mathrm{y} 74, \mathrm{y} 75, \mathrm{y} 76, \mathrm{y} 77, \mathrm{y} 78, \mathrm{y} 79, \mathrm{y} 80, \mathrm{y} 81, \mathrm{y} 82, \mathrm{y} 83$ $, \mathrm{y} 84, \mathrm{y} 85, \mathrm{y} 86, \mathrm{y} 87]$

end

$[A 29(j, 1), A 29(j, 2)]=$ curveintersect $(m(:, j), n(:, j), x 12, y 12)$;

for $j=1: 45$

$\mathrm{m}=[\mathrm{y} 88, \mathrm{x} 44, \mathrm{x} 45, \mathrm{x} 46, \mathrm{x} 47, \mathrm{x} 48, \mathrm{x} 49, \mathrm{x} 50, \mathrm{x} 51, \mathrm{x} 52, \mathrm{x} 53, \mathrm{x} 54, \mathrm{x} 55, \mathrm{x} 56, \mathrm{x} 57, \mathrm{x} 58, \mathrm{x} 59, \mathrm{x} 60, \mathrm{x} 61, \mathrm{x} 62$, $x 63, x 64, x 65, x 66, x 67, x 68, x 69, x 70, x 71, x 72, x 73, x 74, x 75, x 76, x 77, x 78, x 79, x 80, x 81, x 82, x 83$ $, \times 84, \times 85, \times 86, x 87]$

$\mathrm{n}=[\mathrm{x} 88, \mathrm{y} 44, \mathrm{y} 45, \mathrm{y} 46, \mathrm{y} 47, \mathrm{y} 48, \mathrm{y} 49, \mathrm{y} 50, \mathrm{y} 51, \mathrm{y} 52, \mathrm{y} 53, \mathrm{y} 54, \mathrm{y} 55, \mathrm{y} 56, \mathrm{y} 57, \mathrm{y} 58, \mathrm{y} 59, \mathrm{y} 60, \mathrm{y} 61, \mathrm{y} 62$, $\mathrm{y} 63, \mathrm{y} 64, \mathrm{y} 65, \mathrm{y} 66, \mathrm{y} 67, \mathrm{y} 68, \mathrm{y} 69, \mathrm{y} 70, \mathrm{y} 71, \mathrm{y} 72, \mathrm{y} 73, \mathrm{y} 74, \mathrm{y} 75, \mathrm{y} 76, \mathrm{y} 77, \mathrm{y} 78, \mathrm{y} 79, \mathrm{y} 80, \mathrm{y} 81, \mathrm{y} 82, \mathrm{y} 83$ $, \mathrm{y} 84, \mathrm{y} 85, \mathrm{y} 86, \mathrm{y} 87] ;$

end

$[A 30(j, 1), A 30(j, 2)]=$ curveintersect $(m(:, j), n(:, j), x 11, y 11)$;

for $j=1: 45$

$m=[y 88, x 44, x 45, x 46, x 47, x 48, x 49, x 50, x 51, x 52, x 53, x 54, x 55, x 56, x 57, x 58, x 59, x 60, x 61, x 62$, $x 63, x 64, x 65, x 66, x 67, x 68, x 69, x 70, x 71, x 72, x 73, x 74, x 75, x 76, x 77, x 78, x 79, x 80, x 81, x 82, x 83$ $, \times 84, \times 85, \times 86, x 87]$;

$\mathrm{n}=[\mathrm{x} 88, \mathrm{y} 44, \mathrm{y} 45, \mathrm{y} 46, \mathrm{y} 47, \mathrm{y} 48, \mathrm{y} 49, \mathrm{y} 50, \mathrm{y} 51, \mathrm{y} 52, \mathrm{y} 53, \mathrm{y} 54, \mathrm{y} 55, \mathrm{y} 56, \mathrm{y} 57, \mathrm{y} 58, \mathrm{y} 59, \mathrm{y} 60, \mathrm{y} 61, \mathrm{y} 62$, $\mathrm{y} 63, \mathrm{y} 64, \mathrm{y} 65, \mathrm{y} 66, \mathrm{y} 67, \mathrm{y} 68, \mathrm{y} 69, \mathrm{y} 70, \mathrm{y} 71, \mathrm{y} 72, \mathrm{y} 73, \mathrm{y} 74, \mathrm{y} 75, \mathrm{y} 76, \mathrm{y} 77, \mathrm{y} 78, \mathrm{y} 79, \mathrm{y} 80, \mathrm{y} 81, \mathrm{y} 82, \mathrm{y} 83$ $, \mathrm{y} 84, \mathrm{y} 85, \mathrm{y} 86, \mathrm{y} 87]$

$[A 31(j, 1), A 31(j, 2)]=$ curveintersect $(m(:, j), n(:, j), x 10, y 10)$;

end

for $j=1: 45$

$m=[y 88, x 44, x 45, x 46, x 47, x 48, x 49, x 50, x 51, x 52, x 53, x 54, x 55, x 56, x 57, x 58, x 59, x 60, x 61, x 62$, $x 63, x 64, x 65, x 66, x 67, x 68, x 69, x 70, x 71, x 72, x 73, x 74, x 75, x 76, x 77, x 78, x 79, x 80, x 81, x 82, x 83$ $, \times 84, x 85, x 86, x 87]$

$\mathrm{n}=[\mathrm{x} 88, \mathrm{y} 44, \mathrm{y} 45, \mathrm{y} 46, \mathrm{y} 47, \mathrm{y} 48, \mathrm{y} 49, \mathrm{y} 50, \mathrm{y} 51, \mathrm{y} 52, \mathrm{y} 53, \mathrm{y} 54, \mathrm{y} 55, \mathrm{y} 56, \mathrm{y} 57, \mathrm{y} 58, \mathrm{y} 59, \mathrm{y} 60, \mathrm{y} 61, \mathrm{y} 62$, $\mathrm{y} 63, \mathrm{y} 64, \mathrm{y} 65, \mathrm{y} 66, \mathrm{y} 67, \mathrm{y} 68, \mathrm{y} 69, \mathrm{y} 70, \mathrm{y} 71, \mathrm{y} 72, \mathrm{y} 73, \mathrm{y} 74, \mathrm{y} 75, \mathrm{y} 76, \mathrm{y} 77, \mathrm{y} 78, \mathrm{y} 79, \mathrm{y} 80, \mathrm{y} 81, \mathrm{y} 82, \mathrm{y} 83$ $, \mathrm{y} 84, \mathrm{y} 85, \mathrm{y} 86, \mathrm{y} 87] ;$

$[\mathrm{A} 32(j, 1), \mathrm{A} 32(j, 2)]=$ curveintersect $(m(:, j), \mathrm{n}(:, j), \mathrm{x} 9, \mathrm{y} 9)$;

end

for $j=1: 45$

$m=[y 88, x 44, x 45, x 46, x 47, x 48, x 49, x 50, x 51, x 52, x 53, x 54, x 55, x 56, x 57, x 58, x 59, x 60, x 61, x 62$, $x 63, x 64, x 65, x 66, x 67, x 68, x 69, x 70, x 71, x 72, x 73, x 74, x 75, x 76, x 77, x 78, x 79, x 80, x 81, x 82, x 83$ $, \times 84, x 85, x 86, x 87]$

$\mathrm{n}=[\mathrm{x} 88, \mathrm{y} 44, \mathrm{y} 45, \mathrm{y} 46, \mathrm{y} 47, \mathrm{y} 48, \mathrm{y} 49, \mathrm{y} 50, \mathrm{y} 51, \mathrm{y} 52, \mathrm{y} 53, \mathrm{y} 54, \mathrm{y} 55, \mathrm{y} 56, \mathrm{y} 57, \mathrm{y} 58, \mathrm{y} 59, \mathrm{y} 60, \mathrm{y} 61, \mathrm{y} 62$, $\mathrm{y} 63, \mathrm{y} 64, \mathrm{y} 65, \mathrm{y} 66, \mathrm{y} 67, \mathrm{y} 68, \mathrm{y} 69, \mathrm{y} 70, \mathrm{y} 71, \mathrm{y} 72, \mathrm{y} 73, \mathrm{y} 74, \mathrm{y} 75, \mathrm{y} 76, \mathrm{y} 77, \mathrm{y} 78, \mathrm{y} 79, \mathrm{y} 80, \mathrm{y} 81, \mathrm{y} 82, \mathrm{y} 83$ $, \mathrm{y} 84, \mathrm{y} 85, \mathrm{y} 86, \mathrm{y} 87]$

$[A 33(j, 1), A 33(j, 2)]=$ curveintersect $(m(:, j), n(:, j), x 8, y 8)$;

end

for $j=1: 45$

$m=[y 88, x 44, x 45, x 46, x 47, x 48, x 49, x 50, x 51, x 52, x 53, x 54, x 55, x 56, x 57, x 58, x 59, x 60, x 61, x 62$, $x 63, x 64, x 65, x 66, x 67, x 68, x 69, x 70, x 71, x 72, x 73, x 74, x 75, x 76, x 77, x 78, x 79, x 80, x 81, x 82, x 83$ $, \times 84, x 85, x 86, x 87]$

$\mathrm{n}=[\mathrm{x} 88, \mathrm{y} 44, \mathrm{y} 45, \mathrm{y} 46, \mathrm{y} 47, \mathrm{y} 48, \mathrm{y} 49, \mathrm{y} 50, \mathrm{y} 51, \mathrm{y} 52, \mathrm{y} 53, \mathrm{y} 54, \mathrm{y} 55, \mathrm{y} 56, \mathrm{y} 57, \mathrm{y} 58, \mathrm{y} 59, \mathrm{y} 60, \mathrm{y} 61, \mathrm{y} 62$, $y 63, y 64, y 65, y 66, y 67, y 68, y 69, y 70, y 71, y 72, y 73, y 74, y 75, y 76, y 77, y 78, y 79, y 80, y 81, y 82, y 83$ $, \mathrm{y} 84, \mathrm{y} 85, \mathrm{y} 86, \mathrm{y} 87]$

end

$[A 34(j, 1), A 34(j, 2)]=$ curveintersect $(m(:, j), n(:, j), x 7, y 7)$;

for $j=1: 45$

$m=[y 88, x 44, x 45, x 46, x 47, x 48, x 49, x 50, x 51, x 52, x 53, x 54, x 55, x 56, x 57, x 58, x 59, x 60, x 61, x 62$, $x 63, x 64, x 65, x 66, x 67, x 68, x 69, x 70, x 71, x 72, x 73, x 74, x 75, x 76, x 77, x 78, x 79, x 80, x 81, x 82, x 83$ $, \times 84, x 85, x 86, x 87]$

$\mathrm{n}=[\mathrm{x} 88, \mathrm{y} 44, \mathrm{y} 45, \mathrm{y} 46, \mathrm{y} 47, \mathrm{y} 48, \mathrm{y} 49, \mathrm{y} 50, \mathrm{y} 51, \mathrm{y} 52, \mathrm{y} 53, \mathrm{y} 54, \mathrm{y} 55, \mathrm{y} 56, \mathrm{y} 57, \mathrm{y} 58, \mathrm{y} 59, \mathrm{y} 60, \mathrm{y} 61, \mathrm{y} 62$, $\mathrm{y} 63, \mathrm{y} 64, \mathrm{y} 65, \mathrm{y} 66, \mathrm{y} 67, \mathrm{y} 68, \mathrm{y} 69, \mathrm{y} 70, \mathrm{y} 71, \mathrm{y} 72, \mathrm{y} 73, \mathrm{y} 74, \mathrm{y} 75, \mathrm{y} 76, \mathrm{y} 77, \mathrm{y} 78, \mathrm{y} 79, \mathrm{y} 80, \mathrm{y} 81, \mathrm{y} 82, \mathrm{y} 83$ $\left., \mathrm{y}^{84}, \mathrm{y} 85, \mathrm{y} 86, \mathrm{y} 87\right] ;$

end

$[A 35(j, 1), A 35(j, 2)]=$ curveintersect $\left(m(:, j), n(:, j), x 6, y^{6}\right)$;

for $j=1: 45$

$m=[y 88, x 44, x 45, x 46, x 47, x 48, x 49, x 50, x 51, x 52, x 53, x 54, x 55, x 56, x 57, x 58, x 59, x 60, x 61, x 62$, $x 63, x 64, x 65, x 66, x 67, x 68, x 69, x 70, x 71, x 72, x 73, x 74, x 75, x 76, x 77, x 78, x 79, x 80, x 81, x 82, x 83$ $, \times 84, \times 85, \times 86, \times 87]$ 
$\mathrm{n}=[\mathrm{x} 88, \mathrm{y} 44, \mathrm{y} 45, \mathrm{y} 46, \mathrm{y} 47, \mathrm{y} 48, \mathrm{y} 49, \mathrm{y} 50, \mathrm{y} 51, \mathrm{y} 52, \mathrm{y} 53, \mathrm{y} 54, \mathrm{y} 55, \mathrm{y} 56, \mathrm{y} 57, \mathrm{y} 58, \mathrm{y} 59, \mathrm{y} 60, \mathrm{y} 61, \mathrm{y} 62$, $\mathrm{y} 63, y 64, y 65, y 66, y 67, y 68, y 69, y 70, y 71, y 72, y 73, y 74, y 75, y 76, y 77, y 78, y 79, y 80, y 81, y 82, y 83$ $, \mathrm{y} 84, \mathrm{y} 85, \mathrm{y} 86, \mathrm{y} 87]$

end

$[A 36(j, 1), A 36(j, 2)]=$ curveintersect $(m(:, j), n(:, j), x 5, y 5)$;

for $j=1: 45$

$\mathrm{m}=[\mathrm{y} 88, \mathrm{x} 44, \mathrm{x} 45, \mathrm{x} 46, \mathrm{x} 47, \mathrm{x} 48, \mathrm{x} 49, \mathrm{x} 50, \mathrm{x} 51, \mathrm{x} 52, \mathrm{x} 53, \mathrm{x} 54, \mathrm{x} 55, \mathrm{x} 56, x 57, x 58, x 59, x 60, x 61, x 62$, $x 63, x 64, x 65, x 66, x 67, x 68, x 69, x 70, x 71, x 72, x 73, x 74, x 75, x 76, x 77, x 78, x 79, x 80, x 81, x 82, x 83$ $, \times 84, x 85, x 86, x 87]$

$\mathrm{n}=[\mathrm{x} 88, \mathrm{y} 44, \mathrm{y} 45, \mathrm{y} 46, \mathrm{y} 47, \mathrm{y} 48, \mathrm{y} 49, \mathrm{y} 50, \mathrm{y} 51, \mathrm{y} 52, \mathrm{y} 53, \mathrm{y} 54, \mathrm{y} 55, \mathrm{y} 56, \mathrm{y} 57, \mathrm{y} 58, \mathrm{y} 59, \mathrm{y} 60, \mathrm{y} 61, \mathrm{y} 62$, $\mathrm{y} 63, \mathrm{y} 64, \mathrm{y} 65, \mathrm{y} 66, \mathrm{y} 67, \mathrm{y} 68, \mathrm{y} 69, \mathrm{y} 70, \mathrm{y} 71, \mathrm{y} 72, \mathrm{y} 73, \mathrm{y} 74, \mathrm{y} 75, \mathrm{y} 76, \mathrm{y} 77, \mathrm{y} 78, \mathrm{y} 79, \mathrm{y} 80, \mathrm{y} 81, \mathrm{y} 82, \mathrm{y} 83$ $, \mathrm{y} 84, \mathrm{y} 85, \mathrm{y} 86, \mathrm{y} 87]$

end

$[A 37(j, 1), A 37(j, 2)]=$ curveintersect $(m(:, j), n(:, j), x 4, y 4) ;$

for $j=1: 45$

$m=[y 88, x 44, x 45, x 46, x 47, x 48, x 49, x 50, x 51, x 52, x 53, x 54, x 55, x 56, x 57, x 58, x 59, x 60, x 61, x 62$, $x 63, x 64, x 65, x 66, x 67, x 68, x 69, x 70, x 71, x 72, x 73, x 74, x 75, x 76, x 77, x 78, x 79, x 80, x 81, x 82, x 83$ $, \times 84, \times 85, x 86, x 87]$

$\mathrm{n}=[\mathrm{x} 88, \mathrm{y} 44, \mathrm{y} 45, \mathrm{y} 46, \mathrm{y} 47, \mathrm{y} 48, \mathrm{y} 49, \mathrm{y} 50, \mathrm{y} 51, \mathrm{y} 52, \mathrm{y} 53, \mathrm{y} 54, \mathrm{y} 55, \mathrm{y} 56, \mathrm{y} 57, \mathrm{y} 58, \mathrm{y} 59, \mathrm{y} 60, \mathrm{y} 61, \mathrm{y} 62$, $\mathrm{y} 63, \mathrm{y} 64, \mathrm{y} 65, \mathrm{y} 66, \mathrm{y} 67, \mathrm{y} 68, \mathrm{y} 69, \mathrm{y} 70, \mathrm{y} 71, \mathrm{y} 72, \mathrm{y} 73, \mathrm{y} 74, \mathrm{y} 75, \mathrm{y} 76, \mathrm{y} 77, \mathrm{y} 78, \mathrm{y} 79, \mathrm{y} 80, \mathrm{y} 81, \mathrm{y} 82, \mathrm{y} 83$ $, \mathrm{y} 84, \mathrm{y} 85, \mathrm{y} 86, \mathrm{y} 87]$

$[A 38(j, 1), A 38(j, 2)]=$ curveintersect $(m(:, j), n(:, j), x 3, y 3)$;

end

for $j=1: 45$

$m=[y 88, x 44, x 45, x 46, x 47, x 48, x 49, x 50, x 51, x 52, x 53, x 54, x 55, x 56, x 57, x 58, x 59, x 60, x 61, x 62$, $x 63, x 64, x 65, x 66, x 67, x 68, x 69, x 70, x 71, x 72, x 73, x 74, x 75, x 76, x 77, x 78, x 79, x 80, x 81, x 82, x 83$ $, \times 84, x 85, x 86, x 87]$;

$\mathrm{n}=[\mathrm{x} 88, \mathrm{y} 44, \mathrm{y} 45, \mathrm{y} 46, \mathrm{y} 47, \mathrm{y} 48, \mathrm{y} 49, \mathrm{y} 50, \mathrm{y} 51, \mathrm{y} 52, \mathrm{y} 53, \mathrm{y} 54, \mathrm{y} 55, \mathrm{y} 56, \mathrm{y} 57, \mathrm{y} 58, \mathrm{y} 59, \mathrm{y} 60, \mathrm{y} 61, \mathrm{y} 62$, $\mathrm{y} 63, \mathrm{y} 64, \mathrm{y} 65, \mathrm{y} 66, \mathrm{y} 67, \mathrm{y} 68, \mathrm{y} 69, \mathrm{y} 70, \mathrm{y} 71, \mathrm{y} 72, \mathrm{y} 73, \mathrm{y} 74, \mathrm{y} 75, \mathrm{y} 76, \mathrm{y} 77, \mathrm{y} 78, \mathrm{y} 79, \mathrm{y} 80, \mathrm{y} 81, \mathrm{y} 82, \mathrm{y} 83$ $, \mathrm{y} 84, \mathrm{y} 85, \mathrm{y} 86, \mathrm{y} 87]$

end

$[A 39(j, 1), A 39(j, 2)]=$ curveintersect $(m(:, j), n(:, j), x 2, y 2) ;$

for $j=1: 16$

$m=[y 88, x 44, x 45, x 46, x 47, x 48, x 49, x 50, x 51, x 52, x 53, x 54, x 55, x 56, x 57, x 58]$;

$\mathrm{n}=[\mathrm{x} 88, \mathrm{y} 44, \mathrm{y} 45, \mathrm{y} 46, \mathrm{y} 47, \mathrm{y} 48, \mathrm{y} 49, \mathrm{y} 50, \mathrm{y} 51, \mathrm{y} 52, \mathrm{y} 53, \mathrm{y} 54, \mathrm{y} 55, \mathrm{y} 56, \mathrm{y} 57, \mathrm{y} 58] ;$

$[A 40(j, 1), A 40(j, 2)]=$ curveintersect $(m(:, j), n(:, j), x 1, y 1)$;

end

for $j=1: 45$

$m=[y 88, x 44, x 45, x 46, x 47, x 48, x 49, x 50, x 51, x 52, x 53, x 54, x 55, x 56, x 57, x 58, x 59, x 60, x 61, x 62$, $x 63, x 64, x 65, x 66, x 67, x 68, x 69, x 70, x 71, x 72, x 73, x 74, x 75, x 76, x 77, x 78, x 79, x 80, x 81, x 82, x 83$ $, x 84, x 85, x 86, x 87]$

$\mathrm{n}=[\mathrm{x} 88, \mathrm{y} 44, \mathrm{y} 45, \mathrm{y} 46, \mathrm{y} 47, \mathrm{y} 48, \mathrm{y} 49, \mathrm{y} 50, \mathrm{y} 51, \mathrm{y} 52, \mathrm{y} 53, \mathrm{y} 54, \mathrm{y} 55, \mathrm{y} 56, \mathrm{y} 57, \mathrm{y} 58, \mathrm{y} 59, \mathrm{y} 60, \mathrm{y} 61, \mathrm{y} 62$, $\mathrm{y} 63, \mathrm{y} 64, \mathrm{y} 65, \mathrm{y} 66, \mathrm{y} 67, \mathrm{y} 68, \mathrm{y} 69, \mathrm{y} 70, \mathrm{y} 71, \mathrm{y} 72, \mathrm{y} 73, \mathrm{y} 74, \mathrm{y} 75, \mathrm{y} 76, \mathrm{y} 77, \mathrm{y} 78, \mathrm{y} 79, \mathrm{y} 80, \mathrm{y} 81, \mathrm{y} 82, \mathrm{y} 83$ $, \mathrm{y} 84, \mathrm{y} 85, \mathrm{y} 86, \mathrm{y} 87]$ end

$[A 41(j, 1), A 41(j, 2)]=$ curveintersect $(m(:, j), n(:, j), x 88, y 88) ;$ o\# ok<*AGROW>

$\mathrm{A} 42=[5,0 ; 0,5 ; 4.9,0 ; 0,4.9 ; 4.8,0 ; 0,4.8 ; 4.7,0 ; 0,4.7 ; 4.6,0 ; 0,4.6 ; 4.5,0 ; 0,4.5 ; 4.4,0 ; 0,4$. $4 ; 4.3,0 ; 0,4.3 ; 4.2,0 ; 0,4.2 ; 4.1,0 ; 0,4.1 ; 4,0 ; 0,4 ; 3.9,0 ; 0,3.9 ; 3.8,0 ; 0,3.8 ; 3.7,0 ; 0,3.7 ; 3$ $.6,0 ; 0,3.6 ; 3.5,0 ; 0,3.5 ; 3.4,0 ; 0,3.4 ; 3.3,0 ; 0,3.3 ; 3.2,0 ; 0,3.2 ; 3.1,0 ; 0,3.1 ; 3,0 ; 0,3 ; 2.9$, $0 ; 0,2.9 ; 2.8,0 ; 0,2.8 ; 2.7,0 ; 0,2.7 ; 2.6,0 ; 0,2.6 ; 2.5,0 ; 0,2.5 ; 2.4,0 ; 0,2.4 ; 2.3,0 ; 0,2.3 ; 2.1$ , $0 ; 0,2.2 ; 0,2.1 ; 0,2 ; 0,1.9 ; 0,1.8 ; 0,1.7 ; 0,1.6 ; 0,1.5 ; 0,1.4 ; 0,1.3 ; 0,1.2 ; 0,1.1 ; 0,1 ; 1,0 ; 1$. 41,$0 ; 1.874,0 ; .984, .174 ; .174, .984 ; .965, .259 ; .259, .965 ; .939, .342 ; .342, .939 ; .906, .423 ;$ $.423, .906 ; .866, .5 ; .5, .866 ; .819, .573 ; .573, .819 ; .766, .643 ; .643, .766 ; .707, .707 ; .996, .0$ $87 ; .087, .996] ;$

$\mathrm{A}=[\mathrm{A} 1 ; \mathrm{A} 2 ; \mathrm{A} 3 ; \mathrm{A} 4 ; \mathrm{A} 5 ; \mathrm{A} 6 ; \mathrm{A} 7 ; \mathrm{A} 8 ; \mathrm{A} 9 ; \mathrm{A} 10 ; \mathrm{A} 11 ; \mathrm{A} 12 ; \mathrm{A} 13 ; \mathrm{A} 14 ; \mathrm{A} 15 ; \mathrm{A} 16 ; \mathrm{A} 17 ; \mathrm{A} 18 ; \mathrm{A} 19 ; \mathrm{A} 20 ; \mathrm{A} 21 ; \mathrm{A} 22 ; \mathrm{A}$ 23;A24;A25;A26;A27;A28;A29;A30;A31;A32;A33;A34;A35;A36;A37;A38;A39;A40;A41;A42]

plot $\left([A(:, 1),-A(:, 1), A(:, 1),-A(:, 1)],[A(:, 2),-A(:, 2),-A(:, 2), A(:, 2)], m^{*}\right)$

function dydx=ODE1 $(\mathrm{x}, \mathrm{y})$

$r=\operatorname{sqrt}(x \cdot \wedge 2+y \cdot \wedge 2) ; t=a \operatorname{atan}(y \cdot / x) ; t 1=-2 ; t 2=-1 ;$

$\operatorname{sr}=(t 1 / 2) *(1-(1 /(r \cdot \wedge 2)))+(t 1 / 2) *(1+(3 /(r \cdot \wedge 4))-(4 /(r \cdot \wedge 2))) * \cos (2 * t)+(t 2 / 2) *(1-$

$\left.\left(1 /\left(r \wedge^{\wedge} 2\right)\right)\right)-(t 2 / 2) *\left(1+\left(3 /\left(r \wedge^{\wedge} 4\right)\right)-\left(4 /\left(r \wedge^{\wedge} 2\right)\right)\right){ }^{*} \cos (2 * t) ;$

$s t=(t 1 / 2) *(1+(1 /(r \cdot \wedge 2)))-$

$(t 1 / 2) *\left(1+\left(3 /\left(r \cdot \wedge^{\wedge}\right)\right)\right){ }^{*} \cos (2 * t)+(t 2 / 2) *\left(1+\left(1 /\left(r \cdot{ }^{\wedge} 2\right)\right)\right)+(t 2 / 2) *(1+(3 /(r . \wedge 4))){ }^{*} \cos (2 * t)$

$\operatorname{srt}=-(t 1 / 2) *\left(1-(3 /(r \cdot \wedge 4))+\left(2 /\left(r \cdot^{\wedge} 2\right)\right)\right) * \sin (2 * t)+(t 2 / 2) *(1-$

$(3 /(r \cdot \wedge 4))+(2 /(r \cdot \wedge 2))) * \sin (2 * t) ;$

$s x=s r .{ }^{*}(\cos (t)) \cdot{ }^{\wedge} 2-2^{*} \operatorname{srt} .{ }^{\star} \cos (t){ }^{*} \sin (t)+s t .{ }^{*}(\sin (t)) \cdot{ }^{\wedge} 2$;

$\operatorname{sy}=\operatorname{st} .{ }^{*}(\cos (t)) \cdot{ }^{\wedge} 2+\operatorname{sr} .{ }^{*}(\sin (t)) \cdot{ }^{\wedge} 2+\operatorname{srt} .{ }^{*} \sin (2 * t)$;

txy $=0.5^{\star}\left(\left(2 * \operatorname{srt} \cdot{ }^{*} \cos (2 * t)+(\operatorname{sr}-s t) \cdot{ }^{*} \sin (2 * t)\right)\right) ;$

$d y d x=-(\operatorname{sx}-s y) /(2 * t x y)-\operatorname{sqrt}\left(1+((\operatorname{sx}-\mathrm{sy}) /(2 * \operatorname{txy}))^{\wedge} 2\right)$;

if $\operatorname{abs}(d y d x)>10$ 


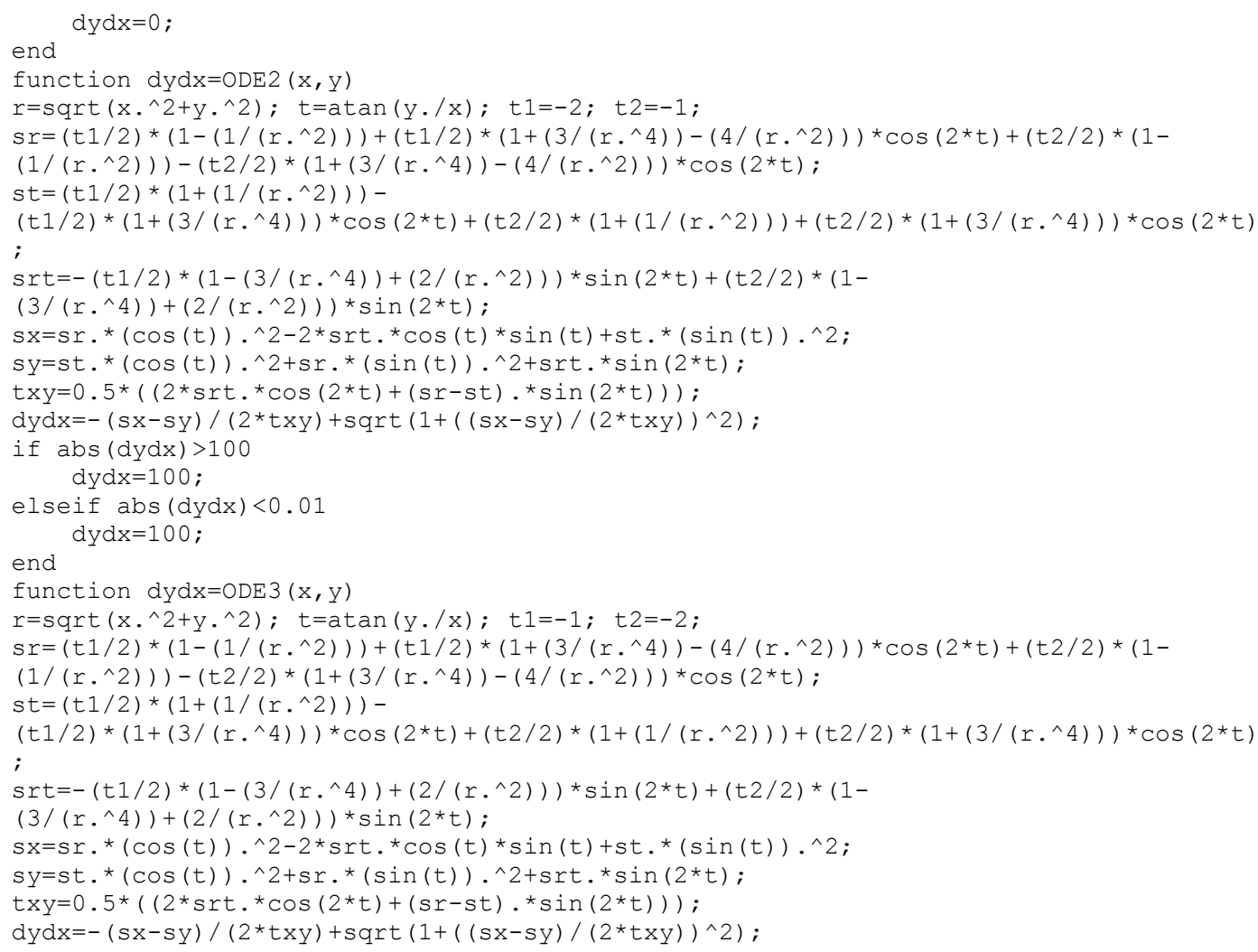

\section{Random Meshes}

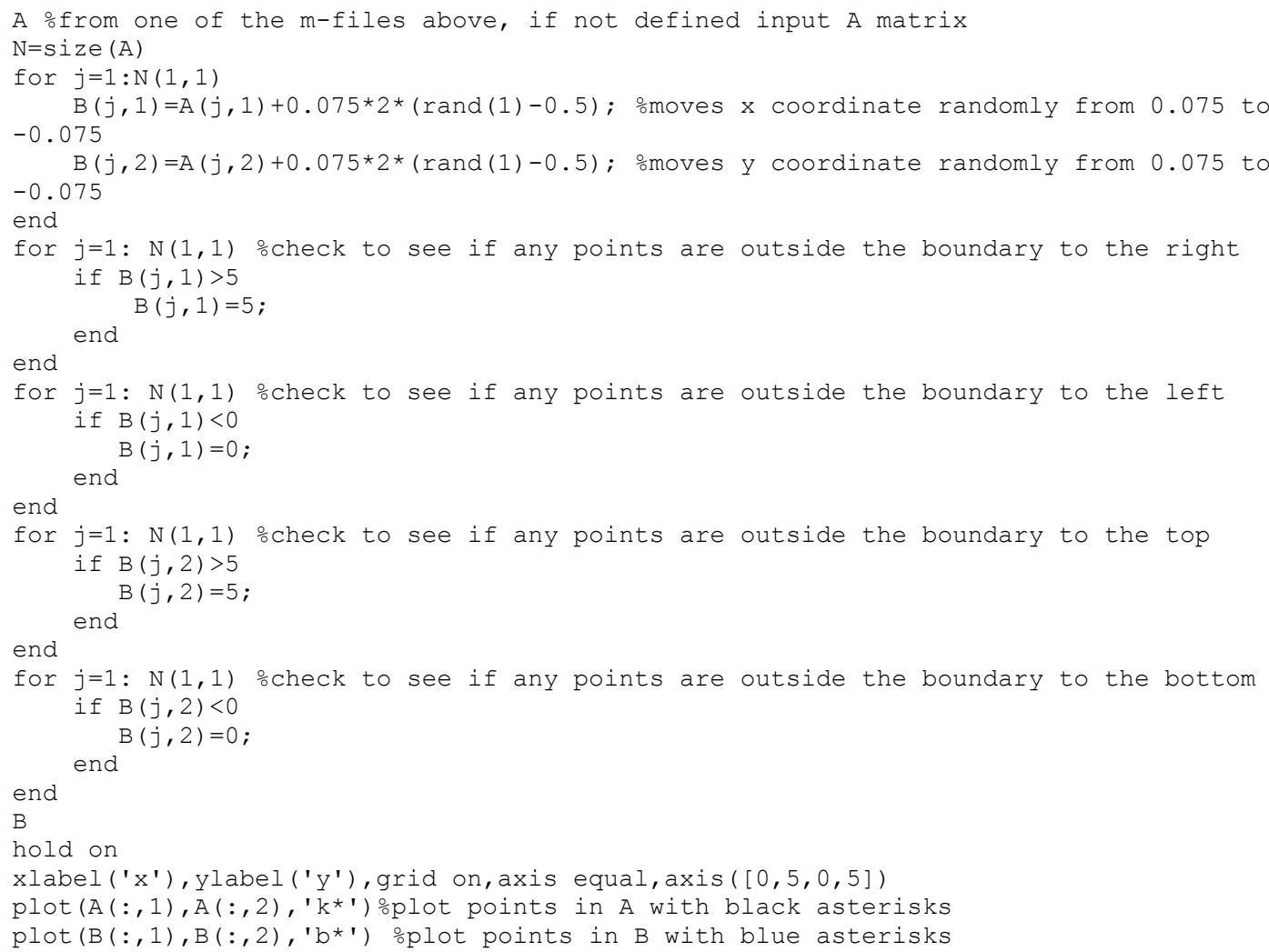




\section{BIBLIOGRAPHY}

Alkhader, M., \& Vural, M. (2008). Mechanical response of cellular solids: Role of cellualr topology and microstructural irregularity. International Journal of Engineering Science, 46, 1035-1051.

Almeida Perira, O., \& Moitinho de Almeida, J. (1994). Automatic Drawing of Stress Trajectories in Plane Systems. Computers \& Structures , 473-476.

Andrews, E. W., \& Gibson, L. J. (2001). The influence of cracks, notches and holes on the tensile strength of cellular solids. Acta Materialia , 49, 2975-2979.

Ashby, M. F., \& Gibson, L. J. (1997). In Cellular solids: Structures and properties. Cambridge: Cambridge University Press.

Blumenfeld, R. (2003). Stress transmission in planar disordered solid foams. Journal of Physics: Mathematical and General , 2399-2411.

Budynas, R. (1999). Advanced Strength and AppliedStress Analysis. Boston: WCB/McGraw-Hill.

Chan, A. S. (1960). The Design of Michell Optimum Structures. Cranfield: College of Aeronautics Reports.

Composite Honeycombs. (2010, June 01). Retrieved from http://en.wikipedia.org/wiki/Composite_honeycomb

Gere, J. M., \& Goodno, B. J. (2009). Mechanics of Materials. Mason: Cengage Learning.

Gibson, L. J., \& Ashby, M. F. (1997). Cellular Solids: Structures and Properties. Cambridge: Cambridge University Press.

Gilat, A. (2008). MATLAB: An Introduction with Applications (3rd ed.). Hoboken, NJ: Wiley.

Hibbeler, R. C. (2009). Structural Analysis. Upper Saddle River: Pearson Prentice Hall.

Hoff, N. J. (1951). Structural Problems of Future Aircraft. London: The Royal Aeronautical Society.

Holz, S. (2005, November 02). Matlab Central, 2. Retrieved November 09, 2011 , from Curve Intersect 2: http://www.mathworks.com/matlabcentral/fileexchange/8908curve-intersect-2 
Irsa, J., \& Galybin, A. (2010). Stress trajectories element method for stress determination from discrete data on principal directions. Engineering Analysis with Boundary Elements , 423-432.

Jones, Richard. (2011). Mechanical Behavior of Functionally Graded Materials Around a Graded Hole. MS Thesis, University of Rhode Island.

Kelly, D., \& Tosh, M. (2000). Interpreting load paths and stress trajectories in elasticity. Engineering Computations, 117-135.

Kiessling, G. C. (1961). Modern Packaging. 35 (3A), 287.

Kumar, R. S., \& McDowell, D. L. (2004). Generalized continuum modeling of 2-D periodic cellular solids. International Journal of Solids and Structures , 41, 73997422.

Megson, T. (2005). Structural and Stress Analysis. Burlington: Elsevier ButterworthHeinimann.

Molleda, F., Mora, J., Molleda, F., Carrillo, E., \& Mellor, B. (2005). Stress trajectories for Mode I fracture. Materials Characterization , 9-12.

Onch, P. R. (2003). Scale effects in cellular metals. MRS Bulletin, 279-283.

Petrucci, G., \& Restivo, G. (2007). Automated stress separation along stress trajectories. Experimental Mechanics, 733-743.

Ramamurthy, R., \& Farouki, R. T. (1999). Voronoi diagram and medial axis algorithm for planar domains with curved boundaries I. Theoretical foundations. Journal of Computational and Applied Mechanics , 102, 119-141.

Reddy, J. N. (2006). An Introduction to the Finite Element Method. Boston: McGrawHill.

Sadd, M. H. (2009). Elasticity: Theory, Applications, and Numerics (2 ed.). Burlington, MA: Academic Press.

Schaffner, G., Guo, X.-D. E., Silva, M. J., \& Gibson, L. J. (2000). Modelling fatigue damage accumulation in two-dimensional Voronoi honeycombs. Internation Journal of Mechanical Sciences , 42, 645-656.

Silva, M. J., \& Gibson, L. J. (1997). The effects of non-periodic microstructure and defecs on the compressive strength of two-dimensional cellular solids. International Journal of Mechanical Sciences , 39, 549-563.

Skedros, J. G., \& Baucom, S. L. (2007). Mathematical analysis of trabecular 'trajectories' in apparent trajectorial structures: The unfortunate historical emphasis on the human proximal femur. Journal of Theoretical Biology , 15-45. 
Taggart, D. G., \& Dewhurst, P. (2010). Development and validation of a numerical topology optimization scheme for two and three dimensional structures. Advances in Engineering Software, 910-915.

Taggart, D. G., Dewhurst, P., Dobrot, L., \& Gill, D. D. (2008). Development of a Beta Function Based Topology Optimization Procedure. Abaqus Users Conference.

Taggart, D. G., Jahns, D., Nair, A., \& Dewhurst, P. (2010). Application of the PMR Topology Optimization Scheme to Dual Material Structures. Vancouver: ASME 2010 International MEchanical Engineering Congress and Exposition.

Thamm, F. (2000). The Role of the Stress Trajectories as an Aid in the Choice of the Suitable Shape of Load-bearing Structural Elements of Engines and Structures. Periodica Polytechnica Ser mechanical Engineering , 171-183.

Thompson, D. (1961). On Growth and Form. Cambridge: Cambridge University Press.

Tsubota, K.-i., Adachi, T., \& Tomita, Y. (2002). Functional adaptation of cancellous bone in human proximal femur predicted by trabecular surface remodeling simulation toward uniform stress state. Jornal of biomechanics , 1541-1551.

Whittle, P. (2007). Structural optimisation: Michell structures. Cambridge: Cambridge University Press. 